


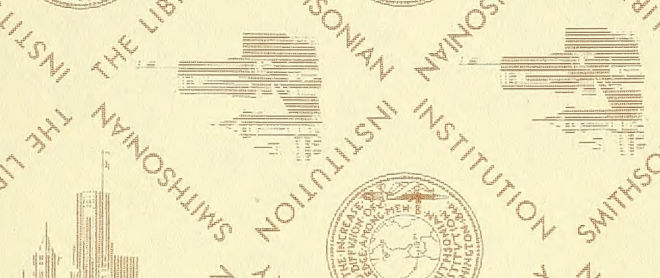

(I)

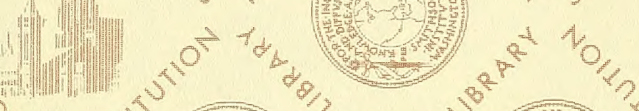

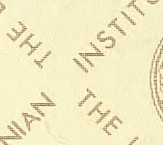

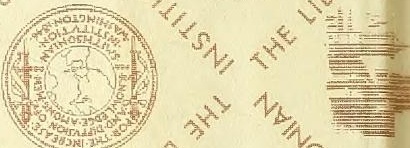

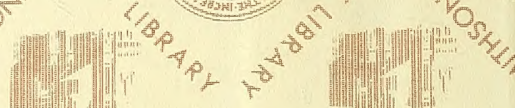

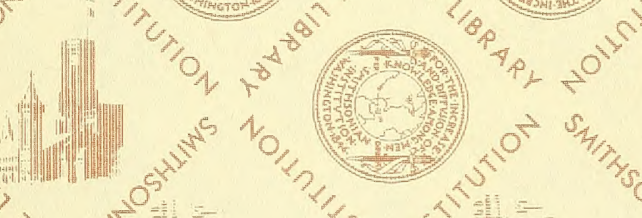

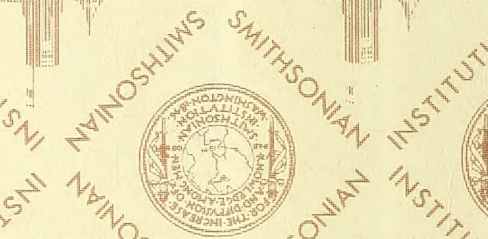

0
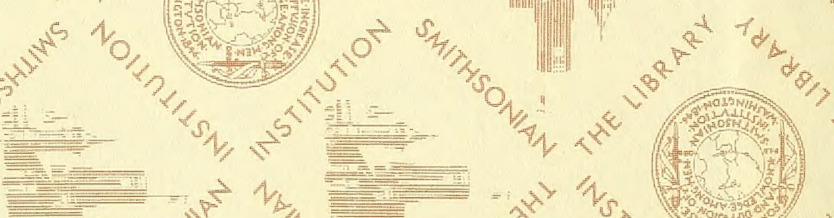

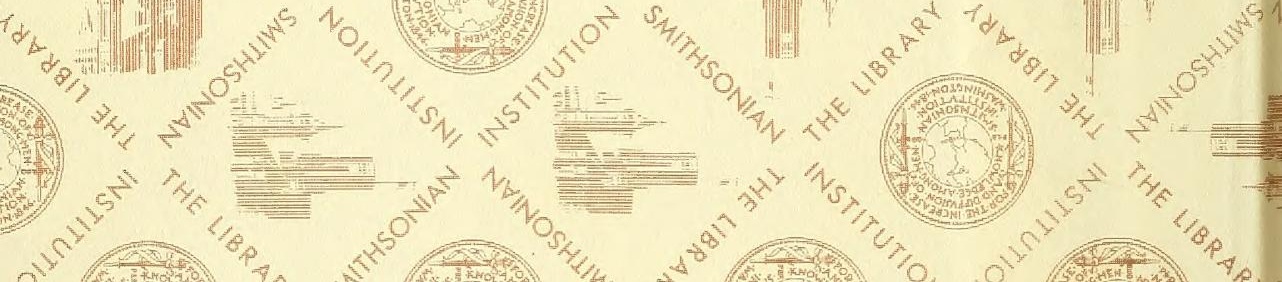

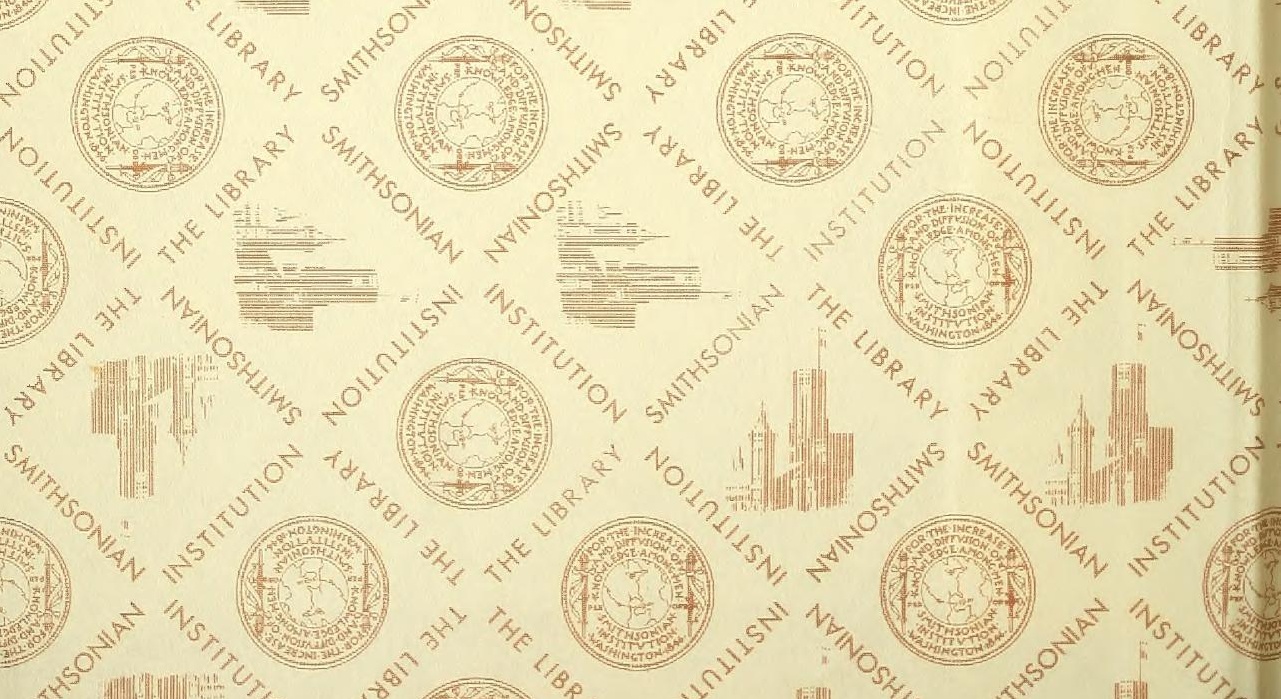




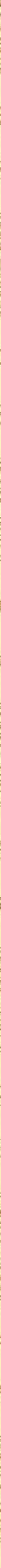








\section{THE METRIC SYSTEM}

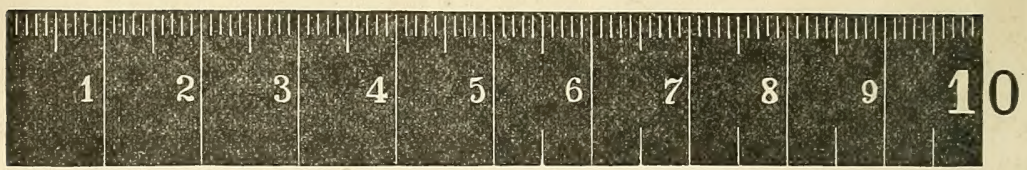

\section{CENTIMETER RULE}

The upper edge is in millimeters, the lower in centimeters, and half centimeters.

UNITS.

The most commonly used divisions and multiples.

THE METER FOR (Centimeter (cm.), o.or Meter; Millimeter (mm.), o.oor Meter: Micron $(\mu)$, LENGTH. 0.00 I Millimeter; the Micron is the unit in Micrometry (? I66).

LENGTH. - ' (Kilometer, IOoo Meters; used in measuring roads and other long distances.

THE GRAM FOR ( Milligram (mg.), o.oor Gram.

WEIGHT. . . Kilogram, rooo Grams, used for ordinary masses, like groceries, etc.

THE LITER FOR f Cubic Centimeter (cc.), o.00I Liter. This is more common than the correct CAPACITY. . $\{$ form, Milliliter.

Divisions of the Units are indicated by the Latin prefixes: deci, o.r; centi, o.or ; Milli, $0.00 \mathrm{I}$; Micro, one millionth (0.00000I) of any unit.

Multiples are designated by the Greek prefixes: deka, to times; hecto, roo times; kilo, Iooo timse; myria. I0.000 times: Mega, one million ( $1,000,000)$ times any unit.

Meter $(\mathrm{U}$

$39.37 \mathrm{C}$

Centimete

Millimete

Micron (

liontl

$\operatorname{Vard}=3 \mathrm{f}$

Foot $=\mathrm{I} 2$

Inch $=1$

Liter (U

Cubic cen

Fluid oun

Gram (U

Kilogram

Ounce avi

Ounce Tr

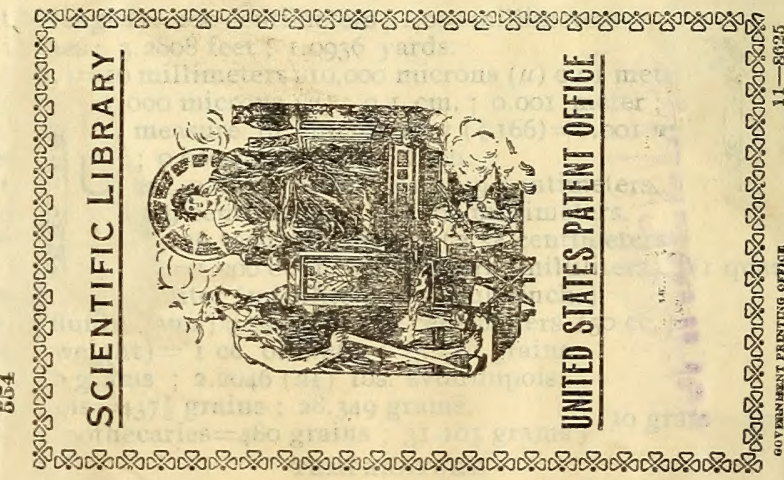

ns $(\mu)$

To $\mathrm{cl}$

to find the equiv

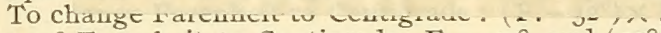

to reduce $50^{\circ}$ Farenheit to Centigrade, $\mathrm{F} .=50^{\circ}$, and $\left(50^{\circ}-32^{\circ}\right) \times \frac{5}{9}=\mathrm{IOC}$. ; or -40 Farenheit to Centigrade, $\mathrm{F} .=-40^{\circ}\left(-40^{\circ}-32^{\circ}\right)=-72^{\circ}$, whence $-72^{\circ} \times \frac{5}{9}^{\frac{5}{9}}$ $=-40^{\circ} \mathrm{C}$.

Address of American Opticians: For the price of microscopes and microscopical supplies the student is advised to obtain a catalog of one or more of the opticians. Nearly all of them import foreign apparatus. For all institutions entitled to duty free importation, American microscopes are sold at duty free rates. For the foreign opticians see the table of tube-length p. I4.

The Bausch and Lomb Optical Co., Eimer and Amend,

The Franklin Laboratory Supply Co

The Gundlach Optical Company,

Wm. Krafft (Representative of Leitz in America) Harcourt St., Boston, Mass. Rochester, N. Y

Edward Pennock,

Queen \& Company, West 59 th St, New York. Richards \& Co Spencer I ens Company, Williams, Brown \& Earle, G. S. Woolman (Queen \& Co. in New York), _._._................... II6 Fulton St., New York. Joseph Zentmayer, 226-228 South I5th St., Philadelphia, $\mathrm{Pa}$

Besides the names here given, nearly every large city has one or more dealers in microscopes and microscope supplies. 


\section{THE MICROSCOPE}

$\mathrm{AN}$

INTRODUCTION TO MICROSCOPIC ME'THODS AND TO HISTOLOGY

BY SIMON HENRY GAGE C PROFESSOR OF MICROSCOPY, HISTOLOGY AND EMBRYOLOGY IN CORNELL UNIVERSITY, AND THE NEW YORK STATE VETERINARY COLLEGE.

EIGHTH
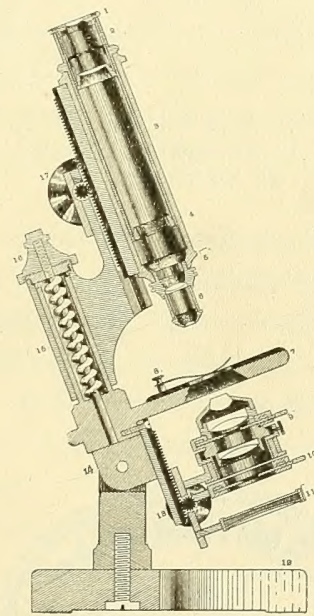

EDITION

REVISED, ENLARGED AND ILLUSTRATED BY OVER TWO HUNDRED FIGURES

COMSTOCK PUBLISHING COMPANY ITHACA, NEW YORK I9OI 


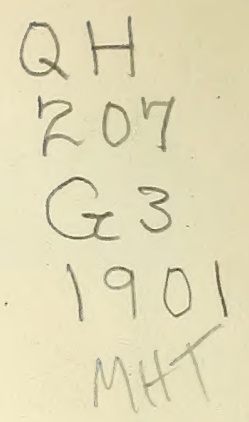

Copyright, I90I.

By Simon Henry Gage.

All Rights Reserved. Ithaca, N. Y. 


\section{(a)}

$\Lambda \mathrm{S}$ the preface to the sixth edition of this work expresses accurately what A should be said to-day it is appended :

"The rapid advance in microscopical knowledge, and the great strides in the sciences employing the microscope as an indispensable tool, have reacted upon the microscope itself, and never before were microscopes so excellent, convenient and cheap. Indeed, the financial reason for not possessing a microscope can no longer be urged by any high school or academy, or by any person whose profession demands it.

Naturally, to get the greatest good from instruments, tools, or machines of any kind, the one who uses them must understand the principles upon which their action depends, their possibilities and limitations.

That the student may acquire a just comprehension of some of the fundamental principles of the microscope, and gain a working acquaintance with it and its applications, this book has been prepared. It is a growth of the laboratory, and has been modified from time to time to keep pace with optical improvements and advancing knowledge."

In rewriting this edition the different chapters have been recast, new figures added and in most cases the matter considerably increased. A new chapter has been added upon class demonstrations. The general availability of the constant electric current, and the improvement in apparatns have made micro-projection practicable and satisfactory. It has served the writer so well in his teaching of histology and embryology that it seemed worth while to give the benefit of his experience to his fellow workers.

It is hoped that the book as it now stands will serve more completely than ever before the needs of the class-room and of the laboratory.

"Simply reading a work on the microscope, and looking a few times into an instrument completely adjusted by another, is of very little value in giving real knowledge. In order that the knowledge shall be made alive, it must become a part of the student's experience by actual experiments carried out by the student himself. Consequently, exercises illustrating the principles of the microscope and the methods of its employment have been made an integral part of the work.

"In considering the real greatness of the microscope, and the truly splendid service it has rendered, the fact has not been lost sight of that the microscope is, after a11, only an aid to the eye of the observer, only a means of getting a larger image on the retina than would be possible without it; but the appreciation of 
the retinal image, whether it is made with or without the aid of a microscope, must always depend upon the character and training of the seeing and appreciating brain behind the eye. The microscope simply aids the eye in furnishing raw material, so to speak, for the brain to work upon. (From $3 \mathrm{~d}$ ed.)

Grateful acknowledgment is made to the opticians and instrument makers for the loan of cuts and for courteous and complete answers to numerous question ; to the directors of laboratories in different parts of the country, to his colleagues in the departments of Physics, Chemistry and Electrical Engineering in Cornell University; and finally to his pupils past and present who have given their support and encouragement.

In closing I would like to urge those who are interested in Microscopy to take same microscopical journal, and if possible to become a member of some microscopical club or society. One can do very little alone, but by helping others and being helped in return, the workers in any field of human endeavor can accomplish great things.

\section{SIMON HENRY GAGE,}

\section{CORNELL, UNIVERSITY,}

October I, I9от. ITHACA, N. Y., U. S. A. 


\section{CONTEN'TS}

\section{CHAPTER I}

PAGE

\% I- 59-The Microscope and its parts

CHAPTER II

?. 60-I28-Lighting and Focusing; Manipulation of Dry, Adjustable and Immersion Objectives; Care of the Microscope and of the Eyes. Laboratory Microscopes.

CHAPTER III

\&. I29-I53-Interpretation of Appearances.

$90-102$

CHAPTER IV

\& I54-I76-Magnification and Micrometry

$103^{-1} 2 \mathrm{I}$

CHAPTER V

3 I77-IS7-Drawing with the Microscope

I 22-1 33

CHAPTER VI

3. I88-233-The Microspectroscope and Polariscope; Micro-Chemistry; Textile Fibers and Food Products ; Micro-Metallography $134-160$

\section{CHAPTER VII}

\& 234-335-Slides and Cover-Glasses; Mounting; Isolation; Sectioning by the Collodion and the Paraffin Methods; Serial Sections ; Labeling and Storing Microscopical Preparations; Reagents and their Preparation

\section{CHAPTER VIII}

\& 336-392-Photographing objects with a Vertical Camera; Photographing Large Transparent Objects; Photographing with a Microscope; (A) Transparent Objects ; (B) Opaque Objects, and the Surfaces of Metals and Alloys; Enlarging Negatives; Photographing Petri Dishes and Culture Tubes

\section{CHAPTER IX}

393-426-Class-Room Demonstrations with the Microscope; With the Projection Microscope; with the Episcope

CHAP'TER $\mathrm{X}$

\% 427-438-The Abbe Test-Plate and Apertometer; Equivalent Focus of Objectives and Oculars; Drawings for Photo-Engravings ; Wax Models; Some Apparatus for Imbedding and Sectioning 


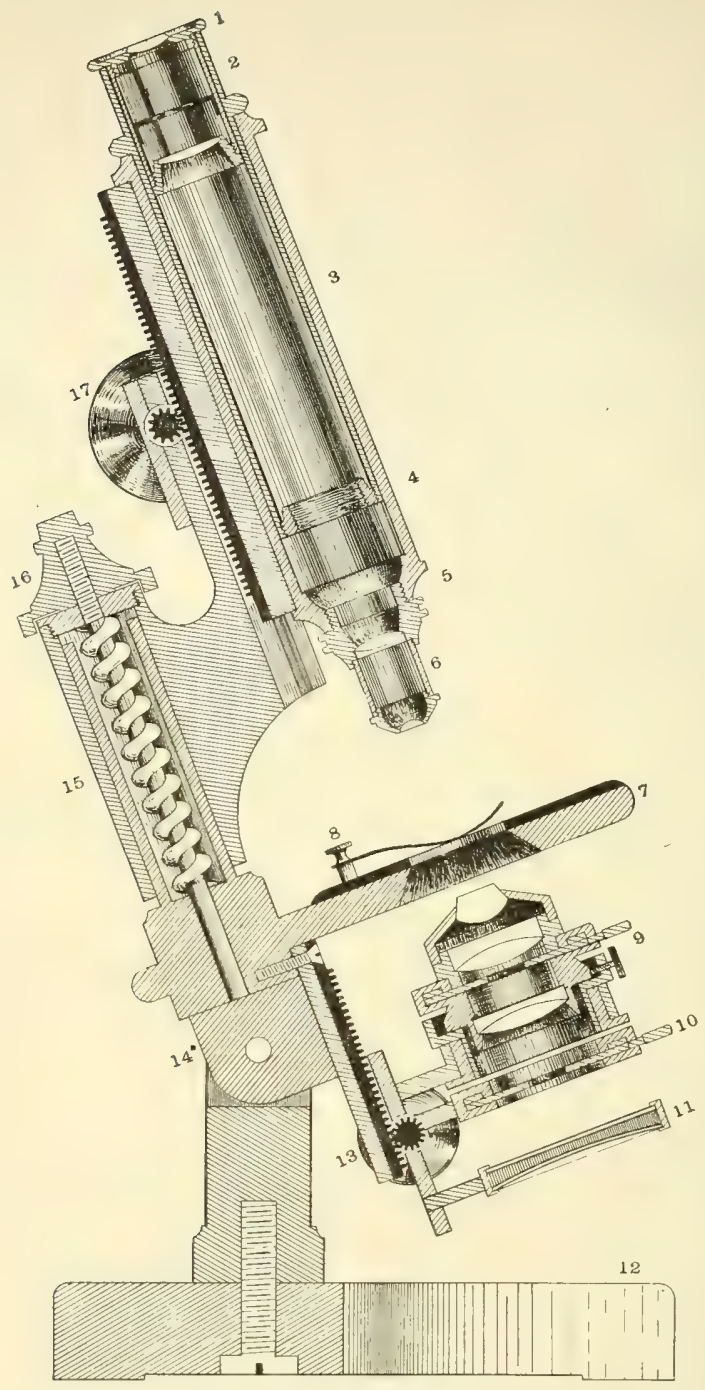

THE MICROSCOPE IN SECTION.

I. Huygenian ocular (see p. I02 for positive ocular).

2. Draw-tube by which the tube is lengtheued or shortened.

3. Main tube or body containing the drawtube, and attached to the pillar by the arm

4. Society screw in the lower end of the draw-tube.

5. Society screw in the lower end of the tube.

6. Objective in position.

7. Stage, under which is the substage with the substage condenser.

S. Spring clip for holding the specimen.
9. Screw for centering, and handle for the iris diaphragm in the achromatic condenser (see Fig. 4I).

Io. Iris diaphragm outside the principal focus of the condenser for use in centering ( $38 \mathrm{I})$

II. Mirror with plane and concave faces.

12. Horse-shoe base.

13. Rack and pinion for the substage condenser.

14. Jointed pillar.

15. Part of pillar with spiral spring of fine adjustment.

16. Screw of fine adjustment.

17. Milled head of coarse adjustment. 


\title{
THE MICROSCOPE
}

\author{
AND \\ MICROSCOPICAL METHODS
}

\section{CHAPTER I}

\author{
THE MICROSCOPE AND ITS PARTS
}

APPARATUS AND MATERIAL FOR THIS CHAPTER

A simple microscope $(3 / 3$, I I ) ; A compound microscope with nose-piece (Figs. 70-80); eye-shade (Fig. 60), achromatic (\&20), apochromatic (\&22), dry (\&I7), immersion ( $\%$ IS), unadjustable and adjustable objectives $(\xi 23,24)$; Huygenian or negative $(\% 38)$, positive $(3.37)$ and compensation oculars $(3,39)$; stage micrometer (Ch. IV); homogeneous immersion liquid (?. IS) ; mounted letters or figures ( 8.53$)$; ground-glass and lens paper ( 853$)$.

\section{A MICROSCOPE}

I. A Microscope is an optical apparatus with which one may obtain a clear image of a near object, the image being always larger than the object; that is, it enables the eye to see an object under a greatly increased visual angle, as if the object were brought very close to the eye without affecting the distinctness of vision. Whenever the microscope is used for observation, the eye of the observer forms an integral part of the optical combination (Figs. I6, 2r).

3,2. A Simple Microscope.-With this an enlarged, erect image of an object may be seen. It always consists of one or more converging lenses or lens-systems (Figs. I6-20), and the object must be placed within the principal focus ( $\xi$ II). The simple microscope may be held in the hand or it may be mounted in some way to facilitate its use (Figs $17-20$ ). 

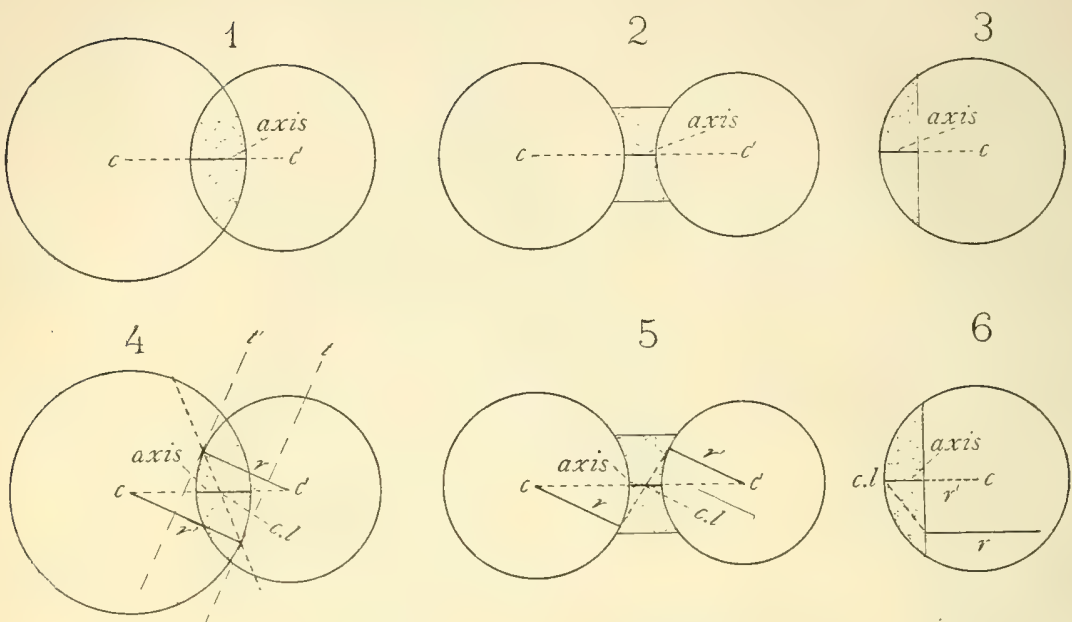

6
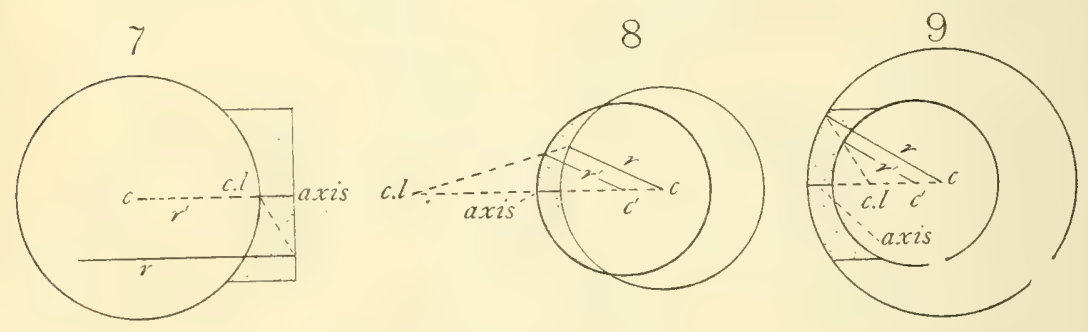

FIGS. I-9, showing the Principal Optic Axis and the Optical Center of various forms of Lenses.

Axis. The Principal Optic Axis. $c-c^{\prime}$. Centers of curvature of the two surfaces of the lens. c.l. Optical center of the lens. $r-r^{\prime}$. Radii of curvature of the two lens surfaces. $t-t^{\prime}$. Tangents in Fig. 4 .

8 3. Principal Optic Axis.-In spherical lenses, i. e., lenses whose surfaces are spherical, the Axis is a line joining the centers of curvature and indefinitely extended. In the lens it is the unbroken part of the line $c-c^{\prime}$ in the figures. In lenses with one plane surface (Figs. $3,6,7$ ) the radius of the plane surface is any line at right angles to it, but in determining the axis it must be the one which is continuous with the radius of the curved surface, consequently the axis in such lenses is on the radius of the curved surface which meets the plane surface at right angles.

3. Optical Center.-The optical center of a lens is the point through which rays pass without angular deviation, that is, the emergent ray is parallel to the 
incident ray. It is determined geometrically by drawing parallel radii of the curved surfaces, $r-r^{\prime}$ in Figs. $4-9$, and joining the peripheral ends of the radii. The optical center is the point on the axis cut by the line joining the peripheral ends of the parallel radii of the two lens surfaces. In Figs. 4-5 it is within the lens; in $6-7$ it is at the curved surface, and in the meniscus $(8,9)$ it is wholly outside the lens, being situated on the side of the greater curvature.

In determining the center in a lens with a plane surface, the conditions can be satisfied only by using the radius of the curved surface which is continuous with the axis of the lens, then any line at right angles to the plane surface will be parallel with it, and may be considered part of the radius of the plane surface. (That is, a plane surface may be considered part of a sphere with infinite radius, hence any line meeting the plane surface at right angles may be considered as the peripheral part of the radius.) In Figs. $6,7,\left(\boldsymbol{r}^{\prime}\right)$ is the radius of the curved surface and $(r)$ of the plane surface; and the point where a line joining the ends of these radii crosses the axis is at the curved surface in each case.

By a study of Fig. 4 it will be seen that if tangents be drawn at the peripheral ends of the parallel radii, the tangents will also be parallel and a ray incident at one tangential point and traversing the lens and emerging at the other tangential point acts as if traversing, and is practically traversing a piece of glass which has parallel sides at the point of incidence and emergence, therefore the emergent ray will be parallel with the incident ray. This is true of all rays traversing the center of the lens.

Thick Lenses. - In all of the diagrams of lenses and the course of rays through them in this book the lenses are treated as if they were infinitely thin. In thick lenses like those figured, while there would be no angular deviation for rays traversing the center of the lens, there would be lateral displacement. This is shown in Fig. 57 illustrating the effect of the cover-glass.

\%. 5econdary Axis.-Every ray traversing the center of the lens, except the principal axis, is a secondary axis; and every secondary axis is more or less oblique to the principal axis. In Fig. I4, line (2), is a secondary axis, and in Fig. 15, line (I). See also Fig. 58.

FIGS, IO, II.-Sectional views of $a$ concave or diverging and a convex or converging lens to show that in the concave lens the principal focus is virtual as indicated by the dotted lines, while with the conviex lens the focus is real and on the side of the lens opposite to that from which the light comes.

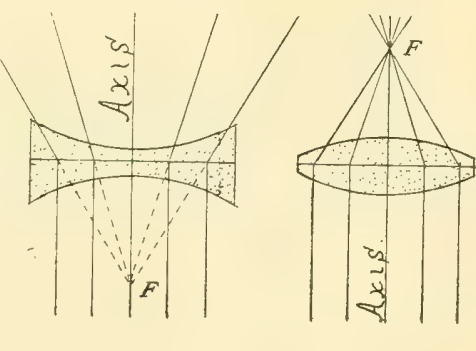

IO

II

3. 6. Principal Focus.-This is the point where rays parallel with the axis and traversing the lens cross the axis; and the distance from the focus to the center of the lens measured along the axis is the Principal Focal Distance. In the diagrams, Fig. Io is seen to be a diverging lens and the rays cross the axis only by being projected backward. Such a focus is said to be virtual, as it has no real existence. In 
Fig. I I the rays do cross the axis and the focus is said to be real. If the light came from the opposite direction it would be seen that there is a principal focus on the other side, that is there are two principal foci, one on each side of the lens. These two foci are both principal foci, but they will be equally distant from the center of the lens only when the curvature of the two lens surfaces are equal. There may be foci on secondary axes also, each focus on a secondary axis has its conjugate. In the formation of images the image is the conjugate of the object and conversely the object is the conjugate of the image.

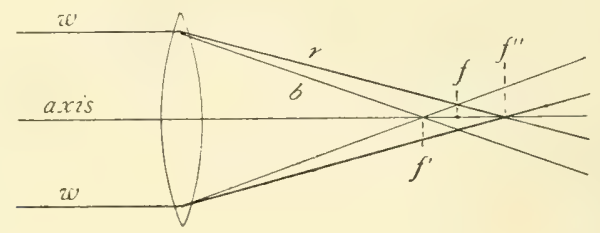

FIg. I2.-Double Convex Lens, Showing Chromatic Aberration.

The ray of white light $(w)$ is represented as dividing into the short waved, blue. $(b)$ and the long waved, red $(r)$ light. The blue $(b)$ ray comes to a focus nearer the lens and the red ray ( $r$ ) farther from the lens than the principal focus $(f)$. Principal focus $(f)$ for rays very near the axis; $f^{\prime}$ and $f^{\prime \prime}$, foci of blue and red light coming from near the edge of the lens. The intermediate wave lengths would have foci all the way between $f^{\prime}$ and $f^{\prime \prime}$.

8. Chromatic Aberration - This is due to the fact that ordinary light consists of waves of varying length, and as the effect of a lens is to change the direction of the waves, it changes the direction of the short waves more markedly than the long waves. Therefore, the short waved, blue light will cross the axis sooner than the long waved, red light, and there will result a superposition of colored images, none of which are perfectly distinct (Fig. 12).

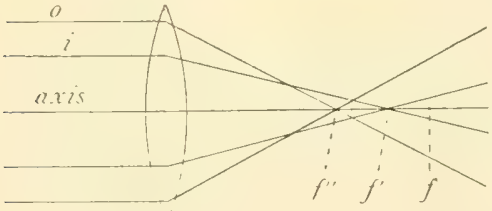

FIG. 13. Double Convex Lens, showing Spherical Aberration.

FIG. I3. The ray (o) near the edge of the lens is brought to a focus nearer the lens than the ray (i). Both are brought to a focus sooner than rays very near the axis. ( $f$ ) Principal focus for rays very near the axis; ( $\left.f^{\prime}\right)$ Focus for the ray (i), and ( $f^{\prime \prime}$ ) Focus for the ray (o). Intermediate rays would cross the axis all the way from ( $f^{\prime \prime}$ to $f$ ).

S. Spherical Aberration. - This is due to the unequal turning of the light in different zones of a lens. The edge of the lens refracts proportionally too much and hence the light will cross the axis or come to a focus nearer the lens than a ray which is nearer the middle of the lens. Thus, in Fig. I3, if the focus 
of parallel rays very near the axis is at $f$, rays $(o i)$, nearer the edge, would come to a focus nearer the lens, the focus of the ray nearest the edge being nearest the lens.

8. 9. Correction of Chromatic and of Spherical Aberration.-Every simple lens has the defect of both chromatic and spherical aberration, and to overcome this, kinds of glass of different refractive power and different dispersive power are combined, concave lenses neutralizing the defects of convex lenses. If the concave lens is not sufficiently strong to neutralize the aberrations of the convex lens, the combination is said to be under-corrected, while if it is too strong and brings the marginal rays or the blue rays to a focus beyond the true principal focus, the combination is over-corrected.

In Newton's time there was supposed to be a direct proportion between the refractive power of any transparent medium and its dispersive power $(i . e$. its power to separate the light into colors). If this were true then the contention of Newton that it would be impossible to do away with the color without at the same time doing away with the refraction would be true and useful achromatic combinations would be impossible. It was found by experiment, however, that there is not a direct ratio between the refractive and dispersive powers for the different colors in different forms of glass, so that it is possible to do away largely with chromatic aberration and retain sufficient refraction to make the combination serve for the production of images. (See also the discussion under apochromatic objectives $\$ 22$ )

Probably no higher technical skill is used in any art than is requisite in the preparation of microscopical objectives, oculars and illuminators.

Figs. I4 AND I5. I4. Convex lens showing the position of the object $(A-B)$ outside the principal focus $(F)$, and the course of the rays in the formation of real images. To avoid confusion the rays are drazen from only one point.

$A B$. Object outside the principal focus. $B^{\prime} A^{\prime}$. Real, enlarged image on the opposite side of the lens.

Axis. Principal opticaxis. $I, 2,3$. Rays after traversing the lens. They are converging, and consequently form a real image. The dotted line and the line (2) give the direction of the rays as if unaffected by the lens. $(F)$. The principal focus.

FIG. I5.-Convex lens, showing the position of the object ( $A B$ ) within the principal focus and the course of rays in the formation of a virtual image.

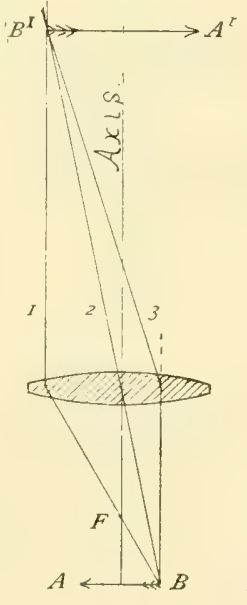

I4

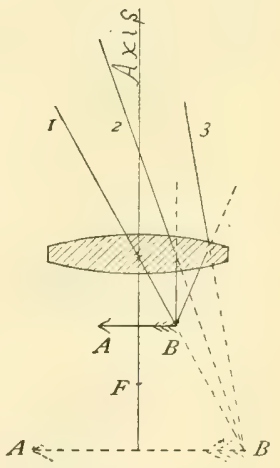

I5

$A B$. The object placed between the lens and its focus; $A^{\prime} B^{\prime}$ virtual image formed by tracing the rays backward. It appears on the same side of the lens as the object, and is erect $(\xi I I)$.

Axis. The principal optic axis of the lens. F. The principal focus.

$I, 2,3$. Rays from the point $B$ of the object. They are diverging after traversing the lens, but not so divergent as if no lens were present, as is shown by the 
dotted lines. Ray $(I)$ traverses the center of the lens, and is therefore not deflected. It is a secondary axis ( 8,5$)$.

3 10. Geometrical Construction of Images.-As shown in Figs. I4-I 5, for the determination of any point of an image, or the image being known, to determine the corresponding part of the object, it is necessary to know the position of the principal focus (and there is one on each side of the lens, \& 6), and the optical center (Figs. I-9 of the lens). Then a secondary axis (2) in Fig. I4, (I) in Fig. I5, is drawn from the extremity of the object and prolonged indefinitely above the lens, or below it for virtual images. A second line is drawn from the extremity of the object, (3) in Fig. I4, (2) in Fig. 15, to the lens parallel with the principal axis. After traversing the lens it must be drawn through the principal focal point. If now it is prolonged it will cross the secondary axis above the lens for a real image and below for a virtual image. The crossing point of these lines determines the position of the corresponding part of the image. Commencing with any point of the object the corresponding point of the image may be determined as just described, and conversely commencing with the image, corresponding points of the object may be determined.

\section{SIMPLE MICROSCOPE : EXPERIMENTS}

S r I. Employ a tripod or other simple microscope, and for object a printed page. Hold the eye about two centimeters from the upper surface of the magnifier, then alternately raise and lower the magnifier until a clear image may be seen. (This mutual arrangement of microscope and object so that a clear image is seen, is called focusing). When a clear image is seen, note that the letters appear as with the unaided eye except that they are larger, and the letters appear erect or right side up, instead of being inverted, as with the compound microscope (§ I2).

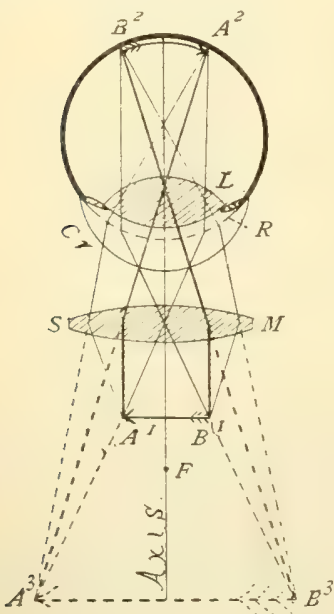

Fig. I6. Diagram of the simple microscope showing the course of the rays and all the images, and that the eye forms an integral part of it.

$A^{\mathrm{r}} B^{\mathrm{T}}$. The object within the principal focus. $A^{3}$ $B$. The virtual image on the same side of the lens as the object. It is indicated with dotted lines, as it has no actual existence.

$B^{2} A^{2}$. Retinal image of the object $\left(A^{\mathrm{x}} B^{\mathrm{x}}\right)$. The viriual image is simply a projection of the retinal image in the field of vision.

Axis. The principal optic axis of the microscope and of the eye. Cr. Cornea of the eye. $L$. Crystalline lens of the eye. $R$. Ideal refracting surface at which all the refractions of the eye may be assumed to take place. 
Hold the simple microscope directly toward the sun and move it away from and toward a piece of printed paper until the smallest bright point on the paper is obtained. This is the burning point or focus, and as the rays of the sun are nearly parallel, the burning point represents approximately the principal focus (Fig. I I). Without changing the position of the paper or the magnifier, look into the magnifier and note that the letters are very indistinct or invisible. Move the magnifier a centimeter or two farther from the paper and no image can be seen. Now move the magnifier closer to the paper, that is, so that it is less than the focal distance from the paper, and the letters will appear distinct. This shows that in order to see a distinct image with a simple microscope, the object must always be nearer to it than its principal focal point. Or, in other words, the object must be within the principal focus. Compare $(\$ 53)$.

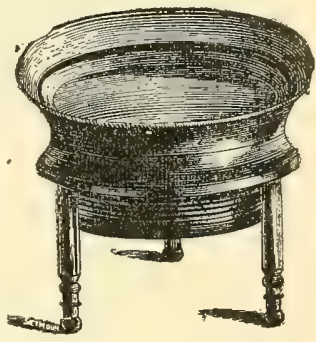

FIG. I7.

After getting as clear an image as possible with a simple microscope, do not change the position of the microscope but move the eye nearer and farther from it, and note that when the eye is in one position, the largest field may be seen. This position corresponds to the eye-point (Fig. 30) of an ocular, and is the point at which the largest number of rays from the microscope enter the eye. Note that the image appears on the same side of the magnifier as the object.

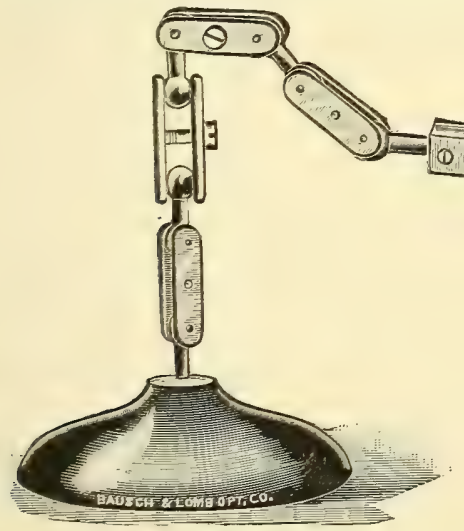

Fig. I9. Lens-holder (The Bausch है Lomb Optical Co.)

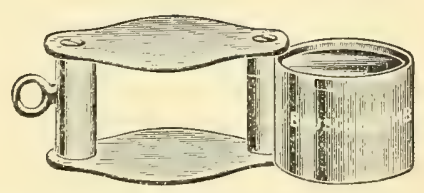

Fig. IS. The Hastings Aplanatic Triplet. (The Bausch \& Lomb Optical Co.) 


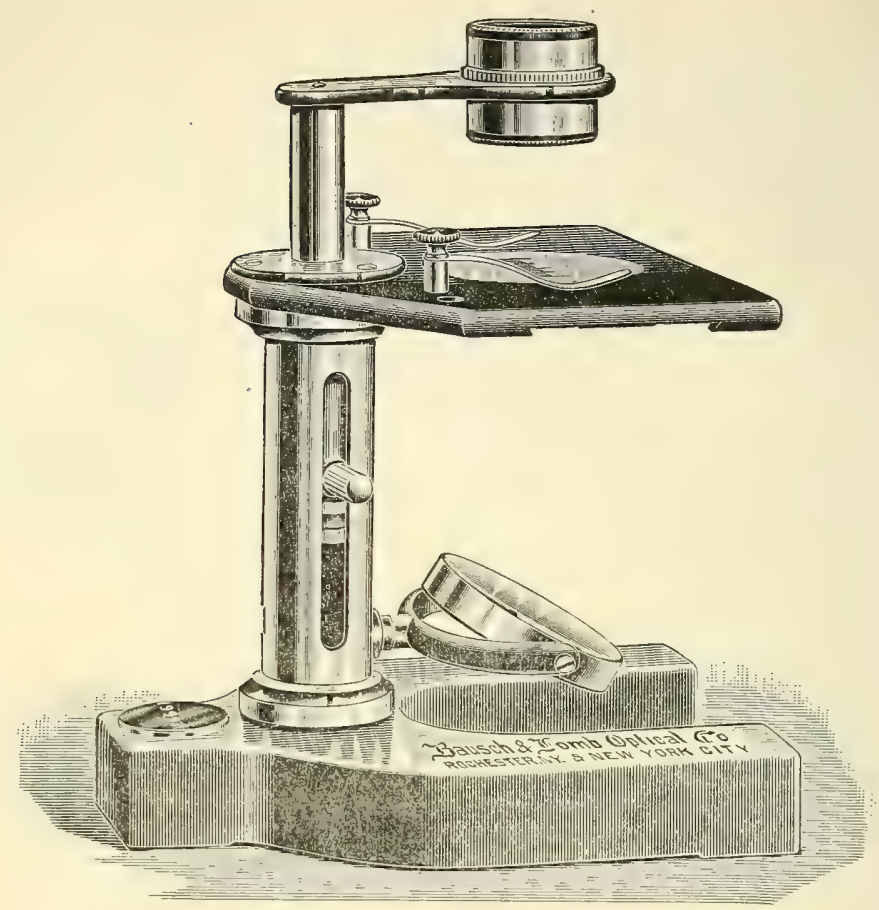

FIG. 20. Dissecting Microscope. This is simply a device for holding the lens and the object to be observed. (The Bausch \& Lomb Optical Co.)

Simple microscopes are very convenient when only a small magnification ( $\mathrm{Ch} . \mathrm{IV}^{\top}$ ) is desired, as for dissecting. Achromatic triplets are excellent and convenient for the pocket. For use in conjunction with a compound microscope, the tripod magnifier (Fig. I7) is one of the best forms. For many purposes a special mechanical mounting is to be preferred.

\section{COMPOUND MICROSCOPE}

8 r2. A Compound Microscope.-This enables one to see an enlarged, inverted image. It always consists of two optical parts-an objective, to produce an enlarged, inverted, real image of the object, and an ocular acting in general like a simple microscope to magnify this real image (Fig. 2I). There is also usually present a mirror, or both a mirror and some form of condenser or illuminator for lighting the object. The stand of the microscope consists of certain mechanical arrangements for holding the optical parts and for the more satisfactory use of them. (See frontispiece.)

\&. I3. The Mechanical Parts of a laboratory, compound microscope are shown in the frontispiece, and are described in the explanation of that figure. The stu- 
dent should study the figure with a microscope before him and become thoroughly familiar with the names of all the parts. See also the cuts of microscopes at the end of Ch. II.

\section{OPTICAL PARTS}

3. I4. Microscopic Objective.-This consists of a converging lens or of one or more converging lens-systems, which give an enlarged, inverted, real image of the object (Figs. I4, 2I). And as for the formation of real images in all cases, the object must be placed outside the principal focus, instead of within it, as for the simple microscope. (See $z$ I I, 53, Figs. I6, 21.)

Modern microscopic objectives usually consist of two or more systems or combinations of lenses, the one next the object being called the front combination or lens, the one farthest from the object and nearest the ocular, the back combination or system. There may be also one or more intermediate systems. Each combinatiơn is, in general, composed of a convex and a concave lens. The combined action of the system serves to produce an image free from color and from spherical distortion. In the ordinary achromatic objectives the convex lenses are of crown and the concave lenses of flint glass (Figs. 22, 23).

FIG. 21. Diagram showing the principle of a compound microscope with the course of the rays from the object ( $A B$ ) through the objective to the real image ( $\left.B^{\prime} A^{\prime}\right)$, thence through the ocular and into the eye to the retinal image $\left(A^{2} B^{2}\right)$, and the projection of the retinal image into the field of vision as the virtual image ( $\left.B^{3} A^{3}\right)$.

$A B$. The object. $A^{2} B^{2}$. The retinal image of the inverted real image, $\left(B^{\mathrm{I}} A^{\mathrm{I}}\right)$, formed by the objective. $B^{3} A^{3}$. The inverted virtual image, a projection of the retinal image.

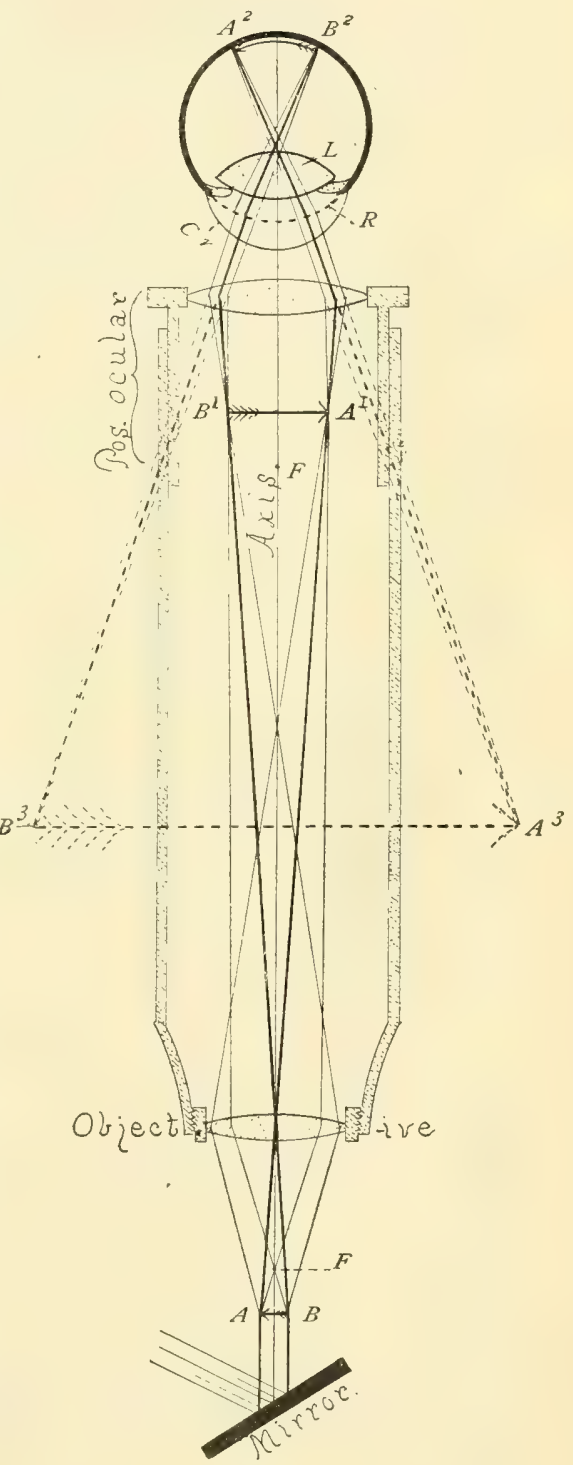


Axis. The principal optic axis of the microscope and of the eye.

$C r$. Cornea of the eye. L. Crystalline lens of the eye. R. Single, ideal, refracting surface at which all the refractions of the eye may be assumed to take place.

F. F. The principal focus of the positive ocular and of the objective.

Mirror. The mirror reflecting parallel rays to the object. The light is central. See Ch. II.

Pos. Ocular. An ocular in which the real image is formed outside the ocular. Compare the positive ocular with the simple microscope (Fig. I6).

\section{NOMENCLATURE OR TERMINOLOGY OF OBJECTIVES}

3. I5. Equivalent Focus.-In America, England, and sometimes also on the Continent, objectives are designated by their equivalent focal length. This length is given either in inches (usually contracted to in.) or in millimeters (mm.) Thus: An objective designated $\frac{1}{1}$ in. or $2 \mathrm{~mm}$., indicates that the objective produces a real image of the same size as is produced by a simple converging lens whose principal focal distance is $\frac{1}{12}$ inch or 2 millimeters (Fig. II). An objective marked 3 in. or $75 \mathrm{~mm}$., produces approximately the same sized real image as a simple converging lens of 3 inches or 75 millimeters focal length. And in accordance with the law that the relative size of object and image vary directly as their distance from the center of the lens (Figs. I4, I5, see Ch. IV,) it follows that the less the focal distance of the simple lens or of the equivalent focal distance of the objective, the greater is the size of the real image, as the tube-length remains constant and the image in all cases is found at about 160 or $250 \mathrm{~mm}$. from the objective.

\& I6. Numbering or Lettering Objectives.-Instead of designating objectives by their equivalent focus, many Continental opticians use letters or figures for this purpose. With this method the smaller the number, or the earlier in the alphabet the letter, the lower is the power of the objective. (See further in Ch. IV, for the power or magnification of objectives). This method is entirely arbitrary and does not, like the one above, give direct information concerning the objective.

\& I7. Air or Dry Objectives. - These are objectives in which the space between the front of the objective and the object or cover-glass is filled with air (Fig. 22). Most objectives of 1 ow and medium power (i. $e, \frac{1}{8}$ in. or $3 \mathrm{~mm}$. and lower powers) are dry.

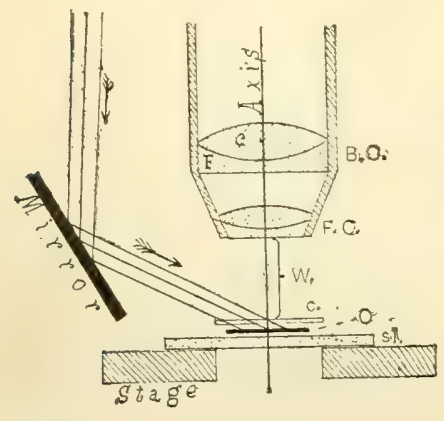

FIG. 22. Section of a dry objective showing working distance and lighting by reflected light.

Axis. The principal optic axis of the objective.

$B C$. Back Combination, composed of a plano-concave lens of fint glass $(F)$, and a double convex lens of crown glass $(c)$.

$F C$. Front Combination.

$C, O$, sl. The cover-glass, object and slide.

Mirror. The mirror is represented as above the stage, and as reflecting parallel rays from its plane face upon the object.

Stage. Section of the stage of the microscope. 
W. The Working Distance, that is the distance from the front of the objective to the object when the objective is in focus.

?. IS. Immersion Objectives.-An immersion objective is one with which there is some liquid placed between the front of the objective and the object or cover-glass. The most common immersion objectives are those $(A)$ in which water is used as the immersion fluid, and (B) where some liquid is used having the same refractive and dispersive power as the front lens of the objective. Such a liquid is called homogeneous, as it is optically homogeneous with the front glass of the objective. It may consist of thickened cedar wood oil or of glycerin containing some salt, as stannous chlorid in solution. When oil is used as the immersion fluid the objectives are frequently called oil immersion objectives. The disturbing effect of the cover-glass (Fig. 57) is almost wholly eliminated by the use of homogeneous immersion objectives, as the rays undergo very little or no refraction on passing from the cover-glass through the immersion medium and into the objective; and when the object is mounted in balsam there is practically no refraction in the ray from the time it leaves the balsam till it enters the objective.

FIG. 23. Sectional view of an Immersion, Adjustable Objective, and the object lighted zith axial or central and with oblique light.

Axis. The principal optic axis of the objective.

$B C, M C, F C$. The back, middle and front combination of the objective. In this case the front is not a combination, but a single planoconvex lens.

$A, B$. Parallel rays reflected by the mirror axially or centrally upon the object.

C. Ray reflected to the object obliquely.

I. Immersion fluid between the front of the objective and the cover glass or object $(O)$.

Mirror. The mirror of the microscope.

$O$. Object. It is represented without a coverglass. Ordinarily objects are covered whether examined with immersion or with dry objectives.

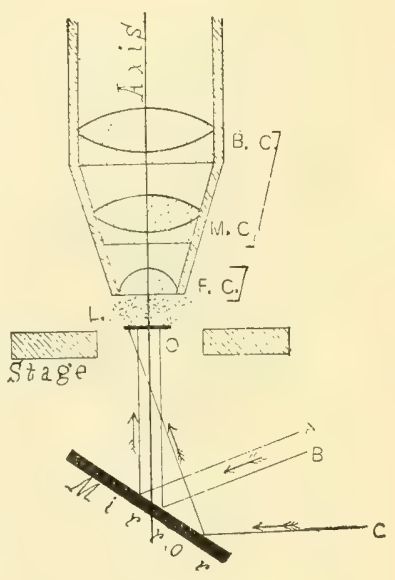

Stage. Section of the stage of the microscope.

3. I9. Non-Achromatic Objectives.-These are objectives in which the chromatic aberration is not corrected, and the image produced is bordered by colored fringes. They show also spherical aberration and are used only on very cheap microscopes. ( $8,87,8$, Figs. I2, I3).

\% 20. Achromatic Objectives.-In these the chromatic and the spherical aberration are both largely eliminated by combining concave and convex lenses of different kinds of glass "so disposed that their opposite aberrations shall correct each other." All the better forms of objectives are achromatic and also aplanatic. That is the various spectral colors come to the same focus.

21. Aplanatic Objectives, etc.-These are objectives or other pieces of optical apparatus (oculars, illuminators, etc.), in which the spherical distortion is 
wholly or nearly eliminated, and the curvatures are so made that the central and marginal parts of the objective focus rays at the same point or level. Such pieces of apparatus are usually achromatic also.

\%. 22. Apochromatic Objectives.-A term used by Abbe to designate a form of objective made by combining new kinds of glass with a natural mineral (Calcium fluorid, Fluorite, or Fluor spar). The name, Apochromatic, is used to indicate the higher kind of achromatism in which rays of three spectral colors are combined at one focus, instead of rays of two colors as in the ordinary achromatic objectives. At the present time (Igor) several opticians make apochromatic objectives without using the fluorite. Some of the early apochromatics deteriorated rather quickly in hot moist climates. Those now made are quite permanent.

The special characteristics of these objectives, when used with the "compensating oculars" are as follows :

(x) Three rays of different color are brought to one focus, leaving a small tertiary spectrum only, while with objectives as formerly made from crown and flint glass, only two different colors could be brought to the same focus.

(2) In these objectives the correction of the spherical aberration is obtained for two different colors in the brightest part of the spectrum, and the objective shows the same degree of chromatic correction for the marginal as for the central part of the aperture. In the old objectives, correction of the spherical aberration was confined to rays of one color, the correction being made for the central part of the spectrum, the objective remaining under-corrected spherically for the red rays and over-corrected for the blue rays ( 89 ).

(3) The optical and chemical foci are identical, and the image formed by the chemical rays is much more perfect than with the old objectives, hence the new objectives are well adapted to photography.

(4) These objectives admit of the use of very high oculars, and seem to be a considerable improvement over those made in the old way with crown and flint glass. According to $\operatorname{Dippel~(Z.w.~M.~IS86,~p.~300)~dry~apochromatic~objectives~}$ give as clear images as the same power water immersion objectives of the old form.

\%. 23. Non-Adjustable or Unadjustable Objectives.-Objectives in which the lenses or lens systems are permanently fixed in their mounting so that their relative position always remains the same. Low power objectives and those with homogenous immersion are mostly non-adjustable. For beginners and those unskilled in manipulating adjustable objectives $(\% 24)$, non-adjustable ones are more satisfactory, as the optician bas put the lenses in such a position that the most satisfactory results may be obtained when the proper thickness of cover-glass and tube-length are employed. (See table of tube-length and thickness of cover-glass below, p. I4.)

\& 24. Adjustable Objectives. - An adjustable objective is one in which the dis. tance between the systems of lenses (usually the front and the back systems) may be changed by the observer at pleasure. The object of this adjustment is to correct or compensate for the displacement of the rays of light produced by the mounting medium and the cover-glass after the rays have left the object. It is also to compensate for variations in "tube-length." See \& 29. As the displacement of the rays by the cover-glass is the most constant and important, these objectives are usually designated as having cover-glass adjustment or correction. (Fig. 23. See also practical work with adjustable objectives, Ch. II). 
8. 25. Parachromatic, Pantachromatic and Semi-apochromatic Objectives.These are trade names for objectives, most of them containing one or more lenses of the new glass $\left(z_{2} 22\right)$. They are said to approximate much more closely to the apochromatics than to the ordinary objectives.

8 26. Variable Objective.-This is a low power objective of 36 to $26 \mathrm{~mm}$. equivalent focus, depending upon the position of the combinations. By means of a screw collar the combinations may be separated, diminishing the power, or approximated and thereby increasing it.

3. 27. Projection Objectives.-These are designed especially for projecting an image on a screen and for photo-micrography. They are characterized by having a flat, sharp field brilliantly lighted. (See Ch. IV, IX.)

3. 28. Illuminating or Vertical Illuminating Objectives.-These are designed for the study of opaque objects with good reflecting surfaces, like the rulings on metal bars and broken or polished and etched surfaces of metals employed in micro-metallography. The light enters the side of the tube or objective and is reflected vertically downward through the objective and thereby is concentrated upon the object. The object reflects part of the light back into the microscope thus enabling one to see-a clear image.

3. 29. Tube-Length and Thickness of Cover-Glasses.- "In the construction of microscopic objectives, the corrections must be made for the formation of the image at a definite distance, or in other words the tube of the microscope on which the objective is to be used must have a definite length. Consequently the microscopist must know and use this distance or 'microscopical tube-length' to obtain the best results in using any objective in practical work." Unfortunately different opticians have selected different tube-lengths and also different points between which the distance is measured, so that one must know what is meant by the tube-length of each optician whose objectives are used. See table.

The thickness of cover-glass used on an object (See Ch. VII, on mounting), except with homogeneous immersion objectives, has a marked effect on the light passing from the object (Fig. 57). To compensate for this the position of the systems composing the objective are closer together than they would be if the object were uncovered. Consequently, in non-adjustable objectives some standard thickness of cover-glass is chosen by each optician and the position of the systems arranged accordingly. With such an objective the image of an uncovered object would be less distinct than a covered one, and the same result would follow the use of a cover-glass much too thick. 
Length in Millimeters and Parts included in "Tube-Length" by Various Opticians.*

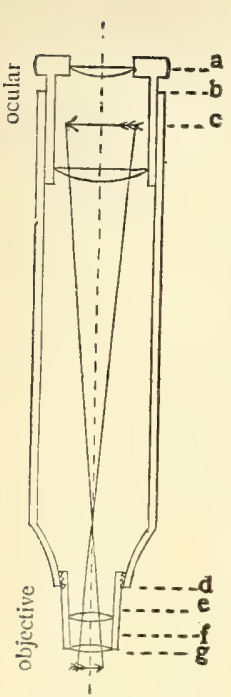

FIG. 24 .
Pts. included in "Tube-

length.' See Diagram
'Tube-Length" in Millimeters. a-d

E. Leitz, Wetzlar

Natchet et Fils, Paris

Powell and Lealand, London

C. Reichert, Vienna

Spencer Lens Co., Buffalo

W. Wales, New York

Bausch \& Lomb Opt. Co., Rochester_._ 160 or $216 \mathrm{~mm}$. Bézu, Hausser et Cie, Paris

b-d Klönne und Müller, Berlin

W. \& H. Seibert, Wetzlar

Swift \& Son, London

C. Zeiss, Jena

R. Winkel, Göttingen

a-g . Gundlach Optical Co., Rochester

c-d . Ross \& Co., London

c-d . Queen \& Co., Philadelphia.

c-e . R. \& J. Beck, London

c-f . Hartnack, Potsdam, Germany

Vérick (Stiassnie) Paris

Watson \& Sons, London

J. Zentmayer, Philadelphia
160 tin.

$254 \mathrm{~mm}$.

160 to $180 \mathrm{~mm}$ $160 \mathrm{~mm}$. I $80 \mathrm{~mm}$.

I 60-I 80 or $254 \mathrm{~mm}$. $170 \mathrm{~mm}$.

I65 to $2281 / 2 \mathrm{~mm}$.

I 60 or $250 \mathrm{~mm}$.

$192 \mathrm{~mm}$.

$254 \mathrm{~mm}$.

254 or $160 \mathrm{~mm}$.

$160 \mathrm{~mm}$.

254 or $160 \mathrm{~mm}$.

I6o and I $80 \mathrm{~mm}$.

I60-200 $\mathrm{mm}$.

I $60-235 \mathrm{~mm}$.
$254 \mathrm{~mm}$.

\footnotetext{
施 $\mathrm{mm}$. $\quad\{$ Powell and Lealand, London.

W. Wales, New York.

$\frac{20}{100} \mathrm{~mm}$. Watson \& Sons, London.

It $\mathrm{mm}$. $\mathrm{E}$. Leitz, Wetzlar.

I $655 \mathrm{~mm}$. Ross \& Co., London.

Klönne und Müller, Berlin.

Spencer Lens Co., Buffalo.

$\frac{18}{100} \mathrm{~mm}$.

$\frac{15}{10} \frac{20}{10} \mathrm{~mm}$. C. Zeiss, Jena.

Bausch \& Lomb Optical Co., Rochester.

Queen \& Co., Philadelphia.

$\frac{15}{10} \frac{18}{0} \mathrm{~mm}$. C. Reichert, Vienna.

$150 \mathrm{~mm}$.

Gundlach Optical Co., Rochester.

IV. and H. Seibert, Wetzlar.

( R. and J. Beck, London.

$\frac{12}{10} \frac{1}{0} \mathrm{~mm}$.

$\frac{10^{\top} 115}{100} \mathrm{~mm}$.

$\frac{10}{100} \mathrm{~mm}$.

J. Zentmayer, Philadelphia.

$\{$ Nachet et Fils, Paris.

Bézu, Hausser et Cie, Paris.

Swift and Son, London.

$1 \frac{6}{10} \frac{1}{8} \mathrm{~mm}$.

E. Hartnack, Potsdam, Germany,
}

*The information contained in these tables was very kindly furnished by the opticians named, or obtained by consulting catalogs. In most of the later catalogs the information is definite, and many makers now not only put their names and the equivalent focal length on their objectives, but they add the numerical aperture ( $\& 3 \mathrm{I}$ ) and the tube-length for which the objective is corrected. This is in accordance with the recommendations of the author in the original paper on "tubelength," (Proc. Amer. Soc. Micr., Vol. IX., p. I68, also by Bausch, Vol. XII, p. 43). If the table in this edition is compared with the original table or with that in the previous edition of this book some differences will be noted, the changes being 
Owing to information received after the table on p. I4 was printed it is necessary to replace that table by one containing the latest information. In this revised table tube-length $\mathrm{b}$ - $\mathrm{d}$ of the diagram greatly preponderates, and the great majority of unadjustable objectives are corrected for a thickness of cover-glass falling between fifteen and twenty one hundredths of a millimeter (O. I5-0.20 mm.).

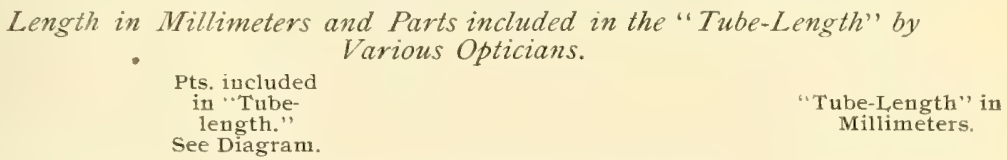

"Tube-L,ength" in villimeters.

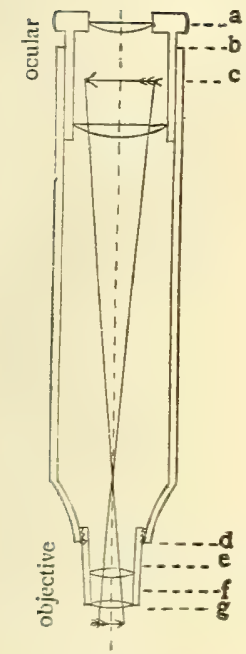

FIG. 24.

Chas. Baker, London, England 150 or $250 \mathrm{~mm}$ The Bausch \& Lomb Optical Co.

Rochester, N. Y. .... I60 or $216 \mathrm{~mm}$

R. \& J. Beck, London, England ..... I60 or $220 \mathrm{~mm}$ Bézu, Hausser \& Cie, Paris, France _..._._. ISo mm.

Klonne und Müller, Berlin, Germany _-_ I 60 or $250 \mathrm{~mm}$.

b-d Queen \& Co., Incorporated, Phila., Pa. - $170 \mathrm{~mm}$.

Ross, Ltd, London, England ..... I60 or $254 \mathrm{~mm}$.

W. und H. Seibert, Wetzlar, Germany

Swift \& Son, London, England _........ 160 or $228 \mathrm{~mm}$

Watson \& Sons, London, England _._._. 160 or $250 \mathrm{~mm}$

R. Winkel, Goettingen, Germany _... $192 \mathrm{~mm}$.

Carl Zeiss, Jena, Germany ............. _ I60 or $250 \mathrm{~mm}$.

Ernst Leitz, Wetzlar, Germany _._..... $170 \mathrm{~mm}$.

Nachet et Fils, Paris, France _..._.... I60 mm.

a-d Powell \& Lealand, London, England ... $254 \mathrm{~mm}$.

C. Reichert, Vienna, Austria ........... I60-I $80 \mathrm{~mm}$.

Spencer Lens Company, Buffalo, N. Y. _ I $60 \mathrm{~mm}$.

W. Wales, New York .............. $254 \mathrm{~mm}$.

The Gundlach Opt. Co., Rochester, N. Y. 254 mm.

E. Hartnack, Potsdam, Germany

I $60 \mathrm{~mm}$.

Dollond \& Co., London, England ........ $165,240 \mathrm{~mm}$.

Vérick (Stiassnie) Paris, France _... _... I60-200 mm.

P. Waechter, Berlin-Friedenau, Germany _ $160 \mathrm{~mm}$.

J. Zentmayer, Philadelphia, Pa. ....... I60 or $235 \mathrm{~mm}$.

Thickness of Coner-Glass for Which Non-Adjustable Objectizes are Corrected by Various Opticians

$0.18 \mathrm{~mm}$.

The Bausch \& Lomb Optical Co., Rochester, N. Y.

Klonne und Müller, Berlin, Germany.

Queen \& Co., Incorporated, Philadelphia, Pa.

The Spencer Lens Co., Buffalo, N. Y.

$0.17 \mathrm{~mm}$.

( Ernst Leitz, Wetzlar, Germany.

P. Wächter, Berlin-Friedenau, Germany.

( R. Winkel, Goettingen, Germany.

Chas. Baker, London, England.

o. $15 \mathrm{~mm}$.

R. \&. J. Beck, Ltd., London, England.

Gundlach Optical Co., Rochester, N. Y.

W. und H. Seibert, Wetzlar, Germany.

$0.15-0.18 \mathrm{~mm}$.

f E. Hartnack, Potsdam, Germany.

C. Reichert, Vienna, Austria.

( Ross, Ltd., London, England.

O. $15-0.20 \mathrm{~mm}$

Vérick (Stiassnie), Paris, France.

( Carl Zeiss, Jena, Germany.

O. $12-0.17 \mathrm{~mm}$.

J. Zentmayer, Philadelphia, $\mathrm{Pa}$.

O. IO-O. $5 \mathrm{~mm}$.

Dollond \& Co., London, England.

1 Nachet et Fils, Paris, France.

O. IO-O. $2 \mathrm{~mm}$.

Bézu Hausser \& Cie, Paris, France.

$0.10 \mathrm{~mm}$.

f Powell \& Lealand, London, England.

( Swift \& Son, London, England.

$0.20 \mathrm{~mm}$.

Watson \& Sons, London, England.

$0.25 \mathrm{~mm}$.

W. Wales, New York. 

$\$ 30$. Aperture of Objectives. - The angular aperture or angle of aperture of an objective is the "angle contained, in each case, between the most diverging of the rays issuing from the axial point of an object [i.e., a point in the object situated on the optic axis of the microscope], that can enter the objective and take part in the formation of an image." (Carpenter).

in the direction of uniformity and in general in the direction recommended by the writer and Mr. Bausch and the committee of the American Microscopical Society. The recommendations of the committee, published in the Proceedings, Vol. XII., p. 250, are as follows :

"Believing in the desirability of a uniform tube-length for microscopes, we unanimously recommend: I. That the parts of the microscope included in the tube-length should be the same by all opticians, and that the parts included should be those between the upper end of the tube where the ocular is inserted and the lower end of the tube where the objective is inserted:

2. That the actual extent of tube length as defined in section $\mathrm{I}-\mathrm{Be}$, for the short or continental tube, $160 \mathrm{~mm}$., or 6.3 inches, and $216 \mathrm{~mm}$., or $8 \frac{1}{2}$ inches, for the long tube, and that the draw tube of the microscope possess two special marks indicating these standard lengths.

3. That oculars be made par-focal, and that the par-focal plane be coincident with that of the upper end of the tube.

4. That the mounting of all objectives of $6 \mathrm{~mm}$. ( $/ / 4 \mathrm{inch}$ ) and shorter focus should be such as to bring the optical center of the objective $\mathrm{I} / 2$ inches below the shoulder, and that all objectives be marked with the tube-length for which they are corrected.

5. That non-adjustable objectives be corrected for cover-glass from $\frac{15}{100}$ to $\frac{20}{100}$ mm. ( $\frac{1}{170}$ to $\frac{1}{130}$ inch) in thickness.

These recommendations give a distance of Io inches ( $254 \mathrm{~mm}$.) between the par-focal plane of the ocular and the optical center of the objective for the long tube, and are essentially in accord with the actual practice of opticians.

At the request of the committee, a joint conference was held with the opti-

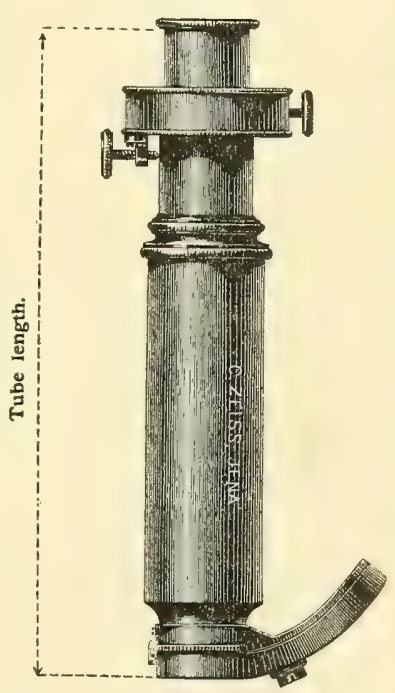

FIG. 25. The tube of a microscope with ocular micrometer and nose piece in position to show that in measuring tube-length one must measure from the eye lens to the place where the objective is attached. (Zeiss' Catalog.) cians belonging to the Society and present at the meeting. They expressed their belief in the entire practicability of the above recommendations and a willingness to adopt them."

(Signed)

SIMON H. GAGE,

A. CIIFFORD MERCER,

CHARLES E. BARR. 
In general the angle increases with the size of the lenses forming the objective and the shortness of the equivalent focal distance ( 8 I 5$)$. If all objectives were dry or all water or all homogeneous immersion a comparison of the angular aperture would give one a good idea of the relative number of image forming rays

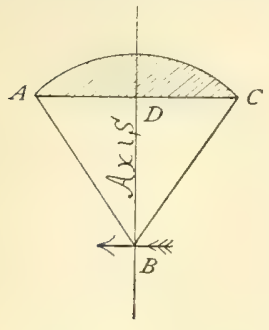

FIG. 26. Diagram illustrating the angular aperture of a microscopic objective. Only the front lens of the objective is shown.

\section{Axis. The principal optic axis of the objective.}

$B A, B C$, the most divergent rays that can enter the objective, they mark the angular aperture. $A B D$ or $C B$ $D$ half the angular aperture. This is designated by $u$ in making Numerical Aperture computations. See the table, \& 33 .

transmitted by different objectives; but as some are dry, others water and still others homogeneous immersion, one can see at a glance that, other things being equal, the dry objective (Fig. 27) receives less light than the water immersion, and the water immersion (Fig. 28) less than the homogeneous immersion (Fig. 29). In order to render comparison accurate between different kinds of objectives, Professor Abbe takes into consideration the rays actually passing from the back combination of the objectives to form the real image; he thus takes into account the medium in front of the objective as well as the angular aperture. The term "Numerical Aperture," ( $N$. A.) was introduced by Abbe to indicate the capacity of an optical instrument "for receiving rays from the object and transmitting them to the image.

3. 3r. Numerical Aperture (abbreviated N. A. ), as now employed for microscope objectives, is the ratio of the semi-diameter of the emergent pencil to the focal length of the lens. Or as the factors are more readily obtainable it is simpler to utilize the relationship shown in the La Grange-Helmholtz-Abbe formula, and indicate the aperture by the expression: N. A. $=n \sin u$. In this formula $n$ is the index of refraction of the medium in front of the objective (air, water or homogeneous liquid), and $\sin u$ is the sine of half the angle of aperture (Fig. 26, D B A). For the mathematical discussion showing that the expressions semi-diameter of emergent pencil

focal length of the lens - $\mathrm{n}$ sin $\mathrm{u}$, the student is referred to the Joumal of the Royal Microscopical Society, I88 r, pp. 392-395, i898, p. 363 .

For example, take three objectives each of $3 \mathrm{~mm}$. equivalent focus, one being a dry, one a water immersion, and one a homogeneous immersion. Suppose that the dry objective has an angular aperture of $106^{\circ}$, the water immersion of $94^{\circ}$ and the homogeneous immersion of $90^{\circ}$. Simply compared as to their angular aperture, without regard to the medium in front of the objective, it would look as if the dry objective would actually take in and transmit a wider pencil of light than either of the others. However, if the medium in front of the objective is considered, that is to say, if the numerical instead of the angular apertures are compared, the results would be as follows: Numerical Aperture of "a dry objective of $106^{\circ}, X . A . \ldots n \sin u$. In the case of dry objectives the medium in front of the objective being air, the index of refraction is unity, whence $n=1$. Half the angular aperture is $100^{\circ}=53^{\circ}$. By consulting a table of natural sines it will be found that the sine of $53^{\circ}$ is 0.799 , whence $X . A .=n$ or $1 \times \sin u$ or $0.799=0.799$.* $^{*}$

*\% 32. Interpolation.-In practice, as in solving problems similar to those on the following pages and those in refraction if one cannot find a sine exactly 


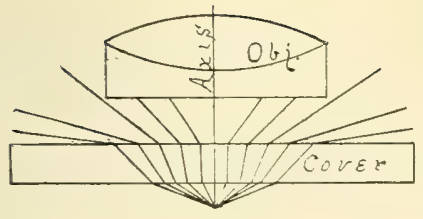

FIGs. 27-29 are somewhat modified from Ellenberger, and are introduced to illustrate the relative amount of utilized light, withdry, water immersion and homogeneous immer-

27 sion objectives of the same equivalent focus. The point from which the rays emanate is in air in each case. If Canada balsam were beneath the cover-glass in place of the air there would be practically no refraction of the rays on entering the cover glass ( $\&$ I8).

FIG. 27. Showing the course of the rays 28 passing through a cover glass from an axial point of the object, and the number that finally enter the front of a dry objective.

FIG. 28. Rays from the axial point of the object traversing a cover of the same thickness as in Fig. 27, and entering the front lens of a water immersion objective.

FIG. 29. Rays from an axial point of the 29 object traversing a cover glass and entering the front of a homogeneous immersion objective.

With the water immersion objective the medium in front is water, and its index of refraction is $\mathrm{I} .33$, whence $n=\mathrm{I} .33$. Half the angular aperture is $\frac{94}{2}=47^{\circ}$, and by the table the sine of $47^{\circ}$ is found to be $0.73 \mathrm{I}, i . e_{.}, \sin u=0.73 \mathrm{I}$, whence $\mathrm{N}$. A. $=n$ or $\mathrm{I} .33 \times \sin u$ or $0.73 \mathrm{I}=0.972$.

corresponding to a given angle; or if one has an angle which does not correspond to any sine or angle given in the table, the sine or angle may be closely approximated by the method of interpolation, as follows: Find the sine in the table nearest the sine whose angle is to be determined. Get the difference of the sines of the angles greater and less than the sine whose angle is to be determined. That will give the increase of sine for that region of the arc for 15 minutes. Divide this increase by $I_{5}$ and it will give with approximate accuracy the increase for I minute. Now get the difference between the sine whose angle is to be determined and the sine just below it in value. Divide this difference by the amount found necessary for an increase in angle of I minute and the quotient will give the number of minutes the sine is greater than the next lower sine whose angle is known. Add this number of minutes to the angle of the next lower sine and the sum will represent the desired angle of the sine. Or if the sine whose angle is to be found is nearer in size to the sine just greater, proceed exactly as before, getting the difference in the sines, but subtract the number of minutes of difference and the result will give the angle sought. For example take the case in Section 97 where the sine of the angle of $28^{\circ} 54^{\prime}$ is given as 0.48327 . If one consults the table the nearest sines found are 0.48099 , the sine of $28^{\circ} 45^{\prime}$, and $0.4848 \mathrm{I}$, the sine of $29^{\circ}$. Evidently 
With the oil immersion in the same way N. A. $=n \sin u ; n$ or the index of refraction of the homogeneous fluid in front of the objective is $1.5^{2}$, and the semi-angle of aperture is $\frac{900^{\circ}}{2}=45^{\circ}$. The sine of $45^{\circ}$ is 0.707 , whence $\mathrm{N} . \mathrm{A} .=n$ or $152 \times \sin u$ or $0.707=\mathrm{I} .074$.

By comparing these numerical apertures: Dry 0.799, water 0.972 , homogeneous immersion $\mathrm{I} .074$, the same idea of the real light efficiency and image power of the different objectives is obtained, as in the graphic representations shown in Figs. 27-29.

If one knows the numerical aperture (N. A.) of an objective the angular aperture is readily determined from the formula; and one can determine the equivalent angles of objectives used in different media (i.e., dry or immersion). For example, suppose each of three objectives has a numerical aperture $(N$. A.) of 0.80 , what is the angular aperture of each? Using the formula of $\mathrm{N} . \mathrm{A} .=n \sin u$, one has N. A. $=0.80$ for all the objectives.

For the dry objective $n=$ I (Refractive index of air).

water immersion objective $n=\mathrm{r} .33$. (Refractive index of water).

" homogeneous immersion objective $n=\mathrm{r} .52$ (Refractive index of homogeneous liquid). And $2 u$ is to be found in each case.

For the dry objective, substituting the known values the formula becomes $0.80=\mathrm{I} \sin u$, or $\sin u=0.80$. By inspecting the table of natural sines ( 3 d page of corer) it will be found that 0.80 is the sine of 53 degrees and 8 minutes. As this is half the angle the entire angular aperture of the dry objective must be $53^{\circ} 8^{\prime} \times 2=106^{\circ} 16^{\prime}$.

For the water immersion objective, substituting the known values in the formula as before: $0.80=\mathrm{I} .33 \sin u$, or $\sin u=\frac{0.80}{\mathrm{I} .33}=0.60 \mathrm{I} 5$. Consulting the table of sines as before, it will be found that 0.6015 is the sine of $36^{\circ} 59^{\prime}$ whence the angular aperture (water angle) is $36^{\circ}$ $59^{\prime} \times 2=73^{\circ} 58^{\prime}$.

For the homogeneous immersion objective, substituting the known values, the formula becomes: $0.80=\mathrm{I} .5^{2} \sin u$ whence $\sin u=$ $\frac{0.80}{I .52}=0.5263$. And by consulting the table of sines it will be found

then the angle sought must lie between $28^{\circ} 45^{\prime}$, and $29^{\circ}$. If the difference between

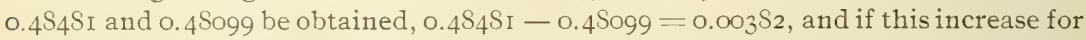
$15^{\prime}$ be divided by I5 it will give the increase for I minute; $0.00382 \div 15=0.000254$. Now the difference between the sine whose angle is to be found and the next lower sine is $0.4 S_{327}-0.4 S_{099}=0.0022 \mathrm{~S}$. If this difference be divided by the amount found necessary for I minute it will give the total minutes above $28^{\circ} 45^{\prime}$; $0.00228 \div 0.000254=9$. That is, the angle sought is 9 minutes greater than $2 \mathrm{~S}^{\circ}+5^{\prime}=2 \mathrm{~S}^{\circ} 54^{\prime}$. 
that this is the sine of $3 \mathrm{I}^{\circ} 45^{\frac{1^{\prime}}{2}}$ whence $2 u$ or the entire angle (balsam or oil angle) is $63^{\circ} 3 \mathrm{I}^{\prime}$.

That is, three objectives of equal resolving powers, each with a numerical aperture of 0.80 would have an angular aperture of $106^{\circ} 16^{\prime}$ in air, $73^{\circ} 58^{\prime}$ in water and $63^{\circ} 3^{I^{\prime}}$ in homogeneous immersion liquid.

For the apparatus and method of determining aperture, see appendix.

\$33. Table of a Group of Objectives with the Numerical Aperture (N. A) and the method of obtaining it. Half the angular aperture is designated by $u$ and the index of refraction of the medium in front of the objective by $n$. For dry objectives this is air and $n=I$, for water immersions $n=I .33$, and for homogeneous immersions $n=I .52$. (For a table of natural sines, see third page of cover.)

\begin{tabular}{|c|c|c|c|c|c|}
\hline ОВJECTIVE. & 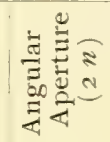 & $\begin{array}{l}\text { NATURAL, Sine } \\
\text { of half the angular } \\
\text { aperture } \\
(\sin u .)\end{array}$ & $\begin{array}{c}\text { Index of } \\
\text { Refraction } \\
\text { of the medi- } \\
\text { um in front } \\
\text { of the objec- } \\
\text { tive }(n) \text {. }\end{array}$ & \multicolumn{2}{|c|}{$\begin{array}{l}\text { NUMERICAL, APERTURE, } \\
\qquad \text { (N. A.) }=n \sin u\end{array}$} \\
\hline $\begin{array}{l}25 \mathrm{~mm} . \\
\text { (Dry.) }\end{array}$ & $20^{\circ}$ & $\operatorname{Sin} \frac{20}{2}=0.1736$ & $n=\mathbf{I}$ & N.A. $=$ & $\mathrm{I} \times 0.1736=0 . \mathrm{I} 73$ \\
\hline $\begin{array}{l}25 \mathrm{~mm} . \\
\text { (Dry.) }\end{array}$ & $40^{\circ}$ & $\sin \frac{40}{2}=0.3420$ & $n=\mathrm{I}$ & N.A. $=$ & I $\times 0.3420=0.342$ \\
\hline $\begin{array}{l}\text { I2 } 2 / 2 \mathrm{~mm} . \\
\text { (Dry.) }\end{array}$ & $42^{\circ}$ & $\operatorname{Sin} \frac{4^{2}}{2}=0.35^{84}$ & $n=\mathrm{I}$ & N.A. $=$ & $\mathrm{I} \times 0.35^{8} 3=0.35^{8}$ \\
\hline $\begin{array}{l}\text { I2 } 2 / 2 \mathrm{~mm} . \\
\text { (Dry.) }\end{array}$ & $100^{\circ}$ & $\operatorname{Sin} \frac{100}{2}=0.7660$ & $n=\mathrm{I}$ & N.A. $=$ & $\mathrm{I} \times 0.7660=0.766$ \\
\hline $\begin{array}{l}6 \mathrm{~mm} . \\
\text { (Dry.) }\end{array}$ & $75^{\circ}$ & $\operatorname{Sin} \frac{75}{2}=0.6087$ & $n=\mathrm{I}$ & N.A. $=$ & $\mathrm{I} \times 0.6087=0.609$ \\
\hline $\begin{array}{l}6 \mathrm{~mm} \text {. } \\
\text { (Dry.) }\end{array}$ & $136^{\circ}$ & $\operatorname{Sin} \frac{136}{2}=0.9272$ & $n=\mathbf{I}$ & N. A. $=$ & $I \times 0.9272=0.927$ \\
\hline $\begin{array}{l}3 \mathrm{~mm} . \\
\text { (Dry.) }\end{array}$ & $I 15^{\circ}$ & $\operatorname{Sin} \frac{\operatorname{II} 5}{2}=0.8434$ & $n=\mathrm{I}$ & N.A. $=$ & $\mathrm{I} \times 0.8434=0.843$ \\
\hline $\begin{array}{l}3 \mathrm{~mm} \text {. } \\
\text { (Dry.) }\end{array}$ & $163^{\circ}$ & $\operatorname{Sin} \frac{163}{2}=0.9890$ & $n=\mathrm{I}$ & N. A. $=$ & $\mathrm{I} \times 0.9890=0.989$ \\
\hline $\begin{array}{l}2 \text { mm. } \\
\text { Water. } \\
\text { Immersion. }\end{array}$ & $96^{\circ} 12^{\prime}$ & $\sin \frac{9^{6} 12^{\prime}}{2}=0.7443$ & $n=\mathrm{I} .33$ & N. A. $=\mathrm{I}$ & $.33 \times 0.7443=0.99$ \\
\hline $\begin{array}{l}2 \mathrm{~mm} \text {. } \\
\text { Homogeneous } \\
\text { Immersion. }\end{array}$ & $\mathrm{IIO}^{\circ} \mathrm{S}^{\prime}$ & $\operatorname{Sin} \frac{110^{\circ}}{2} \frac{38^{\prime}}{2}-0.8223$ & $n=1.52$ & N. A. $=$ & $.52 \times 0 . S_{223}=1.25$ \\
\hline $\begin{array}{l}2 \mathrm{~mm} \text {. } \\
\text { Homogeneous } \\
\text { Immersion. }\end{array}$ & $134^{\circ} \mathrm{IO}^{\prime}$ & $\operatorname{Sin} \frac{134^{\circ} \mathrm{IO}^{\prime}}{2}=0.92 \mathrm{II}$ & $n=\mathrm{I} .52$ & N. A. $=$ & $.52 \times 0.92 \mathrm{IO}=\mathrm{I} .4 \mathrm{O}$ \\
\hline
\end{tabular}


$\$$ 34. Significance of Aperture.-As to the real significance of aperture in microscopic objectives, it is now an accepted doctrine thatthe corrections in spherical and chromatic aberration being the same(I) Objectives vary directly as their numerical aperture in their ability to define or make clearly visible minute details (resolving power). For example an objective of $4 \mathrm{~mm}$. equivalent focus and a numerical aperture of 0.50 would define or resolve only half as many lines to the millimeter or inch as a similar objective of I.OO N.A. So also an objective of $2 \mathrm{~mm}$. focus and I.4O N.A. would resolve only twice as many lines to the millimeter as a $4 \mathrm{~mm}$. objective of $0.70 \mathrm{~N}$.A. Thus it is seen that defining power is not a result of magnification but of aperture, otherwise the $2 \mathrm{~mm}$. objective would resolve far more than twice as many lines as the $4 \mathrm{~mm}$. objective.

Taking the results of the researches of Abbe as a guide to visibility with the microscope, one has the general formula $2 \lambda \times \mathrm{N}$.A. That is twice the number of wave lengths of the light used multiplied by the numerical aperture of the objective. From this general statement it will be seen that the shorter the wave lengths of the light, the more there will be in an inch or centimeter and therefore the greater the number of lines visible in a given space. That is the kind of light used is one element and the objective the other in determining the number of lines visible under the microscope.

Following Mr. E. M. Nelson (Jour. Roy. Micr. Soc., I893, p. 15) it is beliered that not more than $3 / 4$ ths of the numerical aperture of an objectire is really available for microscopic study, with a central, solid cone of light. To determine the number of lines visible in a given space with a giren light the formula would become $2 \lambda \times 3 / 4$ ths N.A. $=3 / 2 \lambda N$. A To determine the working-resolving power of any objective it is only necessary to know the number of light waves in a given space, say an inch or a centimeter and to multiply this number by $3 / 2 \mathrm{~N}$. A. For example suppose one uses ordinary daylight and assumes the average wave length is I 46666 in., then there must be 46,666 per inch and $46,666 \times 32=70,000$ approximately. If the $\mathrm{N}$. A. is $\mathrm{I}$, then the objective will resolve or make visible 70,000 lines to the inch, or approximately 28,000 to the centimeter. If blue light were used the number rould be 32,000 per centimeter, or 80,000 per inch. It will be seen that the number of lines here given is smaller than that in the table of Carpenter-Dallinger, because in the latter the full aperture is supposed to be employed and the light is of the greatest available obliquity. 
(2) The illuminating power of an objective of a given focus is found to vary directly as the square of the numerical aperture (N.A.) ${ }^{2}$. Thus if two 4 mm. objectives of N.A. O. 20 and N.A. O. 40 were compared as to their illuminating power it would be found from the above that they would vary as $0.20^{2}: 0.40^{2}=0.0400: 0.1600$ or $1: 4$. That is the objective of $0.20 \mathrm{~N}$.A. would have but $1 / 4$ th the illuminating power of the one of 0.40 N.A.

(3) The penetrating power, that is the power to see more than one plane, is found to vary as the reciprocal of the numerical aperture $\frac{\mathrm{I}}{\mathrm{N} . \mathrm{A} \text {. }}$ so that in an objective of a given focus the greater the aperture the less the penetrating power.

Of course when equivalent focus and numerical aperture both differ the problem becomes more complex.

While all microscopists are agreed that the fineness of detail which can be seen depends directly on the numerical aperture of the objective used, the general theory of microscopic vision has two interpretations:

(A.) That it is as with the unaided eye, the telescope and the photographic camera. This is the original view and the one which many are favoring at the present day (see Mercer, Proceedings of the Amer. Micr. Soc. I 896, pp. 321-396).

(B) The other view originated with Professor Abbe, and in the words of Carpenter-Dallinger, pp. 62, 43: "What this is becomes explicable by the researches of Abbe. It is demonstrated that microscopic vision is sui generis. There is and can be, no comparison between microscopic and macroscopic vision. The images of minute objects are not delineated microscopically by means of the ordinary laws of refraction; they are not dioptrical results, but depend entirely on the laws of diffraction. These come within the scope of and demonstrate the undulatory theory of light, and involve a characteristic change which material particles or fine structural details, in proportion to their minuteness, effect in transmitted rays of light. The change consists generally in the breaking up of an incident ray into a group of rays with large angular dispersion within the range of which periodic alternations of dark and light occur."

For a consideration of the aperture question, its history and significance, see J. D. Cox, Proc. Amer. Micr. Soc., I884, pp. 5-39; Jour. Roy. Micr. Soc., I881, pp. 303, 348, 365, 388 ; I882, pp. 300, 460 ; I883, p. 790 ; I884, p. 20 ; I896, p. 68I ; 1897, p. 7 I ; I898.pp. 354, 362, 592 ; Mercer, Proceedings Amer. Micr. Soc., I 896, pp. 32 I- 
396 ; Lewis Wright, Philos. Mag., June, I898, pp. 480-503; Carpenter-Dallinger, Chapter II ; Nelson, Jour. Quekett Micr. Club, VI, pp. I $4-38$.

\section{THE OCULAR}

\% 35. A Microscopic Ocular or Eye-Piece consists of one or more converging lenses or lens systems, the combined action of which is, like that of a simple microscope, to magnify the real image formed by the objective.

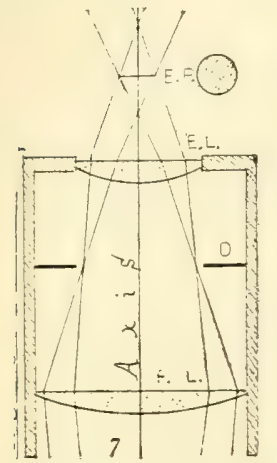

FIG. 30. Sectional view of a Huygenian ocular to show the formation of the Eye-Point.

Axis . Optic axis of the ocular. D. Diaphragm of the ocular. E.L. Eye-Lens. F. L. Field-Lens.

E. P. Eye-point. As seen in sectron, it appears something like an hour-glass. When seen as looking into the ocular, i.e., in transection, it appears as a circle of light. It is at the point where the most rays cross.

Depending upon the relation and action of the different lenses forming oculars, they are divided into two great groups, negative and positive.

2. 36. Negative Oculars are those in which the real, inverted image is formed within the ocular, the lower or field-lens serving to collect the image-forming rays somewhat, so that the real image is smaller than as if the field-lens were absent (Fig. 2I). As the field-lens of the ocular aids in the formation of the real image it is considered by some to form a part of the objective rather than of the ocular. The upper or eye-lens of the ocular magnifies the real image.

3. 37. Positive Oculars are those in which the real, inverted image of the object is formed outside the ocular, and the entire system of ocular lenses magnifies the real image like a simple microscope (Fig. I6).

Positive and negative oculars may be readily distinguished, as a positive ocular may be used as a simple microscope, while a negative ocular cannot be so used when its field-lens is in the natural position toward the object. By turning the eye-lens toward the object and looking into the field-lens an image may be seen, however.

In works and catalogs concerning the microscope and microscopic apparatus, and in articles upon the microscope in periodicals, various forms of oculars or eyepieces are so frequently mentioned, without explanation or definition, that it seems worth while to give a list, with the French and German equivalents, and a brief statement of their character.

Achromatic Ocular ; Fr. Oculaire achromatique ; Ger. achromatisches Okular. Oculars in which chromatic aberration is wholly or nearly eliminated. - Aplanatic Ocular ; Fr. Oculaire aplanatique ; Ger. aplanatisches Okular (see \& 2I).-Binocular, stereoscopic Ocular; Fr. Oculaire binoculaire stereoscopique; Ger. stereoskopisches Doppel-Okular. An ocular consisting of two oculars about as far apart as the two eyes. These are connected with a single tube which fits a monocular microscope. By an arrangement of prisms the image forming rays are divided, half 
being sent to each eye. The most satisfactory form was worked out by Tolles and is constructed on true stereotomic principles, both fields being equally illuminated. His ocular is also erecting.-Campani's Ocular (see Huygenian Ocular).-Compound Ocular; Fr. Oculaire composé ; Ger. zusammengesetztes Okular. An ocular of two or more lenses, e.g., the Huygenian (see Fig. 30).-Continental Ocular. An ocular mounted in a tube of uniform diameter as in Fig. 31.-Deep Ocular, see high ocular.-Erecting Ocular; Fr. Oculaire redresseur; Ger. bildumkehrendes Okular. An ocular with which an erecting prism is connected so that the image is erect as with the simple microscope. Such oculars are most common on dissecting microscopes. - Filar micrometer Ocular; Screw m. o., Cobweb m. o., Ger. Okular-Schraubenmikrometer. A modification of Ramsden's Telescopic Cobweb micrometer ocular.-Goniometer Ocular; Fr. Oculaire à goniomètre ; Ger. Goniometer-Okular. An ocular with goniometer for measuring the angles of minute crystals. - High Ocular, sometimes called a deep ocular. One that magnifies the real image considerably, $i$. e., Io to 20 fold.-Huygenian Ocular, Huygens' O., Campani's O., Airy's O.; Fr. Oculaire d'Huygens, o. de Campani; Ger. Huygens'sches Okular, Campaniches Okular, see ?.38.-Index Ocular; Ger. SpitzenO. An ocular with a minute pointer or two pointers at the level of the real image. The points are movable and serve for indicators and also, although not satisfactorily, for micrometry.-Kellner's Ocular, see orthoscopic ocular-Low ocular, also called shallow ocular. An ocular which magnifies the real image only moderately, i.e., 2 to 8 fold.-Micrometer or micrometric Ocular; Fr. Oculaire micrometrique ou à micromètre; Ger. Mikrometer-Okular, Mess Okular, Bénèches $O$. Jackson m. o., see 3,4r.-Microscopic Ocular; Fr. Oculaire microscopique ; Ger. mikroskopisches Okular. An ocular for the microscope instead of one for a telescope. -Negative Ocular, see $36 .-$ Nelson's screw-micrometer ocular. A modification of the Ramsden's screw or cob-web micrometer in which positive compensating oculars may be used.-Orthoscopic Oculars; also called Kellner's Ocular; Fr. Oculaire orthoscopique; Ger. Kellner'sches oder orthoskopisches Okular. An ocular with an eye-lens like one of the combinations of an objective (Figs. 22, 23) and a double convex field lens. The field-lens is in the focus of the eye-lens and there is no diaphragm present. The field is large and flat.-Par-focal Oculars, a series of oculars so arranged that the microscope remains in focus when the oculars are interchanged (Pennock, Micr. Bulletin, vol. iii, p. 9, 3I).-Periscopic Ocular; Fr. Ocuiaire periscopique; Ger. periskopisches Okular. A positive ocular devised by Gundlach. It consists of a double convex field-lens and a triplet eye-lens. It gives a large, flat field.-Positive Ocular, see \& 37.-Projection Ocular; Fr. Oculaire de projection; Ger. Projections-Okular, see \& 4o.-Ramsden's Ocular; Fr. Oculaire de Ramsden; Ger. Ramsden'sches Okular. A positive ocular devised by Ramsden. It consists of two plano-convex lenses placed close together with the convex surfaces facing each other. Only the central part of the field is clear. Searching Ocular; Fr. Oculaire d'orientation; Ger. Sucher-Okular, see $\S 39$, Shallow Ocular, see low ocular. - Solid Ocular, holosteric O.; Fr. Oculaire holostère; Ger. holosterisches Okular, Vollglass-Okular. A negative eye-piece devised by Tolles. It consists of a solid piece of glass with a moderate curvature at one end for a field-lens, and the other end with a much greater curvature for an eye-lens. For a diaphragm, a groove is cut at the proper level and filled with black pigment. It is especially excellent where a high ocular is desired. - Spectral 
or spectroscopic Ocular; Fr. Oculaire spectroscopique; Ger, Spectral-Okular, see Microspectroscope, Ch. VI.-Stauroscopic Ocular; Fr. Oculaire Stauroscopique. Ger. Stauroskop-Okular. An ocular with a Bertrand's quartz plate for mineralogical purposes.-Working Ocular: Fr. Oculaire de travail; Ger. Arbeits-Okular, see $\& 39$.

8. 38. Huygenian Ocular - A negative ocular designed by Huygens for the telescope, but adapted also to the microscope. It is the one now most commonly employed. It consists of a field-lens or collective (Fig. 30), aiding the objective in forming the real image, and an eye-lens which magnifies the real image. While

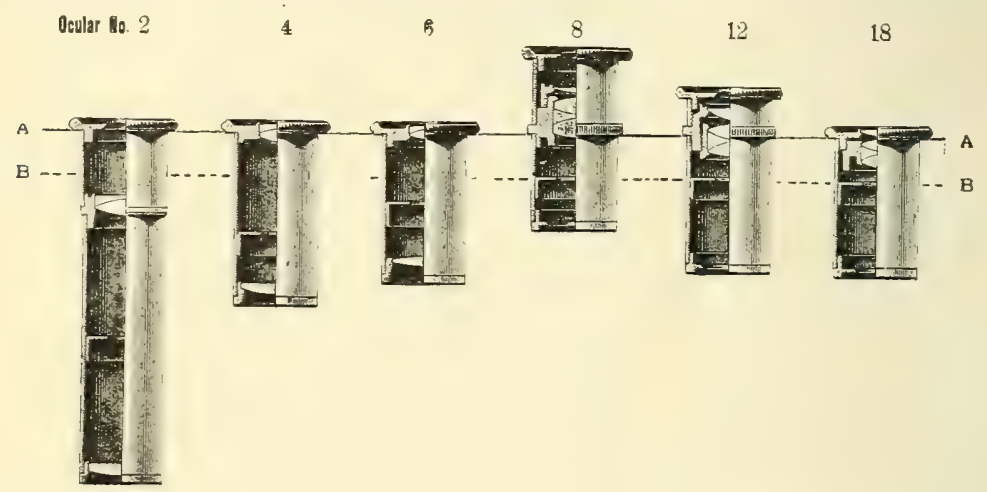

FIG. 3I. Compensating Oculars of Zeiss, with section removed to show the construction. The line $A-A$ is at the level of the upper end of the tube of the microscope while $B-B$ represents the lower focal points. It will be seen that the mounting is so arranged that the lower focal points in all are in the same plane and therefore the microscope remains in focus upon changing oculars. (The oculars are par-focal). The lower oculars, 2, 4 and 6 are negatize, and the higher ones, 8,12 , 18 , are positive. The numbers 2, 4, 6, 8, I2, 18 , indicate the magnification of the ocular. From Zeiss' Catalog.)

the field-lens aids the objective in the formation of the real, inverted image, and increases the field of view, it also combines with the eye-lens in rendering the image achromatic. (See \& 46).

3. 39. Compensating Oculars. - These are oculars specially constructed for use with the apochromatic objectives. They compensate for aberrations outside the axis which could not be so readily eliminated in the objective itself. An ocular of this kind, magnifying but twice, is made for use with high powers, for the sake of the large field in finding objects; it is called a searching ocular; those ordinarily used for observation are in contradistinction called working oculars. Part of the compensating oculars are positive and part negative. (Fig. 3I.)

\% 40. Projection Oculars.-These are oculars especially designed for projecting a microscopic image on the screen for class demonstrations, or for photographing with the microscope. While they are specially adapted for use with apochromatic objectives, they may also be used, with ordinary achromatic objectives of large numerical aperture. 
FIG. 32. Projection Oculars with section removed to show the construction. Below are shown the upper ends with graduated circle to indicate the amount of rotation found necessary to focus the diaphragm on the screen. No. 2, No.4. The numbers indicate the amount the ocular magnifies the image formed by the objective as with the compensation oculars. (Zeiss' Catalog.)

3. 4I. Micrometer Ocular.-This is an ocular connected with an ocular micrometer. The micrometer may be removable, or it may be permanently in connection with the ocular, and arranged with a spring and screw, by which it may be moved back and forth across the

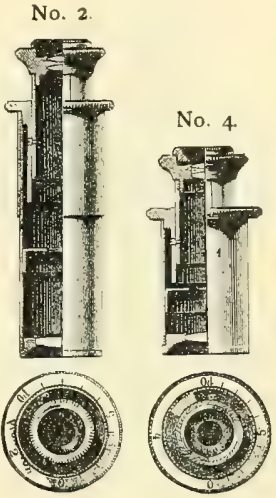
freld. (See Ch. IV.)

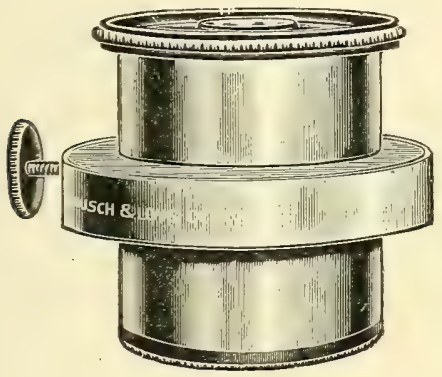

FIG. 33

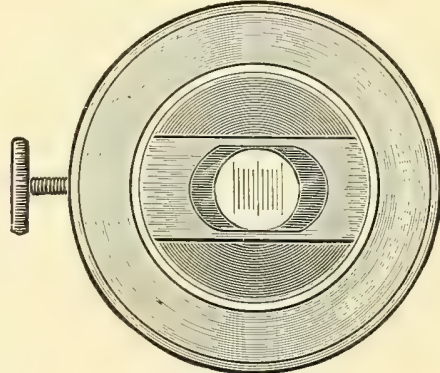

FIG. 34

FIGS. 33-34. Ocular Micrometer and movable scale. Fig. 33 is a side view of the ocular while Fig. 34 gives a sectional end view, and shows the ocular micrometer in position. In both the screw which moves the micrometer is shown at the left. (From Bausch oै Lomb Opt. Co.)

\% 42. Spectral or Spectroscopic Ocular.-(See Micro-Spectroscope, Ch. VI).

\section{DESIGNATION OF OCULARS}

\% 43. Equivalent Focus.-As with objectives, some opticians designate the oculars by their equivalent focus ( $\left.\xi_{1} 1_{5}\right)$. With this method the power of the ocular, as with objectives, varies inversely as the equivalent focal length, and therefore the greater the equivalent focal length the less the magnification. This seems as desirable a mode for oculars as for objectives and is coming more and more into use by the most progressive opticians. It is the method of designation advocated by Dr. R. H. Ward for many years, and was recommended by the committee of the American Microscopical Society, (Proc. Amer. Micr. Soc., I883, p. I75, I884, p. 228). 


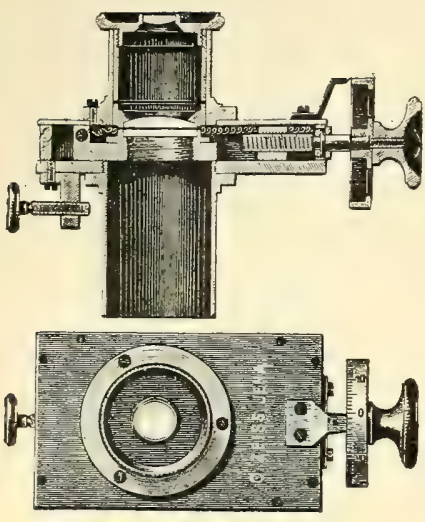

Fig. 35. Ocular Screw-Micrometer with compensation ocular 6. The upper figure shows a sectional view of the ocular and the screw for moving the micrometer at the right. At the left is shown a clamping screw to fasten the ocular to the upper part of the microscope tube. Below is a face view, showing the graduation on the wheel. An ocular micrometer like this is in general like the cob-web micrometer and may be used for measuring objects of varying sizes very accurately. With the ordinaryocular micrometer very small objects frequently fill but a part of an interval of the micrometer, but with this the movable cross lines traverse the object (or rather its real image) regardless of the minuteness of the object. (Zeiss' Catalog).

\&. 44. Numbering and Lettering.-Oculars like objectives may be numbered or lettered arbitrarily. When so designated, the smaller the number, or the earlier the letter in the alphabet, the lower the power of the ocular.

\& 45. Magnification.-The compensating oculars are marked with the amount they magnify the real image. Thus an ocular marked $\times 4$, indicates that the real image of the objective is magnified four fold by the ocular.

The projection oculars are designated simply by the amount they multiply the real image of the objective. Thus for the short or $160 \mathrm{~mm}$. tube-length they are, $\times 2, \times 4$; and for the long or $250 \mathrm{~mm}$. tube, they are $\times_{3}$ and $\times 6$. That is, the final image on the screen or the ground glass of the photographic camera will be $2,3,4$, or 6 times greater than it would be if no ocular were used. See Ch. VIII.

3. 46. Standard Size Oculars.-The Royal Microscopical Society of London took a very important step (Dec. 20, IS99) in establishing standard sizes for oculars and sub-stage condensers. To quote from the Journal of the Royal Microscopical Society for I900, p. I47 :

Resoli'ed, "That the standard size for the inside diameter of the substage fitting be $1.527 \mathrm{in} .=38.796 \mathrm{~mm}$. That the gauges for standardizing eye-pieces be the internal diameters of the draw-tubes, the tightness of the fit being left to the discretion of the manufacturers."

The sizes for oculars are four in number, I and 2 being most common.

(I) $0.9173 \mathrm{inch}=23.300 \mathrm{~mm}$. This is the Continental size.

(2) 1.04 inch $=26.416 \mathrm{~mm}$. This is the size used by the English Opticians for student and small microscopes.

(3) 1.27 inch $=32.258 \mathrm{~mm}$. Medium size binoculars (English.)

(4) $\mathrm{r} .4 \mathrm{I}$ inch $=35.8 \mathrm{I} 4 \mathrm{~mm}$. Long tube binoculars.

For the history of the Huygenian Ocular, and a discussion of formulae for its construction, see Nelson, J. R. M. S., I900, p. I62-I69.

\section{EXPERIMENTS}

$\$ 47$. Putting an Objective in Position and Removing it.Elevate the tube of the microscope by means of the coarse adjustment 
(frontispiece) so that there may be plenty of room between its lower end and the stage. Grasp the objective lightly near its lower end with two fingers of the left hand, and hold it against the nut at the lower end of the tube. With two fingers of the right hand take hold of the milled ring near the back or upper end of the objective and screw it into the tube of the microscope. Reverse this operation for removing the objective. By following this method the danger of dropping the objective will be avoided.

$\$ 48$. Putting an Ocular in Position and Removing it.-Elevate the body of the microscope with the coarse adjustment so that the objective will be $2 \mathrm{~cm}$. or more from the object-grasp the ocular by the milled ring next the eye-lens (Fig. 2I ), and the coarse adjustment or the tube of the microscope and gently force the ocular into position. In remoring the ocular, reverse the operation. If the above precautions are not taken, and the oculars fit snugly, there is danger in inserting them of forcing the tube of the microscope downward and the objective upon the object.

$\$$ 49. Putting an Object under the Microscope.-This is so placing an object under the simple microscope, or on the stage of the compound microscope, that it will be in the field of view when the microscope is in focus $(\$ 50)$.

With low powers, it is not difficult to get an object under the microscope. The difficulty increases, however, with the power of the microscope and the smallness of the object. It is usually necessary to move the object in various directions while looking into the microscope, in order to get it into the field. Time is usually saved by getting the object in the center of the field with a low objective before putting the high objective in position. This is greatly facilitated by using a nose-piece, or revolver. (See Figs. 36-36a, and the pictures of microscopes, Ch. II.)
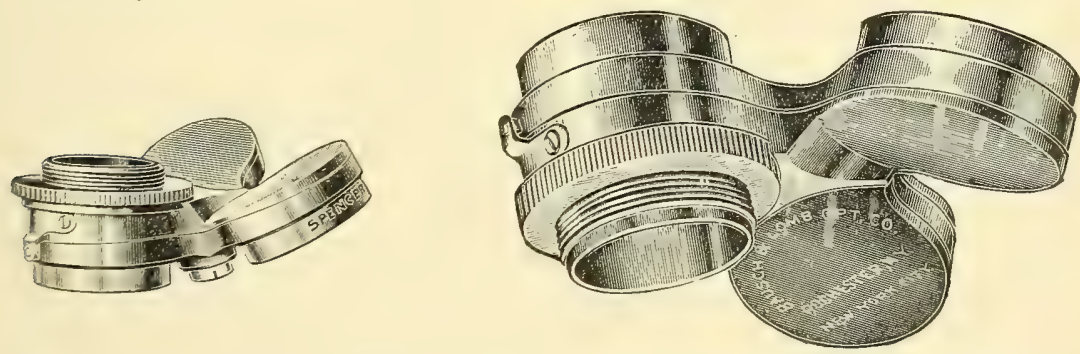

FIG. 36. Triple nose-piece or revolier for quickly changing objectives. (The Spencer Lens Co.)

FIG. 36a, Triple nose-piece or revolver for quickly changing objectives. (The Bausch \& Lomb Optical Co.) 
$\$ 50$. Field or Field of View of a Microscope.-This is the area visible through a microscope when it is in focus. When properly lighted and there is no object under the microscope, the field appears as a circle of light. When examining an object it appears within the light circle, and by moving the object, if it is of sufficient size, different parts are brought successively into the field of view.

In general, the greater the magnification of the entire microscope, whether the magnification is produced mainly by the objective, the ocular, or by increasing the tube length, or by a combination of all three (see Ch. IV, under magnification), the smaller is the field.

The size of the field is also dependent, in part, without regard to magnification, upon the size of the opening in the ocular diaphragm. Some oculars, as the orthoscopic and periscopic, are so constructed as to eliminate the ocular diaphragm, and in consequence, although this is not the sole cause, the field is considerably increased. The exact size of the field may be read off directly by putting a stage micrometer under the microscope and noting the number of spaces required to measure the diameter of the light circle.
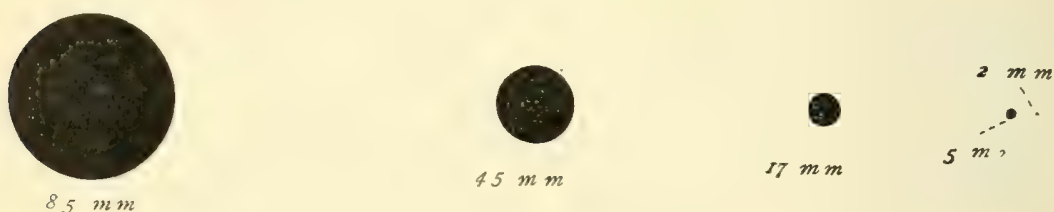

FIG. 37. Figures showing approximately the actual size of the field with objectives of $85 \mathrm{~mm}$., $45 \mathrm{~mm}$., $17 \mathrm{~mm} ., 5 \mathrm{~mm}$, and $2 \mathrm{~mm}$., equivalent focus, and ocular of $37 \mathrm{1} / 2 \mathrm{~mm}$., equivalent focus in each case. This figure shows graphically what is also very clearly indicated in the table ( 852$)$.

5 I. The size of the field of the microscope as projected into the field of vision of the normal human eye (i. e., the virtual image) may be determined by the use of the camera lucida with the drawing surface placed at the standard distance of 250 millimeters (Ch. IV.)

$\$ 52$. Table showing the actual size in millimeters of the field of a group of commonly' used objectives and oculars. Compare with the graphic representation in Fig. 37. See also \$ 50. 


\begin{tabular}{|c|c|c|c|}
\hline $\begin{array}{l}\text { Equivalent } \\
\text { Focus and } \\
\text { N. A. of } \\
\text { Objective }\end{array}$ & $\begin{array}{l}\text { Diameter } \\
\text { of Field } \\
\text { in mum. }\end{array}$ & $\begin{array}{l}\text { Equivalent } \\
\text { Focus of } \\
\text { Ocular }\end{array}$ & $\begin{array}{l}\text { Kind of } \\
\text { Ocular }\end{array}$ \\
\hline $\mathrm{S}_{5} \mathrm{~mm} . . .$. & $\begin{array}{r}15.4 \\
10.6 \\
8.3\end{array}$ & $\begin{array}{l}37^{1} / 2 \mathrm{~mm} . \\
25 \\
121 / 2\end{array}$ & Huygenian \\
\hline $45 \mathrm{~mm}$ & $\begin{array}{l}7.0 \\
5.0 \\
4.0\end{array}$ & $\begin{array}{lll}37^{1} / 2 & \mathrm{~mm} . \\
25 & . \\
12^{1 / 2} & \cdots\end{array}$ & Huygenian \\
\hline I7 mm..... & $\begin{array}{l}3.0 \\
2.0 \\
1.6\end{array}$ & $\begin{array}{l}37 \mathrm{r} / 2 \mathrm{~mm} . \\
25 \\
\mathrm{I} 21 / 2\end{array}$ & Huygenian \\
\hline N. A. $=0.25$ & $\begin{array}{l}5.7 \\
2.8 \\
1.4 \\
0.97\end{array}$ & $\begin{array}{rc}\text { I8O } & \text { mm. } \\
45 & ، \\
\text { I5 } & ، \\
\text { IO } & \text { “' }\end{array}$ & Compensation \\
\hline $5 \mathrm{~mm} . \ldots$ & $\begin{array}{l}0.541 \\
0.371 \\
0.290\end{array}$ & $\begin{array}{l}37 \mathrm{r} / 2 \mathrm{~mm} . \\
25 \\
\mathrm{I} 2 \mathrm{x} / 2\end{array}$ & Huygenian \\
\hline N. A. $=0.92$ & $\begin{array}{l}0.850 \\
0.501 \\
0.250 \\
0.173\end{array}$ & $\begin{array}{rc}\text { I } 80 & \text { mm. } \\
45 & \text { " } \\
\text { I5 } & \text { " } \\
\text { I0 } & \text { " }\end{array}$ & Compensation \\
\hline $2 \mathrm{~mm}$. & $\begin{array}{l}0.270 \\
0.186 \\
0.147\end{array}$ & $\begin{array}{l}37 \mathrm{r} / 2 \mathrm{~mm} . \\
25 \mathrm{r} \\
\mathrm{I} 2 \mathrm{r} / 2\end{array}$ & Huygenian \\
\hline N. A. $=I .25$ & $\begin{array}{l}0.450 \\
0.25 \mathrm{I} \\
0.125 \\
0.088\end{array}$ & $\begin{array}{rc}\mathrm{r} 8 \mathrm{o} & \mathrm{mm} . \\
45 & " 6 \\
15 & 6 \\
10 & " 6\end{array}$ & Compensation \\
\hline
\end{tabular}

FUNCTION OF AN OBJECTIVE

55. Put a $50 \mathrm{~mm}$. objective on the microscope or screw off the front combination of a $16 \mathrm{~mm} .,(2 / 3-i n$.$) , and put the back combination$ on the microscope for a low objective.

Place some printed letters or figures under the microscope, and light well. In place of an ocular put a screen of ground glass, or a piece of lens paper, over the upper end of the tube of the microscope.*

*Ground glass may be very easily prepared by placing some fine emery or carborundum between two pieces of glass, wetting it with water and then rubbing the glasses together for a few minutes. If the glass becomes too opaque, it may be rendered more translucent by rubbing some oil upon it. 
Lower the tube of the microscope by means of the coarse adjustment until the objective is within $2-3 \mathrm{~cm}$. of the object on the stage. Look at the screen on the top of the tube, holding the head about as far from it as for ordinary reading, and slowly elevate the tube by means of the coarse adjustment until the image of the letter appears on the screen.

The image can be more clearly seen if the object is in a strong light and the screen in a moderate light, $i . e_{\text {. }}$, if the top of the microscope is shaded.

The letters will appear as if printed on the ground glass or paper, but will be inverted (Fig. 2I).

If the objective is not raised sufficiently, and the head is held too near the microscope, the objective will act as a simple microscope. If the letters are erect, and appear to be down in the microscope and not on the screen, hold the head farther from it, shade the screen, and raise the tube of the microscope until the letters do appear on the ground glass.

To demonstrate that the object must be outside the principal focus with the compound microscope, remove the screen and turn the tube of the microscope directly toward the sun. More the tube of the microscope with the coarse adjustment until the burning or focal point is found $(\$ 6)$. Measure the distance from the paper object on the stage to the objective, and it will represent approximately the principal focal distance (Figs. Io, II). Replace the screen over the top of the tube, no image can be seen. Slowly raise the tube of the microscope and the image will finally appear. If the distance between the object and the objective is now taken, it will be found considerably greater than the principal focal distance (compare $\S$ II).

$\$ 54$. Aerial Image.-After seeing the real image on the groundglass, or paper, use the lens paper over about half of the opening of the tube of the microscope. Hold the eye about $250 \mathrm{~mm}$. from the microscope as before and shade the top of the tube by holding the hand between it and the light, or in some other way. The real image can be seen in part as if on the paper and in part in the air. More the paper so that the image of half a letter will be on the paper and half in the air. Another striking experiment is to have a small hole in the paper placed orer the center of the tube opening, then if a printed word extends entirely across the diameter of the tube its central part may be seen in the air, the lateral parts on the paper. The adrantage of the paper over part of the opening is to enable one to accommodate the 
eyes for the right distance. If the paper is absent the eyes adjust themselves for the light circle at the back of the objective, and the aerial image appears low in the tube. Furthermore it is more difficult to see the aerial image in space than to see the image on the groundglass or paper, for the eye must be held in the right position to receive the rays projected from the real image, while the granular surface of the glass and the delicate fibres of the paper reflect the rays irregularly, so that the image may be seen at almost any angle, as if the letters were actually printed on the paper or glass.

$\$ 55$. The Function of an Objective, as seen from these experiments, is to form an enlarged, inverted, real image of an object, this image being formed on the opposite side of the objective from the object (Fig. 2I).

FUNCTION OF AN OCULAR

56 . Using the same objective as for $\$ 53$, get as clear an image of the letters as possible on the lens paper screen. Look at the image with a simple microscope (Fig. I 7 or 18 ) as if the image were an object.

Observe that the image seen through the simple microscope is merely an enlargement of the one on the screen, and that the letters remain inverted, that is they appear as with the naked eye ( $\$$ I I). Remove the screen and observe the aerial image with the tripod.

Put a $50 \mathrm{~mm}$. (A, No. I or 2 in.), ocular $i$. e., an ocular of low magnification) in position ( $\$ 48$ ). Hold the eye about to to 20 millimeters from the eye-lens and look into the microscope. The letters will appear as when the simple microscope was used (see above), the image will become more distinct by slightly raising the tube of the microscope with the coarse adjustment.

57 . The Function of the Ocular, as seen from the above, is that of a simple microscope, viz.: It magnifies the real image formed by the objective as if that image were an object. Compare the image formed by the ocular (Fig. 2r), and that formed by a simple microscope (Fig. 38).

It should be borne in mind, however, that the rays from an object as usually examined with a simple microscope, extend from the object in all directions, and no matter at what angle the simple microscope is held, provided it is sufficiently near and points toward the object, an image may be seen. The rays from a real image, however, are continued in certain definite lines and not in all directions; hence, in order to see this aerial image with an ocuiar or simple microscope, or 
in order to see the aerial image with the unaided eye, the simple microscope, ocular or eye must be in the path of the rays (Fig. 2I.)

FIG. 38. Diagram of the simple microscope showing the course of the rays and all the images, and that the eye forms an integral part of it.

$A^{\mathrm{I}} B^{\mathrm{r}}$. The object within the principal focus. $A^{3} B^{3}$. The virtual image on the same side of the lens as the object. It is indicated by dotted lines, as it has no actual existence.

$B^{2} A^{2}$. Retinal image of the object $\left(A^{\mathrm{x}} B^{\mathrm{r}}\right)$. The virtual image is simply a projection of the retinal image into the field of vision.

Axis. The principal optic axis of the microscope and of the eye. Cr. Cornea of the eye. $L$. Crystalline lens of the eye. $R$. Ideal refracting surface at athich all the refractions of the eye may be assumed to take place.

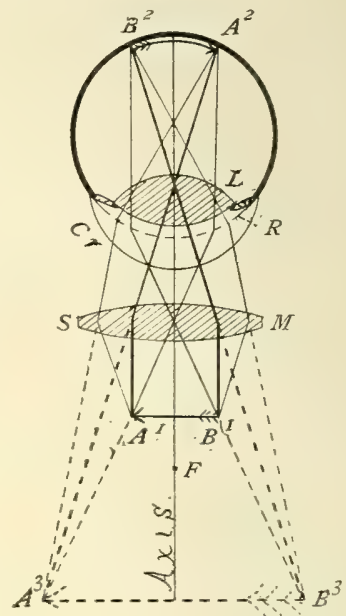

58 . The field-lens of a Huygenian ocular makes the real image smaller and consequently increases the size of the field; it also makes the image brighter by contracting the area of the real image. (Fig. 30.) Demonstrate this by screwing off the field-lens and using the eye-lens alone as an ocular, refocusing if necessary. Note also that the image is bordered by a colored haze $(\$ 7)$.

When looking into the ocular with the field-lens removed, the eye should not be held so close to the ocular, as the eye-point is considerably farther away than when the field-lens is in place.

59. The eye-point.-This is the point above the ocular or simple microscope where the greatest number of emerging rays cross. Seen in profile, it may be likened to the narrowest part of an hour glass. Seen in section (Fig. 30), it is the smallest and brightest light circle above the ocular. This is called the eye-point, for if the pupil of the eye is placed at this level, it will receive the greatest number of rays from the microscope, and consequently see the largest field.

Demonstrate the eye-point by having in position an objective and ocular as above 53 ). Light the object brightly, focus the microscope, shade the ocular, then hold some ground-glass or a piece of the lens paper abore the ocular and slowly raise and lower it until the smallest circle of light is found. By using different oculars it will be 
seen that the eye-point is nearer the eye-lens in high than in low oculars, that is the eye-point is nearer the eye-lens for an ocular of small equivalent focus than for one of greater focal length.

\section{REFERENCES FOR CHAPTER I}

In chapter $\mathrm{X}$ will be given a bibliography, with full titles, of the works and periodicals referred to.

For the subjects considered in this chapter, general works on the microscope may be consulted with great advantage for different or more exhaustive treatment. The most satisfactory work in English is Carpenter-Dallinger, 8th Ed. For the history of the microscope, Mayall's Cantor Lectures on the microscope are very satisfactory. For a continuation of the history begun by Mayall in the Cantor Lectures see Nelson, Journal of the Queckett Micr. Club, and the Jour. Roy. Hicr. Soc., IS97-IgoI-- Carpenter-Dallinger, 8th Ed. Petri, Das Mikroskop.

The following special articles in periodicals may be examined with advantage: Apochromatic Objectives, etc. Dippel in Zeit. wiss. Mikr., I886, p. 303 ; also in the Jour. Roy. Micr. Soc., I886, pp. 316, 849, II Io, ; same, I89o, p. 480 ; Zeit. f. Instrumentenk., I89o, pp. I-6 ; Micr. Bullt., I89i, pp. 6-7.

Tube-length, etc. Gage, Proc. Amer. Soc. Micrs., I887, pp. I68-I72 ; also in the Microscope, the Jour. Roy. Micr. Soc., and in Zeit, wiss. Mikr., r887-8. Bausch, Proc. Amer. Soc. Micrs., ISgo, pp. 43-49; also in the Microscope, I8go, pp. 289-296.

Aperture. J. D. Cox, Presidential Address, Proc. Amer. Soc. Micrs., r884, pp, 5-39, Jour. Roy. Micr. Soc., I88I, pp. 303, 348, 365, 388 ; I882, pp. 300, 460 ; 1883, p. $790 ; 1884$, p. 20. Czapski, Theorie der optischen Instrumente nach Abbe. See also references in 3.34 .

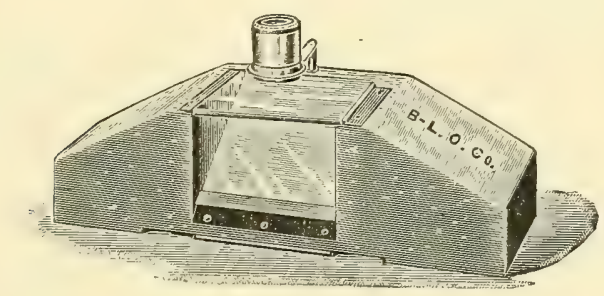

The Barnes Dissecting Microscope (The Bausch \& Lomb Optical Company). 


\section{CHAPTER II}

\section{LIGHTING AND FOCUSING; MANIPULATION OF DRY, ADJUSTABLE AND IMMERSION OBJECTIVES ; CARE OF THE MICROSCOPE AND OF THE EYES ; LABORATORY MICROSCOPES}

\section{APPARATUS AND MATERIAL, FOR THIS CHAPTER}

Microscope supplied with plane and concave mirror, Achromatic and Abbe condensers, dry, adjustable and immersion objectives, oculars, triple nose-piece. Microscope lamp and movable condenser (bull's eye or other form, Fig. 53), Homogeneous immersion liquid; Benzin, alcohol, distilled water ; Mounted preparation of fly's wing ( 870$)$; Mounted preparation of Pleurosigma $(\xi 77,78)$. Stage or ocular micrometer ( 892 ); Glass slides and cover-glasses (Ch. VII); ro per ct. solution of salicylic acid in 95 per ct. alcohol ( 892$)$; Preparation of stained bacteria ( $\&$ IOS); Vial of equal parts olive or cotton seed oil or liquid vaselin and benzin ( $\&$ I I2): Double eye shade (Fig. 60); Screen for whole microscope (Fig. 59).

\section{FOCUSING}

\& 6o. Focusing is mutually arranging an object and the microscope so that a clear image may be seen.

With a simple microscope ( $?$ II ) either the object or the microscope or both may be moved in order to see the image clearly, but with the compound microscope the object more conveniently remains stationary on the stage, and the tube or body of the microscope is raised or lowered (frontispiece).

In general, the higher the power of the whole microscope whether simple or compound, the nearer together must the object and objective be brought. With the compound microscope, the higher the objective, and the longer the tube of the microscope, the nearer together must the object and the objective be brought. If the oculars are not par-focal, the higher the magnification of the ocular, the nearer must the object and objective be brought.

\& 6I. Working Distance.-By this is meant the space between the simple microscope and the object, or between the front lens of the compound microscope and the object, when the microscope is in focus. This working distance is always considerably less than the equivalent focal length of the objective. For example, the front-lens of a $6 \mathrm{~mm}$. or $1 / 4$ th in, objective would not be 6 millimeters or $1 / 4$ th inch from the object when the microscope is in focus, but considerably less than that distance. If there $\pi$ ere no other reason than the limited working distance of high objectives, it would be necessary to use a very thin cover-glass over the 
object. (See 824,29 .) If too thick covers are used it may be impossible to get an objective near enough an object to get it in focus. For objects that admit of examination with high powers it is always better to use thin covers.

\section{LIGHTING WITH DAYLIGHT}

\%62. Unmodified sunlight should not be employed except in special cases. North light is best and most uniform. When the sky is covered with white clouds the light is most favorable. To avoid the shadows produced by the hands in manipulating the mirror, etc., it is better to face the light; but to protect the eres and to shade the stage of the microscope some kind of screen should be used. The one figured in (Fig. 62) is cheap and efficient. If one dislikes to face the window or lamp it is better to sit so that the light will come from the left as in reading.

It is of the greatest importance and advantage for one who is to use the microscope for serious work that he should comprehend and appreciate thoroughly the various methods of illumination, and the special appearances due to different kinds of illumination.

Depending on whether the light illuminating an object traverses the object or is reflected upon it, and also whether the object is symmetrically lighted, or lighted more on one side than the other, light used in microscopy is designated as reflected and transmitted, axial and oblique.

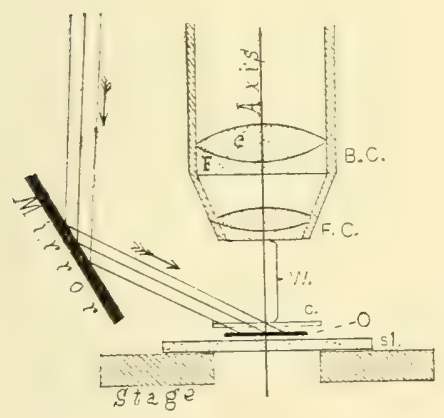

39

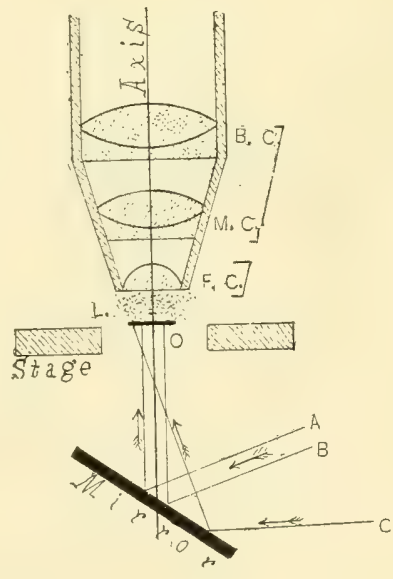

40

FIGS. 39-40. For full explanation see Figs. 22 and 23.

\&63. Reflected, Incident or Direct Light.-By this is meant light reflected upon the object in some way and then irregularly reflected from the object to the microscope. By this kind of light objects are ordinarily seen by the unaided eye, and the objects are mostly opaque. In Vertebrate Histology, reflected light is but 
little used; but in the study of opaque objects, like whole insects, etc., it is used a great deal. For low powers, ordinary daylight that naturally falls upon the object, or is reflected or condensed upon it with a mirror or condensing lens, answers very well. For high powers and for special purposes, special illuminating apparatus has been devised $(\& 28)$. (See also Carpenter-Dallinger, Ch. IV).

8.64. Transmitted Light.-By this is meant light which passes through an object from the opposite side. The details of a photographic negative are in many cases only seen or best seen by transmitted light, while the print made from it is best seen by reflected light.

Almost all objects studied in Vertebrate Histology are lighted by transmitted light, and they are in some way rendered transparent or semi-transparent. The light traversing and serving to illuminate the object in working with a compound microscope is usually reflected from a plane or concave mirror, or from a mirror to a condenser ( $\xi 88$ ), and thence transmitted to the object from below (Figs. 48-5I).

8 65. Axial or Central Light.-By this is understood light reaching the object, the rays of light being parallel to each other and to the optic axis of the microscope, or a diverging or converging cone of light whose axial ray is coincident with the optic axis of the microscope. In either case the object is symmetrically illuminated.

3. 66. Oblique Light.-This is light in which parallel rays from a plane mirror form an angle with the optic axis of the microscope (Fig. 40). Or if a concave mirror or a condenser is used, the light is oblique when the axial ray of the cone of light forms an angle with the optic axis (Fig. 40).

\section{DIAPHRAGMS}

2.67. Diaphragms and their Proper Employment.-Diaphragms are opaque disks with openings of various sizes, which are placed between the source of light or mirror and the object. In some cases an iris diaphragm is used, and then the same one is capable of giving a large range of openings. The object of a diaphragm in general, is to cut off all adventitious light and thus enable one to light the ohject in such a way that the light finally reaching the microscope shall all come from the object or its immediate vicinity. The diaphragms of a condenser serve to vary its aperture to the needs of each object and each objective.

3.68. Size and Position of Diaphragm Opening.-When no condenser is used the size of the opening in the diaphragm should be about that of the front lens of the objective. For some objects and some objectives this rule may be quite widely departed from; one must learn by trial.

When lighting with a mirror the diaphragm should be as close as possible to the object in order, (a) that it may exclude all adventitious light from the object ; (b) that it may not interfere with the most efficient illumination from the mirror by cutting off a part of the illuminating pencil. If the diaphragm is a considerable distance below the object, ( I ) it allows considerable adventitious light to reach the object and thus injures the distinctness of the microscope image; (2) it prevents the use of very oblique light unless it swings with the mirror; (3) it cuts off a part of the illuminating cone from a concave mirror. On the other hand, even with a small diaphragm, the whole field will be lighted. 
With an illuminator or condenser (Figs. 4I, 48), the diaphragm serves to narrow the pencil to be transmitted through the condenser, and thus to limit the aperture (see 8 84). Furthermore, by making the diaphragm opening eccentric, oblique light may be used, or by using a diaphragm with a slit around the edge (central stop diaphragm), the center remaining opaque, the object may be lighted with a hollow cone of light, all of the rays having great obliquity. In this way the socalled dark-ground illumination may be produced ( $\& 92$; Fig. 5I).

\section{ARTIFICIAL, ILLUMINATION}

8. 69. For evening work and for certain special purposes, artificial illumination is employed. A good petroleum (kerosene) lamp with flat wick has been found very satisfactory, also an incandescent electric or Welsbach light, but for brilliancy and for the actinic power necessary for very rapid photo-micrography (see Ch. VIII) the electric arc lamp or an acetylene lamp serves well. Whatever source of artificial light is employed, the light should be brilliant and steady.

\section{LIGHTING AND FOCUSING: EXPERIMENTS}

\$ 70. Lighting with a Mirror.-As the following experiments are for mirror lighting only, remove the substage condenser if present (see $\$ 79$, for condenser). Place a mounted fly's wing under the microscope, put the $16 \mathrm{~mm} .(2 / 3 \mathrm{in}$.) or other low objective in position, also a low ocular. With the coarse adjustment lower the tube of the microscope to within about I cm. of the object. Use an opening in the diaphragm about as large as the front lens of the objective; then with the plane mirror try to reflect light up through the diaphragm upon the object. One can tell when the field $(\S 50)$ is illuminated, by looking at the object on the stage, but more satisfactorily by looking into the microscope. It sometimes requires considerable manipulation to light the field well. After using the plane side of the mirror turn the concave side into position and light the field with it. As the concave mirror condenses the light, the field will look brighter with it than with the plane mirror. It is especially desirable to remember that the excellence of lighting depends in part on the position of the diaphragm (\$68). If the greatest illumination is to be obtained from the concave mirror, its position must be such that its focus will be at the level of the object. This distance can be very easily determined by finding the focal point of the mirror in full sunlight.

$\$ 7$ I. Use of the Plane and of the Concave Mirror.-The mirror should be freely movable, and have a plane and a concave face. The concave face is used when a large amount of light is needed, the plane face when a moderate amount is needed or when it is necessary to have parallel rays or to know the direction of the rays. 
$\$ 72$. Focusing with Low Objectives.-Place a mounted fly's wing under the microscope; put the $16 \mathrm{~mm} .(2 / 3$ in.) objective in position, and also the lowest ocular. Select the proper opening in the diaphragm and light the object well with transmitted light $(\$ 64,68)$.

Hold the head at about the level of the stage, look toward the window, and between the object and the front of the objective; with the coarse adjustment lower the tube until the objective is within about half a centimeter of the object. Then look into the microscope and slowly elevate the tube with the coarse adjustment. The image will appear dimly at first, but will become very distinct by raising the tube still higher. If the tube is raised too high the image will become indistinct, and finally disappear. It will again appear if the tube is lowered the proper distance.

When the microscope is well focused try both the concave and the plane mirrors in various positions and note the effect. Put a high ocular in place of the low one $(\$ 43)$. If the oculars are not parfocal it will be necessary to lower the tube somewhat to get the microscope in focus. *

Pull out the draw-tube $4-6 \mathrm{~cm}$., thus lengthening the body of the microscope ; it will be found necessary to lower the tube of the microscope somewhat. (For reason, see Fig. 58.)

\$ 73. Pushing in the Draw-Tube.-To push in the draw-tube, grasp the large milled ring of the ocular with one hand, and the milled head of the coarse adjustment with the other, and gradually push the draw-tube into the tube. If this were done without these precautions the objective might be forced against the object and the ocular thrown out by the compressed air.

74 . Focusing with High Objectives.-Employ the same object as before, elevate the tube of the microscope and, if no revolving nose-piece is present, remove the $16 \mathrm{~mm}$. ( $23 \mathrm{in}$.) objective as indicated. Put the $3 \mathrm{~mm}$. ( $1 / 8$ in.) or a higher objective in place, and use a low ocular.

Par-focal oculars are so constructed, or so mounted, that those of different powers may be interchanged without the microscopic image becoming wholly out of focus (Fig. 3I). When high objectives are used, while the image may be seen after changing oculars, the instrument nearly always needs slight focusing. With low powers this may not be necessary.

Objectives are also now commonly mounted in the triple or double revolving nose-pieces (Figs. 36, 36 a) so that if one of the objectives is in focus either of the others will be approximately in focus when turned into position. This is a very great convenience. 
Light well, and employ the proper opening in the diaphragm, etc. (\$68). Look between the front of the objective and the object as before $(\$ 72)$, and lower the tube with the coarse adjustment till the objective almost touches the cover-glass over the object. Look into the microscope, and with the coarse adjustment, raise the tube very slowly until the image begins to appear, then turn the milled head of the fine adjustment (frontispiece), first one way and then the other, if necessary, until the image is sharply defined.

In practice it is found of great advantage to move the preparation slightly while focusing. This enables one to determine the approach to the focal point either from the shadow or the color, if the object is colored. With high powers and scattered objects there might be no object in the small field (see $\$ 5$, Fig. 37 for size of field). By moving the preparation an object will be moved across the field and its shadow gives one the hint that the objective is approaching the focal point. It is sometimes desirable to focus on the edge of the cement ring or on the little ring made by the marker (see Figs. 6I-66).

Note that this high objective must be brought nearer the object than the low one, and that by changing to a higher ocular (if the oculars are not par-focal) or lengthening the tube of the microscope it will be found necessary to bring the objective still nearer the object, as with the low objective. (For reason see Fig. 58.)

75. Always Focus Up, as directed above. If one lowers the tube only when looking at the end of the objective as directed above, there will be no danger of bringing the objective in contact with the object, as may be done if one looks into the microscope and focuses down.

When the instrument is well focused, move the object around in order to bring different parts into the field. It may be necessary to re-focus with the fine adjustment every time a different part is brought into the field. In practical work one hand is kept on the fine adjustment constantly, and the focus is continually varied.

76. Determination of Working Distance.-As stated in $\$ 6 \mathbf{I}$, this is the distance between the front lens of the objective and the object when the objective is in focus. It is always less than the equivlent focal length of the objective.

Make a wooden wedge Io $\mathrm{cm}$. long which shall be exceedingly thin at one end and about $20 \mathrm{~mm}$. thick at the other. Place a slide on the stage and some dust on the slide. Do not use a cover-glass. Focus the dust carefully first with the low then with the high objective. When 
the objective is in focus push the wedge under the objective on the slide until it touches the objective. Mark the place of contact with a pencil and then measure the thickness of the wedge with a rule opposite the point of contact. This thickness will represent very closely the working distance. For measuring the thickness of the wedge at the point of contact for the high objective use a steel scale ruled in $\frac{1}{5}$ ths $\mathrm{mm}$. and the tripod to see the divisions. Or one may use a cover-glass measure ( $\mathrm{Ch}$. VIII) for determining the thickness of the wedge.

For the higher powers if one has a microscope in which the fine adjustment is graduated, the working distance may be readily determined when the thickness of the cover-glass over the specimen is known, as follows: Get the object in focus, lower the tube of the microscope, until the front of the objective just touches the cover-glass. Note the position of the micrometer screw and slowly focus up with the fine adjustment until the object is in focus. The distance the objective was raised plus the thickness of the cover-glass represents the working distance. For example, a $3 \mathrm{~mm}$. objective after being brought in contact with the cover-glass was raised by the fine adjustment a distance represented by 16 of the dirisions on the head of the micrometer screw. Each division represented. or mm., consequently the objective was raised. $16 \mathrm{~mm}$. As the cover-glass on the specimen used was. I $5 \mathrm{~mm}$. the total working distance is . $16+.15=.31 \mathrm{~mm}$.

?. 76a. Free Working Distance.-In the microscope catalog of Zeiss there is given a table of the size of the field and also of the "free working-distance." This free working-distance is the space between the lower end of the objective and the cover glass of $r_{10}^{15} \mathrm{~mm}$. thickness, when the objective is in focus on an object immediately under the cover. This is exceedingly practical information for a possessor of a microscope, and it is hoped that the other opticians will adopt the suggestion. Naturally, however, the free working-distance for each optician should be reckoned from the top of the cover for which his unadjustable objectives are corrected. If, for example, the thickness of cover for which an objective is corrected is ${ }_{1}^{25} \mathrm{~mm}$. then the free working-distance should be that between the top of this and the objective when the objective is in focus on an object under the cover. (See the table of cover-glass thickness, p. 14).

\section{CENTRAL AND OBLIQUE LIGHT WITH A MIRROR}

$\S 77$. Axial or Central Light $(\$ 65)$.- - Remove the condenser or any diaphragm from the substage, then place a preparation containing minute air bubbles under the microscope. The preparation may be easily made by beating a drop of mucilage on a slide and covering 
it (see Ch. III). Use a $3 \mathrm{~mm} .,(\mathrm{I} / 8 \mathrm{in}$.) or No. 7 objective and a medium ocular. Focus the microscope and select a very small bubble, one whose image appears about $\mathrm{I} \mathrm{mm}$. in diameter, then arrange the plane mirror so that the light spot in the bubble appears exactly in the center. Without changing the position of the mirror in the least, replace the air bubble preparation by one of Pleurosigma angulatum or some other finely marked diatom. Study the appearance very carefully.

$\$ 78$. Oblique Light ( $\$ 66)$. - - Swing the mirror far to one side so that the rays reaching the object may be very oblique to the optic axis of the microscope. Study carefully the appearance of the diatom with the oblique light. Compare the appearance with that where central light is used. The effect of oblique light is not so striking with histological preparations as with diatoms.

It should be especially noted in $\$ \$ 77,78$, that one cannot determine the exact direction of the rays by the position of the mirror. This is especially true for axial light $(\$ 77)$. To be certain the light is axial some such test as that given in $\$ 77$ should be applied. (See also Ch. III, under Air-bubbles.)

\section{CONDENSERS OR ILLUMINATORS*}

$\$ 79$. These are lenses or lens-systems for the purpose of illuminating with transmitted light the object to be studied with the microscope.

For the highest kind of investigation their value cannot be overestimated. They may be used either with natural or artificial light, and should be of sufficient numerical aperture to satisfy objectives of the widest angle.

*No one has stated more clearly, or appreciated more truly the value of correct illumination and the methods of obtaining it than Sir David Brewster, I82O, I83I. He says of illumination in general: "The art of illuminating microscopic objects is not of less importance than that of preparing them for observation." "The eye should be protected from all extraneous light, and should not receive any of the light which proceeds from the illuminating center, excepting that portion of it which is transmitted through or reflected from the object." So likewise the value and character of the substage condenser was thoroughly understood and pointed out by him as follows: "I have no hesitation in saying that the apparatus for illumination requires to be as perfect as the apparatus for vision, and on this account I would recommend that the illuminating lens should be perfectly free of chromatic and spherical aberration, and the greatest care be taken to exclude all extraneous light both from the object and from the eye of the observer." See Sir David Brewster's treatise on the Microscope, I837, pp. 136, I38, I46, and the Edinburgh Journal of Science, new series, No. II (I83I) p. 83. 
It is of the greatest advantage to have the sub-stage condenser mounted so that it may be easily moved up or down under the stage. The iris diaphragm is so convenient that it should be furnished in all cases, and there should be marks indicating the $\mathrm{N}$. A. of the condenser utilized with different openings. Finally the condenser should be supplied with central stops for dark-ground illumination $(\$ 92)$ and with blue and neutral tint glasses to soften the glare when artificial light is used $(\$ 89,93)$.

Condensers or Illuminators fall into two great groups, the Achromatic, giving a large aplanatic cone, and Non-achromatic, giving much light, but a relatively small aplanatic cone of light.

\$ 8o. Achromatic Condenser.-It is still believed by all expert microscopists that the contention of Brewster was right, and the condenser to give the greatest aid in elucidating microscopic structure must approach in excellence the best objectives. That is, it should be as free as possible from spherical and chromatic aberration, and therefore would transmit to the object a very large aplanatic cone of light. Such condensers are especially recommended for photo-micrography by a1l, and those who believe in getting the best possible image in every case are equally strenuous that achromatic condensers should be used for all work. Unfortunately good condensers like good objectives are expensive, and student microscopes as well as many others are usually supplied with the non-achromatic condensers or with none.

Many excellent achromatic condensers have been made, but the most perfect of all seems to be the apochromatic of Powell and Lealand (Carpenter-Dallinger, p. 302). To attain the best that was possible many workers have adopted the plan of using objectives as condensers. A special substage fitting is provided with the proper screw and the objective is put into position, the front lens being next the object. As will be seen below $\left(\$ 8_{3}-84\right)$, the full aperture of an objective can rarely be used, and for histological preparations perhaps never, so that an objective of greater equivalent focus, $i . e$., lower power is used for the condenser than the one on the microscope. It is much more convenient, however, to have a special condenser with iris diaphragm or special diaphragms so that one may use any aperture at will, and thus satisfy the conditions necessary for lighting different objects for the same objective and for lighting with objectives of different apertures. An excellent condenser of this form has been produced by Zeiss (Fig. 4I). It has a total numerical aperture of I.00, and an aplanatic aperture of 0.65 . 
Fis. 4I. Zeiss' Achromatic Condenser. c.s. c. s. Centering screws for changing the position of the condenser and making its axis continuous with that of the microscope. A segment of the condenser is cut away to show the combinations of lenses. For very low powers the upper lens is sometimes screwed off. There is an iris diaphragm between the middle and lower combinations. (Zeiss' Catalog).

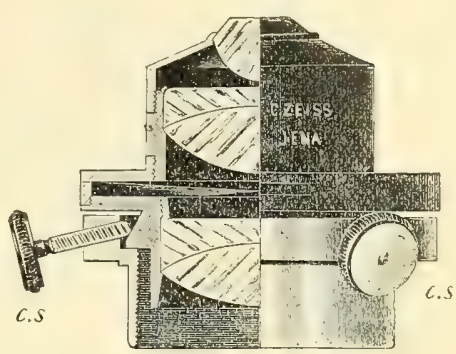

Si. Centering the Condenser.-To get the best possible illumination for bringing out in the clearest manner the minute details of a microscopic object two conditions are necessary, viz.: The principal optic axis of the condenser must be continuous with that of the microscope (see frontispiece) and the object must be in the focus of the condenser, $i . e .$, at the apex of the cone of light given by the condenser.

The centering is most conveniently accomplished as follows although dáylight may be used with almost equal facility. A very small diaphragm is put below the condenser. (If the Zeiss achromatic condenser is used, the diaphragm of the Abbe illuminator serves for this. If there is no pin-hole diaphragm one can be made of stiff, black paper. Care must be taken, however, to make the opening exactly central. This is best accomplished by putting the paper disc over the iris or metal diaphragms and then making the hole in the center of the small circle uncovered by the metal diaphragm. For the hole a fine needle is best). Light well and lower the objective so that it is at about its working-distance from the top of the condenser. If now the condenser is lowered or racked away from the objective the image of the diaphragm will appear. If the opening is not central it should be made so by using the centering screws of the condenser.

A better plan than to lower the condenser to focus the image of the diaphragm, is to raise the body of the microscope slowly with the coarse adjustment. It is almost impossible to make apparatus so accurate that two parts like the body of the microscope and the substage, each working on different sliding surfaces, shall continue in exactly the same plane. So one will find that if the condenser be accurately centered with the condenser lowered, and then the condenser be racked up close to the stage and the image of the diaphragm opening brought again into focus by racking up the body of the microscope, it will not be 
accurately centered in most cases. For this reason it is advised that the condenser be left in position close to the stage and the tube of the microscope be used to focus the diaphragm exactly as in ordinary work.

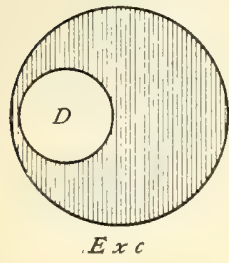

FIG. 42

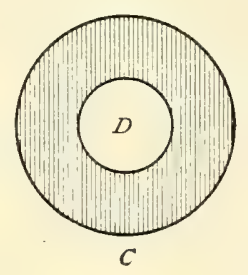

FIG. 43

FIG. 42. Showe that the optic axis of the condenser does not coincide with that of the microscope. (D). Image of the diaphragm of the condenser shown at one side of the field of view.

FIG. 43. Shows the image of the diaphragm $(D)$ in the center of the field of the microscope, and thus the coincidence of the axis of the condenser with that of the microscope.

82. Centering the Image of the Source of Illumination.For the best results it is not only necessary that the condenser be properly centered, but that the object to be studied should be in the image of the source of illumination and that this should also be centered (Figs. 44, 45). After the condenser itself is centered the iris diaphragm is opened to its full extent or the diaphragm carrier turned wholly aside. A transparent specimen like the fly's wing is put under the microscope and focused. The condenser is then turned up and down until the image of the flame is apparently on the specimen. If this cannot be accomplished the relative position of the lamp and condenser is not correct and should be so changed that the image of the edge of the flame is sharply defined. This image must also be centered. This is easily accomplished by manipulation of the mirror or, if a lamp is used, by changing the position of the lamp or of the bull's eye (Fig. 53).

\$3. Proper Numerical Aperture of the Condenser.-As stated above, the aperture of the condenser should have a range by means of properly selected diaphragms to meet the requirements of all objectives from the lowest to those of the highest aperture. It is found in practice that for diatoms, etc., the best images are obtained when the object is lighted with a cone which fills about three-fourths of the diameter of the back lens of the objective with light but for histological and other preparations of lower refractive power only onehalf or one-third the aperture often gives the most satisfactory images (§34). 
FIG. 44. Shows the image of the flame (Fl.) in the center (C) of the field of the microscope and illuminating the object.

FIG. 45. Shows the image of the flame ( $F l$. ) at one side of the centre (Exc.) and not properly illuminating the object.

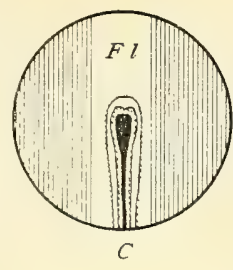

FIG. 44.

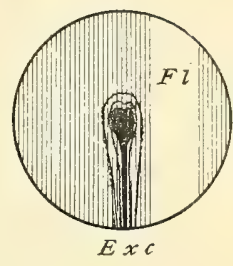

FIG. 45 .

To determine this in any case focus upon some very transparent object, take out the ocular, look down the tube at the back lens. If less than three-fourths of the back lens is lighted, increase the opening in the diaphragm-if more than three-fourths diminish it. For some objects it is advantageous to use less than three-fourths of the aperture. Experience will teach the best lighting for special cases.

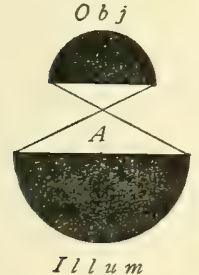

FIG. 46.
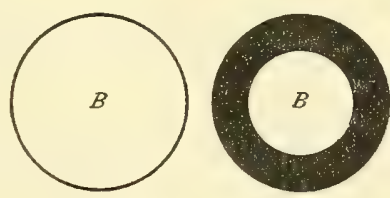

FIG. 47 .

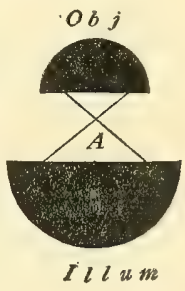

$l \ell \ell u m$

FIGS. 46-47. Figures showing the dependence of the objective upon the illum inating cone of the condenser (Nelson).

FIG. $46(A)$. The illuminating cone from the condenser (Illum). This is seen to be just sufficient to fill the objective (Obj).

$(B)$. The back lens of the objective entively filled with light, showing that the numerical aperture of the illuminator is equal to that of the objective.

FIG. $47(A)$. In this figure the illuminating cone frome the condenser (Illum.) is seen to be insufficient to fill the objective $(O b j)$.

$(B)$. The back lens of the objective only partly filled with light, due to the restricted aperture of the illuminator.

\section{Aperture of the Illuminating Cone and the Field.-It} is to be remarked that with a very small source of light the entire aperture of the objective may be filled if a proper illuminator or condenser is used. The aperture depends on the diaphragm used with the condenser. And the size of the diaphragm must be directly as the aperture of the objective. That is, it is just the reverse of the rule for diaphragms where no condenser is used $(\$ 67)$; for there the diaphragm 
is made large for low powers, and consequently low apertures, while with the condenser the diaphragm is made small for low and large for high powers as the aperture is greater in the high powers of a given series of objectives. It is very instructive to demonstrate this by using a $16 \mathrm{~mm}$. objective and opening the diaphragm of the condenser till the back lens is just filled with light. Then if one uses a 3 or $4 \mathrm{~mm}$. objective it will be seen that the back lens of the higher objective is only partly filled with light and to fill it the diaphragm must be much more widely opened.

With a condenser, then, the diaphragm has simply to regulate the aperture of the illuminating cone, and has nothing to do with lighting a large or a small field.

With the condenser there are two conditions that must be fulfilled, - the proper aperture must be used, and that is determined by the diaphragm, and secondly the whole field must be lighted. The latter is accomplished by using a larger source of light, as the face instead of the edge of a lamp flame, or by lowering or raising the condenser so that the object is not in the focus of the condenser, but above or below it, and therefore lighted by a converging or diverging beam where the light is spread over a greater area (Figs. 48-5I, § 88).

$\$ 8$. Non-Achromatic Condenser.- Of the non-achromatic condensers or illuminators, the Abbe condenser or illuminator is the one most generally used. From its cheapness it is also much more commonly used than the achromatic condenser. It consists of two or three very large lenses and transmits a cone of light of I.20 N. A. to I.40 N. A., but the aberrations, both spherical and chromatic, are very great in both forms. Indeed, so great are they that in the best form of three lenses with an illuminating cone of $\mathrm{r} .40 \mathrm{~N}$. A., the aplanatic cone transmitted is only 0.5 , and it is the aplanatic cone which is of real use in microscopic illumination where details are to be studied. There is no doubt, however, that the results obtained with a non-achromatic condenser like the Abbe are much more satisfactory than with nocondenser. The highest results cannot be attained with it, however. (Carpenter-Dallinger, p. 309).

$\S 86$. Arrangement of the Condenser. - The proper position of the illuminator for high objectives is one in which the beam of light traversing it is brought to a focus on the object. If parallel rays are reflected from the plane mirror to it, they will be focused only a few millimeters above the upper lens of the condenser; consequently the illuminator should be about on the level of the top of the stage and 
therefore almost in contact with the lower surface of the slide. For some purposes when it is desirable to avoid the loss of light by reflection or refraction, a drop of water or homogeneous immersion fluid is put between the slide and condenser, forming the so-called immersion illuminator. This is necessary only with objectives of high power and large aperture or for dark-ground illumination.

$\$ 87$. Centering the Condenser.-The illuminator should be centered to the optic axis of the microscope, that is the optic axis of the condenser and of the microscope should coincide. Unfortunately there is extreme difficulty in determining when the Abbe illuminator is centered. Centering is approximated as follows: Put a pin-hole diaphragm-that is a diaphragm with a small central hole-over the end of the condenser (Fig. 52), the central opening should appear to be in the middle of the field of the microscope. If it does not the condenser should be moved from side to side by loosening the centering screws until it is in the center of the field. In case no pin-hole diaphragm accompanies the condenser, one may put a very small drop of ink, as from a pen-point, on the center of the upper lens and look at it with the microscope to see if it is in the center of the field. If it is not, the condenser should be adjusted until it is. When the condenser is centered as nearly as possible remove the pin-hole diaphragm or the spot of ink. The microscope and illuminator axes may not be entirely coincident eren when the center of the upper lens appears in the center of the field, as there may be some lateral tilting of the condenser, but the above is the best the ordinary worker can do, and unless the mechanical arrangements of the illuminator are very deficient, it will be very nearly centered.

It is to be hoped that the opticians will devise some kind of mounting for this the most commonly used condenser whereby it may be centered as described for the achromatic condenser instead of by the crude methods described above. If the condenser mounting regularly possessed centering screws as in the microscope of Watson \& Sons and there were a centering diaphragm in the proper position so that its image could be projected into the field of view, the operation would be very simple. If, further, the condensers of Powell and Lealand were selected as models the condensers need not be so bulky, and would still retain all their efficiency.

Fortunately the Royal Microscopical Society of London which has done so much toward standardizing microscopical apparatus has recently proposed as a standard size for the substage fitting for the condenser of I.527 in. $=38.786 \mathrm{~mm}$. (see \& 46). 
\$ 88. Mirror and Light for the Abbe Condenser.-It is best to use light with parallel rays. The rays of daylight are practically parallel; it is best theretore to employ the plane mirror for all but the lowest powers. If low powers are used the whole field might not be illuminated with the plane mirror when the condenser is close to the object; furthermore, the image of the window frame, objects outside the building, as trees, etc., would appear with unpleasant distinctness in the field of the microscope. To overcome these defects one can lower the condenser and thus light the object with a diverging cone of light, or use the concave mirror and attain the same end when the condenser is close to the object (Fig. 48).

$\$ 89$. Artificial Light.-If one uses lamp light, it is recommended that a large bull's eye be placed in such a position between the light and the mirror that parallel rays fall upon the mirror or in some cases an image of the lamp flame. If one does not have a bull's eye the concave mirror may be used to render the rays less divergent. It may be necessary to lower the illuminator somewhat in order to illuminate the object in its focus.

\section{ABBE CONDENSER : EXPERIMENTS}

90. Abbe Condenser, Axial and Oblique Light.-Use a diaphragm a little larger than the front lens of the $3 \mathrm{~mm}$. ( $\mathrm{I} / 8 \mathrm{in}$.) objective, have the illuminator on the level, or nearly on the level of the upper surface of the stage, and use the plane mirror. Be sure that the diaphragm carrier is in the notch indicating that it is central in position. Use the Pleurosigma as object. Study carefully the appearance of the diatom with this central light, then make the diaphragm eccentric so as to light with oblique light $(\$ 78)$. The differences in appearance will probably be even more striking than with the mirror alone.

9I. Lateral Swaying of the Image.-Frequently in studying an object, especially with a high power, it will appear to sway from side to side in focusing up or down. A glass stage micrometer or fly's wing is an excellent object. Make the light central or axial and focus up and down and notice that the lines simply disappear or grow dim. Now make the light oblique, either by making the diaphragm opening eccentric or if simply a mirror is used, by swinging the mirror sidewise. On focusing up and down, the lines will sway from side to side. What is the direction of apparent movement in focusing down 
with reference to the illuminating ray? What in focusing up? If one understands the experiment it may sometimes save a great deal of confusion. (See under testing the microscope for swaying with central light \& II9.)

$\$$ 92. Dark-Ground Illumination.-When an object is lighted with rays of a greater obliquity than can get into the front lens of the objective, the field will appear dark (Fig. 5I). If now the object is

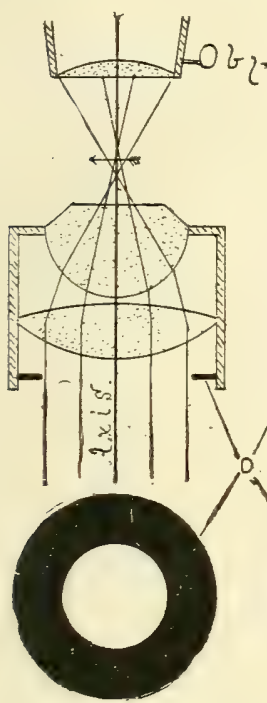

48.

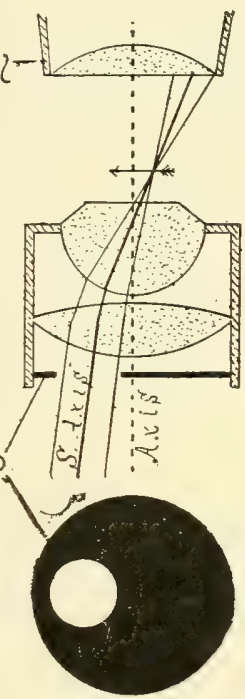

49.

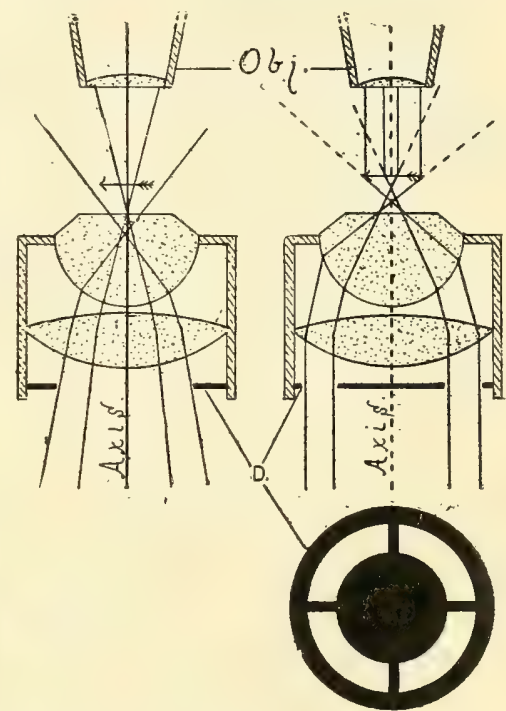

50.

$5 \mathrm{I}$.

FIGS. 48-51. Sectional views of the Abbe Illuminator of $1.20 N$. A. showing various methods of illumination $(\xi 90)$. Fig. 48, axial light with parallel rays. Fig. 49, oblique light. Fig. 50, axial light with converging beam. Fig. 5I, darkground illumination with a central stop diaphragm.

Axis. The optic axis of the illuminator and of the microscope. The illuminator is centered, that is its optic axis is a prolongation of the optic axis of the microscope.

S. Axis. Secondary axis. In oblique light the central ray passes along a secondary axis of the illuminator, and is therefore oblique to the principal axis.

D. D. Diaphragms. These are placed in sectional and in face views. The diaphragm is placed between the mirror and the illuminator. In Fig. 49 the opening is eccentric for oblique light, and in Fig. 5I the opening is a narrow ring, the central part being stopped out, thus giving rise to dark-ground illumination ( 8 92).

Obj. Obj. The front of the objective.

composed of fine particles, or is semi-transparent, it will refract or reflect the light which meets it, in such a way that a part of the very 
oblique rays will pass into the objective, hence as light reaches the objective only from the object, all the surrounding field will be dark and the object will appear 'like a self-luminous one on a dark back-

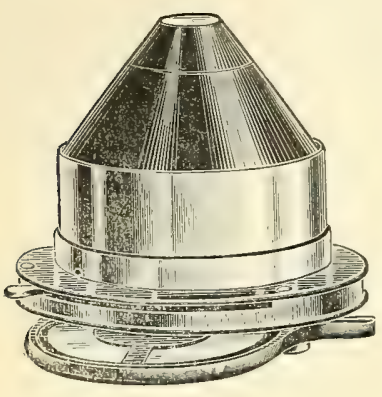

FIG. 52. An Abbe Condenser in its mounting (The Bausch \& Lomb Optical Company).

ground. This form of illumination is most successful with low powers. It is well to make the illuminator immersion for this experiment, (see $\$$ I05).

(A) With the Mirror-Remove all the diaphragms so that very oblique light may be used, employ a stage micrometer in which the lines have been filled with graphite, use a $16 \mathrm{~mm}$. ( $2 / 3 \mathrm{in}$.) objective, and when the light is sufficiently oblique the lines will appear something like streaks of silver on a black back-ground. A specimen like that described below in (B) may also be used.

(B) With the Abbe Condenser.-Hare the illuminator so that the light is focused on the object (see $\$ 86$ ) and use a diaphragm with the annular opening (Fig. 5I); employ the same objective as in (A). For object place a drop of Io \% solution of salicylic acid in $95 \%$ alcohol on the middle of a slide; it will crystallize. The crystals will appear brilliantly lighted on a dark back-ground. Put in an ordinary diaphragm and make the light oblique by making the diaphragm eccentric. The same specimen may also be tried with a mirror and oblique light. In order to appreciate the difference between this darkground and ordinary transmitted-light illumination, use an ordinary diaphragm and observe the crystals.

A very striking and instructive experiment may be made by adding a very small drop of the solution to the dried preparation, putting it under the microscope quickly, lighting for dark-ground illumination and then watching the crystallization.

\section{ARTIFICIAL ILLUMINATION}

\$93. For evening work and for regions where daylight is not sufficiently brilliant, artificial illumination must be employed. Furthermore, for the most critical investigation of bodies with fine markings like diatoms, artificial light has been found superior to daylight.

A petroleum (kerosene) lamp with flat wick gives a satisfactory light. It is recommended that instead of the ordinary glass chimney, 
one made of metal with a slit-opening covered with an oblong coverglass is more satisfactory, as the source of light is more restricted. Tery excellent results may be obtained, however, with the ordinary bed-room lamp furnished with the usual glass chimney.

The new acetylene light promises to be excellent for microscopic observation and for photo-micrography. (See under photomicrography.)

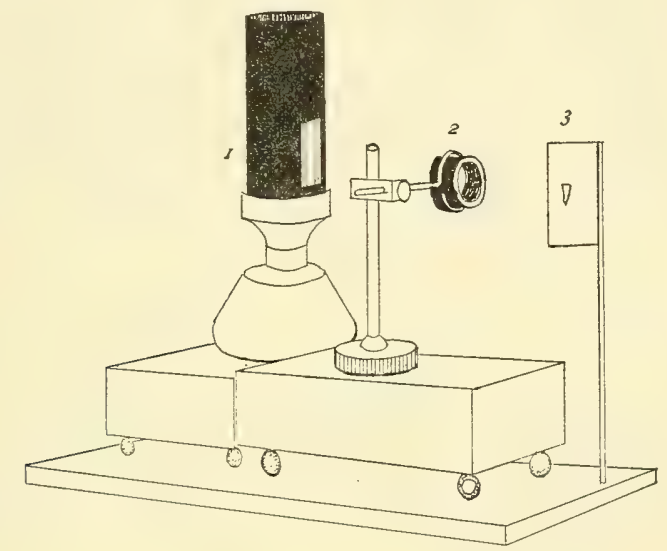

FIG. 53. I. Lamp with slit-opening in metal chimney. 2. Bull's eye on separate stand. 3. Screen showing image of flame.

Whenever possible the edge of the flame is turned toward the microscope, the advantage of this arrangement is the great brilliancy, due to the greater thickness of the flame in this direction.

$\$ 94$. Mutual Arrangement of Lamp, Bull's Eye and Microscope.-To fulfill the conditions given above, namely, that the object be illuminated by the image of the source of illumination the lamp must be in such a position that the condenser projects a sharp image of the flame upon the object (Fig. 53), and only by trial can this position be determined. In some cases it is found advantageous to discard the mirror and allow the light from the bull's eye to pass directly into the condenser. This method is especially excellent in photomicrography (see Ch. VIII).

\$95. Illuminating the Entire Field.-With low objectives and large objects, the entire object might not be illuminated if the above method were strictly followed; in this case turn the lamp so that the flame is oblique, or if that is not sufficient, continue to turn the lamp until the full width of the flame is used. If necessary the 
condenser may be lowered, and the concave mirror used. (See also $\$ 84$.)

\author{
REFRACTION AND COLOR IMAGES
}

8. 96. Refraction Images are those mostly seen in studying microscopic objects. They are the appearances produced by the refraction of the light on entering and on leaving an object. They therefore depend (a) on the form of the object, (b) on the relative refractive powers of object and mounting medium. With such images the diaphragm should not be too large (see 8.83 ).

If the color and refractive index of the object were exactly like the mounting medium it could not be seen. In most cases both refractive index and color differ somewhat, there is then a combination of color and refraction images which is a great advantage. This combination is generally taken advantage of in histology. The air bubble in $z_{7} 77$ is an example of a purely refractive image.

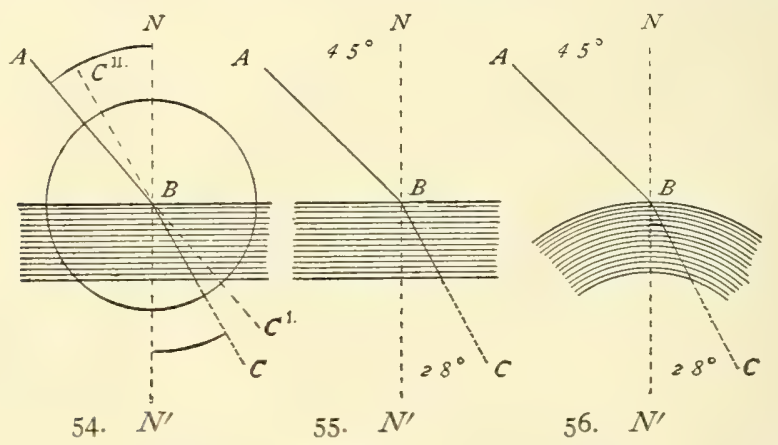

FIGS. 54-56. Diagrams illustrating refraction in different media and at plane and curied surfaces. In cach case the denser medium is represented by line shading and the perpendicular or normal to the refracting surface is represented by the dotted line $N-N^{\prime}$, the refracted ray by the bent line $A C$.

37. Refraction.-Lying at the basis of microscopical optics is refraction, which is illustrated by the above figures. It means that light passing from one medium to another is bent in its course. Thus in Fig. 54 light passing from air into water does not continue in a straight line but is bent toward the normal $\mathrm{N}^{-\mathrm{N}^{\prime}}$, the bending taking place at the point of contact of the air and water; that is, the ray of light $A B$ entering the water at $B$ is bent out of its course, extending to $C$ instead of $C^{\prime}$.

Conversely, if the ray of light is passing from water into air, on reaching the air it is bent from the normal, the ray C B passing to $\mathrm{A}$ and not in a straight line to $C^{\prime \prime}$. By comparing Figs. 55, 56 in which the denser medium is crown glass instead of water, the bending of the rays is seen to be greater as crown glass is denser than water.

It has been found by physicists that there is a constant relation between the angle taken by the ray in the rarer medium and that taken by the ray in the denser medium. The relationship is expressed thus: Sine of the angle of inci- 
dence divided by the sine of the angle of refraction equals the index of refraction. In the figures, $\frac{\operatorname{Sin} A B N}{\operatorname{Sin} C B N^{\prime}}=$ index of refraction. Worked out completely in Fig. 54, $A B N=40^{\circ}, C B N^{\prime}=28^{\circ} 54^{\prime}$ and $\frac{\operatorname{Sin} 40^{\circ}}{\operatorname{Sin} 28^{\circ} 54^{\prime}}=\frac{0.64279}{0.48327}=$ I.33, i.e., the index of refraction from air to water is I.33. (See \& 33.) In Figs. 55-56, illustrating refraction in crown glass, the angles being given, the problem is easily solved as just illustrated. (For table of natural sines see third page of cover ; for interpolation,, 32 ).

3. 98. Absolute Index of Refraction.-This is the index of refraction obtained when the incident ray passes from a vacuum into a given medium. As the index of the vacuum is taken as unity, the absolute index of any substance is always greater than unity. For many purposes, as for the object of this book, air is treated as if it were a vacuum, and its index is called unity, but in reality the index of refraction of air is about 3 ten-thousandths greater than unity. Whenever the refractive index of a substance is given, the absolute index is meant unless otherwise stated. For example, when the index of refraction of water is said to be $I .33$, and of crown glass $1.5^{2}$, etc., these figures represent the absolute index, and the incident ray is supposed to be in a vacuum.

\% 99. Relative Index of Refraction.-This is the index of refraction between two contiguous media, as for example between glass and diamond, water and glass, etc. It is obtained by dividing the absolute inflex of refraction of the substance containing the refracted ray, by the absolute index of the substance transmitting the incident ray. For example, the relative index from water to glass is I.52 divided by I.33. If the light passed from glass to water it would be, I.33 divided by 1.52 .

By a study of the figures showing refraction, it will be seen that the greater the refraction the less the angle and consequently the less the sine of the angle, and as the refraction between two media is the ratio of the sines of the angles of incidence and refraction $\left(\frac{\sin i}{\sin r}\right)$, it will be seen that whenever the sine of the angle of refraction is increased by being in a less refractive medium, the index of refraction will show a corresponding decrease and vice versa. That is the ratio of the sines of the angles of incidence and refraction of any two contiguous substances is inversely as the refractive indices of those substances. The formula is :

$\left(\begin{array}{l}\text { Sine of angle of incident ray } \\ \text { Sine of angle of refracted ray }\end{array}\right)=\left(\begin{array}{l}\text { Index of refraction of refracting medium } \\ \text { Index of refraction of incident medium }\end{array}\right)$ Abbreviated $\left(\frac{\sin i}{\sin r}\right)=\left(\frac{\text { index } r}{\text { index } i}\right)$. By means of this general formula one can solve any problem in refraction whenever three factors of the problem are known. The universality of the law may be illustrated by the following cases :

(A) Light incident in a vacuum or in air, and entering some denser medium, as water, glass, diamond, etc.

$\left(\begin{array}{c}\text { Sine of angle made by the ray in air } \\ \text { Sine of angle made by ray in denser medium }\end{array}\right)=\left(\frac{\text { Index of ref.of denser med. }}{\text { Index of ref. of air (I) }}\right)$ If the dense substance were glass $\left(\frac{\sin i}{\sin r}\right)=\left(\frac{\mathrm{I} .5^{2}}{\mathrm{I}}\right)$. If the two media were 
water and glass, the incident light being in water the formula would be: $\left(\frac{\sin i}{\sin r}\right)=\left(\frac{\mathrm{I} .5^{2}}{\mathrm{I} .33}\right)$. If the incident ray were in glass and the refracted ray in water: $\left(\frac{\sin i}{\sin r}\right)=\left(\frac{\mathrm{I} .33}{\mathrm{I} .52}\right)$. And similarly for any two media; and as stated above if any three of the factors are given the fourth may be readily found.

3. Ioo. Critical Angle and Total Reflection.-In order to understand the Wollaston camera lucida (Ch. IV) and other totally reflecting apparatus, it is necessary briefly to consider the critical angle.

The critical angle is the greatest angle that a ray of light in the denser of two contiguous media can make with the normal and still emerge into the less refractive medium. On emerging it will form an angle of $90^{\circ}$ with the normal, and if the substances are liquids, the refracted ray will be parallel with the surface of the denser medium.

Total Reflection.- In case the incident ray in the denser medium is at an angle with the normal greater than the critical angle, it will be totally reflected at the surface of the denser medium, that surface acting as a perfect mirror. By consulting the figures it will be seen that there is no such thing as a critical angle and total reflection in the rarer of two contiguous media.

To find the critical angle in the denser of two contiguous media :-

Make the angle of refraction ( $i . e$. , the angle in the rarer of the two media) $90^{\circ}$ and solve the general equation: $\left(\begin{array}{l}\sin i \\ \sin r\end{array}\right)=\left(\frac{\text { index } r}{\text { index } i}\right)$. Let the two substances be water and air, then the sine of $r\left(90^{\circ}\right)$ is $I$, the index of air is $I$, that of water $\mathrm{I} .33$, whence $\left(\frac{\sin i}{\mathrm{I}}\right)=\left(\frac{\mathrm{I}}{\mathrm{I} .33}\right)$ or $\sin i=75 \mathrm{I}+$. This is the sine of $48^{\circ}+$, and whenever the ray in the water is at an angle of more than $48^{\circ}$ it will not emerge into the air, but be totally reflected back into the water.

The case of a ray passing from crown glass into the water :

$$
\left(\frac{\sin i}{\sin r\left(\sin 90^{\circ}=\mathrm{I}\right)}\right)=\left(\frac{\text { index water }(\mathrm{I} .33)}{\text { index } \operatorname{glass}\left(\mathrm{I} .5^{2}\right)}\right) \text { or }\left(\frac{\sin i}{\mathrm{I}}\right)=\left(\frac{\mathrm{I} .33}{\mathrm{I} .5^{2}}\right) \text {. }
$$

whence $\sin i=.875$ sine of critical angle in glass covered with water. The corresponding angle is approximately $6 \mathrm{I}^{\circ}$.

3. IOI. Color Images.-These are images of objects which are strongly colored and lighted with so wide an aperture that the refraction images are drowned in the light. Such images are obtained by removing the diaphragm or by using a larger opening. This method of illumination is especially applicable to the study of deeply stained bacteria. (See below. \& I08).

\section{ADJUSTABLE WATER AND HOMOGENEOUS OBJEC'TIVES}

\section{EXPERIMENTS}

₹. I02. Adjustment for Objectives.-As stated above ( 824$)$, the aberration produced by the cover-glass (Fig. 57), is compensated for by giving the combinations in the objective a different relative position than they would have if the objective were to be used on uncovered objects. Although this relative position cannot be changed in unadjustable objectives, one can secure the best results of 
which the objective is capable by selecting covers of the thickness for which the objective was corrected. (See table p. I4.) Adjustment may be made also by increasing the tube-length for covers thinner than the standard and by shortening the tube-length for covers thicker than the standard (Fig. $5^{8}$ ).

FIG. 57. Effect of the cover-glass on the rays from the object to the objective (Ross).

Axis. The projection of the optic axis of the microscope.

F. Focal or axial point of the objective.

$F^{\prime}$ and $F^{\prime \prime}$. Points on the axis where rays 2 and 3 appear to originate if traced backward after emerging from the upper side of the cover-glass.

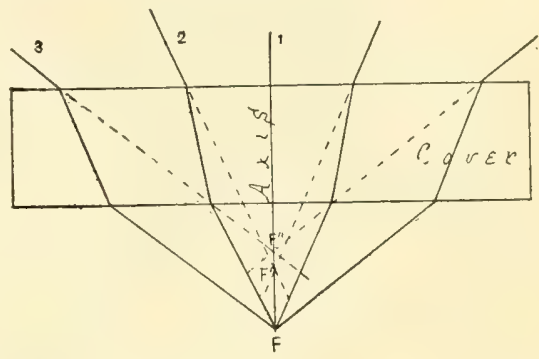

In learning to adjust objectives, it is best for the student to choose some object whose structure is well agreed upon, and then to practice lighting it, shading the stage and adjusting the objective, until the proper appearance is obtained. The adjustment is made by turning a ring or collar which acts on a screw and increases or diminishes the distance between the systems of lenses, usually the front and the back systems (Fig. 40).

S. I03. General Directions.-(A) The thinner the cover-glass, the further must the system of lenses be separated, $i . e .$, the adjusting collar is turned nearer the zero or the mark "'uncovered,' and conversely; (B) the thicker the cover-glass the closer together are the systems brought by turning the adjusting collar from the zero mark. This also increases the magnification of the objective (Ch. IV).

The following specific directions for making the cover-glass adjustment are given by Mr. Wenham (Carpenter, 7 th Ed., p. I66). "Select any dark speck or opaque portion of the object, and bring the outline into perfect focus; then lay the finger on the milled-head of the fine motion, and move it briskly backwards and forwards in both directions from the first position. Observe the expansion of the dark outline of the object, both when within and when without the focus. If the greater expansion or coma is when the object is without the focus, or farthest from the objective $[i$. e., in focusing up], the lenses must be placed further asunder, or toward the mark uncovered [the adjusting collar is turned toward the zero mark as the cover-glass is too thin for the present adjustment]. If the greater expansion is when the object is within the focus, or nearest the objective [i.e., in focusing down], the lenses must be brought closer together, or toward the mark covered $[i$. e., the adjusting collar should be turned away from the zero mark, 
the cover-glass being too thick for the present adjustment]." In most objectives the collar is graduated arbitrarily, the zero $(O)$ mark representing the position for uncovered objects. Other objectives have the collar graduated to correspond to the various thicknesses of cover-glasses for which the objective may be adjusted. This seems to be an admirable plan; then if one knows the thickness of the cover-glass on the preparation (Ch. VIII) the adjusting collar may be set at a corresponding mark, and one will feel confident that the adjustment will be approximately correct. It is then only necessary for the observer to make the slight adjustment to compensate for the mounting medium or any variation from the standard length of the tube of the microscope. In adjusting for variations of the length of the tube from the standard it should be remembered that: (A) If the tube of the microscope is longer than the standard for which the objective was corrected, the effect is approximately the same as thickening the cover-glass, and therefore the system of the objective must be brought closer together, i.e., the adjusting collar must be turned away from the zero mark. (B) If the tube is shorter than the standard for which the objective is corrected, the effect is approximately the same as diminishing the thickness of the cover-glass, and the systems must therefore be separated (Fig. 40).

In using the tube-length for cover correction Shorten the tube for too thick covers and Lengthen the tube for too thin covers.

Furthermore, whatever the interpretation by different opticians of what should be included in "tube-length," and the exact length in millimeters, its importance is very great; for each objective gives the most perfect image of which it is capable with the "tube-length" for which it is corrected, and the more perfect the objective the greater the illeffects on the image of varying the "tube-length" from this standard. The plan of designating exactly what is meant by "tube-length," and engraving on each objective the "tube-length" for which it is corrected, is to be commended, for it is manifestly difficult for each worker with the microscope to find out for himself for what "tube-length" each of his objectives was corrected. (See Ch. X).

SIO4. Water Immersion Objectives. - Put a water immersion objective in position $(\$ 47)$ and the fly's wing for object under the microscope. Place a drop of distilled water on the cover-glass, and with the coarse adjustment lower the tube till the objective dips into the water, then light the field well and turn the fine adjustment one way and another till the image is clear. Water immersions are exceedingly convenient in studying the circulation of the blood, and for 
many other purposes where aqueous liquids are liable to get on the cover-glass. If the objective is adjustable, follow the directions given in $\S 103$.

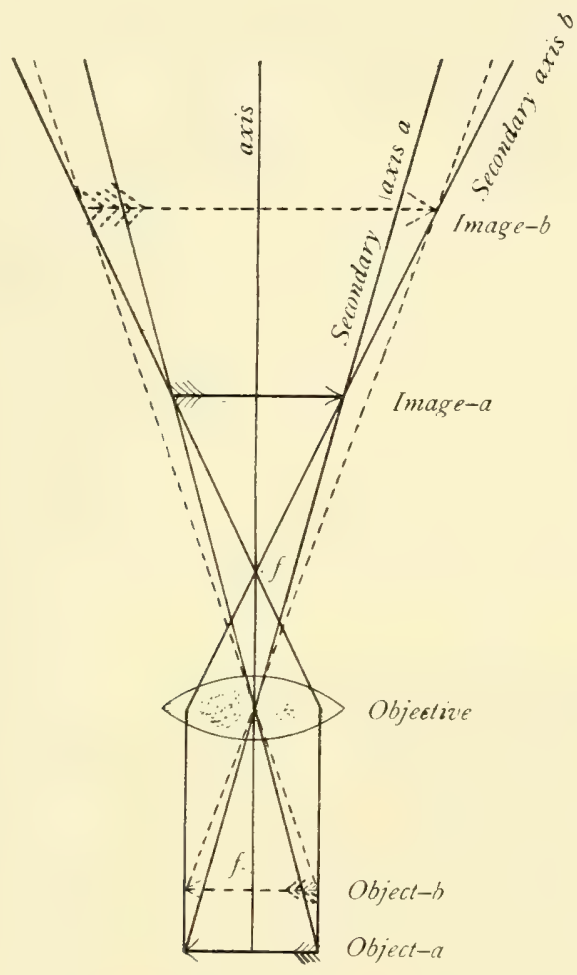

FIG. 58. Figure to show that in lengthening the tube of the microscope the object must be brought nearer the principal focus or center of the lens. It will be seen by consulting the figure that in shortening the tube of the microscope the object must be removed farther from the center of the lens. By consulting the figure showing the effect of the cover-glass (Fig. 57) it will be seen that the effect of the cover-glass is to bring the object nearer the objective, and the thicker the cover the nearer is the object brought to the objective. As shortening the tube serves to remove the object, it neutralizes the effect of the thick cover, and if the cover is so thin that it does not elevate the object enough for the corrections of the objective, then an increase in the tube-length will correct the defect. 
When one is through using a water immersion objective, remove it from the microscope and with some lens paper wipe all the water from the front lens. Unless this is done dust collects and sooner or later the front lens will be clouded. It is better to use distilled water to avoid the gritty substances that are liable to be present in natural waters, as these gritty particles might scratch the front lens.

\section{HOMOGENEOUS IMMERSION OBJECTIVES : EXPERIMENTS}

I05. As stated above, these are objectives in which a liquid of the same refractive index as the front lens of the objective is placed between the front lens and the cover-glass.

$\$$ I06. Tester for Homogeneous Liquid.-In order that full advantage be derived from the homogeneous immersion principle, the liquid employed must be truly homogeneous. To be sure that such is the case, one may use a tester like that constructed by the Gundlach Optical Co., then if the liquid is too dense it may be properly diluted and vice versa. For the cedar oil immersion liquid, the density may be diminished by the addition of pure cedar wood oil. The density may be increased by allowing it to thicken by evaporation. (See H. L. Smith, Proc. Amer. Soc. Micr., I 885, p. 83, and Ch. X).

\$I07. Refraction Images. - Put a $2 \mathrm{~mm}$. ( $\frac{1}{12}$ th in.) homogeneous immersion objective in position, employ an illuminator. Use some histological specimen like a muscular fiber as object, make the diaphragm opening about $3 \mathrm{~mm}$. in diameter, add a drop of the homogeneous immersion liquid and focus as directed in $\$ 74$. The object will be clearly seen in all details by the unequal refraction of the light traversing it. The difference in color between it and the surrounding medium will also increase the sharpness of the outline. If an air bubble preparation ( 57 ) were used, one would get pure, refraction images.

$\$$ I08. Color Images.-Use some stained bacteria as Bacillus tuberculosis for object. Put a drop of the immersion liquid on the cover-glass or the front lens of the homogeneous objective. Remove the diaphragms from the illuminator or in case the iris diaphragm is used, open to its greatest extent. Focus the objective down so that the immersion fluid is in contact with both the front lens and the coverglass, then with the fine adjustment get the bacteria in focus. They will stand out as clearly defined colored objects on a bright field. 
FIG. 59. Screen for shading the microscope and the face of the observer. This is very readily constructed as shown in the figure by supporting a wire in a disc of lead, iron, or heavy wood. The screen is then completed by hanging over the bent'wire, cloth or manilla paper $30 x 40 \mathrm{~cm}$. The lower edge of the screen should be a little below the stage of the microscope and the upper edge high enough to screen the eyes of the observer.

$\S$ Iog. Shading the Object.-To get the clearest image of an object no light should reach the eye except from the object. A handkerchief or a dark cloth wound around the

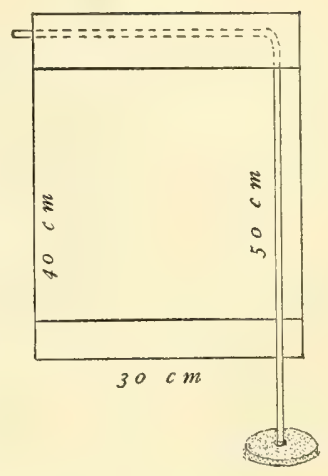
objective will serve the purpose. Often the proper effect may be obtained by simply shading the top of the stage with the hand or with a piece of bristol board. Unless one has a very favorable light the shading of the object is of the greatest advantage, especially with homogeneous immersion objectives. The screen (Fig. 59) is the most satisfactory means for this purpose, as the entire microscope above the illuminating apparatus is shaded.

\$IIO. Cleaning Homogeneous Objectives.-After one is through with a homogeneous objective, it should be carefully cleaned as follows: Wipe off the homogeneous liquid with a piece of the lens paper ( $\$$ I I 4$)$, then if the fluid is cedar oil, wet one corner of a fresh piece in benzin or chloroform and wipe the front lens with it. Immediately afterward wipe with a dry part of the paper. The cover-glass of the preparation can be cleaned in the same way. If the homogeneous liquid is a glycerin mixture proceed as above, but use water to remove the last traces of glycerin.

\section{CARE OF THE MICROSCOPE}

$\$$ III. The microscope should be handled carefully and kept perfectly clean. The oculars and objectives should never be allowed to fall.

When not in use keep it in a place as free as possible from dust.

All parts of the microscope should be kept free from liquids, especially from acids, alkalies, alcohol, benzin, turpentine and chloroform.

$\$$ II2. Care of the Mechanical Parts. - To clean the mechanical parts put a small quantity of some fine oil (olive oil or liquid vas- 
elin and benzin, equal parts), on a piece of chamois leather or on the lens paper, and rub the parts well, then with a clean dry piece of the chamois or paper wipe off most of the oil. If the mechanical parts are kept clean in this way a lubricator is rarely needed. Where opposed brass surfaces "cut," i. $e$, when from the introduction of some gritty material, minute grooves are worn in the opposing surfaces, giving a harsh movement, the opposing parts should be separated, carefully cleaned as described above and any ridges or prominences scraped down with a knife. Where the tendency to "cut" is marked, a very slight application of equal parts of beeswax and tallow, well melted together, serves a good purpose.

In cleaning lacquered parts, benzin alone answers well, but it should be quickly wiped off with a clean piece of the lens paper. Do not use alcohol as it dissolves the lacquer.

$\$$ I I 3. Care of the Optical Parts.-These must be kept scrupulously clean in order that the best results may be obtained.

Glass surfaces should never be touched with the fingers, for that will soil them.

The glass of which the lenses are made is quite soft, consequently it is necessary that only soft, clean cloth or paper be used in wiping them.

Whenever an objective is left in position on a microscope, or when several are attached by means of a revolving nose-piece, an ocular should be left in the upper end of the tube to prevent dust from falling down upon the back lens of the objective.

\$ Ir 4. Lens Paper.-The so-called Japanese filter paper, which from its use with the microscope, I have designated lens paper, has been used in the author's laboratory for the last sixteen years for cleaning the lenses of oculars and objectives, and especially for removing the fluid used with immersion objectives. Whenever a piece is used once it is thrown away. It has proved more satisfactory than cloth or chamois, because dust or sand is not present; and from its bibulous character it is very efficient in removing liquid or semi-liquid substances.

$\$$ II5. Dust may be removed witk a camel's hair brush, or by wiping with the lens paper.

Cloudiness may be removed from the glass surfaces by breathing on them, then wiping quickly with a soft cloth or the lens paper.

Cloudiness on the inner surfaces of the ocular lenses may be removed by unscrewing them and wiping as directed above. A high objective should never be taken apart by an inexperienced person. 
If the cloudiness cannot be removed as directed above, moisten one corner of the cloth or paper with 95 per cent. alcohol, wipe the glass first with this, then with the dry cloth or the paper.

Water may be removed with soft cloth or the paper.

Glycerin may be removed with cloth or paper saturated with distilled water ; remove the water as above.

Blood or other albuminous material may be removed while fresh with a moist cloth or paper, the same as glycerin. If the material has dried on the glass, it may be removed more readily by adding a small quantity of ammonia to the water in which the cloth is moistened, (water Ioo cc., ammonia I cc).

Canada Balsam, damar, paraffin, or any oily substance may be removed with a cloth or paper wet with chloroform, benzin or xylene. The application of these liquids and their removal with a soft dry cloth or paper should be as rapid as possible, so that none of the liquid will have time to soften the setting of the lenses.

Shellac Cement may be removed by the paper or a cloth moistened in 95 per cent. alcohol.

Brunswick Black, Gold Size, and all other substances soluble in chloroform, etc., may be removed as directed for balsam and damar.

In general, use a solvent of the substance on the glass and wipe it off quickly with a fresh piece of the lens paper.

It frequently happens that the upper surface of the back combination of the objective becomes dusty. This may be removed in part by a brush, but more satisfactorily by using a piece of the soft paper loosely twisted. When most of the dust is removed some of the paper may be put over the end of a pine stick (like a match stick) and the glass surface carefully wiped.

\section{CARE OF THE EYES}

5 I 6. Keep both eyes open, using the eye-screen if necessary (Figs. $60,60 a)$; and divide the labor between the two eyes, $i . e_{\text {. }}$, use one eye for observing the image awhile and then the other. In the beginning it is not advisable to look into the microscope continuously for more than half an hour at a time. One never should work with the microscope after the eyes feel fatigued. After one becomes accustomed to microscopic observation he can work for several hours with the microscope without fatiguing the eyes. This is due to the fact that the eyes become inured to labor like the other organs of the body by judicious exercise. It is also due to the fact that but very slight accommodation is required of the eyes, the eyes remaining nearly in a condition of rest as for distant objects. The fatigue incident upon 
using the microscope at first is due partly at least to the constant effort on the part of the observer to remedy the defects of focusing the microscope by accommodation of the eyes. This should be avoided and the fine adjustment of the microscope used instead of the muscles of accommodation. With a microscope of the best quality, and suitable light - that is light which is steady and not so bright as to dazzle the eyes nor so dim as to strain them in determining details-microscopic work should improve rather than injure the sight.

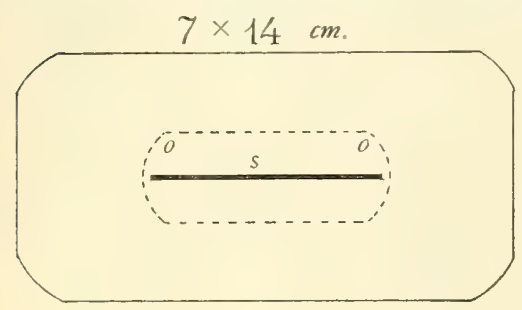

FIG. 6o. Double Eye Shade. This is readily made by taking some thick bristol board $7 x$ I4 centimeters and making an oblong opening with rounded ends $(o-0)$ and of such a diameter that it goes readily over the tube of the microscope. This is then covered on both sides with velveteen and a central slit (s) made in the cloth. This admits the tube of the microscope and holds the screen in position. It may readily be pulled from side to side and thus serves for either eye, or for the use of the eyes alternately.

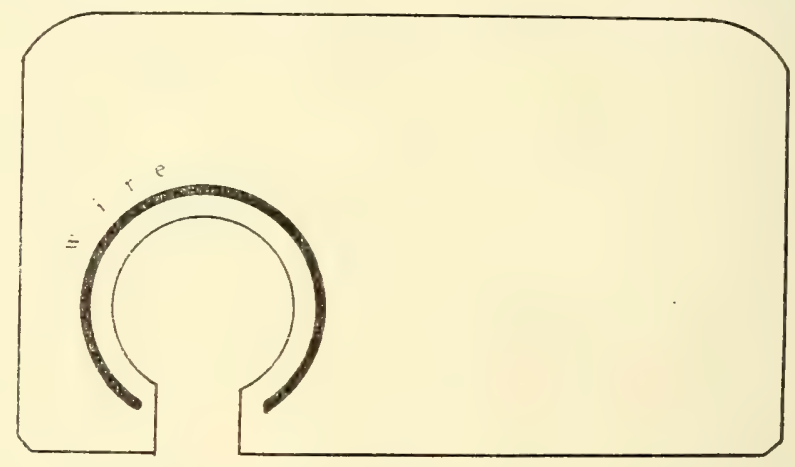

FiIG. 60a. Adjusting Eye-Shade. This is prepared like the preceding by covering a card about 6 x 12 centimeters with black velveteen. A copper wire about $3 \mathrm{~mm}$. (1/s in.) and of the right length is curved as shoun in the figure. Its ends are rounded, and finally it is put under the cloth and served carefully all around. The card and cloth are then cut as shown. The flexible wire makes it possible to put the screen on the tube at any level.

SI7. Position and Character of the Work-Table.-The work-table should be very firm and large ( $60 \times$ I $20 \mathrm{~cm}$; $24 \times 48$ in.), so that the necessary apparatus and material for work may not be too crowded. The table should also be of the right height to make work by it comfortable. An adjustable stool, something like a piano stool is convenient, then one may vary the height corresponding to the neces- 
sities of special cases. It is a great advantage to sit facing the window if daylight is used, then the hands do not constantly interfere with the illumination. To avoid the discomfort of facing the light a screen like that shown in Fig. 59 is very useful (see also under lighting, $\$ 62)$.

\section{TESTING THE MICROSCOPE}

\% II8. Testing the Microscope.-To be of real value this must be accomplished by a person with both theoretical and practical knowledge, and also with an unprejudiced mind. Such a person is not common, and when found does not show over anxiety to pass judgment. Those most ready to offer advice should as a rule be avoided, for in most cases they simply "have an ax to grind," and are sure to commend only those instruments that conform to the "fad" of the day. From the writer's experience is seems safe to say that the inexperienced can do no better than to state clearly what he wishes to do with a microscope and then trust to the judgment of one of the optical companies. The makers of microscopes and objectives guard with jealous care the excellence of both the mechanical and optical part of their work, and send out only instruments that have been carefully tested and found to conform to the standard. This would be done as a matter of business prudence on their part, but it is believed by the writer that microscope makers are artists first and take an artist's pride in their work, they therefore have a stimulus to excellence greater than business prudence alone could give.

\% I I9. Mechanical Parts. - All of the parts should be firm, and not too easily shaken. Bearings should work smoothly. The mirror should remain in any position in which it is placed.

Focusing Adjustments. - The coarse or rapid adjustment should be by rack and pinion, and work so smoothly that even the highest power can be easily focused with it. In no case should it work so easily that the body of the microscope is liable to run down and plunge the objective into the object. If any of the above defects appear in a microscope that has been used for some time, a person with moderate mechanical instinct will be able to tighten the proper screw, etc.

The Fine Adjustment is more difficult to deal with. From the nature of its purpose unless it is approximately perfect, it would be better off the microscope entirely. It has been much improved recently.

It should work smoothly and be so balanced that one cannot tell by the feeling when using it whether thescrew is going up or down. Then there should be absolutely no motion except in the direction of the optic axis, otherwise the image will appear to sway even with central light. Compare the appearance when using the coarse and when using the fine adjustment. There should be no swaying of the image with either if the light is central ( 877$)$.

/2 I 20. Testing the Optical Parts.-As stated in the beginning, this can be done satisfactorily only by an expert judge. It would be of very great advantage to the student if he eould have the help of such a person. In no case is a microscope to be condemned by an inexperienced person. If the beginner will bear in mind that his failures are due mostly to his own lack of knowledge and lack of skill; and will truly endeavor to learn and apply the principles laid down in this and in the standard works referred to, he will learn after a while to estimate at their true value all the pieces of his microscope. (See Ch. $\mathrm{X}$ ). 


\section{LABORATORY AND HIGH-SCHOOL COMPOUND MICROSCOPES .}

\&. I2I. Optical Parts.-A great deal of beginning work with the microscope in biological laboratories is done with simple and inexpensive apparatus. Indeed if one contemplates the large classes in the high schools, the universities and medical schools, it can be readily understood that microscopes costing from $\$ 25-50$ each and magnifying from 25 to 500 diameters, are all that can be expected. But for the purpose of modern histological investigation and of advanced microscopical work in general, a microscope should have something like the following character : Its optical outfit should comprise, (a) dry objectives of $50 \mathrm{~mm}$. ( $2 \mathrm{in}$.), I6-I $8 \mathrm{~mm}$. ( $2 / 3$ in.) and $3 \mathrm{~mm}$. ( $1 / 8 \mathrm{in}$.) equivalent focus. There should be present also a $2 \mathrm{~mm}$. ( $\frac{1}{12}$ in.) or $1.5 \mathrm{~mm}$. ( $\frac{1}{16}$ in.) homogeneous immersion objective. Of oculars there should be several of different power. A centering substage condenser, and an Abbe camera Iucida are also necessities, and a micro-spectroscope and a micro-polarizer are very desirable.

Even in case all the optical parts cannot be obtained in the beginning, it is wise to secure a stand upon which all may be used when they are finally secured.

As to the objectives. The best that can be afforded should be obtained. Certainly at the present, the apochromatics stand at the head, although the best achromatic objectives approach them very closely.

\%, I22. Mechanical Parts or Stand.-The stand should be low enongh so that it can be used in a vertical position on an ordinary table without inconvenience ; it should have a jointed (flexible) pillar for inclination at any angle to the horizontal. The adjustments for focusing should be two,- - coarse adjustment or rapid movement with rack and pinion, and a fine adjustment by means of a micrometer screw. Both adjustments should move the entire tube of the microscope. The body or tube should be short enough for objectives corrected for the short or I6o millimeter tube-length. It is an advantage to have the draw-tube graduated in centimeters and millimeters. The lower end of the draw tube and of the tube should each possess a standard screw for objectives (frontispiece). The stage should be quite large for the examination of slides with serial sections and other large objects. The substage fittings should be so arranged as to enable one to use the condenser or to dispense entirely with diaphragms. The condenser mounting should allow up and down motion.

\section{STANDARD SIZES RECOMMENDED BY THE ROYAL MICROSCOPICAL SOCIETY}

8 I23. Society Screw.-Owing to the lack of uniformity in screws for microscope objectives, the Royal Microscopical Society of London, in I857, made an earnest effort to introduce a standard size. The specifications of this standard are 
as follows: "Whitworth thread, $i$. e., a V shaped thread, sides of thread inclined to angle of $55^{\circ}$ to each other, one-sixth of the $\mathrm{V}$ depth of the thread being rounded off at the top of the thread, and one-sixth of the thread being rounded off at the bottom of the thread. Pitch of screw, 36 to the inch; length of thread on object-glass, O.I25 inch; plain fitting above thread of object-glass, O.I 5 inch long, to be about the size of the bottom of male thread; length of thread of nose-piece [on the lower end of the tube of the microscope], not less than 0.25 inch; diameter of the object-glass screw at the bottom of the screw, 0.7626 inch; diameter of the nose-piece screw at the bottom of the thread, o. 8 inch."

In order to facilitate the introduction of this universal screw, or as it soon came to be called "The Society Screw," the Royal Microscopical Society undertook to supply standard taps. From the mechanical difficulty in making these taps perfect there soon came to be considerable difference in the "Society Screws," and the object of the society in providing a universal screw was partly defeated.

In I884 the American Microscopical Society appointed Mr. Edward Bausch and Prof. William A. Rogers upon a committee to correspond with the Royal Microscopical Society, with a view to perfecting the standard "Society Screw," or of adopting another standard and of perfecting methods by which the screws of all makers might be truly uniform. Although this matter was earnestly considered at the time by the Royal Microscopical Society, the mechanical difficulties were so great that the improvements were abandoned.

Fortunately, however, during the year (1896) that society again took hold of the matter in earnest, and the "Society Screw" is now accurate, and facilities for obtaining the standard are so good that there is a reasonable certainty that the unicersal screw for microscopic objectives may be realized. It is astonishing to see how widely the "Society Screw" has been adopted. Indeed there is not a maker of first-class microscopes in the world who does not supply the objectives and stands with the "Society Screw," and an objective in England or America which does not have this screw should be looked upon with suspicion. That is, it is either old, cheap, or not the product of one of the great opticians. For the Standard, or "Society Screw," see: Trans. Roy. Micr. Soc., I857, pp. 39-4I ; I859, pp. 92-97; 1860, pp. I03-104. (All to be found in Quar. Jour. Micr. Sci., o. s., vols. VI, VII and VIII). Proc. Amer. Micr. Soc. 1884 , p. 274 ; I886, p. I99; I893, p. 38. Journal of the Royal Microscopical Society, August, 1896.

In this last paper of four pages the matter is very carefully gone over and full specifications of the new screw given. It conforms almost exactly with the original standard adopted by the society, but means have been devised by which it may be kept standard.

3. 24. Standard Size Oculars and Substage Condensers.-For a consideration of these, with measurements, see $\xi 46,87$.

\section{MARKERS AND MECHANICAL STAGES}

Markers are devices to facilitate the finding of some object or part which it is especially desired to refer to again or to demonstrate to a class. The mechanical stage makes it much easier to follow out a series of objects, to move the slide when using high powers, and for complete exploration of a preparation. Most of the mechanical stages have scales or scales and verniers by which an object once recorded may be readily found again. 
3 125. Marker for Preparations. (Figs. 6r-66). - This instrument consists of an objective-like attachment which may be screwed into the nose:piece of the microscope. It bears on its lower end (Figs. 6I-3) a small brush and the brush can be made more or less eccentric and can be rotated, thus making a larger or smaller circle. In using the marker the brush is dipped in colored shellac or other cement and when the part of the preparation to be marked is found and put exactly in the middle of the field the objective is turned aside and the marker turned into position. The brush is brought carefully in contact with the cover-glass and rotated. This will make a delicate ring of the colored cement around the object. Within this very small area the desired object can be easily found on any microscope. The brush of the marker should be cleaned with $95 \%$ alcohol after it is used. (Proc. Amer. Micr. Soc., I894, pp. I I 2-I I8).

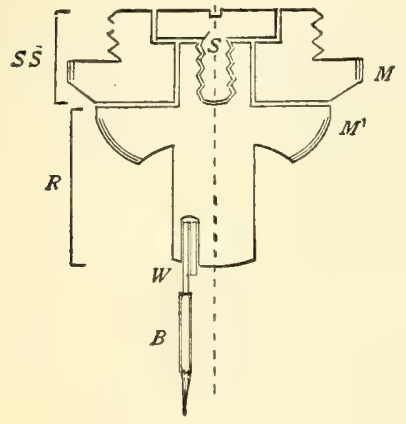

$6 \mathrm{r}$.

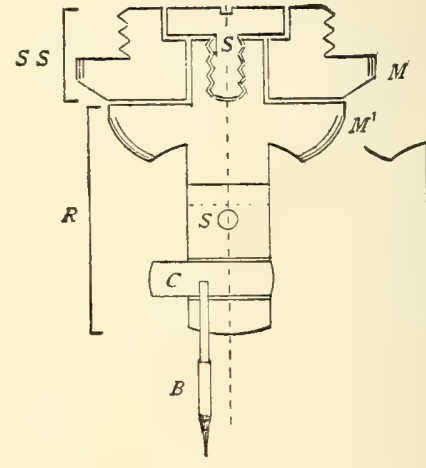

62.

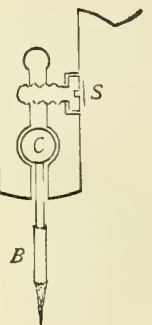

63.

Figs. 6I-63. Sectional Views of the two Forms of the Marker.

FIG. 6r. The simplest form of marker. It consists of the part SS with the milled edge $(M)$. This part bears the society or objective screw for attaching the marker to the microscope. R. Rotating part of the marker. This bears the eccentric brush $(B)$ at its lower end. The brush is on a wire $(W)$. This wire is eccentric, and may be made more or less so by bending the wire. The central dotted line coincides with the axis of the microscope. The revolving part is connected with the "Society Screw" by the small screw $(S)$.

FIG. 62. SS, R, and B. All parts same as with Fig. 61, except that the brush is carried by a sliding cylinder the end view being indicated in Fig. 63.

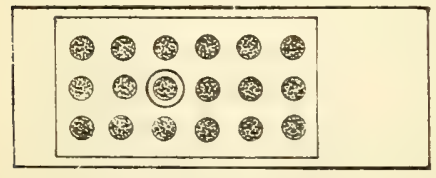

64

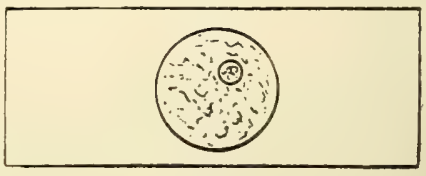

65 


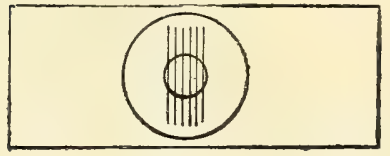

66

FIGs. 64, 65, 66. Specimens Showing the Use of the Marker.

In Fig. 64 a section of a series is marked to indicate that this section shows something especially well. In Fig. 65 some blood corpuscles showing ingested carbon very satisfactorily are surrounded by a minute ring, and in Fig. 66 the lines of a micrometer are ringed to facilitate finding the lines.

\& I26. Pointer in the Ocular. - The Germans have a pointer ocular (SpitzenOkular), an ocular with one or two delicate rods or pointers at the level of the real image, that is, at the level of the diaphragm (Figs. 2I, 30 D). For the purposes of demonstrating any particular structure or object in the field, a temporary pointer may be easily inserted in any ocular as follows: Remove the eye-lens and with a little mucilage or Canada Balsam fasten an eye-lash (cilium) to the diaphragm (Fig. 3o D) so that it will project about half way across the opening. If one uses this ocular, the pointer will appear in the field and one can place the specimen so that the pointer indicates it exactly, as in using a pointer on a diagram or on the black-board. It is not known to the author who devised this method. It is certainly of the greatest advantage in demonstrating objects like amoebas or white blood corpuscles to persons not familiar with them, as the field is liable to have in it many other objects which are more easily seen.

\&. 127. Mechanical Stage.-For High School and ordinary laboratory work a mechanical stage is not needed; but for much work, especially where high objectives are used a mechanical stage is of great advantage. It is also advantageous if the mechanical stage can be easily removed (see Figs. 67 to 70 ). The one found on the most expensive American and English microscopes for the last twenty years and the one now present on the larger continental microscopes, is excellent for high powers and preparations of moderate dimensions, but for the study of serial sections and large sections or preparations in general, mechanical stages like those shown in Figs. 68-69 are more useful. This form of mechanical stage has the advantage of giving great lateral and forward and backward motion. It is a modification of the mechanical stage of Tolles. The modification consists in doing away with the thin plate and having a clamp to catch the ends of the glass slide. The slide is then moved on the face of the stage proper. This modification was first made by Mayall. It has since been modified by Reichert, Zeiss, Leitz, and others in Europe and by the Bausch \& Lomb Optical Co., Queen \& Co., and the Spencer Lens Co., in America.-Jour. Roy. Micr. Soc., I885, p. I22. See also Zeit. Wiss. Mikroskopie (II), I885, pp. 289-295; I887 (IV, pp. 25-30).

Those figured below have the great advantage of ready removal from the stage of the microscope, thus leaving it free. They have also the very excellent feature that with them one can explore an entire slide full of serial sections, as the sections are ordinarily mounted, $i$. $e_{\text {. }}$, under a cover-glass $24 \times 50 \mathrm{~mm}$. 


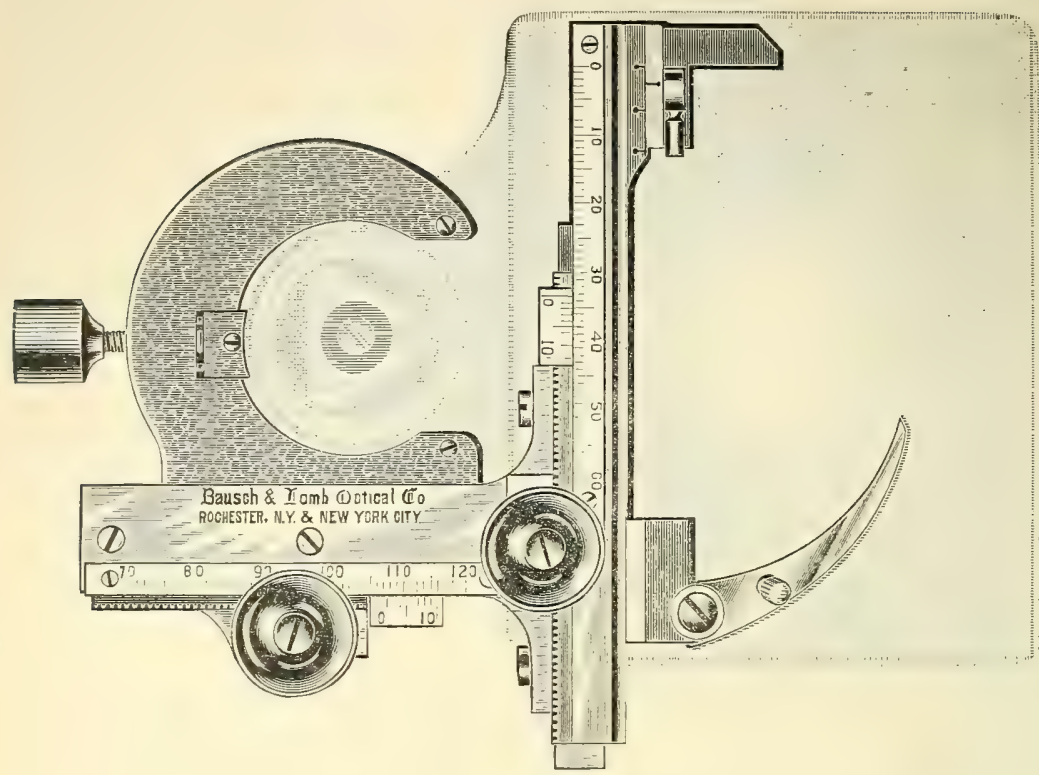

FIG 67 .

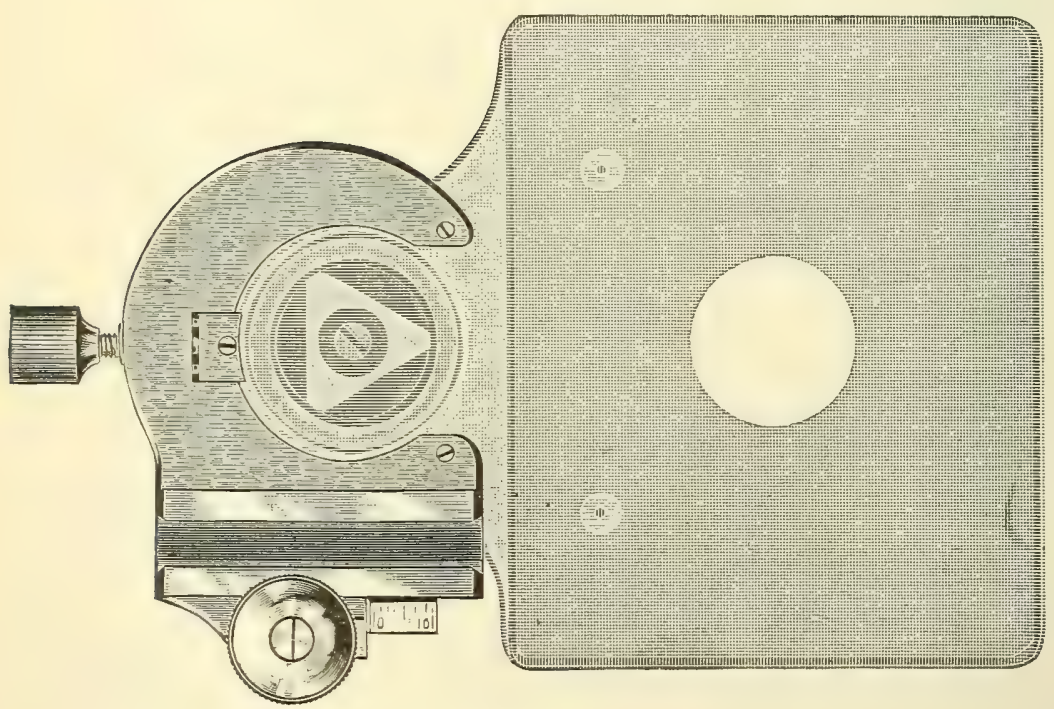

FIG. $67 \mathrm{a}$.

FIGs. 67, 67a. The remoz'able mechanical stage of the Bausch \& Lomb Optical Company. In the upper figure it is in position on the stage of the microscope; in the lower figure only' the clamping part is in position, the rest having been removed to leave the stage of the microscope free. 


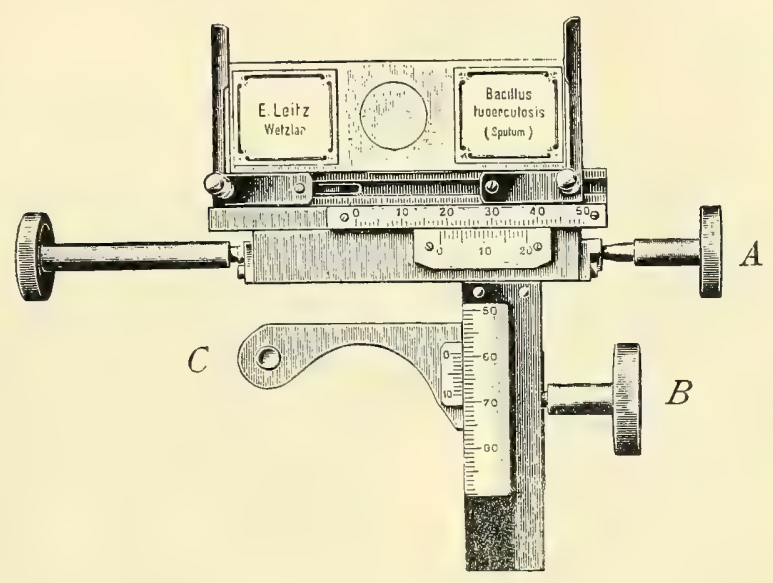

FIG. 68.

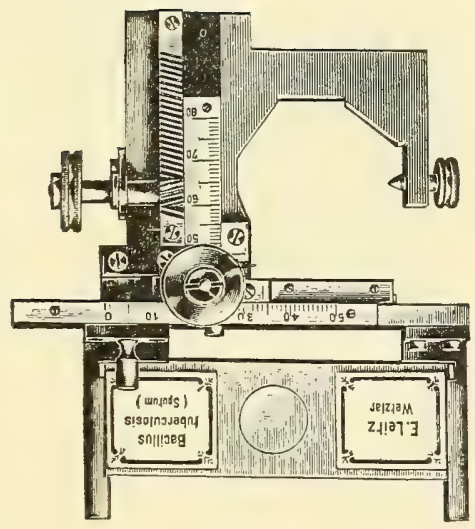

FIG. 68a.

FIG. 68, 68a. Two forms of removable mechanical stage by Leitz. 68 is somewhat more complex and expensive. Both have the desirable features mentioned in \& 127 . 


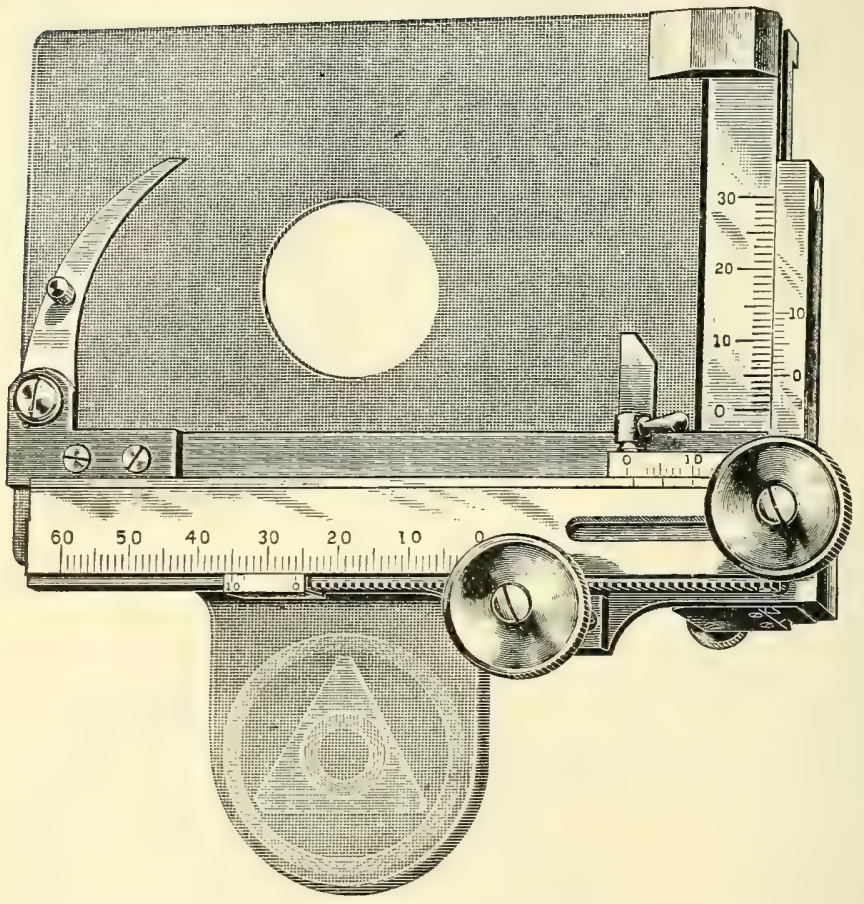

FIG. 69. The removable mechanical stage of the Spencer Lens Co. It is a modification of a form der'ised by Winkel. Besides the general features mentioned in \& 127 it has the adiantage of fitting any square stage. It is fastened to the stage by the clamps shown at the right. Another form is made having ont screw on the side. 
FIG. 7o. Krauss' Method of Marking Objectives on a Revolving. Nose-Piece.

As seen in the figure, the equivalent focus of the objective is engraved on the diaphragm above the back lens and may be very readily seen in rotating the nose-piece. This is of great advantage, as one can see what objective is coming into place without trouble. It is also an advantage in showing where each objective belongs when the microscope comes from the manufacturers. The method is coming into general use.

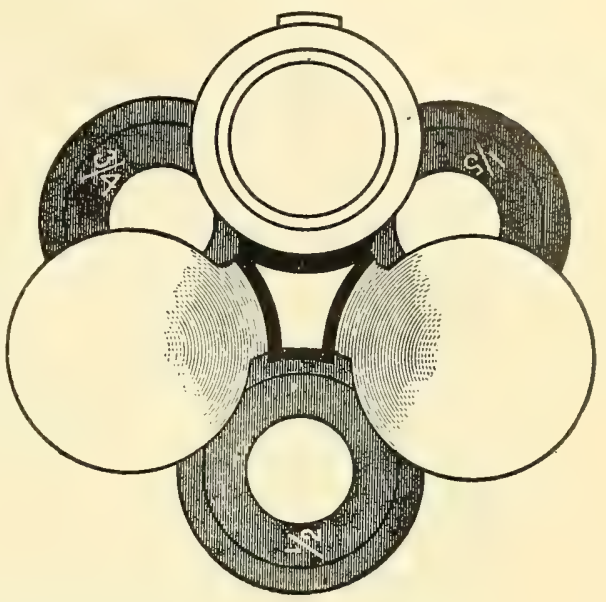

\section{FIGURES OF LABORATORY AND HIGH SCHOOL MICROSCOPES}

In order that teachers and students may get a good general idea of the appearance of microscopes of various makers for high school and advanced laboratory work a few pictures are appended of the microscopes most used in the United StatesThis has been rendered possible by the courtesy of the manufacturers or importers. It will be seen that the microscope for the advanced laboratory work is on the left and the cheaper one for school and less advanced laboratory work is on right. The microscopes are arranged in alphabetical order.

Laboratory microscopes which will answer nearly all the requirements for work in Biology, including Histology, Embryology, Pathology and Bacteriology, are lister in the makers catalogs at about \$100.00. The less expensive microscopes shown on the right are listed at $\$ 25$ to $\$ 45$. There is usually a discount of IO $1 / 1$, or more from these prices. Fortunately in the State of New York the State pays half for high school apparatus, so that there is no reason why every high school should not be properly equipped with microscopes of a good grade. To avoid misunderstanding it should be added that the quality of the oculars and objectives on the high school microscopes figured is the same as for the best laboratory microscopes. The mechanical work also is of excellent quality.

During the last ten years great vigor has been shown in the microscopical world. This has been stimulated largely by the activity in biological science and the widespread appreciation of the microscope, not only as a desirable, but as a necessary instrument for study and research. The production of the new kinds of glass, (Jena glass), and the apochromatic objectives has been a no less potent factor in promoting progress. The stadent is advised to write to one or more of the opticians for complete catalogs. (See list, p. 2 of cover). 


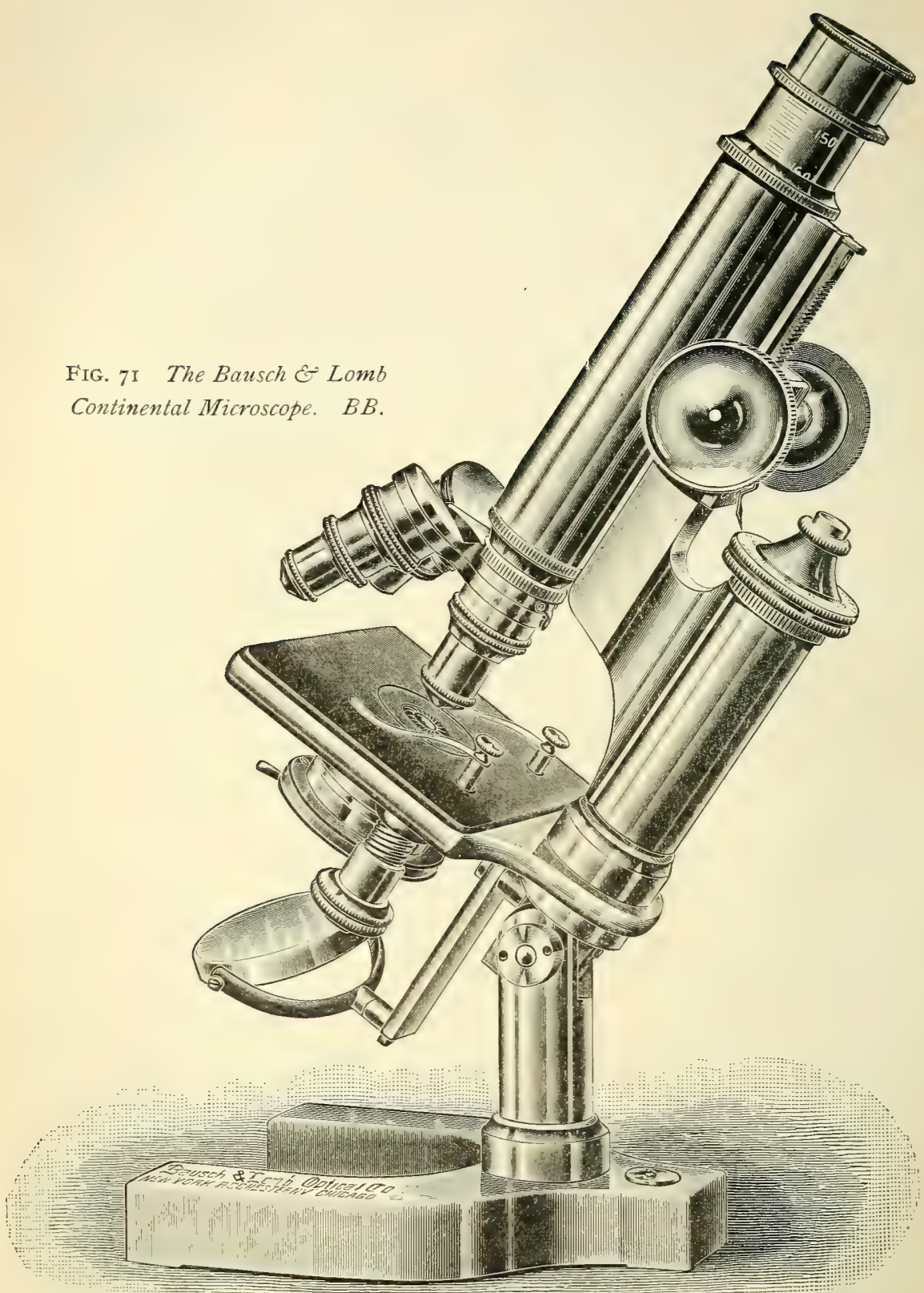


FIG. 72. The Bausch \& Lomb Continental Microscope. B. 


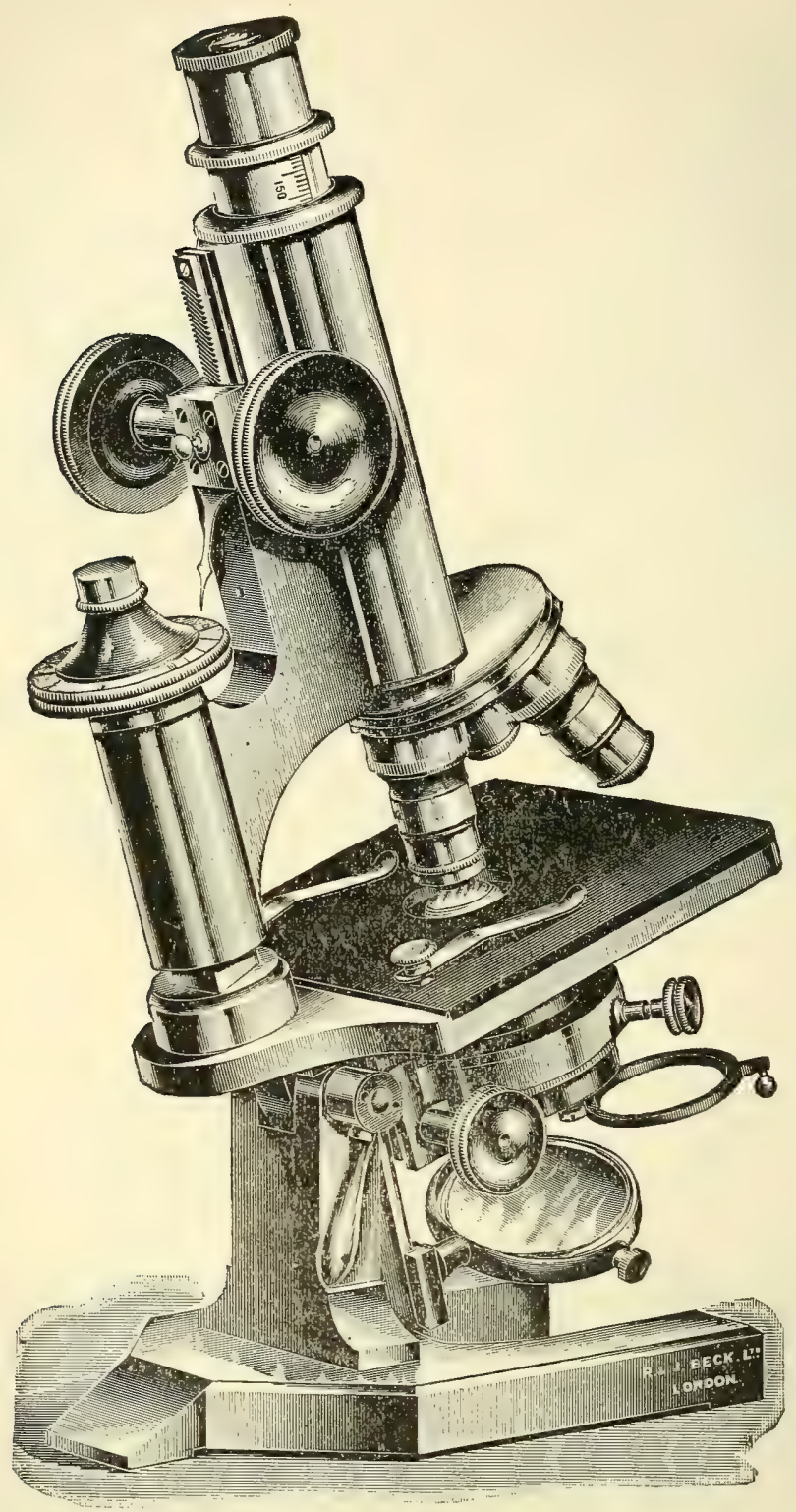

FIG. 73. R. G J. Beck's New Continental Microscope, No. I152 (Williams, Brown \& Earle, Philadelphia). 


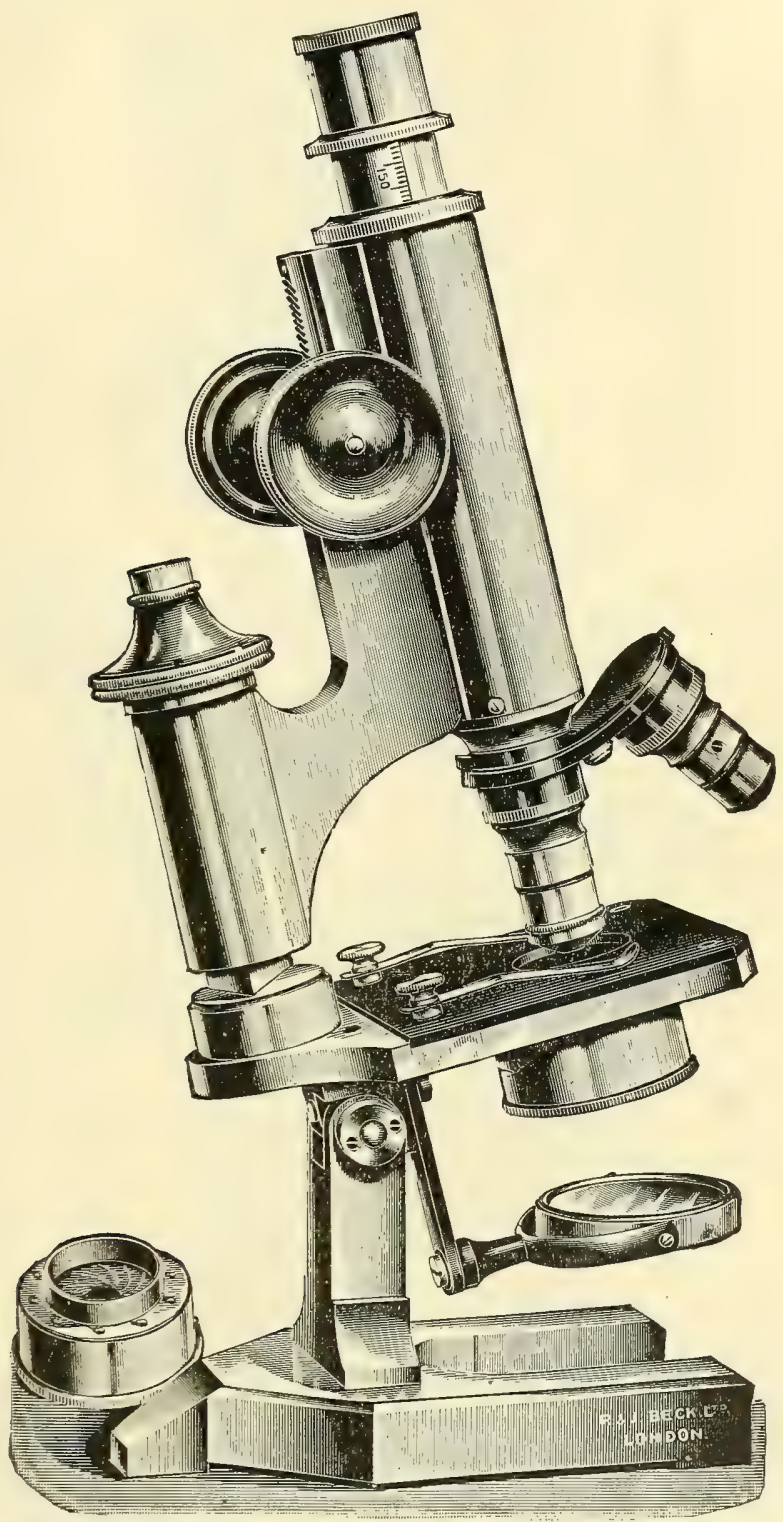

Fig. 74. R. \& J. Beck's Nerw Continental Microscope, No. I125 (Williams, Brown \& Earle, Philadelphia). 


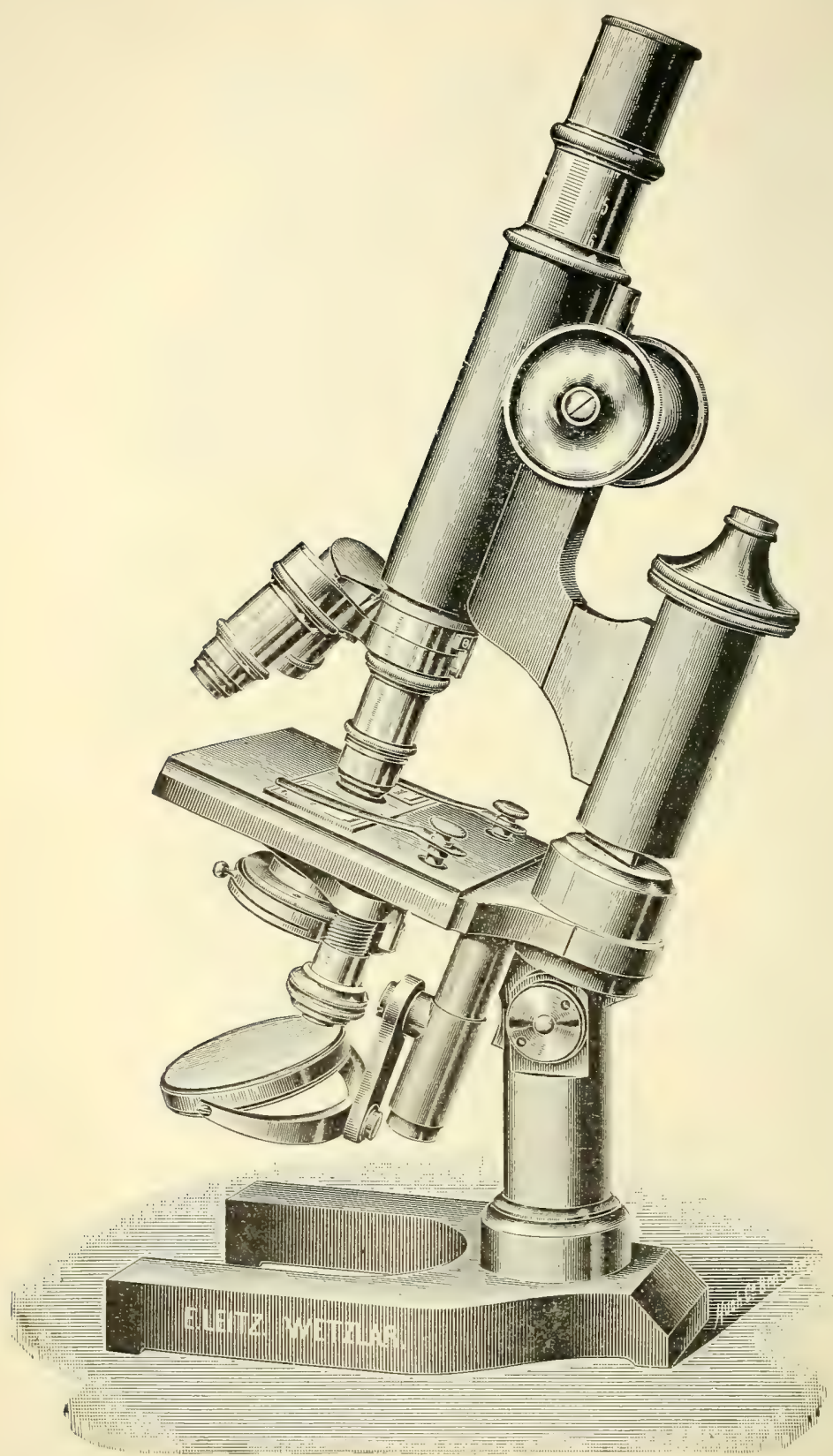

Fig. 75. E. Leitz Microscope IIC. (Wm. Krafft, New York). 


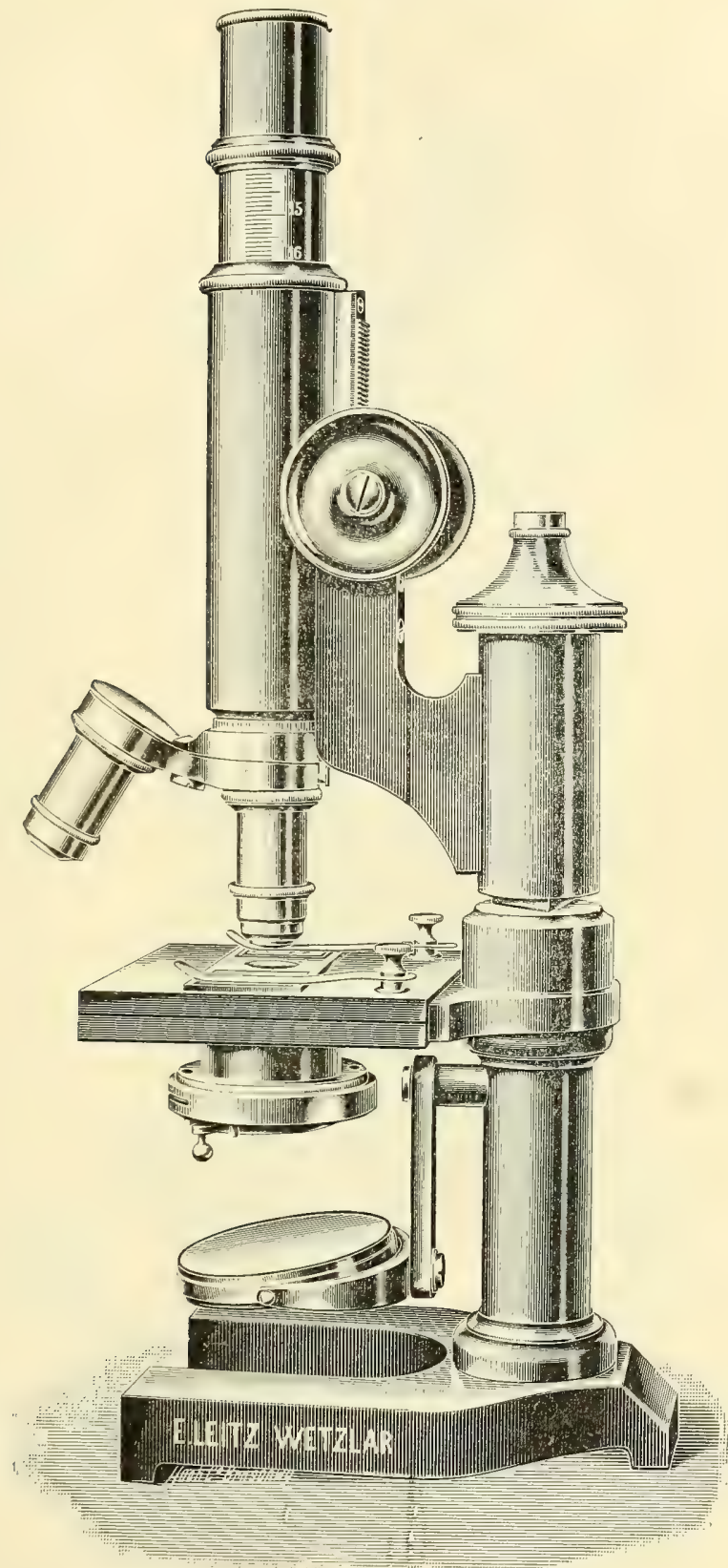

FIG. 76. Leitz Microscope II E. It will be noticed that this microscope has no joint for inclination ( Wm. Krafft, $N . Y$.). 


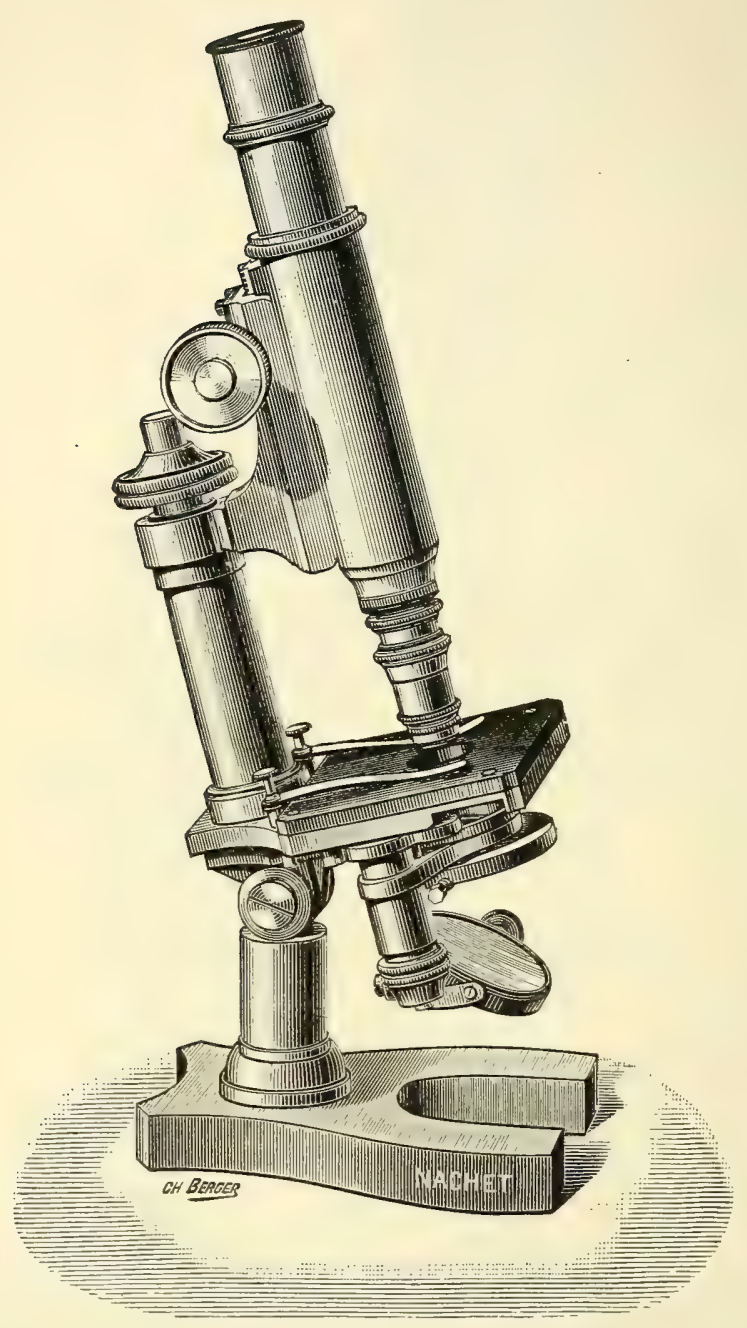

Fig. 77. Nachet's Microscope 6 bis. Old model. Nachet is a successor to Hartnack who introduced the present "Continental Model" Mayall, Cantor Lectures, p. 68 (Franklin Laboratory Supply Co., Boston). 


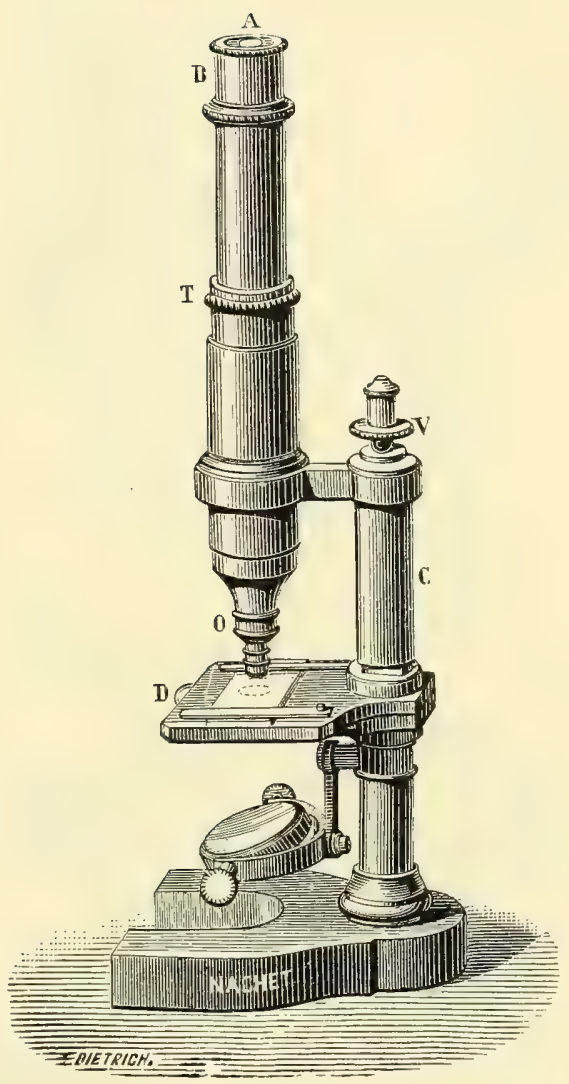

Frg. 78. Nachet's Microscope, No. II (Franklin Laboratory Supply Co., Boston).

This microscope has no joint for inclination, and no rack and pinion for coarse adjustment. For coarse adjustment, the tube is pulled up and down with the hands. This kind of coarse adjustment was much more common ten years ago than now. (See also Fig. 82.) 

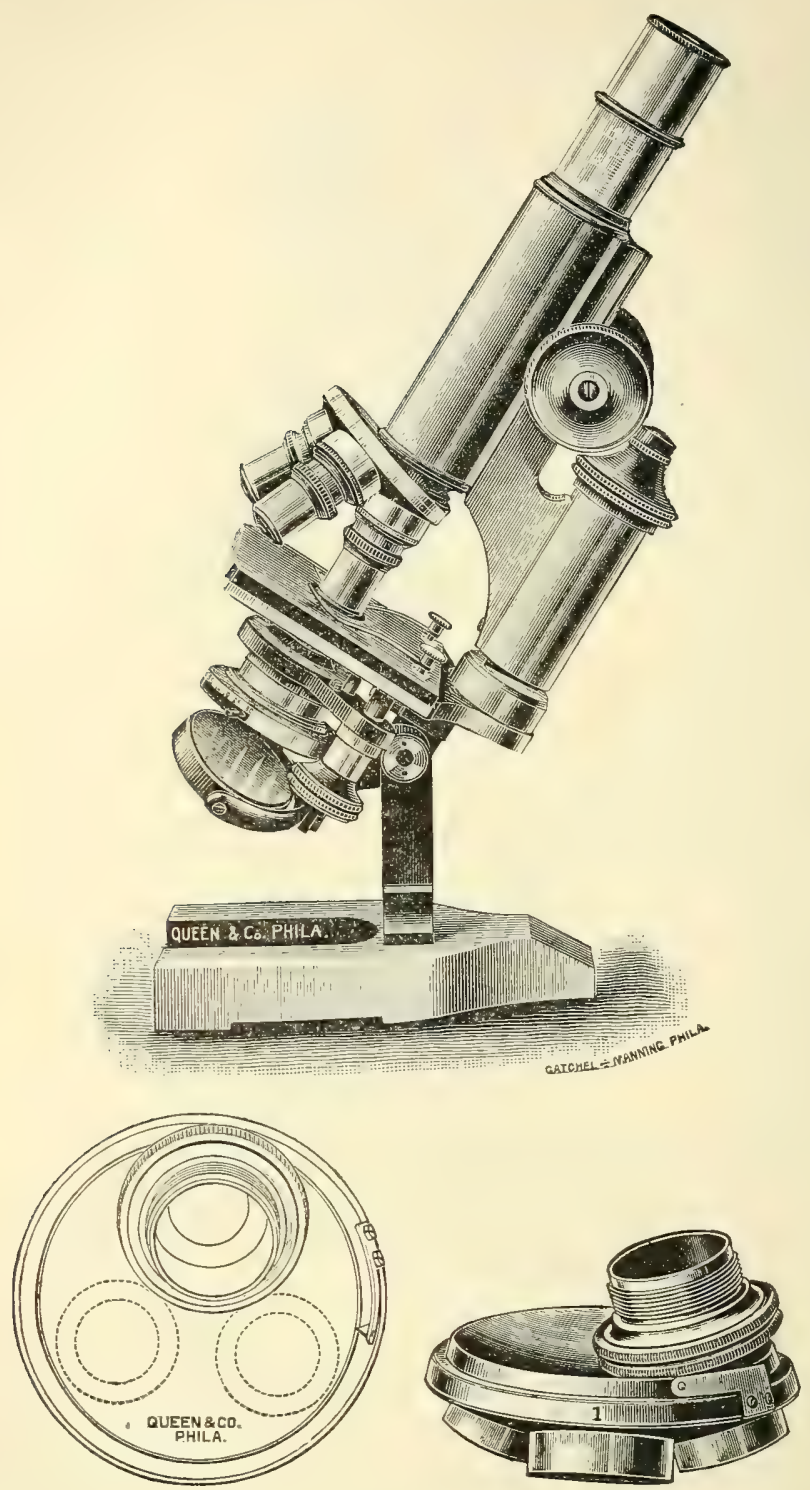

FiG. 79. A. Queen \&ै Co's Continental Microscope, No. II. B. Dust-proof, triple nose-piece. The difference between this and the ordinary form can be seen by comparing with Fig. 36 . This form of revolving nose-piece has been made for many years by Winkel of Goettingen. 


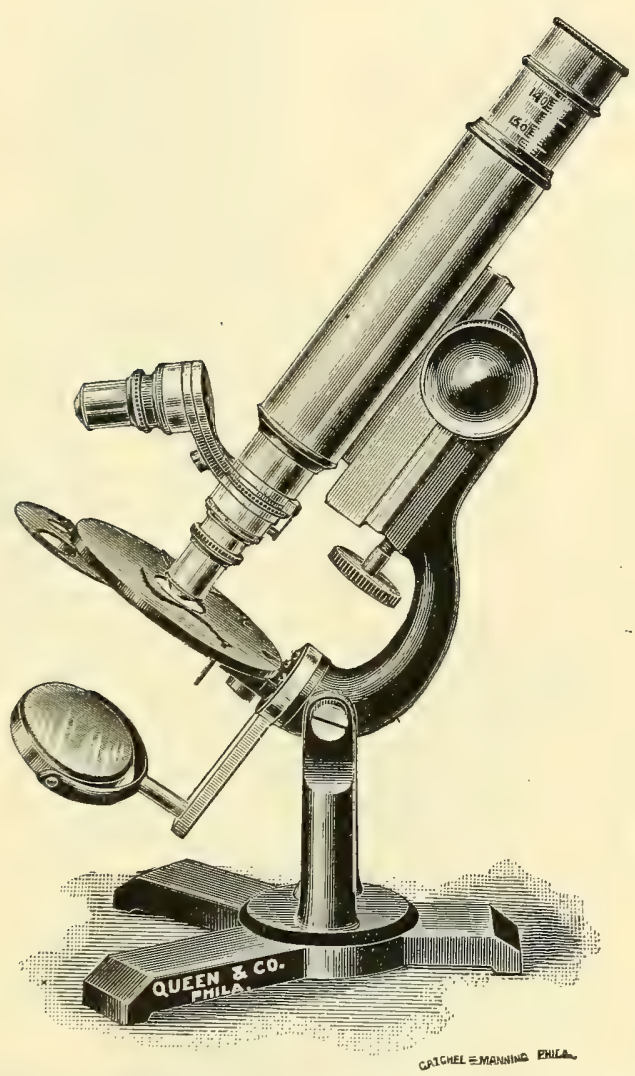

Fig. 8o. Queen \&ै Co's Acme Microscope for Schools 
FIG. 8I. Reichert's New Microscope, No. III B. (Richards \& Co., N. Y. and Chirago).

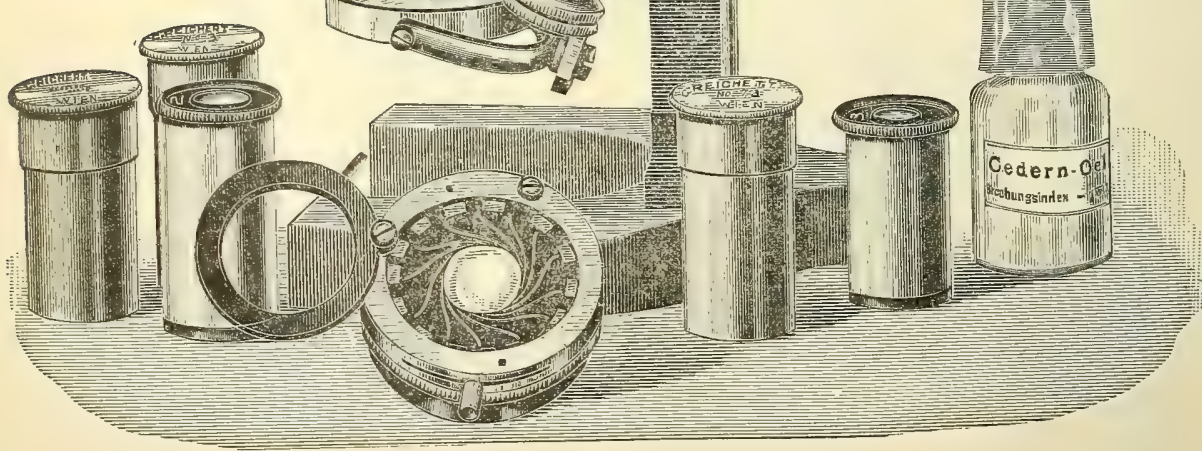




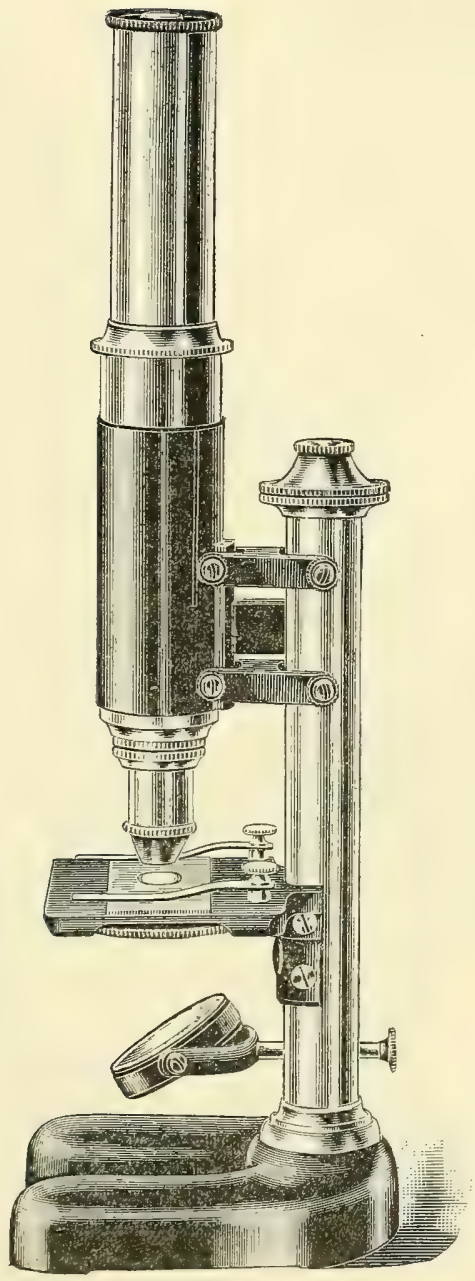

FIG. 82. Reichert's Microscope, No. $V$. This has no joint for inclination. (Richards of Co., New York and Chicago). 
FIG. 83. The Spencer Lens Company's Microscope No. $I$. This has the standard size, Continental oculars, and the objectives and oculars have the equivalent focus in millimeters.

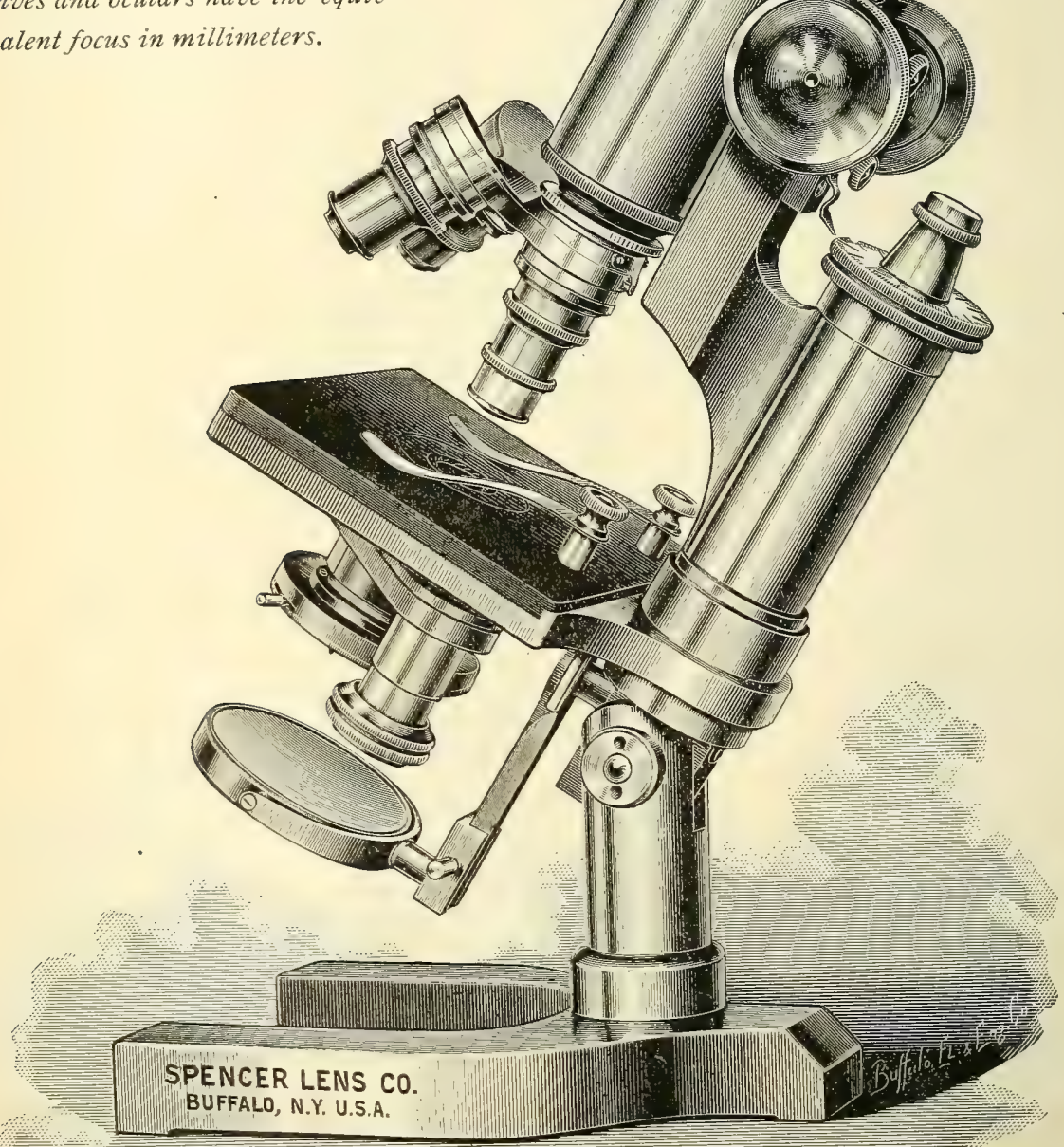


Fig. 84. The Spencer Lens

Company's Microscope, No. 2.

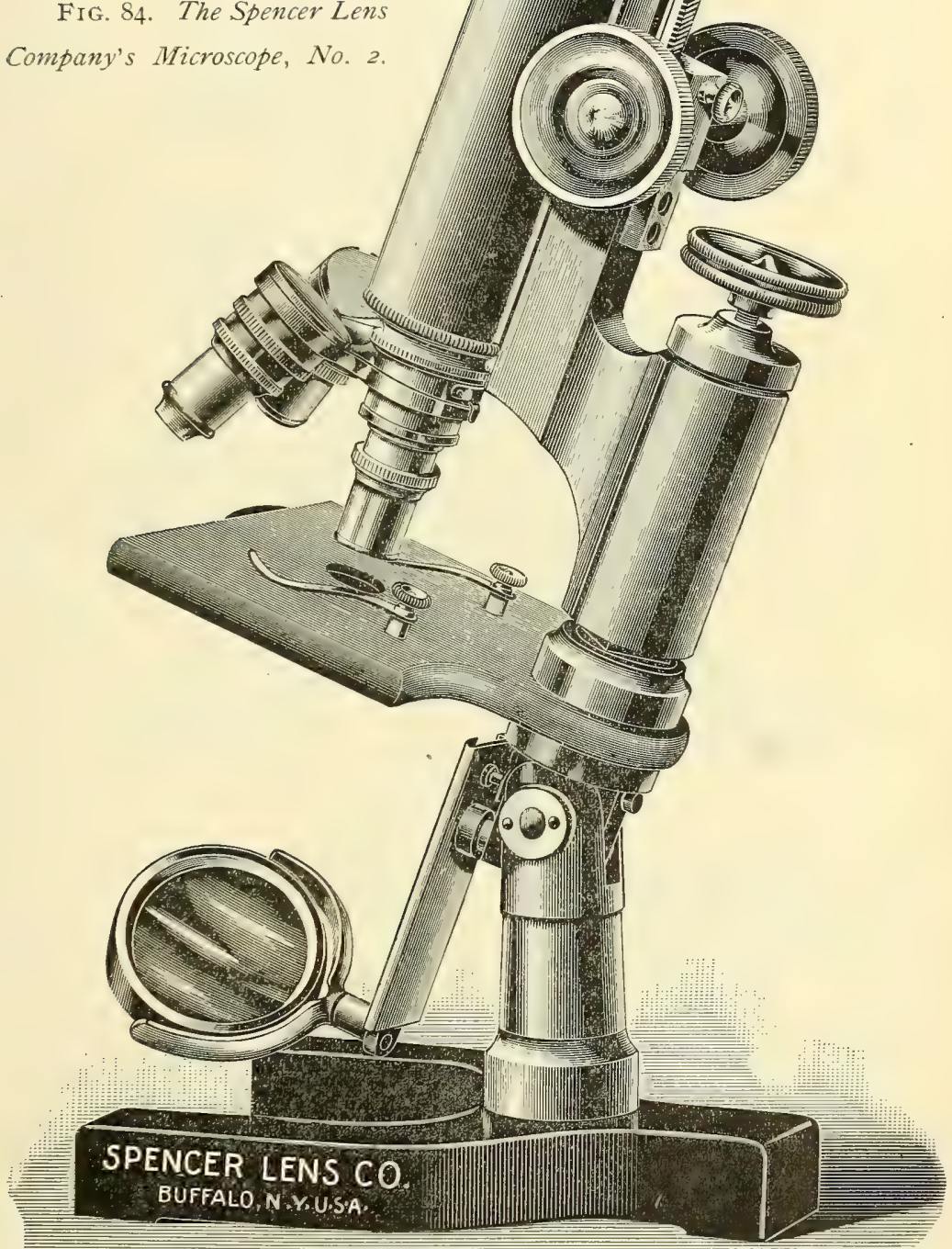




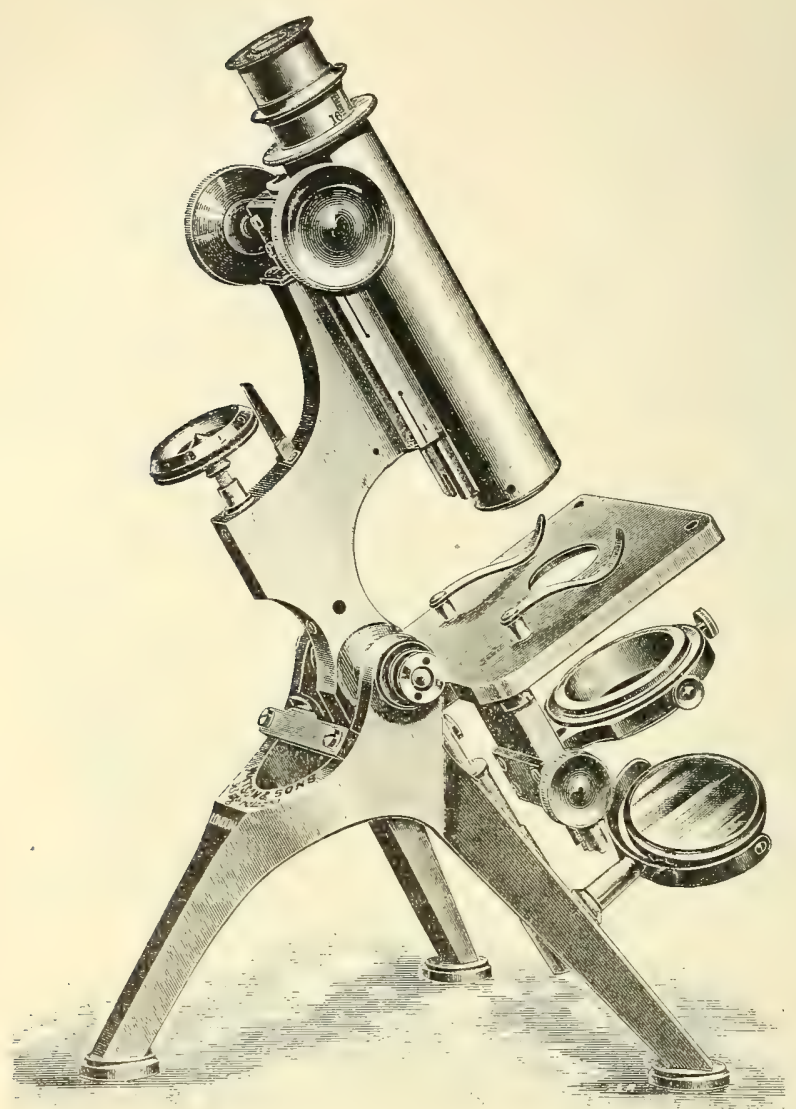

Fig. 85. Watson G Sons, Edinburgh Students' Microscope (Stand G). This is a good representative of the tripod-base, English models of to-day. It is in general like the Powell and Lealand stands which have held their position with the foremost English microscopists for the last 40 years. (See Carpenter-Dallinger, p. 172 .)

Microscopes with tripod base something after this pattern are now being made on the Continent.

Attention is called to the sub-stage for the condenser. It possesses centering screws so that any apparatus used in it may be accurately cenlered. It is to be hoped that all microscopes of this grade will soon be supplied with a centering sub-stage. 


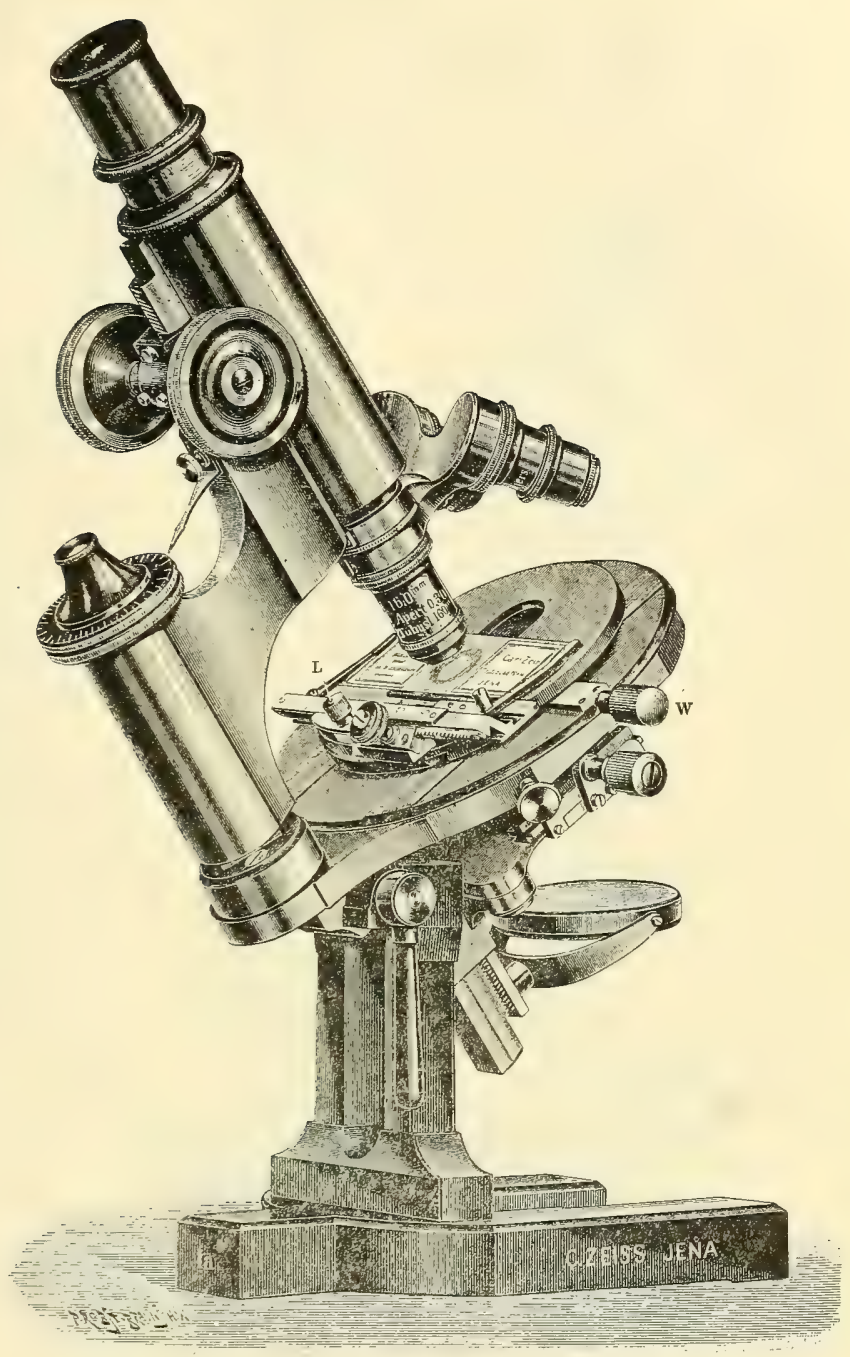

FIG. 86. Zeiss Microscope- $I^{\mathrm{a}}$ with Mechanical Stage. This figure from Zeiss' Catalog No. 3o, represents the Continental Model of Microscope in its most perfect form.

K. Milled head of the screw for the lateral movements of the stage.

$L$. Screw for fixing the laterally moving mechanism of the stage. By unscrewing this the laterally moving part may be removed, leaving the plain stage.

$W$. Screw for moving the stage forward and backward. 


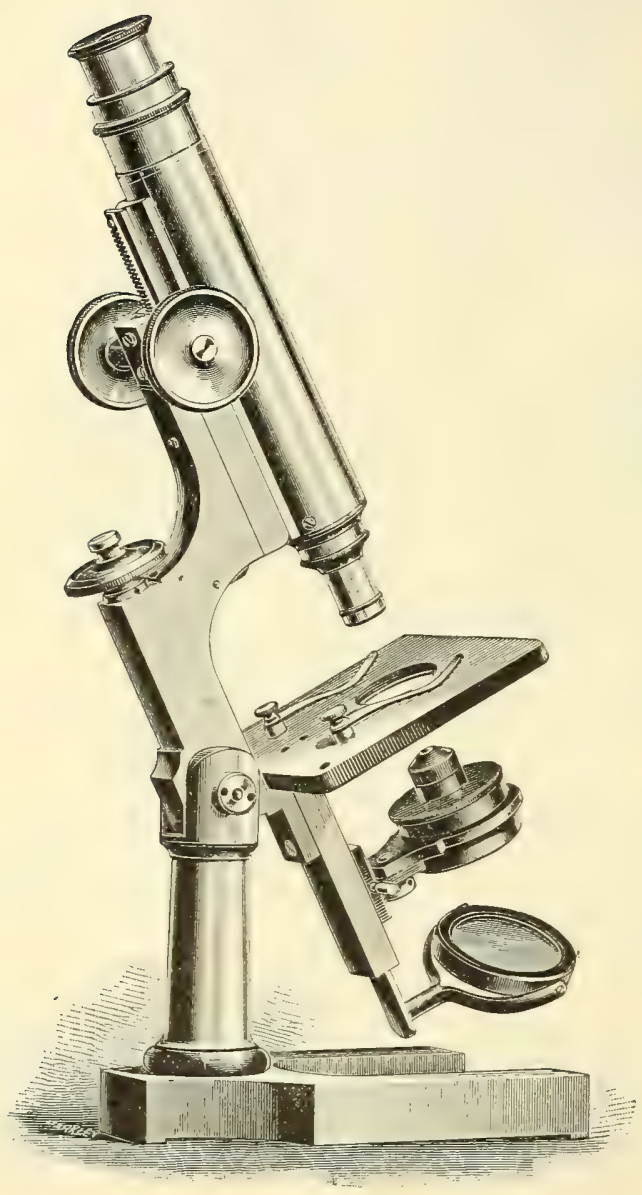

FIG. 87, Zentmeyer's Microscope, No. $V$. 


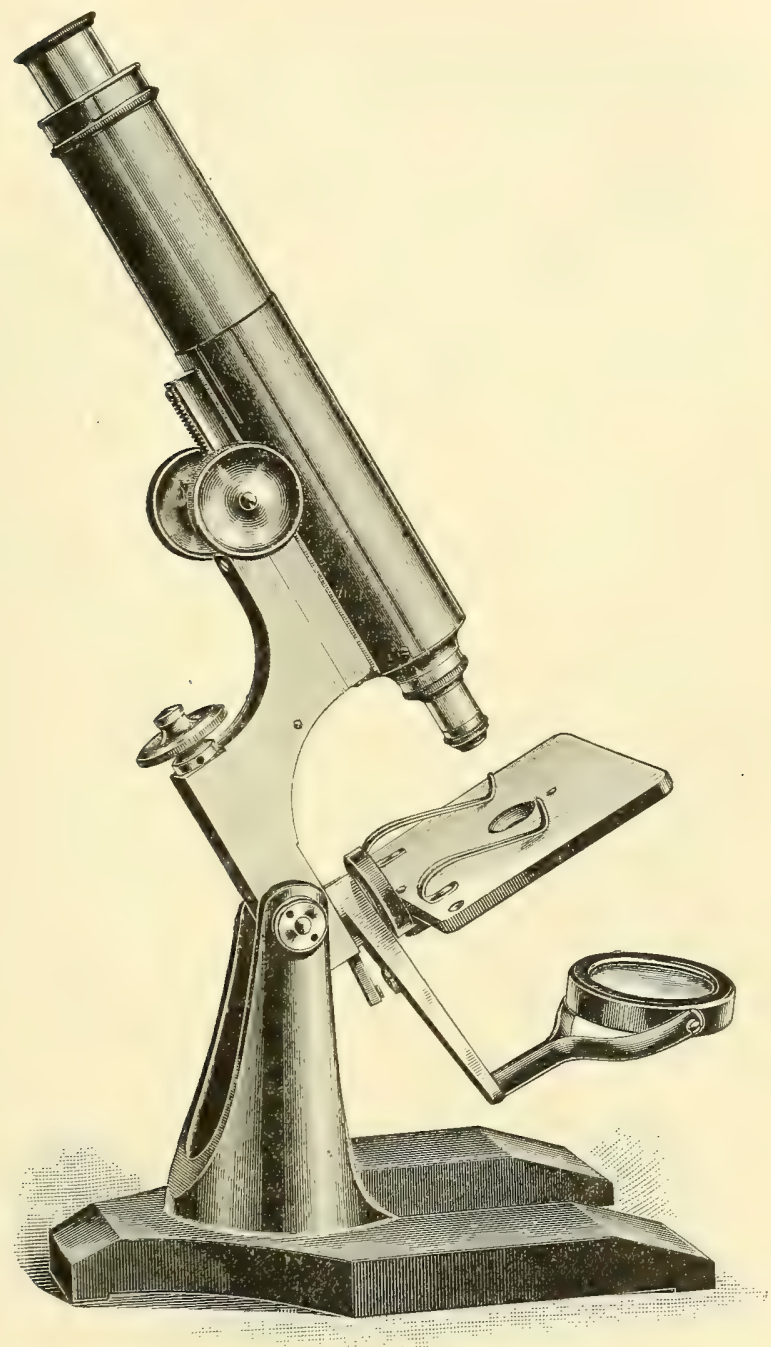

Fig. 88. Zentmayer's Microscope, No. $I V$. 


\section{CHAPTER III}

\section{INTERPRE'TATION OF APPEARANCES}

\section{APPARATUS AND MATERIAL FOR CHAPTER III}

A laboratory, compound microscope ( $\xi$ I II); Preparation of fly's wing ; 50 per cent. glycerin; Slides and covers; Preparation of letters in stairs (Fig. 89); Mucilage for air-bubbles and olive or clove oil for oil-globules ( 8 I36-I39). Solid glass rod, and glass tube ( 8 I $44-146)$; Collodion ( $\%$ I 46$)$; Carmine, India ink, or lamp black ( 8 I48-I50); Frog, castor oil and micro-polariscope ( 8 I 52).

\section{INTERPRETATION OF APPEARANCES UNDER THE MICROSCOPE}

- I29. General Remarks.-The experiments in this chapter are given secondarily for drill in manipulation, but primarily so that the student may not be led into error or be puzzled by appearances which are constantly met with in microscopical investigation. Anyonecan look into a microscope, but it is quite another matter to interpret correctly the meaning of the appearances seen.

It is especially important to remember that the more of the relations of any object are known, the truer is the comprehension of the object. In microscopical investigation every object should be scrutinized from all sides and under all conditions in which it is likely to occur in nature and in microscopical investigation. It is best also to begin with objects of considerable size whose character is well known, to look at them carefully with the unaided eye so as to see them as wholes and in their natural setting; then a low power is used, and so on, step by step until the highest power arailable has been employed. One will in this way see less and less of the object as a whole, but every increase in magnification will give increased proninence to detail, detail which might be meaningless when taken alone and independent of the object as a whole. The pertinence of this advice will be appreciated when the student undertakes to solve the problems of histology ; for even after all the years of incessant labor spent in trying to make out the structure of man and the lower animals, many details are still in doubt, the same visual appearances being quite differently interpreted by eminent observers. 
Appearances which seem perfectly unmistakable with a low power may be found erroneous or very inadequate, for details of structure that were indistinguishable with the low power may become perfectly evident with a higher power or a more perfect objective. Indeed the problems of microscopic structure appear to become ever more complex, for difficulties overcome by improvements in the microscope simply give place to new difficulties, which in some cases render the subject more obscure than it appeared to be with the less perfect appliances.

The need of the most careful observation and constant watchfulness lest the appearances may be deceptive are thus admirably stated by Dallinger (see Carpenter-Dallinger, p. 427): "The correctness of the conclusions which the microscopist will draw regarding the nature of any object from the visual appearances which it presents to him when examined in the various modes now specified will necessarily depend in a great degree upon his previous experience in microscopic observation and upon his knowledge of the class of bodies to which the particular specimen may belong. Not only are observations of any kind liable to certain fallacies arising out of the previous notions which the observer may entertain in regard to the constitution of the objects' or the nature of the actions to which his attention is directed, but even the most practiced observer is apt to take no note of such phenomena as his mind is not prepared to appreciate. Errors and imperfections of this kirid can only be corrected, it is obvious, by general advance in scientific knowledge ; but the history of them affords a useful warning against hasty conclusions drawn from a too cursory examination. If the history of almost any scientific investigation were fully made known it would generally appear that the stability and completeness of the conclusions finally arrived at had been only attained after many modifications, or even entire alterations, of doctrine. And it is therefore of such great importance as to be almost essential to the correctness of our conclusions that they should not be finally formed and announced until they have been tested in every conceivable mode. It is due to science that it should be burdened with as few false facts [artifacts] and false doctrines as possible. It is due to other truth-seekers that they should not be misled, to the great waste of their time and pains, by our errors. And it is due to ourselves that we should not commit our reputation to the chance of impairment by the premature formation and publication of conclusions which may be at once reversed by other observers better informed than ourselves, or may be proved fallacious at some future time, perhaps even by our 
own more extended and careful researches. The suspension of the judgment whenever there seems room for doubt is a lesson inculcated by all those philosophers who have gained the highest repute for practical wisdom ; and it is one which the microscopist cannot too soon learn or too constantly practice."

For these experiments no condenser is to be used except where specifically indicated.

SI30. Dust or Cloudiness on the Ocular.-Eimploy the I6 mm. ( $2 / 3$ in.) objective, low ocular, and fly's wing as object.

Unscrew the field-lens and put some particles of lint from dark cloth on its upper surface. Replace the field-lens and put the ocular in position $(\$ 48)$. Light the field well and focus sharply. The image will be clear, but part of the field will be obscured by the irregular outline of the particles of lint. Move the object to make sure this appearance is not due to it.

Grasp the ocular by the milled ring, just above the tube of the microscope, and rotate it. The irregular objects will rotate with the ocular. Cloudiness or particles of dust on any part of the ocular may be detected in this way.

S I I. Dust or Cloudiness on the Objective.-Employ the same ocular and objective as before and the fly's wing as object. Focus and light well, and observe carefully the appearance. Rub glycerin on one side of a slide near the end. Hold the clean side of this end close against the objective. The image will be obscured, and cannot be made clear by focusing. Then use a clean slide and the image may be made clear by elevating the tube slightly. The obscurity produced in this way is like that caused by clouding the front-lens of the objective. Dust would make a dark patch on the image that would remain stationary while the object or ocular is moved.

If a small diaphragm is employed and it is close to the object, only the central part of the field will be illuminated, and around the small light circle will be seen a dark ring (Fig. 42). If the diaphragm is lowered or a sufficiently large one employed the entire field will be lighted.

$\$$ I32. Relative Position of Objects or parts of the same object. The general rule is that objects highest up come into focus last in focusing up, first in focusing down.

$\$$ I33. Objects having Plane or Irregular Outlines.-As object use three printed letters in stairs mounted in Canada balsam (Fig. 89). The first letter is placed directly upon the slide, and covered with a 
small piece of glass about as thick as a slide. The second letter is placed upon this and covered in like manner. The third letter is placed upon the second thick cover and covered with an ordinary cover-glass. The letters should be as near together as possible, but not over-lapping. Employ the same ocular and objective as above ( $\$$ I 30 ).

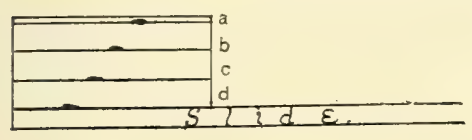

sectional view. Slide. The glass slide on which the letters are mounted.

Lower the tube till the objective almost touches the top letter, then look into the microscope, and slowly focus up. The lowest letter will first appear, and then, as it disappears, the middle one will appear, and so on. Focus down, and the top letter will first appear, then the middle one, etc. The relative position of objects is determined exactly in this way in practical work.

For example, if one has a micrometer ruled on a cover-glass i $5^{-25}$ hundredths mm. thick, it is not easy to determine with the naked eye which is the ruled surface. But if one puts the micrometer under a microscope and uses a $3 \mathrm{~mm}$. ( $1 / 8$ in.) objective, it is easily determined. The cover should be laid on a slide and focused till the lines are sharp. Now, without changing the focus in the least turn the cover over. If it is necessary to focus up to get the lines of the micrometer sharp, the lines are on the upper side. If one must focus down, the lines are on the under surface. With a thin cover and delicate lines this method of determining the position of the rulings is of considerable practical importance.

I34. Determination of the Form of Objects.-The procedure is exactly as for the determination of the form of large objects. That is, one must examine the various aspects. For example, if one were placed in front of a wall of some kind he could not tell whether it was a simple wall or whether it was one side of a building unless in some way he could see more than the face of the wall. In other words, in order to get a correct notion of any body, one must examine more than one dimension, - - two for plane surfaces, three for solids. So for microscopic objects, one must in some way examine more than one face. To do this with small bodies in a liquid the bodies may be made to roll over by pressing on one edge of the cover-glass. And in rolling over the various aspects are presented to the observer. With solid bodies, 
like the various organs, correct notions of the form of the elements can be determined by studying sections cut at right angles to each other. The methods of getting the elements to roll over, and of sectioning in different planes are in constant use in Histology, and the microscopist who neglects to see all sides of the tissue elements has a very inadequate and often a very erroneous conception of their true form.

$\$$ 135. Transparent Objects having Curved Outlines.-The success of these experiments will depend entirely upon the care and skill used in preparing the objects, in lighting, and in focusing.

Employ a $3 \mathrm{~mm}$. ( $1 / 8$ in.) or higher objective and a high ocular for all the experiments. It may be necessary to shade the object ( $\$$ I09) to get satisfactory results. When a diaphragm is used the opening should be small and it should be close to the object.

S i36. Air Bubbles.-Prepare these by placing a drop of thin mucilage on the center of a slide and beating it with a scalpel blade until the mucilage looks milky from the inclusion of air bubbles. Put on a cover-glass but do not press it down.

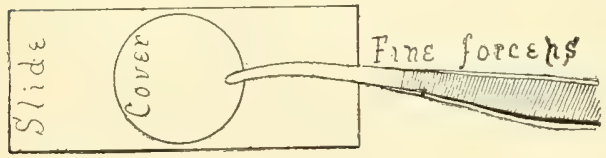

FIG. 90. Diagram showing how to place a coverglass upon an object with the forceps.

\$37. Air Bubbles with Central Illumination.-Shade the object; and with the plane mirror, light the field with central light (Fig. 23).

Search the preparation until an air bubble is found appearing about I $\mathrm{mm}$. in diameter, get it into the center of the field, and if the light is central the air bubble will appear with a wide, dark, circular margin and a small bright center. If the bright spot is not in the center, adjust the mirror until it is.

This is one of the simplest and surest methods of telling when the light is central or axial when no condenser is used $(\$ 65)$.

Focus both up and down, noting that, in focusing up, the central spot becomes very clear and the black ring very sharp. On elevating the tube of the microscope still more the center becomes dim, and the whole bubble loses its sharpness of outline.

I38. Air Bubbles with Oblique Illumination.- Remore the sub-stage of the microscope and all the diaphragms. Sring the mirror so that the rays may be sent rery obliquely upon the object (Fig. 23, 
C). The bright spot will appear no longer in the center but on the side away from the mirror (Fig. yI).

$\S$ I39. Oil Globules. - Prepare these by beating a small drop of clove oil with mucilage on a slide and covering as directed for air bubbles ( $\S \mathrm{r} 37$ ), or use a drop of milk.

$\S$ I 40. Oil Globules with Central Illumination.-Use the same diaphragm and light as above (\$ I 37 ). Find an oil globule appearing about I $\mathrm{mm}$. in diameter. If the light is central a bright spot will appear in the center as with air. Focus up and down as with air, and note that the bright center of the oil globule is clearest last in focusing up.

FIG. 9I. Very small Globule of Oil (O) and an Air Bubble (A) seen by Oblique Light. The arrow indicates the direction of the light rays.

\section{$\S$ I 4 I. Oil Globules with Oblique Illumination.-} Remove the sub-stage, etc., as above, and swing the mirror to one side and light with oblique light. The bright spot will be eccentric, and will appear to be on the same side as the mirror (Fig. 9I).

$\S$ I42. Oil and Air Together.-Make a preparation exactly as described for air bubbles ( $\$$ 1 36 ), and add at one edge a little of the mixture of oil and mucilage ( $\$$ I39); cover and examine.

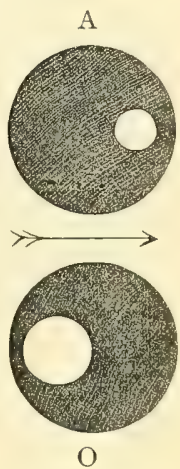

$\mathrm{O}$

The sub-stage need not be used in this experiment. Search the preparation until an air bubble and an oil globule, each appearing about I mm. in diameter, are found in the same field of view. Light first with central light, and note that, in focusing up, the air bubble comes into focus first and that the central spot is smaller than that of the oil globule. Then, of course, the black ring will be wider in the air bubble than in the oil globule. Make the light oblique. The bright spot in the air bubble will move away from the mirror while that in the oil globule will move toward it. See Fig. 9r.*

I43. Air and Oil by Reflected Light.-Cover the diaphragm or mirror so that no transmitted light $(\$ 64)$ can reach the preparation, using the same preparation as in $\$ 42$. The oil and air will appear like globules of silver on a dark ground. The part that was darkest in

"It should be remembered that the image in the compound microscope is inverted (Fig. 2I), hence the bright spot really moves toward the mirror for air, and away from it for oil. 
each will be lightest, and the bright central spot will be somewhat dark.*

$\S$ I44. Distinctness of Outline.-In refraction images this depends on the difference between the refractive power of a body and that of the medium which surrounds it. The oil and air were very distinct in outline as both differ greatly in refractive power from the medium which surrounds them, the oil being more refractive than the mucilage and the air less. (Figs. 54-56.)

Place a fragment of a cover-glass on a clean slide, and cover it (see under mounting). The outline will be distinct with the unaided eye. Use it as object and employ the $16 \mathrm{~mm}$. (2/3 in.) objective and high ocular. Light with central light. The fragment will be outlined by a dark band. Put a drop of water at the edge of the cover-glass. It will run in and immerse the fragment. The outline will remain distinct, but the dark band will be somewhat narrower. Remove the cover-glass, wipe it dry, and wipe the fragment and slide dry also. Put a drop of $50 \%$ glycerin on the middle of the slide and mount the fragment of cover-glass in that. The dark contour will be much narrower than before.

Draw a solid glass rod out to a fine thread. Mount one piece in air, and the other in 50\% glycerin. Put a cover-glass on each. Employ the same optical arrangement as before. Examine the one in air first. There will be seen a narrow, bright band, with a wide, dark band on each side.

The one in glycerin will show a much wider bright central band, with the dark borders correspondingly narrow (Fig. 92, b). The dark contour depends also on the numerical aperture of the objective-being wider with low apertures. This can be readily understood when it is remembered that the greater the aperture the more oblique the rays of light that can be received, and the dark band simply represents an area in which the rays are so greatly bent or refracted (Figs. 54-56) that they cannot enter the objective and contribute to the formation of the image; the edges are dark simply because no light from them reaches the observer.

*It is possible to distinguish oil and air optically, as described above, only when quite high powers are used and very small bubbles are selected for observation. If a $16 \mathrm{~mm}$. ( $2 / 3 \mathrm{in}$.) is used instead of a $3 \mathrm{~mm}$. ( $1 / 8$ in.) objective, the appearances will vary considerably from that given above for the higher power. It is well to use a low as well as a high power. Marked differences will also be seen in the appearances with objectives of small and of large aperture. 
FIG. 92. Solid glass rod showing the
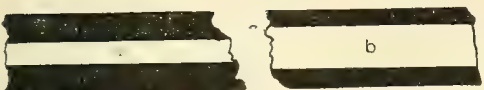
appearance when viewed with transmitted, central light, and with an objective of medium aperture.

a. Mounted in air. b. Mounted in 50 per cent. glycerin.

If the glass rod or any other object were mounted in a medium of the same color and refractive power, it could not be distinguished from the medium.*

A very striking and satisfactory demonstration may be made by painting a zone or band of eosin or other transparent color on a solid glass rod, and immersing the rod in a test tube or vial of cedar oil, clove oil or turpentine. Above the liquid the glass rod is very evident, as it is also at the colored zone, but at other levels it can hardly be seen in the liquid.

\$ 45 . Highly Refractive.-This expression is often used in describing microscopic objects, (medullated nerve fibers, for example), and means that the object will appear to be bordered by a wide, dark margin when it is viewed by transmitted light. And from the above (\$ I44), it would be known that the refractive power of the object, and the medium in which it was mounted must differ considerably.

I46. Doubly Contoured.-This means that the object is bounded by two, usually parallel dark lines with a lighter band between them. In other words, the object is bordered by (I) a dark line, (2) a light band, and (3) a second dark line (Fig. 93).

This may be demonstrated by coating a fine glass rod ( $\$$ I 44) with one or more coats of collodion or celloidin and allowing it to dry, and then mounting in $50 \%$ glycerin as above. Employ a $3 \mathrm{~mm}$. ( $1 / 8 \mathrm{in}$.) or higher objective, light with transmitted light, and it will be seen that where the glycerin touches the collodion coating there is a dark linenext this is a light band, and finally there is a second dark line where the collodion is in contact with the glass rod.* (Fig. 93).

FIG. 93. Solid glass rod coated with collodion to show a double contour. Toward one end the collodion had gathered in a fusiform drop.

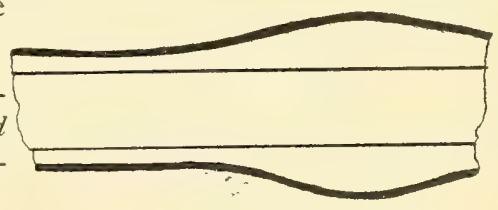

* Some of the rods have air bubbles in them, and then there results a capillary tube when they are drawn out. It is well to draw out a glass tube into a fine thread and examine it as described. The central cavity makes the experiment much more complex. 
\$ 147 . Optical Section.-This is the appearance obtained in examining transparent or nearly transparent objects with a microscope when some plane below the upper. surface of the object is in focus. The upper part of the object which is out of focus obscures the image but slightly. By changing the position of the objective or object, a different plane will be in focus and a different optical section obtained. The most satisfactory optical sections are obtained with high objectives having large aperture.

Nearly all the transparent objects studied may be viewed in optical section. A striking example will be found in studying mammalian red blood-corpuscles on edge. The experiments with the solid glass rods (Fig. 92) furnish excellent and striking examples of optical sections.

\$ I48. Currents in Liquids.-Employ the $16 \mathrm{~mm} .(2 / 3$ in.) objective, and as object put a few particles of carmine on the middle of a slide, and add a drop of water. Grind the carmine well with a scalpel blade, and then cover it. If the microscope is inclined, a current will be produced in the water, and the particles of carmine will be carried along by it. Note that the particles seem to flow up instead of downwhy is this?

Lamp-black rubbed in water containing a little mucilage answers well for this experiment.

S I49. Velocity Under the Microscope. - In studying currents or the movement of living things under the microscope, one should not forget that the apparent velocity is as unlike the real velocity as the apparent size is unlike the real size. If one consults Fig. 37 it will be seen that the actual size of the field of the microscope with the different objectires and oculars is inrersely as the magnification. That is, with great magnification only a small area can be seen. The field appears to be large, however, and if any object moves across the field it may appear to move with great rapidity, whereas if one measures the actual distance passed and notes the time, it will be seen that the actual motion is quite slow. One should keep this in mind in studying the circulation of the blood. The truth of what has just been said can be easily demonstrated in studying the circulation in the gills

*The collodion used is a $6 \%$ solution of gun cotton in equal parts of sulphuric ether and $95 \%$ alcohol. It is well to dip the rod two or three times in the collodion and to hold it vertically while drying. The collodion will gather in drops, and one will see the difference between a thick and a thin membranous covering. (Fig. 93). 
of Necturus, or in the frog's foot, by using first a low power in which the field is actually of considerable diameter (Fig. 37, Table, $\S 5 \mathrm{I}$ ) and then using a high power. With the high power the apparent motion will appear much more rapid. For spiral, serpentine and other forms of motion, see Carpenter-Dallinger, p. 433.

I 50 . Pedesis or Brownian Movement.-Employ the same object as above, but a $3 \mathrm{~mm}$. ( $1 / 3 \mathrm{in}$.) or higher objective in place of the $16 \mathrm{~mm}$. Make the body of the microscope vertical, so that there may be no currents produced. Use a small diaphragm and light the field well. Focus and there will be seen in the field large motionless masses, and between them small masses in constant motion. This is an indefinite, dancing or oscillating motion.

This indefinite but continuous motion of small particles in a liquid is called $P \bar{e}-d \bar{e}$ sis or Brownian movement. Also, but improperly, molecular movement, from the smallness of the particles.

The motion is increased by adding little gum arabic solution or a slight amount of silicate of soda or soap ; sulphuric acid and various saline compounds retard or check the motion. One of the best objects is lamp-black ground up with a little gum arabic. Carmine prepared in the same way, or simply in water, is excellent; and very finely powdered pumice-stone in water has for many years been a favorite object.

Pedesis is exhibited by all solid matter if it is finely enough dirided and in a suitable liquid. In the minds of most, no adequate explanation has yet been offered. See Carpenter-Dallinger, p. 43I ; Beale, p. I95; Jerons in Quart. Jour. Science, n. s., Vol. VIII (1878), p. 167. In I894, Meade Bache published a paper in the Proc. Amer. Philos. Soc., Vol. XXXIII, pp. I63-I67, entitled "The Secret of the Brownian Movement." This paper is suggestive if not very satisfactory.

For the orginal account of this see Robert Brown, "Botanical appendix to Captain King's voyage to Australia,"' Vol. II, p. 534. (1826).

See also Dr. C. Aug. Sigm. Schultze, "Mikroskopische Untersuchungen uiber des Herren Robert Brown Entdeckung lebender, selbst im Feuer unzerstörbarer Theilchen in allen Körpern." From "Die Gesellschaft für Betörderung der Naturwissenschaften zu Freiburg." I 828.

Compare the pedetic motion with that of a current by slightly inclining the tube of the microscope. The small particles will continue 
their independent leaping movements while they are carried along by the current. The pedetic motion makes it difficult to obtain good photographs of milk globules and other small particles. The difficulty may be overcome by mixing the milk with a very weak solution of gelatin and allowing it to cool (see Ch. IX).

$\S$ I 5 I. Demonstration of Pedesis with the Polarizing Microscope (Ch. VI).- The following demonstration shows conclusively that the pedetic motion is real and not illusive. (Ranvier, p. I73.)

Open the abdomen of a dead frog (an alcoholic or formalin specimen is satisfactory). Turn the viscera to one side and observe the small, whitish masses at the emergence of the spinal nerves. With fine forceps remove one of these and place it on the middle of a clean slide. Add a drop of water, or of water containing a little gum arabic. Rub the white mass around in the drop of liquid and soon the liquid will have a milky appearance. Remove the white mass, place a coverglass on the milky liquid and seal the cover by painting a ring of castor oil all around it, half the ring being on the slide and half on the cover-glass. This is to avoid the production of currents by evaporation.

Put the preparation under the microscope and examine with, first a low then a high power $(3 \mathrm{~mm}$. or $1 / 8 \mathrm{in}$.). In the field will be seen multitudes of crystals of carbonate of lime; the larger crystals dre motionless but the smallest ones exhibit marked pedetic movement.

Use the micro-polariscope, light with great care and exclude all adventitious light from the microscope by shading the object ( $\$$ IO9) and also by shading the eye. Focus sharply and observe the pedetic motion of the small particles, then cross the polarizer and analyzer, that is, turn one or the other until the field is dark. Part of the large motionless crystals will shine continuously and a part will remain dark, but small crystals between the large ones will shine for an instant, then disappear, only to appear again the next instant. This demonstration is believed to furnish absolute proof that the pedetic movement is real and not illusory.

S I52. Muscae Volitantes. - These specks or filaments in the eyes due to minute shreds or opacities of the vitreous sometimes appear as part of the object as they are projected into the field of vision. They may be seen by looking into the well lighted microscope when there is no object under the microscope. They may also be seen by looking at brightly illuminated snow or other white surface. By studying them carefully it will be seen that they are somewhat movable and float 
across the field of vision, and thus do not remain in one position as do the objects under observation. Furthermore, one may, by taking a little pains, familiarize himself with the special forms in his own eyes so that the more conspicuous at least may be instantly recognized.

S I53. In addition to the above experiments it is very strongly recommended that the student follow the advice of Beale, p. 248, and examine first with a low then with a higher power, mounted dry, then in water, lighted with reflected light, then with transmitted light, the following: Potato, wheat, rice, and corn starch, easily obtained by scraping the potato and the grains mentioned; bread crumbs ; portions of feather. Portions of feather accidentally present in histological preparations have been mistaken for lymphatic vessels (Beale, 288). Fibers of cotton, linen and silk. Textile fibers accidentally present have been considered nerve fibers, etc. Human and animal hairs. Study with especial care hairs from various parts of the body of the animals used for dissection in the laboratory where you work. These are liable to be present in histological preparations, and unless their character is understood there is chance for much confusion and erroneous interpretation. The scales of butterflies and moths, especially the common clothes moth. The dust swept from carpeted and wood floors. Tea leaves and coffee grounds. Dust found in iiving rooms and places not frequently dusted. In the last will be found a regular museum of objects.

For figures (photo-micrographs, etc.) of the various forms of starch, see Bulletin No. ${ }_{3} 3$ of the Chemical Division of the U. S. Department af Agriculture. For Hair and Wool, see Bulletin of the National Association of Wool Growers, 1875, p. 470, Proc. Amer. Micr. Soc., I884, pp. 65-68. Herzfeld, translated by Salter.-The technical testing of yarns and textile fabrics, London, I898.

For different appearances due to the illuminator, see Nelson, in Jour. Roy, Micr. Soc., I89I, pp. 90-IO5; and for the illusory appearances due to diffraction phenomena, see Carpenter-Dallinger, p. 434. Mercer. Trans. Amer. Micr. Soc., pp. $321-396$.

If it is necessary to see all sides of an ordinary gross object, and to observe it with varying illumination and under various conditions of temperature, moisture, etc., in order to obtain a fairly accurate and satisfactory knowledge of it, so much the more is it necessary not to be satisfied in microscopical observation until every means of investigation and verification has been called into service, and then of the image that falls upon the retina, only such details will be noted as the brain behind the eye is ready to appreciate. 
To summarize this chapter and leave with the beginning student the result of the experience of many eminent workers :

I. Get all the information possible with the unaided eye. See the whole object and all sides of it, so far as possible.

2. Examine the preparation with a simple microscope in the same thorough way for additional detail.

3. Use a low power of the compound microscope.

4. Use a higher power.

5. Use the highest power available and applicable. In this way one sees the object as a whole and progressively more and more details. Then as the object is viewed from two or more aspects, something like a correct notion may be gained of its form and structure.

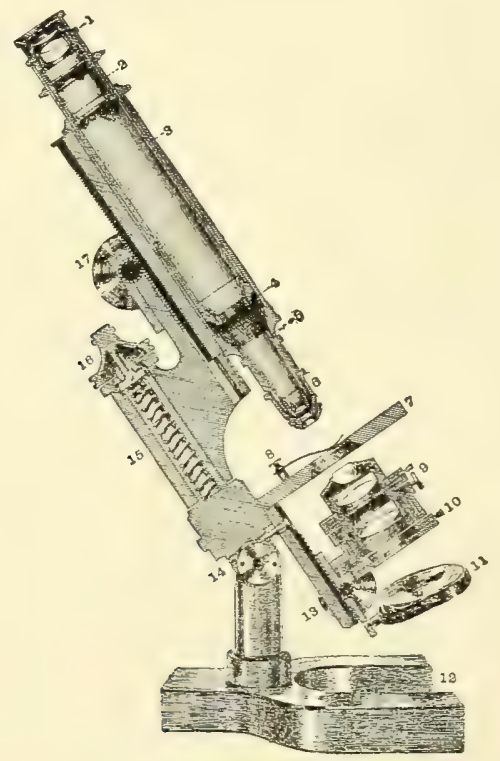

THE MICROSCOPE IN SECTION

I. Positive ocular.

2. Draw-tube.

. Main tube or body.

4-5. Society screws in the

draw-tube and body.

6. Objective in position.

7. Stage.

Spring for holding slides.

9. Sub-stage condenser.

Io. Iris diaphragm.
II. Plaue and concave mirror.

12. Horse-shoe base

13. Rack and pinion for condenser.

I4. Flexible pillar

15. Spiral spring of fine adjustment.

16. Fine adjustment

17. Coarse adjustment. 


\section{CHAPTER IV}

\section{MAGNIFICATION AND MICROMETRY}

\section{APPARATUS AND MATERIAL FOR THIS CHAPTER}

Simple and compound microscope ( $\left(8,56,5_{5} 8\right)$; Steel scale or rule divided to millimeters and $\frac{1}{5}$ ths ; Block for magnifier and compound microscope (?. I56, I60); Dividers ( 8 I56, I60); Stage micrometer (? I59); Wollaston camera lucida ( 8 r60); Ocular screw-micrometers (Figs. I06-IO7); Micrometer ocular (Figs. IO4-IO5). Abbe camera lucida (Fig. IOI). Necturus red blood corpuscles ( 8 I68).

\section{$\S$ I 54. The Magnification, Amplification or Magnifying Power} of a simple or compound microscope is the ratio between the real and the apparent size of the object examined. The apparent size is obtained by measuring the virtual image (Figs. 21, 38). The object for determining magnification must be of known length and is designated a micrometer (S I59). In practice a virtual image is measured by the aid of some form of camera lucida (Figs. 97, IOI), or by double vision ( I $_{5} 6$ ). As the length of the object is known, the magnification is easily determined by dividing the apparent size of the image by the actual size of the object. For example, if the virtual image measures $40 \mathrm{~mm}$. and the object magnified, $2 \mathrm{~mm}$., the amplification must be $4 \mathrm{O} \div 2=2 \mathrm{O}$, that is, the apparent size is 20 fold greater than the real size.

Magnification is expressed in diameters or times linear, that is, but one dimension is considered. In giving the scale at which a microscopical or histological drawing is made, the word magnification is frequently indicated by the sign of multiplication thus: $\times 450$, upon a drawing would mean that the figure or drawing is $45^{\circ}$ times as large as the object.

$\$$ I55. Magnification of Real Images. - In this case the magnification is the ratio between the size of the real image and the size of the object, and the size of the real image can be measured directly. By recalling the work on the function of an objective $(\$ 53)$, it will be remembered that it forms a real image on the ground glass placed on the top of the tube, and that this real image could be looked at with the 
eye or measured as if it were an actual object. For example, suppose the object were three millimeters long and its image on the ground glass measured $\mathrm{I} 5 \mathrm{~mm}$., then the magnification must be, $15 \div 3=5$, that is, the real image is 5 times as long as the object. The real images seen in photography are mostly smaller than the objects, but the magnification is designated in the same way by dividing the size of the real image measured on the ground glass by the size of the object. For example, if the object is 400 millimeters long and its image on the ground glass is $25 \mathrm{~mm}$. long, the ratio is $25 \div 400=\frac{1}{16}$. That is, the image is $\frac{1}{16}$ th as long as the object and is not magnified but reduced. In marking negatives, as with drawings, the sign of multiplication is put before the ratio, and in the example the designation would be $\times \frac{1}{16}$ th.

MAGNIFICATION OF A SIMPLE MICROSCOPE

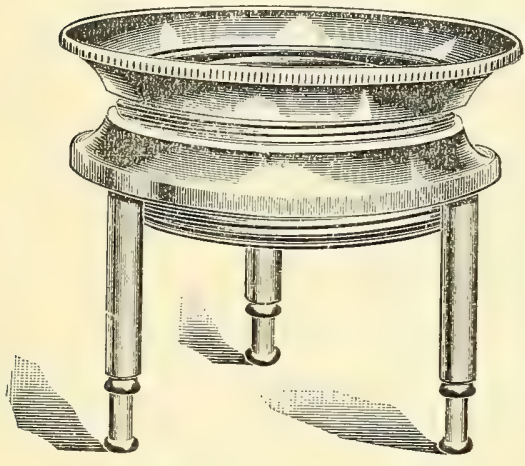

$\S$ I56. The Magnification of a Simple Microscope is the ratio between the object magnified (Fig. I6, $\left.A^{\prime} B^{\prime}\right)$, and the virtual image $\left(\mathrm{A}^{3} \mathrm{~B}^{3}\right)$. To obtain the size of this virtual image place the tripod magnifier near the edge of a support of such a height that the distance from the upper surface of the magnifier to the table is 250 millimeters.

FIG. 94. Tripod Magnifier.

As object, place a scale of some kind ruled in millimeters on the support under the magnifier. Put some white paper on the table at the base of the support and on the side facing the light.

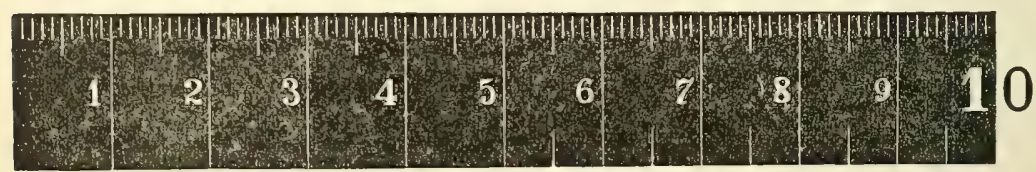

FIG. 95. Ten Centimeter Rule. The upper edge is divided into millimeters, the lower into centimeters at the left and half centimeters at the right.

Close one eye, and hold the head so that the other will be near the upper surface of the lens. Focus if necessary to make the image clear 
( $\$$ I I ). Open the closed eye and the image of the rule will appear as if on the paper at the base of the support. Hold the head very still, and with dividers get the distance between any two lines of the image. This is the so-called method of double vision in which the microscope image is seen with one eye and the dividers with the other, the two images appearing to be fused in a single visual field.

5 I57. Measuring the Spread of Dividers.-This should be done on a steel scale divided to millimeters and $\frac{1}{5}$ ths.

As $\frac{1}{5} \mathrm{~mm}$. cannot be seen plainly by the unaided eye, place one arm of the dividers at a centimeter line, and with the tripod magnifier count the number of spaces on the rule included between the points of the dividers. The magnifier simply makes it easy to count the spaces on the rule included between the points of the dividers-it does not, of course, increase the number of spaces or change their value.

As the distance between any two lines of the image of the scale gives the size of the virtual image (Fig. I6, $\mathrm{A}^{3} \mathrm{~B}^{3}$ ), and as the size of the object is known, the magnification is determined by dividing the size of the image by the size of the object. Thus, suppose the distance between the two lines of the image is measured by the dividers and found on the steel scale to be i 5 millimeters, and the actual size of the space between the two lines of the object is 2 millimeters, then the magnification must be $15 \div 2=71 / 2$. That is, the image is $7 \frac{1}{2}$ times as long or wide as the object. In this case the image is said to be magnified $7 \frac{1}{2}$ diameters, or $7 \frac{T}{2}$ times linear.

The magnification of any simple magnifier may be determined experimentally in the way described for the tripod.

\section{MAGNIFICATION OF A COMPOUND MICROSCOPE}

I 58. The Magnification of a Compound Microscope is the ratio between the final or virtual image (Fig. $2 \mathrm{I}, \mathrm{B}^{3} \mathrm{~A}^{3}$ ), and the object magnified (A B).

The determination of the magnification of a compound microscope may be made as with a simple microscope (\$ I 56 ), but this is very fatiguing and unsatisfactory.

S 59. Stage, Object or Objective Micrometer.-For determining the magnification of a compound microscope and for the purpose of micrometry, it is necessary to have a finely divided scale or rule on glass or on metal. Such a finely divided scale is called a micrometer, and for ordinary work one mounted on a glass slide ( $\times 3$ in, $25 \times 76 \mathrm{~mm}$.) is most convenient. 
The spaces between the lines should be $\frac{1}{10}$ and $\frac{1}{100} \mathrm{~mm}$. (or if in inches, $\frac{1}{100}$ and $\frac{1}{1000}$ in.) Micrometers are sometimes ruled on the slide, but more satisfactorily on a cover-glass of known thickness, preferably 0.15-0. I $8 \mathrm{~mm}$. The covers should be perfectly clean before the ruling, and afterwards simply dusted off with a camel's hair duster, and then mounted, lines downward over a shellac or other good cell. (See Ch. VII). If one rubs the lines the edges of the furrow made by the diamond are liable to be rounded and the sharpness of the micrometer is lost. If the lines are on the slide and uncovered one cannot use the micrometer with an oil immersion, as the oil obliterates the lines. Cleaning the slide makes the lines less sharp as stated. If the lines are very coarse, it is an advantage to fill them with plumbago. This may be done either with some very fine plumbago on the end of a soft cork, or by using an exceedingly soft lead pencil. Lines properly filled may be covered with balsam and a coverglass as in ordinary balsam mounting (Ch. VII).

$\$$ I60. Determination of Magnification.--This is most readily accomplished by the use of some form of camera lucida (Ch. V), that of Wollaston being most convenient as it may be used for all powers, and the determination of the standard distance of 250 millimeters at which to measure the images is very readily determined (Fig. 97, $\$ 162$ ).

Employ the $16 \mathrm{~mm}$. ( $2 / 3 \mathrm{in}$.) objective and a $37 \mathrm{~mm}$. (or $\times 8$ ocular with a stage micrometer as object. For this power the $\frac{1}{10} \mathrm{~mm}$. spaces of the micrometer should be used as object. Focus sharply.

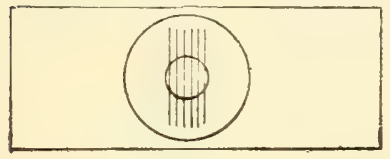

micrometer lines (Fig. 96). The light must also be carefully regulated. If too much light is used, $i . e_{\text {. }}$, too large an aperture, the lines will be drowned in the light. In focusing with the high powers be very careful. Remember the micrometers are expensive, and one cannot afford to break them. As suggested in $\$ 74$, focus on the edge of the cement ring enclosing the lines, then in focusing down to find the lines, move the preparation very slightly, back and forth.

After the lines are sharply focused, and the slide clamped in position make the tube of the microscope horizontal, by bending the flexible pillar, being careful not to bring any strain upon the fine adjustment (frontispiece). 
FIG. 97. Wollaston's Camera Lucida, showing the rays from the microscope and from the drawing surface, also the position of the pupil of the eye.

Axis, Axis. Axial rays from the microscope and from the drawing surface ( $C h . V)$.

Camera Lucida. A section of the quadrangular prism showing the course of the rays in the prism from the microscope to the eye. As the rays are twice reflected, they have the same relation on entering the eye that they would have by looking directly into the ocular.

A. B. The lateral rays from the microscope and their projection upon the drawing surface.

C. $D$ Rays from the drazing surface to ths eye.

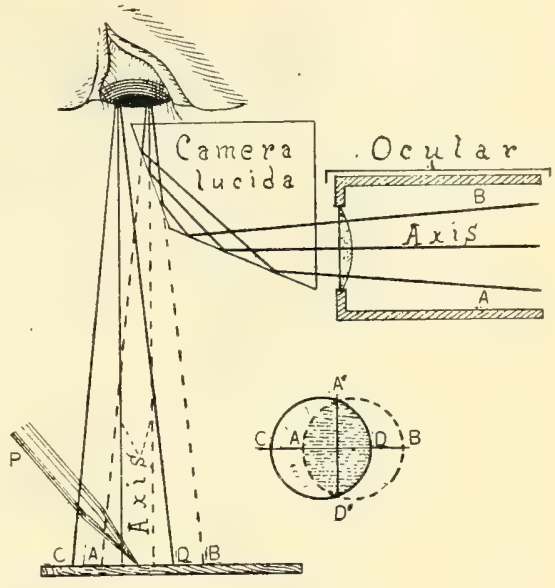

FIG. 97.

A. D. $A^{\prime} D^{\prime}$. Overlapping portions of the two fields, where both the microscopic image and the drawing surface, pencil, etc., can be seen. It is represented by the shaded part of the overlapping circles at the right.

Ocular. The ocular of the microscope.

$P$. The drawing pencil. Its point is shown in the overlapping fields.

Put a Wollaston camera 1ucida (Fig. 97 and Ch. V) in position, and turn the ocular around if necessary so that the broad flat surface may face directly upward, as shown in Fig. 97. Elevate the microscope by putting a block under the base, so that the perpendicular distance from the upper surface of the camera lucida to the table is $25^{\circ}$ $\mathrm{mm}$. (\$ I62). Place some white paper on the work-table beneath the camera lucida.

Close one eye, and hold the head so that the other may be very close to the camera lucida. Look directly down. The image will appear to be on the table. It may be necessary to readjust the focus after the camera lucida is in position. If there is difficulty in seeing dividers and image consult $\mathrm{Ch}$. V. Measure the image with dividers and obtain the power exactly as above $\left(\S_{156-1} 57\right)$.

Thus: Suppose two of the $\frac{1}{10}$ th $\mathrm{mm}$., spaces were taken as object, and the image is measured by the dividers, and the spread of the dividers is found on the steel rule to be $9 \frac{2}{\overline{5}}$ millimeters. If now the object is $\frac{2}{10}$ ths of a millimeter and the magnified image is $9 \frac{2}{5}$ millimeters, the magnification (which is the ratio between size of object 
and image) must be $9 \frac{2}{5} \div \frac{2}{10}=47$. That is, the magnification is 47 diameters, or 47 times linear. If the fractional numbers in the above example trouble the student, both may be reduced to the same denomination, thus: If the size of the image is found to be $9 \frac{2}{5} \mathrm{~mm}$. this number may be reduced to tenths mm., so it will be of the same

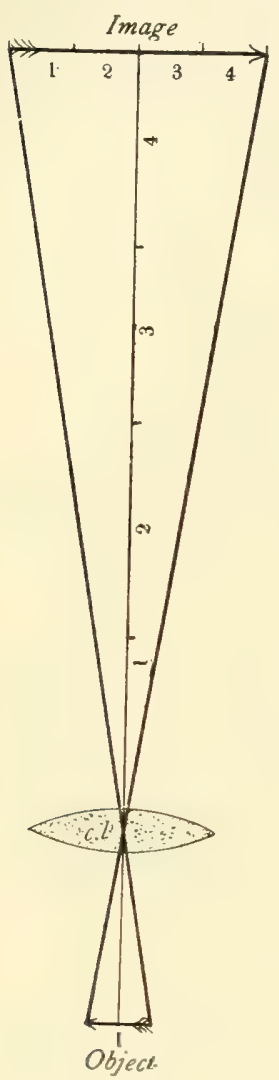

FIG. 98.

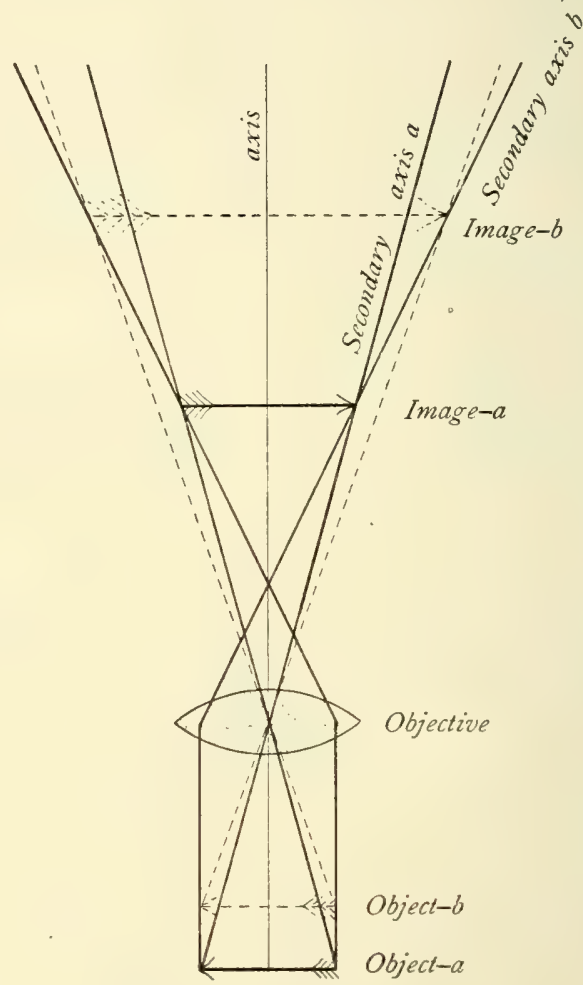

FIG. 99.

FIGS, 98-99. Figures showing that the size of object and image very directly as their distance from the center of the lens. In Fig. 99 one can also see why it is necessary to focus down, i. e., bring the object and objective nearer together when the tube is lengthened. See also fig. 58 .

denomination as the object. In $9 \mathrm{~mm}$. there are 30 tenths, and in $\frac{2}{5}$ there are 4 tenths, then the whole length of the image is $90+4=94$ tenths of a millimeter. The object is 2 tenths of a millimeter, then 
there must have been a magnification of $94 \div 2=47$ diameters in order to produce an image 94 tenths of a millimeter long.

Put the $25 \mathrm{~mm}$. ( $\mathrm{I}$ in., C, or $\times$ I2) ocular in place of one of 37 $\mathrm{mm}$. focus, and then put the camera lucida in position. Measure the size of the image with dividers and a rule as before. The power will be considerably greater than when the low ocular was used. This is because the virtual image (Fig. $2 \mathrm{I}, \mathrm{B}^{3} \mathrm{~A}^{3}$ ) seen with the high ocular is larger than the one seen with the low one. The real image (Fig. 2I, $\mathrm{A}^{1} \mathrm{~B}^{1}$ ) remains nearly the same, and would be just the same if positive, par-focal oculars $(\$ 37,72$, note) were used.

Lengthen the tube of the microscope $50-60 \mathrm{~mm}$. by pulling out the draw-tube. Remove the camera lucida, and focus, then replace the camera and obtain the magnification. It will be greater than with the shorter tube. This is because the real image (Fig. 99) is formed farther from the objective when the tube is lengthened, and the objective must be brought nearer the object. The law is: The size of object and image varies directly as their distance from the center of the lens. The truth of this statement is illustrated by Figs. 98 and 99.

$\$$ I6I. Varying the Magnification of a Compound Microscope.-It will be seen from the above experiments ( $\$$ I 60 ) that independently of the distance at which the microscopic image is measured ( $\$ 162)$, there are three ways of varying the power of a compound microscope. These are named below in the order of desirability.

(I) By using a higher or lower objective.

(2) By using a higher or lower ocular.

(3) By lengthening or shortening the tube of the microscope (Fig. 99).*

162. Standard Distance of 250 Millimeters at which the Virtual Image is Measured.-For obtaining the magnification of both the simple and the compound microscope the directions were to measure the virtual image at a distance of 250 millimeters. This is not

*Amplifier. - In addition to the methods of varying the magnification given in \% I6r, the magnification is sometimes increased by the use of an amplifier, that is a diverging lens or combination placed between the objective and ocular and serving to give the image-forming rays from the objective an increased divergence. An effective form of this accessory was made by Tolles, who made it as a small achromatic concavo-convex lens to be screwed into the lower end of the draw-tuibe (frontispiece) and thus but a short distance above the objective. The divergence given to the rays increases the size of the real image about two-fold. 


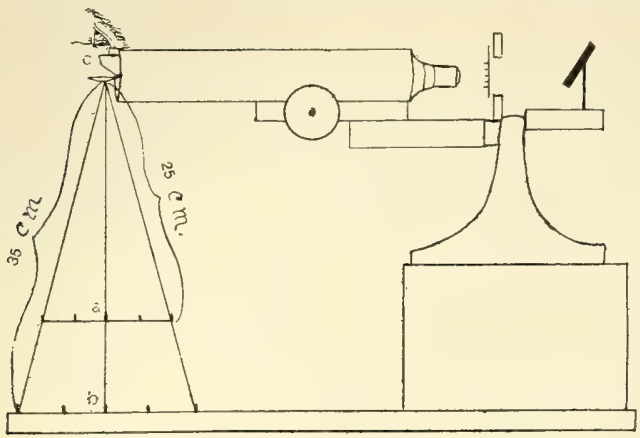

FIG. 100. Figure showing the position of the microscope, the camera lucida, the eye, and the difference in size of the image depending upon the distance at which it is projected from the eye. (a) The size at $25 \mathrm{~cm} . ;$ (b) at $35 \mathrm{~cm}$., (\& I62).

that the image could not be seen and measured at any other distance, but because some standard must be selected, and this is the most common one. The necessity for the adoption of some common standard will be seen at a glance in Fig. Ioo, where is represented graphically the fact

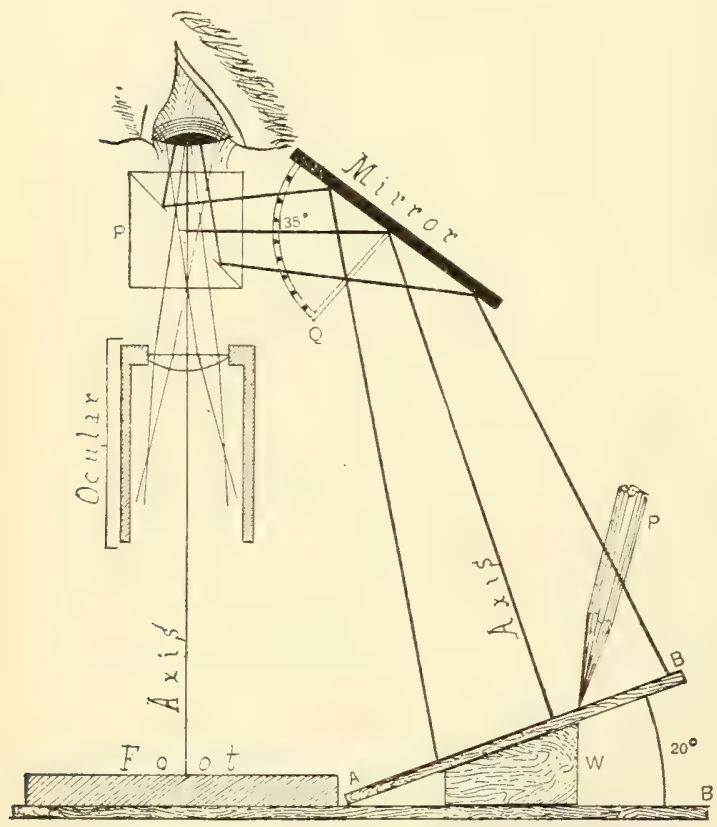

Fig. Ior. Sectional view of the Abbe Camera Lucida to show that in measuring the standard distance of 250 millimeters, one must measure along the axis from the point $P$, at the left of the prism, to the mirror, and from the mirror to the drawing surface. For a full explanation of this camera lucida, see next chapter.

FIG. IOI.

that the size of the rirtual image depends directly on the distance at which it is projected, and this size is directly proportional to the vertical distance from the apex of the triangle, of which it forms a base. The distance of 250 millimeters has been chosen on the supposition that it is the distance of most distinct vision for the normal human eye. 
Demonstrate the difference in magnification due to the distance at which the image is projected, by raising the microscope so that the distance will be 350 millimeters, then lowering to 50 millimeters.

In preparing drawings it is often of great convenience to make them at a distance somewhat less or somewhat greater than the standard. In such a case the magnification must be determined for the special distance. (See the next chapter, $\S$ I8I.)

For discussion of the magnification of the microscope, see: Beale, pp. +I, 355 ; Carpenter-Dallinger, p. 288 ; Nägeli and Schwendener, p. I76 ; Ranvier, p. 29 ; Robin, p. I26 ; Amer. Soc. Micrs., I884, p. I83; I889, p. 22 ; Amer. Jour. Arts and Sciences, I890, p. 50 ; Jour. Roy. Micr. Soc., I888, I889.

\begin{tabular}{|c|c|c|c|c|c|}
\hline & $\begin{array}{r}0 C \\
37 \circ\end{array}$ & $\begin{array}{l}\mathrm{LAR} \\
\mathrm{O} \mathrm{mm} .\end{array}$ & & $\begin{array}{r}\text { OCULA } \\
25 \mathrm{~mm}\end{array}$ & \\
\hline OBJECTIVE. & $\begin{array}{l}\text { TUBE } \\
\text { IN }\end{array}$ & $\begin{array}{l}\text { TUBE } \\
\text { OUT } \\
\text { - MM. }\end{array}$ & $\underset{\text { IN }}{\text { TuBp }}$ & $\begin{array}{l}\text { TUBE: } \\
\text { OUT } \\
- \text { MM. }\end{array}$ & $\begin{array}{l}\text { OCULAR MICROMETER } \\
\text { VALUATION. } \\
\text { TUBE IN. OUT- MM. }\end{array}$ \\
\hline & x & $x$ & $x$ & $x$ & \\
\hline & x & $x$ & $x$ & x & \\
\hline & x & $x$ & $x$ & $\times$ & \\
\hline & $x$ & x & $x$ & $x$ & \\
\hline & x & X & $x$ & $x$ & \\
\hline & X & $x$ & $x$ & X & \\
\hline SIMPLE Mid & ROSCOP & $x$ & & & \\
\hline
\end{tabular}

FIG. IO2.

I63. Table of Magnifications and of the Valuations of the Ocular Micrometer. - The above table should be filled out by each student. In using it for Micrometry and Drawing it is necessary to keep clearly in mind the exact conditions under which the determinations were 
made, and also the ways in which variations in magnification and the valuation of the ocular micrometer may be produced (\$ I61. I62, I72, I76).

\section{MICROMETRY}

$\S$ I64. Micrometry is the determination of the size of objects by the aid of a microscope:

\section{MICROMETRY WITH THE SIMPLE MICROSCOPE}

\$ 165 . With a simple microscope (A), the easiest and best way is to use dividers and then with the simple microscope determine when the points of the dividers exactly include the object. The spread of the dividers is then obtained as above ( $\$ 157)$. This amount will be the actual size of the object, as the microscope was only used in helping to see when the divider points exactly enclosed the object, and then for reading the divisions on the rule in getting the spread of the dividers.

(B) One may put the object under the simple microscope and then, as in determining the power $(\$ 156)$, measure the image at the standard distance. If the size of the image so measured is divided by the magnification of the simple microscope, the quotient will give the actual size of the object.

Use a fly's wing or some other object of about that size, and try to determine the width in the two ways described above. If all the work is accurately done the results will agree.

\section{MICROMETRY WITH THE COMPOUND MICROSCOPE}

There are several ways of varying excellence for obtaining the size of objects with the compound microscope, the method with the ocular micrometer $\left(\$ 175^{-17} 6\right)$ being most accurate.

S i66. Unit of Measure in Micrometry.-As most of the objects measured with the compound microscope are smaller than any of the originally named divisions of the meter, and the common or decimal fractions necessary to express the size are liable to be unnecessarily cumbersome, Harting, in his work on the microscope (I 859), proposed the one thousandth of a millimeter $\left(\frac{1}{1000} \mathrm{~mm}\right.$. or $0.001 \mathrm{~mm}$.) or one millionth of a meter ( $\frac{1}{1000000}$ or 0.00000 I meter $)$ as the unit. He named this unit micro-millimeter and designated it mmm. In I869, Listing (Carl's Repetorium für Experimental-Physik, Bd, X, p. 5) 
farored the thousandth of a millimeter as unit and introduced the name Mikron or micrum. In English it is most often written Micron (plural micra or microns, pronunciation M̆̌k'rŏn or Mìk'rŏn). By universal consent the sign or abbreviation used to designate it is the Greek $\mu$. Adopting this unit and sign, one would express five thousandths of a millimeter $\left(\frac{5}{1000}\right.$ or $0.005 \mathrm{~mm}$. ) thus, $5 \mu$.*

3. 167. Micrometry by the use of a stage micrometer on which to mount the object.-In this method the object is mounted on a micrometer and then put under the microscope, and the number of spaces covered by the object is read off directly. It is exactly like putting any large object on a rule and seeing how many spaces of the rule it covers. The defect in the method is that it is impossible to properly arrange objects on the micrometer. Unless the objects are circular in outline they are liable to be oblique in position, and in every case the end or edges of the object may be in the middle of a space instead of against one of the lines, consequently the size must be estimated or guessed at rather than really measured.

S I68. Nicrometry by dividing the size of the image by the magnification of the microscope.-For example, employ the $3 \mathrm{~mm}$. ( $1 / 8 \mathrm{in}$.) objective, $25 \mathrm{~mm}$. ( $\mathrm{I}$ in.) ocular, and a Necturus' red blood-corpuscle preparation as object. Obtain the size of the image of the long and short axes of three corpuscles with the camera lucida and dividers, exactly as in obtaining the magnification of the microscope ( $\$ 60)$. Divide the size of the image in each case by the magnification, and the result will be the actual size of the blood-corpuscles. Thus, suppose the image of the long axis of the corpuscle is $18 \mathrm{~mm}$. and the magnification of the micro-

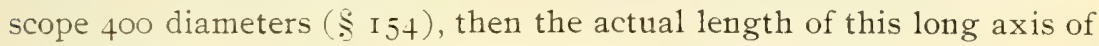
the corpuscle is $18 \mathrm{~mm} . \div 400=0.045 \mathrm{~mm}$. or $45 \mu\left(\S_{166}\right)$.

FIG. I03. Preparation of blood with a ring around a group of blood corpuscles.

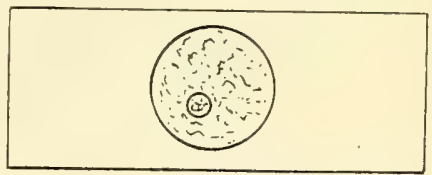

As the same three blood-corpuscles are to be measured in three ways, it is an advantage to put a delicate ring around a group of three or more corpuscles, and make a sketch of the whole enclosed group,

*The term micromillimeter, abbreviation mmm., is very cumbersome, and besides is entirely inappropriate since the adoption of the definite meanings for the prefixes micro and mega, meaning respectively one-millionth and one million times the unit before which it is placed. A micromillimeter would then mean one-millionth of a millimeter, not one-thousandth. The term micron has been adopted by the great microscopical societies, the international commission on weights and measures, and by original investigators, and is, in the opinion of the Writer, the best term to employ. Jour. Roy. Micr. Soc., I888, p. 502 ; Nature, Vol. XXXVII (1888), p. 388. 
marking on the sketch the corpuscles measured (Figs. 6I-66). The different corpuscles vary considerably in size, so that accurate comparison of different methods of measurement can only be made when the same corpuscles are measured in each of the ways.

$\$ 169$. Micrometry by the use of a Stage Micrometer and a Camera Lucida.-Employ the same object, objective and ocular as before. Put the camera lucida in position, and with a lead pencil make dots on the paper at the limits of the image of the bloodcorpuscle. Measure the same three that were measured in $\S$ I68.

Remove the object, place the stage micrometer under the microscope, focus well, and draw the lines of the stage micrometer so as to include the dots representing the limits of the part of the image to be measured. As the value of the spaces on the stage micrometer is known, the size of the object is determined by the number of spaces of the micrometer required to include it.

This simply enables one to put the image of a fine rule on the image of a microscopic object. It is theoretically an excellent method, and nearly the same as measuring the spread of the diriders with a simple microscope (§ I57, I76).

OCULAR MICROMETER

$\$$ I 70. Ocular Micrometer, Eye-Piece Micrometer.-This, as the name implies, is a micrometer to be used with the ocular. It is a micrometer on glass, and the lines are sufficiently coarse to be clearly seen by the ocular. The lines should be equidistant and about $\frac{1}{10}$ th or $\frac{1}{2}$ th $\mathrm{mm}$. apart, and every fifth line should be longer and heavier to facilitate counting. If the micrometer is ruled in squares (net micrometer) it will be very convenient for many purposes.

The ocular micrometer is placed in the ocular, no matter what the

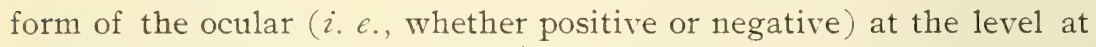
which the real image is formed by the objective, and the image appears to be immediately upon or under the ocular micrometer, and hence the number of spaces on the ocular micrometer required to measure the real image may be read off directly. This, however, is measuring the size of the real image, and the actual size of the object can only be determined by determining the ratio between the size of the real image and the object. In other words, it is necessary to get the valuation of the ocular micrometer in terms of a stage micrometer.

I7I. Valuation of the Ocular Micrometer.-This is the value of the dirisions of the ocular micrometer for the purposes of 
micrometry, and is entirely relative, depending upon the magnification of the real image formed by the objective, consequently it changes with every change in the magnification of the real image, and must be especially determined for every optical combination (i.e., objective and ocular), and for every change in the length of the tube of the microscope. That is, it is necessary to determine the ocular micrometer valuation for every condition modifying the real image of the microscope $(\S \mathrm{I} 6 \mathrm{I})$.

Any Huygenian ocular (Fig. 30) may, however, be used as a micrometer ocular by placing the ocular micrometer at the level of the ocular diaphragm, where the real image is formed. If there is a slit in the side of the ocular, and the ocular micrometer is mounted in some way it may be introduced through the opening in the side. When no side opening exists the mounting of the eye-lens may be unscrewed and the ocular micrometer, if on a cover-glass can be laid on the upper side of the ocular diaphragm.

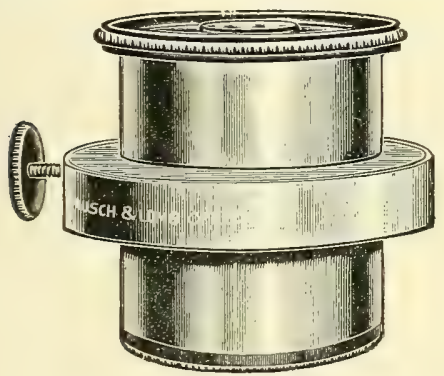

FIG. I04.

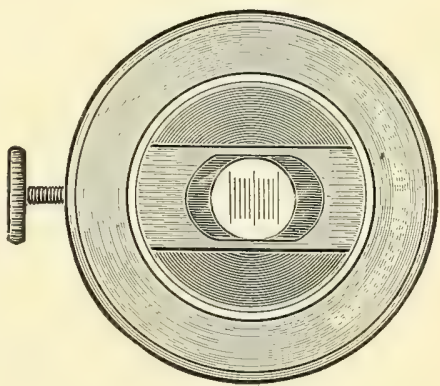

FIG. 105.

FIGS. I04-I05. Ocular Micrometer with movable scale. Fig. I04 is a side view of the ocular while Fig. IO5 gives a sectional end view, and shows the ocular micrometer in position. In both the screw which moves the micrometer is shown at the left. (Bausch \& Lomb Opt. Co.)

I72. Obtaining the Ocular Micrometer Valuation for an Ocular Micrometer with Fixed Lines (Figs. I04-105).-Use the stage micrometer as object. Light the field well and look into the microscope. The lines of the ocular micrometer should be very sharply defined. If they are not, raise or lower the eye-lens to make them so ; that is, focus as with the simple magnifier.

When the lines of the ocular micrometer are distinct, focus the microscope $(\$ 72,74,75)$ for the stage micrometer. The image of the stage micrometer will appear to be directly under or upon the ocular micrometer.

Make the lines of the two micrometers parallel by rotating the ocular or changing the position of the stage micrometer or both if necessary, 
and then make any two lines of the stage micrometer coincide with any two on the ocular micrometer. To do this it may be necessary to pull out the draw-tube a greater or less distance. See how many spaces are included in each of the micrometers.

Divide the value of the included space or spaces on the stage micrometer by the number of divisions on the ocular micrometer required to include them, and the quotient so obtained will give the valuation of the ocular micrometer in fractions of the unit of measure of the stage micrometer. For example, suppose the millimeter is taken as the unit for the stage micrometer and this unit is divided into spaces of $\frac{1}{10}$ th and $\frac{1}{100}$ th millimeter. If now, with a given optical combination and tubelength, it requires Io spaces on the ocular micrometer to include the real image of $\frac{1}{10}$ th millimeter on the stage micrometer, obviously one space on the ocular micrometer would include only one-tenth as much, or $\frac{1}{10}$ th $\mathrm{mm} . \div \mathrm{IO}=\frac{1}{100}$ th $\mathrm{mm}$. That is, each space on the ocular micrometer would include $\frac{1}{10} 0$ th of a millimeter on the stage micrometer, or $\frac{1}{10}$ th millimeter of the length of any object under the microscope, the conditions remaining the same. Or, in other words, it would require Ioo spaces on the ocular micrometer to include I millimeter on the stage micrometer, then as before, I space of the ocular micrometer would have a valuation of $\frac{1}{10}$ th millimeter for the purposes of micrometry. The size of any minute object may be determined by multiplying this valuation of one space by the number of spaces required to include it. For example, suppose the fly's wing or some part of it covered 8 spaces on the ocular micrometer, it would be known that the real size of the part measured is $\frac{1}{100}$ th $\mathrm{mm} . \times 8=\frac{8}{100} \mathrm{~mm}$. or $80 \mu(\S \mathrm{I} 66)$. See Mark, Jour. Applied Microscopy, Vol. I, p. 4.

I73. Micrometry with the Ocular Micrometer.-Use the 3 $\mathrm{mm}$. ( $\frac{1}{8} \mathrm{in}$.) objective with the preparation of Necturus blood-corpuscles as object. Make certain that the tube of the microscope is of the same length as when determining the ocular micrometer valuation. In a word, be sure that all the conditions are exactly as when the valuation was determined, then put the preparation under the microscope and find the same three red corpuscles that were measured in the other ways ( $\$ 168-169)$.

Count the divisions on the ocular micrometer required to enclose or measure the long and the short axis of each of the three corpuscles, multiply the number of spaces in both cases by the valuation of the ocular micrometer for this objective, tube-length and ocular, and the results will represent the actual length of the axes of the corpuscles in each case. 
The same corpuscle is, of course, of the same actual size, when measured in each of the three ways, so that if the methods are correct and the work carefully enough done, the same results should be obtained by each method. (\$ I 76).*

FIG. I06. Ocular Screw-Micrometer with compensation ocular $\times 6$. The upper figure shows a sectional view of the ocular and the screw for moving the micrometer at the right. At the left is shown a clamping screw to fasten the ocular to the upper part of the microscope tube. Below is a face view, showing the graduation on the wheel. An ocular micrometer like this is in general like the cob-zveb micrometer and may be used for measuring objects of varying sizes very accurately. With the ordinary ocular micrometer very small objects frequently fill but a part of an interval of the micrometer, but with this the movable cross lines traverse the object (or rather its real image) regardless of the minuteness of the object. (Zeiss' Catalog).
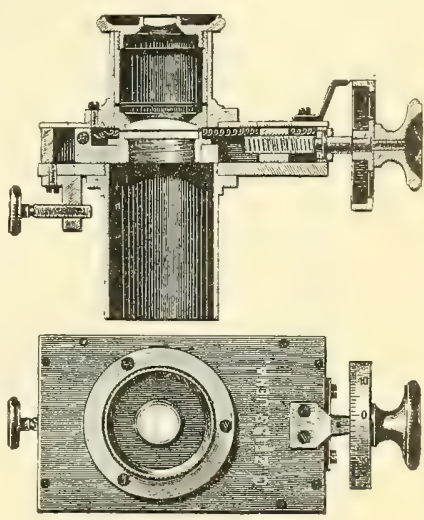

\& I74. Obtaining the Valuation of the Screw or Filar Micrometer.-This micrometer (Fig. IO6-IO7) usually consists of a Ramsden's ocular and cross lines. As seen in Fig. IO7 $A$ there are three lines. The horizontal and one vertical line are fixed. One vertical line may be moved by the screw back and forth across the field.

For obtaining the valuation of this ocular micrometer an accurate stage micrometer must be used. Carefully focus the $\frac{1}{10}$ th $\mathrm{mm}$. spaces. The lines of the ocular micrometer should also be sharp. If they are not focus them by moving the top of the ocular up or down $(\& I 72)$. Make the vertical lines of the filar micrometer parallel with the lines of the stage micrometer. Take the precautions regarding the width of the stage micrometer lines given in \% 176 (see also Fig. IOS). Note the position of the graduated wheel and of the teeth of the recording comb, and then rotate the wheel until the movable line traverses one space on the stage micrometer. Each tooth of the recording comb indicates a total revolution of the wheel, and by noting the number of teeth required and the graduations on the wheel, the revolutions and parts of revolution required to measnre the $\frac{1}{10} 0$ th $\mathrm{mm}$, of the stage micrometer can be easily noted. Measure in like manner 4 or 5 spaces and get the average. Suppose this average is $I \frac{1}{4}$ th revolutions or 125 graduations on the wheel, to measure the $\frac{1}{10}$ th $\mathrm{mm}$. or IO $\mu$ ( of the graduations on the wheel would measure Io $\mu$ divided by $125=.08 \mu$. In using this valuation for actual measurement, the tube of the microscope and the objective must be exactly as when obtaining the valuation (see $\&$ I 75 ).

Example of Measurement.-Suppose one uses the red blood corpuscles of a dog or monkey, etc., every condition being as when the valuation was determined, one notes very accurately how many of the graduations on the wheel are required to make the movable line traverse the object from edge to edge. Suppose it requires 
94 of the graduations to measure the diameter, the actual size of the corpuscle would be $94 \times .08 \mu=7.52 \mu$.

The advantage of the filar micrometer is that the valuation of one graduation being so small, even the smallest object to be measured would require several graduations to measure it. In ocular micrometers with fixed lines, small objects like bacteria might not fill even one space, therefore estimations, not measurements, must be made. For large objects, like most of the tissue elements, the ocular micrometers with fixed lines answer very well, for the part which must be estimated is relatively small and the chance of error is correspondingly small.

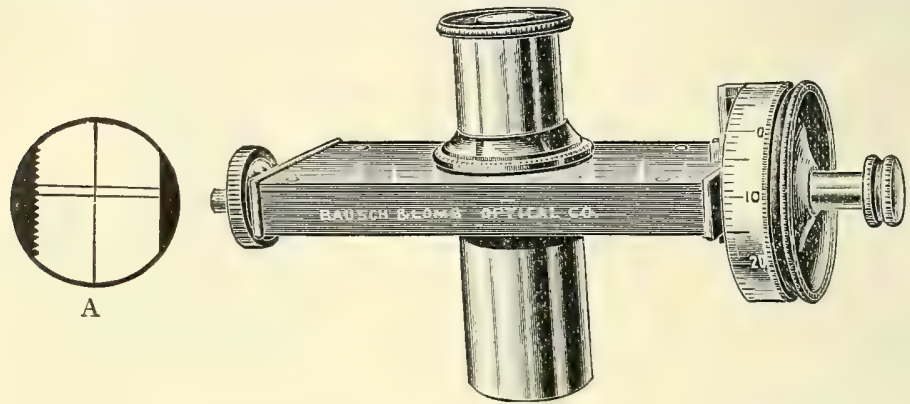

FIG. I07. Filar Ocular Micrometer with Field A (Bausch \& Lomb, Optical Co.).

\section{I75. Varying the Ocular Micrometer Valuation.-Any} change in the objective, the ocular or the tube-length of the microscope, that is to say, any change in the size of the real image, produces a corresponding change in the ocular micrometer valuation (\$ I6I, I7I, I 76 ).

\& I76. Remarks on Micrometry.-In using adjustable objectives (\&24, I03), the magnification of the objective varies with the position of the adjusting collar, being greater when the adjustment is closed as for thick cover-glasses than when open, as for thin ones. This variation in the magnification of the objective produces a corresponding change in the magnification of the entire microscope, and the ocular micrometer valuation-therefore it is necessary to determine the magnification and ocular micrometer valuation for each position of the adjusting collar.

While the principles of micrometry are simple, it is very difficult to get the exact size of microscopic objects. This is due to the lack of perfection and uni-

*There are three ways of using the ocular micrometer, or of arriving at the size of the objects measured with it :

(A) By finding the value of a division of the ocular micrometer for each optical combination and tube-length used, and employing this valuation as a multiplier. This is the method given in the text, and "the one most frequently employed. Thus, suppose with a given optical combination and tube-length it required five divisions on the ocular micrometer to include the image of $\frac{2}{10}$ th millimeter of the stage micrometer, then obviously one space on the ocular micrometer would include $\frac{1}{5}$ th of $\frac{2}{10}$ ths $\mathrm{mm}$. or $\frac{1}{25}$ th $\mathrm{mm}$; the size of any unknown object under the 
formity of micrometers, and the difficulty of determining the exact limits of the object to be measured. Hence, all microscopic measurements are only approximately correct, the error lessening with the increasing perfection of the apparatus and the skill of the observer.

microscope would be obtained by multiplying the number of divisions on the ocular micrometer required to include its image by the value of one space, or in this case, $\frac{1}{2}$ th $\mathrm{mm}$. Suppose some object, as the fly's wing, required 15 spaces of the ocular micrometer to include some part of it, then the actual size of this part of the wing would be $15 \times \frac{1}{25}=\frac{3}{5}$ ths, or $0.6 \mathrm{~mm}$.

(B) By finding the number of divisions on the ocular micrometer required to include the image of an entire millimeter of the stage micrometer, and using this number as a divisor. This number is also sometimes called the ocular micrometer ratio. Taking the same case as in (A), suppose five divisions of the ocular micrometer are required to include the image of $\frac{2}{10}$ ths $\mathrm{mm}$., on the stage micrometer, then evidently it would require $5 \div \frac{2}{1} \overline{0}=25$ divisions on the ocular micrometer to include a whole millimeter on the stage micrometer, and the number of divisions of the ocular micrometer required to measure an object divided by 25 would give the actual size of the object in millimeters or in a fraction of a millimeter. Thus, suppose it required $\mathrm{I}_{5}$ divisions of the ocular micrometer to include the image of some part of the fly's wing, the actual size of the part included would be $15 \div 25$ $=\frac{3}{5}$ or $0.6 \mathrm{~mm}$. This method is really exactly like the one in (A), for dividing by 25 is the same as multiplying by $\frac{1}{25}$ th.

(C) By having the ocular micrometer ruled in millimeters and divisions of a millimeter, and then getting the size of the real image in millimeters. In employing this method a stage micrometer is used as object and the size of the image of one or more divisions is measured by the ocular micrometer, thus: Suppose the stage micrometer is ruled in $\frac{1}{10}$ th and $\frac{1}{10}$ th $\mathrm{mm}$. and the ocular micrometer is ruled in millimeters and $\frac{7}{10}$ th $\mathrm{mm}$. Taking $\overline{1}^{2} \mathrm{t}$ th $\mathrm{mm}$. on the stage micrometer as object, as in the other cases, suppose it requires Io of the $\frac{1}{10}$ th $\mathrm{mm}$. spaces or I $\mathrm{mm}$. to measure the real image, then the real image must be magnified $\frac{10}{10} \div \frac{2}{10}=5$ diameters, that is, the real image is five times as great in length as the object, and the size of an object may be determined by putting it under the microscope and getting the size of the real image in millimeters with the ocular micrometer and dividing it by the magnification of the real image, which in this case is 5 diameters.

Use the fly's wing as object, as in the other cases, and measure the image of the same part. Suppose that it required 30 of the $\frac{1}{10} \mathrm{~mm}$. divisions $=\frac{30}{10} \mathrm{~mm}$. or 3 $\mathrm{mm}$. to include the image of the part measured, then evidently the actual size of the part measured is $3 \mathrm{~mm}, \div 5=\frac{3}{3} \mathrm{~mm}$., the same result as in the other cases.

In comparing these methods it will be seen that in the first two (A and B) the ocular micrometer may be simply ruled with equidistant lines without regard to the absolute size in millimeters or inches of the spaces. In the last method the ocular micrometer must have its spaces some known division of a millimeter or inch.' In the first two methods only one standard of measure is required, viz, the stage micrometer; in the last method two standards must be used,-a stage micrometer and an ocular micrometer. Of course, the ocular micrometer in the first two cases must have the lines equidistant as well as in the last case, but ruling lines equidistant is quite a different matter from getting them an exact division of a millimeter or of an inch apart. 
A difficulty when one is using high powers is the width of the lines of the micrometer. If the micrometer is perfectly accurate half the width of each line belongs to the contiguous spaces, hence one should measure the image of the space from the centers of the lines bordering the space, or as this is somewhat difficult in using the ocular micrometer, one may measure from the inside of one bordering line and from the outside of the other. If the lines are of equal width this is as accurate as measuring from the center of the lines. Evidently it would not be right to measure from either the inside or the outside of both lines (Fig. Io8).

It is also necessary in micrometry to use an objective of sufficient power to enable one to see all the details of an object with great distinctness. The necessity of using sufficient amplification in micrometry has been especially remarked upon by Richardson, Monthly Micr. Jour., I574, I875, ; Rogers, Proc. Amer. Soc. Microscopists, I882, p. 239; Ewell, North American Pract., I89o, pp. 97, I73.

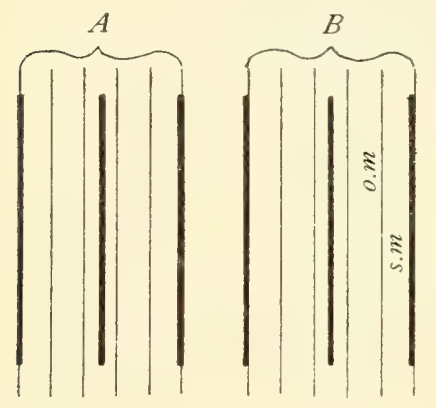

FIG. I08. The appearance of the coarse stage micrometer and of the fine ocular micrometer lines when using a high objective.

(A). The method of measuring the spaces by putting the fine ocular micrometer lines opposite the center of the coarse stage micrometer lines.

(B). Method of measuring the spaces of the stage micrometer by putting one line of the ocular micrometer $(0 . m$.) at the inside and one at the outside of the coarse stage micrometer lines (s. m.).

FIG, IOS.

As to the limit of accuracy in micrometry, one who has justly earned the right to speak with authority expresses himself as follows: "I assume that $0.2 \mu$ is the limit of precision in microscopic measures beyond which it is impossible to go with certainty." W. A. Rogers Proc. Amer. Soc. Micrs., r883, p. I98.

In comparing the methods of micrometry with the compound microscope given above $(\% 167,168,169,175)$, the one given in ? 167 is impracticable, that given in \& I68 is open to the objection that two standards are required, - the stage micrometer, and the steel rule; it is open to the further objection that several different operations are necessary, each operation adding to the probability of error. Theoretically the method given in \& I69 is good, but it is open to the very serious objection in practice that it requires so many operations which are especially liable to introduce errors. The method that experience has found most safe and expeditious, and applicable to all objects, is the method with the ocular micrometer. If the valuation of the ocular micrometer has been accurately determined, then the only difficulty is in deciding on the exact limits of the objects to be measured and so arranging the ocular micrometer that these limits are inclosed by some divisions of the micrometer. Where the object is not exactly included by whole spaces on the ocular micrometer, the chance of error comes in, in estimating just how far into a space the object reaches on the side not in contact with one of the micrometer 
lines. If the ocular micrometer has some quite narrow spaces, and others considerably larger, one can nearly always manage to exactly include the object by some two lines. The ocular screw micrometers (Fig. I06-IO7) obviates this entirely as the cross hair or lines traverse the object or its real image, and whether this distance be great or small it can be read off on the graduated wheel, and uo estimation or guess work is necessary.

For those especially interested in micrometry, as in its relation to medical jurisprudence, the following references are recommended. These articles consider the problem in a scientific as well as a practical spirit: The papers of Prof. Wm. A. Rogers on micrometers and micrometry, in the Amer. Quar. Micr. Jour., Vol. I, pp. 97, 208 ; Proceedings Amer. Soc. Microscopists, I882, I883, I887. Dr. M. D. Ewell, Proc. Amer. Soc. Micrs., I8go ; The Microscope, 1889, pp. 43-45; North Amer. Pract., I89o, pp. 97, I73. Dr. J. J. Woodward, Amer. Jour. of the Med. Sci., I875. M. C. White, Article "Blood-stains," Ref. Hand-Book Med. Sciences, ISS5. Medico-Legal Journal, Vol. XII. For the change in magnification due to a change in the adjustment of adjustable objectives, see Jour. Roy. Micr. Soc., I8So, p. 702 ; Amer. Monthly Micr. Jour., r88o, p. 67. Carpenter-Dallinger, p. 270.

If one consuits the medico-legal journals; the microscopical journals, the Index Medicus, and the Index Catalog of the Library of the Surgeon General's Office, under Micrometry, Blood, and Jurisprudence, he can get on track of the main work which has been and is being done.
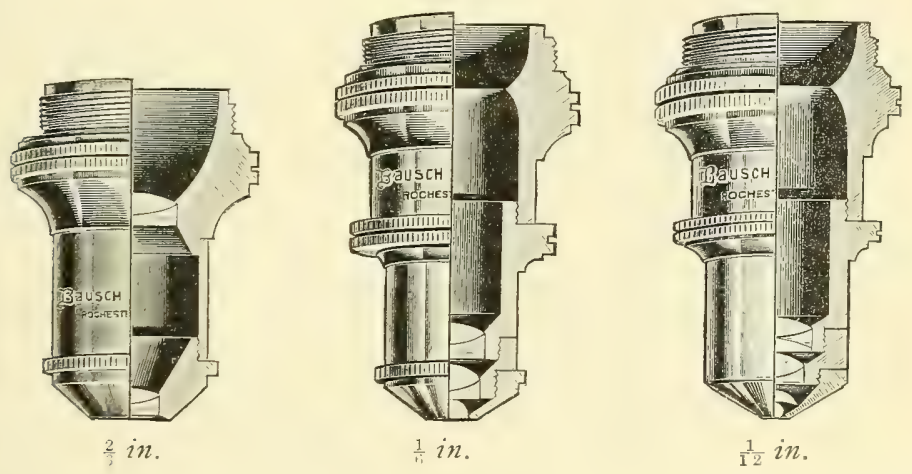

Dry objectives of $16 \mathrm{~mm}$. ( $\frac{2}{3} \mathrm{in}$.), $4 \mathrm{~mm}$. ( $\frac{1}{6} \mathrm{in}$.) and homogeneous immersion

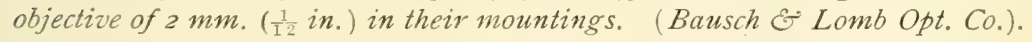




\title{
CHAPTER V
}

\section{DRAWING WITH THE MICROSCOPE}

\author{
APPARATUS AND MATERIAL FOR THIS CHAPTER
}

Microscope, Abbe and Wollaston's camera lucidas, drawing board, thumb tacks, pencils, paper, and microscope screen, (Fig. 59), microscopic preparations.

\section{DRAWING MICROSCOPIC OBJECTS}

\$ 777. Microscopic objects may be drawn free-hand directly from the microscope, but in this way a picture giving only the general appearance and relations of parts is obtained. For pictures which shall have all the parts of the object in true proportions and relations, it is necessary to obtain an exact outline of the image of the object, and to locate in this outline all the principal details of structure. It is then possible to complete the picture free-hand from the appearance of the object under the microscope. The appliance used in obtaining outlines, etc., of the microscope image is known as a camera lucida.

$\$$ I 78. Camera Lucida.-This is an optical apparatus for enabling one to see objects in greatly different situations, as if in one field of vision, and with the same eye. In other words it is an optical device for superimposing or combining two fields of view in one eye.

As applied to the microscope, it causes the magnified virtual image of the object under the microscope to appear as if projected upon the table or drawing board, where it is visible with the drawing paper, pencil, dividers, etc., by the same eye, and in the same field of vision. The microscopic image appears like a picture on the drawing paper (see note to $\$ \mathrm{I} 8 \mathrm{I}$ ). This is accomplished in two distinct ways:

(A) By a camera lucida reflecting the rays from the microscope so that their direction when they reach the eye coincides with that of the rays from the drawing paper, pencil, etc. In some of the camera lucidas from this group (Wollaston's, Fig. I I2), the rays are reflected twice, and the image appears as when looking directly into the microscope. In others the rays are reflected but once, and the image has the inversion produced by a plane mirror. For drawing purposes this 
inversion is a great objection, as it is necessary to similarly invert all the details added free-hand.

(B) By a camera lucida reflecting the rays of light from the drawing paper, etc., so that their direction when they reach the eye coincides with the direction of the rays from the microscope (Fig. 58, I09). In all of the camera lucidas of this group, the rays from the paper are twice reflected and no inversion appears.

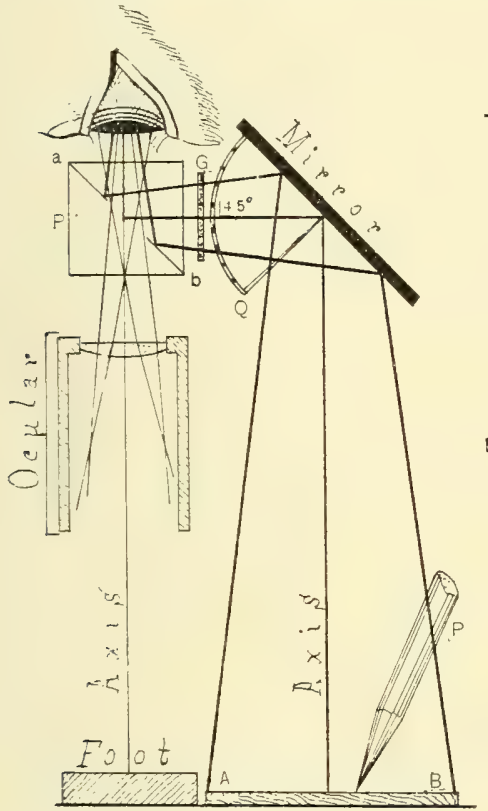

FiG. I09.

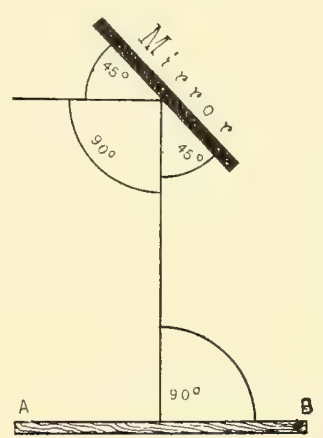

FIG. IIO.

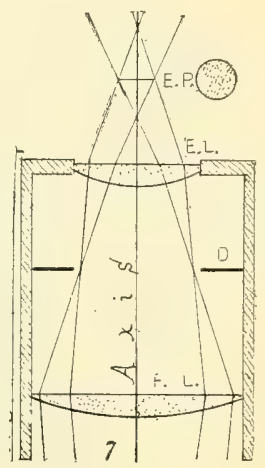

FIG. III.

FIG. I09: Abbe Camera Lucida with the mirror at $45^{\circ}$, the drawing surface horizontal, and the microscope vertical.

Axis, Axis. Axial ray from the microscope and from the drawing surface. $A, B$. Marginal rays of the field on the drawing surface. a b. Sectional view of the silvered surface on the upper of the triangular prisms composing the cubical prism $(P)$. The silvered surface is shown as incomplete in the center, thus giving passage to the rays from the microscope.

Foot. Foot or base of the microscope.

$G$. Smoked glass seen in section. It'is placed between the mirror and the prism to reduce the light from the drawing surface.

Mirror. The mirror of the camera lucida. A quadrant ( $Q$ ) has been added to indicate the angle of inclination of the mirror, which in this case is $45^{\circ}$.

Ocular. Ocular of the microscope over which the prism of the camera lucida is placed.

$P, P$. Drawing pencil and the cubical prism over the ocular.

FIG. IIO. Geometrical figure showing the angles made by the axial ray with the drawing surface and the mirror.

$A, B$. The drawing surface.

FIG. III. Ocular showing eye-point, E. P. It is at this point both horizontally and vertically that the hole in the silvered surface should be placed (\&, 182). 
The better forms of camera lucidas (Wollaston's, Grunow's, Abbe's, etc.), may be used for drawing both with low and with high powers. Some require the microscope to be inclined (Fig. IOo) while others are designed to be used on the microscope in a vertical position. As in biological work, it is often necessary to have the microscope rertical, the form for a vertical microscope is to be preferred; but see Figs. I I5I I6.

S I79. Avoidance of Distortion.--In order that the picture drawn by the aid of a camera lucida may not be distorted, it is necessary that the axial ray from the image on the drawing surface shall be at right angles to the drawing surface (Figs. II 2, I I4.)

\&. ISo. Wollaston's Camera Lucida.-This is a quadrangular prism of glass put in the path of the rays from the microscope, and it serves to change the direction of the axial ray 90 degrees. In using it the microscope is made horizontal, and the rays from the microscope enter one-half of the pupil while rays from the drawing surface enter the other half of the pupil. As seen in the figure (Fig. II2), the fields partly overlap, and where they do so overlap, pencil or dividers and microscopic image can be seen together.

In drawing or using the dividers with the Wollaston camera lucida it is necessary to have the field of the microscope and the drawing surface about equally lighted. If the drawing surface is too brilliantly lighted the pencil or dividers may be seen very clearly, but the microscopic image will be obscure. On the other hand, if the field of the microscope has too much light the microscopic image will be very definite, but the pencil or dividers will not be visible. It is necessary, as with the Abbe camera lucida (\% I 82$)$, to have the Wollaston prism properly arranged with reference to the axis of the microscope and the eye-point. If it is not, one will be unable to see the image well, and may be entirely unable to see the pencil and the image at the same time. Again, as rays from the microscope and from the drawing surface must enter independent parts of the pupil of the same eye, one must hold the eye so that the pupil is partly over the camera lucida and partly over the drawing surface. One can tell the proper position by trial. This is not a very satisfactory camera to draw with, but it is a very good form to measure the vertical distance of $250 \mathrm{~mm}$. at which the drawing surface should be placed when determining magnification ( 8 I 62 ).

S I8I. *Abbe Camera Lucida.-This consists of a cube of glass cut into two triangular prisms and silvered on the cut surface of the

FFor some persons the image and drawing surface, pencil, etc., do not appear on the drawing board as stated above, but under the microscope, according to the general principle that "objects appear in space where they could be touched along a perpendicular to the retinal surface stimulated,"-that is in the line of rays entering the eye. This is always the case with the Wollaston camera lucida. The explanation of the apparent location of the image, etc., on the drawing board with the Abbe camera lucida is that the attention is concentrated upon the drawing surface rather than upon the object under the microscope (Dr. W. B. Pillsbury ). 
upper one. A small oval hole is then cut out of the center of the silrered surface and the two prisms are cemented together in the form of the original cube with a perforated 45 degree mirror within it (Fig. I09, a b). The upper surface of the cube is covered by a perforated metal plate. This cube is placed over the ocular in such a way that the light from the microscope passes through the hole in the silvered face and thence directly to the eye. Light from the drawing surface is reflected by a mirror to the silvered surface of the prism and reflected by this surface to the eye in company with the rays from the microscope, so that the two fields appear as one, and the image is seen as if on the drawing surface (Figs. Iog, II4). It is designed for use with a vertical microscope, but see $\S$ I 84 .

FIG. II2. Wollaston's Camera Lucida, showning the rays from the microscope and from the drawing surface, and the position of the pupil of the eye.

For full explanation see Fig. 97.

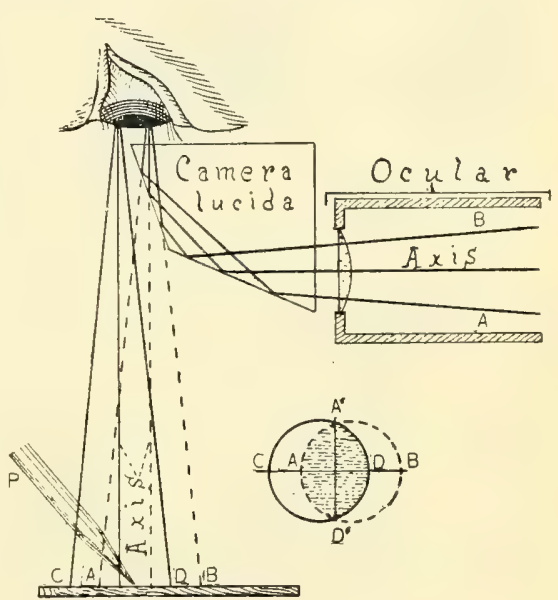

I 82. Arrangement of the Camera Lucida Prism.-In placing this camera lucida over the ocular for drawing or the determination of magnification, the center of the hole in the silvered surface is placed in the optic axis of the microscope. This is done by properly arranging the centering screws that clamp the camera to the microscope tube or ocular. The perforation in the silvered surface must also be at the level of the eye-point. In other words the prism must be so arranged vertically and horizontally that the hole in the silvered surface will be in the axis of the microscope and coincident with the eye-point of the ocular. If it is above or below, or to one side of the eye-point, part or all of the field of the microscope will be cut off. As stated above, the centering screws are for the proper horizontal arrangement of the prism. The prism is set at the right height by the makers for the eye-point of a medium ocular. If one desires to use an ocular with the eye-point farther away or nearer, as in using high or low oculars the position of the eye-point may be determined dis directd in 
$\$ 59$ and the prism loosened and raised or lowered to the proper level ; but in doing this one should avoid setting the prism obliquely to the mirror.

In the latest and best forms of this camera lucida special arrangements have been made for raising or lowering the prism so that it may be used with equal satisfaction on oculars with the eye-point at different levels, and the prism is hinged to turn aside without disturbing the mirror.

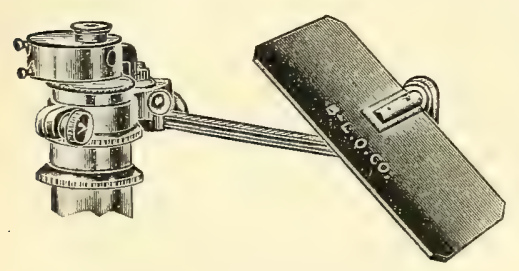

FIG. II3. One of the latest and best forms of the Abbe Camera Lucida (Bausch \& Lomb Optical Co.).

One can determine when the camera is in a proper position by looking into the microscope through it. If the field of the microscope appears as a circle and of about the same size as without the camera lucida, then the prism is in a proper position. If one side of the field is dark, then the prism is to one side of the center; if the field is considerably smaller than when the prism is turned off the ocular, it indicates that it is not at the correct level, i. e., it is above or below the eyepoint.

\section{$\$ 183$. Arrangement of the Mirror and the Drawing Surface.} -The Abbe camera lucida was designed for use with a vertical microscope (Fig. Iog). On a rertical microscope if the mirror is set at an angle of $45^{\circ}$, the axial ray will be at right angles with the table top or a drawing board which is horizontal, and a drawing made under these conditions will be in true proportion and not distorted. The stage of most microscopes, however, extends out so far at the sides that with a $45^{\circ}$ mirror the image appears in part on the stage of the microscope. In order to avoid this the mirror may be depressed to some point below $45^{\circ}$, say at $40^{\circ}$ or $35^{\circ}$ (Fig. I 4 ). But as the axial ray from the mirror to the prism must still be reflected horizontally, it follows that the axial ray will no longer form an angle of 90 degrees with the drawing surface, but a greater angle. If the mirror is depressed to $35^{\circ}$, then the axial ray must take an angle of $I 10^{\circ}$ with a horizontal drawing surface (see the geometrical figure Fig. I I $4, \mathrm{~A}$ ). To make the angle $90^{\circ}$ again, so that there shall be no distortion, the drawing board must be raised toward the microscope $20^{\circ}$. The general rule is to raise the drawing board twice as many degrees toward the microscope as the mirror is depressed below $45^{\circ}$. Practically the field for drawing 
B

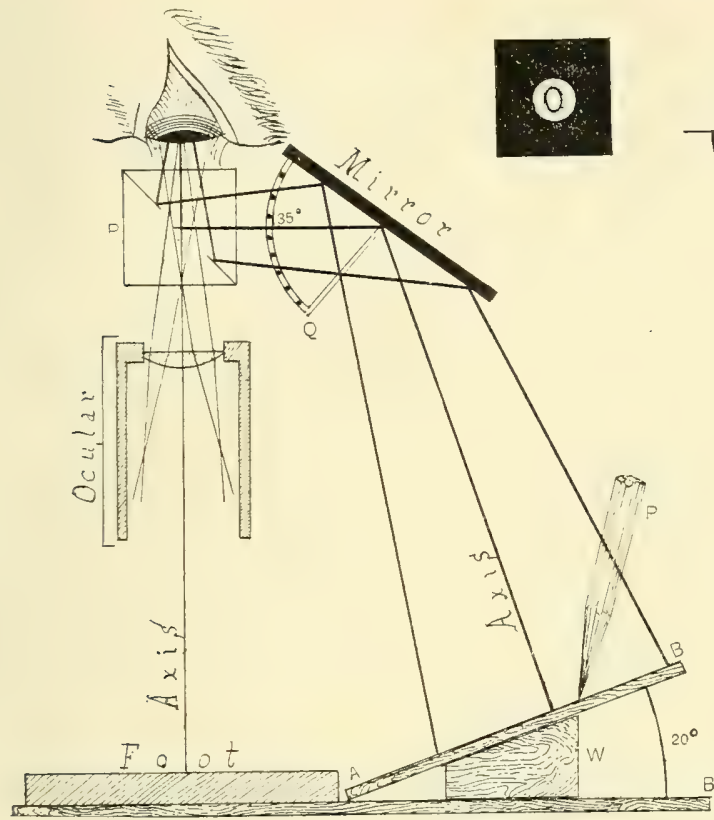

FIG. II 4 .
A 
can always be made free of the stage of the microscope, at $45^{\circ}$, at $40^{\circ}$, or at $35^{\circ}$. In the first case $\left(45^{\circ}\right.$ mirror) the drawing surface should be horizontal, in the second case $\left(40^{\circ}\right.$ mirror) the drawing surface should be elevated $10^{\circ}$, and in the third case ( $35^{\circ}$ mirror) the drawing board should be elevated $20^{\circ}$ toward the microscope. Furthermore it is necessary in using an elevated drawing board to have the mirror bar project directly laterally so that the edges of the mirror will be in planes parallel with the edges of the drawing board, otherwise there will be front to back distortion, although the elevation of the drawing board would avoid right to left distortion. If one has a micrometer ruled in squares (net micrometer) the distortion produced by not having the axial ray at right angles with the drawing surface may be very strikingly shown. For example, set the mirror at $35^{\circ}$ and use a horizontal drawing board. With a pencil make dots at the corners of some of the squares, and then with a straight edge connect the dots. The figures will be considerably longer from right to left than from front to back. Circles in the object would appear as ellipses in the drawings, the major axis being from right to left.

The angle of the mirror may be determined with a protractor, but that is troublesome. It is much more satisfactory to have a quadrant attached to the mirror and an indicator on the projecting arm of the mirror. If the quadrant is graduated throughout its entire extent, or preferably at three points, $45^{\circ}, 40^{\circ}$ and $35^{\circ}$, one can set the mirror at a known angle in a moment, then the drawing board can be hinged and the elevation of $10^{\circ}$ and $20^{\circ}$ determined with a protractor. The drawing board is very conveniently held up by a broad wedge. By marking the position of the wedge for $10^{\circ}$ and $20^{\circ}$ the protractor need be used but once, then the wedge may be put into position at any time for the proper elevation.

I84. Abbe Camera and Inclined Microscope.-It is very fatiguing to draw continuously with a vertical microscope, and many mounted objects admit of an inclination of the microscope, when one can sit and work in a more comfortable position. The Abbe camera is as perfectly adapted to use with an inclined as with a vertical microscope. All that is requisite is to be sure that the fundamental law is observed regarding the axial ray of the image and the drawing surface, viz., that they should be at right angles. This is very easily accomplished as follows: The drawing board is raised toward the microscope twice as many degrees as the mirror is depressed below $45^{\circ}$ ( $\$$ I 83 ), then it is raised exactly as many degrees as the microscope is inclined, 
and in the same direction, that is, so the end of the drawing board shall be in a plane parallel with the stage of the microscope. The mirror must have its edges in planes parallel with the edges of the drawing board also (Figs. I 5 , I 16 . )

FIG. II5. Arrangement of the drawing board for using the microscope in an inclined position with the Abbe camera lucida (designed by Mrs. S. P. Gage, I887.)

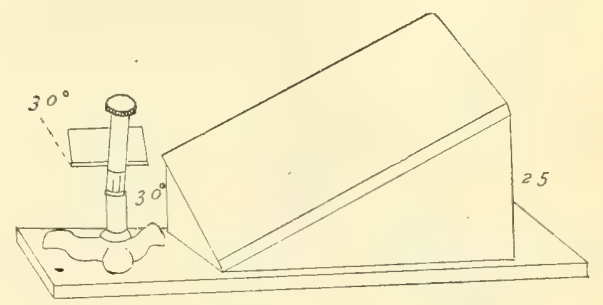

S I85. Drawing with the Abbe Camera Lucida.-(A) The light from the microscope and from the drawing surface should be of nearly equal intensity, so that the image and the drawing pencil can be seen with about equal distinctness. This may be accomplished with very low powers ( $6 \mathrm{~mm}$. and lower objectives) by covering the mirror of the microscope with white paper when transparent objects are to be drawn. For high powers it is best to use a substage condenser. Often the light may be balanced by using a larger or smaller opening in the diaphragm. One can tell which field is excessively illuminated, for it is the one in which objects are most distinctly seen. If it is the microscopic, then the image of the microscopic object is very distinct and the pencil is invisible or very indistinct. If the drawing surface is too brilliantly lighted the pencil can be seen clearly, but the microscopic image will be obscure.

When opaque objects, that is objects which must be lighted with reflected light $(\$ 63)$, like dark colored insects, etc., are to be drawn the light must usually be concentrated upon the object in some way. The microscope may be placed in a very strong light and the drawing board shaded or the light may be concentrated upon the object by means of a concave mirror or a bull's eye condenser (Fig. 53).

If the drawing surface is too brilliantly illuminated, it may be shaded by placing a book or a ground glass screen between it and the window, also by putting one or more smoked glasses in the path of the rays from the mirror (Fig. Iog $\mathrm{G}$ ). If the light in the microscope is too intense, it may be lessened by using white paper over the mirror, or by a ground glass screen between the microscope mirror and the source of light (Piersol, Amer. M. M. Jour., I888, p. I03). It is also an excellent plan to blacken the end of the drawing pencil with carbon 
ink. Sometimes it is easier to draw on a black surface, using a white pencil or style. The carbon paper used in manifolding letters, etc., may be used, or ordinary black paper may be lightly rubbed on one side with a moderately soft lead pencil. Place the black paper over white paper and trace the outlines with a pointed style of ivory or bone. A corresponding dark line will appear on the white paper beneath. (Jour. Roy. Micr. Soc., I883, p. 423).

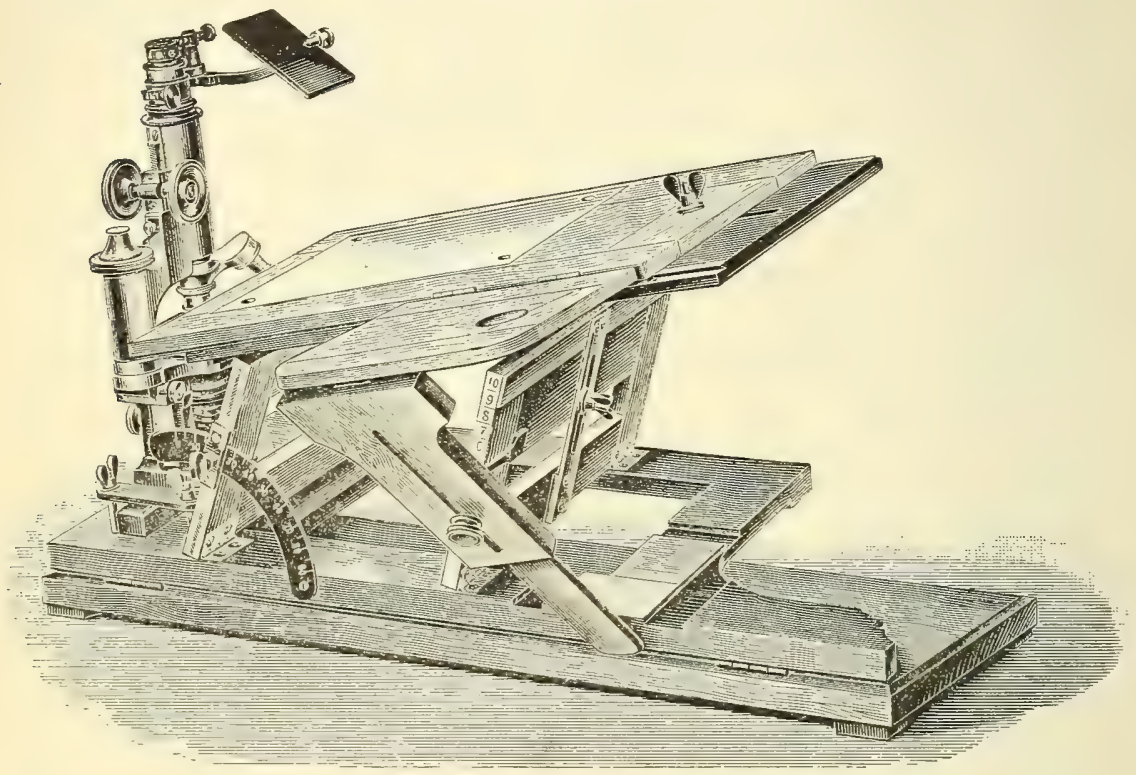

FIG. 'Ir6. Bernhard's Drawing Board for the Abbe Camera Lucida. This drawing hoard is adjustable vertically, and the board may be inclined to prevent distortion. It is also arranged for use with an inclined microscope, having the base board hinged." Microscope and drawing surface are then inclined together. (Zeit. wiss. Mikroskopie, vol. vi (I894, p. 298). (Zeiss Catalog).

(A) It is desirable to have the drawing paper fastened with thumb tacks, or in some other way. (B) The lines made while using the camera lucida should be very light, as they are liable to be irregular. (C) Only outlines are drawn and parts located with a camera lucida. Details are put in free-hand. (D) It is sometimes desirable to draw the outline of an object with a moderate power and add the details with a higher power. If this is done it should alway's be clearly stated. It is adrisable to do this only with objects in which the same structure is many times duplicated, as a nerve or a muscle. In such an object all 
the different structures could be shown, and by omitting some of the fibers the others could be made plainer without an undesirable enlargement of the entire figure.

(E) If a drawing of a given size is desired and it cannot be obtained by any combination of oculars, objectives and lengths of the tube of the microscope, the distance between the camera lucida and the table may be increased or diminished until the image is of the desired size. This distance is easily changed by the use of a book or a biock, but more conveniently if one has a drawing board with adjustable drawing surface like that shown in Fig. I 6 . The image of a few spaces of the micrometer will give scale of enlargement, or the power may be determined for the special case ( $\$ 186-187$ ).

(F) It is of the greatest advantage, as suggested by Heinsius (Zeit. II. Mikr., I 889, p. 367 ), to have the camera lucida hinged so that the prism may be turned off the ocular for a moment's glance at the preparation, and then returned in place without the necessity of loosening screws and readjusting the camera. This form is now made by several opticians, and the quadrant is added by some. (Fig. I I4.) Any skilled mechanic can add the quadrant.

I86. Magnification of the Microscope and size of Drawings with the Abbe Camera Lucida.-In determining the standard distance of 250 millimeters at which to measure the image in getting the magnification of the microscope, it is necessary to measure from the point marked $\mathrm{P}$ on the prism (Fig. I09) to the axis of the mirror and then vertically to the drawing board.

In getting the scale to which a drawing is enlarged the best way is to remove the preparation and put in its place a stage micrometer, and to trace a few ( 5 or IO) of its lines upon one corner of the drawing. The value of the spaces of the micrometer being given, thus :

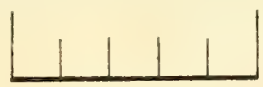

${ }_{1}^{1} \frac{1}{60}$ th $\mathrm{mm}$.

FIG. I17. Showing the method of indicating the scale at which a drawing zuas made.

The enlargement of the figure can then be accurately determined at any time by measuring with a steel scale the length of the image of the micrometer spaces and dividing it by their known size.

Thus, suppose the 5 spaces of the scale of enlargement given with a drawing were found to measure 25 millimeters and the spaces on the 
micrometer were $\bar{I}^{1}$ th millimeter, then the enlargement would be $25 \div \frac{\overline{5}}{100}=500$. That is, the image was drawn at a magnification of 500 diameters.

If the micrometer scale is added to every drawing, there is no need of troubling one's self about the exact distance at which the drawing is made, convenience may settle that, as the special magnification in each case may be determined from the scale accompanying the picture. It should be remembered, however, that the conditions when the scale is drawn must be exactly as when the drawing was made.

I87. Drawing at Slight Magnification.-Some objects are of considerable size and for drawings should be enlarged but a few diameters, -5 to 20 . By using sufficiently low objectives and different oculars a great range may be obtained. Frequently, however, the range must be still further increased. For a moderate increase in size the drawing surface may be put farther off, or, as one more commonly needs less rather than greater magnification, the drawing surface may be brought nearer the mirror of the camera lucida by piling books or other objects on the drawing board. If one takes the precaution to draw a scale on the figure under the same conditions, its enlargement can be readily determined ( $\$ \mathrm{I} 86)$.

A very satisfactory way to draw at low magnifications is to use a simple microscope and arrange a camera lucida over it as over the ocular. In this way one may get drawings at almost any low magnification.

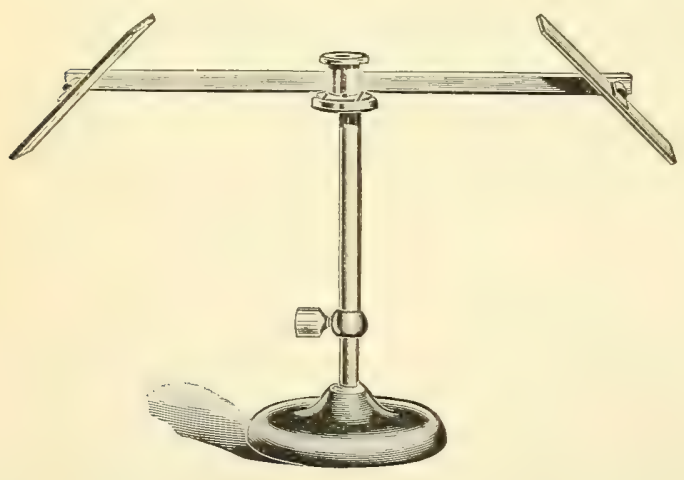

If one has many large objects to draw at a low magnification, then some form of embryograph is very convenient. (Jour. Roy. Micr., Soc., I899, p. 223.) The writer has made use of a photographic camera and different photographic objectives for the purpose. The object is

FIG. Ir8. Camera lucida for drawing objects natural size. (H. Bausch Jour. Applied Microscopy, vol. iii (Igoo, p. 89I).

illuminated as if for a photograph and in place of the ground glass a plain glass is used and on this some tracing paper is stretched. Noth- 
ing is then easier than to trace the outlines of the object. See also Ch. VIII.

\section{REFERENCES}

Beale, 3I, 355 ; Behrens, Kossel and Schiefferdecker, 77 ; Carpenter-Dallinger, 278 ; Van Heurck, 91 ; American Naturalist, 1886, p. IO7I, I887, pp. IO4O-IO43; Amer. Monthly Micr. Jour., I888, p. I03, I89o, p. 94 ; Jour. Roy. Micr. Soc., I88r,

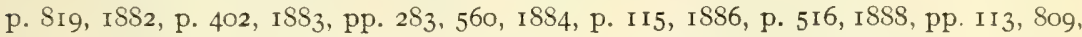
798 ; Zeit. wiss. Mikroskopie, I884, pp. I-2I, I889, p. 367, I893, pp. 289-295. Here is described an excellent apparatus made by Winkel. Consult also the latest catalogs of the opticians.

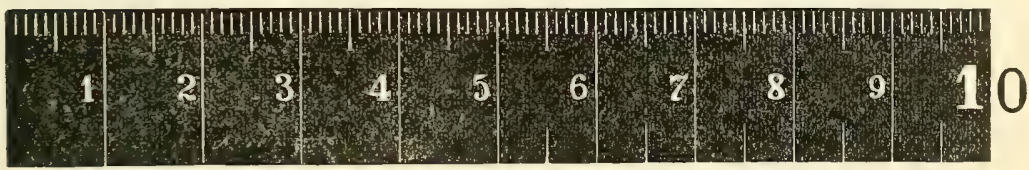

\section{CENTIMETER RULE}

The upper edge is in millimeters, the lower in centimeters, and half centimeters.

\section{THE METRIC STSTEM}

UNTTS.

The most commonly used divisions and multiples.

THE METER FOR (Centimeter (c.m.), I-Iooth Meter; Millimeter (m.m.), I-Ioooth Meter: Micron $(\mu)$, I-Ioooth Millimeter; the Micron is the unit in Micrometry (3 I66).

LENGTH. - (Kilometer, I000 Meters; used in measuring roads and other long distances.

THE GRAM FOR (Milligram (m.g.), I-IOooth Gram.

WEIGHT. . Kilogram, 1000 Grams, used for ordinary masses, like groceries, etc.

THE LITER FOR, Cubic Centimeter(c.c.), I-IOooth Liter. This is more common than the correct CAPACITY. . $\{$ form, Milliliter.

Divisions of the Units are indicated by the Latin prefixes : deci, I-Ioth; centi, I-Iooth ; Milli, I-loooth; Micro, I-I,000,000th of any unit.

Multiples are designated by Greek prefixes : deka, ro times; hecto, 100 times; kilo, Iooo times myria, 10,000 times: Mega, 1,000,000 times any unit. 


\title{
CHAPTER VI
}

\author{
MICRO-SPECTROSCOPE AND POLARISCOPE, \\ MICRO-CHEMISTRY, MICRO-MATALLOGRAPHY, \\ TEXTILE FIBERS
}

APPARATUS AND MATERIÀL REQUIRED FOR THIS CHAPTER

Compound microscope; Micro-spectroscope (? ISS); Watch-glasses and small vials, slides and covers ( $\$ 207)$; Various substances for examination (as blood and ammonium sulphide, permanganate of potash, chlorophyll, some colored fruit, etc., ( $8.208-217)$; Micro-polarizer ( 8.218$)$; Selenite plate ( 8.227$)$; Various doubly refracting objects, as crystals, textile fibers, starch, section of bone; Various chemicals, metals, etc.

\section{MICRO-SPECTROSCOPE}

\%. IS8. A Micro-Spectroscope, Spectroscopic or Spectral Ocular, is a direct vision spectroscope in connection with a microscope ocular. The one devised by Abbe and made by Zeiss consists of a direct vision spectroscope prism of the Amici pattern, and of considerable dispersion, placed over the ocular of the microscope. This direct vision or Amici prism consists of a single triangular prism of heavy flint glass in the middle and one of crown glass on each side, the edge of the crown glass prisms pointing toward the base of the flint glass prism, $i . \ell$., the edge of the crown and flint glass prisms point in opposite directions. The flint glass prism serves to give the dispersion or separation into colors, while the crown glass prisms serve to make the emergent rays approximately parallel with the incident rays, so that one looks directly into the prism along the axis of the microscope.

The Amici prism is in a special tube which is hinged to the ocular and held in position by a spring. It may be swung free of the ocular. In connection with the ocular is the slit mechanism and a prism for reflecting horizontal rays vertically for the purpose of obtaining a comparison spectrum ( $/ 201$ ). Finally near the top is a lateral tube with mirror for the purpose of projecting an Angstrom scale of wave lengths upon the spectrum ( $\%$ 202, Figs. I I9-I2O.)

3. ISg. Apparent Reversal of the Position of the Colors in a Direct Vision Spectroscope.-In accordance with the statements in 388 the dispersion or separation into colors is given by the flint glass prism or prisms and in accordance with the general law that the waves of shortest length, blue, etc., will be bent most, the colors have the position indicated in the top of Fig. I23, also above Fig. IIg. But if one looks into the direct vision spectroscope or holds the eye close to the single prism (Fig. I24), the colors will appear reversed as if the red were more bent. The explanation of this is shown in Fig. I24, where it can be 
readily seen that if the eye is placed at $\mathrm{E}$, close to the prism, the different colored rays appear in the direction from which they reach the eye and consequently are crossed in being projected into the field of vision and the real position is inverted. The same is true in looking into the micro-spectroscope. The actual

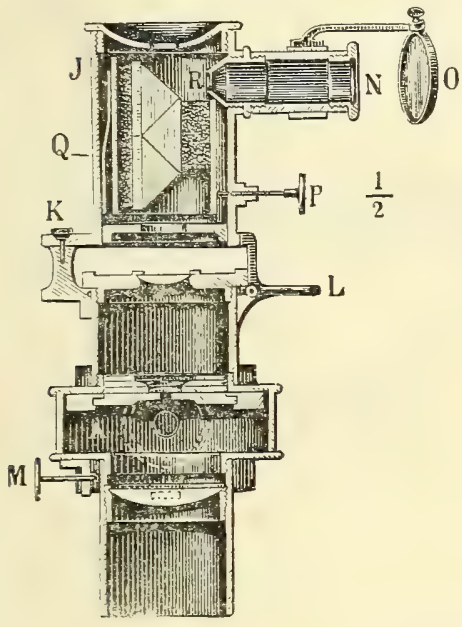

FIG. IIg.

Longitudinal Section of the whole instrument.

$$
\text { (1/2 Full size.) }
$$

Abbe's Micro-spectroscope.

Slit Mechanism separately. (Plan view, Full size.)

"The eye lens is adjustable so as to accurately focus on the slit situated between the lenses. The mechanism for contracting and expanding the slit is actuated by the screw $F$ and causes the laminae to move symmetrically (Merz's movement). The slit may be made sufficiently wide so as to include the whole visual field. The screw $H$ serves to limit the length of the slit so as to completely fill the latter with the imnage of the object under investigation when the comparison prism is inserted. The comparison prism is provided with a lateral frame and clips to hold the object and the illuminating mirror. All these parts are encased in a drum on the ocular.

Above the eye-piece is placed an Amici prism of great dispersion which may be turned aside about the pivot $K$, so as to allow of the adjustment of the object. The prism is retained in its axial position by the spring catch $L$. A scale is projected on the spectrum by means of a scale tube and mirror attached to the prism casing. The divisions of the scale indicate in decimals of a micron the wave length of the respective section of the spectrum. The screw $P$ serves to adjust the scale relative to the spectrum.

The instrument is inserted in the tube in place of the ordinary eye-piece and is clamped to the former by means of the screw $M$ in such a position that the mirrors $A$ and $O$, which respectively serve to illuminate the comparison prism and the scale of wave lengths are simultaneously illuminated." (Cut loaned by Wm. Krafft, N. Y.) 
position of the different colors may be determined by placing some ground glass or some of the lens-paper near the prism and observing with the eye at the distance of distinct vision.*

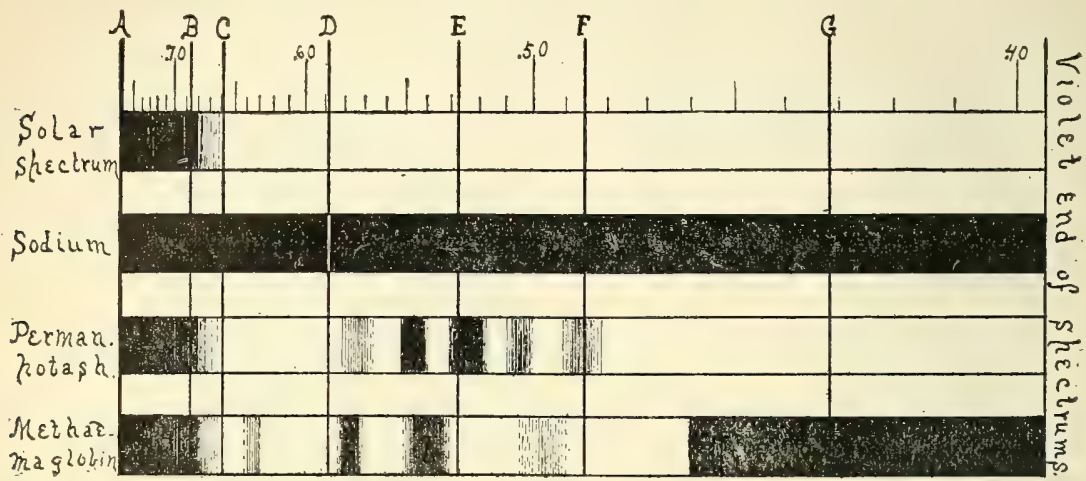

FIG. I2I. Various Spectrums.-All except that of sodium were obtained by diffused day-light with the slit of such a width as gave the most distinct Fraunhofer lines.

It frequently occurs that with a substance giving several absorption bands (e. $g$. chlorophyll) the density or thickness of the solution must be varied to show all the different bands clearly.

Solar Spectrum.- With diffused day-light and a narrow slit the spectrum is not visible much beyond the fixpd line $B$. In order to extend the visible spectrum in the red to the line $A$, one should use direct sunlight and a piece of ruby glass in place of the watch-glass in Fig. 123 .

Sodium Spectrum. - The line spectrum (\&I9I) of sodium obtained by lighting the microscope with an alcohol fame in which some salt of sodium is glowing. With the micro-spectroscope the sodium line seen in the solar spectrum and with the incandescent sodium appears single, except under very favorable circumstances. (\&.192). By using a comparison spectrum of day-light with the sodium spectrum the light and dark D-lines will be seen to be continwous as here shown.

Permanganate of Potash. - This spectrum is characterized by the presence of fire absorption bands in the middle of the spectrum and is best shown by using $a \frac{1}{10}$ per cent. solution of permanganate in water in a watch-glass as in Fig. 123.

Met-hemoglobin. - The absorption spectrum of met-hemoglobin is characterized by a considerable darkening of the blue end of the spectrum and of four absorption bands, one in the red near the line $C$ and two between $D$ and $E$ nearly in the place of the two bands of oxy-hemoglobin; finally there is a somewhat faint, wide, band near F. Such a met-hemoglobin spectrum is best obtained by making a solution of blood in water of such a concentration that the two oxy-hemoglobin bands run together $\left(\frac{2}{6} 2 I I\right)$, and then adding three or four drops of a $\frac{1}{10}$ per cent. aqueous solution of pernanganate of potash or a few drops of hydrogen dioxid $\left(\mathrm{H}_{2} \mathrm{O}_{2}\right)$. Soon the bright red will change to a brownish color, when it may be examined.

"The author wishes to acknowledge the aid rendered by Professor E. L. Nichols in giving the explanation offered in this section. 


\section{VARIOUS KINDS OF SPECTRA}

By a spectrum is meant the colored bands appearing when the light traverses a dispersing prism or a diffraction grating, or is affected in any way to separate the different wave lengths of light into groups. When daylight or some good artificial light is thus dispersed one gets the appearance so familiar in the rainbow.

\& Igo. Continuous Spectrum.-In case a good artificial light as the electric light is used the various rainbow or spectral colors merge gradually into one another in passing from end to end of the spectrum. There are no breaks or gaps.

\& I9I. Line Spectrum.-If a gas is made incandescent, the spectrum it produces consists, not of the various rainbow colors, but of sharp, narrow, bright lines, the color depending on the substance. All the rest of the spectrum is dark. These line spectra are very strikingly shown by various metals heated till they are in the form of incandescent vapor.

\& I92. Absorption Spectrum.-By this is meant a spectrum in which there are dark lines or bands in the spectrum. The most striking and interesting of the absorption spectra is the Solar Spectrum, or spectrum of sunlight. If this is examined carefully it will be found to be crossed by dark lines, the appearance being as if one were to draw pen marks across a continuous spectrum at various levels, sometimes apparently between the colors and sometimes in the midst of a color. These dark lines are the so-called Fraunhofer Lines. Some of the principal ones have been lettered with Roman capitals, A, B, C, D, E, F, G, H, commencing at the red end. The meaning of these lines was for a long time enigmatical, but it is now known that they correspond with the bright lines of a line spectrum (§ I9I). For example, if sodium is put in the flame of a spirit lamp it will vaporize and become luminous. If this light is examined there will be seen one or two bright yellow bands corresponding in position with D of the solar spectrum (Fig. I2I). If now the spirit-lamp flame, colored by the incandescent sodium, is placed in the path of the electric light, and it is examined as before, there will be a continuous spectrum, except for dark lines in place of the bright sodium lines. That is, the comparatively cool yellow light of the spirit lamp cuts off or absorbs the intensely hot yellow light of the electric light; and although the spirit flame sends a yellow light to the spectroscope it is so faint in comparison with the electric light that the sodium lines appear dark. It is believed that in the sun's atmosphere there are incandescent metal vapors (sodium, iron, etc.), but that they are so cool in comparison with the rays of their wave length in the sun that the cooler light of the incandescent metallic vapors absorb the light of corresponding wave length, and are, like the spirit lamp-flame, unable to make up the loss, and therefore the presence of the dark lines.

393. Absorption Spectra from Colored Substances.-While the solar spectrum is an absorption spectrum, the term is more commonly applied to the spectra obtained with light which has passed through or has been reflected from colored objects which are not self-luminous.

It is the special purpose of the micro-spectroscope to investigate the spectra of colored objects which are not self-luminous, $i . e_{.}$, blood and other liquids, various minerals, as monazite, etc. The spectra obtained by examining the light reflected from these colored bodies or transmitted through them, possess, like the solar 
spectrum dark lines or bands, but the bands are usually much wider and less sharply defined. Their number and position depend on the substance or its constitution (Fig. I22), and their width, in part, upon the thickness of the body. With some colored bodies, no definite bands are present. The spectrum is simply restricted at one or both ends and various of the other colors are considerably lessened in intensity. This is true of many colored fruits.

\&. 194. Angström and Stokes' Law of Absorption Spectra.-The waves of light absorbed by a body when light is transmitted through some of its substance

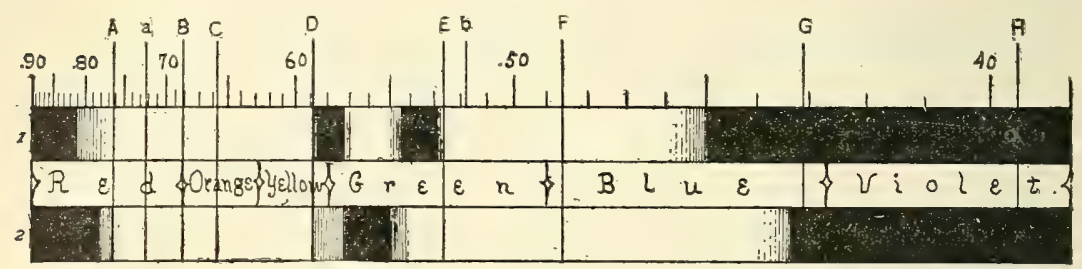

FIG. I22. Absorption spectrum of Oxy-hemoglobin or arterial blood $(I)$ and of Homoglobin or venous blood (2). (From Gamgee and McMunn).

$A, B, C, D, E, F, G, H$. Some of the Prinipal Fraunhofer lines of the solar spectrum $(\&$ I 192$)$.

$.90, .80, .70, .60, .50, .40$. Wave lengths in microns, as shown in Angström's scale $(\& 202)$. It will be seen that the wave lengths increase toward the red and decrease toward the violet end of the spectrum.

Red, Yellow, Orange, etc. Color regions of the spectrum. Indigo should come between the blue and the violet to complete the seven colors usually given. It was omitted through inadvertence.

are precisely the waves radiated from it when it becomes self-luminous. For example, a piece of glass that is yellow when cool, gives out blue light when it is hot enough to be self-luminous. Sodium vapor absorbs two bands of yellow light (D lines); but when light is not sent through it, but itself is luminous and examined as a source of light its spectrum gives bright sodium lines, all the rest of the spectrum being dark (Fig. I2I).

\& I95. Law of Color.-The light reaching the eye from a colored, solid, liquid or gaseous body lighted with white light, will be that due to white light less the light waves that have been absorbed by the colored body. Or in other words, it will be due to the wave lengths of light that finally reach the eye from the object. For example, a thin layer of blood under the microscope will appear yellowish green, but a thick layer will appear pure red. If now these two layers are examined with a micro-spectroscope, the thin layer will show all the colors, but the red end will be slightly, and the blue end considerably restricted, and some of the colors will appear considerably lessened in intensity. Finally there may appear two shadow-like bands, or if the layer is thick enough, two well-defined dark bands in the green ( 8 , 2 ro $)$.

If the thick layer is examined in the same way, the spectrum will show only. red with a little orange light, all the rest being absorbed. Thus the spectroscope shows which colors remain, in part or wholly, and it is the mixture of this remaining or unabsorbed light that gives color to the object. 
\% I96. Complementary Spectra.-While it is believed that Angström's law ( 3 I94) is correct, there are many bodies on which it cannot be tested, as they change in chemical or molecular constitution before reaching a sufficiently high temperature to become luminous. There are compounds, however, like those of didymium, erbium and terbium, which do not change with the heat necessary to render them luminous, and with them the incandescence and absorption spectra are mutually complementary, the one presenting bright lines where the other presents dark ones (Daniell).

\section{ADJUSTING THE: MICRQ-SPECTROSCOPE}

$\$$ I97. The micro-spectroscope, or spectroscopic ocular, is put in the place of the ordinary ocular in the microscope, and clamped to the top of the tube by means of a screw for the purpose.

$\S$ I98. Adjustment of the Slit.-In place of the ordinary diaphragm with circular opening, the spectral ocular has a diaphragm composed of two movable knife edges by which a slit-like opening of greater or less width and length may be obtained at will by the use of screws for the purpose. To adjust the slit, depress the lever holding the prism-tube in position over the ocular, and swing the prism aside. One can then look into the ocular. The lateral screw should be used and the knife edges approached till they appear about half a millimeter apart. If now the Amici prism is put back in place and the microscope well lighted, one will see a spectrum by looking into the upper end of the spectroscope. If the slit is too wide, the colors will overlap in the middle of the spectrum and be pure only at the red and blue ends; and the Fraunhofer or other bands in the spectrum will be faint or invisible. Dust on the edges of the slit gives the appearance of longitudinal streaks on the spectrum.

$\S$ Ig9. Mutual Arrangement of Slit and Prism.-In order that the spectrum may appear as if made up of colored bands going directly across the long axis of the spectrum, the slit must be parallel with the refracting edge of the prism. If the slit and prism are not thus mutually arranged, the colored bands will appear oblique, and the whole spectrutn may be greatly narrowed. If the colored bands are oblique, grasp the prism tube and slowly rotate it to the right or to the left until the various colored bands extend directly across the spectrum.

5. 200. Focusing the Slit.-In order that the lines or bands in the spectrum shall be sharply defined, the eye-lens of the ocular should be accurately focused on the slit. The eye-lens is movable, and when the prism is swung aside it is very easy to focus the slit as one focused 
for the ocular micrometer ( $\$ \mathrm{I}_{2}$ ). If one now uses daylight there will be seen in the spectrum the dark Fraunhofer lines (Fig. I 2 I E. F., etc.).

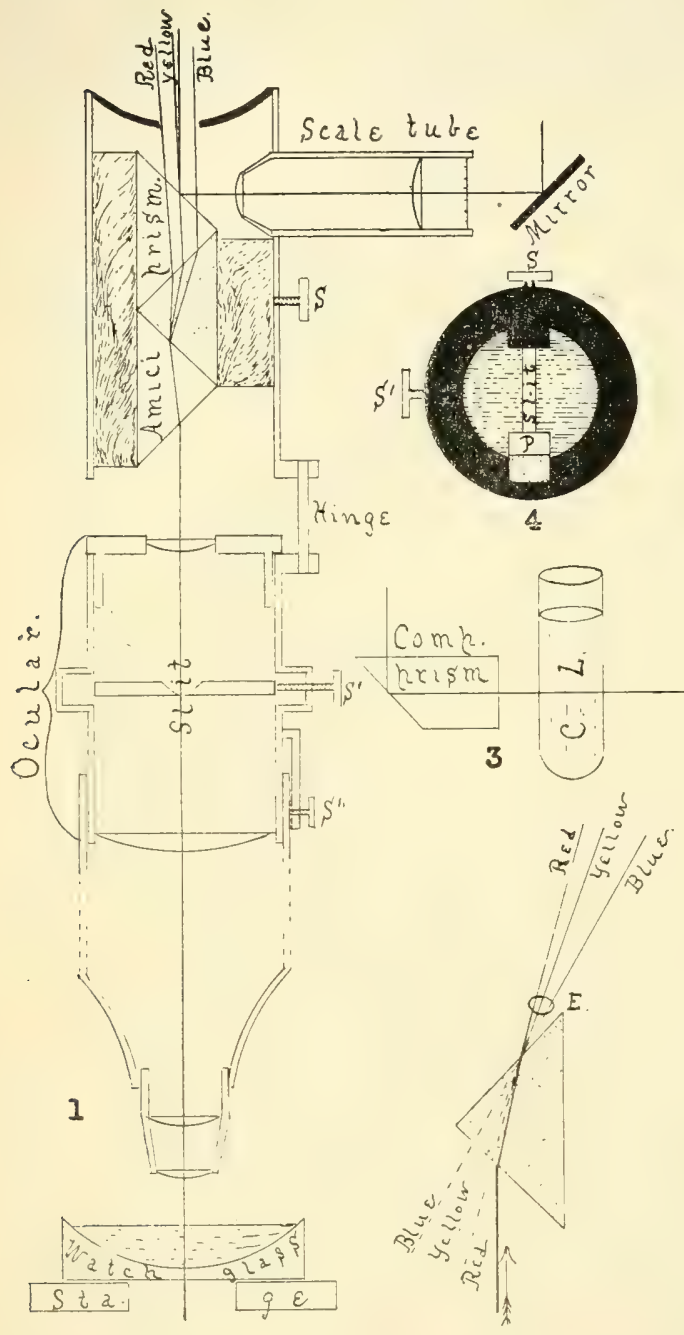

FIG. 123.
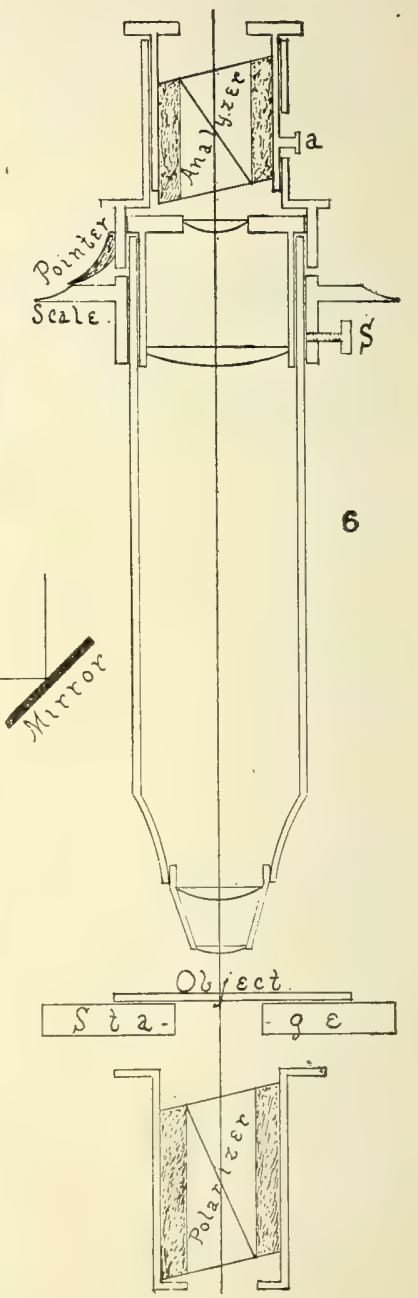

FIG. I25.

FIG. I23. (I). Section of the tube and stage of the microscope with the spectral ocular or micro-spectroscope in position.

Amici Prism (\& ISS). - The direct vision prism of Amici in which the central shaded prism of fint glass gizes the dispersion or separation into colors, while the 
end prisms of crown glass cause the rays to emerge approximately parallel with the axis of the microscope. A single ray is represented as entering the prism and this is divided into three groups (Red, Yellow, Blue), which emerge from the prism, the red being least and the blue most bent toward the base of the flint prism (see Fig. I24). ocular.

Hinge.-The hinge on which the prism tube turns when it is swung off the

Ocular (3 I88)-The ocular in which the slit mechanism takes the place of the diaphragm (\$ I98). The eye-lens is movable as in a micrometer ocular, so that the slit may be accurately focused for the different colors ( 8200$)$.

$S$. Screw for setting the scale of wave lengths (\& 202).

$S^{\prime}$. Screw for regulating the width of the slit ( \& 198).

$S^{\prime \prime}$. Screw for clamping the micro-spectroscope to the tube of the microscope.

Scale Tube.-The tube near the upper end containing the Angström scale and the lenses for projecting the image upon the upper face of the Amici prism, whence it is reflected upward to the eye with the different colored rays. At the right is a special mirror for lighting the scale.

Slit.-The linear opening between the knife edges. Through the slit the light passes to the prism. It must be arranged parallel with the refracting edge of the prism, and of such a width that the Fraunhofer or Fixed Lines are very clearly and sharply defined when the eye-lens is properly focused (\&. I98-200).

Stage. - The stage of the microscope. This supports a watch-glass with sloping sides for containing the colored liquid to be examined.

(3) Comparison Prism with tube for colored liquid (C. L.), and mirror. The prism reflects horizontal rays vertically, so that when the prism is made to cover part of the slit two parallel spectra may be seen, one from light sent directly through the entire microscope and one from the light reflected upward from the comparison prism.

(4) View of the Slit Mechanism from below.-Slit, the linear space between the knife edges through which the light passes.

$P$. Comparison prism beneath the slit and covering part of it at will.

S. $S^{\prime}$. Screzes for regulating the length and width of the slit.

FIG. 124. Flint-Glass Prism showing the separation or dispersion of white light into the three groups of colored rays (Red, Yellow, Blue). the blue rays being bent the most from the refracting edge (\& 189$)$.

FIG. 125. Sectional View of a Microscope with the Polariscope in position $(8218-227)$.

Analyzer and Polarizer.-They are represented with corresponding faces parallel so that the polarized beam could traverse freely the analyzer. If either Nicol were rotated $90^{\circ}$ they would be crossed and no light would traverse the analyzer unless some polarizing substance were used as object. (a) Slot in the analyzer tube so that the analyzer may be raised or lowered to adjust it for difference of level of the eye-point in different oculars $(\$ 59,200)$.

Pointer and Scale. - The pointer attached to the analyzer and the scale or divided circle clamped (by the screw $S$ ) to the tube of the microscope. The pointer and scale enable one to determine the exact amount of rotation of the analyzer (\& 220).

Object-The object whose character is to be investigated by polarized light. 
To show the necessity of focusing the slit, move the eye-lens down or up as far as possible, and the Fraunhofer lines cannot be seen. While looking into the spectroscope move the ocular lens up or down, and when it is focused the Fraunhofer lines will reappear. As the different colors of the spectrum have different wave lengths, it is necessary to focus the slit for each color if the sharpest possible pictures are desired.

It will be found that the eye-lens of the ocular must be farther from the slit for the sharpest focus of the red end than for the sharpest focus of the lines at the blue end. This is because the wave length of red is markedly greater than for blue light.

Longitudinal dark lines of the spectrum may be due to irregularity of the edge of the slit or to the presence of dust. They are most troublesome with a very narrow slit.

$\$ 201$. Comparison or Double Spectrum.-In order to compare the spectra of two different substances it is desirable to be able to examine their spectra side by side. This is provided for in the better forms of micro-spectroscopes by a prism just below the slit, so placed that the light entering it from a mirror at the side of the drum shall be totally reflected in a vertical direction, and thus parallel with the rays from the microscope. The two spectra will be side by side with a narrow dark line separating them. If now the slit is well focused and daylight be sent through the microscope and into the side to the reflecting or comparison prism, the colored bands and the Fraunhofer dark lines will appear directly continuous across the two spectra. The prism for the comparison spectrum is movable and may be thrown entirely out of the field if desired. When it is to be used, it is moved about half way across the field so that the two spectra shall have about the same width.

$\$ 202$. Scale of Wave Lengths. - In the Abbe micro-spectroscope the scale is in a separate tube near the top of the prism and at right angles to the prism-tube. A special mirror serves to light the scale, which is projected upon the spectrum by a lens in the scale-tube. This scale is of the Angström form, and the wave lengths of any part of the spectrum may be read off directly, after the scale is once set in the proper position, that is, when it is set so that any given wave length on the scale is opposite the part of the spectrum known by previous investigation to have that particular wave length. The point most often selected for setting the scale is opposite the sodium line where the wave length is, according to Angström, $0.5892 \mu$. In ad- 
justing the scale, one may focus very sharply the dark sodium line of the solar spectrum and set the scale so that the number 0.589 is opposite the sodium or D line, or a method that is frequently used and serves to illustrate $\$$ I $9 \mathrm{I}-2$, is to sprinkle some salt of sodium (carbonate of sodium is good) in an alcohol lamp flame and to examine this flame. If this is done in a darkened place with a spectroscope, a narrow bright band will be seen in the yellow part of the spectrum. If now ordinary daylight is sent through the comparison prism, the bright line of the sodium will be seen to be directly continuous with the dark line at $\mathrm{D}$ in the solar spectrum (Fig. I2I). By reflecting light into the scale-tube the image of the scale will appear on the spectrum, and by a screw just under the scale-tube but within the prism-tube, the proper point on the scale $\left(0.5^{89} \mu\right)$ can be brought opposite the sodium band. All the scale will then give the wave lengths directly. Sometimes the scale is oblique to the spectrum. This may be remedied by turning the prism-tube slightly one way or the other. It may be due to the wrong position of the scale itself. If so, grasp the milled ring at the distal end of the scale-tube and, while looking into the spectroscope, rotate the tube until the lines of the scale are parallel with the Fraunhofer lines. It is necessary in adjusting the scale to be sure that the larger number, 0.70 , is at the red end of the spectrum.

The numbers on the scale should be very clearly defined. If they do not so appear, the scale-tube must be focused by grasping the outer tube of the scale-tube and moving it toward or from the prism-tube until the scale is distinct. In focusing the scale, grasp the outer scaletube with one hand and the prism-tube with the other, and push or pull in opposite directions. In this way one will be less liable to injure the spectroscope.

203. Designation of Wave Length.-Wave lengths of light are designated by the Greek letter $\lambda$, followed by the number indicating the wave length in some fraction of a meter. With the Abbe micro-spectroscope the micron is taken as the unit as with other microscopical measurements ( $\$ 66)$. Various units are in use, as the one hundred thousandth of a millimeter, millionths or ten millionths of a millimeter. If these smaller units are taken, the wave lengths will be indicated either as a decimal fraction of a millimeter or as whole numbers. Thus, according to Angström, the wave length of sodium light is 5892 ten millionths mm., or 589.2 millionths, or 58.92 one hundred thousandths, or 0.5892 one thousandth $\mathrm{mm}$., or $0.5^{892} \mu$. The last would be indicated thus, $\lambda \mathrm{D}=0.5892 \mu$. 


\section{$\$ 204$. Lighting for Micro-spectroscope.-For opaque objects} a strong light should be thrown on them either with a concave mirror or a condensing lens. For transparent objects the amount of the substance and the depth of color must be considered. As a general rule it is well to use plenty of light, as that from an Abbe illuminator with a large opening in the diaphragm, or with the diaphragm entirely open. For very small objects and thin layers of liquids it may be better to use less light. One must try both methods in a given case, and learn by experience.

The direct and the comparison spectra should be about equally illuminated. One can manage this by putting the object requiring the greater amount of illumination on the stage of the microscope and lighting it with the Abbe illuminator. In lighting it is found in general that for red or yellow objects, lamp-light gives very satisfactory results. For the examination of blood and blood crystals the light from a petroleum lamp is excellent. For objects with much blue or violet, daylight or artificial light rich in blue light is best.

Furthermore, one should be on his guard against confusing the ordinary absorption bands with the Fraunhofer lines when daylight is used. With lamp-light the Fraunhofer lines are absent and, therefore, not a source of possible confusion.

205. Objectives to Use with the Micro-spectroscope.-If the material is of considerable bulk, a low objective ( 16 to $50 \mathrm{~mm}$.) is to be preferred. This depends on the nature of the object under examination, however. In case of individual crystals one should use sufficient magnification to make the real image of the crystal entirely fill the width of the slit. The length of the slit may then be regulated by the screw on the side of the drum, and also by the comparison prism. If the object does not fill the whole slit the white light entering the spectroscope with the light from the object might obscure the absorption bands. For opaque objects illuminating objectives are useful $(\$ 28,233$. $)$

In using high objectires with the micro-spectroscope one must very carefully regulate the light ( $\mathrm{Ch}$. II) and sometimes shade the object.

206. Focusing the Objective.-For focusing the objective the prism-tube is swung aside, and then the slit made wide by turning the adjusting screw at the side. If the slit is open one can see objects when the microscope is focused as with an ordinary ocular. After an object is focused, it may be put exactly in position to fill the slit of the 
spectroscope, then the knife edges are brought together till the slit is of the right width ; if the slit is then too long it may be shortened by using one of the mechanism screws on the side, or if that is not sufficient, by bringing the comparison prism farther over the field. If one now replaces the Amici prism and looks into the microscope, the spectrum is liable to have longitudinal shimmering lines. To get rid of these focus up or down a little so that the microscope will be slightly out of focus.

\section{$\$ 207$. Amount of Material Necessary for Absorption Spectra} and its Proper Manipulation.-The amount of material necessary to give an absorption spectrum varies greatly with different substances, and can be determined only by trial. It a transparent solid is under investigation it is well to have it in the form a wedge, then successive thicknesses can be brought under the microscope. If a liquid substance is being examined, a watch glass with sloping sides forms an excellent vessel to contain it, then successive thicknesses of the liquid can be brought into the field as with the wedge-shaped solid. Frequently only a very weak solution is obtainable; in this case it can be placed in a homcopathic vial, or in some glass tubing sealed at the end, then one can look lengthwise through the liquid and get the effect of a more concentrated solution. For minute bodies like crystals or blood corpuscles, one may proceed as described in the previous section.

\section{MICRO-SPECTROSCOPE-EXPERIMENTS*}

208. Put the micro-spectroscope in position, arrange the slit and the Amici prism so that the spectrum will show the various spectral colors going directly across it (\$ I98-I99) and carefully focus the slit. This may be done either by swinging the prism-tube aside and proceeding as for the ocular micrometer $(\S \mathrm{I} 72$ ), or by moving the eye-lens of the ocular up and down while looking into the microspectroscope until the dark lines of the solar spectrum are distinct. If they cannot be made distinct by focusing the slit, then the light is too feeble or the slit is too wide $(\$ 198)$. With the lever move the comparison prism across half the field so that the two spectra shall be of about equal width. For lighting, see $\$ 204$.

*If one does not possess a micro-spectroscope, quite satisfactory results may be obtained by using a microscope with a 16 to $12 \mathrm{~mm}$. objective and a pocket, directvision spectroscope in place of the eye-piece. (Bleile, Trans. Amer. Micr. Soc. IgoO, p. 8). 
$\S$ 209. Absorption Spectrum of Permanganate of Potash.Make a solution of permanganate of potash in water of such a strength that a stratum 3 or $4 \mathrm{~mm}$. thick is transparent. Put this solution in a watch-glass with sloping sides, and put it under the microscope. Use a $50 \mathrm{~mm}$. or $16 \mathrm{~mm}$. objective, and use the full opening of the illuminator. Light strongly. Look into the spectroscope and slowly move the watch-glass into the field. Note carefully the appearance with the thin stratum of liquid at the edge and then as it gradually thickens on moving the watch-glass still farther along. Count the absorption bands and note particularly the red and blue ends. Compare carefully with the comparison spectrum (Figs. I21, I 22). For strength of solution see $\$ 207$.

\$2 IO. Absorption Spectrum of Blood.-Obtain blood from a recently killed animal, or flame a needle, and after it is cool prick the finger two or three times in a small area, then wind a handkerchief or a rubber tube around the base of the finger, and squeeze the finger with the other hand. Some blood will ooze out of the pricks. Rinse this off into a watch-glass partly filled with water. Continue to add the blood until the water is quite red. Place the watch-glass of diluted blood under the microscope in place of the permanganate, using the same objective, etc. Note carefully the spectrum. It would be advantageous to determine the wave length opposite the center of the dark bands. This may easily be done by setting the scale properly as described in $\S 202$. Make another preparation, but use a homeopathic vial instead of a watch-glass. Cork the vial and lay it down upon the stage of the microscope. Observe the spectrum. It will be like that in the watchglass. Remove the cork and look through the whole length of the vial. The bands will be much darker, and if the solution is thick enough only red and a little orange will appear. Re-insert the cork and incline the vial so that the light traverses a very thin layer, then gradually elevate the vial and the effect of a thicker and thicker layer may be seen. Note especially that the two characteristic bands unite and form one wide band as the stratum of liquid thickens. Compare with the following :

Add to the vial of diluted blood a drop or two of ammonium sulphide, such as is used for a reducing agent in chemical laboratories. Shake the bottle gently and then allow it to stand for ten or fifteen minutes. Examine it and the two bands will have been replaced by a single, less clearly defined band in about the same position. The blood will also appear somewhat purple. Shake the vial vigorously 
and the color will change to the bright red of fresh blood. Examine it again with the spectroscope and the two bands will be visible. After five or ten minutes another examination will show but a single band. Incline the bottle so that a thin stratum may be examined. Note that the stratum of liquid must be considerably thicker to show the absorption band than was necessary to show the two bands in the first experiment. Furthermore, while the single band may be made quite black on thickening the stratum, it will not separate into two bands with a thinner stratum. In this experiment it is very instructive to have a second vial of fresh diluted blood, say that from the watchglass, before the opening of the comparison prism. The two banded spectrum will then be in position to be compared with the spectrum of the blood treated with the ammonium sulphide.

The two banded spectrum is that of oxy-hemoglobin, or arterial blood, the single banded spectrum of hemoglobin (sometimes called reduced hemoglobin) or venous blood, that is, the respiratory oxygen is present in the two banded spectrum but absent from the single banded spectrum. When the bottle was shaken the hemoglobin took up oxygen from the air and became oxy-hemoglobin, as occurs in the lungs, but soon the ammonium sulphide took away the respiratory oxygen, thus reducing the oxy-hemoglobin to hemoglobin. This may be repeated many times (Fig. I 22).

$\S 2$ II. Met-Hemoglobin.-The absorption spectrum of methemoglobin is characterized by a considerable darkening of the blue end of the spectrum and of four absorption bands, one in the red near the line $\mathrm{C}$ and two between $\mathrm{D}$ and $\mathrm{E}$, nearly in the place of the two bands of oxy-hemoglobin; finally there is a somewhat faint, wide band near F. Such a met-hemoglobin spectrum is best obtained by making a solution of blood in water of such a concentration that the two oxy-hemoglobin bands run together ( $\$ 210$ ), and then adding three or four drops of a $\frac{1}{10}$ per cent. aqueous solution of permanganate of potash. Soon the bright red will change to a brownish color, when it may be examined (Fig. I2I). Instead of the permanganate one may use hydrogen dioxide $\left(\mathrm{H}_{2} \mathrm{O}_{2}\right)$.

$\$ 2$ I2. Carbon Monoxide Hemoglobin (CO-Hemoglobin).To obtain this, kill an animal in illuminating gas, or one may allow illuminating gas to bubble through some blood already taken from the body. The gas should bubble through a minute or two. The oxygen will be displaced by carbon monoxide. This forms quite a stable compound with hemoglobin, and is of a bright cherry-red color. Its 
spectrum is nearly like that of oxy-hemoglobin, but the bands are farther toward the blue. Add several drops of ammonium sulphide and allow the blood to stand some time. No reduction will take place, thus forming a marked contrast to solutions of oxy-hemoglobin. By the addition of a few drops of glacial acetic acid a dark brownish red color is produced.

2I3. Carmine Solution.-Make a solution of carmine by putting $\frac{1}{10}$ th gram of carmine in $100 \mathrm{cc}$. of water and adding ro drops of strong ammonia. Put some of this in a watch-glass or in a small vial and compare the spectrum with that of oxy-hemoglobin or carbon monoxide hemoglobin. It has two bands in nearly the same position, thus giving the spectrum a striking similarity to blood. If now several drops, 15 or 20 , of glacial acetic acid are added to the carmine, the bands remain and the color is not markedly changed, while with either oxy-hemoglobin or CO-hemoglobin the color would be decidedly changed from the bright red to a dull reddish brown, and the spectrum, if any could be seen, would be markedly different. Carmine and O-hemoglobin can be distinguished by the use of ammonium sulphide, the carmine remaining practically unchanged while the blood shows the single band of hemoglobin ( $\$ 210$ ). The acetic acid serves to differentiate the CO-hemoglobin as well as the O-hemoglobin.

\section{S 2I4. Colored Bodies not giving Distinctly Banded Absorp-} tion Spectra.--Some quite brilliantly colored objects, like the skin of a red apple, do not give a banded spectrum. Take the skin of a red apple, mount it on a slide, put on a cover-glass and add a drop of water at the edge of the corer. Put the preparation under the microscope and observe the spectrum. Although no bands will appear, in some cases at least, yet the ends of the spectrum will be restricted and various regions of the spectrum will not be so bright as the comparison spectrum. Here the red color arises from the mixture of the unabsorbed waves, as occurs with other colored objects. In this case, however, not all the light of a given wave length is absorbed, consequently there are no clearly defined dark bands, the light is simply less brilliant in certain regions and the red rays so predominate that they give the prevailing color.

$\$ 215$. Nearly Colorless Bodies with Clearly Marked Absorption Spectra.-In contradistinction to the brightly colored objects with no distinct absorption bands are those nearly colorless bodies and solutions which give as sharply defined absorption bands as could be desired. The best examples of this are afforded by solutions 
of the rare earths, didymium, etc. These in solutions that give hardly a trace of color to the eye give absorption bands that almost rival the Fraunhofer lines in sharpness.

$\$ 2$ I6. Absorption Spectra of Minerals.-As example take some monazite sand on a slide and either mount it in balsam (see \$256), or cover and add a drop of water. The examination may be made also with the dry sand, but it is less satisfactory. Light well with transmitted light, and move the preparation slowly around. Absorption bands will appear occasionally. Swing the prism tube off the ocular, open the slit and focus the sand. Get the image of one or more grains directly in the slit, then narrow and shorten the slit so that no light can reach the spectroscope that has not traversed the grain of sand. The spectrum will be satisfactory under such conditions. It is frequently of great service in determining the character of unknown mineral sands to compare the spectra with known minerals. If the absorption bands are identical, it is strong evidence in favor of the identity of the minerals. For proper lighting see $\$ 204$.

$\$ 217$. While the study of absorption spectra gives one a great deal of accurate information, great caution must be exercised in drawing conclusions as to the identity or even the close relationship of bodies giving approximately the same absorption spectra. The rule followed by the best workers is to have a known body as control and to treat the unknown body and known body with the same reagents, and to dissolve them in the same medium. If all the reactions are identical then the presumption is strong that the bodies are identical or very closely related. For example, while one might be in doubt between a solution of oxy- or CO-hemoglobin and carmine, the addition of ammonium sulphide serves to change the double to a single band in the O-hemoglobin, and glacial acetic acid enables one to distinguish between the CO-blood and the carmine, although the ammonium sulphide would not enable one to make the distinction. Furthermore it is unsafe to compare objects dissolved in different media. The same objects as "cyanine and aniline blue dissolved in alcohol give a very similar spectrum, but in water a totally different one." "'Totally different bodies show absorption bands in exactly the same position (solid nitrate of uranium and permanganate of potash in the blue)." (MacMunn). The rule given by MacMunn is a good one: "The recognition of a body becomes more certain if its spectrum consists of several absorption bands, but even the coincidence of these bands with those of another body is not sufficient to enable us to infer chemical identity; 
what enables us to do so with certainty is the fact: that the two solutions give bands of equal intensities in the same parts of the spectrum which undergo analogous changes on the addition of the same reagent."

\section{REFERENCES TO THE MICRO-SPECTROSCOPE AND}

\section{SPECTRUM ANALYSIS}

The micro-spectroscope is playing an ever-increasingly important role in the spectrum analysis of animal and vegetable pigments, and of colored mineral and chemical substances, therefore a somewhat extended reference to literature will be given. Full titles of the books and periodicals will be found in the Bibliography at the end.

Angström, Recherches sur le spectre solaire, etc. Also various papers in periodicals. See Royal Soc's Cat'1 Scientific Papers ; Anthony \& Brackett ; Beale, p. 269 ; Behrens, p. 139; Kossel und Schiefferdecker, p. 63; Carpenter, p. 323 ; Browning, How to Work with the Spectroscope, and in Monthly Micr. Jour., II, p. 65 ; Daniell, Principles of Physics. The general principles of spectrum analysis are especially well stated in this work, pp. 435-455; Davis, p. 342 ; Dippel, p. 277 ; Frey; Gamgee, p. 91 ; Halliburton; Hogg, p. I22; also in Monthly Micr. Jour., Vol. II, on colors of flowers; Jour, Roy. Micr. Soc., I880, 1883, and in various other vols.; Kraus; Lockyer; M'Kendrick; MacMunn; and also in Philos, Trans. R. S., I886; various vols. of Jour. Physiol.; Nägeli und Schwendener; Proctor; Ref. Hand-Book Med. Science, Vol. I, p. 577, VI, p. 5I6, VII, p. 426; Roscoe; Schellen; Sorby, in Beale, p. 269 ; also Proc. R. S., 1874, p. 3 I, I867, p. 433 ; see also in the Scientific Review, Vol. V, p. 66, Vol. II, p. 4I9. The larger works on Physiology, Chemistry and Physics may also be consulted with profit.

Vogel, Spectrum analysis; also in Nature, Vol. xix, p. 495, on absorption spectra. The bibliography in MacMunn is excellent and extended.

For hemochromogen in medico-legal cases see Bleile, Trans. Amer. Micr. Soc., I900, p. 9.

\section{MICRO-POLARISCOPE}

\&.2IS. The micro-polariscope, or polarizer, is a polariscope used in connection with a microscope.

The most common and typical form consists of two Nicol prisms, that is, two somewhat elongated rhombs of Iceland spar cut diagonally and cemented together with Canada balsam. These Nicol prisms are then mounted in such a way that the light passes through them lengthwise, and in passing is divided into two rays of plane polarized light. The one of these rays obeying most nearly the ordinary law of refraction is called the ordinary ray, the one departing farthest from the law is called the extra-ordinary ray. These two rays are not only polarized, but polarized in planes almost exactly at right angles to each other. The Nicol prism totally reflects the ordinary ray at the cemented surface as it meets that surface at an angle greater than the critical angle, and only the extraordinary or less refracted ray is transmitted. 
\& 2r9. Polarizer and Analyzer.-The polarizer is one of the Nicol prisms. It is placed beneath the object and in this way the object is illuminated with polarized light. The analyzer is the other Nicol and is placed at some level above the object, very conveniently above the ocular.

When the corresponding faces of the polarizer and analyzer are parallel $i . e_{\text {., }}$ when the faces through which the oblique section passes are parallel, light passes freely through the analyzer to the eye. If these corresponding faces are at right angles, that is, if the Nicols are crossed, then the light is entirely cut off and the two transparent prisms become opaque to ordinary light. There are then, in the complete revolution of the analyzer, two points, at $0^{\circ}$ and $180^{\circ}$, where the corresponding faces are parallel and where light freely traverses the analyzer. There are also two crossing points of the Nicols, at $90^{\circ}$ and $270^{\circ}$, where the light is extinguished. In the intermediate points there is a sort of twilight.

\& 220. Putting the Polarizer and Analyzer in Position.-Swing the diaphragm carrier of the Abbe illuminator out from under the illuminator, remove the disk diaphragm or open widely the iris diaphragm and place the analyzer in the diaphragm carrier, then swing it back under the illuminator. Remove the ocular, put the graduated ring on the top of the tube and then replace the ocular and put the analyzer over the ocular and ring. Arrange the graduated ring so that the indicator shall stand at $o^{\circ}$ when the field is lightest. This may be done by turning the tube down so that the objective is near the illuminator, then shading the stage so that none but polarized light shall enter the microscope. Rotate the analyzer until the lightest possible point is found, then rotate the graduated ring till the index stands at $o^{\circ}$. The ring may then be clamped to the tube by the side screw for the purpose. Or, more easily, one may set the index at $0^{\circ}$, clamp the ring to the microscope, then rotate the draw-tube of the microscope till the field is lightest.

3. 22I. Adjustment of the Analyzer.-The analyzer should be capable of moving up and down in its mounting, so that it can be adjusted to the eye-point of the ocular with which it is used. If on looking into the analyzer with parallel Nicols the edge of the field is not sharp, or if it is colored, the analyzer is not in a proper position with reference to the eye-point, and should be raised or lowered till the edge of the field is perfectly sharp and as free from color as the ocular with the analyzer removed.

\% 222. Objectives to Use with the Polariscope.-Objectives of the lowest powers may be used, and also all intermediate forms up to a $2 \mathrm{~mm}$. homogeneous immersion. Still higher objectives may be used if desired. In general, however, the lower powers are somewhat more satisfactory. A good rule to follow in this case is the general rule in all microscopic work,-use the power that most clearly and satisfactorily shows the object under investigation.

3. 223. Lighting for Micro-Polariscope Work.-Follow the general directions given in Chapter II. It is especially necessary to shade the object so that no unpolarized light can enter the objective, otherwise the field cannot be sufficiently darkened. No diaphragm is used over the polarizer for most examinations. Direct sunlight may be used to advantage with some objects, and as a rule the object would best be very transparent.

₹ 224. Mounting Objects for the Polariscope.-So far as possible objects should be mounted in balsam to render them transparent. In many cases objects mounted in water do not give satisfactory polariscope appearances. For example, 
if starch is mounted dry or in water, the appearances are not so striking as in a balsam mount (Davis, p. 337 ; Suffolk).

8. 225. Purpose of a Micro-Polariscope.-The object of a micro-polariscope is to determine, in microscopic masses, one or more of the following points: (A) Whether the body is singly refractive, mono-refringent, or isotropic, that is, optically homogeneous, as are glass and crystals belonging to the cubical system ; (B) Whether the object is doubly refractive or anisotropic, uniaxial or biaxial; (C) Pleochroism; (D) The rotation of the plane of polarization, as with solutions of sugar, etc. ; (E) To aid in petrology and mineralogy ; ( $\mathrm{F}$ ) To aid in the determination of very minute quantities of crystallizable substances; (G) For the production of colors.

For petrological and mineralogical investigations the microscope should possess a graduated, rotating stage so that the object can be rotated, and the exact angle of rotation determined. Fig. I26. It is also found of advantage in investigating object swith polarized light where colors appear, to combine a polariscopic and spectroscope (Spectro-Polariscope).

MICRO-POLARISCOPE-EXPERIMENTS

$\$ 226$. Arrange the polarizer and analyzer as directed above $(\$ 220)$ and use a $16 \mathrm{~mm}$. objective except when otherwise directed.

(A) Isotropic or Singly Refracting Objects.-Light the microscope well and cross the Nicols, shade the stage and make the field as dark as possible ( $\$ 2 \mathrm{Ig}$ ). As an isotropic substance, put an ordinary glass slide under the microscope. The field will remain dark. As an example of a crystal belonging to the cubical system and hence isotropic, make a strong solution of common salt (sodium chloride) put a drop on a slide and allow it to crystallize, put it under the microscope, remove the analyzer, focus the crystals and then replace the analyzer and cross the Nicols. The field and the crystals will remain dark.

(B) Anisotropic or Doubly Refracting Objects.-Make a fresh preparation of carbonate of lime crystals like that described for pedesis ( I 5I), or use a preparation in which the crystals have dried to the slide, use a 5 or $3 \mathrm{~mm}$. objective, shade the object well, remove the analyzer and focus the crystals, then replace the analyzer. Cross the Nicols. In the dark field will be seen multitudes of shining crystals, and if the preparation is a fresh one in water, part of the smaller crystals will alternately flash and disappear. By observing carefully, some of the larger crystals will be found to remain dark with crossed Nicols, others will shine continuously. If the crystals are in such a position that the light passes through them parallel with the optic 


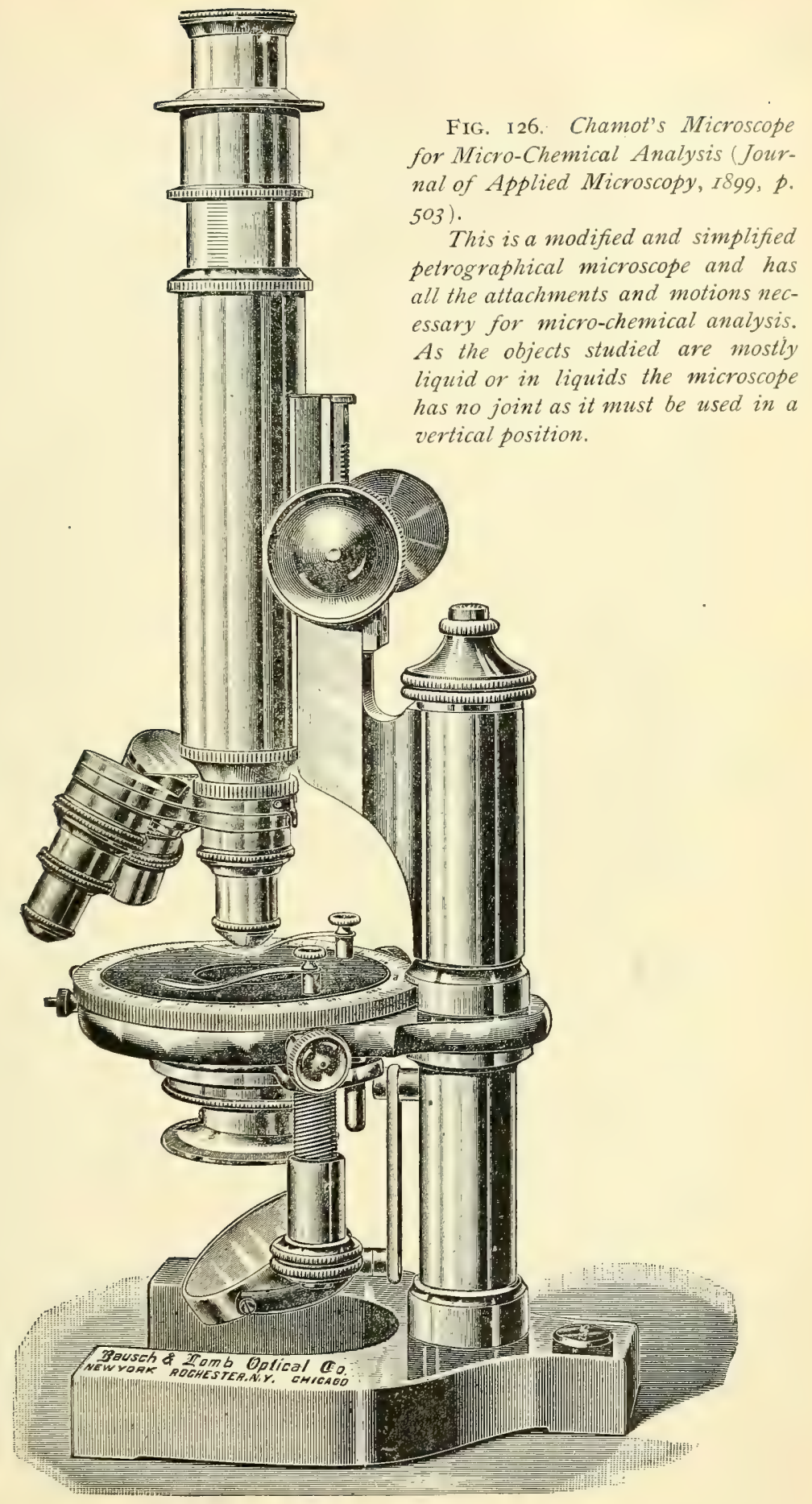


axis, * the crystals are isotropic like salt crystals and remain dark. If, however, the light traverses them in any other direction the ray from the polarizer is divided into two constituents vibrating in planes at right angles to each other, and one of these will traverse the analyzer, hence such crystals will appear as if self-luminous in a dark field. The experiment with these crystals from the frog succeeds well with a $2 \mathrm{~mm}$. homogeneous immersion.

As a further illustration of anisotropic objects, mount some cotton fibers in balsam ( $\S 256)$, also some of the lens paper ( $\S$ I I 4 ). These furnish excellent examples of vegetable fibers.

Striated muscle fibers are also very well adapted for polarizing objects.

As examples of biaxial crystals, allow some borax solution to dry and crystallize on a slide; use the crystals as objects. As all doubly refracting objects restore the light with crossed Nicols, they are sometimes called depolarizing.

(C) Pleochroism.-This is the exhibition of different tints as the analyzer is rotated. An excellent subject for this will be found in blood crystals.

$\$ 227$. Production of Colors.-For the production of gorgeous colors, a plate of selenite giving blue and yellow colors is placed between the polarizer and the object. If properly mounted, the selenite is very conveniently placed on the diaphragm carrier of the Abbe illuminator, just above the polarizer. A thin plate or film of mica also answers well.

It is not necessary to use selenite or mica for the production of the most glorious colors in many objects. One of the most beautiful preparations, and one of the most instructive also, may be prepared as follows: Heat some xylene balsam on a slide until the xylene is nearly eraporated. Add some crystals of the medicine, sulphonal and warm till the sulphonal is melted and mixes with the balsam. While the balsam is still melted put on a cover-glass. If one gets perfect crystals there will be shown not only the most beautiful colors, but the black cross with perfection. (Clark).

*The optic axis of doubly refracting crystals is the axis along which the crystal is not doubly refracting, but isotropic like glass. When there is but one such axis, the crystal is said to be uniaxial, if there are two such axes the crystal is said to be bi-axial.

The crystals of carbonate of lime from the frog (see $\% 151$ ) are uniaxial crystals. Borax crystals are bi-axial. 
It is very instructive and interesting to examine many organic and inorganic substances with a micro-polarizer.

REFERENCES TO THE POLARISCGPE AND TO THE USE OF POLARIZED LIGHT.

Anthony \& Brackett, I 33 ; Behrens ; Behrens, Kossel und Schiefferdecker ; Carnoy, 6I ; Carpenter-Dallinger, 3I7, I097 ; Clark ; Daniell, 494 ; Davis ; v. Ebener ; Gamgee; Halliburton, 36, 272 ; Hogg, I33, 729 ;.Lehmann ; M'Kendrick ; Nägeli und Schwendener, 299; Quekett; Suffolk, I25; Valentin. Physical Review, I., p. 127. Daniell, Physics for Medical Students. Nichols, Physics.

\section{MICRO-CHEMISTRY}

$\$ 228$. During the last decade the microscope has become one of the necessities of the expert chemist, and the signs of the times indicate that in every research laboratory of chemistry the microscope will become as familiar as it now is in research laboratories of biology. Its proper place in chemistry has been admirably stated by Chamot:

"It is rather remarkable how slow American chemists have been in realizing the importance of the microscope as an adjunct to every chemical laboratory. This is, perhaps, largely due to the fact that few of our students in chemistry become familiar with the construction and manipulation of this instrument, just as few of them become sufficiently familiar with the spectroscope and its manifold uses; and doubtless also because of the prevailing impression that a microscope is primarily an instrument for the biologist and is of necessity a most expensive luxury. The fact is, however, that this instrument is now far from being a luxury to the chemist, and the time is not far distant when it will be conceded to be as much a necessity in every analytical laboratory as is the balance.

"Nor is the apprenticeship to its use in chemical work long nor intricate.

"Micro-chemical analysis should appeal to every chemist because of its neatness, wonderful delicacy, in which it is not excelled even by the spectroscope, and the expedition with which an analysis can be made. A complete analysis, intricate though it may be, is a matter of a few minutes rather than of a few hours.

"While there is no good reason to believe, as do some enthusiasts, that this new system is to displace the old analysis in the wet way, every chemist should, nevertheless, familiarize himself with the microscope, its accessories, and the elegant and time-saving methods of micro-analysis, thus enabling him to examine qualitatively the most minute amounts of material with a rapidity and accuracy which is truly marvelous; not to speak of the many substances for which no other method of identification is known.

"At present the greatest bar to its general use is the absence of any well defined scheme, and the absolute necessity of being well grounded in general chemistry. There are no tables which can be followed in a mechanical way by the student, but on the contrary he is obliged to exercise his knowledge and judgment at every step. For this very reason the introduction of this subject into the list of those now taught is greatly to be desired." 
It will be seen by the last paragraph that in chemistry as in biology, the microscope is only an aid to investigation; it cannot take the place of thorough training in the fundamentals of the subject under investigation.

$\$ 229$. The following list of substances is suggested by Dr. Chamot for beginning practice as the results given are definite and easily obtained :

\section{SUGGESTIONS FOR STUDY ON CRYSTAL SYSTEMS}

\section{"Isometric.}

Sodium chlorid, potassium chlorid, potassium iodid.

Alums, crystallize in octahedra, cubes or combinations of the two. It is well to recall that the alums have the general formula, $\mathrm{M}_{2}\left(\mathrm{SO}_{4}\right)_{3} \cdot \mathrm{N}_{2} \mathrm{SO}_{4} 24$ $\mathrm{H}_{2} \mathrm{O}$, where $-\mathrm{M}-\mathrm{can}$ be $\mathrm{Al}, \mathrm{Cr}, \mathrm{Mn}, \mathrm{Fe}, \mathrm{In}, \mathrm{Ga}, \mathrm{Tl}$, and $-\mathrm{N}-\mathrm{Na}, \mathrm{K}, \mathrm{Rb}$, $\mathrm{Cs}, \mathrm{NH}, \mathrm{Ag}$, or Tl. All alums are isomorphous.

Tetragonal.

Potassium copper chlorid. Ammonium copper chlorid.

Nickel sulfate $6 \mathrm{H}_{2} \mathrm{O}$. This salt is dimorphic, crystallizing also in the monoclinic system. Nickel sulfate $7 \mathrm{H}_{2} \mathrm{O}$ is orthorhombic.

Orthorhombic.

Mercuric chlorid. Silver nitrate. Potassium sulfate. Potassium nitrate.

Magnesium sulfate $7 \mathrm{H}_{2} \mathrm{O}$. Potassium chromate. Sodium nitrate (also Hexagonal).

Monoclinic.

Potassium chlorate (sodium chlorate is Isomet. or Tetrag.)

Lead acetate. Copper acetate $\mathrm{H}_{2} \mathrm{O}$. Oxalic acid.

Ferrous sulfate, this salt forms normally with $7 \mathrm{H}_{2} \mathrm{O}$ and is then Monoclinic, but in presence of zinc sulfate becomes Orthorhombic, and in presence of copper sulfate, Triclinic.

Sodium sulfate $\mathrm{IOH}_{2} \mathrm{O}$.

Triclinic.

Copper sulfate ${ }_{5} \mathrm{H}_{2} \mathrm{O}$. Boric acid.

Hexagonal.

Lead iodid. Sodium nitrate (also @rthorhombic).

8. 230. Before performing analytical tests, it is necessary that the student be familiar with the properties of crystals and also thoroughly familiar with the appearance of crystals of the chlorides, nitrates, and sulfates of Sodium, Potassium, and Ammonium; since some of these salts are sure to appear in almost every test drop examined. For this reason the following experiments should be carefully performed." 


\section{AN EXERCISE FOR PRACTICE}

I. "Sodium chlorid. Isometric system

a. Take a fragment of the salt the size of this period (.) or a little smaller. Dissolve at the corner of a slide in a minute drop of distilled water. Heat over the "micro" flame so as to cause rapid evaporation. When dry examine residue (after cooling) with $1 / 2$ inch obj. and $2 \mathrm{in}$. eyepiece.

Breathe on the preparation several times, allow the moisture to evaporate spontaneously. There should now be obtained well developed crystals instead of a mere crystalline mass. Too rapid crystallization is always to be avoided. The object of the experiment is to emphasize this fact.

Select one of the best crystals. Measure its angles. Try its behavior between crossed Nicols. (\& 2 I9. )

b. Place a small drop of water in the usual position add carefully Ferric - Chlorid till the drop when held over white paper appears distinctly yellow. Stir. Add two or three tiny fragments of Sodium Acetate at the center of the drop. Place on the stage of the microscope. There is formed Ferric acetate, Sodium chlorid and possibly a double chlorid of iron and sodium. Notice the following points. I. Tendency toward formation of double salt. 2. That the type crystal of $\mathrm{NaCl}$ is a cube. 3, Cubes may so grow as to present the appearance of a rectangular prism. 4. In certain positions cubes have the appearance of a pyramid. 5. In other positions they exhibit a hexagonal outline, thus simulating a polyhedron of many faces. 6. There is scarcely any tendency in this case toward the formation of the hoppers observed in the first experiment $(a)$. 7. The crystals often develop fastest along the diagonal planes so that the regular faces are replaced by pyramidal depressions."

FIG. I27. Czapski's Ocular Iris-diaphragm with cross hairs for examining and accurately determining the axial images of small crystals. The iris diaphragm enables the observer to make the field as large or small as desired.

A. Longitudinal section.

$B$. Transection, showing the cross lines and the iris diaphragm with the projecting part at the left, by which the diaphragm is opened and closed. (Zeiss' Catalog).

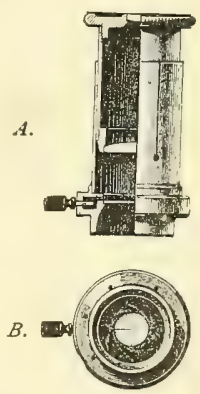

For directions and hints in micro-chemical work and crystallography, consult the various volumes of the Journal of the Roy. Micr. Soc.; Zeitschrift für physiologische Chemie, and other chemical journals; Wormly; Klément \& Renard; Carpenter-Dallinger; Hogg ; Behrens, Kossel und Schiefferdecker ; Frey ; Dana, and other works on mineralogy; Davis. Behrens, T. H.-Anleitung zur microchemischen Analyse der wichtigsten organischen Verbindungen. Hamburg, 1895-1897. Microchemische Technik, 2d edition, Hamburg, r9oo. A manual of microchemical analysis with an introdnctory chapter by J. W. Judd, London, 
I894. Especial attention is also called to the articles by Dr. E. M. Chamot in the Journal of Applied Microscopy beginning with vol. ii. p. 502, and continued in vol. iii and iv.

\section{TEXTILE FIBERS, FOOD AND PHARMACOLOGICAL PRODUCTS}

$\S 23 \mathrm{I}$. The microscope is coming more and more into use for the determination of the character of textile fibers, both in the raw state and after manufacture. As the textile fibers have distinctive characters it is not difficult to determine mixtures in fabrics of various kinds. The student is advised to study carefully known fibers, as of cotton, wool, linen, silk, jute, etc., so that he is certain of the appearances, and then to determine of what fibers different fabrics are composed. He will be astonished at the amount of "Alabama wool" in supposedly all wool goods.

For works and articles upon textile fibers see: Herzfeld, J. Translated by Salter. The technical testing of yarns and textile fabrics with reference to official specifications. London, I898. E. A. Posselt-The structure of fibers, yarns and fabrics. Philadelphia and and London, I89I. Dr. C. Rougher-Des filaments végétaux employés dans l'industrie. Paris, 1873. Wm. P. Wilson and E. Fahring-, The conditioning of wool and other fabrics in the technological laboratories of the Philadeiphia Commercial Museum. Journal of Applied Microscopy, Vol. II, (I899) pp. 290-292, 457-460. Bulletin of the National Association of Wool Growers, I875, p. 470. Proceedings of the Amer. Micr. Soc., I884, pp. 65-68. Besides these references one is liable to find pictures and discussions of various fibers in general works on the microscope, and in technical and general cyclopædias.

$\$ 232$. From the nature of food and pharmacological products adulterations are in many cases most accurately and easily determined by microscopic examination. The student will find constant reference to the microscopical characters of the genuine and spurious substances in medicines and other pharmacological products in works on pharmacy or pharmacology ; also in pharmacological journals and in druggists reports, e. g. the excellent and well illustrated article by Burt E. Nelson of the chemical laboratory of the Binghamton State Hospital on the Microscopical examination of tea, coffee, spices and condiments. This appeared in Merck's Report, Oct. 15, Dec. I5, I898. The microscopical Journals also contain occasional articles bearing upon this subject. See also Food Products in bulletins of the U. S. Dep't Agr. Macé, E.-Les substances alimentaire, etc., Paris, r89I. Schimper, 
A. F. W. Anleitung, etc. Jena, I9oo. Hugh Galt,-The Microscopy of the starches, illustrated by photo-micrographs, London, I900.

THE MICROSCOPE IN ME'TALLOGRAPHY

$\S 233$. In the modern investigation of metals and alloys much light has been thrown upon the structural peculiarities which render some mixtures satisfactory and others unsatisfactory. There are two great methods: First, that of studying fractured surfaces without recourse to any reagents. Second, to polish a metallic surface carefully with emery or carborundum and finally with rouge or diamantine and then etch it with some acid for a longer or shorter time. For either method reflected light must be used. For low powers that obtained at a good window or by a lamp or a lamp and bulls eye are good. The illuminating objectives $(\$ 28), i . e$. objectives in which a prism in the side of the objective reflects light down through the lenses which act as a condenser, are preferable for most work and indeed necesssary if one uses high powers. For special microscope see Fig. I26 A.

Elaborate arrangements have been devised for holding the piece of metal on the stage, but some beeswax, or some clay made plastic with glycerin answers well. For pictures of the appearances seen in studying metallic surfaces, see the journals of engineering and metallurgy, especially the Metallographist, a quarterly publication devoted to the study of metals with special reference to their physics and micro-structure, etc. In twenty-five or more of the great metal manufacturing establishments special laboratories for microscopic examination and investigation have been established. This is an illustration of what has frequently occurred-great manufacturing interests have outrun the universities in the appreciation and application of methods of reasearch. Fortunately, however, laboratories are already springing up in connection with the universities, and probably within ten years every great technical school will have its laboratory of micro-metallography where students will have opportunity to perfect themselves in the preparation, photography and microscopic study of the metals and alloys.

Beside the sources of information given above, see Dr. H. Ost und Dr. Fr. Kolbeck, Lehrbuch der chemischen Technologie mit einem Schlussabschnitt "Metallurgie." Hannover, Igor. Behrens, T. H.Das mikroskopische Gefüge der Matalle, etc. Hamburg, I894. For an excellent bibliography of 188 titles; see the Metallographist, Vol. $\mathrm{I}$, I898, and appended to the special papers in all the volumes. Also in Iron Age, Jan. 27, 1898. Carpenter-Dallinger, p. 264. 


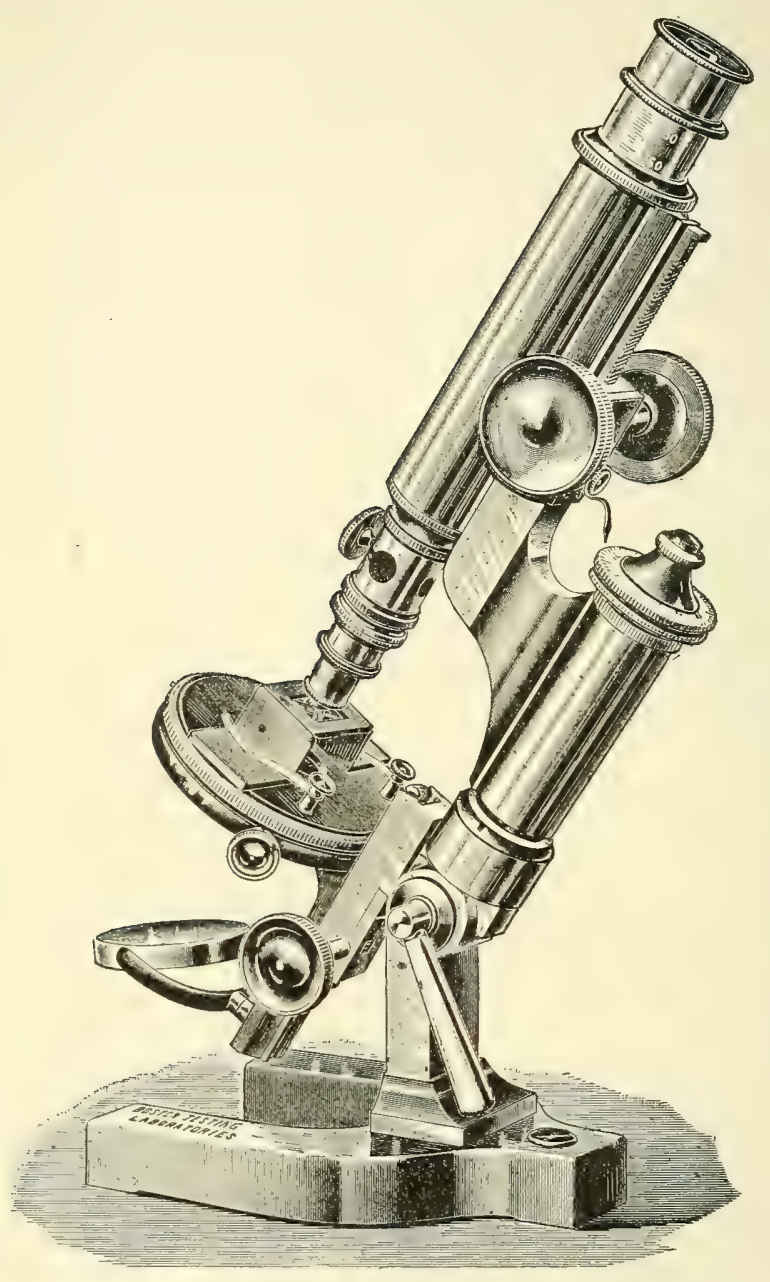

FIG. I26 A. Microscope especially constructed for the study of metals and alloys. (The Boston Testing Laboratories).

The stage is rotary, and may be raised or lowered by rack and pinion. Above the objective is the arrangement for illumination (see Ch. VII). 


\section{CHAP'TER VII}

\section{SLIDES AND COVER-GLASSES ; MOUNTING ; ISOLATION ; SECTIONING BY THE COLLODION AND THE PARAF- FIN METHODS; LABELING AND STORING MICRO- SCOPICAL PREPARATIONS ; REAGENTS}

\section{SLIDES AND COVER-GLASSES}

\% 234. Slides, Glass Slides or Slips, Microscopic Slides or Slips.-These are strips of clear flat glass upon which microscopic specimens are usually mounted for preservation and ready examination. The size that has been almost universally adopted for ordinary preparations is $25 \times 76$ millimeters ( $1 \times 3$ inches). For rock sections, slides $25 \times 45 \mathrm{~mm}$. or $32 \times 32 \mathrm{~mm}$. are used; for serial sections, slides $25 \times 76 \mathrm{~mm}$., $50 \times 76 \mathrm{~mm}$. or $37 \times 87 \mathrm{~mm}$. are used. For special purposes, slides of the necessary size are employed without regard to any conventional standard.

Whatever size of slide is used, it should be made of clear glass and the edges should be ground. It is altogether false economy to mount microscopic objects on slides with unground edges. It is unsafe also as the unground edges are liable to wound the hands.

For micro-chemical work Dr. Chamot recommends slides of half the length of those used in ordinary microscopic work. From the rapidity with which they are destroyed, he thinks the ground edges are unnecessarily expensive. He adds further: "It is a great misfortune that the colorless glass slips used in America and so excellent for ordinary microscopic work should be easily attacked by all liquids; even water extracts a relatively enormous amount of alkalies and alkaline earths. The slips of greenish glass, while not as neat or desirable for general microscopy, seem to be decidedly more resistant, and are therefore preferable." Transparent celluloid slides are recommended by Behrens for work where hydrofluoric acid and its derivatives are to be examined. (Chamot, Jour. Appl. Micr. vo1. iii, p. 793 ).

3. 235. Cleaning Slides.-For new slides a thorough rinsing in clean water with subsequent wiping with a soft cloth like glass toweling, or thin cotton cloth like bleached cheese cloth (bunting or gauze, or absorbent surgical gauze), usually fits them for ordinary use. If they are not satisfactorily cleaned in this way, soak them a short time in $50 \%$ or $75 \%$ alcohol, let them drain for a few moments on a clean towel or on blotting paper, and then wipe with a soft cloth. In handling the slides grasp them by their edges to avoid soiling the face of the slide. After the slides are cleaned they should be stored in a place as free as possible from dust. For storing, covered glass dishes are best. Use museum jars or glass boxes (Fig. I50). 


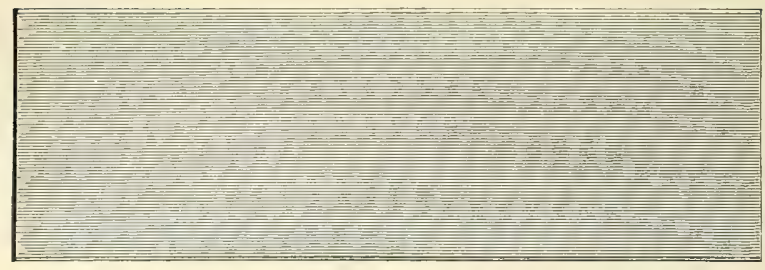

FIG. I28. Glass slide or slip of the ordinary size for microscopic work ( $3 x \mathrm{I}$ in., $76 \times 25 \mathrm{~mm}$.). (Cut loaned by the Spencer Lens Company).

For old slides, if only water, glycerin or glycerin jelly has been used on them, they may be cleaned with water, or preferably, warm water and then with alcohol if necessary. Where balsam, or any oily or gummy substance has been used upon the slides, they may be freed from the balsam, etc., by soaking them for a week or more in one of the cleaning mixtures for glass. If they are first soaked in xylene, benzin or turpentine to dissolve the balsam, then soaked in the cleaning mixture, the time required will be much shortened ( 8242$)$. After all foreign matter is removed the slides should be thoroughly rinsed in water to remove all the cleaning mixture. They may then be treated as directed for new slides.

If slides with large corers, as in mounted series, are put into the cleaning mixture, the swelling of the balsam is liable to break the covers. Dissolving away the balsam with turpentine, avoids this, and greatly shortens the time necessary for cleaning the old slides and covers.

Another excellent method for balsam mounts is to heat the slides until the balsam is soft and then remove the cover-glasses. The turpentine cleaning mixture, etc., can then act on the entire surface. It should be said, however, that at the present price of slides and cover-glasses it costs nearly as much as the slides and covers are worth to clean those that have been used in balsam mounting.

\& 236. Cover-Glasses or Covering Glasses.-These are circular or quadrangular pieces of thin glass used for covering and protecting microscopic objects. They should be very thin, 0.10 to 0.25 millimeter (see table, \& 29). It is better never to use a cover-glass over $0.20 \mathrm{~mm}$. thick, then the preparation may be studied with a $2 \mathrm{~mm}$. oil immersion as well as with lower objectives. Except for objects wholly unsuited for high powers, it is a great mistake to use cover-glasses thicker than the rorking distance of a homogeneous objective ( $\xi 61$ ). Indeed, if one wishes to employ high powers, the thicker the sections the thinner should be the cover-glass (see $\& 240$ ).

The cover-glass should always be considerably larger than the object over which $2 t$ is placed.

\%. 237. Cleaning Cover-Glasses.-New cover-glasses should be put into a glass dish of some kind containing one of the cleaning mixtures $(\% 242)$ and allowed to remain a day or longer. In putting them in, push one in at a time and be sure that each is entirely immersed, otherwise they adhere very closely and the cleaning mixture is unable to act freely. Soiled covers should be left a week or more in the cleaning mixture. An indefinite sojourn in the cleaner does not seem 
to injure the slides or covers. After one day or longer, pour off the cleaning mixture into another glass jar, and rinse the cover-glasses, moving them around with a gentle rotary motion. Continue the rinsing until all the cleaning mixture is remored. One may rinse them occasionally, and in the meantime allow a very gentle stream of water to flow on them, or they may be allowed to stand quietly and have the water renewed from time to time. When the cleaning mixture is removed rinse the covers well with distilled water, and then cover them with $50 \%$ to $75 \%$ alcohol.
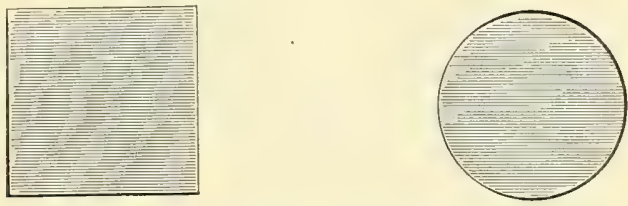

Figs. I29-I30. Figures of square and of circular cover-glasses. See also Fig. 162 for covers on serial sections.

8. 238. Wiping the Cover-Glasses.-When ready to wipe the cover-glasses, remove several from the alcohol and put them on a soft, dry cloth, or on some of the lens or filter paper to let them drain. Grasp a cover-glass by its edges, cover the thumb and index finger of the other hand with a soft, clean cloth or some of the the lens paper. The bleached cheese cloth $(\% 235)$ is good for wiping covers. Grasp the cover between the thumb and index and rub the surfaces. In doing this it is necessary to keep the thumb and index well opposed on directly opposite faces of the cover so that no strain will come on it, otherwise the cover is liable to be broken.

When a cover is well wiped, hold it up and look through it toward some dark object. The cover will be seen partly by transmitted and partly by reflected light, and any cloudiness will be easily detected. If the cover does not look clear, breathe on the faces and wipe again. If it is not possible to get a cover clear in this way it should be put again into the cleaning mixture.

As the covers are wiped put them in a clean glass box or Petri dish. Handle them always by their edges, or use fine forceps. Do not put the fingers on the faces of the covers, for that will surely cloud them.

FIG. I3I. Glass dish for holding covers (Whitall, Tatum \& Co.).

8 239. Cleaning Large Cover-Glasses.-For serial sections and especially large sections, large quadrangular covers are used (Fig. I62). These are to be put one by one into a cleaning

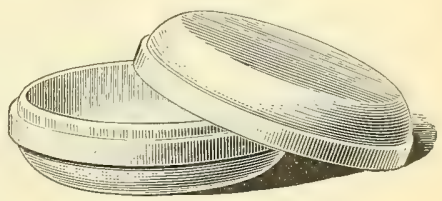
mixture as for the smaller covers and treated in every way the same. In wiping them one may proceed as for the small covers, but special care is necessary to avoid breaking them. It is especially desirable that these large covers should be thin-not over 0 . I 5-0.20 mm. otherwise high objectives cannot be used in studying the preparations.

\%, 240. Measuring the Thickness of Cover-Glasses.-It is of the greatest advantage to know the exact thickness of the cover-glass on an object; for, (a) 
In studying the preparation one would not try to use objectives of a shorter working distance than the thickness of the cover ( 8 6I); (b) In using adjustable objectives with the collar graduated for different thicknesses of cover, the collar might be set at a favorable point without loss of time; (c) For unadjustable objectives the thickness of cover may be selected corresponding to that for which the objective was corrected (see table, $; 29$ ). Furthermore, if there is a variation from the standard, one may remedy it, in part at least, by lengthening the tube if the cover is thinner, and shortening it if the cover is thicker than the standard (\& to2, Fig. I33.)

In the so called No. I cover-glasses of the dealers in microscopical supplies, the writer has found covers varying from o. $10 \mathrm{~mm}$ : to $0.35 \mathrm{~mm}$. To use coverglasses of so wide a variation in thickness without knowing whether one has a thick or thin one is simply to ignore the fundamental principles by which correct microscopic images are obtained.

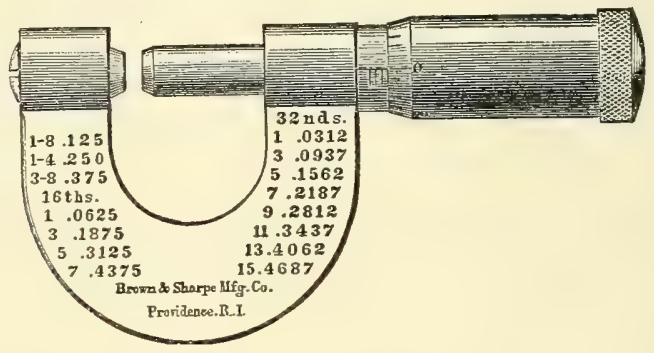

FIG. I32. Micrometer Calipers (Brown and Sharpe). Pocket Calipers, graduated in inches or millimeters, and well adapted for measuring cover-glasses.

It is then strongly recommended that every preparation shall be covered with a cover-glass whose thickness is known, and that this thickness should be indicated in some way on the preparation.

3. 24 I. Cover-Glass Measurers, Testers or Gauges. For the purpose of measuring cover-glasses there are three very excellent pieces of apparatus. The micrometer calipers (Fig. I32) used chiefly in the mechanic arts, are convenient and from their size easily carried in the pocket. The two cover-glass measurers specially designed for the purpose are shown in Figs. I33-I34. With either of these the covers may be more rapidly measured than with the calipers.

With all of these measures or gauges one should be certain that the index stands at zero when at rest. If the index does not stand at zero it should be adjusted to that point, otherwise the readings will not be correct.

As the covers are measured the different thicknesses should be put into different boxes and properly labeled. Unless one is striving for the most accurate possible results, cover-glasses not varying more than $0.06 \mathrm{~mm}$. may be put in the same box. For example, if one takes $0.15 \mathrm{~mm}$. as a standard, covers varying 0.03 $\mathrm{mm}$. on each side may be put into the same box. In this case the box would contain covers of $0.12,0.13,0.14,0.15,0.16,0.17$ and $0.18 \mathrm{~mm}$. 


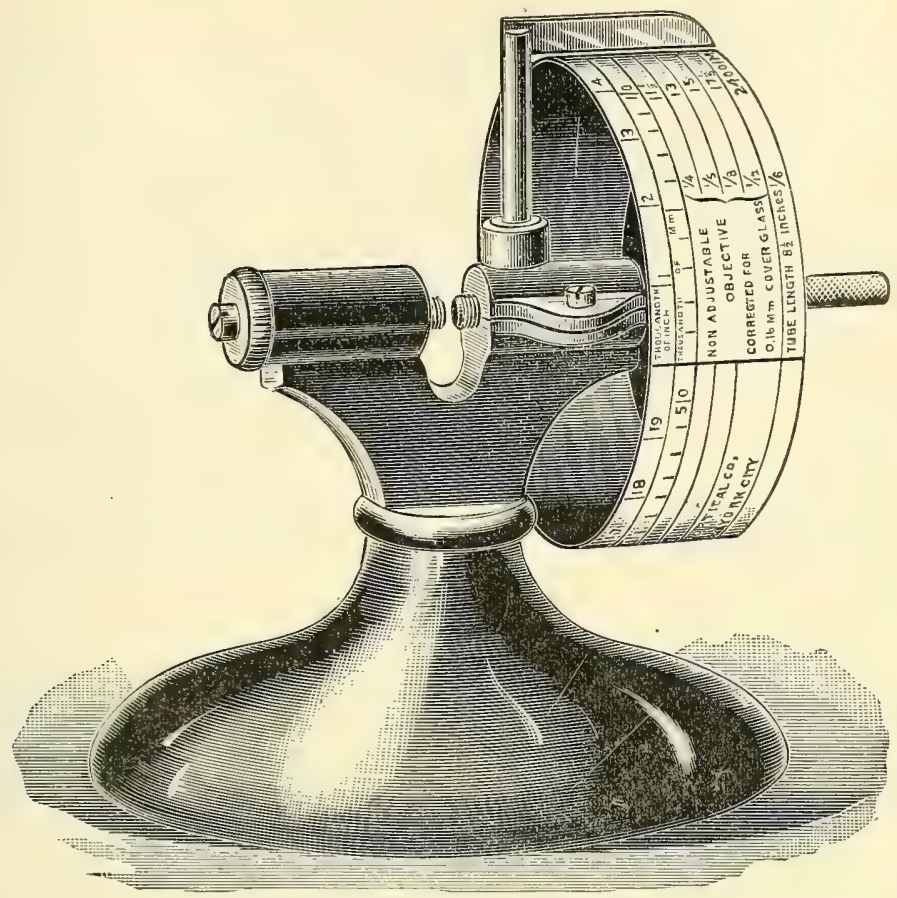

FIG. I33. Cover-Glass Measurer (Edward Bausch).

The cover glass is placed in the notch between the two screws, and the drum is turned by the milled head at the right till the cover is in contact with the screws. The thickness is then indicated by the knife edge on the drum and may be read off directly in 0.001 of a millimeter or inch. In other columns is given the proper tube-length for various unadjustable objectives $\left(\frac{1}{4}, \frac{1}{5}, \frac{1}{8}\right.$, and $\frac{1}{12} \mathrm{in}$.) (Bausch and Lomb Optical Company).

FIG. 134. Zeiss Cover-Glass Measurer. With this the knife edge jaws are opened by means of a lever and the cover inserted. The thickness may then be read off on the face as the pointer indicates the thickness in hundredths millimeter in the outer circle and in thousandths inch on the inner circle.

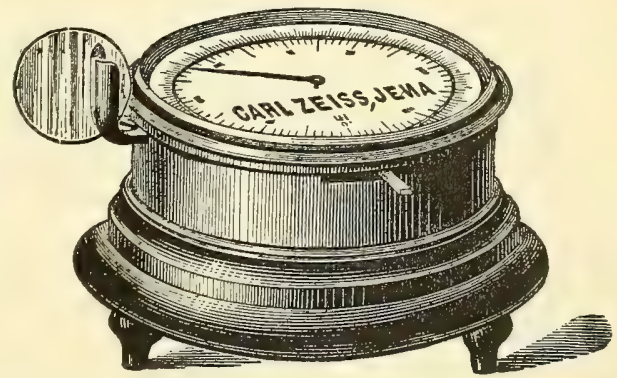

242. Cleaning Mixtures for Glass.-The cleaning mixtures used for cleaning slides and cover-glasses are those commonly used in chemical laboratories: (Dr. G. C. Caldwell's Laboratory Guide in Chemistry). 
(A) Dichromate of Potash and Sulphuric Acid.

Dichromate of potash $\left(\mathrm{K}_{2} \mathrm{Cr}_{2} \mathrm{O}_{7}\right)$

Water, distilled or ordinary

Sulphuric acid $\left(\mathrm{H}_{2} \mathrm{SO}_{4}\right)$

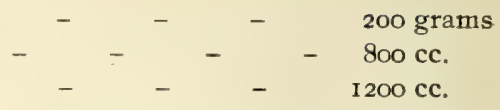

Dissolve the dichromate in the water by the aid of heat, using an agate or other metal dish, then pour it into a heavy iron kettle lined with sheet lead ( $\operatorname{Pr}$. Trans. Amer. Micr. Soc., I899, p. 107). Add the sulphuric acid to the dissolved dichromate in the kettle. The purpose of the lead lined kettle is to avoid breakage from the great heat developed upon the addition of the sulphuric acid. The lead is very slightly affected by the acid, iron would be corroded by it.

For making this mixture, ordinary water, commercial dichromate and strong commercial sulphuric acid may be used. It is not necessary to employ chemically pure materials.

This is an excellent cleaning mixture and is practically odorless. It is exceedingly corrosive and must be kept in glass vessels. It may be used more than once, but when the color changes markedly from that seen in the fresh mixture it should be thrown away.

(B) Sulphuric and Nitric Acid Mixture.

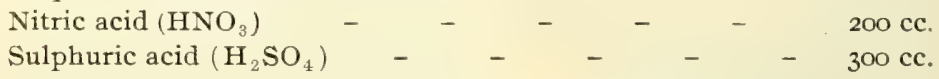

The acids should be strong, but they need not be chemically pure. The two acids are mixed slowly, and kept in a glass stoppered bottle. This is a more corrosive mixture than (A), and has the undesirable feature of giving off stifling fumes, therefore it must be carefully covered. It may be used several times. It acts more rapidly than the dichromate mixture, but on account of the fumes is not so well adapted for general laboratories.

\section{MOUNTING, AND PERMANENT PREPARATION OF MICROSCOPICAL OBJECTS}

8 243. Mounting a Microscopical Object is so arranging it upon some suitable support (glass slide) and in some suitable mounting medium that it may be satisfactorily studied with the microscope.

The cover-glass on a permanent preparation should always be considerably larger than the object; and where several objects are put under one cover-glass it is false economy to crowd them too closely together.

\& 244. Temporary Mounting.-In a great many cases objects do not need to be preserved; they are then mounted in any way to enable one best to study them, and after the study the cover glass is removed, the slide cleaned and made ready for future use. In the study of living objects, of course only temporary preparations are possible. With amoebae, white blood corpuscles, and many other objects both animal and vegetable, the living phenomena can best be studied by mounting them in the natural medium. That is, for amoebae, in the water in which they are found; for the white blood corpuscles, a drop of blood is used and, as the blood soon coagulates, they are in the serum. Sometimes it is not easy or convenient to get the natural medium, then some liquid that has been found to serve in place of the natural medium is used. For many things, water with a 
little common salt (water $100 \mathrm{cc}$, common salt $\frac{6}{10}$ th gram) is employed. This is the so-called normal salt or saline solution. For the ciliated cells from frogs and other amphibia, nothing has been found so good as human spittle. Whatever is used, the object is put on the middle of the slide and a drop of the mounting medium added, and then the cover-glass. The cover is best put on with fine forceps, as shown in Fig. 136 . After the cover is in place, if the preparation is to be studied for some time, it is better to avoid currents and evaporation by painting a ring of castor oil around the cover in such a way that part of the ring will be on the slide and part on the cover (Fig. I65.)

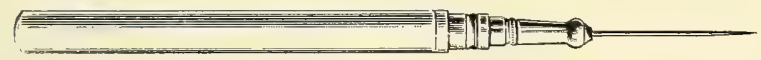

FIG. 135. Needle Holder (Queen \& Co.). By means of the screw clamp or chuck at one end the needle may be quickly changed.

FIG. 136. To show the method of putting a cover-glass upon a microscopic preparation. The cover is grasped by one edge, the opposite edge is then brought down to the slide, and the cover gradually lowered upon the object.

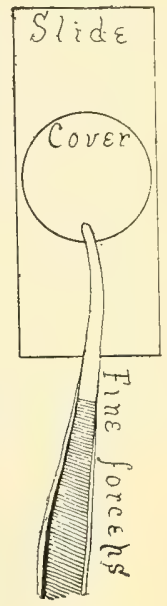

FIG. I36.

245. Permanent Mounting.-For making permanent microscopical preparations, there are three great methods. Special methods of procedure are necessary to mount objects successfully in each of these ways. The best mounting medium and the best method of mounting in a given case can only be determined by experiment. In most cases some previous observer has already made the necessary experiments and furnished the desired information.

The three methods are the following: (A) Dry or in air ( 8 246); (B) In some medium miscible with water, as glycerin or glycerin jelly (\& 250); (C) In some resinous medium like Canada Balsam ( 8255$)$.

3. 246. Mounting Dry or in Air.-The object should be thoroughly dry. If any moisture remains it is liable to cloud the cover-glass, and the specimen may deteriorate. As the specimen must be sealed, it is necessary to prepare a cell slightly deeper than the object is thick. This is to support the cover-glass, and also to prevent the running in by capillarity of the sealing mixture.

\section{$\xi$ 246a. Order of Procedure in Mounting Objects Dry or in Air.}

I. A cell of some kind is prepared. It should be slightly deeper than the object is thick $(\& 248)$.

2. The object is thoroughly dried (dessicated) either in dry air or by the aid of gentle heat.

3. If practicable the object is mounted on the cover-glass; if not it is placed in the bottom of the cell.

4. The slide is warmed till the cement forming the cell wall is somewhat sticky, or a very thin coat of fresh cement is added; the cover is warmed and put 
on the cell and pressed down all around till a shining ring indicates its adherence (\& 249).

5. The cover-glass is sealed $(\& 249)$.

6. The slide is labeled $(\& 308)$.

7. The preparation is cataloged and safely stored ( $8309,3 \mathrm{II})$.

8 247. Example of Mounting Dry, or in Air.-Prepare a shallow cell and dry it $(\& 24 \mathrm{~S})$. Select a clean cover-glass slightly larger than the cell. Pour upon the cover a drop of 10\% solution of salycilic acid in $95 \%$ alcohol. Let it dry spontaneously. Warm the slide till the cement ring or cell is somewhat sticky, then warm the cover gently and put it on the cell, crystals down. Press on the cover all around the edge ( $\& 246)$; seal, label and catalog $(\& 253,308,309)$.

A preparation of mammalian red blood corpuscles may be satisfactorily made by spreading a very thin layer of fresh blood on a cover with the end of a slide. After it is dry, warm gently to remove the last traces of moisture and mount blood side down, precisely as for the crystals. One can get the blood as directed for the Micro-spectroscopic work ( \& 210).

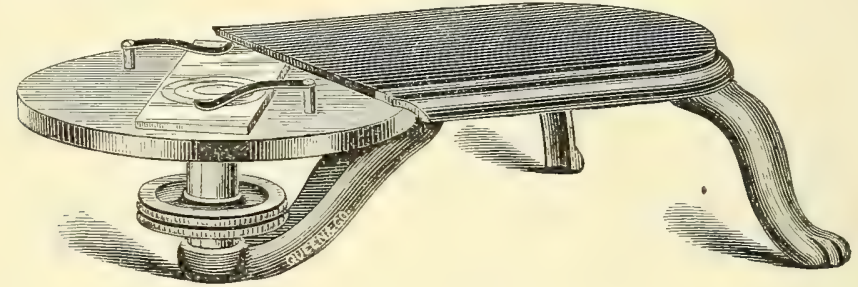

FIG. I37. Turn-Table for sealing cover-glasses and making shallow mounting cells. (Queen $\mathcal{G}$ Co.)

\& 248. Preparation of Mouuting Cells.-(A) Thin Cells. These are most conveniently made of some of the cements used in microscopy. Shellac is one of the best and most generally applicable. To prepare a shellac cell place the slide on a turn-table (Fig. I37) and center it, that is, get the center of the slide over the center of the turn-table. Select a guide ring on the turn-table which is a little smaller than the cover-glass to be used, take the brush from the shellac, being sure that there is not enough cement adhering to it to drop. Whirl the turn-table and hold the brush lightly on the slide just over the guide ring selected. An even ring of the cement should result. If it is uneven, the cement is too thick or too thin, or too much was on the brush. After a ring is thus prepared remove the slide and allow the cement to dry spontaneously, or heat the slide in some way. Before the slide is used for mounting, the cement should be so dry when it is cold that it does not dent when the finger nail is applied to it.

A cell of considerable depth may be made with the shellac by adding successive layers as the previous one drys.

(B) Deep Cells are sometimes made by building up cement cells, but more frequently, paper, wax, glass, hard rubber, or some metal is used for the main part of the cell. Paper rings, block tin or lead rings are easily cut out with gun punches. These rings are fastened to the slide by using some cement like the shellac. 
8. 249. Sealing the Cover-Glass for Dry Objects Mounted in Cells.-When an object is mounted in a cell, the slide is warmed until the cement is slightly sticky or a very thin coat of fresh cement is put on. The cover-glass is warmed slightly also, both to make it stick to the cell more easily, and to expel any remaining moisture from the object. When the cover is put on it is pressed down all around over the cell until a shining ring appears, showing that there is an intimate contact. In doing this use the convex part of the fine forceps or some other blunt, smooth object; it is also necessary to avoid pressing on the cover except immediately over the wall of the cell for fear of breaking the cover. When the cover is in contact with the wall of cement all around, the slide shonld be placed on the turn-table and carefully arranged so that the cover-glass and cell wall will be concentric with the guide rings of the turn-table. Then the turntable is whirled and a ring of fresh cement it painted, half on the cover and half on the cell wall (Fig. I65.) If the cover-glass is not in contact with the cell wall at any point and the cell is shallow, there will be great danger of the fresh cement running into the cell and injuring or spoiling the preparation. When the coverglass is properly sealed, the preparation is put in a safe place for the drying of the cement. It is advisable to add a fresh coat of cement occasionally.

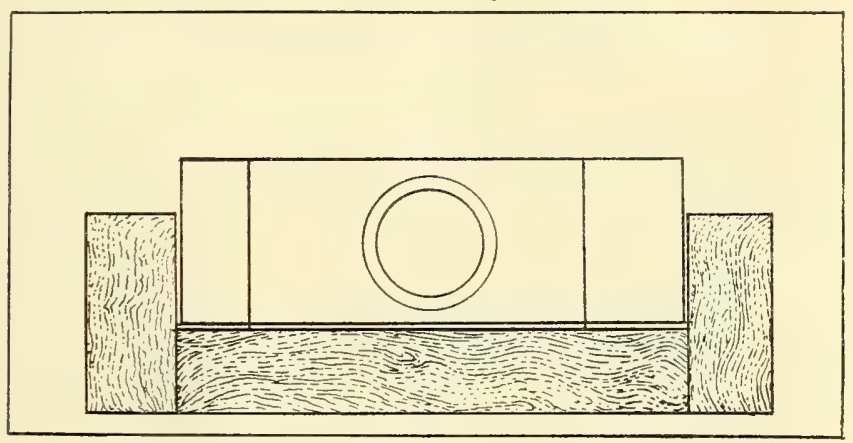

FIG. I38. Centering Card. A card with stops for the slide and circles in the position occupied by the center of the slide. If the slide is put upon such a card it is very easy to arrange the object so that it will be approximately in the center of the slide. The position of the long cover used for serial sections is also shown (Fig. I62). (From the Microscope, December, I886).

\% 250. Mounting Objects in Media Miscible with Water.-Many objects are so greatly modified by drying that they must be mounted in some medium other than air. In some cases water with something in solution is used. Glycerin of various strengths, and glycerin jelly are also much employed. All these media keep the object moist and therefore in a condition resembling the natural one. The object is usually and properly treated with gradually increasing strengths of glycerin or fixed by some fixing agent before being permanently mounted in strong glycerin or either of the other media. 
In all of these different methods, unless glycerin of increasing strengths has been used to prepare the tissue, the fixing agent is washed away with water before the object is finally and permanently mounted in either of the media.

For glycerin jelly no cell is necessary unless the object has a considerable thickness.

\section{\& 25I. Order of Procedure in Mounting Objects in Glycerin.}

I. A cell must be prepared on the slide if the object is of considerable thickness $(\% 248,249)$.

2. A suitably prepared object $(\xi 250)$ is placed on the center of a clean slide, and if no cell is required a centering card is used to facilitate the centering (Fig. I38).

3. A drop of pure glycerin is put upon the object, or if a cell is used, enough to fill the cell.

4. In putting on the cover-glass it is grasped with fine forceps and the under side breathed on to slightly moisten it so that the glycerin will adhere, then one edge of the cover is put on the cell or slide and the cover gradually lowered upon the object (Fig. 136). The cover is then gently pressed down. If a cell is used, a a fresh coat of cement is added before mounting ( 8249$)$.
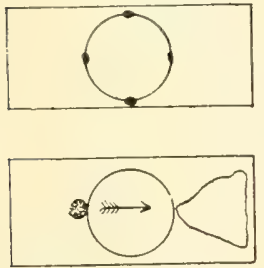

FIG. I39. Slide and cover-glass showing method of anchoring a cover-glass with a glycerin preparation when no cell is used. A cover-glass so anchored is not liable to move when the cover is being sealed (3.253).

FIG. I40. Glass slide with cover-glass, a drop of reagent and a bit of absorbent paper to show method of irrigation $(3,262,263)$.

5. The cover-glass is sealed ( $\& 249)$.

6. The slide is labeled $(\& 308)$.

7. The preparation is cataloged and safely stored $(\& 309,311)$.

8. 252. Order of Procedure in Mounting Objects in Glycerin Jelly.

I. Unless the object is quite thick no cell is necessary with glycerin jelly.

2. A slide is gently warmed and placed on the centering card (Fig. I 38 ) and a drop of warmed glycerin jelly is put on its center. The suitably prepared object is then arranged in the center of the slide.

3. A drop of the warm glycerin jelly is then put on the object, or if a cell is used it is filled with the medium.

4. The cover-glass is grasped with fine forceps, the lower side breathed on and then gradually lowered upon the object (Fig. I36) and gently pressed down.

5. After mounting, the preparation is left flat in some cool place till the glycerin jelly sets, then the superfluous amount is scraped and wiped away and the cover-glass sealed with shellac ( 8253 ).

6. The slide is labeled $(\xi 308)$.

7. The preparation is cataloged and safely stored ( $\& 309,3 \mathrm{II})$.

8. 253. Sealing the Cover-Glass when no Cell is used.-(A) For glycerin mounted specimens. The superfluous glycerin is wiped away as carefully as possible with a moist cloth, then four minute drops of cement are placed at the edge of the cover (Fig. I39), and allowed to harden for half an hour or more. These will 
anchor the cover-glass, then the preparation may be put on the turn-table and a ring of cement put around the edge while whirling the turn-table.
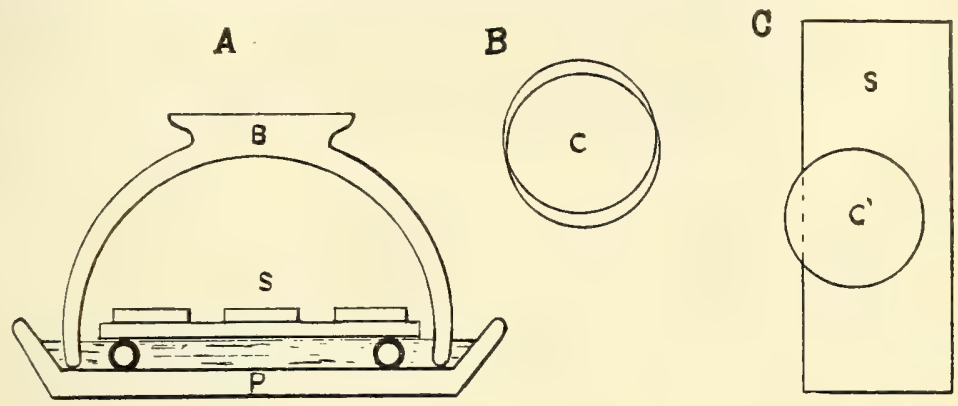

FIG. I4I. A-Simple form of moist chamber made with a plate and bowl. B, bowl serving as a bell jar; $\mathrm{P}$, plate containing the water and over which the bowl is inverted; S, slides on which are mounted preparations which are to be kept moist. These slides are seen endwise and rest upon a bench made by cementing short pieces of large glass tubing to a strip of glass of the desired length and width.

$\mathrm{B}-$ Two cover-glasses (C) made eccentric, so that they may be more easily separated by grasping the projecting edge.

$\mathrm{C}$-Slide (S) with projecting cover-glass (C). Thc projection of the cover enables one to grasp and raise it without danger of moving it on the slide and thus folding the substance under the cover. (From Proc. Amer. Micr. Soc., I89I).

(B) For objects in glycerin jelly, Farrants' solution or a resinous medium. The mounting medium is first allowed to harden, then the superfluous medium is scraped away as much as possible with a knife, and then removed with a cloth moistened with water for the glycerin jelly and Farrants' solution or with alcohol, chloroform or turpentine, etc., if a resinous medium is used. Then the slide is put on a turn-table and a ring of the shellac cement added. (C) Balsam preparations may be sealed with shellac as soon as they are prepared, but it is better to allow them to dry for a few days. One should never use a cement for sealing preparations in balsam or other resinous media if the solvent of the cement is a solvent also of the balsam, etc. Otherwise the cement will soften the balsam and finally run in and mix with it, and partly or wholly ruin the preparation. Shellac is an excellent cement for sealing balsam perparations, as it never runs in. Balsam preparations are rarely sealed.

3, 254. Example of Mounting in Glycerin Jelly.-For this select some stained and isolated muscular fibres or other suitably prepared objects. (See under isolation 3 259). Arrange them on the middle of a slide, using the centering card, and mount in glycerin jelly as directed in $\& 252$. Air bubbles are not easily removed from glycerin jelly preparations, so care should be taken to avoid them.

\%, 255. Mounting Objects in Resinous Media.-While the media miscible with water offer many advantages for mounting animal and vegetable tissues the preparations so made are liable to deteriorate. In many cases, also, they do not produce sufficient transparency to enable one to use high enough powers for the demonstration of minute details. 
By using sufficient care almost any tissue may be mounted in a resinous medium and retain all its details of structure.

For the successful mounting of an object in a resinous medium it must in some way be deprived of all water and all liquids not miscible with the resinous mounting medium. There are two methods of bringing this about: (A) By drying or desiccation ( $\& 256)$, and (B) by successive displacements ( $\left.\& 25^{8}\right)$.

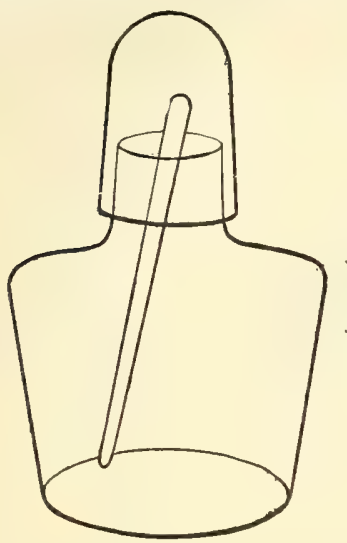

FIG. I42. Small spirit lamp modified into a balsam bottle, a glycerin or glycerin-jelly bottle, or a bottle for homogeneous immersion liquid. For all of these purposes it should contain a glass rod as shown in the figure. By adding a small brush, it answers well for a shellac bottle also (See Fig. I7O).

8. 256. Order of Procedure in Mounting Objects in Resinous Media by Desiccation :

I. The object suitable for the purpose (fly's wings, etc. ) is thoroughly dried in dry air or by gentle heat.

2. The object is arranged as desired in the center of a clean slide on the centering card (Fig. I38).

3. A drop of the mounting medium is put directly upon the object or spread on a cover-glass.

4. The cover-glass is put on the specimen with fine forceps (Fig. I $_{3} 6$ ), but in no case does one breathe on the cover as when media-miscible with water are used.

5. The cover-glass is pressed down gently.

6. The slide is labeled ( 8,308$)$.

7. The preparation is cataloged and safely stored $(\& 309,3$ I I $)$.

8. 257. Example of Mounting in Balsam by Desiccation.-Find a fresh fly, or if in winter, procure a dead one from a window sill or a spider's web. Remove the fly's wings, being especially careful to keep them the dorsal side up. With a camel's hair brush remove any dirt that may be clinging to them. Place a clean slide on the centering card, then with fine forceps put the two wings within one of the guide rings. Leave one dorsal side up, turn the other ventral side up. Spread some Canada balsam on the face of the cover-glass and with the fine forceps place the cover upon the wings (Fig. I36). Probably some air-bubbles will appear in the preparation, but if the slide is put in a warm place these will soon disappear. Label, catalog, etc., (3/307-3II).

3. 258. Mounting in Resinous Media by a Series of Displacements.-For examples of this see the procedure in the paraffin and in the collodion methods 
( $8.280,300)$. The first step in the series is Dehydration, that is, the water is displaced by some liquid which is miscible both with the water and the next liquid to be used. Strong alcohol (95\% or stronger) is usually employed for this. Plenty of it must be used to displace the last trace of water. The tissue may be soaked in a dish of the alcohol, or alcohol from a pipette may be poured upon it. Dehydration usually occurs in the thin objects to be mounted in balsam in 5 to 15 minutes. If a dish of alcohol is used it must not be used too many times, as it loses in strength.

The second step is clearing. That is, some liquid which is miscible with the alcohol and also with the resinous medium is used. This liquid is highly refractive in most cases, and consequently this step is called clearing and the liquid a clearer. The clearer displaces the alcohol, and renders the object more or less translucent. In case the water was not all removed, a cloudiness will appear in parts or over the whole of the preparation. In this case the preparation must be returned to alcohol to complete the dehydration.

One can tell when a specimen is properly cleared by holding it over some dark object. If it is cleared it can be seen only with difficulty, as but little light is reflected from it. If it is held toward the window, however, it will appear translucent.

The third and final step is the displacement of the clearer by the resinous mounting medium.

The specimen is drained of clearer and allowed to stand for a short time till there appears the first sign of dullness from evaporation of the clearer from the surface. Then a drop of the resinous medium is put on the object, and finally a corer-glass is placed over it, or a drop of the mounting medium is spread on the cover and it is then put on the object.

\section{ISOI,ATION OF HISTOLOGICAL ELEMENTS}

3 259. For a correct conception of the forms of the cells and fibers of the various organs of the body, one must see these elements isolated and thus be able to inspect them from all sides. It frequently occurs also that the isolation is not quite complete, and one can see in the clearest manner the relations of the cells or fibers to one another.

The chemical agents or solutions for isolating are, in general, the same as those used for hardening and fixing. But the solutions are only about one-tenth as strong as for fixing, and the action is very much shorter, that is, from one or two hours to as many days. In the weak solution the cell cement or connective tissue is softened so that the cells and fibers may be separated from one another, and at the same time the cells are preserved. In fixing and hardening, on the other hand, the cell cement, like the other parts of the tissue, are made firmer. In preparing the isolating solutions it is better to dilute the fixing agents with normal salt solution ( 833 I) than merely with water.

3 260. Isolation by Means of Formaidehyde.-Formaldehyde in normal salt solution is one of the very best dissociating agents for brain tissue and all the forms of epithelium. It is prepared as follows : $2 \mathrm{cc}$. of formal, (that is, a $40 \%$ solution of formaldehyde) are mixed with Iooo cc. of normal salt solution. This acts quickly and preserves delicate structures like the cilia of ordinary epithelia, 
and also of the endymal cells of the brain. It is satisfactory for isolating the nerve cells of the brain. For the epithelium of the trachea, intestines, etc., the action is sufficient in two hours; good preparations may also be obtained after two days or more. The action on nerve tissue of the brain and myel or spinal cord is about as rapid. For the stratified epithelia, like those of the skin, mouth, etc., it may require two or three days for the most satisfactory preparations.

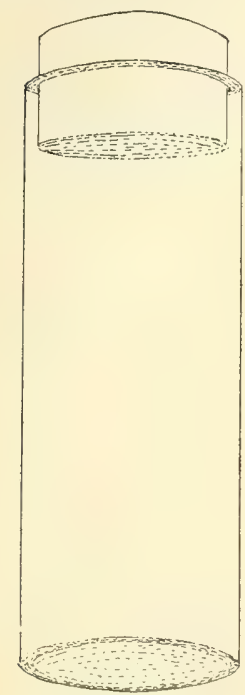

FIG. I43 A.

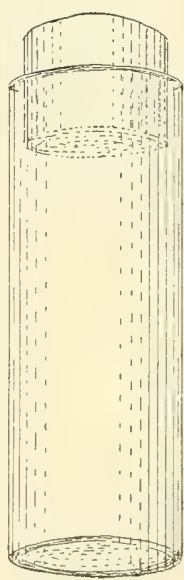

FIG. I43 B.

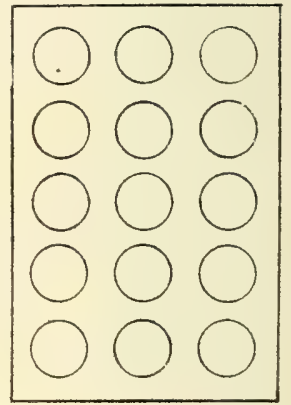

FIG. I44.

FIG. I43. Preparation Vials for Histology and Embryology. This represents the two vials, natural size, that have been found most useful. They are kept in blocks with holes of the proper size.

FIG. I44. Block with holes for containing shell vials.

8. 26I. Example of Isolation.-Place a piece of the trachea of a very recently killed animal, or the roof of a frog's mouth, in the formaldehyde dissociator. After two hours or more, up to two or three days, excellent preparations of ciliated cells may be obtained by scraping the trachea or roof of the mouth and mounting the scrapings on a slide. If one proceeds after two hours, probably most of the cells will cling together, and in the various clumps will appear cells on end showing the cilia or the bases of the cells, and other clumps will show the cells in profile. By tapping the cover gently with a needle holder or other light object the cells will be more separated from one another, and many fully isolated cells will be seen.

2. 262. Staining the Cells.-Almost any stain may be used for the formalin dissociated cells. For example, one may use eosin. This may be drawn under the cover of the already mounted preparation (Fig. I40), or a new preparation may be made and the scrapings mixed with a drop of the eosin before putting on the cover-glass. It is an advantage to study unstained preparations, otherwise one may obtain the erroneous opinion that the structure cannot be seen unless it is 
stained. The stain makes the structural features somewhat plainer; it also accentuates some features and does not affect others so markedly.

3. 263. Permanent Preparations of Isolated Cells. - If one desires to make a permanent preparation of isolated cells it may be done by placing a drop of glycerin at the edge of the cover and allowing it to diffuse under the cover, or the diffusion may be hurried by using a piece of blotting paper, as shown in Fig. I40. One may also make a new preparation and either with or without staining mix the cells with a drop of glycerin on the slide and then cover, or one may use glycerin jelly $(\& 254,326)$.

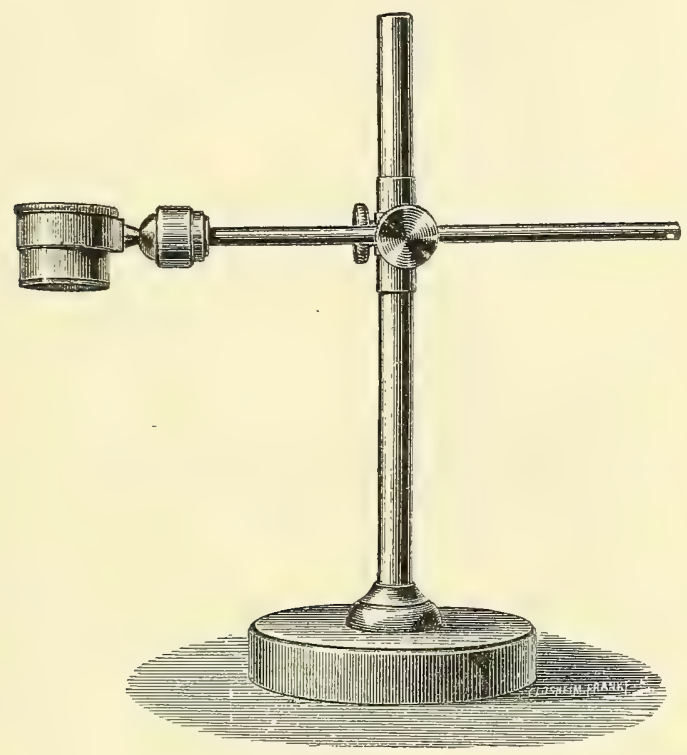

FIG. I45. Adjustable lens holder with universal joint. This is especially useful for gross dissections, and for dissecting the partly isolated elements with needles (Leitz: Wm. Krafft, N. Y.).

\% 264. Isolation of Musculer Fibers.-For this the formal dissociator may be used $(\xi 260,324)$, but the nitric acid method is more successful $(\xi 330)$. The fresh muscle is placed in this in a glass vessel. At the ordinary temperature of a sitting room (2o degrees centigrade) the connective tissue will be so far gelatinized in from one to three days that it is easy to separate the fascicles and fibers either with needles or by shaking in a test tube or reagent vial (Fig. I43) with water. It takes longer for some muscles to dissociate than others, even at the same temperture, so one must try occasionally to see if the action is sufficient. When it is, the acid is poured off and the muscles washed gently with water to remove the acid. If one is ready to make the preparations at once they may be isolated and mounted in water. If it is desired to keep the specimen indefinitely or several days, the water should be poured off and a half saturated solution of alum added ( $\& 3$ I4). The alum solution is also advantageous if the specimens are to be stained. The 
specimens may be mounted in glycerin, glycerin jelly or balsam. Glycerin jelly is the most satisfactory, however.

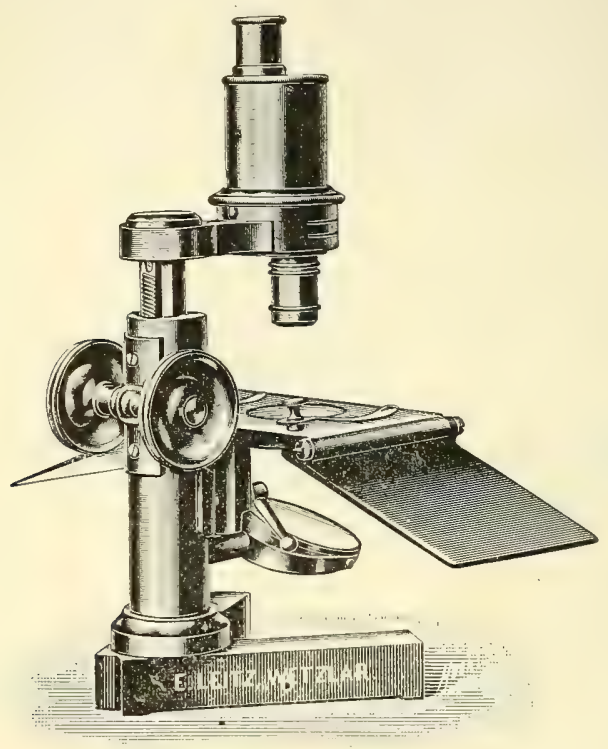

FIG. I46. Pfeiffer's preparation microscope with erecting prism between the objective and ocular (Leitz; Wm. Kraff, New York).

\section{THE PREPARATION OF SECTIONS OF TISSUES AND ORGANS}

8 265. At the present time there are three principal methods of obtaining thin sections of tissues and organs for microscopic study. These methods are: The Collodion Method, the Paraffun Method, and the Freezing Method. Each of these methods has its special application, although the collodion method is perhaps the most generally applicable, and the freezing method the most restricted, and is used mostly in pathological work where rapid diagnosis is necessary and the finest details of structure are not so important. With the paraffin method the thinnest sections may be made, and in some ways it is the most satisfactory of all. A good microtome is of great aid in sectioning.

3. 266. The Collodion Method.-In sectioning by this method the tissues are first hardened properly and then entirely infiltrated with collodion, and the collodion hardened. It is not removed from the tissue, since on account of its transparency it does no harm.

8 267. Fixing and Hardening the Tissue.-Any of the approved methods of hardening and fixing may be employed. A good general method which is applicable to nearly all of the tissues and organs is that by Picric-Alcohol. For the preparation of the solution see $(\xi 333)$. A small piece of tissue or organ not 
containing more than two to three cubic centimeters is placed in 40 or $50 \mathrm{cc}$. of the picric-alcohol and left 6 to 24 hours, when the first picric-alcohol should be thrown away and fresh added. After one or two days more the picricalcohol should be poured off and $67 \%$ alcohol added. In a day or two this is replaced by $75 \%$ or $82 \%$ alcohol $; 82 \%$ is on the whole most satisfactory, and the tissue may be left in this till it is ready for dehydration.

3. 268. Dehydration before Infiltration.-When one is ready to imbed for sectioning, the tissue must first be dehydrated in plenty of $95 \%$ or stronger alcohol. It is better to take only a small piece for this. The smaller the piece the thinner the sections that may be made. The dehydration will usually be completed in 2 to 24 hours. If the alcohol is changed two or three times the dehydration will be hastened.

3. 269. Saturating with Ether-Alcohol. $(8,322)$. - The next step is to remove the tissue from the alcohol and place it in a vial of ether-alcohol $(\& 322)$ for 2 to $2+$ hours. The dehydration becomes somewhat more complete by this step, and the tissue is more perfectly prepared for the reception of the collodion. If the dehydration is very thorough in the alcohol, this step may be ommitted, however, but one is surer of success if the ether-alcohol is used.

3 270. Infiltration with Thin Collodion.-The ether-alcohol is poured off, and a mixture of thin collodion is added ( 3319$)$. Two or three hours will suffice for objects two or three millimeters in thickness. A stay of one or more days does no harm. The larger the object the more time is needed.

3 271. Infiltration with Thick Collodion.-The thin collodion is poured off and thick collodion ( 8 3 I9) added. For very small objects, four or five hours will suffice to infiltrate, but for larger objects a longer time is necessary. The tissue does not seem to be injured at all in the thick collodion, and a stay in it of a day or even a week is more certain to insure a perfect infiltration.

3. 272. Imbedding. - The tissue may be imbedded in a paper box, such as is used for paraffin imbedding, or in any of the other boxes devised for paraffin. It is better, if paper is used, to put a small amount of oil on the paper to prevent the collodion from sticking to it. Vaselin spread over lightly and then removed, so far as possible, with a cloth or with lens paper, gives the right surface. For small objects it is more convenient to imbed immediately on a holder that may be clamped into the microtome. Cylinders or blocks of glass, vulcanite, mood and cork have all been recommended and used. A cork of the proper size is most convenient, and for many purposes answers well. Some collodion is put on the end of the cork and a pin put near one edge. The tissue is transferred from the thick collodion to the cork and leaned against the pin. Drops of the thick collodion are then poured on the tissue, and by moving the cork properly the thick viscid mass may be made to surround and envelop the tissue. Drops of collodion are added at short intervals until the tissue is well surrounded, and then as soon as a slight film hardens on the surface, the cork bearing the tissue is inverted in a wide-mouth vial of considerably larger diameter than the cork (Fig. I43). The vial should contain sufficient chlorform to float the cork. The vial is then tightly corked. In imbedding somewhat larger objects on the end of a cork or other holder, it is frequently advantageous to wind oiled paper around the holder or cork, tie it tightly and have the projecting hollow cylinder sufficiently long to receive the object. The tissue is then put into the cylinder and sufficient 
collodion added to completely immerse it. As soon as a film has formed over the exposed end, the cork may be inverted and immersed in chloroform as described above. For the use of "deck plugs" see \& 274.

3 273. Hardening and Clarifying the Collodion.-After a few hours the collodion is hardened by the chloroform. If it acts long enough, and if no water is present, the imbedding mass is rendered entirely transparent. Whenever the collodion is hard, whether it is clear or not, the chloroform is poured off and the castor-xylene* clarifier $(\% 317)$ added. In a few hours the imbedded mass will become as transparent as glass and the tissue will seem to have nothing around it. The tissue may remain for years in the castor-xylene. Sometimes the collodion remains white and opaque for a considerable time. So far as the writer has been able to judge, this is due to moisture. If one breathes on the mass too much while imbedding, or if it is very damp in the room, opacity may result. Sometimes, in objects of considerable size, this may remain for a week. This is the exception, however, and if the mass seems sufficiently hard and tough, the cutting may proceed even if the clarification is incomplete. $\dagger$

In case the imbedding mass will not clarify after a few days the imbedded object may be placed in 95\% alcohol for a day for dehydration, and then passed through chloroform and into the clarifier. There is usually no trouble in getting the mass perfectly clear in this way.

If one is in a great hurry, the collodion may be hardened in Io or $\mathrm{I}_{5}$ minutes by heating the bottle containing the chloroform in a water bath. The imbedding block of hardened collodion may then be transferred to the castor-xylene clarifier and kept warm. It will soon clear the collodion. One can then cut the sections.

\% 274. Cutting the Sections.-For cutting the sections the collodion block is usually fastened to some form of holder. For small objects cork is fairly good. Blocks of glass, vitrified fiber, etc., have been used. If one uses wood the "deck plugs" of the shipwright are satisfactory. They are about the right length when one plug is made into two holders by sawing in two (Ewing \& Ferguson). To fasten the collodion block to any form of holder, remove it from the castor-xylene, trim as desired, then dry the end on blotting paper, pour some thick collodion on the holder and press the collodion block down into the collodion. The evaporation usually fixes it in two or three minutes, when the holder may be clamped in the jaws of the microtome and the cutting proceed. For collodion sectioning a long, drawing cut is necessary in order to obtain thin, perfect sections. The object is, therefore, put in the jaws of the microtome at the right level, and the knife arranged so that half or more of the blade of the knife is used in cutting the section. It is advantageous also to have the object with its long diameter parallel with the edge of the knife. The surrounding collodion mass should be cut away, as in sharpening a lead pencil, so that there is not more than a thickness of about

*The hydrocarbon xylene $\left(\mathrm{C}_{8} \mathrm{H}_{10}\right)$ is called xylol in German. In English, members of the hydrocarbon series have the termination "ene," while members of the alcohol series terminate in "ol."

† The imbedded object may remain in the castor-xylene clarifier indefinitely without harm. The collodion grows somewhat tougher by a prolonged stay in it. After cutting all the sections desired at one time, the imbedded tissue is returned to the clarifier for future sections. 
two millimeters all around the tissue. This is to render the diameter of the end to be cut as small as possible. The smaller the object the thinner can the sections be made. With an object two or three millimeters thick and not over five millimeters wide, and a good sharp knife, sections $5 \mu$ to $6 \mu$ can be cut without diffculty. When knife and tissue are properly arranged the tissue and the knife are flooded with the clarifier. Make the sections with a steady motion of the knife. Then draw the section up toward the back of the knife with an artist's brush and make the next section. Arrange the sections in serial order on the knife blade till enough are cut to fill the area that the cover-glass will cover. For large objects one can cut thinner sections by a kind of sawing cut.

8. 275. Transferring the Sections to the Slide.-If the clarifier has evaporated so as to leave the sections somewhat dry on the knife, add a small amount. Take a piece of thin absorbent, close-meshed paper about twice the size of a slide and place it directly upon the sections. Press the paper down evenly all around and then pull the paper off the edge of the knife. The sections will adhere to the paper. Place the paper, sections down, on a slide, taking care that the sections are in the desired position on the slide. Use some ordinary lens paper or some absorbent paper, and press it down gently upon the transfer paper. This will absorb the oil, and then the transfer paper may be lifted, with a rolling motion, from the slide. The sections will remain on the slide. (See notes p. I8o).

\& 276. Fastening the Sections to the. Slide.-Drop just enough etheralcohol (equal parts of sulphuric ether and 95\% alchol) on the sections to moisten them. This will melt the collodion and fasten the sections to the slide. Allow the slide to remain in the air till the surface begins to look slightly dull or glazed.

Sometimes, especially when the air is moist, the sections wrinkle badly when the ether-alcohol is put on to fasten them to the slide. The excessive wrinkling can be avoided by using one part aicohol and two parts ether instead of using equal parts of each. Perhaps also it would be advantageous in this case to use absolute alcohol.

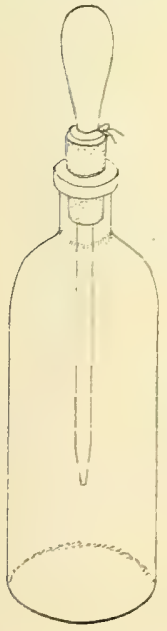

FIG. 147.
FIG. I47. Reagent bottle with combined cork and pipette (This is made by taking a cork of the proper size and making in it a hole with a cork borer for the glass tube. It is advantageous to have a string tied tightly around the rubber bulb as shown).

\& 277. Removing the Oil from the Sections.-As soon as the ether-alcohol has evaporated sufficiently to leave the surface dull, place the slide in a jar of ordinary commercial benzin. It may be left here a day or more without injury to the sections, but if moved around in the jar the oil will be removed in three to five minutes. From the benzin transfer to a jar of $95 \%$ alcohol to wash away the benzin. One may use alcohol in the beginning, but it dissolves the oil far less rapidly than the benzin. The slide may remain in the alcohol half a day or more if one wishes, but a stay of five minutes or a thorough rinsing of half a minute or so by moving the slide around in the alcohol will suffice.

Xylene is to be preferred to benzin for removing the oil, but it is more expensive. 
\& 278. Staining the Sections with an Alcoholic Stain.-If an alcoholic stain containing $50 \%$ or more alcohol (for example, hydrochloric acid carmine in $70 \%$ alcohol) is used, the slide may be removed from the $95 \%$ alcohol, drained somewhat and then the stain poured upon the sections, or preferably, the slide immersed in a jar of the stain. The stain is finally washed away with $67 \%$ or stronger alcohol, the sections dehydrated in $95 \%$ alcohol, cleared and mounted in balsam.

8. 279. Staining the Sections with an Aqueous Dye.-In staining with a watery stain, the slide bearing the sections is transferred from the $95 \%$ alcohol and plunged into a jar of water, and either allowed to remain a few minutes or moved around in the water a moment. Then it is placed horizontally and some of the stain placed on the sectious with a pipette, or preferably, it is immersed in a jar of the stain; in case of immersion the slide should stand vertically or nearly so, then any particles of dust, etc., in the stain will settle to the bottom of the vessel and not settle on the sections. When the sections are stained, usually within five minutes, they are thoroughly washed with water either by the use of a pipette or preferably by immersing in a jar of water. They may then be counterstained for half a minute with some general dye, like eosin or picric acid, or mounted with but the one stain.

*Various forms of paper have been used to handle the collodion sections. It should be moderately strong, fine meshed and not liable to shed lint, and fairly absorbent. One of the first and most successful papers recommended is "closet or toilet paper." Cigarette paper is also excellent. In my own work the heavy white tissue paper has been found almost perfect for the purpose. Ordinary lens paper or thin blotting paper for absorbing the oil may be used with it. ( 8,275$)$.

$\uparrow$ If one is a long time in cutting a series of sections, it sometimes occurs that the xylene evaporates, and while the sections may not look dry, they are practically in castor oil and not easily transferable. In such a case fresh clarifier or even a little xylene to thin the oil on the sections may be used. If the oil is too thick it is viscid and there is difficulty in handling the sections with the paper as they stick rather firmly to the knife. (\$275).

$\neq$ In the past the plan for changing sections from $95 \%$ alcohol to water, for example, has been to run them down gradually, using 75,50 and $35 \%$ alcohol, successively. Each percentage may vary, but the principle of a gradual passing from strong alcohol to water was advocated. On the other hand I have found that the safest method is to plunge the slide directly into water from the $95 \%$ alcohol. The diffusion currents are almost or quite avoided in this way. There is no time for the alcohol and water to mix, the alcohol is washed away almost instantly by the flood of water. So in dehydrating after the use of watery stains, the slide is plunged quickly into a jar of 95\% alcohol. The diffusion currents are avoided in the same way, for the water is removed by the flood of alcohol. This plan has been submitted to the severe test of laboratory work, and has proved itself perfectly satisfactory. 


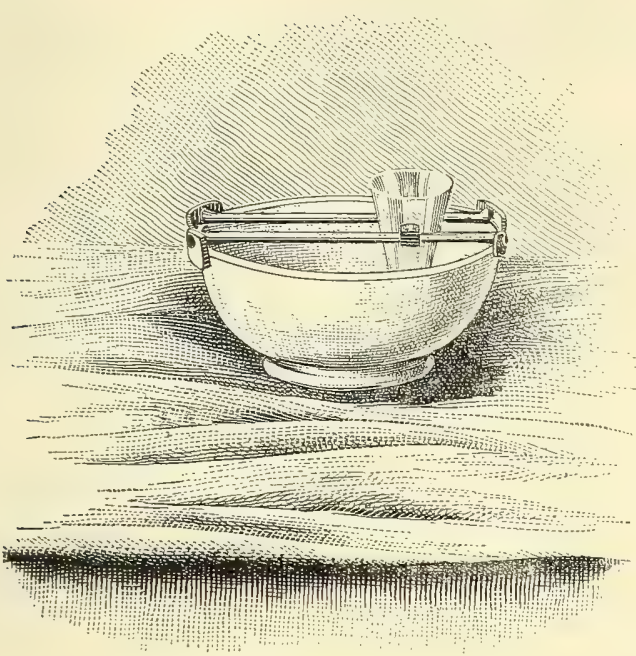

FIG. I48.

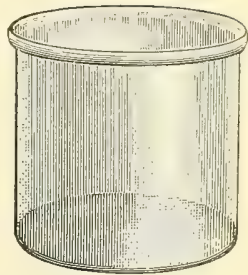

FIG. I49.

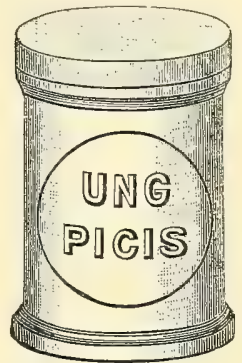

FIG. I50.

FIG. 148. Waste bowl with rack for supporting slides and a small funnel in which the slides stand while draining. This outfit is easily made by any tinsmith. The rack is composed of two brass rods about $4 \mathrm{~mm}$. in diameter. The bent end pieces are sheet lead. The funnel is made of tin, copper or brass. Either copper or brass is preferable to tin. A glass dish like that shown in Fig. I6o is better than a bowl, as it can be more readily and thoroughly cleaned. (Cut loaned by Wm. Wood \& Co. )

FIG. I49. Round glass aquarium. This glass vessel is better than the bowl for all the uses described for the bowl. (Whitall, Tatum \& Co.)

FIG. I50. Glass box or ointment jar with cover. These boxes may be had of various sizes and can be used advantageously for water, and for cleaning mixture for slides and cover glasses ( 8242 ). (Whitall, Tatum $\&$ Co.)

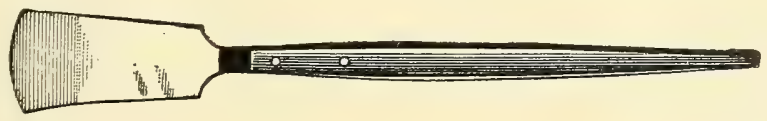

FIG. I5I. Section lifter. This is of thin, springy, flexible metal placed in a handle as shown. These are made of various sizes for large or small sections. Such an instrument is exceedingly helpful in handling loose sections. (Queen $\mathcal{G} \mathrm{Co}$. ) 


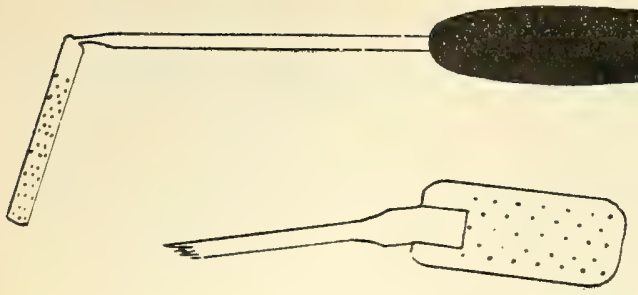

FIG. 152. Perforated section lifter. This is easily made by soldering a wive to some very thin sheet brass or copper, and then perforating this with a coarse needle or fine awl. Any roughness must be removed by using a fine oil stone.

ORDER OF PROCEDURE IN MAKING MICROSCOPICAL PREPARATIONS BY THE COLLODION METHOD

3 280. It will be seen from this table, and sections $267-28 \mathrm{r}$, that it requires about five days to get a microscopical preparation if one commences with the fresh tissue. Other methods of hardening might require as many months. It is evident, therefore, that one must exercise foresight in histology or much time will be wasted.

I. Fixing and hardening the tissue $\mathrm{s}$ $\left(\begin{array}{l}3 \\ 267\end{array}\right), 4$ days or more.

2. Dehydrating the object to be cut in $95 \%$ or stronger alcohol $(\& 268), 2-$ 24 hours.

3. Saturating the tissue in ether-alcohol $(\& 269), 2-24$ hours.

4. Infiltrating with thin collodion $(\& 270), 2$ hours to $z$ days.

5. Infiltrating in thick collodion $(\& 27 \mathrm{I})$, 5 hours to several days.

6. Imbedding the tissue $(8272),{ }_{5}$ to 20 minutes.

7. Hardening the collodion with chloroform (\& 273), 5-24 hours.

8. Clarifying and further hardening the collodion with castor-xylene ( $\&$ 273), Io- 36 hours.

9. Cutting the sections $(\& 274)$, Io minutes to 2 hours.

IO. Transferring the sections to a slide with paper ( $\&$ 275), I minute.

II. Fastening the sections to the slide with ether-alcohol $(\xi 276)$, I or 2 minutes.
I2. Removing the oil from the sections with benzin and alcohol ( $\$ 277$ ), 3-5 minutes, or 24 hours.

13. Staining the sections with an alcoholic dye $(\& 278), 2$ minutes to 24 hours.

I4. Staining the sections with an aqueous dye ( 8 279), 2-Io minutes.

I5. Removing the superfluous dye by washing in water or alcohol $(\& 278-$ 279), 2-5 minutes.

I6. Staining with a general dye ( $\& 279)$, I5-30 seconds.

I7. Washing with water or a $1 \mathrm{cohol}$ ( $8278-279$ ), I to 2 minutes.

18. Dehydrating the sections in $95 \%$ alcohol (\&28I ), 5 min. to 24 hours.

I9. Clearing the sections $(828 \mathrm{I}), 5 \mathrm{~min}-$ utes to 24 hours.

20. Draining the sections, I-2 minutes.

2I. Mounting in Canada balsam ( $\& 28 \mathrm{r}$ ), I-2 minutes.

22. Labeling the preparation $(\& 308), 2$ minutes.

23. Cataloging the preparation ( 8309 ), 5-10 minutes. 
3. 28r. Mounting in Balsam.-After the sections are stained they must be dehydrated and cleared before mounting in balsam. For the dehydration the slide is plunged into a jar of $95 \%$ alcohol. For clearing after the dehydration the slide is drained of alcohol and put down flat and the clearer poured on, or the whole slide is immersed in a jar of clearer ( 8.318$)$. Clearing usually is sufficient in a few minutes; a stay of an hour or even over night does not injure most sections.

In mounting in balsam the clearer is drained away by standing the slide nearly vertically on some blotting paper, or by using the waste bowl and standing it up in the little funnel (Fig. I48). Then the balsam is put on the sections or spread on the cover-glass and that placed over the sections.

For cataloging and labeling, see \& 307-3 I0.

FIG. 153. Small spirit lamp modified into a balsam bottle, or a glycerin or glycerin-jelly bottle, or a bottle for homogeneous immersion liquid. For all of these purposes it should contain a glass rod. See also Fig. 168 .

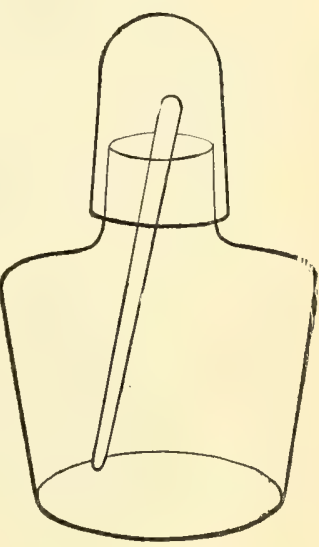

2S2. The Collodion Method with Alcohol,-A good method of procedure for making collodion sections is to do exactly as described, including \& 272 , and then instead of hardening the collodion in chloroform and clarifier, it is hardened in $S_{2}, \%$ alcohol for a day or two before sectioning. In sectioning the knife and tissue are kept wet with $\$ 2 \%$ alcohol and the sections are dehydrated with $95 \%$ alcohol and then fastened to the slide with ether alone or with ether-alcohol. The staining and mounting $(3,27 \mathrm{~S}-2 \mathrm{~S} \mathrm{I})$ are as described. One may preserve the tissue after imbedding for a long time in the $82 \%$ alcohol before sectioning and sections may be made at any time. While this method appears somewhat simpler, the results are not so satisfactory as by the oil method given above.

\section{THE PARAFFIN METHOD}

\$283. As with the collodion method, the tissues are first properly fixed and hardened and then entirely filled with the imbedding mass, but unlike the collodion the mass must be entirely removed before the sections are finally mounted. The tissue thus imbedded and infiltrated is like a homogeneous mass and sections may be cut of extreme thinness.

\& 284. Harden perfectly fresh tissue in picric-alcohol $(\& 333)$ from one to three days. (Any good method for fixing and hardening the elements may be used. One must observe in each case, however, the special conditions necessary 
for each method. The time might be longer or shorter than for the picric-alcohol. (See Lee, the Microtomists' Vade-Mecum.)

If picric-alcohol is used, pour it off after the proper time for fixing has elapsed, and add $67 \%$ alcohol. Leave this on the tissue from one to three days, and if it becomes very yellow it is well to change it two or three times. After two or three days pour off the $67 \%$ alcohol and add $82 \%$. The tissue should remain in this one or two days, and it may remain indefinitely.

In case the alcohol becomes much yellowed, it should be changed.

\% 285. Dehydration and Preparation for Imbedding.-From the pieces of tissue fixed and hardened in any approved manner, cut pieces 5 to 10 millimeters long and 2 to 3 millimeters in breadth. Place one or two pieces in a shell vial (Fig. I43.) and add 95\% alcohol. Change the alcohol after two or three hours, and within 6 to 24 hours, depending on the size of the piece to be dehydrated, the dehydration will be completed. The secret of success is the use of plenty of alcohol and sufficient time. Absolute alcohol for the second change would act more promptly and efficiently, but if plenty of $95 \%$ is used one will succeed, unless the day, or the climate in general, is too damp.

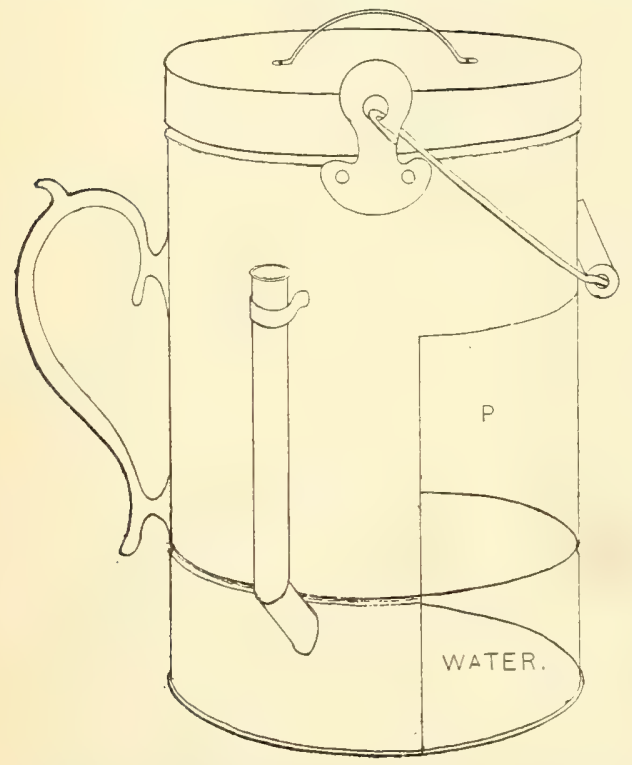

FIG. I54. Copper pail with water bottom for melting paraffin. This also serves as a water bath for large bottles in which saturated solutions of dichromate and other salts are prepared.

(If one is studying organs, then the whole organ may need to be prepared for imbedding, but for minute structure small pieces are preferable, as thinner sections may be made. )

8. 286. Displacing Alcohol and Clearing Tissues with Cedar-wood Oil and Infiltrating with Paraffin.--(Lee, p. 66. Neelson and Schiefferdecker, Arch. für Anat. und Physiol., ISS2, p. 206.) When the tissue is dehydrated it is removed to a vial of cedar-wood oil. When the alcohol used for dehydration is displaced by the oil, the tissue will look clear and translucent. This requires 2 to 24 hours. 
It is hastened by warmth. It is then removed from the cedar-wood oil, drained, and placed in pure, melted paraffin, and this is then put into a paraffin oven and left from 2 to 24 hours (

Paraffin forinfiltrating has usually a somewhat lower melting point than that for imbedding. Equal parts of paraffin of $43 \mathrm{C}$. and 54 C., answer well. For imbedding, the paraffin must be of a melting point which wili give good ribbons in the temperature of the room where the sectioning is to be done. In a room of I9 to $20 \mathrm{C}$. a mixture of I part $43 \mathrm{C}$. paraffin with two parts of $54 \mathrm{C}$. usually answers well.

FIG. I55. Hot filter for paraffin, gelatin, balsam, etc. It is entirely surrounded by a water jacket. The water is heated by placing a burner under the projecting part $H$. The wire basket is to hold the strainer and allow a free flow of the filtered substance on all sides. There is a bail for suspending the filter, and the filtered substance runs out through the narrowed part $F$.

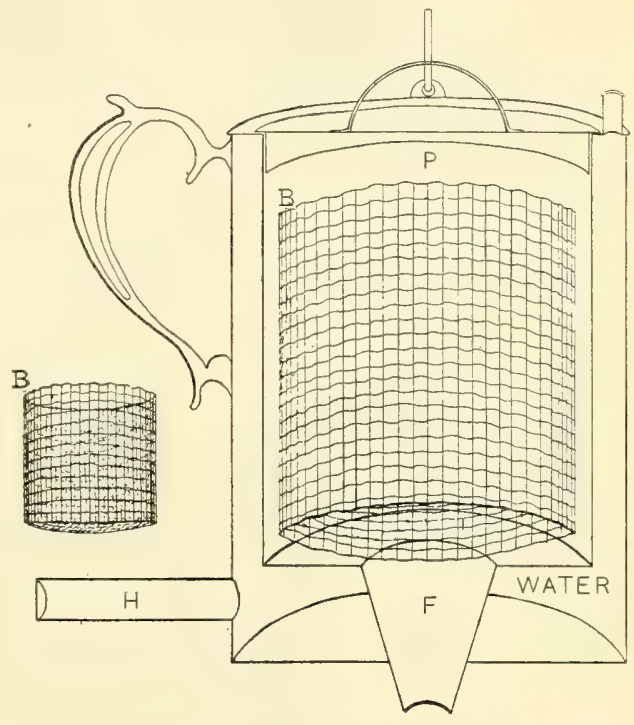

\& 287. Imbedding in Paraffin.-Make a small paper box, fill it nearly full of hot paraffin, place the box for a half minute on some cold water to make a thin solid layer on the bottom, then transfer the tissue to the box and arrange near one end so that the sections may be cut in the desired plane. Now pl ace the paper box on as cold water as possible so that the paraffin may cool quickly. It will be more homogeneous if cooled quickly, and will shrink tightly against the tissue and avoid air spaces.

In imbedding two main things should be looked after: The paraffin should be hot so that it will thoroughly fuse with the paraffin in the tissue. The tissue should be kept from the bottom of the box, either by holding it up in the middle of the box with warmed forceps or a perforated section lifter while a stratum

*Thickened cedar-wood oil like that for oil immersion objectives is recommended by Lee for clearing. This is very expensive, and for most work unnecessary. Any form of cedar-wood oil has been found satisfactory in the writer's laboratory. The great thing is to have the tissue thoroughly dehydrated before putting it into the oil. 
cools on the bottom, or a stratum of the paraffin may be cooled on the bottom before putting the tissue in the box. Cool quickly after the tissue is in place.

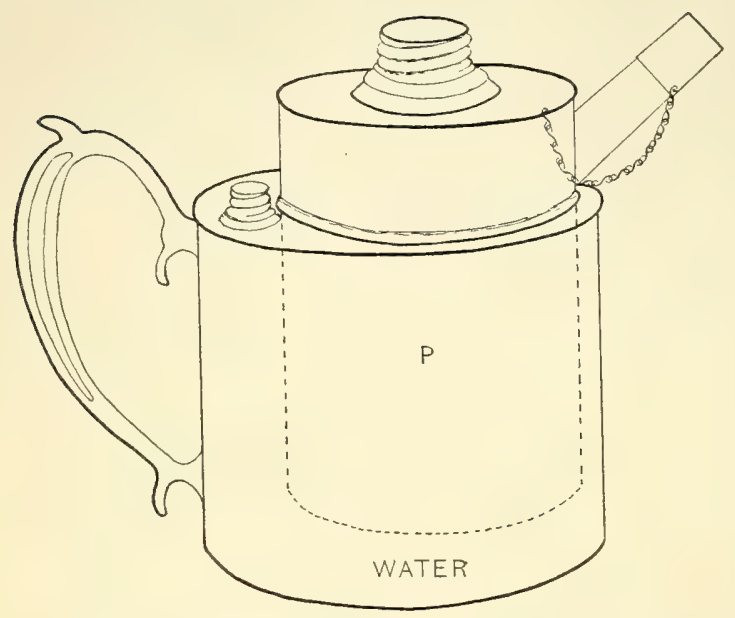

FIG. I56. Paraffin receptacle $P$, with water bath and spout for paraffin imbedding ( 1883$)$.

3. 28S. Cutting the Sections.-After the imbedding mass is well cooled, remove the paper box and trim the end containing the tissue in a pyramidal form. clamp the block of paraffin in the holder of the microtome so that the tissue will be at the proper level for cutting. If a ribbon microtome is used, heat the holder and melt the end of the block upon it. Cool and place the holder in its place in the microtome. Use a very sharp, dry razor for cutting the sections. The sections are made with a rapid, straight cut as in planing. Do not try to section with a drawing cut as in collodion sectioning. If the temperature of the room is right for the paraffin used, the sections will remain flat, and if the opposite sides of the block are parallel, and one edge strikes the knife squarely, the sections will adhere and thus make a ribbon. If the room is too cold for the paraffin the sections will roll. If it is too warm the sections will crumple.

Remember the sections must be very thin, from $3 \mu$ to $15 \mu$ to show fine structural details to good advantage.

The secret of making good ribbons of sections is to have the block of paraffin containing the tissue cut square and properly arranged in the microtome so that the block strikes the edge of the section knife at right angles with the edge; and finally the paraffin must be of a proper melting point for the room in which the sections are to be cut. Remember that the larger the object the thicker must be the sections, and the softer the paraffin. Frequently one may modify the temperature if too cold by a Bunsen burner flame near the microtome. If it is too warm one may go to a basement room.

?. 289. Extending Sections with Warm Water.-Paraffin sections are liable to have fine wrinkles or folds in them. These folds are very annoying and often obscure the structure. To get rid of them the sections are extended or stretched upon warm water. One may put a ribbon of sections on warm water and then cut the ribbon into pieces and transfer the pieces to slides. Practically, however, the 
extension is almost always accomplished on the slide itself. A slide is albumenized $(3,290)$ and the ribbon cut into short pieces and placed on the slide. Distilled or filtered water is then added with a pipette (Fig. I47) until the sections float. Then the slide is moved back and forth over an alcohol or gas flame to warm the water. Care must be taken to avoid melting the paraffin. As the water warms the paraffin containing the sections will flatten and stretch out. One will be surprised at the amount of extension. It is necessary to take pieces considerably shorter than the cover-glass to be used or when extended the sections will not all be covered. After the sections are extended, arrange the ribbons carefully on the slide as shown in Fig. I62 if one is making serial sections. Arrange in the middle of the slide if only one or two sections are on each slide (Fig. I3S). Let the excess water drain off. Now let the slide stand several hours for the water to evaporate completely. The time will depend on the temperature and the dryness of the atmosphere. If there is plenty of time, leave the slides 24 or 48 hours. If one has a register with hot air intake, the slides may be put in the current of hot air. They will dry out in half an hour or an hour. Sections which have been left for a year have given excellent results.
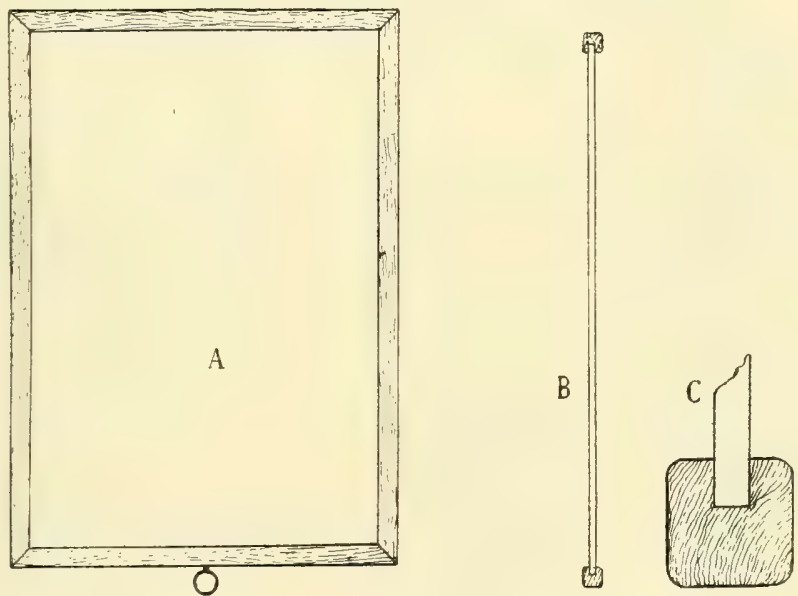

FIG. I57. Trays for slides and for ribbons of sections. The figures shou the construction. It is important to have the bordering frame with rounded corners so that the trays mxy be easily pulled out of a pile or reinserted. The screw eye shown in A makes it easy to pull out a single tray. For ribbons of sections a piece of paper is placed in the tray and the ribbons are placed on it. (A) Face view, (B) Sectional view of the whole tray, (C) Sectional view of one side to show the construction more clearly. Trays of this kind are so cheap (\$15.00 per hundred for those holding 50 to 60 slides), that a laboratory can have a great number. (Trans. Amer. Micr, Soc., I899, p. 107).

The slide trays (Fig. I57) are excellent for drying preparations of all kinds.

2 290. Fastening the Sections to the Slide.-To fasten the sections firmly to the slide, coat the slide with albumen fixative ( 8 3I2) as follows: Put a minute 
drop of the albumen on the center of a slide and with a clean finger spread the albumen over the slide, wiping off all that is possible. Finally beat or tap the slide with the end of the finger. This will make a very thin (it cannot be too thin) and even layer.

The sections are extended and dried as described in \&. 289. When the sections are thoroughly dry they are in optical contact with the slide and have a shining appearance when looking on the back of the slide. When the sections are dry coat them with $3 / 4 \%$ collodion made as follows. Take $3 / 4$ gram of soluble cotton, put it into a bottle and add $60 \mathrm{cc}$. of $95 \%$ or absolute alcohol, and $40 \mathrm{cc}$. of sulphuric ether. Coat the sections with a soft camel's hair brush. The collodion should dry in a minute or less. If one uses too much ether in this fixing collodion, the paraffin will be partly dissolved and will look moist for a long time. The slight excess of alcohol will obviate any such difficulty.
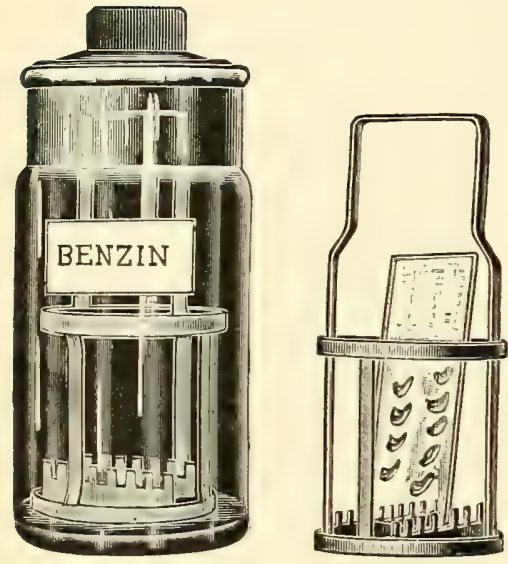

FIG. I58. A slide holder and bottle for containing the same (Mix, Journal of Applied Microscopy, vol. $1,1898, p .169$.

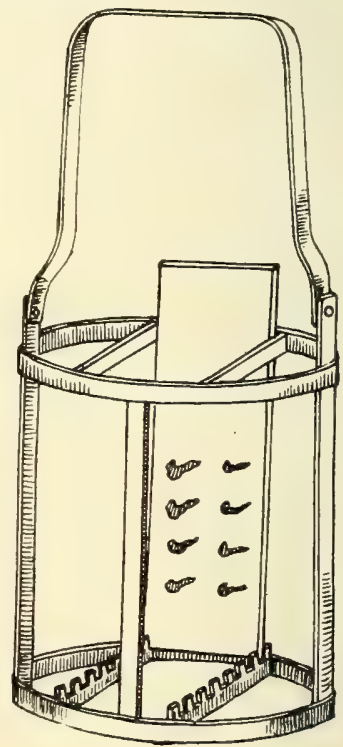

FIG. 159. Slide holder with the bail hinged so that it may be turned aside in inserting or removing the slides.

- When the collodion is dry place the slide in benzin or xylene to dissolve the paraffin (see $\%, 29 I$ ). If the sections are not extended on water, they may be put directly on the albumenized sides, pressed down with the finger and coated with collodion. This is much more rapid, but does not get rid of the fine folds. (See Dr. Agnes Claypole Trans. Amer. Micro. Soc. I894, p. 66, I27.)

8. 291. Removing the Paraffin.-Immerse the slide in a vessel of xylene or benzin. This will dissolve the paraffin. An hour will usually suffice. One can 
hasten the solution of the paraffin by moving the slide in the solvent. In this way it may be dissolved in 5 to Io minutes, or even less. It will do no harm to leave the slide in the benzin or xylene over night. Two or three days even might not do any harm, but it is usually better to proceed at once to the other operations.

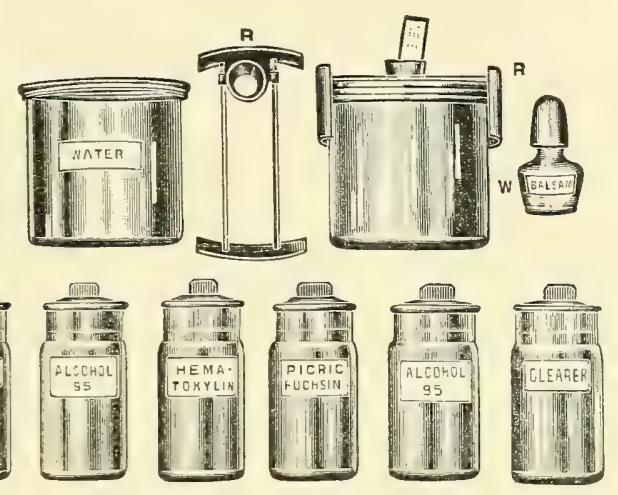

FIG. I60. Apparatus and regents with which the slide holders are used. With this apparatus it is easy to prepare specimens in large numbers very expeditiously. After the sections are fastened to the slide and placed in the holder, the slides need not be touched during all the operations until they are finally ready to be mounted in balsam. Each holder contains from 12 to I4 slides. (\$277-300). The bottles for the reagents are glass stoppered specimen or museum bottles. (Mix, Jour. Ap. Micr. 1898, p. I7I.) Compare Fig. I48.

3. 292. Removing the Xylene or Benzin-From the xylene or benzin plunge the slide bearing the sections into a jar of $95 \%$ alcohol, and leave it for a few minutes, or move it around in the alcohol for half a minute or so.

8 293. Staining the Sections with an Alcoholic Dye.-With an alcoholic stain like hydrochloric acid carmine, remove the slide from the alcohol, and add the stain directly after draining the slide, Do not allow the stain to become dry, for that would injure the tissue. Wash away the stain with $67 \%$ alcohol, then dehydrate with 95\% alcohol, clear and mount in balsam as described below.

8. 294. Staining with as Aqueous Dye.-Wash away the $95 \%$ alcohol from the slide bearing the sections by plunging it into a jar of water and moving it around a moment. Then add the stain to the sections with a pipette, or immerse the slide in a jar of the stain, and allow the stain to act from 5 to ro minutes. Wash thoroughly with water.

3. 295. Staining with a General Dye-Counterstaining.-If it is desired to give a general stain after the nuclear dye (\& 294), carmine stained preparations may be tinted with picric-alcohol for half a minute or more ( 8.333$)$, and the hematoxylin stained specimens with eosin $(\xi 32 \mathrm{I})$. It usually takes less than a minute for this. Wash away the counterstain with water. 
\%. 296. Counterstaining with Picro-fuchsin. -For a general dye to use with hematoxylin, eosin is good, but to differentiate the tissues more completely, especially connective tissue, which is present in practically every section made, it is better to use Van Gieson's mixture of picric acid and acid fuchsin. (Picric acid, saturated aqueous solution $75 \mathrm{cc}$, water $25 \mathrm{cc}$. I $\%$ aqueous solution of acid fuchsin, Io cc.) Sections are first strongly stained with hematoxylin, well washed with water and then stained 3 seconds to 15 minutes in the picro-fuchsin. They are then washed in distilled water; or in tap water, to which has been added a drop or two of glacial acetic acid to Ioo cc. of water. They are then dehydrated, cleared and mounted in acid balsam, that is in balsam which has not been neutralized ( 83 I 5 ). If glycerin or glycerin jelly is used as a mounting medium it should be slightly acid. Unless the mounting medium is slightly acid, the red of the acid fuchsin soon fades. In some cases less acid fuchsin should be used, and in some a greater amount. Acid fuchsin alone without the picric acid is also good for a counterstain. The picro-fuchsin is a very valuable differential stain and combined in different proportions with picric acid will give great assistance in almost every case. It does not seem to be a permanent stain. (See Freeborn, Trans. N. Y. Path, Soc., I893, p. 73. Also studies from the department of pathology of the College of Physicians and Surgeons, Columbia University, N. Y., I $894-1895$ ).

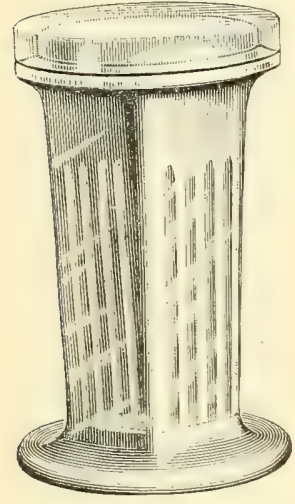

161. Coplin's staining dish. A. The entire dish; $B$. the dish in cross section. This is made of glass and is a very neat piece of apparatus. With it ten slides may be stained at once. (Whitall, Tatum \& Co.)

8 297. Dehydration of the Stained Sections.-Place the slide with the stained sections in a jar of $95 \%$ or absolute alcohol and leave it a few minutes, or wave it around in the alcohol for half a minute or so.

Remember that the larger and thicker (U) the sections the more time it requires to de-

CROSS-SECTION SHOWING SLIDES IN POSITION. liydrate them. In a moist atmosphere, 95\% alcohol is not completely satisfactory, and

one must use a stronger alcohol. When it is dry, 95\% answers very well.

8. 298. Clearing the Sections.-Drain off the alcohol, and place the slide in a jar of clearer $(3.3 \mathrm{IS}, \mathrm{A}$ or $\mathrm{B})$ or put a drop or two of clearer on the sections. The clearing is usually accomplished in two or three minutes.

8 299. Mounting in Balsam.-For this the clearer is drained from the slide, and wiped away with blotting paper, cloth, etc. The balsam is then put upon the sections and the cover added, or a cover-glass is spread with the balsam and then put over the sections. (If the sections show a whitish appearance and are opaque they were not sufficiently dehydrated. If natural balsam is used the sections will clear up in time). 
8. 300. It will be seen from this table and from sections 283 to 299 that it requires from 5 to 7 days to get a microscopical preparation by the paraffin method if one starts with a fresh tissue. Depending on the method of fixing and hardening, the time may be much greater. Much time will be lost in waiting unless one plans ahead in histological work.

I. Fixing and hardening the tissue or organ (\& 284$), 4$ days or more.

2. Dehydrating the object to be cut in $95 \%$ or stronger alcohol $(\& 285)$, I to 24 hours.

3. Displacing the alcohol and clearing tissues with cedar-wood oil. (See \& 286), 2 to 24 hours.

4. Infiltrating the tissue with paraffin in the paraffin oven $(\& 286), 2$ to 24 hours.

5. Imbedding in paraffin $\left(\&_{2} 287\right)$, Io minutes.

6. Cutting the sections $(\& 288)$, IO minutes.

7. Extending the sections with warm Water. (See \& 289.)

8. Fastening the sections to a slide (\& 290), 5 minutes to 24 hours.

9. Removing the paraffin ( $\& 29$ I), IO minutes to 24 hours.

Io. Removing the xylene or benzin (\& 292)
II. Washing with water, note, p. I8o.

12. Staining with an aqueous dye (\& 294), 2 minutes to 24 hours.

I3. Washing away the superfluous stain with water (\& 294).)

I4. Staining with a general dye ( 8 295296), Io seconds to ro minutes.

I5. Washing the sections with water (\& 295-296).

I6. Dehydrating the stained sections in 95\% alcohol $(\& 297), 3$ minutes to 24 hours.

I7. Clearing the sections $(\%, 298) 2$ minutes to 24 hours.

I8. Mounting in Balsam (?.299), I to 5 minutes.

I9. Sealing the cover-glass $\left(\begin{array}{l}\& \\ 253\end{array}\right), 2$ minutes.

20. Labeling the preparation ( $\& 308), 2$ minutes.

2I. Cataloging the preparation ( $\& 309$ ), 5 to to minntes.

\section{SERIAL SECTIONS}

\& 30I. In histological studies it is frequently of the greatest advantage to have the sections in serial order, then an obscure feature in one section is frequently made clear by the following or preceding sections. While serial sections are very desirable in histological study, they are absolutely necessary for the solution of morphological problems presented in complex organs like the brain, in embryos and in minute animals where gross dissection is impossible.

\% 302. Arrangement of Tissues for Sections in Histology.-They should be so arranged that the exact relations of each part to the organ can be readily determined. For example, an organ like the intestine, a muscle or a nerve, should be so arranged that exact transections or longisections can be made. Organs like the liver and other glands, the skin, etc., should be so arranged that sections parallel with the surface or at right angles to it, (surface or vertical sections) may be made. Oblique sections are often very puzzling. 
With cylindrical objects, especially botanical specimens, one may cut tangen-

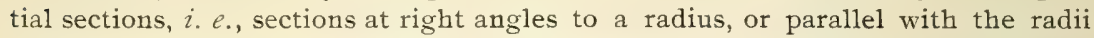
(radial sections), or transections, $i$. e., sections across the long axis.

8. 303. Arrangement of Serial Sections. -The numerical order may be very conveniently like the words on a printed page, $i . e$., beginning at the upper left hand corner and extending from left to right.

The position of the various aspects of the sections should be in general such that when they are under the compound microscope the rights and lefts will correspond with those of the observer. This may be accomplished as follows for sections made in the three cardinal sectional planes, Transections, Frontal Sections, Sagittal Sections:

(C) Transection, $i . e$, sections across the long axis of the embryo or animal dividing it into equal or unequal cephalic and caudal parts.

(a) In accordance with the generally approved method of numbering serial parts in anatomy, the most cephalic section should be first (Fig. I62).

(b) The caudal aspect of the section should face upward toward the coverglass, the cephalic aspect being next the slide.

(c) The dorsal aspect should face toward the upper edge of the slide (Fig. 162).

This arrangement may be easily accomplished for the transections as follows : Imbed the embryo with the right side down, taking the precautions against letting the embryo rest against the bottom of the box $\left(\begin{array}{l}8 \\ 8\end{array} 287\right.$ ).

(I) Mount the block of paraffin containing the embryo so that the tail end will be next the microtome holder. The head will then be cut first.

(2) Place in the microtome so that the right side of the embryo will meet the edge of the knife.

(3) Mount as a printed line and the first or cephalic section will be at the upper left hand corner, and the dorsal aspect of the embryo will be toward the upper edge of the slide.

Under the microscope the rights and lefts will appear as in the observers own body, also the dorsal and ventral aspects so that he can easily locate parts by comparing them with his own body.

(B) Frontal Sections, $i . e$, sections lengthwise of the embryo or animal and from right to left (dextral and sinistral), so that it is divided into equal or unequal dorsal and ventral parts.

Imbed the embryo with the right side down in the imbedding box as before.

(I) Mount the paraffin block so that the ventral side of the embryo is next the microtome holder. The dorsal side will then be cut first.

(2) Let the right side of the embryo meet the edge of the knife.

(3) Mount the first section on the left end of the slide as before and so that the sections will be crosswise on the slide, the tail toward the upper edge. Under the compound microscope the head will appear toward the upper edge and the rights and lefts will be as in the observer's own body.

(C) Sagittal Sections, that is sections lengthwise of the embryo or animal and from the ventral to the dorsal side, thus dividing the body into equal or unequal right and left parts.

For these sections imbed the embryo as in the two preceding cases. 
(I) Put the right side of the embryo next the microtome holder, then the left side will be cut first.

(2) Let the caudal end meet the knife edge.

(3) Put the first section in the upper left hand part of the slide as in the other cases. The sections will be lengthwise of the slide. This will bring the ventral side up and the head to the right on the slide. Under the microscope the head will appear at the left and the dorsal side toward the upper edge of the slide.

3. 304. For serial sections with collodion imbedded objects it is a great advantage to have the imbedding mass unsymmetrically trimmed, so that if a section is accidentally turned over it may be easily noticed and rectified.

Furthermore it is imperatively necessary that the object be so imbedded that the cardinal aspects, dextral and sinistral, dorsal and ventral, cephalic and caudal, shall be known with certainty.

3. 305. Thickness of Cover-Glass and of Serial Sections.-It is a great advantage to use very thin cover-glasses (0.12-0.I $8 \mathrm{~mm}$.) for serial sections, then the cover will not prevent the use of high powers. When the ordinary slides $(25 X$ $76 \mathrm{~mm} ., \mathrm{I} \times 3$ inch) are used cover-glasses $24 \times 50 \mathrm{~mm}$. may be advantageously employed.

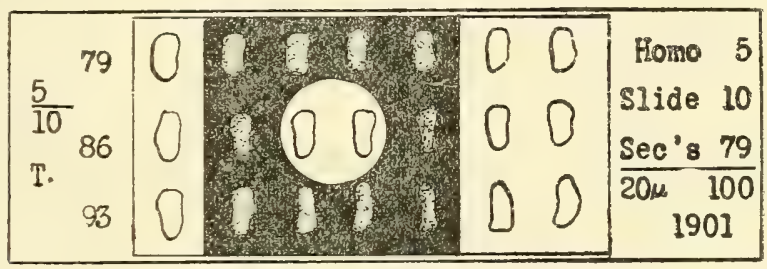

FIG. I62.

FIG. I62. A slide of serial sections showing the position of the sections, the label and the size of the cover-glass $(24 \times 50 \mathrm{~mm}$.). The numbers next the cover at the left indicate the first section of each row, and illustrate the method of placing the sections as if they were wordson a printed page. .T. Transections.

The combined thickness of the sections on a slide is easily determined by noting carefully the position of the microtome screw at the first and last sections and measuring the elevation. With good modern automatic microtomes the successive sections are almost exactly uniform in thickness, hence it is easy to determine the combined thickness of the sections on a slide.

\$306. Labeling Serial Sections.-The label of a slide on which serial sections are mounted should contain at least the following :

The name of the embryo and the number of the series; the number of the slide of that series; the thickness of the sections, and the number of the first and last section on the slide; the date. It is also a convenience to have the information repeated in part on the left end and the number of the first section in each row, as shown in the sample slide of serial sections. (Fig. I62). 


\section{LABELING, CATALOGING AND STORING MICROSCOPICAI, PREPARATIONS}

8. 307. Every person possessing a microscopical preparation is interested in its proper management; but it is especially to the teacher and investigator that the labeling, cataloging and storing of microscopical preparations are of importance. "To the investigator, his specimens are the most precious of his possessions, for they contain the facts which he tries to interpret, and they remain the same while his knowledge, and hence his power of interpretation, increase. They thus form the basis of further or more correct knowledge; but in order to be safe guides for the student, teacher, or investigator, it seems to the writer that every preparation should possess two things: viz., a label and a catalog or history. This catalog should indicate all that is known of a specimen at the time of its preparation, and all of the processes by which it is treated. It is only by the possession -of such a complete knowledge of the entire history of a preparation that one is able to judge with certainty of the comparative excellence of methods, and thus to discard or improve those which are defective. The teacher, as well as the investigator, should have this information in an accessible form, so that not only he, but his students can obtain at any time, all necessary information concerning the preparations which serve him as illustrations and them as examples."

\& 308. Labeling Ordinary Microscopical Preparations.-The label should possess at least the following information (see $\& 306$ for serial sections):

\section{EXAMPLE}

(I) The number of the preparation, the thickness of the cover-glass and of the sections under it.

(2) The name and source of the preparation.

(3) The date of the specimen ( 2 of catalog.)
No. 475. $\quad \begin{aligned} & \text { C. } \frac{.15}{8 \mu} \\ & \text { Secs. }\end{aligned}$

Striated Muscle; transection of the Sartorius of the Cat.

October I5, I 894 .

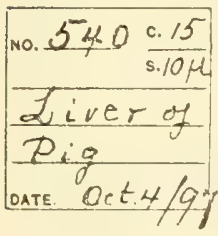

Fig. 163 .

8 309. Cataloging Preparations.-It is believed from personal experience, and from the experience of others, that each preparation (each slide or each series) should be accompanied by a catalog containing at least the information suggested in the following formula. This formula is very flexible, so that the order may be changed, and numbers not applicable in a given case may be omitted. With many objects, especially embryos and small animals, the time of fixing and hardening may be months or even years earlier than the time of imbedding. So, too, an object may be sectioned a long time after it was imbedded, and finally the sections 
may not be mounted at the time they are cut. It would be well in such cases to give the date of fixing under 2 , and under 5,6 and 8 , the dates at which the operations were performed if they differ from the original date and from one another. In brief, the more that is known about a preparation the greater its value.

\section{General Formula for Cataloging $\mathrm{Mi}$ -} croscopical Preparations :

I. The general name and source. Thickness of cover glass and of section.

2. The number of the preparation and the date of obtaining and fixing the specimen; the name of the preparator.

3. The special name of the preparation and the common and scientific name of the object from which it is derived. Purpose of the preparation.

4. The age and condition of the object from which the preparation is derived. Condition of rest or activity; fasting or full fed at the time of death.

5. The chemical treatment, - the method of fixing, hardening, dissociating, etc., and the time required.

6. The mechanical treatment,-imbedded, sectioned, dissected with needles, etc. Date at which done.

7. The staining agent or agents and the time required for staining.

8. Dehydrating and clearing agent, mounting medium, cement used for sealing.

9. The objectives and other accessories (micro-spectroscope, polarizer, etc.,) for studying the preparation.

Io. Remarks, including references to original papers, or to good figures and descriptions in books.
A Catalog Card Written According to this Formula :

\section{Muscular Fibers, Cat.}

C. 15 .

Fibers 20 to $40 \mu$ thick.

2. No. 475. (Drr. IX) Oct. I, I89I. S. H. G., Preparator.

3. Tendinous and intra-muscular terminations of striated muscular fibres from the Sartorius of the cat (Felis domestica).

4. Cat eight months old, heaithy and well nourished. Fasting and quiet for I 2 hours.

5. Muscle pinned on cork with vaselined pins and placed in 20 per cent. nitric acid immediately after death by chloroform. Left 36 hours in the acid; temperature $20^{\circ} \mathrm{C}$. In alum water $(\pi / 2$ sat. aq. sol. ) I day.

6. Fibers separated on the slide with needles, Oct. 3 .

7. Stained 5 minutes with Delafield's hematoxylin.

8. Dehydrated with $95 \%$ alcohol 5 minutes, cleared 5 minutes with carbolturpentine, mounted in xylene balsam ; sealed with shellac.

9. Use a $16 \mathrm{~mm}$. for the general appearance of the fibers, then a 2 or $3 \mathrm{~mm}$. objective for the details of structure. Try the micro-polariscope ( $\&$ 2I 8 ).

Io. The nuclei or muscle corpuscles are very large and numerous; many of the intra-muscular ends are branched. See S. P. Gage, Proc. Amer. Micr. Sci., I89o, p. I32; Ref. Hand-book Med., Sci., Vol. V., p. 59.

\%310. General Remarks cn Catalogs and Labels.-It is especially desirable that labels and catalogs shall be written with some imperishable ink. Some form of water-proof carbon ink is the most available and satisfactory. The water-proof India ink, or the engrossing carbon ink of Higgins, answers well. As purchased, the last is too thick for ordinary writing and should be diluted with one-third its volume of water and a few drops of strong ammonia added. 
If one has a writing diamond it is a good plan to write a label with it on one end of the slide. It is best to have the paper label also, as it can be more easily read.

FIG. I64. Writing diamond for writing numbers and labels on glass slides, cutting cover-glasses, etc. (Queen and Co.)

The author has found stiff cards, $12 \mathrm{I} / 2 \times 7 \mathrm{I} / 2 \mathrm{~cm}$., like those used for cataloging books in public libraries, the most desirable form of catalog. A specimen that is for any cause discarded has its catalog card destroyed. New cards may then be added in alphabetical order as the preparations are made. In fact a catalog on cards has all the flexibility and advantages of the slip system of notes (see Wilder \& Gage, p. 45).

Some workers prefer a book catalog. Very excellent book catalogs have been devised by Alling and by Ward (Jour. Roy. Micr. Soc., I887, pp. I73, 348 ; Amer. Monthly Micr. Jour., I89o, p. 9I ; Amer. Micr. Soc. Proc., I887, p. 233).

The fourth division has been added as there is coming to be a strong belief, practically amounting to a certainty, that there is a different structural appearance in many if not all of the tissue elements depending upon the age of the animal, upon its condition of rest or fatigue ; and for the cells of the digestive organs, whether the animal is fasting or full fed. Indeed as physiological histology is recognized as the only true histology, there will be an effort to determine exact data concerning the animal from which the tissues are derived. (See Minot, Proc. Amer. Assoc. Adv. Science, I89o, pp. 27I-289; Hodge, on nerve cells in rest and fatigue, Jour. Morph., vol. VII. (IS92), pp. 95-I68; Jour. Physiol., vol. XVII., pp. I29-I34; Gage, The processes of life revealed by the microscope; a plea for physiological histology, Proc. Amer. Micr. Soc., vol. XVII. (I895), pp. 3-29; Science, vol. II., Aug. 23, I895, pp. 209-218.

\section{CABINET FOR MICROSCOPICAI, PRE,PARATIONS}

8 3II. While it is desirable that microscopical preparations should be properly labeled and cataloged, it is equally important that they should be protected from injury. During the last few years several forms of cabinets or slide holders have been derised. Some are very cheap and convenient where one has but a few slides. For a laboratory or for a private collection where the slides are numerous the following characters seem to the writer essential :

(I). The cabinet should allow the slides to lie flat, and exclude dust and light.

(2). Each slide or pair of slides should be in a separate compartment. At each end of the compartment should be a groove or bevel, so that upon depressing either end of the slide the other may be easily grasped (Fig. I65). It is also desirable to have the floor of the compartment grooved so that the slide rests only on two edges, thus preventing soiling the slide opposite the object.

(3). Each compartment or each space sufficient to contain one slide of the standard size should be numbered, preferably at each end. If the compartments are made of sufficient width to receive two slides, then the double slides so frequently used in mounting serial sections may be put into the cabinet in any place desired. 
FIG. I65. A part of a cabinet drawer seen from above. In compartment No. 96 is represented a slide lying flat. The label of the slide and the number of the compartment are so placed that the number of the compartment may be seen through the slide. The sealing cement is removed at one place to show that in sealing the cover-glass, the cement is put partly on the cover and partly on the slide (\& 249, 253).

B.-This represents a section of the same part of the drawer. (a) Slide resting as in a. No. 96. The preparation is seen to be above a groove in the floor of the compartment. (b) One end of the slide is seen to be uplifted by depressing the other into the bevel.

(4). The drawers of the cabinet should be entirely independent, so that any drawer may be partly or wholly removed without disturbing any of the others.

(5). On the front of each drawer should be the number of the drawer in Roman numerals, and the number of the first and last com-

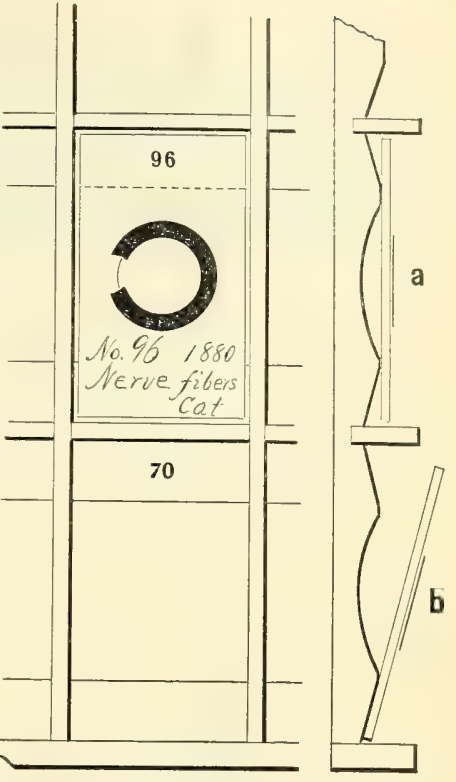

FIG. 165. partment in the drawer in Arabic numerals (Fig. I66).

FIG. I66. Cabinet for Microscopical Specimens, showing the method of arrange. ment and of numbering the drawers and indicating the number of the first and last compartment in each drawer. It is better to have the slides on which the drawers rest somewhat shorter, then the drawer front may be entire and not notched as here shown. (From. Proc. Amer. Micr. Soc., I883).

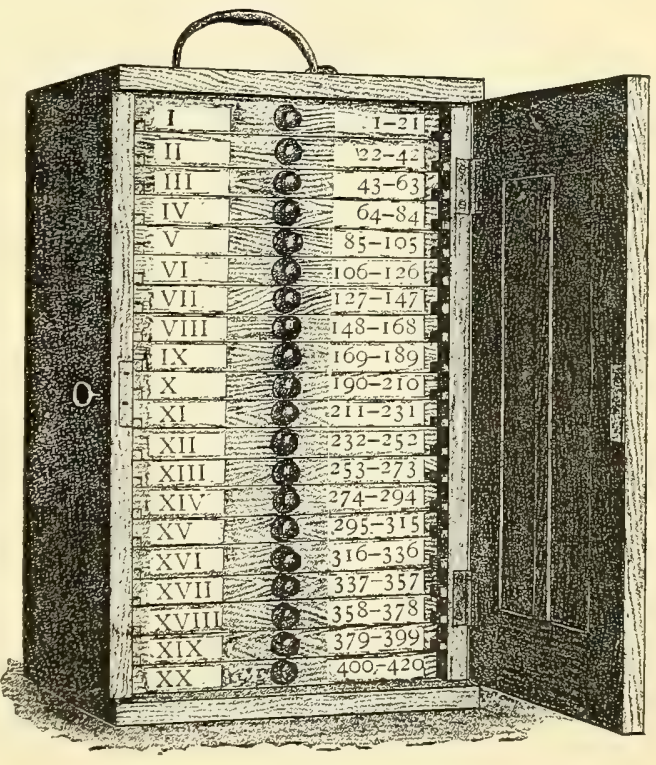




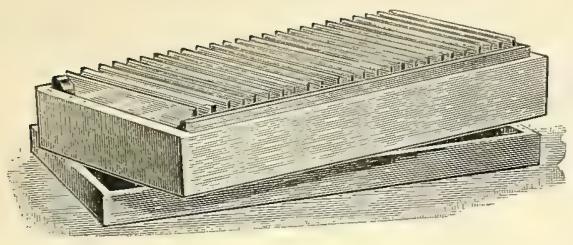

FIG. I67. Slide box for 25 specimens. These are cheap and convenient and may be stood on end like books, thus placing the slides in a horizontal position. Smaller boxes, i. e. for 3,6 and 12 slides are also made, and mailing boxes for a single slide (Bausch $\mathcal{G}$ Lomb Co.).

SOME REAGENTS FOR FIXING, MOUNTING, ETC.

\& 3I2. Albumen Fixative (Mayer's).-This consists of equal parts of wellbeaten white of egg and glycerin. To each $50 \mathrm{cc}$. of this I gram of salicylate of soda is added to prevent putrefactive changes. This must be carefully filtered. For method of use see \&. 290.

\& 313. Alcohol (Ethylic).-Ethyl alcohol is mostly used for histological purposes. (A) absolute alcohol ( $i$. e., alcohol of $\frac{99}{100} \%$ ) is recommended for many purposes, but if plenty of $95 \%$ alcohol is used it answers every purpose in histology, in a dry climate or in a warm, dry room. When it is damp dehydration is greatly facilitated by the use of absolute alcohol.

(B) $82 \%$ alcohol made by mixing 5 parts of $95 \%$ alcohol with I part of water.

(C) $67 \%$ alcohol made by mixing 2 parts of $95 \%$ alcohol with I part of water.

Grades of Alcohol. It has been found by careful tests that quite accurate percentages of alcohol may be obtained by mixing water and alcohol as follows: Pour alcohol into a graduate until the volume of alcohol corresponds to the desired percentage. Add water until the volume in cubic centimeters corresponds to the original percentage of the alcohol used. For example, to get $67 \%$ from $95 \%$ alcohol, pour $67 \mathrm{cc}$. of $95 \%$ into a graduate, and add sufficient water to bring the volume up to $95 \mathrm{cc}$. Far $50 \%$ alcohol from $75 \%$, put $50 \mathrm{cc}$. of $75 \%$ alcohol in a graduate, add sufficient water to make the volume $75 \mathrm{cc}$. From the change in volume it does not answer to mix given volumes of water and alcohol in these cases. In the first case, if one mixed $75 \mathrm{cc}$. of $95 \%$ alcohol and $20 \mathrm{cc}$. of water the resulting mixture would be over $75 \%$; but if sufficient water is added to bring the volume back to the original percentage more than $20 \mathrm{cc}$. of water is added, that is enough more to compenate for the shrinkage, and the result is approximately accurate.

Methyl Alcohol is much cheaper than ethyl, and answers well in most microscopical processes. It has recently been so carefully refined that the disagreeable odor is very little noticeable.

Synthol. This is said to be a synthetic form of alcohol. It seems to serve the purpose of alcohol in histology. Absolute synthol is far cheaper for the private worker than absolute ethyl alcohol.

8.314. Alum Solution.-For muscle dissociated in nitric acid use a saturated solution (i.e., a solution in which the water holds all the alum it can. If one adds an excess so that there will always be some undissolved alum in the vessel he can be sure the solution is saturated after it has stood a few days. An easy way. to get a saturated solution is to take $500 \mathrm{cc}$. of water and add Ioo grams of alum and heat the water in an agate dish. All the alum will be melted, but on 
cooling a part will crystallize out, leaving a cold saturated solution). The saturated solution may be used but, if a half saturated solution is employed, it will answer all the purposes. For a half saturated solution take Ioo cc. of water, and IoO cc. of saturated alum water and mix the two. In case preparations are to be kept some time in alum water, $2 \%$ of chloral hydrate should be added to prevent mold.

8. 3r5. Balsam, Canada Balsam, Balsam of Fir; Xylene Balsam.-This is one of the oldest and most satisfactory of the resinous media used for mounting microscopical preparations.

The natural balsam is most often used, but within the last ten years the belief has arisen that it is better to evaporate the balsam and then dissolve it in xylene or benzole. It certainly dries out more rapidly if so treated. Natural balsam has the advantage of being able to take up a small amount of water so that if sections are not quite dehydrated they will clear up after a time. If xylene balsam is used the dehydration must be almost complete or the preparation will look cloudy.

Filtering Balsam. Balsam is now furnished already filtered through filter paper. If one wishes to filter it himself a hot filter like that shown in Fig. I55 is good. If xylene balsam is used it may be made thin and filtered without heat. For filtering balsam and all resinous and gummy materials, the writer has found a paper funnel the most satisfactory. It can be used once and then thrown away. Such a funnel may be easily made by rolling a sheet of thick writing paper in the form of a cone and cementing the paper where it overlaps, or winding a string several times around the lower part. Such a funnel is best used in one of the rings for holding funnels.

FIG. I68. Vessel for homogeneous immersion liquid (thick cedar-wood oil). This is filled only a little above the lower end of the inner tube. The oil will not then run out if the vessel is tipped over. For applying the oil there is a wire loop attached to the upper cork. The side cork is for the purpose of emptying the bottle, and also for the escape of the air when fulling it. (The Spencer Lens Co.; see also Zeit. wiss. Mikr., 1897, p. 348, and Jour. Roy. Micr. Soc., I898, p. 238).

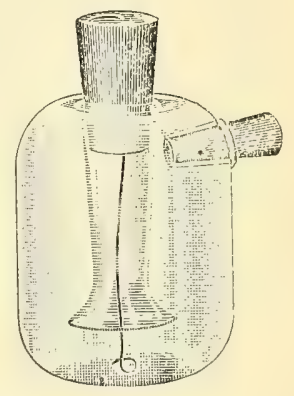

Natural Balsam. All the samples of balsam tested by the author have been found slightly acid. This is an advantage for carmine, and acid fuchsin stains or any other acid stain. Also for preparations injected with carmine or Berlin blue. In these cases the color would fade or diffuse if the medium were not slightly acid. For hematoxylin the acid is detrimental. For example, the slight amount of acid in the balsam will cause the delicate stain in the finest fibers of Weigert preparations to fade. Also the fuchsin and other stains which are faded by acids. To neutralize the balsam add some pure sodium carbonate, set the balsam in a warm place and shake it occassionally. After a month or so the soda will settle and the clear supernatant balsam will be found very slightly alkaline. Use this whenever an acid medium would fade the stain in the specimen. 
\& 3I6. Cedar-Wood Oil,-This is used for oil immersion objectives. It is best kept in a bottle like that shown in Fig. I68. This oil is quite thick.

For penetrating tissues and preparing them for infiltration with paraffin, thick oil is recommended by Lee. The writer has found, however, that any good cedar wood oil gives excellent results in ordinary histological and embryological work. That known as Cedar Wood Oil (Florida) is excellent, also that known as cedar wood oil (true Lebanon). These forms are far less expensive than that used for immersion objectives. The tissues should be thoroughly dehydrated before putting them into cedar-wood oil, and they should remain until they are transparent ( 8 286).

\& 317. Clarifier, Castor-Xylene Clarifier.-This is composed of castor oil I part and xylene* 3 parts. (Trans. Amer. Micr. Soc., I895, p. 36I).

\& 318. Clearing Mixture (\$28I, 298).-(A). One of the most satisfactory and generally applicable clearers is carbol turpentine, made by mixing carbolic acid crystals (Acidum carbolicum. A. phenicum crystallizatum) $40 \mathrm{cc}$. with rectified oil of turpentine (Oleum terebinthinae rectuficatum) 6o cc. If the carbolic acid does not dissolve in the turpentine add $5 \mathrm{cc}$. of $95 \%$ alcohol, or increase the turpentine, thus: carbolic acid $30 \mathrm{cc}$, turpentine $70 \mathrm{cc}$.

(B). Carbol-Xylene Clearer.-Vasale recommends as a clearer, xylene $75 \mathrm{cc}$, carbolic acid (melted crystals) $25 \mathrm{cc}$. It is used in the same way as the preceding.

\& 3I9. Collodion.-This is a solution of soluble cotton $\dagger$ or other form of pyroxylin in equal parts of sulphuric ether and $95 \%$ alcohol. Two solutions are used :

*The hydrocarbon xylene $\left(\mathrm{C}_{8} \mathrm{H}_{10}\right)$ is called xylol in German. In English members of the hydrocarbon series have the termination "ene" while members of the alcohol series terminate in "ol."

†The substance used in preparing collodion goes by various names, soluble cotton or collodion cotton is perhaps best. This is cellulose nitrate, and consists of a mixture of cellulose tetranitrate $\mathrm{C}_{22} \mathrm{H}_{16}\left(\mathrm{NO}_{3}\right)_{4} \mathrm{O}_{6}$, and cellulose pentanitrate, $\mathrm{C}_{12} \mathrm{H}_{15}\left(\mathrm{NO}_{3}\right)_{5} \mathrm{O}_{5}$. Besides the names soluble and collodion cotton, it is called gun cotton and pyroxylin. Pyroxylin is the more general term and includes several of the cellulose nitrates. Celloidin is a patent preparation of pyroxylin, more expensive than soluble cotton, but in no way superior to it for imbedding.

Soluble cotton should be kept in the dark to avoid decomposition. After it is in solution this decomposition is not so liable to occur. The decomposition of the dry cotton gives rise to nitrous acid, and hence it is best to keep it in a box loosely covered so that the nitrous acid may escape.

Cellulose nitrate is explosive under concussion and when heated to $150^{\circ}$ centigrade. In the air, the loose soluble cotton burns without explosion. It is said not to injure the hand if held upon it during ignition and that it does not fire gunpowder if burned upon it. So far as known to the writer, no accident has ever occurred from the use of soluble cotton for microscopical purposes. I wish to express my thanks to Professor W. R. Orndorff, organic chemist in Cornell University, for the above information. Proc. Amer. Micr. Soc., vol. XVII (i895), pp. $36 \mathrm{I}-370$. 
(A). $6 \%$ to $8 \%$ or thick collodion. It is made by mixing $50 \mathrm{cc}$. of sulphuric ether and $50 \mathrm{cc}$. of $95 \%$ alcohol and adding 6 or 8 grams of soluble cotton. If this is shaken repeatedly the solution will be complete in a day or two.

(B). I $1 / 2 \%$ or thin collodion. To prepare this $1 \frac{1}{2}$ grams of soluble cotton are added to Ioo cc. of ether-alcohol ( 8322$)$.

\& 320. $3 / 4 \%$ collodion or cementing collodion. To prepare it $3 / 4$ ths of a gram of soluble cotton is added to alcohol, $60 \mathrm{cc}$, , ether, $40 \mathrm{cc}$.

The excess of alcohol prevents the mixture from softening the paraffin $(8290)$.

\& 321. Eosin.-This is used mostly as a contrast stain with hematoxylin, which is an almost purely nuclear stain. It serves to stain the cell-body, ground substance, etc., which would be too transparent and invisible with hematoxylin alone. If eosin is used alone it gives a decided color to the tissue and thus aids in its study ( 8 I 44 ). Eosin is used in alcoholic and in aqueous solutions. A very satisfactory stain is made as follows : $50 \mathrm{cc}$. of water and $50 \mathrm{cc}$. of $95 \%$ alcohol are mixed and I-Ioth of a gram of dry eosin added.

The eosin is used after the hematoxylin in most cases $\left(\varepsilon_{2} 280\right)$, and, as it is in alcoholic solution, it may be washed off with $95 \%$ alcohol if the object is to be mounted in balsam. If it is to be mounted in glycerin or glycerin jelly, the excess of eosin should be washed away with distilled water.

8 322. Ether, Ether-Alcohol.-Sulphuric ether is meant when ether is mentioned in this book. For the ether-alcohol mentioned in 8269,319 , etc., a mixture of equal volumes of sulphuric ether and 95\% alcohol is meant.

\&323. Farrants' Solution.-Take 25 grams of clean, dry, gum arabic; 25 cc. of a saturated aqueous solution of arsenious acid; $25 \mathrm{cc}$. of glycerin. The gum arabic is soaked for several days in the arsenic water, then the glycerin is added and carefully mixed with the dissolved or softened gum arabic.

This medium retains air bubbles with great tenacity. It is much easier to avoid than to get rid of them in mounting.

\& 324. Formaldehyde Dissociator.-This is composed of $2 \mathrm{cc}$. of a $40 \%$ solution of formaldehyde in $1000 \mathrm{cc}$. of water, to which 6 grams of common table salt (sodium chlorid) have been added. Formaldehyde as bought in the market is a $40 \%$ solution in water, and is called formol, formalin, formalose and formal, the last name being the preferable one. For its use in isolating cells see \& 26o. (Micr. Bulletin and Sci. News, vol. XII. (1895), pp. 4-5).

\& 325. Glycerin.-(A). One should procure pure glycerin for a mounting medium. It needs no preparation, except in some cases it should be filtered through filter paper or absorbent cotton to remove dust, etc.

For preparing objects for final mounting, glycerin $50 \mathrm{cc}$, water $50 \mathrm{cc}$, forms a good mixture. For many purposes the final mounting in glycerin is made in an acid medium, viz., Glycerin 99 cc., Glacial acetic or formic acid, I cc.

By extreme care in mounting and by occasionally adding a fresh coat to the sealing of the cover-glass, glycerin preparations last a long time. They are liable to be disappointing, however. In mounting in glycerin care should be taken to avoid air-bubbles, as they are difficult to get rid of. A specimen need not be discarded, however, unless the air-bubbles are large and numerous.

\& 326. Glycerin Jelly.-Soak 25 grams of the best dry gelatin in cold water in a small agate-ware dish. Allow the water to remain until the gelatin is softened. It usually takes about half an hour. When the gelatin is softened, as may be 
readily determined by taking a little in the fingers, pour off the superfluous water and drain weil to get rid of all the water that has not been imbibed by the gelatin. Warm the softened gelatin over a water bath and it will melt in the water it has absorbed. Add to the melted gelatin about $5 \mathrm{cc}$. of egg albumen, white of egg ; stir it well and then heat the gelatin in the water bath for about half an hour. Do not heat above $75^{\circ}$ or $80^{\circ} \mathrm{C}$., for if the gelatin is heated too hot it will be transformed into meta-gelatin and will not set when cold. The heat will coagulate the albumen and form a kind of floculent precipitate which seems to gather all fine particles of dust, etc., leaving the gelatin perfectly clear. After the gelatin is clarified it should be filtered through a hot flannel filter and mixed with an equal volume of glycerin and 5 grams of chloral hydrate and shaken thoroughly. If it is allowed to remain in a warm place $\left(i . e_{3}\right.$, in a place where the gelatin remains melted) the air-bubbles will rise and disappear.

In case the glycerin jelly remains fluid or semi-fluid at the ordinary temperature $\left(18^{\circ}-20^{\circ} \mathrm{C}\right)$, the gelatin has either been transformed into meta-gelatin by too high a temperature or it contains too much water. The amount of water may be lessened by heating at a moderate temperature over a waterbath in an open vessel. This is a very excellent mounting medium. Air-bubbles should be avoided in mounting as they do not disappear.

8 327. Chloral Hematoxylin.-Hematoxylin is one of the most useful stains employed in histology. An excellent solution for ordinary section staining may be made as follows: Distilled water $200 \mathrm{cc}$, and potash alum $7 \frac{1}{2}$ grams, are boiled together for 5 minutes, in an agate-ware or glass vessel, and sufficient boiled water added to bring the water back to $200 \mathrm{cc}$. After the mixture is cool, 4 grams of chloral hydrate, and $\frac{2}{10}$ ths grams of hematoxylin crystals, previously dissolved in $20 \mathrm{cc}$. of $95 \%$ alcohol, are added. The boiling seems to destroy any fungi present in the alum or water, and the chloral prevents the development of any that may get in afterward, and this solution therefore is quite permanent.

At first the color will be rather faint, but after a week or two it will become a a deep purple. The deepening of the color is more rapid if the bottle is left uncorked in the light and is shaken occasionally. It may be prepared for work at once by the addition of a small amount of hydrogen dioxid $\left(\mathrm{H}_{2} \mathrm{O}_{2}\right)$.

If the stain is too concentrated it may be diluted with freshly distilled water or with a mixture of water, alum and chloral. If the stain is not sufficiently concentrated, more hematoxylin may be added. With hematoxylin of the strength given in the formula, sections are usually sufficiently stained in from one to five minutes.

As may be inferred from what was said above, the boiling is to destroy any living ferments present in the water or alum, and the chloral hydrate is to prevent the development of germs which accidentally reach the solution after it is made.

No precaution is necessary in using this stain for sections, except that applicable to all hematoxylin solutions, viz: It must be filtered occasionally and after staining the surplus stain must be very thoroughly washed away with water; otherwise black granules or needles will appear in or upon the sections. If granules appear in the preparations in spite of the washing, it will be well to boil the solution three to five minutes and filter through paper or absorbent cotton. The addition of one or two per cent. of chloral after the boiling is also advantageous. This stain has not been tried for dyeing in bulk. Other substances than chloral 
were tried, but not with so good success. (Proc. Amer. Micr. Soc, I892, pp. I25-127),

8 328. Hematein. This is used instead of hematoxylin, as it is believed to give more satisfactory results. Prepare as follows: Prepare a 5\% solution of potash alum in distiller water and boil or leave in a steam steralizer an hour or two. While this alum solution is warm add I per cent. of hematein dissolved in a small quantity of alcohol. After the fluid has cooled add 2 grams of chloral for each I00 cc. of solution. (Freeborn, Jour. Ap. Micr., I900, p. I056.)

\& 329. Liquid Gelatin.-Gelatin or clear glue, 75 to 100 grams. Commercial acetic acid (No. 8) Ioo cc., water Ioo cc., or glacial acetic acid $40 \mathrm{cc}$. and water I60 cc., 95\% alcohol Ioo cc., glycerin I 5 to $30 \mathrm{cc}$. Crush the glue and put in into a bottle with the acid, and set in a warm place, and shake occasionally. After three or more days add the other ingredients. This solution is excellent for fastening paper to glass, wood or paper. The brush must be mounted in a quill or wooden handle. For labels, it is best to use linen paper of moderate thickness. This should be coated with the liquid gelatin and allowed to dry. The labels may be cut of any desired size and attached by simply moistening them, as in using postage stamps.

Very excellent blank labels are now furnished by dealers in microscopical supplies, so that it is unnecessary to prepare them one's self, except for special purposes Those like that shown in Fig. I 63 may be had for about $\$ 4$ for 10,000.

3. 330. Nitric Acid Dissociator.-This is prepared by mixing $\delta$ o cc. of water with $20 \mathrm{cc}$. of strong nitric acid. It is used mostly in dissolving the connective tissue of muscle and thus making it possible to separate the fibers. Alum water is used as a restrainer ( $\& 3$ I $_{4}$ and 264). (Proc. Amer. Micr. Soc., Vol. XI, ( IS89), pp. 34-45).

331. Normal Salt Solution or Saline Solution.-Pure water from its differing density from the natural lymph acts injuriously on the tissues. The addition of a little table salt, however, prevents this deleterious action, or greatly lessens it, hence the name of nomal salt solution. It is a $\frac{6}{10} \%$ solution of table salt (sodium chlorid) in water; water $1000 \mathrm{cc}$., salt 6 grams, or water $100 \mathrm{cc}$., salt $\frac{6}{10}$ gram.

\& 332. Paraffin. Paraffin is of various melting points, hence at the ordinary temperature of a laboratory, that melting at the lowest temperature will be moderately soft, hence soft paraffin, while that melting at a higher temperature will be hard. For the best results one usually has to mix hard and soft paraffins. The larger the object to be cut and the thicker the sections the softer should be the paraffin.

533. Picric-Alcohol.-This is an excellent hardener and fixer for almost all tissues and organs. It is composed of $500 \mathrm{cc}$. of water and $500 \mathrm{cc}$. of $95 \%$ alcohol, to which 2 grams of picric acid have been added. (It is a $\frac{1}{5} \%$ solution of picric acid in 50\% alcohol). It acts quickly, in from one to three days. $(\& 267,284)$. (Proc. Amer. Micr. Soc., Vol. XII (I890), pp. I20-I22).

334. Shellac Cement. - Shellac cement for sealing preparations and for making shallow cells $(\% 249)$ is prepared by adding scale or bleached shellac to $95 \%$ alcohol. The bottle should be filled about half full of the solid shellac then enough 95\% alcohol added to fill the bottle nearly full. The bottle is shaken occasionally and then allowed to stand until a clear stratum of liquid appears on the top. This clear, supernatant liquid is then filtered through absorbent cotton, using a paper 
funnel ( $\& 3$ I5), into an open dish or a wide-mouth bottle. To every Ioo cc. of this filtered shellac, $2 \mathrm{cc}$. of castor oil and $2 \mathrm{cc}$. of Venetian turpentine are added to render the shellac less brittle. The filtered shellac will be too thin, and must be allowed to evaporate till it is of the consistency of thin syrup. It is then put into a capped bottle, and for use, into a small spirit lamp (Fig, I53). In case the cement gets too thick add a small amount of $95 \%$ alcohol or some thin shellac. The solution of shellac almost always remains muddy, and in most cases it takes a very long time for the flocculent substance to settle. One can very quickly obtain a clear solution as follows: When the shellac has had time to thoroughly dissolve, $i . e$, in a week or two in a warm place, or in less time if the bottle is frequently shaken, a part of the dissolved shellac is poured into a bottle and about one-fourth as much gasolin or benzin added and the two well shaken. After twenty-four hours or so the flocculent, undissolved substance will separate from the shellac solution and rise with the gasolin to the top. The clear solution may then be siphoned off or drawn off from the bottom if one has an aspirating bottle. (R. Hitchcock, Amer. Monthly Micr. Jour., July, I884, p. I3I).

If one desires to color the shellac, the addition of a strong alcoholic solution of some of the coal tar colors is good, but it is liable to dissolve in the mounting medium when shellac is used for sealing. A small amount of lampblack well rubbed up in very thin shellac and filtered, is good to darken the shellac.

\section{ARRANGING AND MOUNTING MINUTE OBJECTS}

8. 335. Minute objects like diatoms or the scales of insects may be arranged in geometrical figures or in some fanciful way, either for ornament or more satisfactory study. To do this the cover-glass is placed over the guide. This guide for geometrical figures may be a net-micrometer or a series of concentric circles. In order that the objects may remain in place, however, they must be fastened to the cover-glass. As an adhesive substance, mucilage or liquid gelatin ( 8329$)$ thinned with an equal volume of 50\% acetic acid answers well. A very thin coating of this is spread on the cover with a needle, or in some other way and allowed to dry. The objects are then placed on the gelatinized side of the cover and carefully got into position with a mechanical finger, made by fastening a cat's whisker in a needle holder. For most of these objects a simple microscope with stand (Figs. 20, 145, 146) will be found of great advantage. After the objects are arranged, one breathes very gently on the cover-glass to soften the mucilage or gelatin. It is then allowed to dry and if a suitable amount of gelatin has been used, and it has been properly moistened, the objects will be found firmly anchored. In mounting one may use Canada balsam or mount dry on a cell $(\xi 247,255)$. See Newcomer, Amer. Micr. Soc.'s Proc., I886, p. I 28 ; see also E. H. Griffth and H. L. Smith, Amer. Jour. of Micros., iv, I02, v, S7; Amer. Monthly Micr. Jour., i, 66. I07, II3. Cunningham, The Microscope, viii, I888, p. 237.

For additional apparatus for this chapter, see Ch. X. 


\title{
CHAPTER VIII
}

\author{
PHOTOGRAPHING OBJECTS WITH A VERTICAL CAMERA ; \\ PHOTOGRAPHING LARGE TRANSPARENT OBJECTS ; \\ PHOTOGRAPHING WITH A MICROSCOPE: (A) TRANS- \\ PARENT OBJECTS; (B) OPAQUE OBJECTS AND THE \\ SURFACES OF METALS AND ALLOYS*
}

\section{APPARATUS AND MATERIAL FOR THIS CHAPTER}

Tertical camera with photographic objectives (Fig. 169), small vertical camera with special microscope stand for embryos, etc. (Figs. I83-I84); arrangement of camera for large transparent objects (Fig. ISI); photo-micrographic cameras (Figs. IS 3 -IS4, I92); photographic objectives for gross and microscopic work (Figs. I7OI7I, I76-ISo); microscope, microscopic objectives and projection oculars (Figs. I85, IS9, I93); color screens, lamps, dry plates and the chemicals and apparatus necessary for developing, printing, etc.

3. 336. Nothing would seem more natural than that the camera, armed with a photographic objective or with a microscopic objective, should be called into the service of science to delineate with all their complexity of detail, the myriads of forms studied.

For photographing many objects in nature the camera remains horizontal or approximately so, but for a great many of those studied in botany, zoology, mineralogy and in anatomy the specimens cannot be put in a vertical position necessary for a horizontal camera. This difficulty has been overcome by using a mirror or a 45 -degree prism. These are practically alike and have the defect of producing a picture with the inversion of a plane mirror.

\section{VERTICAL CAMERA}

3.337. To meet all the difficulties the object may be left in a horizontal position and the camera made vertical (fig. I69).

For the last twenty-five years such a camera has been in use in the Anatomical Department of Cornell University for photographing all kinds of specimens;

*Papers on this subject were given by the writer at the meeting of the American Association for the Advancement of Science in 1879 , and at the meeting of the Society of Naturalists of the eastern United States in $\mathrm{IS8}_{3}$; and in Science Vol. III, pp. 443, 444. 
among these, fresh brains, and hardened brains have been photographed without the slightest injury to them. Furthermore, as many specimens are so delicate that they will not support their own weight, they may be photographed under alcohol or water with a vertical camera and the result will be satisfactory as a photograph and harmless to the specimen.

A great field is also open for obtaining life-like portraits of water animals. Freshly killed or etherized animals are put into a vessel of water with a contrasting back gruund and arranged as desired, then photographed. The fins have something of their natural appearance and the gills of branchiate salamanders float out in the water in a natural way. In case the fish tends to float in the water a little mercury injected into the abdomen or intestine will serve as ballast.

The photographs obtainable in water are aimost if not quite as sharp as those made in air. Even the corrugations on the scales of such fishes as the sucker (Catostomus teres) show with great clearness. Indeed so good are the results that excellent half tone plates may be produced from the pictures thus made, also excellent photogravures. In those cases, as in anatomical preparations, where the photograph rarely answers the requirements of a scientific figure, still a photograph serves as a most admirable basis for such a figure. The photograph is made of the desired size and all the parts are in correct proportion and in the correct relative position. From this photographic picture may be traced all the outlines upon the drawing paper, and the artist can devote his whole time and energy to giving the proper expression without the tedious labor of making measurements.

"While the use of photography for outlines as bases for figures diminishes the labor of artists about one-half it increases that of the preparator ; and herein lies one of its chief merits. The photographs being exact images of the preparations, the tendency will be to make them with greater care and delicacy, and the result will be less imagination and more reality in published scientific figures; and the objects prepared with such care will be preserved for future reference."

"In the use of photography for figures several considerations arise: (I) The avoidance of distortion; (2) The adjustment of the camera to obtain an image of the desired size ; (3) Focusing ; (4) Lighting and centering the object.

(I). While the camera delineates rapidly, the image is liable to distortion. I believe opticians are agreed, that, in order to obtain correct photographic images, the objective must be properly made, and the plane of the object must be parallel to the plane of the ground glass. Furthermore, as most of the objects in natural history have not plane surfaces, but are situated in several planes at different levels, the whole object may be made distinct by using in the objective a diaphragm with a small opening.

\%33\$. Scale of Sizes and Focusing.-(2). By placing the camera on a long table, and a scale of some kind against the wall, the exact position of the ground glass for various sizes may be determined once for all, and these positions noted in some way.

FIG. I69. Vertical Camera for photographing objects in a horizontal position. The camera is attached to a double frame connected by bent metal pieces fastened to the lower and sliding in a groove in the upper frame. The two frames can then slide over each other without separating. For moving the outer frame a rack work is put on the lower or inner frame and a pinion with a toothed wheel on the outer one. This is tumed by the wheel shown. To prevent the camera run- 
ning down in the vertical position a pazel is held in place by a spring. This may be released by a smaller wheel than that serving to move the pinion. This rack and pinion are fine enough for focusing with the photographic objectives employed.

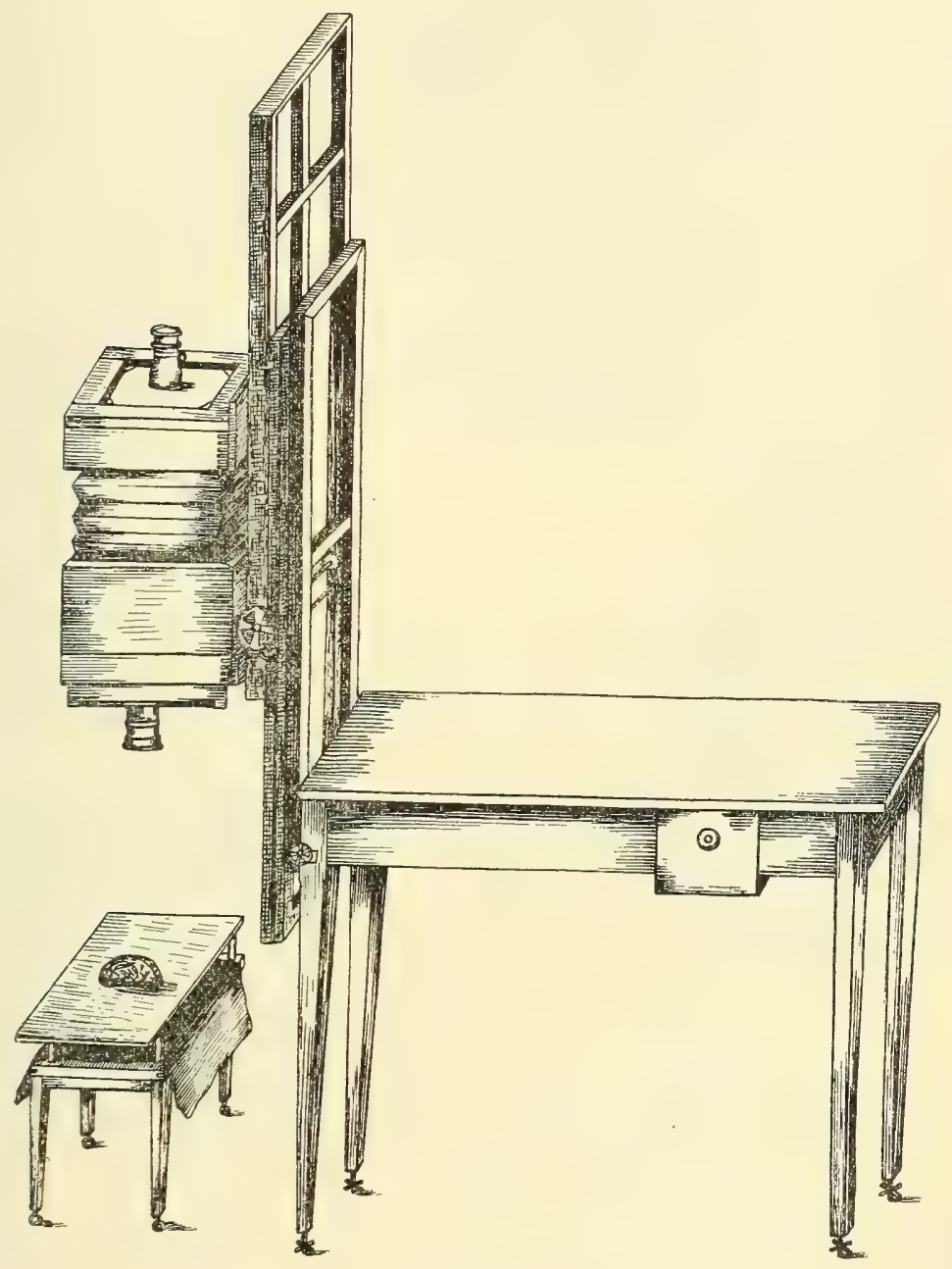

FIG. 169 .

The camera bed is graduated in centimeters so that the exact extent of the bellows can be determined by inspection.

The support on which the specimen rests is of heavy glass on vertical rods about to centimers long. The background is placed on the table top about Io $\mathrm{cm}$. below. This arrangement of support and background serves to avoid the dense shadows which make it diffcult to determine exactly the limits of the specimen. To make the apparatus steady the right hand end of the table is heavily weighted. The tables have leveling screws in the legs. 


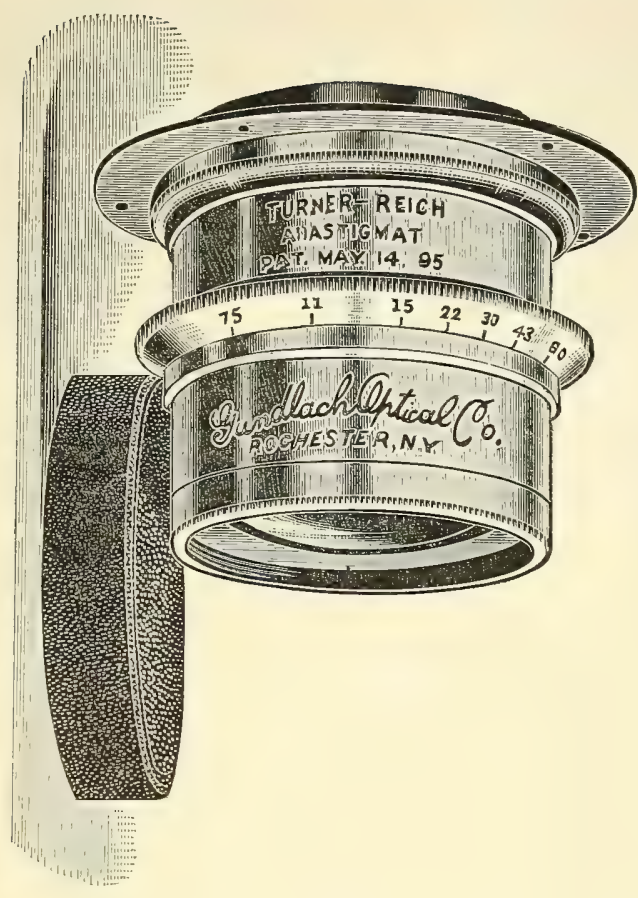

Fig. I70. Turner-Reich anastigmat objective for photography, (Gundlach Opt. Co.)

In the camera here figured, the camera bed is ruled in centimeters so that the position of the ground glass can be determined with accuracy and noted. It takes but a moment to set the ground glass or focusing screen at the right level to give any desired size. In practice it is convenient to have attached to the camera a table giving the position of the ground glass for various sizes, and also the distance of the objective from the object in each case. By having this information it takes but a moment to set the camera and to place it so that it will be approximately in

FIG. I70.

FIG. I7 I. Zeiss anastigmat objective for photography, (Bausch \& Lomb Optical Co.)

focus. The final focusing is then accomplished by the use of the rack and pinion movement. It is an advantage to use a focusing glass and a clear focusing screen or the transparent part of the ordinary screen (Fig. I74), for the final focusing. As many objects have not sharp details which one can focus on, it is helpful to

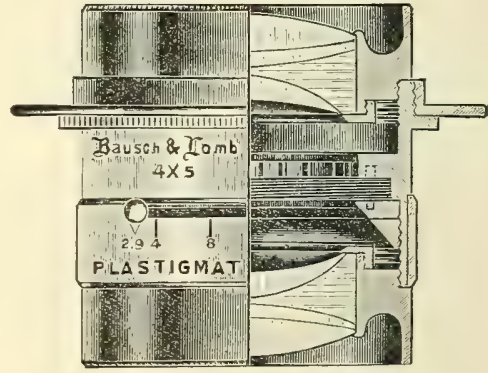

FIG. I7I. place some printed letters on the part to be brought out with the greatest sharpness. Of course these are removed before the exposure is made.

3 339. In lighting the object one should take pains to so arrange it with reference to the light that the details will show with the greatest clearness. Naturally for the vertical camera the light will come from the side and not from a skylight, although good results are obtained with a skylight if one so places the camera that it does not cast objectionable shadows. 
FIG. I72. Tripod magnifier as focusing glass. This is carefully focused on a scratch or pencil mark on the lower or ground surface of the focusing screen. Then whenever the object is sharply focused the focal plane will be at the level of sensitive surface.

As shown in Fig. I69, the object is placed upon a glass support and the background is quite a distance below the support. For a dark object the background should be light, and for a light one dark. Black velveteen is excellent for a back-ground. The advantage of the glass support is that the shadows in the

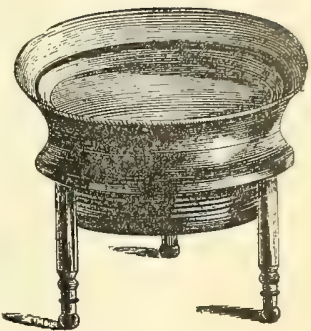

FIG. I72. background which often make it difficult to tell just where the specimen ends and the background begins, is wholly done away with, and that too without at all affecting the proper light and shade of the object itself. (Method of W. E. Rumsey, Canadian Entomologist I896, p. 84).

Fig. I73. Focusing Glass. "It is achromatic, consisting of a double convex crown lens and a negative meniscus flint lens cemented together." It screws into the brass tube and is thus adjustable, enabling one to focus the pencil mark in the clear area of the focusing screen (Fig. I74) with great accuracy. It also serves to focus the image with ease and accuracy. The eye must not be too close to the upper end of the focusing glass or the field will be restricted. (Gundlach Opt. Co.)

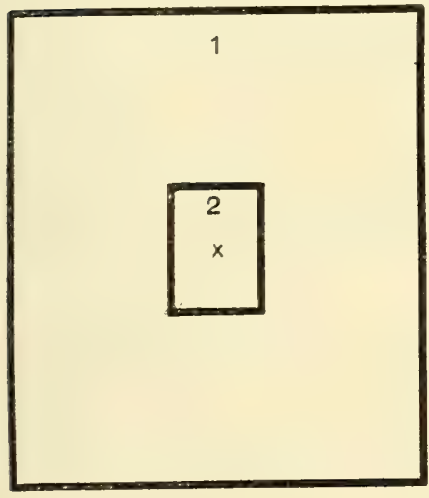

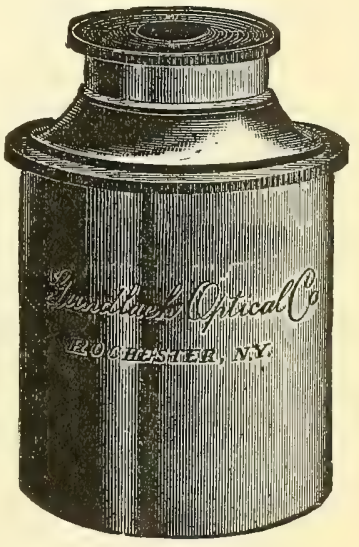

FIG. I73.

FIG. I74. Ground-glass focusing screen with central transparent area for exact focusing with a focusing glass when one does not possess a clear focusing screen. (I) The ground surface; (2) Central part with oblong cover-glass and Canada balsam on the ground surface to render it transparent. $X$. The central point in the entire focusing screen. It is made with a black lead pencil on the ground surface. The focusing glass is focused on this cross, then when the image is in focus it will be at the level of the sensitive coating of the plate. 


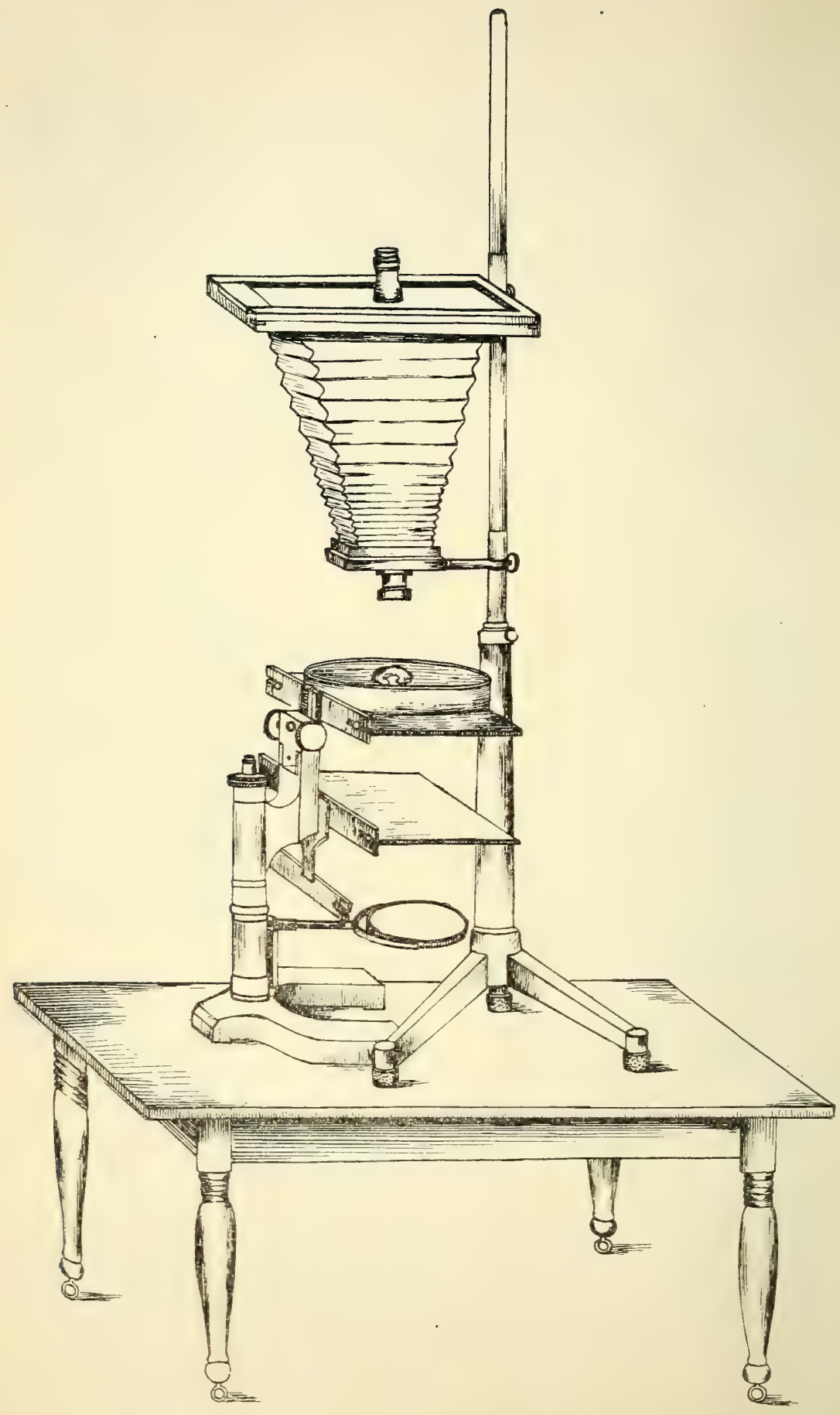


FIG. I75. Vertical Camera and special microscope stand for photographing. embryos and other small specimens in liquids and for photographing large sections. The camera rests on a low table and the operator can stand on the floor while performing all the operations.

The stage of the microscope is attached to the arm in the place of the tube. This stage has two stories. The specimen is shown on the upper and the background on the lower story.

In focusing, the coarse and fine adjustment of the special microscope stand are used. The large mirror is to illuminate embryo chicks mounted entire, and other large transparent preparations. Trans. Amer. Micr. Igor.

\% 340. Prints. - If the photographic prints are to be used solely for outlines, the well-known blue prints so much used in engineering and architecture may be made. If, however, light and shade and fine details are to be brought out with great distinctness, either an aristotype, platinotype or a bromide print is preferable.

$\S 34$. Recording, Storing and Labeling Negatives.- In order to get the greatest benefit from past experience it is necessary to make the results available by means of a careful record. For this purpose the table $(\$ 360)$ has been prepared. If one gives the information called for in this table, whether the result is successful or not, one can after a time work with great exactness, for the elements of success and failure will stand out clearly in the table.

$\$ 342$. Labeling the Negatives.-After the negative is dry the labeling can be done on the gelatin side with carbon ink. Enough data should be given to enable the certain identification of the negative at any future time.

$\$ 343$. Storing Negatives.-This is satisfactorily done by putting each into an envelope and writing a duplicate label on the upper edge, and then the negatives may be placed in drawers in alphabetical order as are the catalog cards of books in a library. One can then find any negative with the same facility that the title of a book can be found in a card catalog.

PHOTOGRAPHING EMBRYOS

For photographing embryos and many other small specimens it is more convenient to use a smaller apparatus than the vertical camera just described. It is necessary also to have a more delicate method of focusing.

344. Camera for Embryos.-This is a vertical camera for photographing with the microscope, with the photographic objective in the end of the camera as for an ordinary camera. This is readily accomplished by having a society screw adapter, and also adapters for the micro-planars or other objectives which one desires to use. The 
magnification usually required varies from natural size $\left(X_{I}\right)$ to five times natural size $\left(\times_{5}\right)$ up to $\times 20$. As with the large camera the position of the ground glass for each magnification and for each objective is determined once for all by using a scale in millimeters. The various positions are accurately noted, then one can set the camera almost instantly for the desired magnification. The supporting rod is divided to half centimeters and therefore the exact position is easily recorded (Fig. I83).

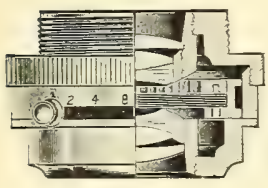

345. Special Microscope Stand.-For the accurate focusing necessary for embryos one should possess a special microscope stand with the stage in two or three stories and attached to the arm in place of the tube of the microscope. The stage proper is absent. This arrangement of the stage permits the use of the coarse and fine adjustment of the microscope to be used for focusing. The position of the camera on a low table ( 45 to $50 \mathrm{~cm}$. high) makes it possible for the operator to stand on the floor while making all the adjustments of the the embryo and for focusing; and all the parts are within reach (Fig. 175).

$\$ 3+6$. Arranging the Embryos. - As usually prepared the embryos are white and therefore require a dark background. This may be attained either by placing the embryos in a dark dish or on some paper blackened with water-proof India ink, or by putting them in a glass ressel like a Petri dish, and a piece of black velveteen on the stage below. The specimens will of course be in a liquid, usually alcohol.

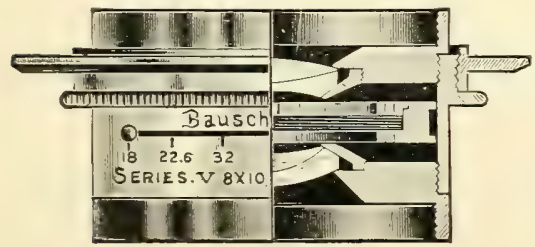

FIG. I77. Wide angle anastigmat objective for photographing at low magnification (Bausch \& Lomb Optical Co.).

If several embryos are to be taken at once, the embryos are arranged in rows something as the words on a line. Arrange them in even rertical as well as horizontal rows so that when the print is made it will be easy to cut them apart. When the embryos are ar- 
ranged, one should be certain that the light brings out the details most desired. For example, if one is photographing an embryo which shows the branchial pockets well, great pains should be taken to so arrange the embryo with reference to the light that the proper shading will be given to bring out the gill pockets most emphatically. One can learn to do this only by practice. It is advantageous to have an assistant, then while the operator is looking into the camera the assistant can turn the embryo in various directions until the appearance is most satisfactory.

$\S 347$. Focusing and Making the Exposure.-For getting a general focus, and for the general arrangement the ground glass screen is used, but for the final focusing it is desirable to use a clear glass screen and a focusing glass. In this way one can focus as satisfactorily as with an ordinary microscope. In daylight with white embryos and a dark ground 30 to 40 seconds is usually sufficient exposure. One must learn this also by trial and it facilitates the obtaining of exact data to make a record of every negative made, whether the negative is good or bad. A table is given in $\$ 360$ to facilitate the record taking. In a short time one can learn to make the correct exposure. If the result is unsatisfactory, try again. The rule adhered to by all first rate workers is to to stick to it until the result is satisfactory.

$\$ 348$. Records of Embryos.-Each specimen or litter of specimens will have its own label giving date and method of preparation. It is an advantage to write this label with water-proof carbon ink, then one can put the label in the dish with the embryos and it will form a part of the picture and serve as a record.

After the picture is satisfactorily made it is wise to number the embryos on the back of the negative with a wax crayon, and later when the negative is dry number on the front with carbon ink. The embryos are placed in separate bottles each with a copy of the original label and the number corresponding with that put on the negative. This is easily accomplished if the embryos are arranged in definite rows as advised in $\$ 346$.

Finally when the embryo is cut into serial sections and mounted, a picture of the whole embryo should accompany the series.

$\$ 349$. Size of the Pictures.-For all embryos it is well to make one picture natural size $\left(X_{I}\right)$ and then for the smallest ones a magnification of at least five times natural size $(\times 5)$. Here, as with the magnification of the microscope, linear magnification is always meant (\$ I 54-155). 
$\S 350$. - Objectives. - For making pictures from one to five times natural size objectives of 60 to $100 \mathrm{~mm}$. focus answer well (Figs. 176I80). Short focus ( 75 to $100 \mathrm{~mm}$. equivalent focus), wide angle photographic objectives are also admirable for this work.

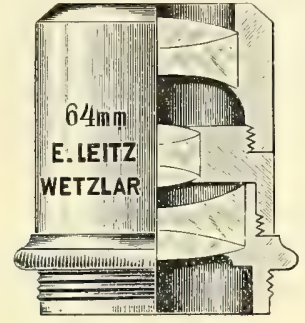

FIG. I 78 .

FIG. I78. Leitz 64 millimeter objective for photography and for projection (Wm. Krafft, N. Y.)

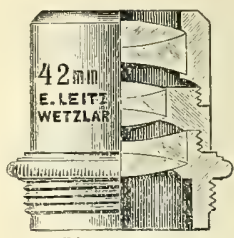

FIG. I79.

FIG. 179. Leitz 42 millimeter objectiv'e for photography and for projection (Wm. Krafft, N.Y.)

FIG. 180. Zeiss' Apochromatic Projection Objective of 70

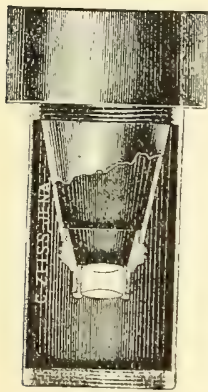
mm. equivalent focus, for photo-micrography. (Zeiss' Catalog.) This, and another of $35 \mathrm{~mm}$. focus, are designed for making pictures of moderate magnification. Usually rather large objects are photographed with them. The object may be illuminated in the ordinary way. They are used without an ocular, like a photographic objective. The one of $35 \mathrm{~mm}$. is screred into the tube of the microscope like an ordinary objective, but the one of $70 \mathrm{~mm}$. here shown, is, by means of a conical adapter, screwed into the ocular end of the tube, Fig. 189.

For illuminating the object, any suitable light may be used, but it is recommended that the light be concentrated by means of a bull's eye or some form of combination like the engraving glass, and that the condenser be so placed that it focuses the light upon the objective, not upon the object. The object is then illuminated with a converging cone of light.

3.5. Record of Negatives. - As indicated in $\$ 34 \mathrm{I}-343$ each negative should have a record, see record blank on p. 219 . On the negative itself should be also written the main facts with carbon ink. The name and magnification, date and any other details which may be thought desirable can be put on the envelope containing the negative, and then the negative stored like a catalog card as described above $(\$ 343)$.

\section{PHOTOGRAPHING LARGE TRANSPARENT OBJECTS}

$\$ 352$. There are many large transparent objects which it is desirable to photograph, e.g., chick embryos mounted whole, large sections of organs like the brain, etc. These must be photographed at a low magnification. 


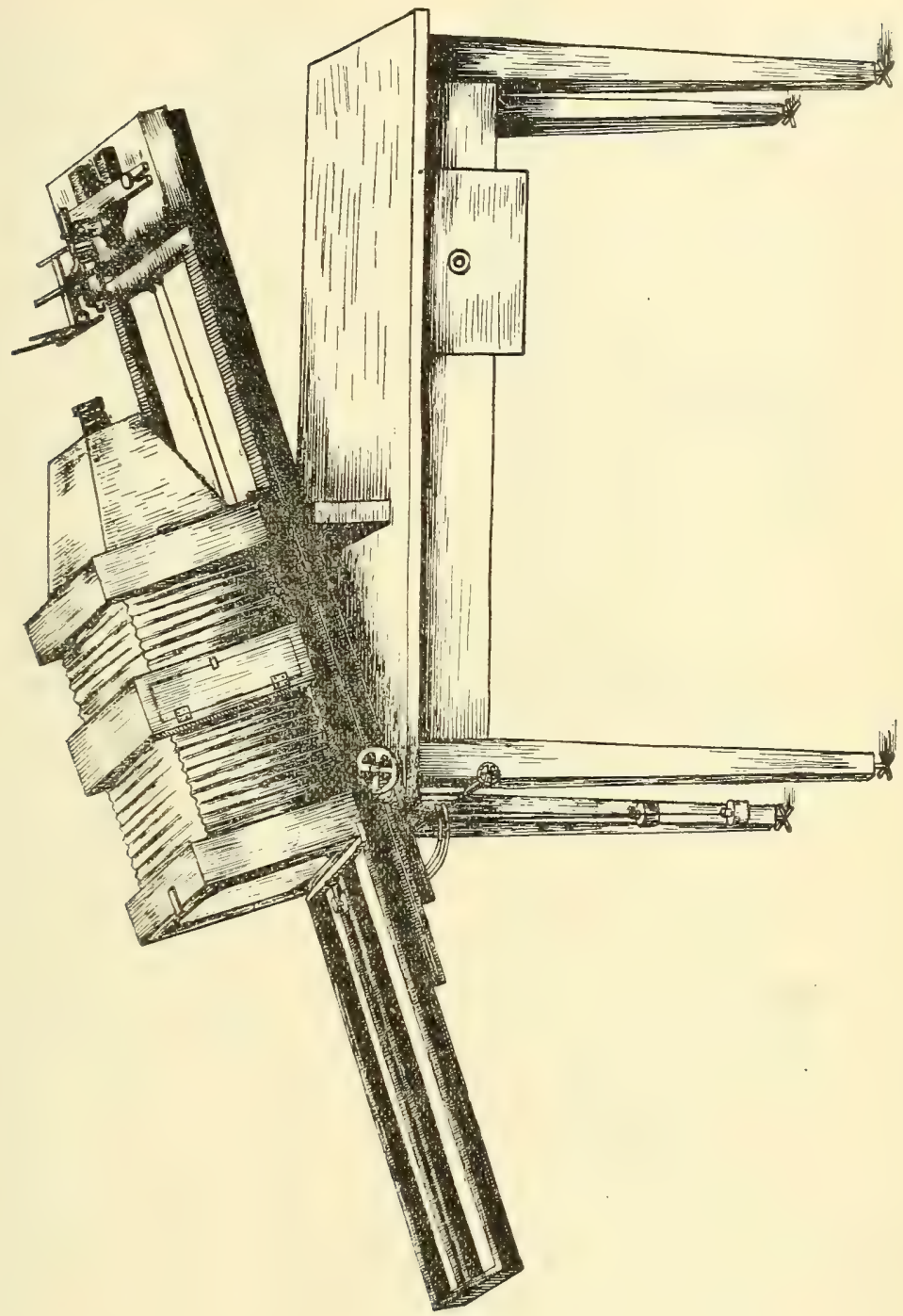

FIG. I8I,

FIG. 18I. Camera and special microscope stand for photographing very large transparent sections. For this the vertical camera is used (Fig. I6g) with the camera reversed on the sliding frame. This frame is elevated sufficiently to utilize the sky as background and illuminant. The special microscope stand is inclined to the horizontal and placed on the fixed frame supporting the camera; the specimen 
placed on the stage. For objective one of the objectives shown in Figs. I76 to I80 is used. The objective is screwed into an adapter in place of the ordinary photographic objective. The focusing is performed roughly by the rack and pinion, and then with great exactness with the focusing glass. For manipulating the fine adjustment of the special microscope the well known device of a cord over the head of the micrometer screw is used. (See also Fig. I75.) (Trans. Amer. Micr: Soc., I9oI.)

Successful photographs require an even lighting and an objective which has sufficient field to take in the whole object. The camera used for embryos (Fig. 175) answers very well for objects of moderate size. For lighting them the specimen is put on the upper stage, and the back-ground shown in the figure is removed. Then the large mirror is used to throw light up through the preparation. If necessary the specimen can be placed on one of the lower stories to bring it nearer the mirror. The lighting and focusing should be as perfect as possible. Lamplight and daylight are both good.

$\$$ 353. Photographing Large Transparent Objects.-For this the large vertical camera (Fig. I69, I8I) is reversed in position on the supporting frames, and elevated only sufficiently to make a sky background; or a 45 degree reflector of white cloth or paper of sufficient size must be used for a horizontal camera. If one has the earth for back-ground the light will be dull and uneven and a very long exposure is necessary, and the final results unsatisfactory.

$\$ 354$. Use of the Special Microscope Stand.-In order to hold the specimen in position and to focus it accurately, it is put on the stage of the special microscope stand (Fig. I75), which is inclined, and fastened to the fixed part of the frame supporting the camera. As the stage of this microscope is mored by the coarse or the fine adjustment, the focusing can be accomplished with the same accuracy as the microscope itself. For the general arrangement of the specimen and the rough focusing the ground glass is used, then this is replaced by a clear-glass focusing screen, and by the aid of a focusing glass the specimen is put in perfect focus. As one cannot reach the fine adjustment while focusing, the well known derice of a cord over the head of the micrometer screw is resorted to. The two ends of the cord should be weighted with about 50 or a hundred grams to keep the cord taut, then whichever one is pulled, the micrometer screw will respond at once. To cut off the light a piece of black velveteen is hung over the end of the objective. This can be removed mithout jarring the apparatus. An exposure of a few seconds ( 3 to ro seconds), will suffice for many preparations, uniess a color screen is used. The color screen increases the time of exposure from three to five times ( $\$ 359$ ). 


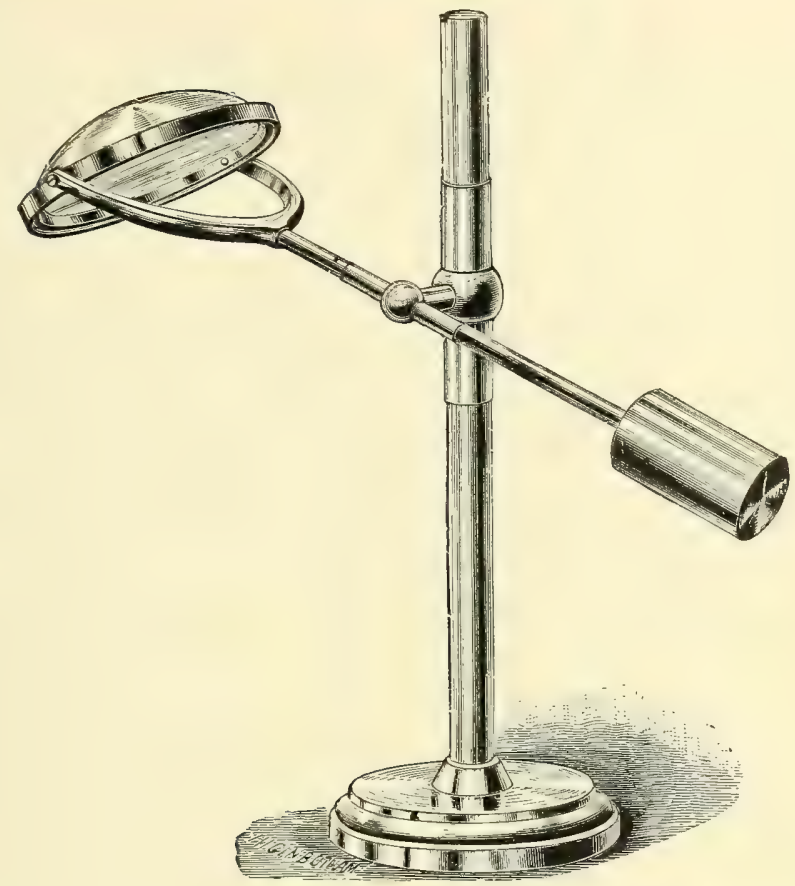

FIG. 182. Bull's eye lens and holder. (Bausch Gै Lomb Opt. Co.)

\section{COLOR-CORRECT PHOTOGRAPHY}

From the fact that the different wave lengths of light affect the photographic plate with different degrees of vigor, the ordinary photographic print of a many colored object or landscape is not satisfactory. All objects whose light is of short wave lengths, as blue, etc., will appear too light and those which are red, yellow and green will be too dark relatively. To obviate this difficulty two methods have been adopted, and for the most complete success they must be combined.

(A) The use of ortho- or iso-chromatic plates and (B) the use of a color screen or light filter.

355. Orthochromatic or Isochromatic Plates.-These are plates which have been rendered much more sensitive than ordinary plates to the long waves of red, orange, yellow and green, they therefore give a much more natural rendering to many-colored objects than ordinary plates. As they are sensitive to red, orange, etc., one must be very careful in exposing them in the dark room even to the light of the developing lantern. The more nearly the plate can be kept from all light, except that acting during the exposure in the camera, the more satisfactory will be the resulting negative.

These color-correct plates are not very enduring, and must be used while they are fresh, or only weak, foggy negatives will result. 
For photographing transparent or translucent objects there is a further diffculty introduced, viz, greater or less transparency. Assuming that all the rays of the spectrum are equally active, there would still be difficulty because the blue and violet stains used in microscopy are liable to be more transparent than those stained with red and orange, consequently a blue stained preparation is liable to lack in contrast since the light reaching the plate from the object and from the space around it produce so nearly equal effects on the plate.

On the other hand a red stain gives too much contrast because the light passing through it has little effect as compared with the light going through the space surrounding the object. So far in the discussion it is assumed that the objects are practically transparent and that pure red and blue are used. As a matter of fact the stain renders the specimen somewhat more opaque so that the specimen would be darker than the background in either case. This is especially true of hematoxylin. While hematoxylin is blue or purple, it renders the tissues more or less opaque, so that with a petroleum lamp and isochromatic plates, freshly stained hematoxylin specimens are very easy to get good pictures of.

\& 356. Color Screens.-As a general statement all color screens which have proved really useful in photographing transparent or translucent microscopic specimens cut off most of the blue end of the spectrum. Others cut off also the red end, leaving only the middle, visually brightest part of the spectrum free. As modern achromatic objectives are corrected for the visual rays, a screen cutting off the blue end of the spectrum serves to obviate any lack of sharpness due to the aberration of the blue rays in such objectives. There is the further advantage that with red and yellow objects, the color being in general of the wave length transmitted by the screen would be in true relative shade or contrast between background and object, and give good detail in the object. For the blue object this form of screen is also good for, as it cuts off the blue rays, the effect will be like photographing a gray object where the light and shade depend on the transparency of different parts. The denser the part the more opaque it is and therefore the darker it appears with transmitted light. The background allowing all the colored light to pass is lighter than the blue object and therefore there will be good contrast and also good detail in the object.

\& 357. Composition of Color Screens.-The most successful color screens are solutions held in parallel sided glass vessels. Colored glass, gelatin, collodion, etc., colored with different chemicals are fairly satisfactory, but not so satisfactory as the solutions.

(I) The most generally used and most generally useful screen is a watery solution of dichromate of potash $\left(\mathrm{K}_{2} \mathrm{Cr}_{2} \mathrm{O}_{7}\right)$. This cuts off the violet, the blue and the bluish green. The amount of light cut out depends upon the density of the solution and the thickness of the stratum through which the light passes.

(2) Zettnow's cupro-chromate color screen allows wave lengths from $0.570 \mu$ to $0.550 \mu$ to pass, that is yellow light. It is made by dissolving in $250 \mathrm{cc}$. of water, I I grams of pure dry cupric nitrate and I gram of chromic acid. The thickness of the stratum of liquid should be about i centimeter.

(3) Gifford's color screen. This is highly spoken of both for observation with the microscope (Carpenter-Dallinger) and for use in photography (J. R. M. S. IS94, p. I64). It is composed of a strong solution of malachit green in water, glycerin, glycerin jelly, etc. Only a thin stratum such as could be mounted be- 


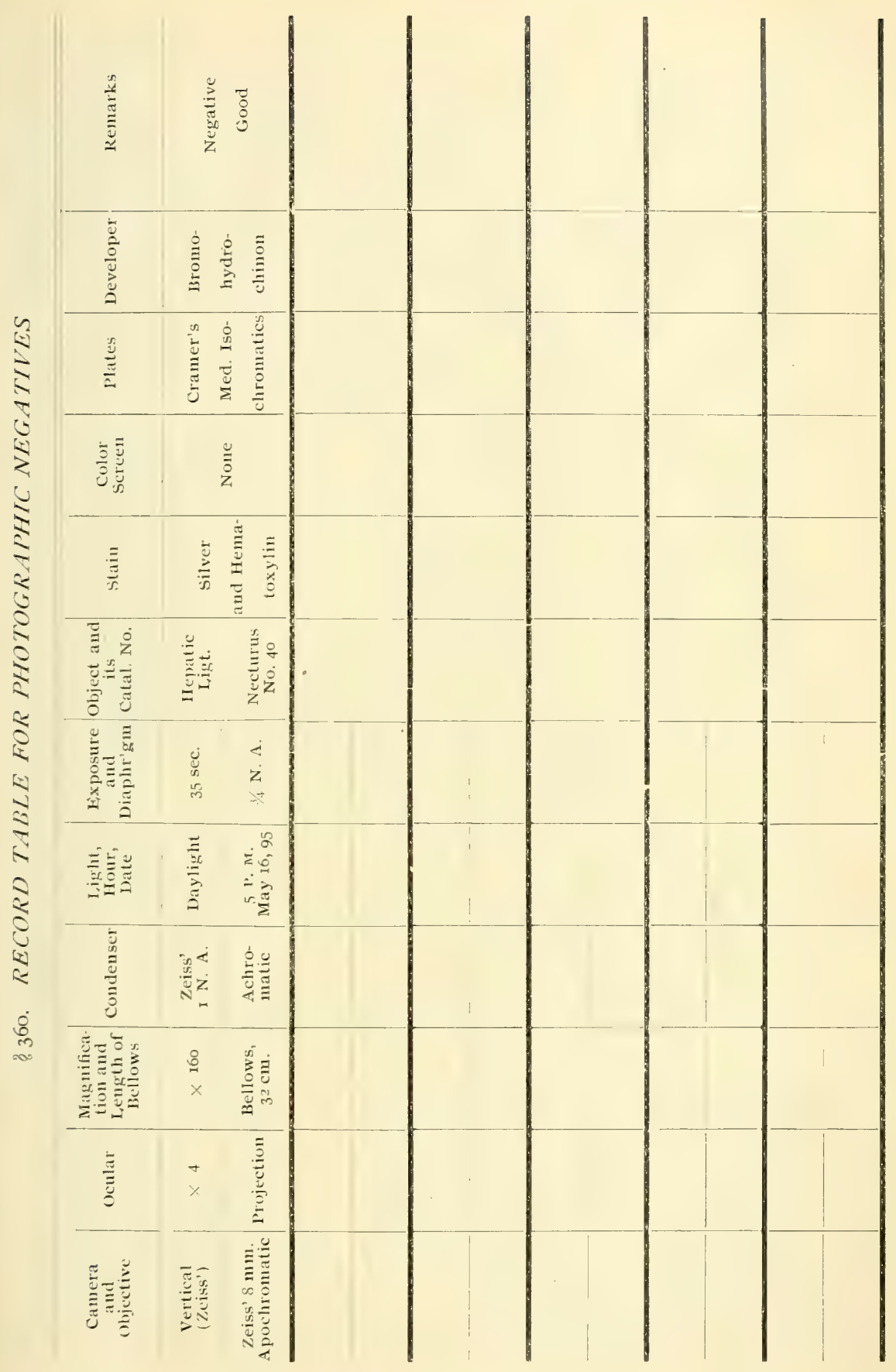


tween two cover-glasses is needed. By combining a little picric acid with the solution or by the use of a thin piece of signal green glass only light between the fixed lines $\mathrm{E}$ and $\mathrm{F}$, is allowed to pass. This is not therefore so generally useful as dichromate.

(4) Bothamley's aurantia color screen is a saturated alcoholic solution of the aurantia added to collodion of 3 to $4 \%$. The collodion is poured on a large coverglass or a glass plate and allowed to dry. Pringle advises several screens of aurantia of different shades. That is easily managed by adding a greater or less amount of the solution to the collodion. This is a good screen and easily used.

Petroleum light serves as a yellow color screen, and one can often get excellent results with such a light when daylight or the electric light without a color screen does not give a good picture. For all photography with the microscope isochromatic or orthochromatic plates are advised. For many objects no color screen is needed if one uses a petroleum lamp.

8. 358. Position of the Screen.-It does not make much difference where the color screen is placed provided no light reaches the object which has not passed through the screen.

8. 359. Exposure with a Color Screen.-The interposition of a color screen increases the time of exposure from three to five times. One can learn the time and whether or not to use a color screen, and the kind of a screen to use only by experiment. To get the full benefit of these experiments for future work, every negative should be carefully recorded $(\xi 360$, table $)$. It would also aid one materially, in the beginning at least, if he were to study the color screen used with the micro-spectroscope and determine the wave lengths which are allowed to pass through it $(\xi 195,202)$. If this study were supplemented by a spectroscopic examination of the object to be photographed, one would learn to choose with great accuracy the color screen which would give the best results.

\section{PHOTOGRAPHING WITH A MICROSCOPE*}

8.36r. The first pictures made on white paper and white leather, sensitized by silver nitrate, were made by the aid of a solar microscope (I802). The pictures

*Considerable confusion exists as to the proper nomenclature of photography with the microscope. In German and French the term micro-photography is very common, while in English photo-micrography and micro-photography mean different things. Thus : A photo-micrograph is a photograph of a small or microscopic object usually made with a microscope and of sufficient size for observation with the unaided eye; while a micro-photograph is a small or microscopic photograph of an object, usually a large object, like a man or woman and is designed to be looked at with a microscope.

Dr. A. C. Mercer, in an article in the Proc. Amer. Micr. Soc., I886, p. I31, says that Mr. George Shadbolt made this distinction. See the Liverpool and Manchester Photographic Journal (now British Journal of Photography), Aug. 15, 1858, p. 203 ; also Sutton's Photographic Notes, Vol. III, IS58, pp. 205-208. On p. 208 of the last, Shadbolt's word "Photomicrography" appears. Dr. Mercer puts the case very" neatly as follows: "A photo-micrograph is a macroscopic photograph of a microscopic object; a micro-photograph is a microscopic photograph of a macroscopic object. See also Medical News, Jan. 27, 1894, p. Io8. 
were made by Wedgewood and Davy, and Davy says: "I have found that images of small objects produced by means of the solar microscope may be copied without difficulty on prepared paper." $\dagger$

Thus among the very first of the experiments in photography the microscope was called into requisition. And naturally, plants and motionless objects were photographed in the beginnings of the art when the time of exposure required was very great.

At the present time photography is used to an almost inconceivable degree in all the arts and sciences and in pure art. Even astronomy finds it of the greatest assistance.

It has also accomplished marvels in the production of colored plates for book illustrations, especially in natural history. For an example see Comstock's Insect Life, 2d edition.

Alhough first in the field, Photo-Micrography has been least successful of the branches of photography. This is due to several causes. In the first place, microscopic objectives have been naturally constructed to give the clearest image to the eye, that is the visual image as it is sometimes called, is for microscopic observation, of prime importance. The actinic or photographic image, on the other hand, is of prime importance for photography. For the majority of microscopic objects transmitted light $(\% 64)$ must be used, not reflected light as in ordinary vision. Finally, from the shortness of focus and the smallness of the lenses, the proper illumination of the object is accomplished with some difficulty, and the fact of the lack of sharpness over the whole field with any but the lower powers, have combined to make photo-micrography less successful than ordinary macrophotography. So tireless, however, have been the efforts of those who believed in the ultimate success of photo-micrography, that now the ordinary achromatic objectives with ortho-chromatic or isochromatic plates and a color screen or petroleum light give good results, while the apochromatic objectives with projection oculars give excellent results, even in hands not especially skilled. The problem of illumination has also been solved by the construction of achromatic and apochromatic condensers and by the electric and other powerful lights now available. There still remains the difficulty of transmitted light and of so preparing the object that structural details stand out with sufficient clearness to make a picture which approaches in definiteness the drawing of a skilled artist.

The writer would advise all who wish to undertake photo-micrography seriousiy, to study samples of the best work that has been produced. Among those who showed the possibilities of photo-micrographs was Col. Woodward of the U.

$\dagger$ In a most interesting paper by A. C. Mercer on "The Indebtedness of Photography to Microscopy," Photographic Times Almanac, I887, it is shown that : "To briefly recapitulate, photography is apparently somewhat indebted to microscopy for the first fleeting pictures of Wedgewood and Davy [1802], the first methods of producing permanent paper prints [Reede, I837-I839], the first offering of prints for sale, the first plates engraved after photographs for the purpose of book illustration [Donné \& Foucalt, 1845], the photographic use of collodion [Archer \& Diamond, $185 \mathrm{I}]$, and finally, wholly indebted for the origin of the gelatino-bromide process, greatest achievement of them all [Dr, R. L. Maddox, I87I]. See further for the history of Photo-micrography, Neuhauss, also Bousfield. 
S. Army Medical Museum. The photo-micrographs made by him and exhibited at the Centennial Celebration at Philadelphia in 1876 , serve still as models, and uo one could do better than to study them and try to equal them in clearness and general excellence. According to the writer's observation no photo-micrographs of histological objects have ever exceeded those made by Woodward, and most of them are vastly inferior. It is gratifying to state, however, that at the present time many original papers are partly or wholly illustrated by photo-micrographs, and no country has produced works with photo-micrographic illustrations superior to those in "Wilson's Atlas of Fertilization and Karyokinesis" and "Starr's Atlas of Nerve Cells," issued by the Columbia University Press.-

In passing the writer would like to pay a tribute to Mr. W. H. Walmsley who has labored in advancing photo-micrography for the last twenty years. His convenient apparatus and abundant experience have been placed freely at the command of every interested worker, and many a beginner has been helped over difficulties by him. His last contribution in "International Clinics," vol. i. ser. II, I2, is encouraging in the highest degree both for its matter and for the illustrations.

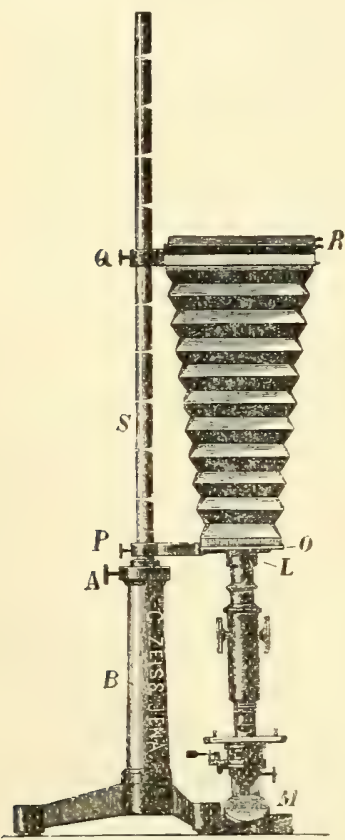

FIG. I83. Zeiss' Vertical Photo-micrographic Camera. A. Set screw holding the $\operatorname{rod}(S)$ in any desired position. $P$; $Q$. Set screws by which the bellows are held in place. $B$. Stand with tripod base in which the supporting $\operatorname{rod}(S)$ is held. This rod is now graduated in centimeters and is a ready means of determining the length of the camera. M. Mirror of the microscope. L. The sleeve serving to make a light-tight connection between the camera and microscope. $O$. The lower end of the camera. $R$. The upper end of the camera where the focusing screen and plate holder are situated. (From Zeiss' Photomicrographic Catalog).

As the difficulties of photo-micrography are so much greater than of ordinary photography, the advice is almost universal that no one should try to learn photography and"photo-micrography at the same time, but that one should learn the 
processes of photography by making portraits, landscapes, copying drawings, etc., and then when the principles are learned one can take up the more difficult subject of photo-micrography with some hope of success.

The advice of Sternberg is so pertinent and judicious that it is reproduced : "Those who have had no experience in making photo-micrographs are apt to expect too much and to underestimate the technical difficulties. Objects which under the microscope give a beautiful picture, which we desire to reproduce by photography may be entirely unsuited for the purpose. In photographing with high powers it is necessary that the objects to be photographed be in a single plane and not crowded together and overlying each other. For this reason photographing bacteria in sections presents special difficulties and satisfactory results can only be obtained when the sections are extremely thin and the bacteria well stained. Even with the best preparations of this kind much care must be taken in selecting a field for photography. It must be remembered that the expert microscopist, in examining a section with high powers, has his finger on the fine adjustment screw and focuses up and down to bring different planes into view. He is in the habit of fixing his attention on the part of the field which is in focus and discarding the rest. But in a photograph the part of the field not in focus appears in a prominent way which mars the beauty of the picture."

\section{APPARATUS FOR PHOTO-MICROGRAPHY}

8 362. Camera.-For the best results with the least expenditure of time one of the cameras especially designed for photo-micrography is desirable but is not by any means indispensable for doing good work. An ordinary photographic camera, especially the kind known as a copying camera, will enable one to get good results, but the trouble is increased, and the difficulties are so great at best, that one would do well to avoid as many as possible and have as good an outfit as can be afforded (Figs. I84, I92).

The first thing to do is to test the camera for the coincidence of the plane occupied by the sensitive plate and the ground glass or focusing screen. Cameras even from the best makers are not always correctly adjusted. By using a straight edge of some kind, one can measure the distance from the inside or ground side of the focusing screen to the surface of the frame. This should be done all around to see if the focusing screen is equally distant at all points from the surface of the frame. If it is not it should be made so. When the focusing screen has been examined, an old plate, but one that is perfectly flat, should be put into the plate holder and the slide pulled out and the distance from the surface of the plate holder determined exactly as for the focusing screen. If the distance is not the same the position of the focusing screen must be changed to correspond with that of the glass in the plate holder, for unless the sensitive surface occupies exactly the position of the focusing screen the picture will not be sharp. no matter how accurately one may focus. Indeed, so necessary is the coincidence of the plane of the focusing screen and sensitive surface that some photo-micrographers put the focusing screen in the plate holder, focus the image and then put the sensitive plate in the holder and make the exposure (Cox). This would be possible with the older forms of plate holders, but not with the double plate holders mostly used at the present day. 
\& 362a. Size of Camera.-The majority of photo-micrographs do not excced 8 centimeters in diameter and are made on plates $8 \times$ II, IOX I 3 or $\mathbf{I} 3$ X I 8 centimeters $\left(3 \frac{1}{4} \times 4^{1 / 4}\right.$ in., $4 \times 5$ in., or $5 \times 7$ in. $)$. Most of the vertical cameras are for plates not exceeding IOX $\mathrm{I}_{3}$ centimeters $(4 \times 5$ in.) but Zeiss' new form will take plates 2 I $\times 2$ I centimeters $\left(8 \frac{1}{4} \times 81 / 4\right.$ in. $)$.

8 363. Work Room.-It is almost self-evident that the camera must be in some place free from vibration. Frequently a basement room where the camera table may rest directly on the cement floor or on a pier is an excellent situation. Such a place is almost necessary for the best work with high powers. For those living in cities, a time must also be chosen when there are no heavy vehicles moving in the streets. For less difficult work an ordinary room in a quiet part of the house or laboratory building will suffice.

364. Arrangement and Position of the Camera and the Microscope.-For much of photo-micrography a vertical camera and microscope are to be preferred (Fig. I $8_{4}$ ). Excellent arrangements were perfected long ago, especially by the French. (See Moitessier.)

Vertical photo-micrographic cameras are now commonly made, and by some firms only vertical cameras are produced. They are exceedingly convenient, and do not require so great a disarrangement of the microscope to make the picture as do the horizontal ones. Van Heurck advises their use, then whenever a structure is shown with especial excellence it is photographed immediately. The variation in size of the picture is obtained by the objective and the projection ocular rather than by length of bellows (see below Fig. I ${ }_{4}$ ): It must not be forgotten, however, that penetration varies inversely as the square of the power, and only inversely as the numerical aperture ( $\% 34)$, consequently there is a real advantage in using a low power of great aperture and a long bellows rather than an objective of higher power with a short bellows. A horizontal camera is more convenient for use with the electric light also (Fig. I92).

For convenience and rapidity of work a microscope with mechanical stage is very desirable. It is also an advantage to have a tube of large diameter so that the field will not be too greatly restricted (Fig. IS9). In some microscopes the tube is removable almost to the nose-piece to avoid interfering with the size of the image. The substage condenser should be movable on a rack and pinion. The microscope should have a flexible pillar for work in a horizontal position. While it is desirable in all cases to have the best and most convenient apparatus that is made, it is not by any means necessary for the production of excellent work. A simple stand with flexible pillar and good fine adjustment will answer.

3 365. Objectives and Oculars for Photo-Micrography. -The belief is almost universal that the apochromatic objectives are most satisfactory for photography. They are employed for this purpose with a special projection ocular. Two very low powers are used without any ocular (Fig. ISo). Some of the best work that has ever been done, however, was done with achromatic objectives (work of Woodward and others). One need not desist from undertaking photo-micrography if he has good achromatic objectives. From a somewhat extended series of experiments with the objectives of many makers the good modern achromatic objectives were found to give excellent results when used without an ocular. Most of them also gave good results with projection oculars, although it must be said that the best results were obtained with the apochromatic objectives and projec- 


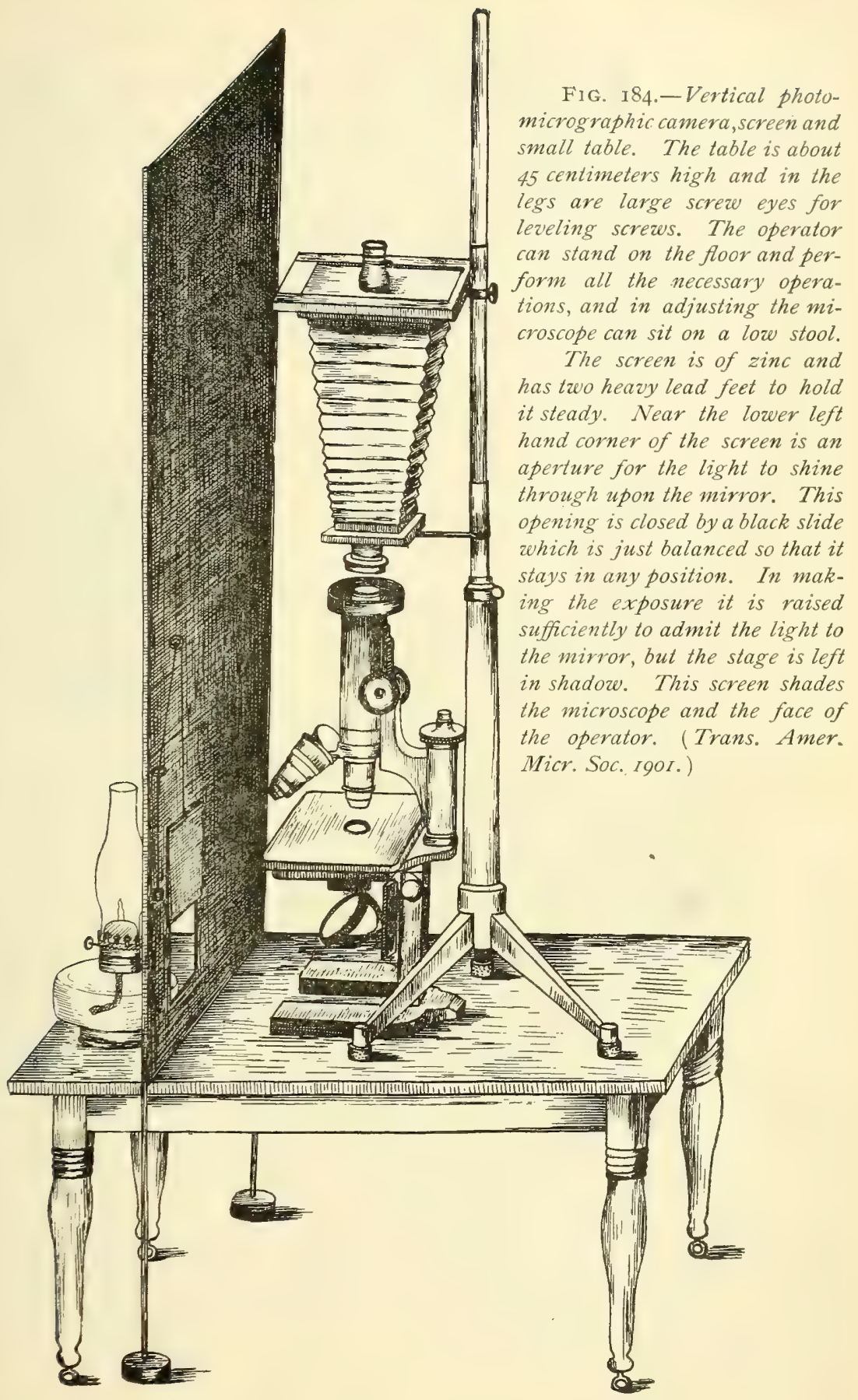


tion oculars. It does not seem to require so much skill to get good results with the apochromatics as with the achromatic objectives. The majority of photomicrographers do not use the Huygenian oculars in photography, although excellent results have been obtained with them. An amplifier is sometimes used in place of an ocular. Considerable experience is necessary in getting the proper mutual position of objective and amplifier. The introduction of oculars especially designed for projection, has led to the discarding of ordinary oculars and of amplifiers. However the projection oculars of Zeiss restrict the field very greatly, hence the necessity of using the objective alone for large specimens.*

FIG. 185. Projection Oculars with section removed to show the construction. Below are shown the upper end with graduated circle to indicate the amount of rotation found necessary to focus the diaphragm on the screen. No.2, No. 4. The numbers indicate the amount the ocular magnifies the image formed by the objective as with the compensation oculars. (Zeiss' Catalog.)

366. Difference of Visual and Actinic Foci.-Formerly there was much difficulty experienced in photo-micrographing on account of the difference in actinic and visual foci. Modern objectives are less faulty in this respect and the apochromatics are practically free

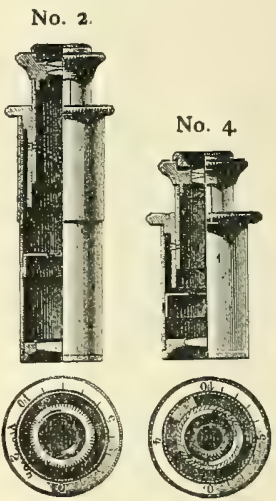
from it. Since the introduction of orthochromatic or isochromatic plates and, in many cases the use of colored screens, but little trouble has arisen from differences in the foci. This is especially true when mono-chromatic light and even when petroleum light is used. In case the two foci are so unlike in an objective, it would be better to discard it for photography altogether, for the estimation of the proper position of the sensitive plate after focusing is only guess work and the result is mere chance. If sharp pictures cannot be obtained with an objective when petroleum light and orthochromatic plates are used the fault may not rest with the objective but with the plate holder and focusing screen. They should be very carefully tested to see if there is coincidence in position of the focusing screen and the sensitive film as described in $\& 362$.

\&. 367. Apparatus for Lighting.-For low power work $35 \mathrm{~mm}$. and longer focus) and for large objects, some form of bull's eye condenser is desirable although fairly good work may be done with diffused light or lamp-light reflected by a mirror. If a bull's eye is used it should be as nearly achromatic as possible. The engraving glass shown in Fig. ISS answers well for large objects. For smaller

*A comparative study both with projection oculars, and without an ocular was made with the achromatic objective $25 \mathrm{~mm}$. ( I inch), I8 $\mathrm{mm}$. ( $\frac{3}{4}$ inch), 5 $\mathrm{mm}$. ( $\frac{1}{3}$ to $\frac{1}{5}$ inch) and $2 \mathrm{~mm}$. ( $\frac{1}{12}$ inch) homogeneous immersion of the Bausch \& Lomb Optical Co.; Gundlach Optical Co.; Leitz; Reichert; Winke1, Zeiss and the Spencer Lens Co. Good results were obtained with all of these objectives both with and without projection oculars. 
objects a Steinheil lens combination gives a more brilliant light and one also more nearly achromatic. For high power work all are agreed that nothing will take the place of an achromatic condenser. This may be simply an achromatic condenser, but preferably it should be an apochromatic condenser. Whatever the form of the condenser it should possess diaphragms so that the aperture of the condenser may be varied depending upon the aperture of the objective. For a long time objectives have been used as achromatic condensers, and they are very satisfactory, although less convenient than a special condenser whose aperture is great enough for the highest powers and capable of being reduced by ueans of diaphragms to the capacity of the lower objectives. It should also be capable of accurate centering. $(\xi 8 \mathrm{I})$.

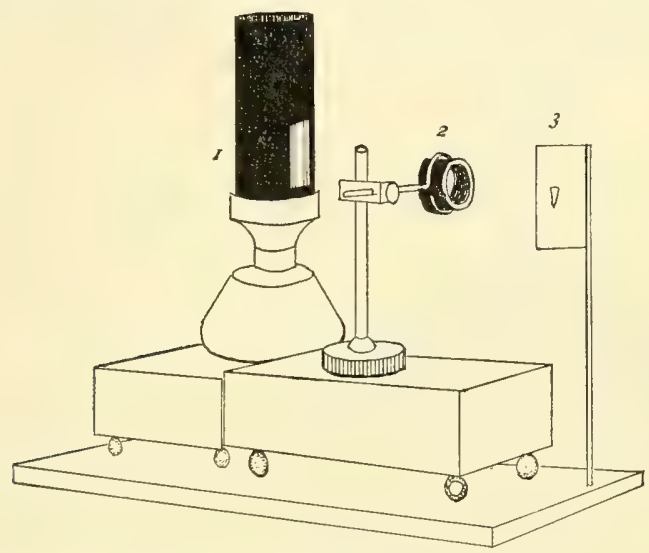

FIG. I86. Arrangement for Artificial Illumination.

I. Lamp with metal chimney, easily made by rolling up some ferrotype plate and making a slit-like opening in one side. This opening should be covered by an oblong cover-glass. A glass slide, being of considerable thickness, breaks too easily. The lamp should have a wick about $40 \mathrm{~mm}$. wide, so that the thickness of the flame, if taken edgervise, will give an intense light. A wide flame also enables one to get a larger image of the flame, and thus to illuminate a larger object than as though a small flame was used.

2. Bull's-eye condenser on a separate stand. The engraving glass shown in Fig. 188, or the tripod magnifier (Fig. I72) answers fairly. The Steinheil lenses are still better.

\section{Screen showing image of the flame inverted.}

The lamp and bull's-eye stand are on blocks with screw-eyes as leveling screws.

\& 368. Objects Suitable for Photo-micrographs.-While almost any large object may be photographed well with the ordinary camera and photographic objective, only a small part of the objects mounted for microscopic study can be photomicrographed satisfactorily. Many objects that give beautiful images when looking into the microscope and constantly focusing with the fine adjustment, appear almost without detail on the screen of the photo-micrographic camera and in the photo-micrograph. 


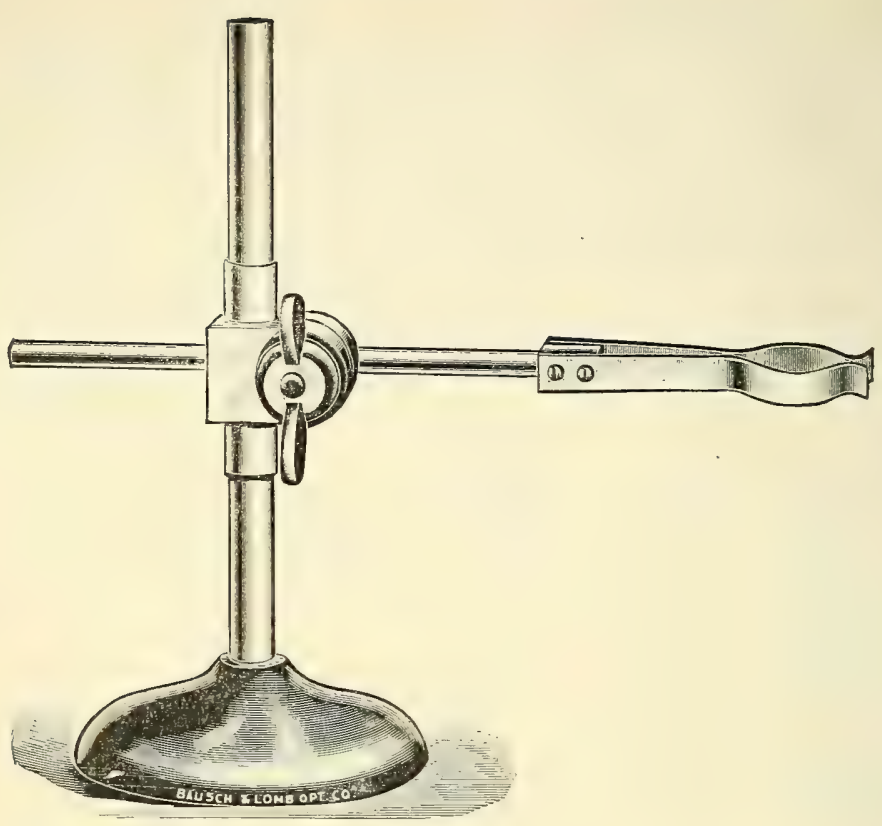

FIG. 187. Adjustable lens holder. This lens holder will take magnifiers of iarious sizes, and from its adjustable mechanism is very convenient for dissecting, or for holding a Steinheil and other lenses for illumination (The Bausch \& Lomb Opt. Co.).

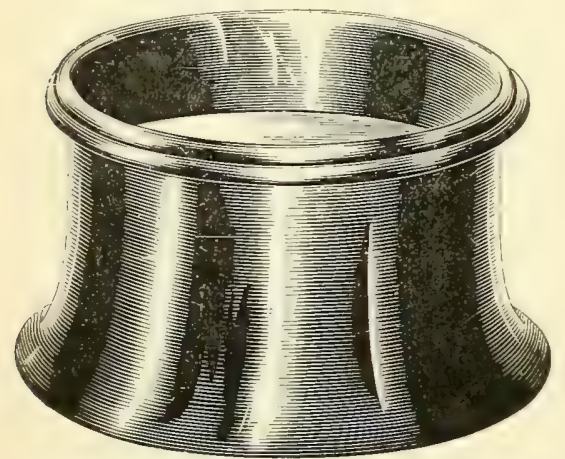

FIG. 188. Engraving glass to serve as a condenser and for a dissecting lens. (Bausch \& Lomb Opt. Co.)

If one examines a series of photo-micrographs the chances are that the greater number will be of diatoms, plant sections or preparations of insects. That is, they are of objects having sharp details and definite outlines, so that contrast and definiteness may be readily obtained. Stained microbes also furnish favorable objects when mounted as cover-glass preparations. 
Preparations in animal histology must approximate as nearly as possible to the conditions more easily obtained with vegetable preparations. That is, they must be made so thin and be so prepared that the cell outlines will have something of the definiteness of vegetable tissue. It is useless to expect to get a clear photograph of a section in which the details are seen with difficulty when studying it under the microscope in the ordinary way.

Many sections which are unsatisfactory as wholes, may nevertheless have parts in which the structural details show with satisfactory clearness. In such a case the part of the section showing details satisfactorily should be surrounded by a delicate ring by means of a marker (see Figs. 6I-66). If one's preparations have been carefully studied and the special points in them thus indicated, they will be found far more valuable both for ordinary demonstration and for photography. The amount of time saved by marking one's specimens can hardly be overestimated. The most satisfactory material for making the rings is shellac colored with lampblack.

Ten years ago many histologic preparations could not be satisfactorily photographed. But now with improved section cutters, better staining and mounting methods, and with the color screens $(\xi 356)$ and isochromatic plates $(8355)$ almost any preparation which shows the elements clearly when looking into the microscope can be satisfactorily photographed. Good photographs cannot, however, be obtained from poor preparations.

8.369. Light.-The strongest available light is sunlight. That has the defect of not always being available, and of differing greatly in intensity from hour to hour, day to day and season to season. The sun does not shine in the evening when many workers find the only opportunity for work. Following the sunlight the electric light is the most intense of the available lights. Then come magnesium, acetylene, the lime light, the gas-glow or Wellsbach light, and lastly, petroleum light. The last is excellent for the majority of low and moderate power work. And even for $2 \mathrm{~mm}$. homogeneous immersion objectives, the time of exposure is not excessive for many specimens (40 seconds to 3 minutes). This light is also cheapest and most available and has the advantage of being somewhat yellow, and therefore in many cases makes the use of a color screen unnecessary if one uses isochromatic plates. Acetylene light is excellent and may be used where the arc light is not available.

A lamp with flat wick about $40 \mathrm{~mm}$. ( $13 / 4 \mathrm{in}$.) wide has been found most generally serviceable. For large objects and low powers the flame may be made large and the face turned toward the mirror. This will light a large field. For high powers the edge toward the mirror gives an intense light. The ordinary glass chimney answers well, especially where a metal screen is used as shown in Fig. 184.

\section{EXPERIMENTS IN PHOTO-MICROGRAPHY}

370. The following experiments are introduced to show practically just how one would proceed to make photo-micrographs with various powers, and be reasonably certain of fair success. If one consults prints or the published figures made directly from photo-micrographs it will be seen that, excepting the bacteria, the magnification ranges mostly between io and I5O diameters. 


\section{$\$ 37$ I. Focusing Screen for Photo-Micrography.-One cannot} expect a picture sharper than the image seen on the focusing screen. Hence the greatest care must be taken in focusing. The general focusing may be done with the unaided eye on the ground glass, but for the final focusing a clear screen and a focusing glass must be used. (Figs. I 72, I73). See $\$ 347$. With the clear focusing screen one cannot at first see the image without using a focusing glass, but with a little experience the aerial image may be seen as with the microscope $(\$ 54)$.

$\S 372$. Photo-micrographs of 20 to 50 Diameters.-For pictures under 15 or 20 diameters it is better to use the camera for embryos with the objective in the end of the camera, and the special microscope stand for focusing (Fig. I75).

For pictures at 25 to 50 diameters one may use the microscope with a low objective, 25 to $35 \mathrm{~mm}$. equivalent focus, and no ocular (Fig. I 84). The object is placed on the stage of the microscope, and focused as in ordinary observation. If a vertical microscope is used the light from the petroleum lamp or other artificial light, is reflected upward by the mirror. It may take some time to get the whole field lighted evenly. Refer back to $\S 95$ for directions. In some cases it may be advisable to discard the condenser and use the mirror only. For some purposes one will get a better light by placing the bull's eye or other condenser between the lamp and the mirror to make the rays parallel or even to make a sharp image of the lamp flame on the mirror. Remember also that in many cases it is necessary to have a color screen between the source of light and the object $(\$ 356)$.

For a horizontal camera it is frequently better to swing the mirror entirely out of the way and allow the light to enter the condenser directly or after traversing the bull's eye (Figs. I82, 186). If the object is small an achromatic combination like a Steinheil magnifier or an engraving glass is excellent (Fig. I88). When the light is satisfactory as seen through an ordinary ocular, remove the ocular.

(A) Photographing without an Ocular.-After the removal of the ocular put in the end of the tube a lining of black velvet to avoid reflections. Connect the microscope with the camera, making a lighttight joint and focus the image on the focusing screen. One may make a light-tight connection by the use of black velveteen or more conveniently by the Zeiss' double metal hood which slips over the end of the tube of the microscope, and into which fits a metal cylinder on the lower end of the camera (Figs. I $84,189,183$ ). In the last figure the connection has been made. 


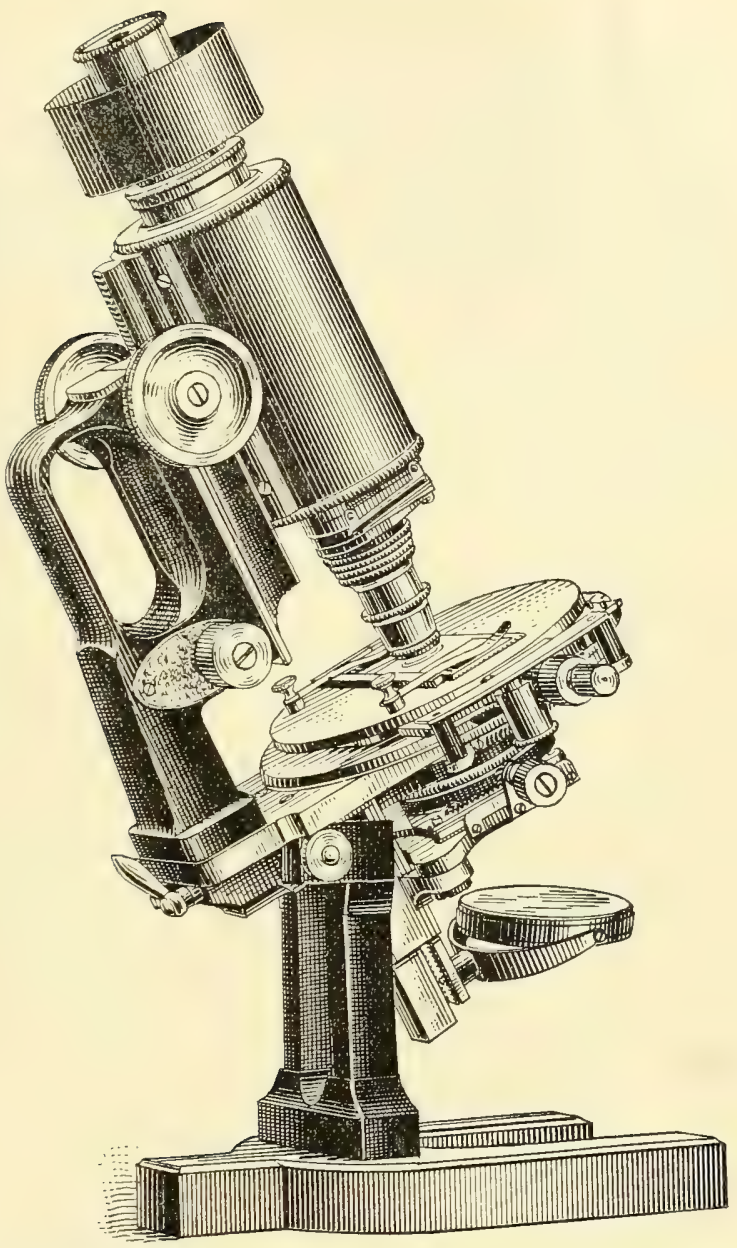

FIG. I89. Zeiss' special photo-micrographic stand. It has a very large tube, a slow acting fine adjustment, mechanical stage and all appliances for the most satisfactory work. (Cut loaned by Eimer and Amend).

It will be necessary to focus down considerably to make the image clear. Lengthen or shorten the bellows to make the image of the desired size, then focus with the utmost care. In case the field is too much restricted on account of the tube of the microscope, remove the draw-tube. When all is in readiness it is well to wait for three to five minutes and then to see if the image is still sharply focused. If it has got out of focus simply by standing, a sharp picture could not be ob- 
FIGS. Igo-I9I. Fine tint, half-tone reproductions of photo-micrographs of sections made by Mrs. Gage, to show the possibilities of photo-micrography with photographic objectives and with low microscopic objectives without a projection ocular.

I. Frontal section of the head of a large red Diemyctylus viridescens (red newt) at the level of the portae of the brain, magnified Io diameters. Negative made with a Gundlach perigraphic objective of about $90 \mathrm{~mm}$. equivalent focus.

2. Frontal section of a lavval Diemyctylus about 10 millimeters in length. Negative made with a Winkel objective of 22 millimeters equivalent focus; no ocular. Magnified 50 diameiers. (Mrs. Susanna Phelps Gage, the Wilder Quarter Century Book).

tained. If it does not remain in focus, something is faulty. When the image remains sharp after focusing make the exposure. From 20 to 60 seconds will usually be sufficient time with medium plates and the light as described. If a color screen is used it will require $40-300$ seconds, $i$. e., 2 to 5 times as long, for a proper exposure ( $\$ 359$ ).

B. Photographing with a Projection Ocular. - If the object is small enough to be included in the field of a projection ocular (Fig. I85) use that for making the negative as follows: Swing the camera around so that it will leave the microscope free. Use an ordinary ocular, focus and light the object, then insert a projection ocular in place of the ordinary one, and swing the camera back over the microscope. It is not necessary to use an ordinary ocular for the first focusing, but as its field is larger it is easier to find the part to be photographed. The first step is then to focus the diaphragm of the projection ocular sharply on the focusing screen. Bring the camera up close to the microscope and then screw out the eye-lens of the ocular a short distance. Observe the circle of light on the focusing screen to see if its edges are perfectly sharp. If not, continue to screw out the eye lens until it is. If it cannot be made sharp by screwing it out reverse the operation. Unless the edge of the light circle, $i . e$, the diaphragm of the ocular, is sharp, the resulting picture will not be satisfactory.

It should be stated that for the $\times 2$ projection ocular the bellows of the camera must be extended about 30 or 40 centimeters or the diaphragm cannot be satisfactorily focused on the screen. The $\times_{4}$ projection ocular can be focused with the bellows much shorter. For either projection ocular the screen distance can be extended almost indefinitely.

When the diaphragm is sharply focused on the screen, the microscope is focused as though no ocular were present, that is, first with the unaided eye then with the focusing glass. The exposure is also made in the same way, although one must have regard to the greater mag- 



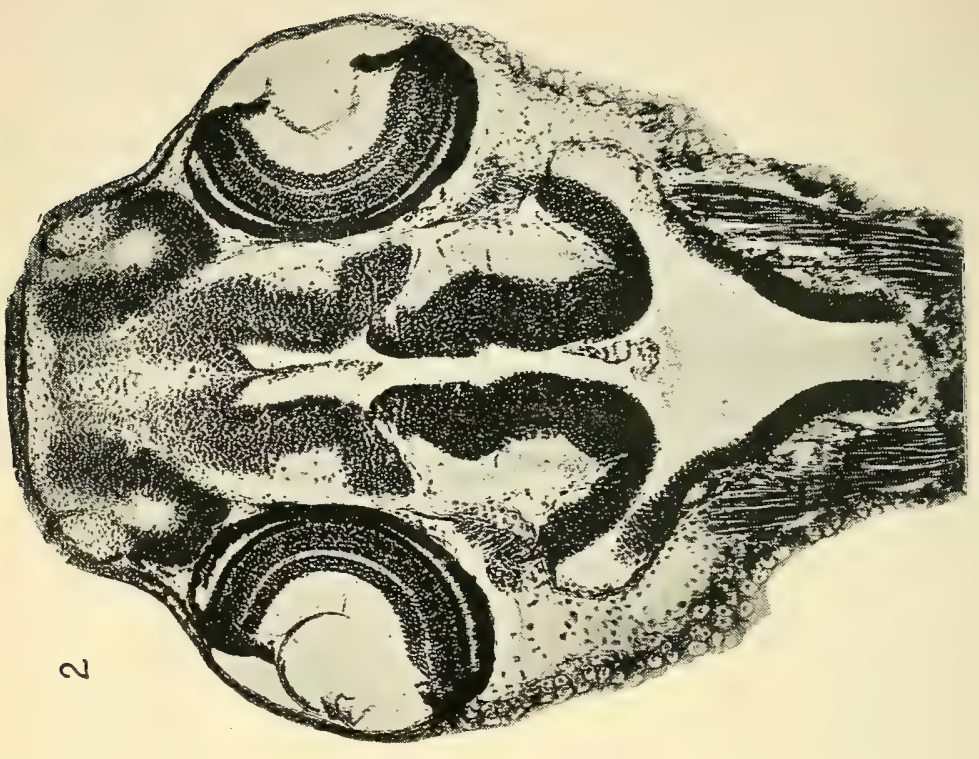

a

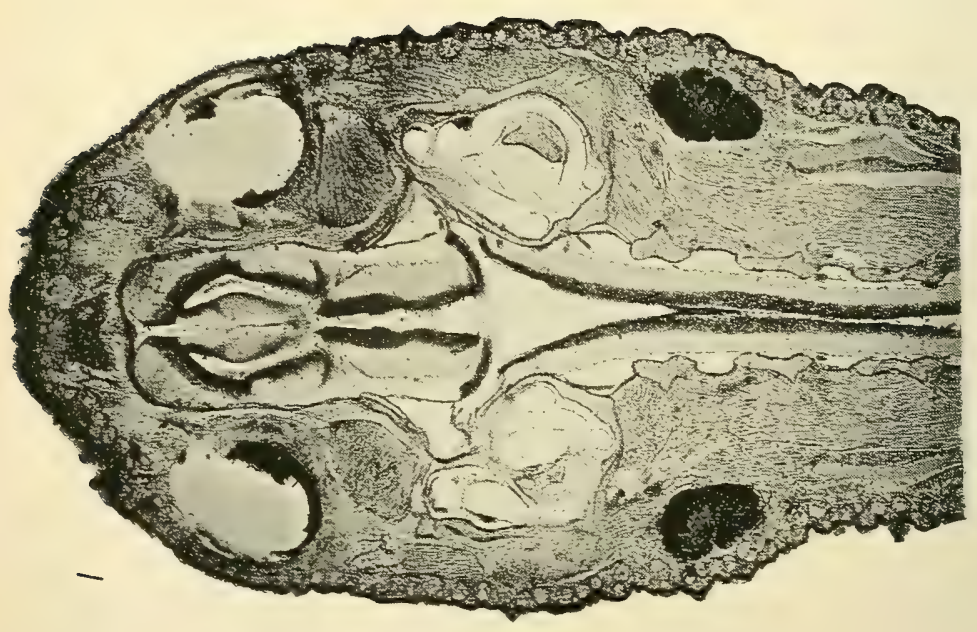

官 
nification produced by the projection ocular and increase the time accordingly; thus when the $x_{4}$ ocular is used, the time should be at least doubled over that when no ocular is employed. The time will be still further increased if a color screen is used $(\$ 359)$.

Zeiss recommends that when the bellows have sufficient length the lower projection oculars be used, but with a short bellows the higher ones. It is also sometimes desirable to limit the size of the field by putting a smaller diaphragm over the eye lens. This also aids in making the field uniformly sharp.

$\$ 373$. Determination of the Magnification of the PhotoMicrograph.-After a successful negative has been made, it is desirable and important to know the magnification. This is easily determined by removing the object and putting in its place a stage micrometer. If the distance between two or more of the lines of the image on the focusing screen is obtained with dividers and the distance measured on one of the steel rules, the magnification is found by dividing the size of the image by the known size of the object (\$ I 54). If now the length of the bellows from the tube of the microscope is noted, say on a record table like that in section 360 , one can get a close approximation to the power at some other time by using the same optical combination and length of bellows.

For obtaining the magnification at which negatives are made it is a great advantage to have one micrometer in half millimeters ruled with coarse lines for use with the lower powers, and one in O.I and o. OI millimeter ruled with fine lines for the higher powers.

$\$ 374$. Photo-Micrographs at a Magnification of 100 to 150 Diameters. - For this, the simple arrangements given in the preceding section will answer, but the objectives must be of shorter focus, 8 to 3 $\mathrm{mm}$. It is better, however, to use an achromatic condenser instead of the engraving glass or the Steinheil lens.

$\$$ 375. Lighting for Photo-Micrography with Moderate and High Powers.--( Ioo to 2,500 diameters). No matter how good one's apparatus, successful photo-micrographs cannot be made unless the object to be photographed is properly illuminated. The beginner can do nothing better than to go over with the greatest care the directions for centering the condenser, for centering the source of illumination, and the discussion of the proper cone of light and lighting the whole field, as given on $\mathrm{pp} .4 \mathrm{I}-52$. Then for each picture the photographer must take the necessary pains to light the object properly. An achromatic condenser is almost a necessity $(\S 80)$. Whether a color-screen should 
be used depends upon judgment and that can be attained only by experience. In the beginning one may try without a screen, and with different screens and compare results.

A plan used by many skilled workers is to light the object and the field around it well and then to place a metal diaphragm of the proper size in the camera very close to the plate holder. This will insure a clean, sharp margin to the picture. This metal diaphragm must be removed while focusing the diaphragm of the projection ocular, as the diaphragm opening is smaller than the image of the ocular diaphragm.

If the young photo-micrographer will be careful to select for his first trials, objects of which really good photo-micrographs have already been made, and then persists with each one until fairly good results are attained, his progress will be far more rapid than as if poor pictures of many different things were made. He should, of course, begin with low magnifications.

$\S 376$. Adjusting the Objective for Cover-Glass.-After the object is properly lighted, the objective, if adjustable, must be corrected for the thickness of cover. If one knows the exact thickness of the cover and the objective is marked for different thicknesses, it is easy to get the adjustment approximately correct mechanically, then the final corrections depend on the skill and judgment of the worker. It is to be noted too that if the objective is to be used without a projection ocular the tube-length is practically extended to the focusing screen and as the effect of lengthening the tube is the same as thickening the cover-glass, the adjusting collar must be turned to a higher number than the actual thickness of the cover calls for (see $\$$ I03).

$\$ 377$. Photographing Without an Ocular.-Proceed exactly as described for the lower power, but if the objective is adjustable make the proper adjustment for the increased tube-length ( $\$$ I03).

$\$ 378$. Photographing with a Projection Ocular.-Proceed as described in $\$ 372 \mathrm{~B}$, only in this case the objective is not to be adjusted for the extra length of bellows. If it is corrected for the ordinary ocular, the projection ocular then projects this correct image upon the focusing screen.

\$379. Photo-Micrographs at a Magnification of 500 to 2000 Diameters.-For this the homogeneous immersion objective is employed, and as it requires a long bellows to get the higher magnification with the objective alone, it is best to use the projection oculars. 
For this work the directions given in $\$ 372 \mathrm{~B}$ must be followed with great exactness. The edge of the petroleum lamp flame is sufficient to fill the field in most cases. With many objects the time required with good lamp light is not excessive; viz., 40 seconds to 3 minutes. The reason of this is that while the illumination diminishes directly as the square of the magnification, it increases with the increase in numerical aperture, so that the illuminating power of the homogeneous immersion is great in spite of the great magnification $(\$ 34)$.

For work with high powers a stronger light than the petroleum lamp is employed by those doing considerable photo-micrography. Good work may be done, however, with the petroleum lamp.

It may be well to recall the statement made in the beginning, that the specimen to be photographed must be of especial excellence for all powers. No one will doubt the truth of the statement who undertakes to make photo-micrographs at a magnification of 500 to 2000 diameters.

If one has a complete outfit with electric arc light (Fig. I92) the time required for photographing objects is much reduced, $i$. e. ranging from I to 20 seconds even with a color screen. As the light is so intense with the arc light it is necessary to soften it greatly for focusing. Several thicknesses of ground glass placed between the lamp and the microscope will answer. These are removed before taking the negative. It is well also to have a water bath on the optical bench to absorb the heat rays. This should be in position constantly (see Ch. IX).

$\$ 380$. Use of Oculars in Photo-Micrography.-There is much diversity of opinion whether or not the ordinary oculars used for observation should be used in photographing. Excellent results have been obtained with them and also without them.

For great magnification Zeiss recommends the use of the compensation oculars with the apochromatics.

The Zeiss projection oculars may be used with achromatic objectives of large aperture as well as with the apochromatics.

\section{PHOTGGRAPHING OPAQUE OBJECTS AND METALLIC SURFACES} WITH A MICROSCOPE

All of the objects cansidered in the first part of this chapter are opaque and some of them were to be photographed somewhat larger than natural size. To meet the needs of modern work, especially with metals and alloys one must be able to examine and photograph prepared surfaces at magnifications ranging from five or ten to five hundred or more diameters.

\$381. Microscope for Opaque Objects.-If one does not need to magnify more than about Ioo diameters, any good microscope will answer. For the higher 


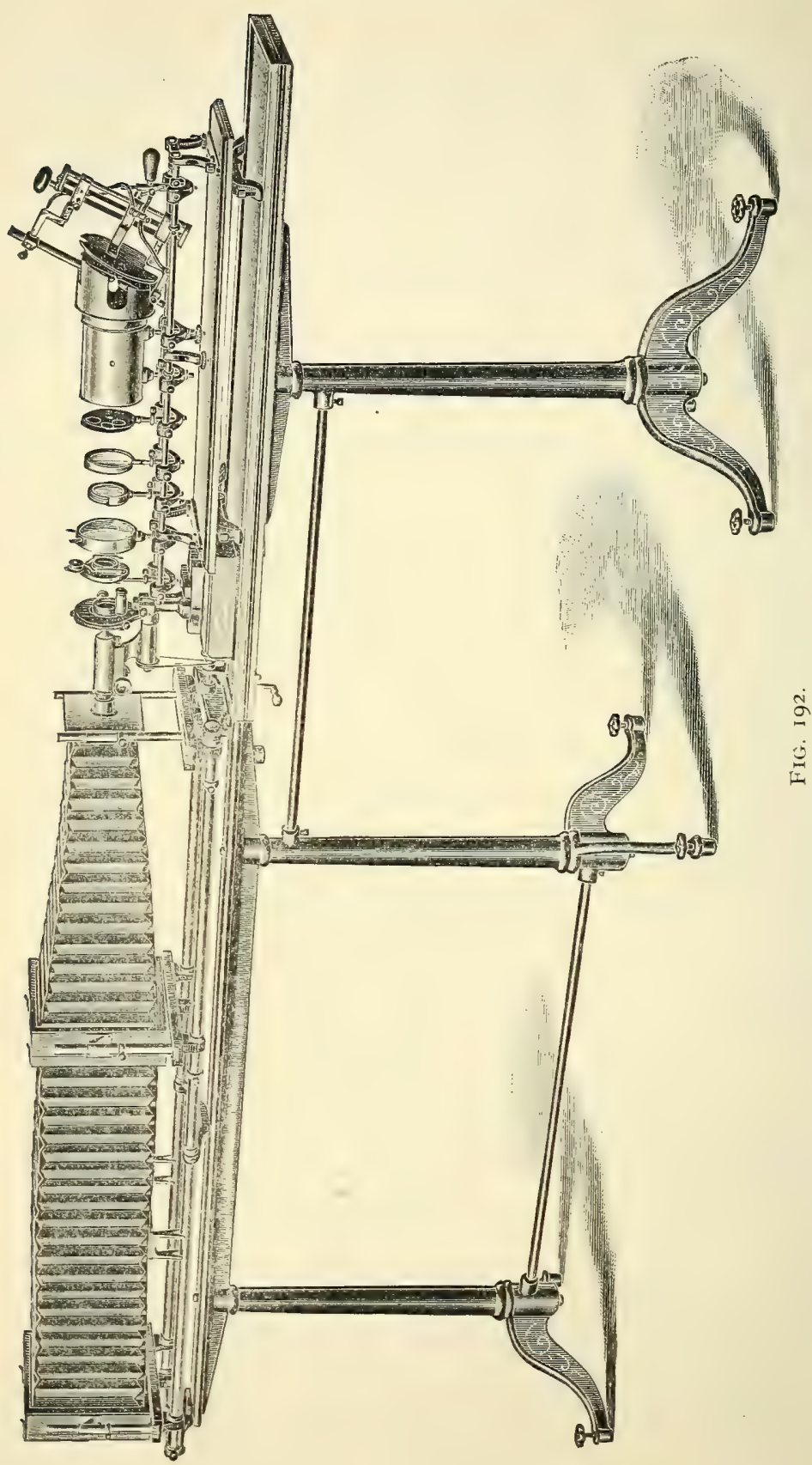


FIG. I92. Buxton's Photo-Micrographic outfit for use with the arc light. (Jour. Ap. Microscopy, I9oI, p. I367). (Cut loaned by the Bausch \& Lomb. Opt. Co.) As will be seen from the figure this apparatus is for work in the horizontal position. The optical bench containing the microscope, water bath, color screen and the electric light, swings sidewise sufficiently for the operator to arrange the specimen exactly as desired. It then swings back into position and is joined to the camera. This is in two sections for either a short or long bellows. This seems to be the most convenient of all the expensive outfits for photo-micrography.

powers it is far more convenient to employ a special microscope for metallography (micro-metalloscope.) (German, Metallmikroskop; French, Microscope pour l'étude des surfaces métalliques et des objets opaque). (Fig. 193.)

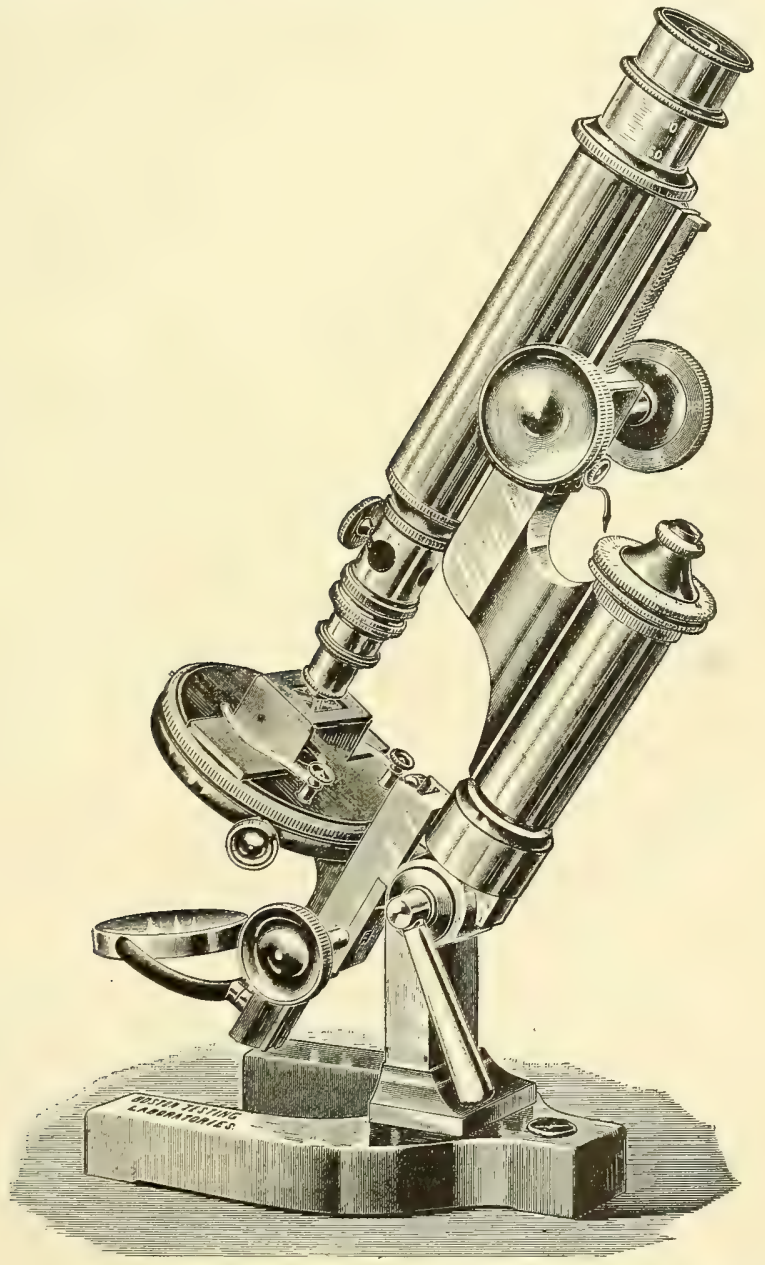

FIG. 193. Special microscope of the Boston Testing Laboratories for the study and photography of metals and alloys ( 8381 ). (Cut loaned by the Boston Testing Laboratories.) 
Such a microscope has the following general characters: The stage is movable up and down with rack and pinion, it is rotary and more or less mechanical by means of centering screws. With some at least the stage may be removed entirely. No substage condenser is present, and a mirror is only present for occasional transparent objects. A revolving nose-piece is not so good as the objective changers.

\& 382. Illumination of Opaque Objects.-(A) for 25 to roo diameters. The directions of Mr. Walmsley are excellent ('Trans. Amer. Micr. Soc., I898, p. I9I). "Altogether the best light for the purpose is diffused daylight. Proper lighting is more easily obtained with a vertical camera. An even illumination avoiding deep shadows is preferable in most cases and is more easily attained with the object in a horizontal position. For many objects it is better not to use a bull's eye or any form of condenser but for others the condenser may be needed, but when the condenser is used one must avoid too much glare. The now little used parabolic reflector and Lieberkühn serve well in many cases, but he adds "the majority yield better results under the most simple forms of illumination," $i$. $e$, with the diffused light from the window. This has been the experience of the writer also.

In case diffused daylight is employed the camera should be near a good sized window, and the object should be somewhat below the window ledge so that the illumination is partly from above and from the side. (This is easily attained with the small table and vertical camera shown in Figs. I75, I84). The vertical illuminator is advantageous for these powers also. See (B.).

(B) For Ioo to 500 diameters,-For the magnifications above 50 it is desirable and for those above Ioo it is necessary to use some form of "vertical illuminator," that is some arrangement by which the light is reflected down through the objective upon the object, the objective acting as a condenser, and from the object back through the objective and ocular to the eye of the observer. This is accomplished in two ways:

(I) By means of a small speculum-metal mirror in the tube of the microscope. This is set at an angle of 45 degrees and the light thrown into the tube upon it is reflected straight down through the objective upon the object. The speculum metal being opaque cuts out a part of the light. Instead of a metal mirror a circular disc of glass is now more frequently used. This allows the major part of the light reflected from the object, up through the objective to reach the eye.

(2) By means of a small glass 45 degree prism inserted into the side of the objective or of a special adapter. The light is from the side of the microscope, and is reflected by the prism straight down through the objective upon the object as before. *

*The idea of the vertical illuminator apparently originated with Hamilton L. Smith. He used the metal reflector. Beck substituted a cover-glass and Powell and Lealand a disc of worked glass; $i$. $e$. glass that had been carefully polishcd and leveled on the two sides. Carpenter-Dallinger, pp. 336-338.

The use of the prism with the objective is due to Tolles (see Jour. Roy. Micr. Soc., vol. iii, r88o, pp. 526, 574).

In Zeiss' catalog the prism form is figured. In the catalog of Nachet both the glass disc and the prism forms are figured. 
For both these devices uncovered objects are most successful or if the object is covered it must be in optical contact with the cover-glass. Naturally good reflecting surfaces like the rulings on polished metal bars give most satisfactory images, hence this method of illumination is especially adapted to micro-metallography. Indeed, without some such adequate method of illumination the study of metals and alloys with high powers would be impossible. So successful is it that oil immersion objectives may be used. (Carpenter-Dallinger, pp. 335-338).

\section{$\S 383$. Light for the Vertical Illuminator.-For moderate} powers one may place the microscope in front of a window, or one may use a petroleum or gas lamp. For the higher powers acetylene or preferably the electric arc light is used. In either case it may be necessary to soften the light somewhat either by a color screen or by some ground glass. The light should be concentrated upon the exposed end of the prism or into the hole leading to the glass disc. Both the prism and the disc should be adjustable for different objectives and different specimens. The cone of light, especially with the electric arc light, should be enclosed in a hollow metal or asbestos cone to avoid the glare in the eyes of the operator, and it may be necessary to soften the light with ground glass before attempting to focus and arrange the specimen. This ground glass would in most cases be removed before making the exposure $(\$ 379)$.

With the electric light and for long exposure or observation a water bath to absorb the heat rays will be necessary to avoid injuring the lenses. (See also under projection in the next chapter).

As it is somewhat difficult to adjust the light in a way to give the best effect, one can see the advantage of the adjustment for raising and lowering the stage. This will serve for all but the finest focusing, and thus avoid moving the tube for focusing enough to throw the lighting out of adjustment. It might be advantageous to have a fine adjustment on the stage also.

3.384. Mounting of Objects.-For observation only and with low powers, the objects may be mounted either in a liquid or dry as seems best. There should be a black background for most objects, then light will reach the eye only from the object. A light background is sometimes desirable, especially where one cares only for outlines.

3.395. Preparation of Metallic Surfaces.-In the first place a flat face is obtained by grinding or filing, and then this is polished. For polishing, finer and finer emery or other polishing powders are used, (rouge or diamantine, or specially prepared alumnina, etc). The aim is to get rid of the scratches so that the surface will be smooth and free from lines.

3.386. Etching.-After the surface is polished it should be etched with some substance. This etching material will corrode the less resistant material, the edges of crystals, etc., so that the structure will appear clearly. For etching, 
tincture of iodine, nitric acid in various degrees of strength, hydrochloric acid, etc., are used or one may use electricity, the metal being immersed in an indifferent liquid. See numerous articles in the Metallographist for methods and micrographs.

After the etching, the surface should be washed well with water to remove the etcher. Le Chatelier recommends that the etched surface when dry be coated with a very thin coating of collodion to avoid tarnishing. The preparation will then last for several months untarnished.

\& 387 . Mounting the Preparations of Metal.-In order to get a satisfactory image the flat, polished and etched face should be at right angles to the optic axis. For preliminary observation one can approximate this by mounting the specimen on a piece of beeswax. (Behrens). Very elaborate arrangements of the stage have also been devised (Reichert). A simple and effective device is shown in Fig. I93 in which the specimen is held against the under side of the plane face of the stage attachment. Rubber bands answer well to support the metal, and only one side need be flat.

\& 388. Photographing Opaque Objects. -The general directions given in \& 347 should be followed with the necessary modifications. The time of exposure is usually considerably greater with opaque objects than with transparent ones. Very few such objects can be photgraphed in less than 30 seconds, even with daylight For metallic surfaces and magnifications of IOO, I5O, 250 to 500 , with the electric arc light as illuminant the time required for favorable objects is $\mathbf{I}, 2$, 4 and 7 seconds; with the Wellsbach lamp the time is 5, IO, 30 and 60 minutes (Sauveur).

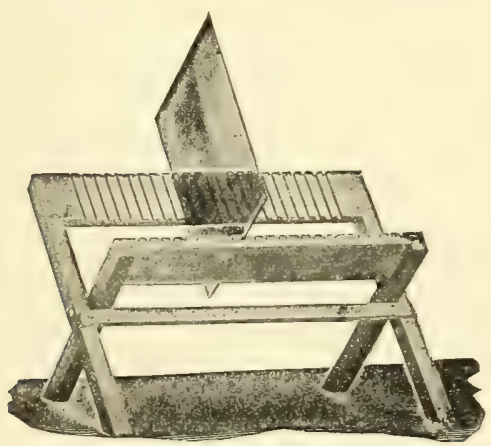

FIG. I94. Rack for drying negatives (Rochester Opt. Co).

FIG. I94.

References to Ch. VIII.

See the works and journals dealing with photography.

For Photo-Micrography see Pringle, Bousfield, Neuhauss, Sternberg, Francotte and the special catalogs on photo-micrography and projection issued by the great opticians. The Journal of the Royal Microscopical Society and of the Quekett Micr. Club; Zeit. wiss. Mikroskopie; the Trans Amer. Micr. Soc.; the Amer. Monthly Micr. Journal; the Journal of Applied Microscopy.

For the photography of metallic surfaces, see the various journals of engineering and metallurgy, but especially Sauveur's journal, the Metallographist, begun in 1898 . 
ENLARGEMENTS ; LANTERN SLIDES ; PHOTOGRAPHING

\section{BACTERIAL CULTURES}

8. 3 S9. Enlargements.-As a low power objective has greater depth of focus or penetration than a higher power $(\xi 34)$, it is desirable in many cases to make a negative of an object with considerable depth at a low magnification, and then to enlarge this picture to the desired size. As a rule negatives will not bear an enlargement of more than five diameters.

For this work the camera shown in Fig. $18 \mathrm{r}$ is excellent, and the special microscope stand shown in this figure and in Fig. I75 serves to enable one to get a very exact focus.

One must select an objective for the enlargement with a field of sufficient size to cover the part of the negative to be enlarged. An objective of 60 to $100 \mathrm{~mm}$. focus will answer in most cases.

For the illumination the camera can be elevated against the sky, or artificial light may be used. It is not easy to light so large a surface evenly by artificial light.

(A) Enlargement on Bromide Paper.-For this the negative is put in place and by pulling out the bellows the proper amount, one gets the right magnification. Focus now as for any other object, using the fine adjustment and focusing glass.

For great exactness one must put a clear glass in the plate holder and focus on the surface away from the objective. Then place the bromide paper on this clear glass and put another over it to hold it flat against the first plate of glass. The sensitive surface will then be in the exact plane of the focus and the picture will be sharp.

For the development and subsequent treatment of the paper, follow the directions of the makers.

(B) Enlargement on a Glass Plate.-One may proceed in enlarging as for making lantern slides and make a positive on a glass plate. If it is then desired to get a negative for printing, place this positive on the microscope stand and make a negative from it as if it were an object. Or one may make a contact impression as is frequently done in lantern slide making. By this method one must make three separate pictures, (I) the original photo-micrographic negative; (2) the enlarged positive from this; (3) a negative from the enlarged positive. With this negative one may print as from the original negative.

\% 390. Lantern Slides from Negatives.-In preparing lantern slides from photo-micrographic or ordinary negatives one may use the contact method, or the camera. With the camera one can enlarge or reduce to suit the particular case. The camera and special microscope stand shown in Fig. I 8 I are admirable for the purpose. For lantern slide work a photographic objective is used and the cone for enlargement removed. One may put the objective in the front of the camera or in the middle segment, making use of the little side door.

391. Photographing Bacterial Cultures in Petri Dishes, -For the successful photographing of these cultures dark ground illumination is employed on the principal stated in $\$ 92$. That is the preparation is illuminated with rays so oblique that none can enter the objective. These striking the culture are reflected 
into the objective. The clear gelatin around the growth or colonies does not reflect the light and therefore the space between the colonies is dark.

For supporting the Petri dishes a hole is made in a front board for the camera. This hole is slightly larger than the dish. Over it is then screwed or nailed a rubber ring slightly smaller than the Petri dish. This will stretch and receive the dish, and grasp it firmly so that it is in no danger of falling out when put in a vertical position. If the camera has two divisions like the one shown the board with the Petri dish is put in the front of the camera, and the objective in the middle division through the side door. Otherwise the board holding the Petri dish must be on a separate support.

The illumination is accomplished by the use of two electric lamps with conical shades. (The cheap tin shades with white enamel paint on the inside are good). The lamps are placed at the sides so that a bright light is thrown on the culture, but at such an angle that none of it enters the objective directly.

A piece of black velveteen is placed io to $20 \mathrm{~cm}$. beyond the culture. This prevents any light from being reflected through the clear gelatin to the objective. Unless some such precaution were taken the background would be gray instead of black.

One may use daylight by putting the culture in a support just outside a window, leaving the camera in the room. The rays from the sky are so oblique that they do not enter the objective. One must use a black non-reflecting background some distance beyond the dish as in using artificial light (Atkinson).

\% 392. Photographing Bacterial Cultures in Test-Tubes.-Here the lighting is as in the preceding section, but a great difficulty is found in getting good results from the refraction and reflections of the curved surfaces. To overcome this one applies the principles discussed in $\%, \mathbf{I} 4$, and the test-tubes are immersed in a bath of water or water and glycerin. The bath must have planesurfaces. Behind it is the black velvet screen, and the light is in front as for the Petri dishes. As suggested by Spitta it is well to employ a bath sufficiently thick in order that streak cultures may be arranged so that the sloping surface will all be in focus at once by inclining the test-tube.

See the works on photo-micrography and photography for the details of lantern slide making. See for the Petri dishes and test-tubes, Atkinson, Botanical Gazette, xviii (I893), p. 333 ; Spitta, Photo-Micrography (I899), p. 26. 


\section{CHAPTER IX}

\section{CLASS DEMONSTRATIONS IN HISTOLOGY AND EMBRYOLOGY}

\section{APPARATUS AND MATERIAL FOR THIS CHAPTER}

Demonstration microscopes, simple and compound (Figs. 195-I96); Traveling microscope (Fig. I97-I98); Indicator ocular (Fig. I99-20I); Marker for putting rings around the parts of specimens to be demonstrated (Fig. 6I); Projection microscope (Fig. 207); Projection objectives (Fig. 2II-2I2); Episcope (Fig. 214).

\section{DEMONSTRATION MICROSCOPES AND INDICATORS}

39.3. Simple Microscope.-The simple microscope held in one hand and the specimen in the other, has always been used for demonstration, but for class demonstration it is necessary to have microscope and specimen together or the part to be observed by the class is frequently missed. Originally blocks of various kinds to hold both microscope and specimen were devised, but within the last few years excellent pieces of apparatus have been devised by several opticians for the purpose. The accompanying figure shows one of the best forms.

FIG. I95. Simple Demonstration Microscope of Leitz ( $W m$. Krafft, N. Y.) As shown in the figure this consists of a handle, a stage and a lens holder which slides up and down for focusing. For observation the student holds it up to the light.

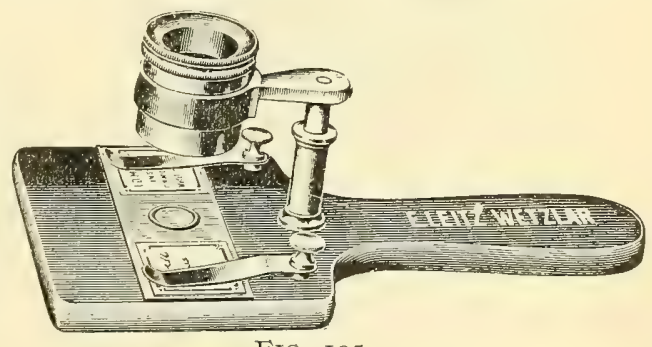

FIG. 195 .

\$394. Compound Demonstration Microscope.-This was originally called a clinical or pocket microscope. It is thus described by Mayall in his Cantor Lectures on the history of the microscope: "A small microscope was devised by Tolles for clinical purposes which seems to me so good in every way that I must ask special attention for 
it. The objective is sciewed into a sliding tube, and for roughly focusing the sliding motion suffices; for fine adjustment, the sheath is made to turn on a fine screw thread on a cylindrical tube, which serves also as a socket-carrier for the stage. The compound microscope is here reduced to the simplest form I have met with to be a really serviceable instrument for the purpose in view; and the mechanism is of thoroughly substantial character. I commend this model to the notice of our opticians."

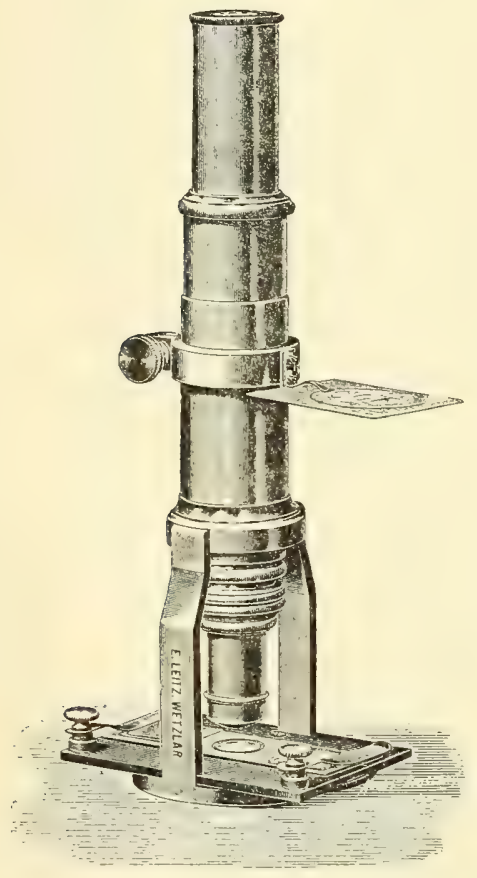

FIG. I96. Demonstration compound microscope of Leitz. Leitz now furnishes a fine adjustment in the form of an intermediate piece between the objective and the tube. This has in it a screw which is turned by a milled ring. For the objectives employed it makes an efficient fine adjustment and renders it possible for each person to adjust the microscope slightly without endangering the loss of the field.

FIG. Ig6.

Since its introduction by Tolles many opticians have produced excellent demonstration microscopes of this type, but most of them have not preserved a special mechanism for fine adjustment. With it one can demonstrate with an objective of $6 \mathrm{~mm}$. satisfactorily. It has a lock so that once the specimen is in the right position and the instrument focused it may be passed around the class. For observation it is only necessary for each student to point the microscope toward a window or a lamp. 


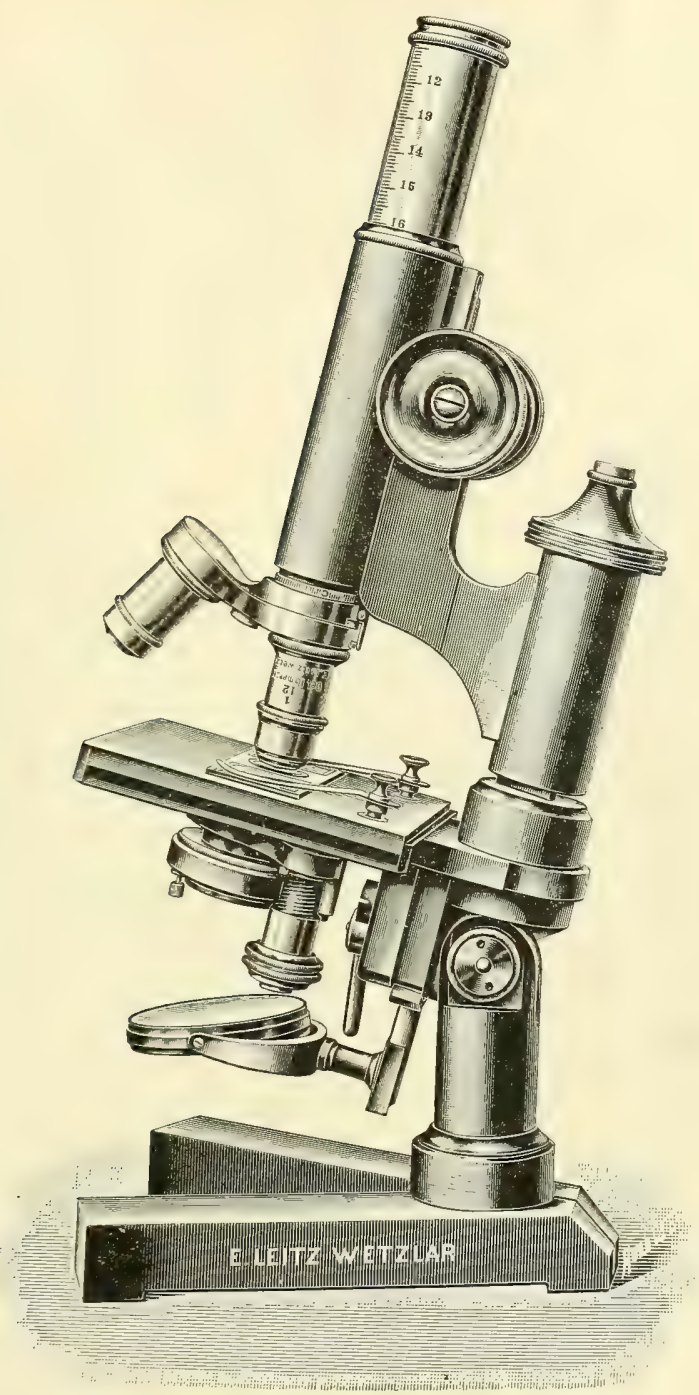

FIG. 197.

FIG. I97. Traveling microscope set up for work (Leitz; from Wm. Krafft, N.Y).

A modification of this clinical microscope was made by Zentmayer in which the microscope was mounted on a board and a lamp for illuminating the object was placed at the right position. 
$\$$ 395. Traveling Microscope.-For many years the French opticians have produced most excellent traveling microscopes. The opticians of other countries have also brought out serviceable instruments. In the one here figured $\mathrm{Mr}$. Leitz has combined in an admirable way a traveling microscope and a laboratory instrument. For the needs of the pathologist and sanitary inspector a microscope must possess compactness and also the qualities which render it usable for nearly all the purposes required in a laboratory. This instrument is a type of such apparatus which has grown up with the needs of advancing knowledge.

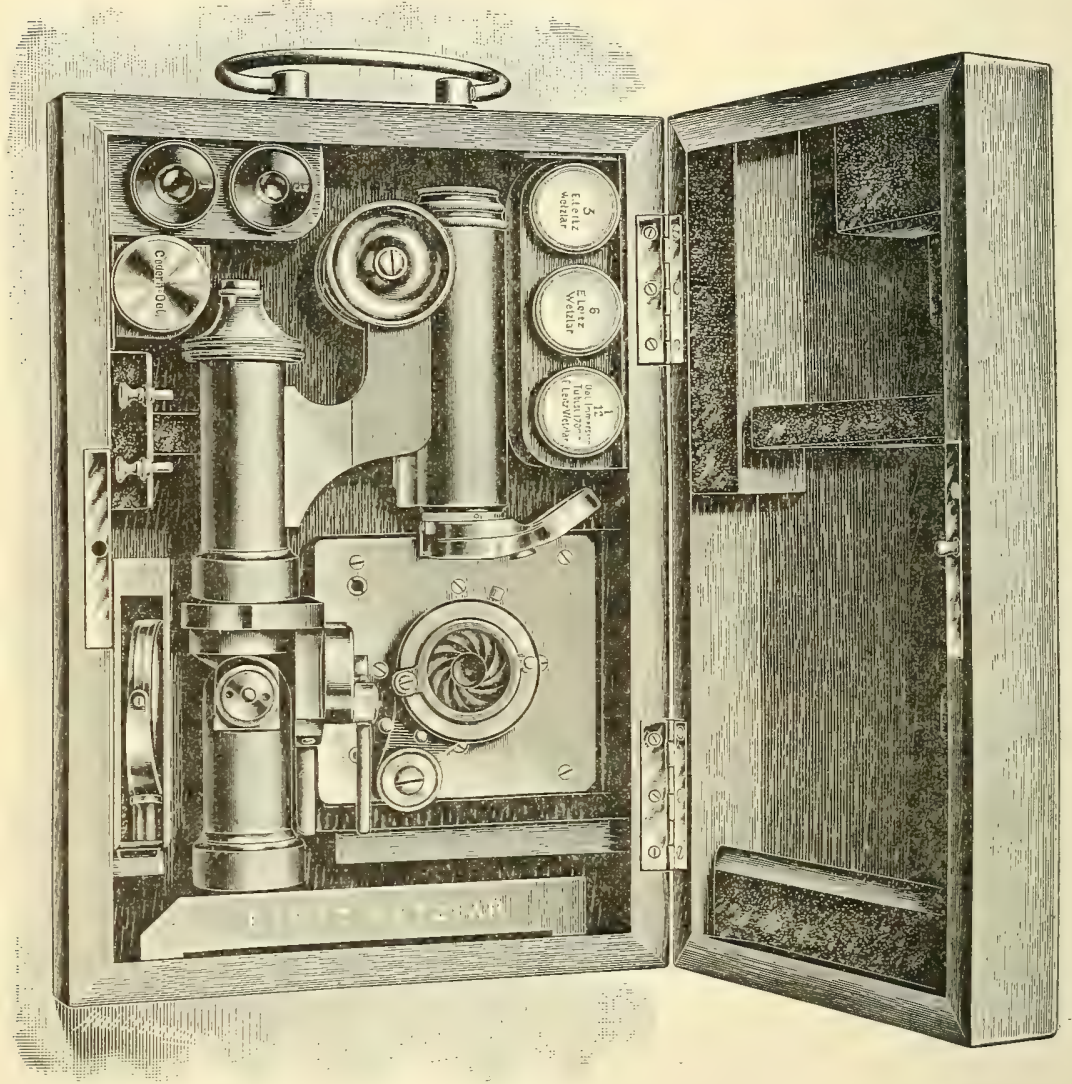

FIG. 198.

FIG. I98. Traveling microscope folded up and in its case (Leitz; from Wm. Kraffl, N.Y.). 
$\$ 396$. Indicator or Pointer Ocular.-This is an ocular in which a delicate pointer of some kind is placed at the level where the real image of the microscope is produced. It is placed at the same level as the ocular micrometer, and the pointer like the micrometer is magnified with the real image and appears as a part of the projected image ( $s$ I $)$. By rotating the ocular or the pointer any part of the real image may be pointed out as one uses a pointer on a wall or blackboard diagram. By means of the indicator eye-piece one can be certain that the student sees the desired object, and is not confused by the multitude of other things present in the field. The method of its use is indicated in Fig. 20I. This device has been invented many times. It illustrates well the adage: "necessity is the mother of invention," for what teacher has not been in despair many times when trying to make a student see a definite object and neglect the numerous other objects in the field. So far as the writer has been able to learn, Quekett was the first to introduce an indicator ocular with a metal pointer which was adjustable and could be turned to any part of the field or wholly out of the field. See Fig. 199, § 126.

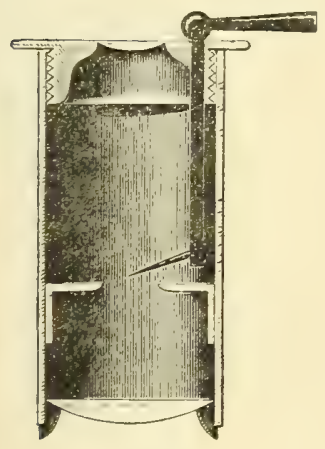

FIG. 199.

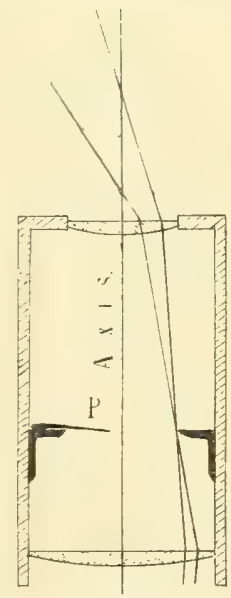

FIG. 200.

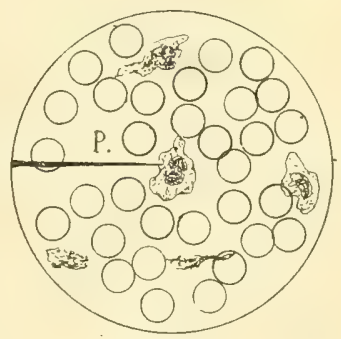

FIG. 20r.

Fig. I99. Indicator ocular with metal pointer like the one devised by Quekett ( Leitz; catalog).

FIG. 200. Indicator ocular with an eyelash (cilium) on the ocular diaphragm to serve as a pointer $(P)$. This projects about half zway across the diaphragm opening. On the opposite side are shown two rays from the microscope to indicate that the real image is formed at the level of the ocular diaphragm.

FIG. 20I. Field of the microscope with a mammalian blood preparation to show the use of the indicator $(P)$ for pointing out a white blood corpuscle. 
It is not known who adopted the simple device of putting the tip of a cat's whisker or an eye-lash on the diaphragm of the ocular as shown in Fig. 200. This may be done with any ocular, positive or negative. One may use a little mucilage, Canada balsam or any other cement, and stick the eyelash on the upper face of the diaphragm so that it projects about half way across the opening. When the eyelens is screwed back in place the hair should be in focus. If it is not screw the eye-lens out a little and look again. If it is not now sharp, the hair is a little too high and should be depressed a little. If it is less distinct on screwing out the ocular it is too low and should be elevated. One can soon get it in exact focus. Of course it may be removed at any time.

$\$ 397$ - Marking the Position of Objects.-In order that one may prepare a demonstration easily and certainly in a short time the specimens to be shown must be marked in some way. A very efficient and simple method is to put rings of black or colored shellac around the part to be demonstrated. For this the Marker, Fig. 6I-62, is employed as described on p. 66.

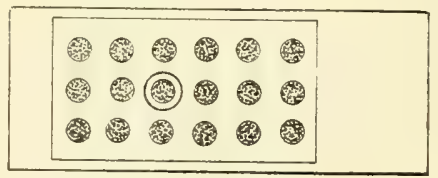

FIG. 202.

FIG. 202. Ring around one of the sections of a series for demonstrating some organ especially well.

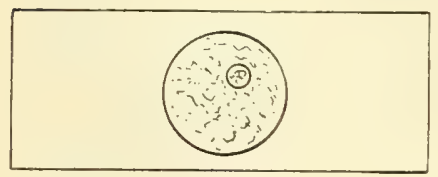

FIG. 203. Figure of a microscopical preparation with a ring around a small part to show the position of some structural feature. 


\section{PROJECTION MICROSCOPE}

One of the most useful and satisfactory means at the disposal of the teacher of Microscopic Anatomy and Embryology for class demonstrations is the Projection Microscope. With it he can show two hundred as well as one person the objects which come within the range of the instrument.

It is far more satisfactory than microscopic demonstrations, for with the projection microscope the teacher can point out on the screen exactly the structural features and organs which he wishes to demonstrate, and he can thus be certain that the students know exactly what is to be studied. Unless one employs a pointer ocular (Fig. 20I), there is no certainty that the student selects from the multitude of things in the microscopic field the one which is meant by the teacher. Like all other means, however, the projection microscope is limited. With it one can show organs both adult and embryonic, and the general morphology. For the accurate demonstration of cells and cell structure the microscope itself must be used by each student personally.

If no ocular is used a $3 \mathrm{~mm}$. or $1 / 8$ inch objective is about as high a power as can be employed in a room holding two hundred. Even with an oil immersion fair demonstrations can be made, but up to the present time really successful demonstrations are usually made with powers below $3 \mathrm{~mm}$. rather than above. If projection oculars are used one can hardly go beyond an $8 \mathrm{~mm}$. objective with real satisfaction. And finally it should be remembered that the Continuous Current Arc Lamp must be employed for satisfactory results. Alternating currents are wholly unsatisfactory for this purpose.

$\$ 398$. Projection Microscope.-This is an arrangement of the microscope so that an image of the object under the microscope is thrown upon a screen of some kind. The picture on the screen is looked at precisely as one looks at the pictures thrown on the screen by an ordinary magic lantern. Indeed the projection microscope is a magic lantern with short focus objectives. One of the first uses of the microscope was to throw the images of various objects on a screen so that they could be seen by several persons at once, and the light used was sunlight. Hence those early projection microscopes were called solar or sun microscopes. If sunlight were available at all times and could be controlled, it would be universally employed; but as it is not 
at all times available and whenever available a heliostat is needed to keep the light fixed in a given position, sunlight is practically discarded and the electric light is employed for illumination.

$\$$ 399. Parts of a Projection Microscope.-These are named in order, beginning with the electric lamp: See Figs. I92, 207-208.

(I) An arc lamp with constant current and rheostat adjustable from about 8 to 20 amperes.

(2) Lamp condenser.

(3) Water bath for removing the heat rays.

(4) Special achromatic condenser for high powers.

(5) Large square stage with opening 6 centimeters in diameter.

(6) Mechanical stage with wide range of movement.

(7) A very wide tube for receiving the objectives and projection oculars. This tube to be connected with coarse and fine adjustment for focusing.

All of these parts should be independent and adjustable so that any one of them can be adjusted or removed without interfering with the others.

In accordance with the suggestion of Dr. Coplin all of the apparatus, including the mountings of the objectives should be dead black to avoid reflections. Reflections are so dazzling that the operator cannot properly focus the image on the screen.

$\$$ 400. The Arc Lamp for the Projection Microscope.-This should have the following characters: (a) The carbons should be tilted so that the crater in the positive carbon is nearly vertical. It then sends the maximum number of rays to the lamp condenser. (b) The lower carbon should be slightly in advance of the upper one as shown in Fig. 204 so that the crater in the positive carbon is on the front of that carbon (Figs. 204-205) that is it should be in position to throw the light toward the condenser.

(c) The lamp must be adjustable vertically and horizontally, and the carbon holders must also be adjustable so that the carbons may be put in line from side to side and front to back. One cannot get satisfactory results unless all these adjustments are possible.

Hand-feed and automatic lamps are both used for projection. The consensus of opinion among experts is that the hand-feed lamp is better for photography and for projection. That has been the experience of the writer also.

$\$ 40$ I. Starting the Lamp.-For starting the lamp with the hand-feed form it is necessary to bring the carbons in contact and then 
when the current is established to separate them slightly (I to $2 \mathrm{~mm}$.) in order to get a satisfactory light. The carbons may be brought in contact before turning on the current or afterwards. An automatic lamp will start as soon as the current is turned on, but here also the carbons must be slightly separated, or one must wait a short time for the carbons to wear away before the best light is obtained.

5 402. Angle of the Carbons.-Originally arc lamps for use with the lantern had the carbons both vertical. The "projector" lamps used at sea, had, however, the carbons inclined at an angle of 30 or 40 degrees from the vertical. Lewis Wright (Optical Projection, p. I63,) states that at his urgent request a projection arc lamp for micro-projection was made with inclined carbons. Certain it is that all projection lamps have now the inclined carbons. The angle of inclination varies with different makers. The lamps furnished by Zeiss and Reichert with their apparatus has the carbons at 40 degrees from the vertical ; Behrens uses aud recommends 45 degrees. Barnard and Carver (J. R. II. S., I898, p. I70) found by a careful series of experiments that an angle of about 27 degrees gave the most satisfactory light. One firm (A.T. Thompson \& Co. of Boston) arranges the carbons at right angles, the upper or positive carbon being horizontal. This gives good results. The purpose of the inclination is to turn the crater toward the condenser (Fig. 205), for it is now appreciated that the arc proper gives very little light comparatively. One will appreciate this by studying the carbons projected on the screen as suggested in $\S 406$.

+03. Adjusting the Carbons. - In many arc lamps for projection there is a metal shelf to show approximately where the two carbons should meet. If one places the carbons in their holders so that the ends are at the level of this shelf they will be nearly in the correct position.

The lower carbon should be slightly in advance of the upper one (Fig. 204). This will insure the formation of the crater on the side facing the condenser.

+04. Length of the Arc. - It has been found by careful investigation (Carhart, Ayrton) that the maximum brilliancy and efficiency of a continuous current arc lamp are obtained when the arc is about I $\mathrm{mm}$. long and the current is about at its maximum for the size of carbon used. If the carbons are too far apart the light becomes purplish. If the current is too weak the lower carbon is blunt, while with a stronger current it becomes more pointed, and hides less light ; it also contributes a share of the illumination from its white tip (Fig. 205). 
In order to see whether the carbons are in the most favorable position, Barnard and Carver (J. R. M. S... 898 , p. I7 I) used a pin-hole camera at right angles to the carbons. This camera has a ground glass with cross lines to serve as guides in maintaining the proper position of the carbons.

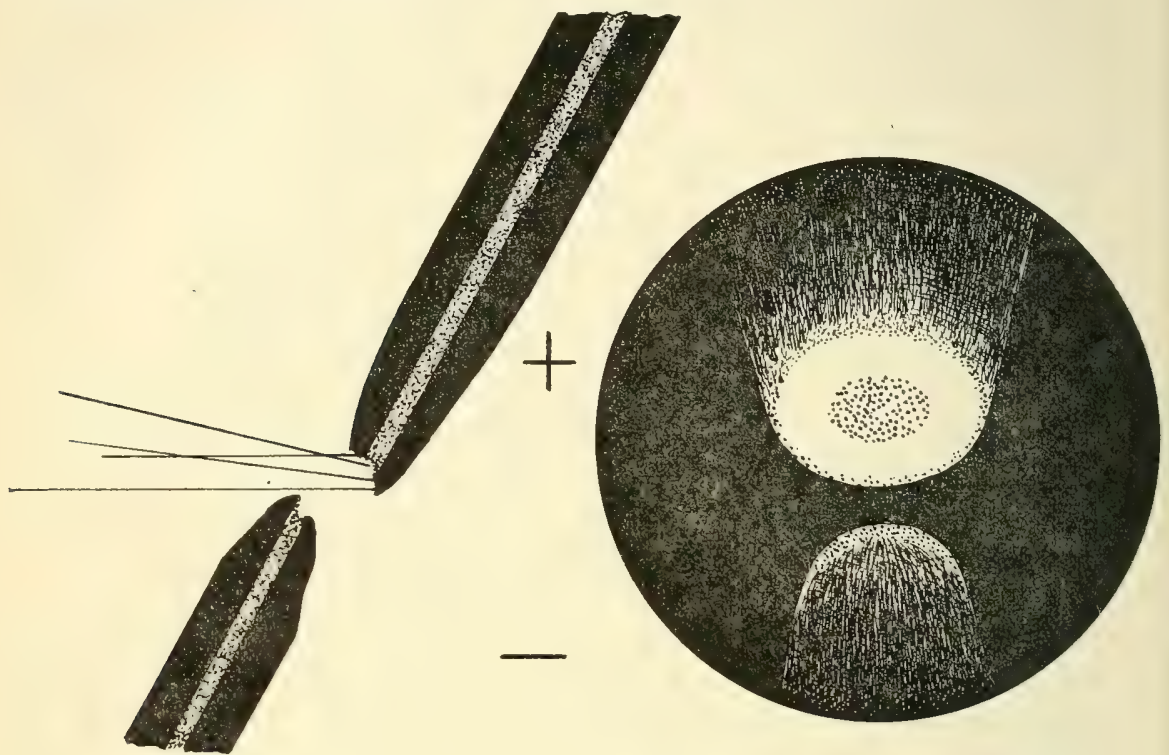

FIG. 204.

FIG. 205

FIGS. 204-205. Front and side views of the carbons of an arc light to give the best illumination. + and - indicate the positive and negative poles

Fig. 204 is a side view showing the carbons in section at an angle of 30 degrees from the vertical and the negatize (-) or lower carbon slightly in front of the positive $(+)$ or upper carbon. The carbons have soft cores.

Fig. 205 is a front view of the carbons as seen projected on the screen with a 42 $m m$. objective. It is a projection of the real image of the carbons formed by the special achromatic condenser next the object $(8.399,4)$. This figure shows that the source of light is the crater in the positize (-) or upper carbon; it shows also that the lower carbon is slightly below the lower carbon as well as slightly in front. This avoids a shadow from the lower carbon.

In the center of the crater is shown a slight shadow. This is due to the pit formed in the soft core of the carbon.

405. Positive and Negative Carbon.-The mechanism of the lantern is arranged so that the upper carbon should be positive $(+)$. In setting up the apparatus one may not be able to insert the wires correctly at first. All one has to do is to make the connections, 
turn on the current and observe which carbon is the more brilliant. As shown in figure 205 the brilliant carbon is at the positive pole. If now the upper carbon is brilliant the wires are properly connected; but if the lower carbon is the brighter, the wires are inserted in the wrong binding posts and should be reversed. In observing the carbons when the current is on, one should use deeply colored glass to avoid injuring the eyes. Another excellent method is to turn the current off after a minute or two and look at the carbons directly. The one at the positive pole will be red or white hot while the other will be black or very dull red.

406. Character of the Carbons and Steadiness of the Light. -One needs a steady light for photography and for projection. To hold the crater in one position and thus render the light steady, a softer core is placed in the carbons (Fig. 204). This serves as a guide, and the crater forms symmetrically around it when the carbons are in a proper position (Fig. 205). Every one who wishes to make a success of micro-projection is urged to study the appearance of the carbons by using a low objective ( 35 to $65 \mathrm{~mm}$.). The real image of the carbons formed by the achromatic condenser next the object $(\$ 399,4)$ can be focused and thrown on the screen as if it were an object and one can study the crater. The image on the screen will be right side up as the achromatic condenser inverts it once and the objective reinverts it (no projection ocular being used).

If the ordinary hard carbons, without soft core, are used the crater shifts its position and thus gives a wavering light. The soft cored carbons give a very steady light as the crater remains constant in position.

Sometimes a small hard carbon is used for the lower or negative side and a large soft cored carbon for the upper or positive pole. This works admirably. The sizes used by Ayrton were for the positive carbon $13 \mathrm{~mm}$. diameter; for the negative I $\mathrm{mm}$.

407. Rapidity of Wear in the Carbons.-If one employs two carbons equal in size and composition the positive carbon wears away twice as fast as the lower one, therefore one will find the feed mechanism in many lamps moves the upper carbon down twice as fast as the lower carbon moves up. This keeps them centered. If one uses a large carbon above and a small one below, and if the sizes of upper and lower carbon are properly selected, the two carbons wear away equally in length and the feed mechanism of the lantern should move the upper carbon down and the lower one up at the same rate to insure constant centering. 
The rapidity of wear in the carbons, irrespective of their position, depends upon the amount of the current. It is uneconomical to use more current than necessary, both on account of the cost of the electricity and the wear of the carbons. It is inconvenient to change the carbons too frequently. It is certainly inconvenient to be compelled to insert new ones during a demonstration.

$\$$ 408. Size of Carbons and Amount of Current.-The size of the carbons must be proportioned to the amount of current used. For an amperage not exceeding 15 , a soft cored carbon of $\mathrm{I} 2 \mathrm{~mm}$. ( $\mathrm{I} / 2$ inch) will answer, but if 20 amperes of current are used then the carbon should be $16 \mathrm{~mm}$. (or $\frac{5}{8}$ inch) in diameter. If one uses too small a carbon for the current, the carbon partly burns, and really less available light is produced for the projection.*

$\$$ 409. Amount of Current for Micro-Projection.-For a lecture room holding 200 and a screen distance of 8 meters ( 26 feet), one can demonstrate almost any suitable preparation with a current of Io to 12 amperes, the voltage being I IO. This serves for objectives as high as $3 \mathrm{~mm}$. when no ocular is used. According to Behrens (Zeit. wiss. Mikr., I 898, pp. 7-23) one cannot make available more than 20 amperes for any micro-projection. The makers of micro-projection apparatus almost invariably make a 20 ampere lamp the limit.

For getting good results it is vastly more important to have all the parts of the apparatus centered and the carbons in the proper relative position than to use a powerful current. The light cannot serve for projection unless it is properly used $(\S 406)$.

One will be surprised to see how excellent the results are with an amperage of Io or 12 when one makes the most of the light. With some preparations one needs more light, and must increase the amperage. Remember that the skill of the operator is of equal account with the amperage. Do not expect the lantern to furnish brains as well as light.

SIIO. Lamp Condenser. -This is a large condenser next the radiant and it serves to collect the light emitted from the crater and

*For the experiments made in preparing this chapter, and for practical use during the last two years, the carbons most employed are designated: "High grade Electra, Nurnberg carbons, soft cored, I $2 \times 190 \mathrm{~mm}$. Other forms were also used in the experiments, sometimes the upper carbon was soft cored and the lower one solid. With study and careful experiment one can get good results with a variety of currents and carbons. The beginner is advised, however to, start with the carbons recommended and furnished by the makers of the arc lamp which he is to employ. 
condense it either upon the object for lantern slides and low objectives, or it narrows the light into a cone of the proper size for further concentration by the achromatic condenser $(\$ 4$ I2). One of the most used, and also one of the best condensers for the arc lamp is composed of two plano-convex lenses with their convex sides facing each other. There is then one plane face next the radiant and one toward the microscope. The lens next the radiant is somewhat smaller than the other. Both are loosely mounted to allow for expansion and the cell in which they are mounted should be freely ventilated. This condenser should be adjustable back and forth and up and down.
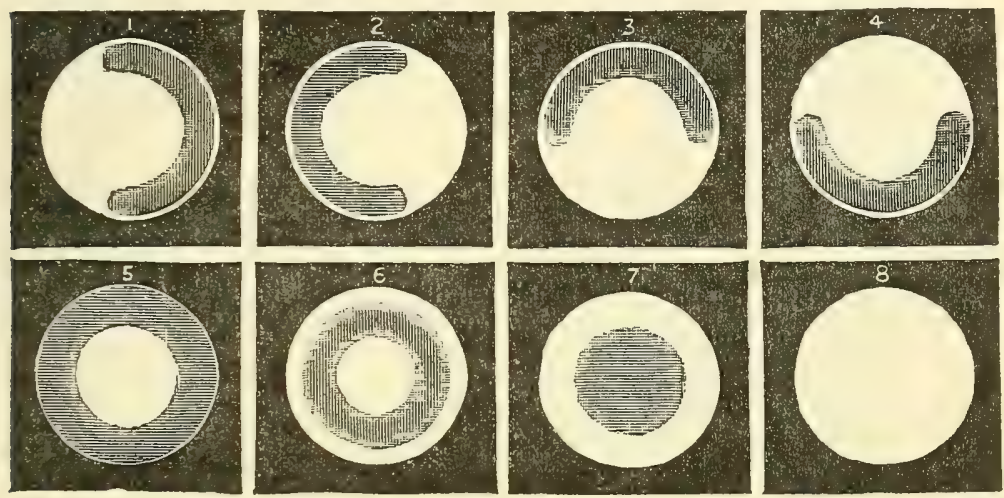

FIG. 206.

FIG. 206. Arrangement and Centering of the Radiant (Leiss).

In (I) The radiant, i. e., the crater (Fig. 205) is too far to the right,

(2) The crater is too far to the left;

(3) The crater is too high;

(4) The crater is too low;

(5) The crater is too far from the lamp condenser;

(6-7) The crater is too near the condenser.

(8) The crater is in the correct position.

As pointed out in the explanation of Fig. 205, there may be a slight central shadow with soft cored carbons when the lamp and condenser are in the best relative position.

$\S 4 \mathrm{II}$. Water Bath for Removing Heat.-This is a cell of some kind with plane glass faces. It should be approximately of a size to allow the light to pass through a stratum of water $50 \mathrm{~mm}$. thick. Even this will allow something over $2 \%$ of the heat to pass. It is a great advantage to have cool or cold water circulate through this constantly. 
The water bath should be free from the condenser mounting so that it may not be heated by conduction (see $\$$ II 3 for special cooler).

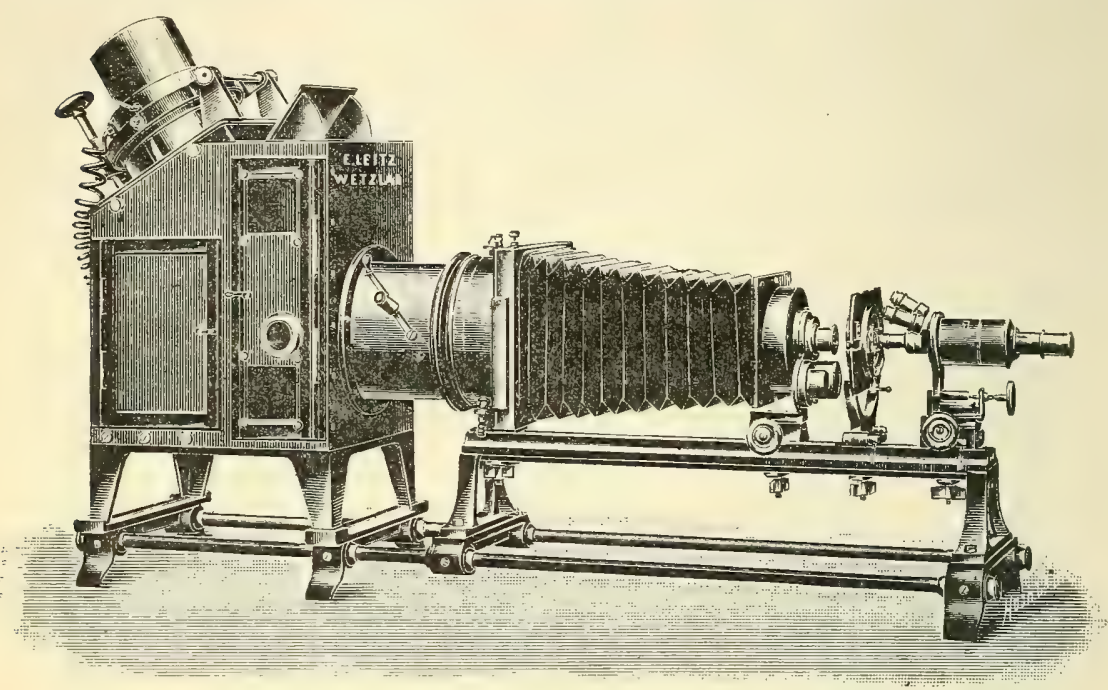

FIG. 207.

FIG. 207. Leitz Large Micro-Projection Apparatus (Leitz Catalog).

In this figure the apparatus is in position for projection with a projection ocular. As here shown it consists of :-

(I) An arc lamp designed for a current up to 20 amperes;

(2) A lamp condenser of three lenses, the two inner ones being adjustabla;

(3) A large water bath for removing the heat;

(4) Bellows. At one end is a space for the insertion of ordinary lantern slides (set the next figure) and at the other end are three condensers of different foci on a revolving nose-piece;

(5) Objective stage with a special cooler (Zoth's \& 4I3), and a special slide carrier serving as a kind of mechanical stage.

(6) An objective carrier. This contains a triple, revolving nose-piece at the end of the large tube. This tube contains an iris diaphragm and receives an adapter for the use of projection oculars. For low objectives and when the projection ocular is not to be used, this adapter is removed. Fine and coarse adjustments are present for focusing.

Each part is independent and capable of special adjustment. (As shown in the next figure, the microscope part may be turned aside, leaving the apparatus for ordinary lantern slide projection.)

\section{\$ 412. Special Achromatic Condenser for High Powers.-} For objectives of $8 \mathrm{~mm}$. and higher there should be a special achromatic condenser next the stage for holding the specimens. This should 
be adjustable and with centering attachment. For objects which would be injured by the heat allowed to pass the large water bath, a special cooler is used next the specimen. For such cases the achromatic condenser must be especially constructed or the light would not be focused on the object, and one would not get sufficient brilliancy for high powers. Special condensers are now made admitting the use of the special cooler, and if one has delicate objects which he desires to project, like infusoria, or if he wishes to demonstrate the circulation of the blood or living objects he should make provision for it in ordering his apparatus.

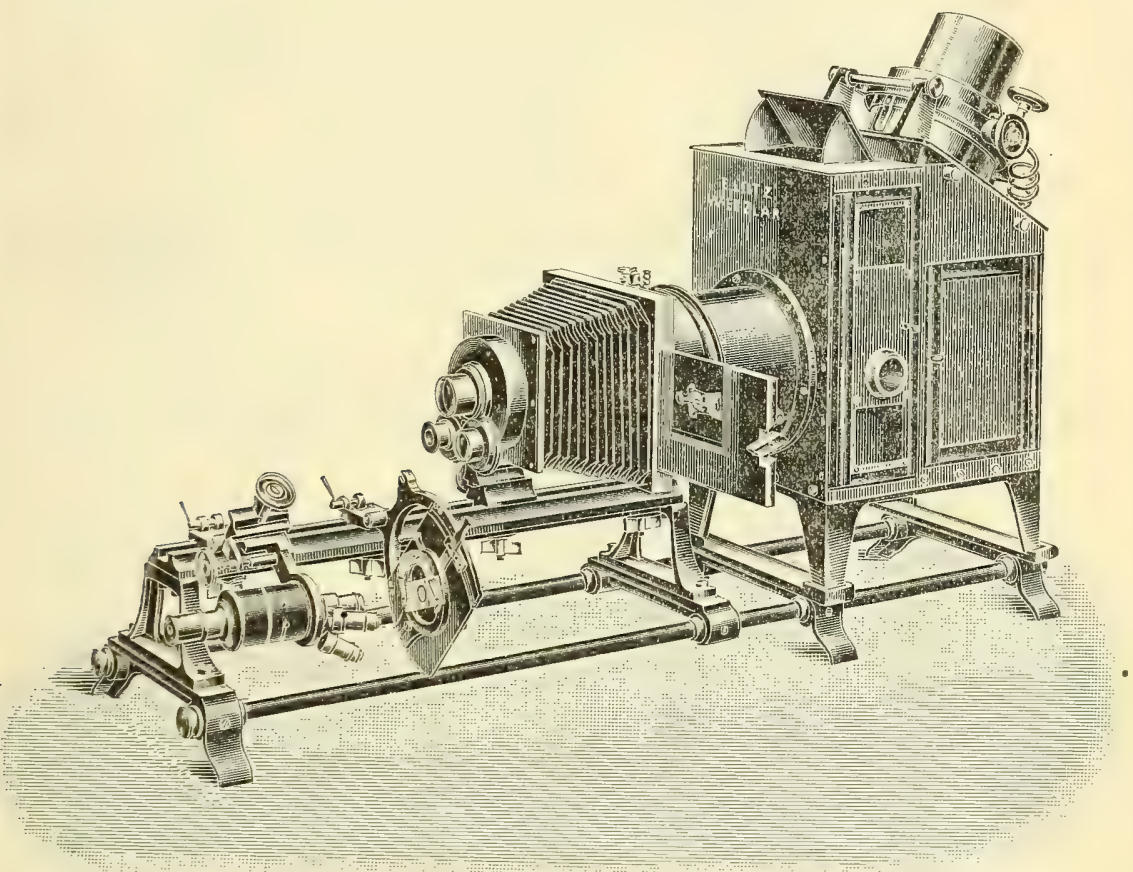

FIG. 208. N. $Y$.)

Fig. 208. Leitz' Large Projection Apparatus (cut loaned by Wm. Krafft,

As here shown the microscope part is tumed aside, and the lozest of the three condensers ( $300 \mathrm{~mm}$. equiv. focus) is in place for projecting ordinarylantern lides.

4I3. Specimen Cooler.-In order to project living objects and delicate preparations the heat passing the large water bath must be still farther eliminated. This is accomplished by a device of Zoth's 
(Zeit. wiss. Mikr., I893, p. I 52, J. R. M. S., I 894, p. I I 2). It consists of a metal box with glass covers. Through this box is circulated cold water, and the specimen rests directly on the cooler. With this the temperature at the focus of the special condenser ( $\$ 4$ I2) rarely exceeds $27^{\circ}$ centigrade.

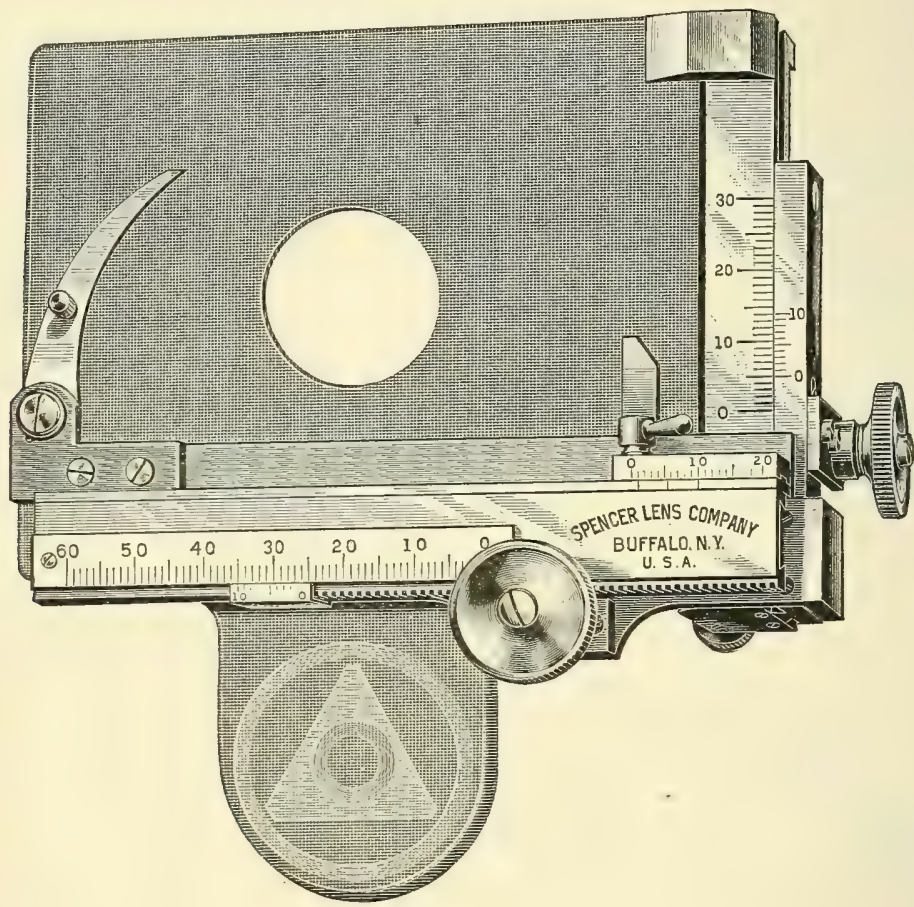

FirG. 209.

FIG 209. Spencer Winkel Mechanical Stage. (Spencer Lens Co.) This stage is convenient for use with the projection microscope as it can be attached to any square stage and has a great range of motion. This large range is especially necessary for series of organs or embryos. For the projection microscope it would be better to have both milled heads for moving the stage on the side as in the next.

$\$ 4$ I4. Stage for Specimens.-This should be large and preferably square or oblong. The central opening should be 50 to $60 \mathrm{~mm}$. in diameter, and have a large iris to lessen this diameter at will. The specimen stage should be on an independent support and adapted for independent motion. This is necessary so that all objects capable of projection may be used on the stage and moved far enough from the 
microscope for focusing and for the best position of the specimen in relation to the other parts of the apparatus $(\$ 420$. ) The stage should be very rigid.

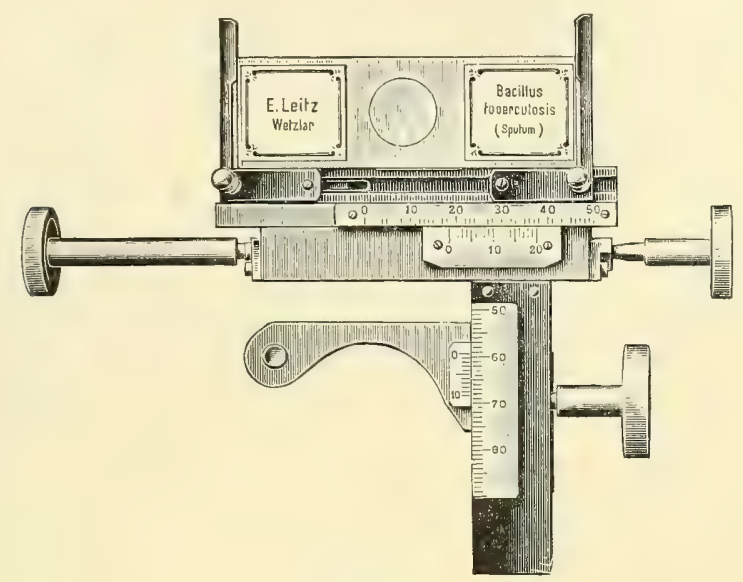

Fig. 210

Fig. 210. Mechanical Stage of Leitz. (Cut loaned by Wm. Krafft, N. Y.) This has the advantage of having both milled heads at the side. It has the disadvantage of not being readily attached to the stage of a projection microscope.

\$ 4I5. Mechanical Stage.-For projection work this is almost a necessity. While one is demonstrating there should be no time and no energy wasted in finding the object. Preferably the milled heads of the stage should be at the side, and the stage should be easy to remove and put back into position.

It should have sufficient range of motion to enable one to demonstrate any section on a slide of serial sections.

$\$ 4$ I6. Objective Carrier.-This like the stage should be on an independent support. There should be both fine and coarse adjustment. The tube should either be very short, or very large to avoid restricting the field. For projection oculars there must be an adapter for using them, and the adapter must be long enough to produce the proper tube-length ( $160 \mathrm{~mm}$.). As with the stage the objective carrier should be very substantial.

$\$ 417$. Objectives to Use in Micro-Projection.-One rarely needs an objective lower than $100 \mathrm{~mm}$. focus or higher than $3 \mathrm{~mm}$. The majority of the work with a screen distance of 5 to 8 meters will be done with objectives of $60-75,25-40,16,8,6$ or $3 \mathrm{~mm}$. focus. For the 
three kept on the nose-piece most constantly a 64,42 and $6 \mathrm{~mm}$ is as good a combination as one can get. Other powers should be available, however, and for special specimens and occasions one may employ the two $\mathrm{mm}$. oil immersion. For powers above $20 \mathrm{~mm}$, ordinary objectives are more satisfactory than most projection objectives.

$\$ 4$ I8. Projection Oculars (Fig. I85). - If one uses the apochromatic objectives of $\mathrm{I} 6$ and $8 \mathrm{~mm}$. focus the projection oculars are used with them. They can also be used with wide angle achromatic objectives. With them one can get an enormous magnification even with the $16 \mathrm{~mm}$. objective. (See the table below, $\S 42 \mathrm{I}$.).

These oculars restrict the field greatly and in the writer's experience it seemed on the whole more desirable for most objects not to use the projoction oculars. They cannot be used advantageously with objectives higher than 8 or $6 \mathrm{~mm}$. and are most satisfactory with one of $16 \mathrm{~mm}$.

In using the projection ocular with a $16 \mathrm{~mm}$. objective one can place the special achromatic condenser next the stage, but if the ocular is not used one must either do without a specimen condenser, or take one of longer focus, if he has two or three as shown in Fig. 207.

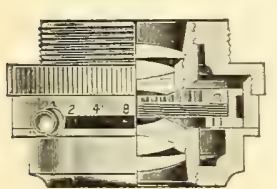

FIG. 211 .

FIG. 2II. Zeiss' Micro-Planar for projection. (Cut loaned by Bausch \& Lomb Optical Co.)

The micro-planars are of $20,35,50,75$, and $100 \mathrm{~mm}$. equivalent focus. When used for projection the special iris in each should be wide open. These are used without a projection ocular.

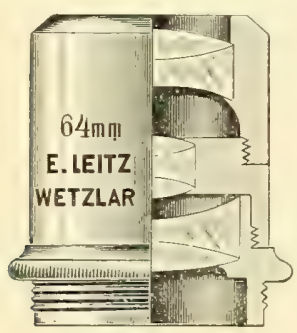

Fig. 2 I2 A.

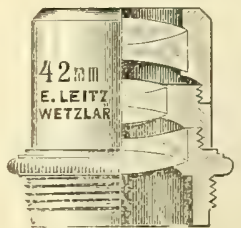

FIG. 2 I2 B.

FIG. 212. Leitz objectives of $64(A)$, and $42, m m . B)$. For micro-projection. (Cut loaned by Wm. Krafft, N. Y.)

These are used without a projection ocular. The iris in the $64 \mathrm{~mm}$. objective should be wide open for micro-projection.

fig. Arrangement of the Parts of the Projection Microscope.-As stated in section 399, all the parts of the apparatus should 
be adjustable. This is because the arrangement needs to be somewhat different for different objectives.

The guiding principle is that the specimen should be lighted by a converging cone of light, and it should be lighted by the entire cone of light traversing the lamp condenser. If one uses a white card it is easy to determine the position and size of the cone of light. If it is too large for the specimen, either the lamp condenser is too near the radiant or the specimen is too close to the lamp condenser.

If one uses an achromatic condenser the lamp condenser and the achromatic condenser should be so arranged that the entire cone of light traversing the lamp condenser can enter the achromatic condenser. If the cone is too large they are too close together, or the lamp condenser is too near the radiant. If the cone is too small then the lamp condenser is too far from the radiant or the achromatic condenser, or perhaps both faults are present. One must remember in all his experiments that a converging cone of light should be used and not a diverging one. The specimen must then not be beyond the focus of the lamp condenser.

If one wishes to make micro-projection a success it will be necessary to give the apparatus the requisite time and thought. Try to understand the conditions of success and continue experimenting until you have learned to make it possible for the machine to do its best for you. The satisfaction of showing a class real things is sufficient reward for all the trouble.

$\$ 420$. Screen and Screen Distance.-For a screen nothing is so good as a dead-white, smooth wall. A lusterless, white cloth screen answers well also. It is an advantage to have this entirely opaque, so that none of the light can pass through it. One must remember that the light passing through the minute lenses of the objective must be spread out over a great space even with low powers, and over a much greater with high powers, so that one cannot afford to have any of the light lost by transmission through the screen. All are agreed that for micro-projection a translucent screen with the projection apparatus behind it is not desirable, although for ordinary lantern slide projection it answers fairly well.

The distance of the screen from the microscope depends largely on the size of one's audience. The writer has found a distance of eight meters (26 feet) good for both low and high power projection. This distance answers well for a class of 200 persons. 
For the minute details of a projected specimen it is recommended that the audience use opera glasses. These are also useful for the operator in focusing the image on the screen.

TABLE SHOWING THE SIZE OF OBJECT, THE MAGNIFICATION AND THE SIZE OF THE SCREEN IMAGE WITH VARIOUS

OBJECTIVES AND PROJECTION OCULARS

Distance of the screen from the stage of the microscope, 8 meters $(26$ feet +$)$. Arc light, 12 ampere current, for illumination.

\begin{tabular}{|c|c|c|c|c|c|c|}
\hline Objective & $\begin{array}{l}\text { Projection } \\
\text { Ocular }\end{array}$ & $\begin{array}{l}\text { Achromatic } \\
\text { Condenser }\end{array}$ & $\begin{array}{c}\text { Actual size of } \\
\text { Field }\end{array}$ & Magnification & \multicolumn{2}{|c|}{$\begin{array}{c}\text { Diameter } \\
\text { of Screen } \\
\text { Image }\end{array}$} \\
\hline $64 \mathrm{~mm}$. & none & none & $20 \mathrm{~mm}$. & $125-$ & 250 & $\mathrm{~cm}$. \\
\hline $42 \mathrm{~mm}$. & none & none & $10 \mathrm{~mm}$. & I 85 & I85 & $\mathrm{cm}$. \\
\hline $35 \mathrm{~mm}$. & none & none & $9 \mathrm{~mm}$. & 230 & 207 & $\mathrm{~cm}$. \\
\hline $24 \mathrm{~mm}$. & none & none & $7 \mathrm{~mm}$. & 335 & 235 & $\mathrm{~cm}$. \\
\hline $\begin{array}{l}\text { IS } \mathrm{mm} . \\
\because \mathrm{mm} . \\
\text { " } \mathrm{mm} .\end{array}$ & $\begin{array}{l}\text { none } \\
\times 2 \\
\times 4\end{array}$ & $\begin{array}{c}\text { none } \\
\text { condenser } \\
\text { condenser }\end{array}$ & $\begin{array}{l}6 \mathrm{~mm} . \\
\text { I. } 5 \mathrm{~mm} . \\
1.5 \mathrm{~mm} \text {. }\end{array}$ & $\begin{array}{r}440 \\
860 \\
1640\end{array}$ & $\begin{array}{l}264 \\
\text { I } 30 \\
245\end{array}$ & $\begin{array}{l}\mathrm{cm} . \\
\mathrm{cm} . \\
\mathrm{cm} .\end{array}$ \\
\hline $\begin{array}{l}\text { I6 } \mathrm{mm} . \\
\text { " } \mathrm{mm} . \\
\mathrm{mm} .\end{array}$ & $\begin{array}{l}\text { none } \\
\times 2 \\
\times 4\end{array}$ & $\begin{array}{c}\text { none } \\
\text { condenser } \\
\text { condenser }\end{array}$ & $\begin{array}{r}4 \mathrm{~mm} \\
\text { I. } 25 \mathrm{~mm} \text {. } \\
\text { I } \mathrm{mm} \text {. }\end{array}$ & $\begin{array}{r}530 \\
1090 \\
2040\end{array}$ & $\begin{array}{l}200 \\
136 \\
204\end{array}$ & $\begin{array}{l}\mathrm{cm} \text {. } \\
\mathrm{cm} \text {. } \\
\mathrm{cm} \text {. }\end{array}$ \\
\hline $\begin{array}{l}8 \mathrm{~mm} . \\
\text { " } \mathrm{mm} . \\
\text { "mm. }\end{array}$ & $\begin{array}{l}\text { none } \\
\times \quad 2 \\
\times 4\end{array}$ & $\begin{array}{l}\text { condenser } \\
\text { condenser } \\
\text { condenser }\end{array}$ & $\begin{array}{l}3+\mathrm{mm} \\
0.60 \mathrm{~mm} \text {. } \\
0.60 \mathrm{~mm} \text {. }\end{array}$ & $\begin{array}{l}1000 \\
2250 \\
4500\end{array}$ & $\begin{array}{l}300 \\
140 \\
270\end{array}$ & $\begin{array}{l}\mathrm{cm} . \\
\mathrm{cm} \text {. } \\
\mathrm{cm} \text {. }\end{array}$ \\
\hline $\begin{array}{l}6 \mathrm{~mm} . \\
\therefore \mathrm{mm} . \\
\text { " } \mathrm{mm} .\end{array}$ & $\begin{array}{l}\text { none } \\
\times 2 \\
\times 4\end{array}$ & $\begin{array}{l}\text { condenser } \\
\text { condenser } \\
\text { condenser }\end{array}$ & $\begin{array}{l}2 \mathrm{~mm} \text {. } \\
0.50 \mathrm{~mm} \text {. } \\
0.50 \mathrm{~mm} \text {. }\end{array}$ & $\begin{array}{l}1200 \\
2550 \\
5000\end{array}$ & $\begin{array}{l}240 \\
\text { I } 30 \\
250\end{array}$ & $\begin{array}{l}\mathrm{cm} \text {. } \\
\mathrm{cm} \text {. } \\
\mathrm{cm} \text {. }\end{array}$ \\
\hline $\begin{array}{l}5 \mathrm{~mm} . \\
\mathrm{mm} .\end{array}$ & $\begin{array}{l}\text { none } \\
\times 2\end{array}$ & $\begin{array}{l}\text { condenser } \\
\text { condenser }\end{array}$ & $\begin{array}{l}\mathrm{I} .80 \mathrm{~mm} \text {. } \\
0.40 \mathrm{~mm} \text {. }\end{array}$ & $\begin{array}{l}1750 \\
3500\end{array}$ & $\begin{array}{l}300 \\
140\end{array}$ & $\begin{array}{l}\mathrm{cm} . \\
\mathrm{cm} .\end{array}$ \\
\hline $4 \mathrm{~mm}$. & none & condenser & I. $50 \mathrm{~mm}$. & 2 I 50 & 320 & $\mathrm{~cm}$. \\
\hline $3 \mathrm{~mm}$. & none & condenser & I $\mathrm{mm}$. & $235^{\circ}$ & 235 & $\mathrm{~cm}$. \\
\hline $2 \mathrm{~mm}$ & none & condenser & $0.60+\mathrm{mm}$ & 4000 & 300 & $\mathrm{~cm}$. \\
\hline
\end{tabular}

$\$+2$ I. This table shows approximately the size of object which each objective will project upon the screen. This was determined exactly as described in $S 50$. For the magnification one simply measures the distance between two or more lines of the image of the micrometer on the screen, and divides the size of the image by the known size of the object ( $\$$ I55). Any good stage micrometer will answer. It is necessary, however, to use one with coarse lines for the low powers (\$ I 59, I 70).

By comparing the magnification with and without the projection oculars, and also comparing the size of object which can be projected 
with and without the oculars one can decide quite accurately the best combination to select. On the whole the writer has found it better to employ a sufficient variety of objectives and not use the projection oculars. It is somewhat easier to obtain a brilliant image without the oculars.

$\$ 422$. Darkening the Room. - It is impossible to succeed in micro-projection unless the room can be made dark, the darker the better. It is especially important that the screen should be free from all light except that projected upon it in forming the image.

$\$ 423$. Enclosing the Projection Apparatus.-It is desirable to have the projection apparatus closed as completely as possible to avoid diffusing light through the room and thus vitiating the most careful darkening of all windows and sky lights. It is also desirable to shut in the light from the apparatus, as it dazzles the eyes of the operator and of those near it in the audience so that the image on the screen cannot be satisfactorily seen. Some forms of apparatus are enclosed in a metal box, others have a frame over them upon which is spread black cloth like silesia. If this is made fireproof by soaking it thoroughly in a solution of alum, borax and sodium tungstate it will not readily catch fire. The cloth should not be too thick, otherwise it will retain too much heat around the apparatus.

One should remember the fundamental law of vision, viz, that other things being equal, the clearest images are obtained when no light reaches the eye except from the object.

424. Preparations Suitable for Micro-Projection.-As a generalization it may be said that any specimen which shows clearly and sharply under the microscope with a $16 \mathrm{~mm}$. objective will also give an excellent projection image. Details which are not visible with the $16 \mathrm{~mm}$. objective are rarely well brought out with sufficient clearness on the screen for one or two hundred people to see.

(A) The stains showing best are those which are very transparent, or pure differential stains like hematoxylin. Admirable results have been obtained with hematoxylin and eosin, and the various carmines when differentiated. Every method of staining which gives either sharply differentiated results or transparent colors produces preparations adapted to projection. A weak, or washed out appearance under the microscope is sure to be even less satisfactory on the screen.

(B) The thickness of the sections may vary from $1 \mu$ to $40 \mu$. But one must remember that thick sections are adapted for low powers 
only, while thin sections, if well stained, may be used with both high and low objectives.

The size of the object which one wishes to project determines the objectives to be used. By consulting the table one can get a fair idea of the size of object which each objective will satisfactorily project. An excellent plan to follow is that for ordinary microscopic study (see p. IO2), that is, use first a low power to show the object as a whole, then a higher one for details.

(C) For minute objects like white blood corpuscles, etc., it is necessary to use a high power and to have a small audience which can be close to the screen, or a somewhat larger audience can see well by using opera glasses.

(D) For the circulation of the blood it is necessary to eliminate the heat rays almost entirely. Nothing has proven so good as the second or specimen cooler $(\$ 4 \mathrm{I} 3)$. The conditions are still more favorable if a circulation of cold water is established in the large water bath also. This is easily done by the use of two large bottles. The cold water can be siphoned or aspirated from an upper one and the warm water allowed to flow off into a lower one. For this it is of much advantage to have a tube in the bottom of the water bath in which to introduce the cold water. The warm water will then flow off through a tube in the top. One must remember that perfectly clean water must be used for the water bath especially when a circulation is established, for opaque particles in the water bath give undesirable shadows in the image.

(B) A practical suggestion is made by Lewis Wright in his book on optical projection, and that is to warm the objective before using it for showing the circulation of the blood or in any case when a moist object is under it. If the objective is cold the vapor from the object will be condensed on the objective and make satisfactory projection impossible.

\$425. Masks for Projection Preparations. - The light used for projection is so brilliant that it is practically impossible to arrange the object under the objective with rapidity and certainty unless there is some kind of guide. The best one found so far is a mask on the back of the slide with an opening for the preparation „to be shown. This mask should be made of black paper. One can cut the holes in it with scissors or with ticket punches. With the specimens properly masked, and the parts of the apparatus lusterless black, as suggested by Dr. 
Coplin, the operator can work with rapidity, certainty and also with comfort. (Fig. 213.)

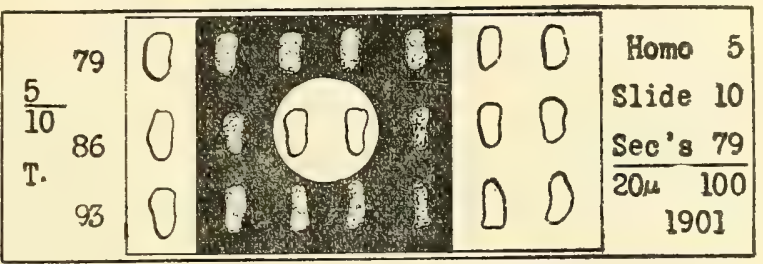

FIG. 2I3. Slide of serial sections with a black mask, perforated over the sections to be demonstrated with the projection microscope. This mask is put on the back of the slide, not on the cover-glass.

Unless one has a mask something like this the light is so dazzling that it is almost impossible to find the proper sections. It is easily removed by placing the slide on wet blotting paper.

$\$ 426$. How to demonstrate with the Micro-Projection Apparatus. - Microscopical preparations are not so easily used as are lantern slides. The writer has found that the most successful method is for the teacher himself to stand by the apparatus, insert the specimens, and find exactly what he wishes his pupils to see. Then to point them out a bamboo fish pole with sharp end is used. This should be 2-3 meters long and if held out in the diverging cone of light leaving the microscope, a sharp shadow will be cast upon the image. With this pointer one can indicate the part to be demonstrated even more satisfactorily than as if he pointed them out directly on the screen. While it is not possible to delegate the finding of the specimen to an assistant he is of great help in keeping the carbons of the lamp in exactly the right position. If the light is kept perfect the teacher has very little trouble with the rest of the manipulation.

$\$ 427$. Cleaning the Glass Surfaces of the Micro-projection Apparatus.-Inasmuch as it is so difficult to make the light sufficiently brilliant for micro-projection, it is of the greatest importance that all glass surfaces be kept as clean as possible. The lenses of the lamp condenser should be carefully wiped occasionally; and the water bath should be opened and the plane glass faces thoroughly cleaned. It is desirable to soak them in the cleaning mixture for glass. There is always a certain amount of deposit on the glass even though distilled water is used. Every grade of opacity renders the image on the screen less excellent. Cleanliness is one of the most important requirements for successful micro-projection. 
Each preparation should be wiped off before it is put in position on the stage. Any particles of dust are painfully evident in the projected image.

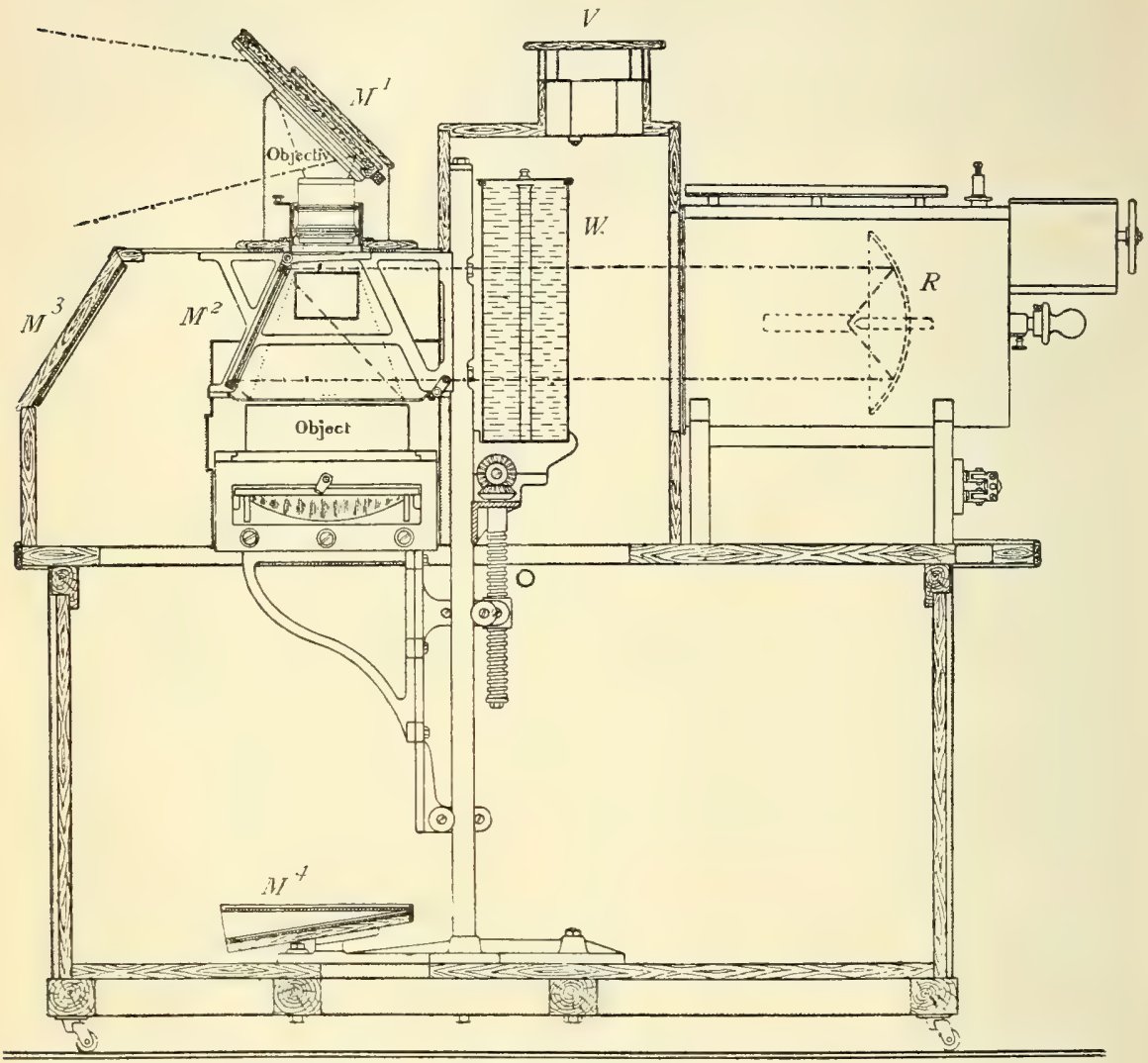

FIG. 2I4. Zeiss Epidiascope for Opaque Objects, and for Transparent Objects in a Horizontal Position (Zeiss' Special Catalog.)

As shown in this figure the apparatus is set up for opaque objects. For transparent objects $M^{2}$ (mirror 2 ) is remoted, when the light striking $M^{3}$ is reflected to $M^{+}$and thence up through the object to $M^{\prime}$ and to the screen.

Commencing at the right: $R$. Parabolic reflector, which projects the light from the crater through $(W)$ the water bath, to $M^{2}$ the mirror which is at the proper angle for reflecting the light doan upon the opaque object. From the opaque object the light is irreguilarly reflected up through the objective to $M^{I}$. $M^{i}$ serves to reflect the rays from the objective to the screen.

$V$. I'entilator. $M 3$ and $.1 \%$ are mirrors for use in reflecting the light through horizontal transparent objects. 
This apparatus is designed to project opaque objects as large as 22 centimeters in diameter, at a magnification of 5 to Io with a 30 ampere current. For a smaller object one may magnify as high as 25 diameters. With a 50 ampere current and a larger reflector the magnification may be from i4 up to 37 diameters.

\section{PROJECTION OF OPAQUE, OBJECTS}

3. 428. Episcope.-For the projection of opaque objects like anatomical preparations, figures in books, coins or indeed any opaque object an apparatus on the principle of the one figured (Fig. 2I4) is used. That is, a powerful light is thrown upon the opaque object and the rays reflected from the object are then projected upon the screen by an objective as for a lantern slide. As the objects are mostly in a horizontal position the objective points directly upward, and the rays from it must be made horizontal by means of a 45 degree mirror or prism.

This apparatus is very old. Its first name was "aphengescope" or opaque lantern. Now it is called an episcope, or megascope, and if for both opaque and transparent objects (Fig. 2I4) it is designated as an epidiascope.

For its satisfactory use exceedingly powerful light must be used. Some opticians employ two radiants, others but one. In any case currents of 30 to 50 amperes are used. One should wear deeply stained glasses in working with it.

The apparatus works well with flat objects, and rather brilliant objects, like the movements of a watch, etc. It is also more satisfactory for objects of slight thickness. For objects like bones, etc, one must focus up and down for the different levels.

\section{REFERENCES TO CHAPTER IX}

Lewis Wright, Optical Projection ; Carpenter-Dallinger ; Leiss, Die optischen Instrumente der Firma R. Fuess; The works on Photo-Micrography; The latest catalogs or special catalogs on projection apparatus issued by the opticians, especialally Zeiss, Reichert and Leitz. The volumes of the microscopical periodicals for the last few years, especially the Journal of the Royal Microscopical Society, and the Zeitschrift für wissentschaftliche Mikroskopie. 


\section{CHAPTER X}

\section{'THE ABBE TEST PLATE AND APERTOMETER; EQUIVA- LENT FOCUS OF OBJECTIVES AND OCULARS; DRAW- INGS FOR PHOTO-ENGRAVING; WAX MODELS}

8. 429. On the Method of Using Abbe's Test-Plate.-This test-plate is intended for the examination of objectives with reference to their corrections for spherical and chromatic aberration and for estimating the thickness of the coverglass for which the spherical aberration is best corrected.

"The test-plate consists of a series of cover-glasses ranging in thickness from $0.09 \mathrm{~mm}$. to $0.24 \mathrm{~mm}$, silvered on the under surface and cemented side by side on a slide. The thickness of each is written on the silver film. Groups of parallel lines are cut through the film and these are so coarsely ruled that they are easily resolved by the lowest powers, yet from the extreme thinness of the silver they form a very delicate test for objectives of even the highest power and widest aperture. To examine an objective of large aperture the plates are to be focused in succession observing each time the quality of the image in the center of the field and the variation produced by using alternately central and very oblique illumination. When the objective is perfectly corrected for spheircal aberration for the particular thickness of cover-glass under examination, the contour of the lines in the center of the field will be perfectly sharp by ohlique illumination without any nebulous doubling or indistinctness of the minute irregularities of the edges. If after exactly adjusting the objective for oblique light, central illumination is used no alteration of the adjustment should be necessary to show the contours with equal sharpness."

"If an objective fulfills these conditions with any one of the plates it is free from spherical aberration when used with cover-glasses of that thickness; on the other hand if every plate shows nebulous doubling or an indistinct appearance of the edges of the silver lines, with oblique illumination, or if the objective requires a different adjustment to get equal sharpuess with central as with oblique light, then the spherical correction is more or less imperfect."

"Nebulous doubling with oblique illumination indicates overcorrection of the marginal zone, want of the edges without marked nebulosity indicates undercorrection of this zone ; an alteration of the adjustment for oblique and central illumination, that is, a difference of plane between the image in the peripheral and central portions of the objective points to an absence of concurrent action of the separate zones, which may be due to either an average under or overcorrection or to irregularity in the convergence of the rays."

"The test of chromatic correction is based on the character of the color bands, which are visible by oblique illumination. With good correction the edges of the 
silver lines in the center of the field should show but narrow color bands in the complementary colors of the secondary spectrum, namely, on one side yellowgreen to apple-green on the other violet to rose. The more perfect the correction of the spherical aberration the clearer this color band appears."

"To obtain obliquity of illumination extending to the marginal zone of the objective and a rapid interchange from oblique to central light Abbe's illuminating apparatus is very efficient, as it is only necessary to move the diaphragm in tuse nearer to or further from the axis by the rack and pinion provided for the purpose. For the examination of immersion objectives, whose aperture as a rule is greater than $180^{\circ}$ in air and those homogeneous-immersion objectives, which considerably exceed this, it will be necessary to bring the under surface of the Test-plate into contact with the upper lens of the illluminator by means of a drop of water, glycerin or oil."

"In this case the change from central to oblique light may be easily effected by the ordinary concave mirror but with immersion lenses of large aperture it is impossible to reach the marginal zone by this method, and the best effect has to be searched for after each alteration of the direction of the mirror."

"For the the examination of objectives of smaller aperture (less than $40^{\circ}-50^{\circ}$ ). we may obtain all the necessary data for the the estimation of the spherical and chromatic corrections by placing the concave mirror so far laterally, that its edge is nearly in the line of the optic axis the incident cone of rays then only filling one-half of the aperture of the objective. The sharpness of the contours and the character of the color bands can be easily estimated. Differences in the thickness of the cover-glass within the ordinary limits are scarcely noticeable with such objectives."

"It is of fundamental importance in employing the test as above described to have brilliant illumination and to use an eye-piece of high power."

"When from practice the eye has learnt to recognize the finer differences in the quality of the contour images this method of investigation gives very trustworthy results. Differences in the thickness of cover glasses of 0.01 or $0.02 \mathrm{~mm}$. can be recognized with objectives of 2 or $3 \mathrm{~mm}$. focus."

"With oblique illumination the light must always be thrown perpendicularly to the direction of the lines.

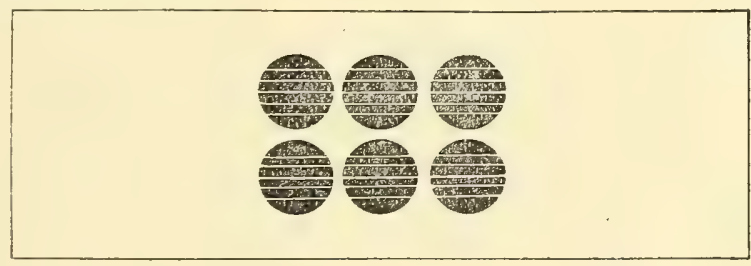

FIG. 215. The Abbe Test Plate.

"The quality of the image outside the axis is not dependent on spherical and chromatic correction in the strict sense of the term. Indistinctness of the contours towards the borders of the field of view arises as a rule, from unequal magnification of the different zones of the objective; color bands in the peripheral 
portion (with good color correction in the middle) are caused by unequal magnification of the different colored images."

"Imperfections of this kind, improperly called "curvature of the field," are shown to a greater or less extent in the best objectives, where the aperture is considerable."

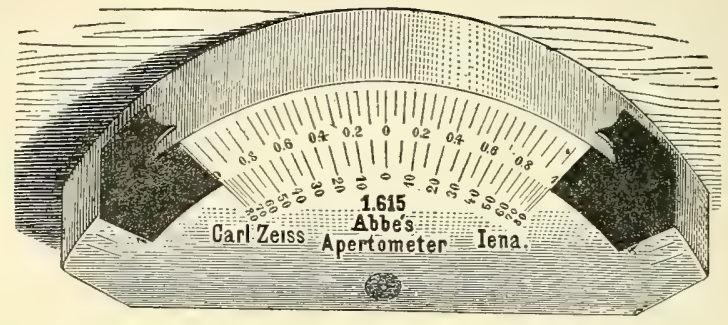

Fig. 2I6. Abbe Apertometer.

\& 430. Determination of the Aperture of Objectives with an Apertometer. Excellent directions for using the Abbe apertometer may be found in the Jour. Roy. Micr. Soc., I878, p. 19, and I8So, p. 20 ; in Dippel, Zimmermann and Czapski. The following directions are but slightly modified from Carpenter-Dallinger, pp. 394-396. The Abbe apertometer involves the same principle as that of Tolles, but it is carried out in a simpler manner; it is shown in Fig. 2J6. As seen by this figure it consists of a semi-circular plate of glass. Along the straight edge or chord the glass is beveled at $45^{\circ}$, and near this straight edge is a small, perforated circle, the perforation being in the center of the circle. To use the apertometer the microscope is placed in a vertical position, and the perforated circle is put under the microscope and accurately focused. The circular edge of the apertometer is turned toward a window or plenty of artificial light so that the whole edge is lighted. When the objective is carefully focused on the perforated circle the draw-tube is removed and in its lower end is inserted the special objective which accompanies the apertometer. This objective and the ocular form a low power compound microscope, and with it the back lens of the objective, whose aperature is to be measured, is observed. The draw-tube is inserted and lowered until the back lens of the objective is in focus. "In the image of the back lens will be seen stretched across, as it were, the image of the circular part of the apertometer. It will appear as a bright band, because the light which enters normally at the surface is reflected by the beveled part of the chord in a vertical direction so that in reality a fan of $180^{\circ}$ in air is formed. There are two sliding screens seen on either side of the apertometer; they slide on the vertical circular portion of the instrument. The images of these screens can be seen in the image of the bright band. These screens should now be moved so that their edges just touch the periphery of the back lens. They act, as it were, as a diaphragm to cut the fan and reduce it, so that its angle just equals the aperature'of the objective and no more." "This angle is now determined by the arc of glass between the screens; thus we get an angle in glass the exact equivalent of the aperature of the objective. As the numerical apertures of these arcs are engraved on the apertometer they can be read off by inspection. Nevertheless a difficulty is experienced, from the fact that it is not easy to 
determine the exact point at which the edge of the screen touches the periphery of the back lens, or as we prefer to designate it, the limit of aperture, for curious as the expression may appear we have found at times that the back lens of an objective is larger than the aperture of the objective requires. 'In that case the edges of the screen refuse to touch the periphery."

In determining the aperture of homogeneous immersion objectives the proper immersion fluid should be used as in ordinary observation. So, also, with glycerin or water immersion objectives.

8 43I. Testing Homogeneous Immersion Liquid.-In order that one shall realize the full benefit of the homogeneous immersion principle it is necessary that the homogeneous immersion liquid shall be truly homogeneous. In order that the ordinary worker may be able to test the liquid used by him, Professor Hamilton L. Smith devised a tester composed of a slip of glass in which was ground accurately a small concavity and another perfectly plain slip to act as cover. (See Proc. Amer. Micr. Soc.; $188_{5}$, p. 83 ). It will be readily seen that this concavity, if filled with air or any liquid of less refractive index than glass, will act as a concave or dispersing lens. If filled with a liquid of greater refractive index than glass, the concavity would act like a convex lens, but if filled with a liquid of the same refractive index as glass, that is, liquid optically homogeneous with glass, then there would be no effect whatever.

In using this tester the liquid is placed in the concavity and the cover put on. This is best applied by sliding it over the glass with the concavity. A small amount of the liquid will run between the two slips, making optical contact on both surfaces. One should be careful not to include air bubbles in the concavity. The surfaces of the glass are carefully wiped so that the image will not be obscured. An adapter with society screw is put on the microscope and the objective is attached to its lower end. In this adapater a slot is cut out of the right width and depth to receive the tester which is just above the objective. As object it is well to employ a stage micrometer and to measure carefully the diameter of the field without the tester, then with the tester far enough inserted to permit of the passage of rays through the glass but not through the concavity, and finally the concavity is brought directly over the back lens of the objective. This can be easily determined by removing the ocular and looking down the tube.

Following Professor Smith's directions it is a good plan to mark in some way the exact position of the tube of the microscope when the micrometer is in focus without the tester, then with the tester pushedin just far enough to allow the light to pass through the plane glass and finally when the light traverses the concavity. The size of the field should be noted also in the three conditions ( $850-52$.)

It will be seen by glancing at the following table that whenever the liquid in the tester is of lower index than glass, that the concavity with the liquid acts as a concave lens, or in other words like an amplifier (p. Iog), and the field is smaller than when no tester is used. It will also be seen that as the liquid in the concavity approaches the glass in refractive index that the field approaches the size when no tester is present. It is also plainly shown by the table that the greater the difference in refractive index of the substance in the concavity and the glass, the more must the tube of the microscope be raised to restore the focus.

If a substance of greater refraction than glass is used in the tester the field would be larger, i.e., the magnification less, and one would have to turn the tube down instead of up to restore the focus. 
The table given below indicates the points with a tester prepared by the Gundlach Optical Co., and used with a $16 \mathrm{~mm}$. apochromatic objective of Zeiss, $X_{4}$ compensation ocular, achromatic condenser, I.oo N. A. (Fig. 4I) :

\begin{tabular}{|c|c|c|}
\hline Tester and Liquid in the Concavity & $\begin{array}{l}\text { Size of the } \\
\text { Field }\end{array}$ & $\begin{array}{l}\text { Elevation of the Tube } \\
\text { necessary to } \\
\text { Restore the Focus }\end{array}$ \\
\hline $\begin{array}{l}\text { less of the tester at one end, } \\
\text { the cavity } \\
5 \text { \% \% alcohol } \\
\text { erosene } \\
\text { mb Opdlach Opt. Co's hom, liquid } \\
\text { iquid. Co.'s hom. liquid } \\
\text { iquid }\end{array}$ & $\begin{array}{l}\text { I. } 825 \mathrm{~mm} .-- \\
\text { I. } 85 \mathrm{~mm} .--- \\
\text { I. } 075 \mathrm{~mm} .-- \\
\text { I. } 5 \mathrm{~mm} .-- \\
\text { I. } 4 \mathrm{~mm} .--- \\
\text { I. } 825 \mathrm{~mm} .-- \\
\text { I. } 825 \mathrm{~mm} .-- \\
\text { I. } 825 \mathrm{~mm} .-- \\
\text { I. } 825 \mathrm{~mm} .--\end{array}$ & 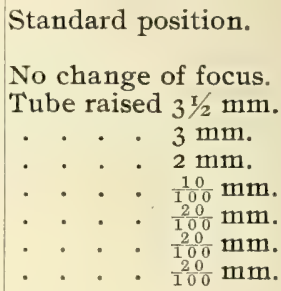 \\
\hline
\end{tabular}

\& 432. Equivalent Focus of Objectives and Oculars.-To work out in proper mathematical form or to ascertain experimentally the equivalent foci of these complex parts with real accuracy would require an amount of knowledge and of apparatus possessed only by an optician or a physicist. The work may be done, however, with sufficient accuracy to supply most of the needs of the working microscopist. The optical law on which the following is based is :- "The size of object and image zaries directly as their distance from the center of the lens."

By referring to Figs. I4, I6, 2I, it will be seen that this law holds good. When one considers compound lens-systems the problem becomes involved, as the centre of the lens systems is not easily ascertainable hence it is not attempted, and only an approximately accurate result is sought.

8. 433. Determination of Equivalent Focus of Objectives. - Look into the upper end of the objective and locate the position of the back lens. Indicate the level.in some way outside of the objective. This is not the center of the objective but serves as an arbitrary approximation. Screw the objective into the tube of the microscope. If a Huygenian ocular is used with the ocular micrometer, screw off the field lens and use the eye-lens only. If a positive ocular is used no change need be made. Pull out the draw-tube until the distance between the ocular micrometer and the back lens is 250 millimeters. Use a stage micrometer as object and focus carefully. Make the lines of the two micrometers parallel (Fig. IOS). Note the number of spaces on the ocular micrometer required to measure one or more spaces on the stage micrometer. Suppose the two micrometers are ruled in $\frac{1}{10} \mathrm{~mm}$. and that it required ro spaces on the ocular micrometer to enciose 2 spaces on the stage micrometer, evidently then 5 spaces would cover one. The image, $\mathrm{A}^{\mathrm{I}} \mathrm{B}^{\mathrm{I}}$ Fig $2 \mathrm{I}$ in this case is five times as long as the object, $\mathrm{A}, \mathrm{B}$. Now if the size of object and image are directly as their distance from the lens it follows that as the size of object is known $\left(\frac{2}{10} \mathrm{~mm}\right.$.), that of the image directly measured ( ${ }_{10}^{1.0} \mathrm{~mm}$.), the distance from the lens to the image also determined in the beginning, there remains to be found the distance between the objective and the object, which will represent approximately the equivalent focus. The general formula is, Object, O:Image, I : equivalent focus, F:250. Supplying the known 
values, $\mathrm{O}=\frac{2}{10}, \mathrm{I}=\frac{10}{10}$ then $\frac{2}{10} \mathrm{~m} .: \mathrm{I} \mathrm{mm} .: \because \mathrm{F}: 250$ whence $\mathrm{F}=50 \mathrm{~mm}$. That is, the equivalent focus is approximately 50 millimeters.

3. 434. Determination of Initial or Independent Magnification of the Objective.-The initial magnification means simply the magnification of the real image $\left(A^{x} B^{x}\right.$, Fig. 2I) unaffected by the ocular. It may be determined experimentally exactly as described in $\% 433$. For example, the image of the object $\left(\frac{2}{10} \mathrm{~mm}\right.$. $)$ measured by the ocular micrometer, at a distance of $250 \mathrm{~mm}$. is $\frac{10}{10} \mathrm{~mm}$., $i$. e., it is five times magnified, hence the initial magnification of the $50 \mathrm{~mm}$. objective is approximately five.

Knowing the equivalent focus of an objective, one can determine its initial magnification by dividing $250 \mathrm{~mm}$. by the equivalent focus in millimeters. Thus the initial magnification of a $5 \mathrm{~mm}$. objective is $\frac{250}{5}=50$; of a $3 \mathrm{~mm}$., $\frac{250}{3}=83.3$; of a $2 \mathrm{~mm} ., \frac{250}{2}=\mathrm{r} 25$, etc.

3. 435. Determining the Equivalent Focus of an Ocular.-If one knows the initial magnification of the objective $(3 / 434)$ the approximate equivalent focus of the ocular can be determined as follows :

The field lens must not be removed in this case. The distance between the position of the real image, a position indicated in the ocular by a diaphragm, and the back lens of the objective should be made $250 \mathrm{~mm}$., as described in $\xi 433,434$, then by the aid of Wollaston's camera lucida the magnification of the whole microscope is obtained, as described in \& I60. As the initial power of the objective is known, the power of the whole microscope must be due to that initial power multiplied by the power of the ocular, the ocular acting like a simple microscope to magnify the real image (Fig. 2I).

Suppose one has a $50 \mathrm{~mm}$. objective, its initial power will be approximately 5 . If with this objective and an ocular of unknown equivalent focus the magnification of the whole microscope is 50 , then the real image or initial power of the objective must have been multiplied ro fold. Now if the ocular multiplies the real image Io fold it has the same multiplying power as a simple lens of $25 \mathrm{~mm}$. focus, for, using the same formula as before : $O=5: I=50:: F: 250$ whence $F=25$. The matter as stated above is really very much more complex than this, but this gives an approximation.

For a discussion of the equivalent focus of compound lens-systems, see modern works on physics; see also C. R. Cross, on the Focal Length of Microscopic Objectives, Franklin Institute Jour., I870, pp. 40I-402; Monthly Micr. Jour., I870, pp. I49-159 J. J. Woodward on the Nomenclature of Achromatic Objectives, Amer. Jour. Science, 1872, pp. 406-414; Monthly Micr. Jour., I872, pp. 66-74. W. S. Franklin, method for determining focal lengths of microscope lenses. Physical Review, Vol. I, I893, p. I42. See pp. III9-II3I of CarpenterDallinger for mathematical formulæ; also Daniell, Physics for medical students ; Czapski, Theorie der optischen Instrumente ; Dippell, Nägeli und Schwendener, Zimmermann. E. M. Nelson, J. R. M. S. I898, p. 362, I900, pp. I62-169. Jour. Quekett Micr. Club, vol. V. pp. 456, 462.

3 436. Drawings for Photo-Engraving.-The inexpensive processes of reproducing drawings bring within the reach of every writer upon scientific subjects the possibility of presenting to the eye by diagrams and drawings the facts discussed in the text. Though artistic ability is necessary for perfect representation of an object, neatness and care will enable anyone to make a simple illustrative drawing, from which an exact copy can be obtained and a plate prepared for printing. 
A careful study of the cuts or plates used to illustrate the same class of facts as one wishes to show will enable one to produce similar effects. Outlines which are transferred to the drawing paper may be obtained by the camera lucida or from a photograph. The drawing should be made so that it can be reduced anywhere from one-eighth to one-half. For ordinary photo-engraving for such line drawings as are used to illustrate this book, use perfectly black carbon ink. A shaded or wash drawing can be reproduced by the half-tone process, also photographs as is illustrated by figures I90-I9I. A crayon drawing on stipple paper with shadows re-enforced by ink lines and high lights scratched out with a sharp knife give admirable results for anatomical figures by the half-tone process. (See for example the work of Max Broedel in Contributions to the Science of Medicine, (Welch Book) Baltimore, rgoo).

For photo-engravings of line work the letters, figures or words used to designate the different parts can be put on the drawing by pasting letters, etc., of the proper size in the right position. In preparing the block the photo-engraver eliminates all shadows and the letters look as if printed on the drawings.

8 437. Wax Models.-Large wax models of the objects which one studies under the microscope are helpful both to the teacher and to the investigator. These models are becoming more and more appreciated for embryologic and morphologic investigations, for, as one can readily appreciate, the effort to produce a representation of the embryo or organ in three dimensions helps to overcome difficulties which are almost insurmountable if studied in the sections alone.

They are made from wax plates, the principle involved being that the diameter of the drawing on the wax plate is as much greater than the object as the wax plate is thicker than the section.

The wax plate is cut with a sharp instrument, following the outlines of the object which has been traced upon it by the aid of a camera lucida or the projection microscope. The sections are piled together, some line or lines obtained from a drawing or photograph of the spceimen before it was imbedded and sectioned being used as a guide by which the correct form of the pile of sections can be tested. Finally the whole is welded into one by the use of hot wax or a hot instrument. Models which illustrate complex internal structures are difficult to prepare, but numerous devices will occur to the worker as the representation of blood vessels and nerves by strings or wires. A large model will need much support which can be given by wire gauze, wires, pins or paper according to the special needs.

A practical method for wax modeling was first published by G. Born, Arch. f. Mikr. Anat., Bd. xxii, $188_{3}$, p. $5 \delta_{4}$. The most detailed statements of improvements of the method have been published by Born (Böhm u. Oppel) I 900 , and by Dr. F. P. Mall and his assistants. See contributions to the Science of Medicine, pp. 926-1045. Proceedings of the Amer. Assoc. Anatomists, I90I, 14th session ( I 900$)$ p. I93.

\% 43S. Some Apparatus for Imbedding and Sectioning.-As a supplement to Chapter VIII, the following figures of imbedding and sectioning apparatus are appended. It will be noticed that the microtomes are complex and consequently expensive. One is figured in which the knife is moved by the hands of the operator (Fig. 217). This form of instrument is excellent, and with it one can do all kinds of work, both with collodion and paraffin. One cannot work so rapidly 
nor with the same precision. For much of the work one may section free-hand, without a microtome. Indeed the great basis of histological and embryological knowledge was gained by studying free-hand sections and dissections. At the present time there is a strong reaction against the exclusive study of sections, and a tendency to combine with the serial sections dissections such as the older anatomists and embryologists made and gained so much from.

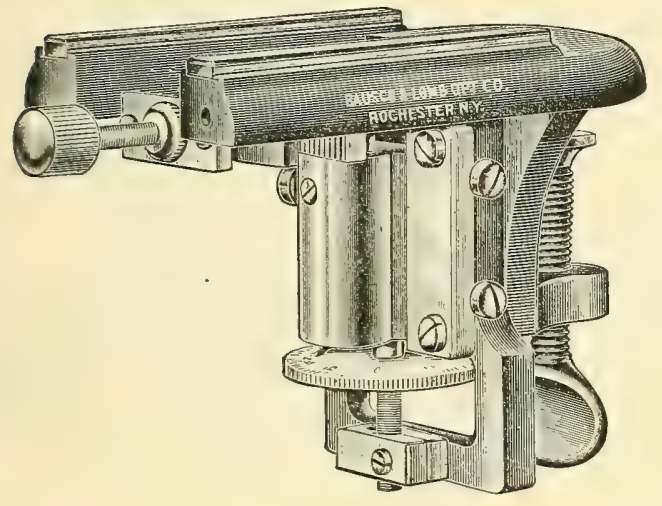

FIG. 217. A Microtome for all kinds of sectioning; the knife is guided by the top of the microtome, but moved by the hands of the operator (Bausch \& Lomb Optical Co.)

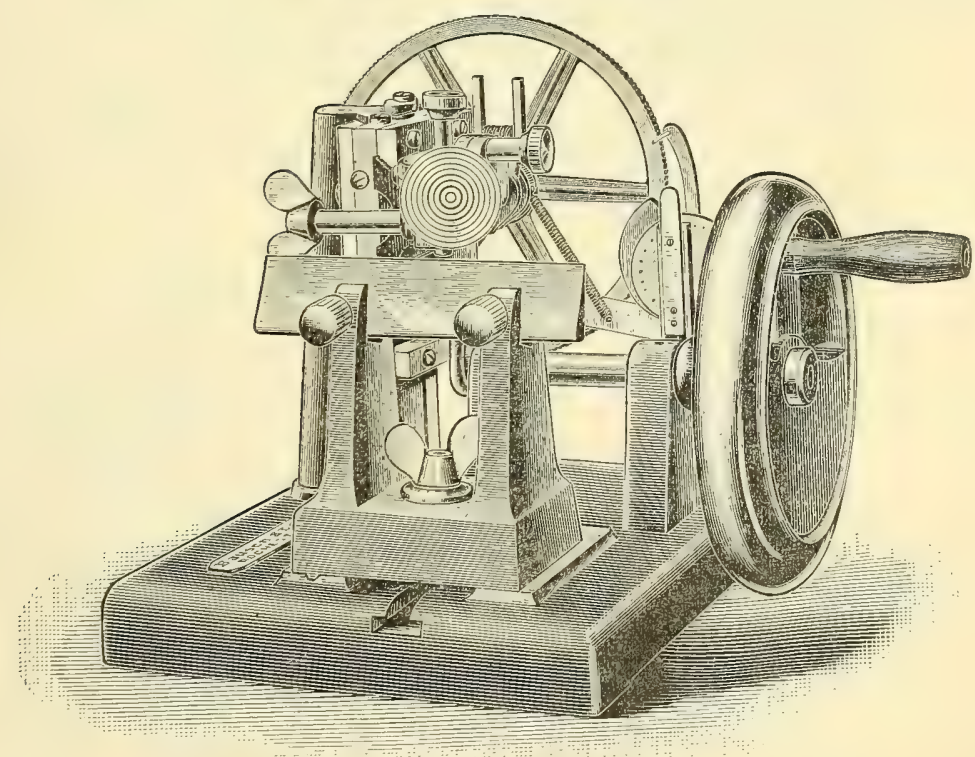

FIG. 2I8. The Minot microtome for ribbon sections as made by Bausch and Lomb Optical Co. It is arranged for sections from $r \mu$ to $25 \mu$ and any intermediate thickness. 


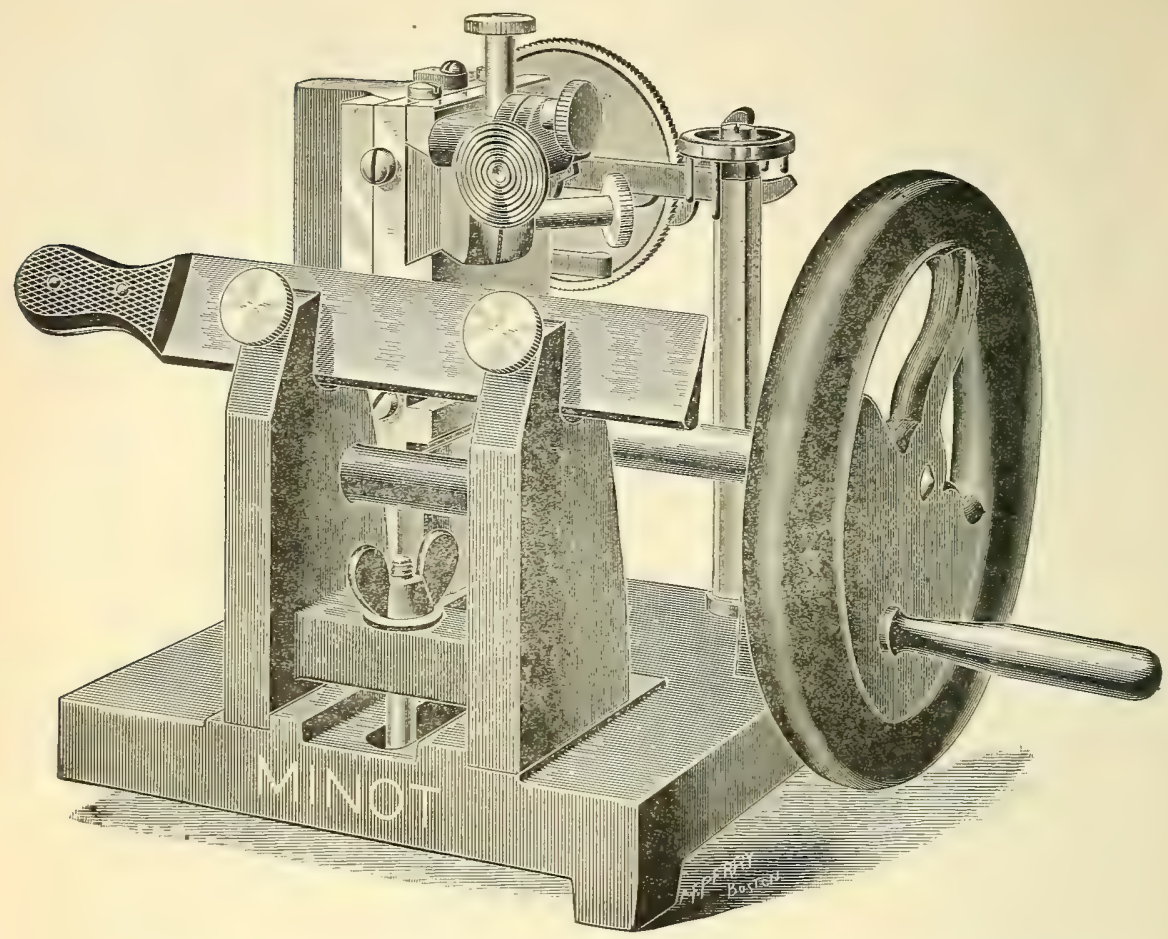

FIG. 219. The Minot microtome for ribbon sections as made by the Franklin Laboratory Supply Co., Boston. This is to be made for $2 \mu, 6 \mu, r o \mu, 14 \mu, 20 \mu$, and zou sections.
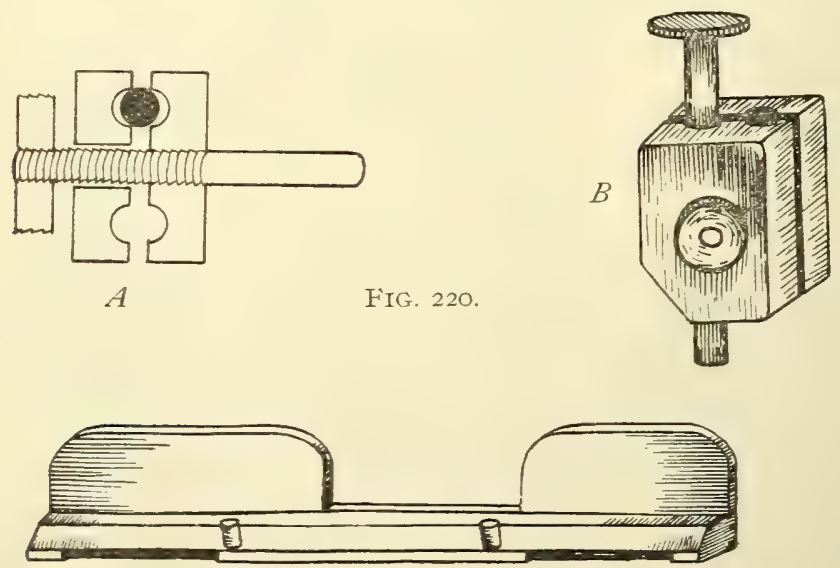

FIG. 22I. $A$. 


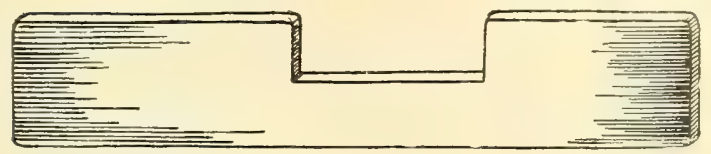

FIG. 22I. B.

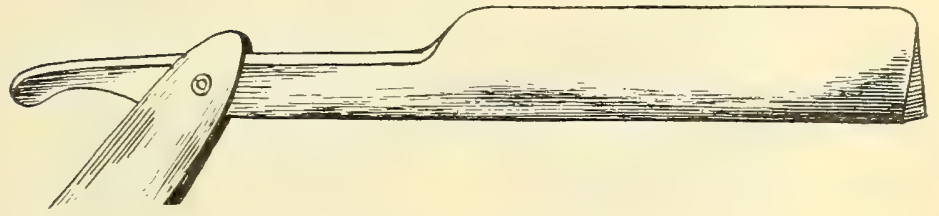

FiG. 22I. C.

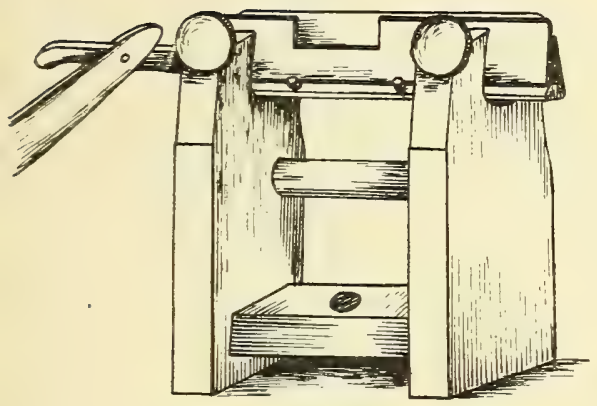

$A$

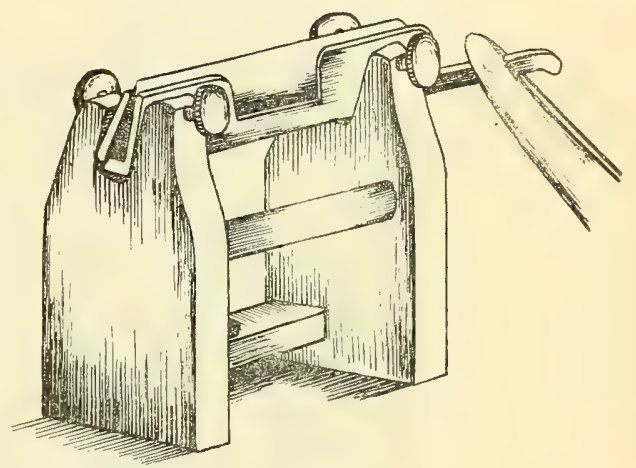

$B$

FIG. 222 .

FIG. 220-222. A paraffin holder clamp and a razor support for the Minot Microtome. (Trans. Amer. Micr. Soc., Igor).

FIG. 220. Clamp for the paraffin block holder. In $A$ it is shown in section, in a side view. With this clamp one can use stove bolts as well as the expensive paraffin holders furnished with the instrument. A laboratory can have as many paraffin block holders as necessary without undue expense.

FIG. 221. Razor Support and Razor.

(A) Support with heavy base and vertical piece. The base should be capable of moving endwise one or two centimeters to bring the opening in the vertical part opposite the paraffin block.

(B) Front piece to the razor (see Fig. $222 A$ ).

(C) Razor with straight back and edge. By moving this back and forth on the support nearly the entive cutting edge can be utilized.

FIG. 222. The knife support of the microtome with the razor support and razor in position.

(A) Front view; $(B)$ Back view. In the inclination of the knife toward the paraffin block is shown. 


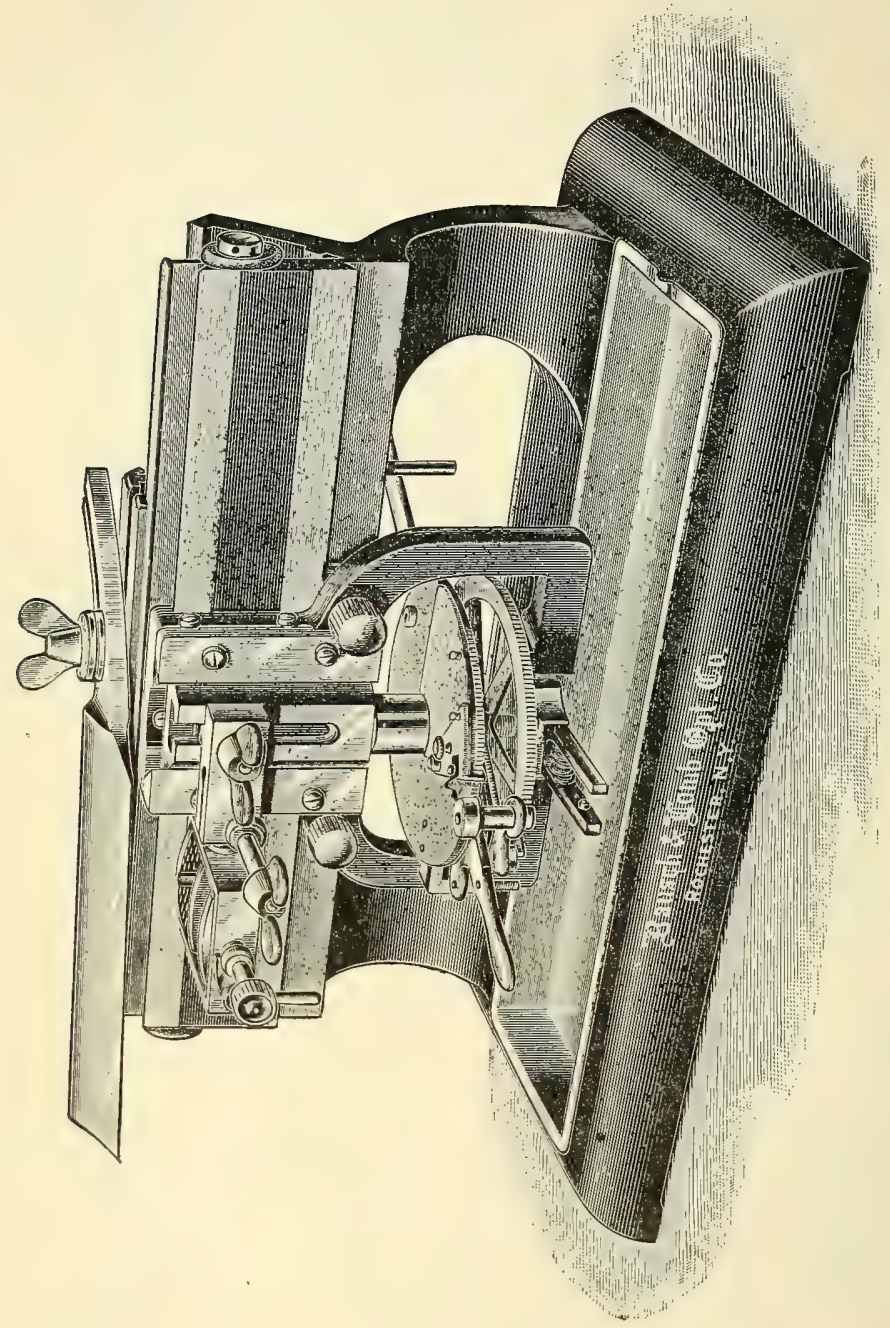

FIG. 223. Sliding microtome adapted especially for collodion sectioning. (The Bausch \& Lomb Optical (o.). 

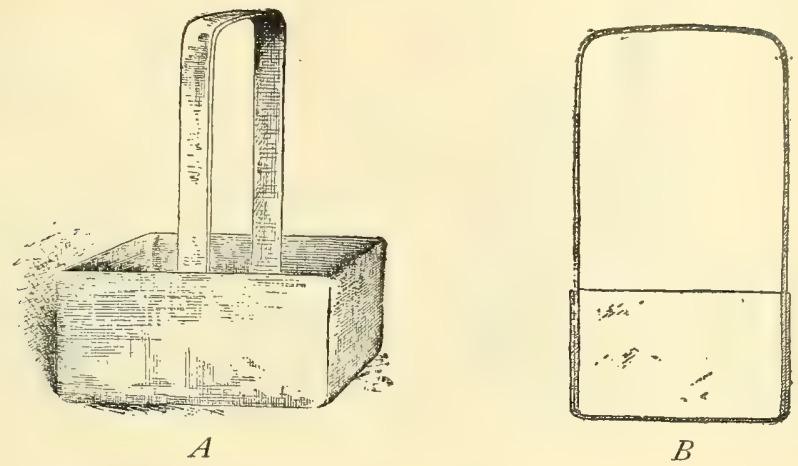

FIG. 224 Paraffin dish for infiltrating in the Lillie oven. It is made of copper and as show'n has a handle for ease in transference. A the whole dish, $B$ the dish in section. (Jour. Appl. Micr. I899, p. 266).

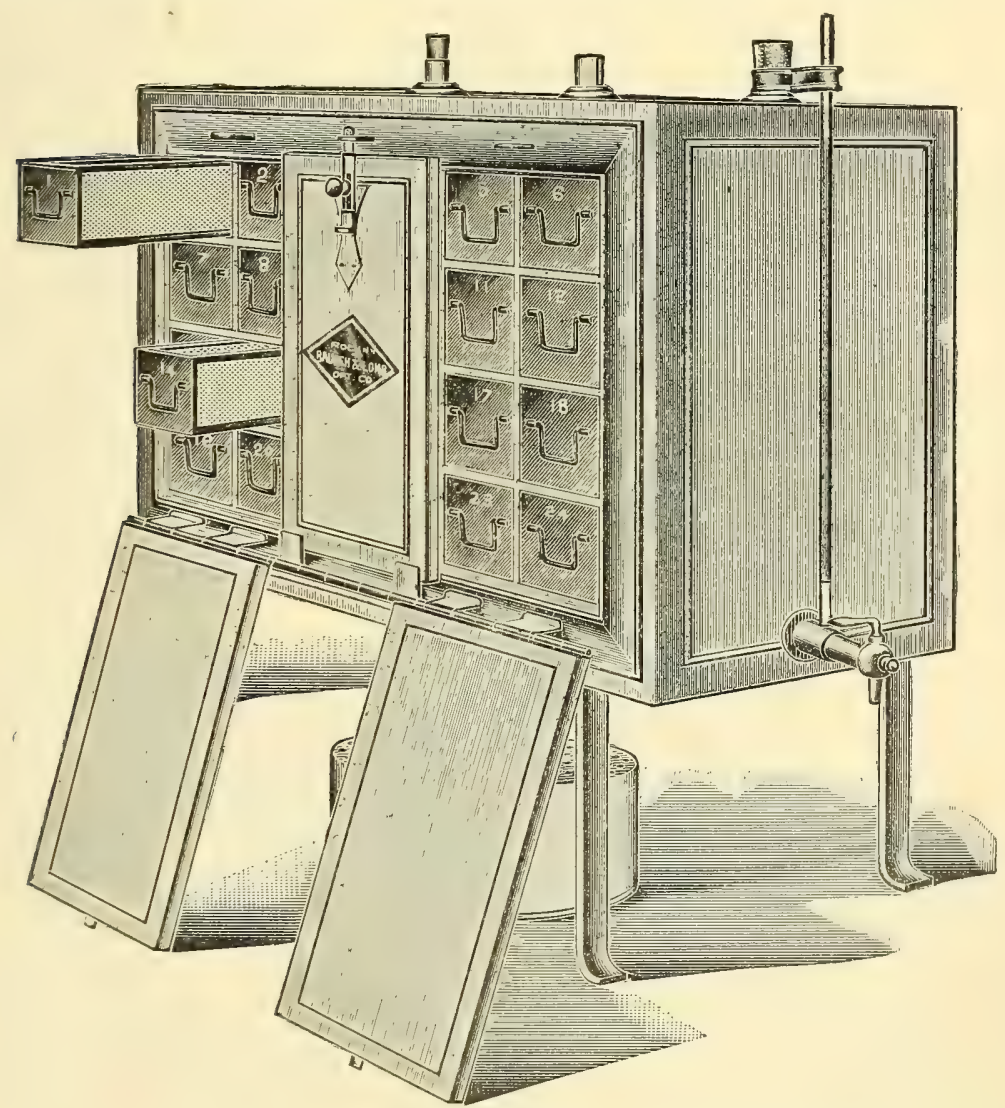

FIG. 225. The Lillie compartment, paraffin oven for infiltrating tissues with paraffin. Various sizes of this are made $(8,16$ and 24 compartments). Except for the largest laporatories the one with 16 compartments and trays will be found of sufficient capacity. (Bausch \& Lomb Optical Co.). 


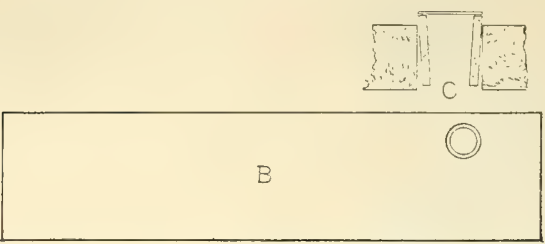

FIG. 226. Circulation board, especially for Necturus. This is prepared from a board about $8 \times 20$ centimeters. Near one edge it has a hole for a perforated cork. On the top of the cork is cemented a thick cover-glass with shellac or rubber cement. The cork can be raised or lowered in the board. The gills of Necturus or the web of a frog's foot can be spread out on glass over the cork. (Jour. Appl. Micr., I898, p. 131 .

FIG. 227. Copper can with screw top for collecting embryologic material and small aquatic animals. It was especially designed for collecting with a bicycle (Jour. Appl. Micr., I898, p. I31).

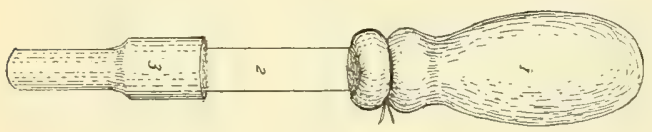

FIG. 228. Egg pipette. This is made by putting a short piece of soft rubber tubing over the end of a glass pipette with rubber bulb. With this one can handle the eggs both fresh and hardened without any degree of injury. (Jour. Appl. Micr. I898, p. I29).

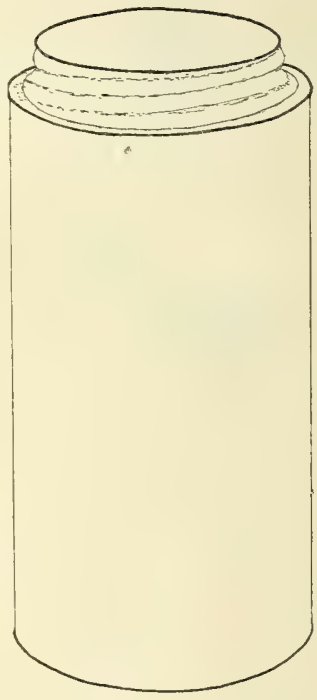

FIG. 227.

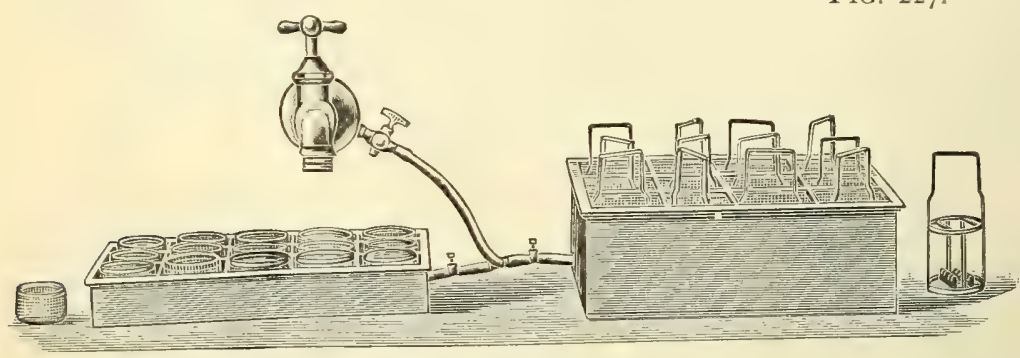

FIG. 229. Washing apparatus for tissues fixed in osmic and chromium mixtures. As shown in the figure the apparatus is connected with the water pipe by a small side cock. It is composed of a double i'ssel, the inner one being made of perforated brass. There are special perforated dishes to insert in the little compartments. This apparatus is conienient for washing cover-glasses, for the washing out for iron hematoxylin, etc. The dieper box at the right answers for the slide baskets or holders (Fig. I59). 


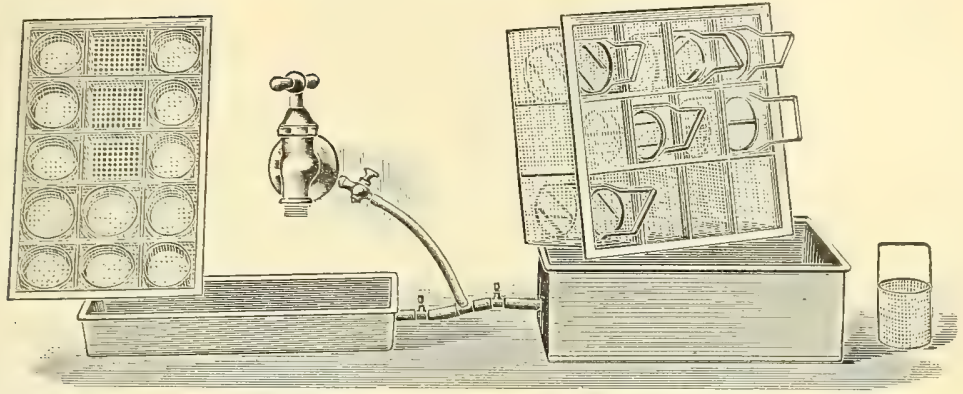

FIG. 229 A. - Same as the preceding with the inner, perforated box on edge.

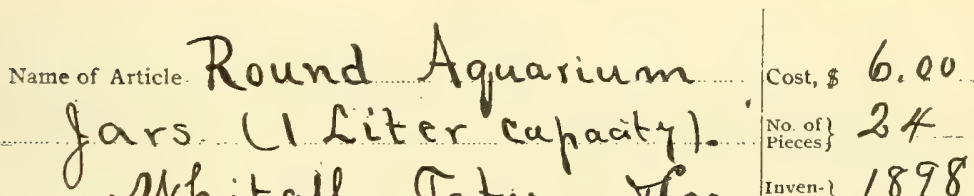

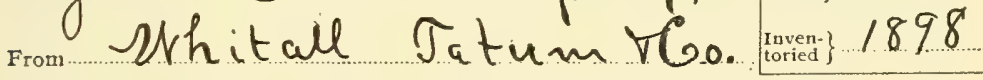
Address 46,48 Barclay st M. Order No 113 Date fune 15,1898 Date of receipt of Articles........ Remarks used for waste fers aud for water

DEx'... Otist ology NEW YORK STATE VETERINARY COLLEGE, CORNELL UNIVERSITY.

FIG. 230. An inventory card for the property in a department. The cards are the standard size for libraries. (Jour. Appl. Micr., 1898, p. 124). 


\section{BIBLIOGRAPHY}

The books and periodicals named below in alphabetical order pertain wholly or in part to the microscope or microscopical methods. They are referred to in the text by recognizable abbreviations.

For current microscopical and histological literature, the Journal of the Royal Microscopical Society, the Zoologischer Anzeiger, and the Zeitschrift für wissenschaftliche Mikroskopie, Anatomischer Anzeiger, Biologisches Centralblatt and Physiologisches Centralblatt, the Journal of Applied Microscopy and Laboratory methods and the smaller microscopical journals taken together furnish nearly a complete record.

References to hooks and papers published in the past may be found in the periodicals just named, in the Index Catalog of the Surgeon General's library; in the Royal Society's Catalog of Scientific Papers, and in the bibliographical references given in special papers. A full list of periodicals may also be found in Vol. XVI of the Index Catalog.

\section{BOOKS.}

Adams, G.-Micrographia illustrata, or the microscope explained, etc. I1lustrated. $4^{\text {th }}$ edition, London, I77I. Also Essays, 1787 .

Angström.-Recherches sur le spectre solaire, spectre normal du soleil. Upsala, I868.

Anthony, Wm. A., and Brackett, C. F.-Elementary text-book of physics. 7 th ed. Pp. 527, I65 Fig. New York, I89I.

Barker-Physics. Advanced course. Pp. 902, 380 Fig. New York, 1892.

Bausch, E,-Manipulation of the Microscope. A manual for the work table and a text-book for the beginners in the use of the microscope, illustrated. New Edition, I9oI. Rochester, N. Y.

Beale, I. S.-How to work with the microscope. 5th ed. Pp. 518, illustrated. London, I88o. Structure and methods.

Beauregard H., et Galippe, V.-Guide de l'élève et du praticien pour les travaux pratiques de micographie, comprenant la technique et les applications du microscope à l'histologie végétale, à la physiologie, à la clinique, à la hygiène et à la médicine légale. Pp. 904, 570 Fig. Paris, 1880.

Behrens, H-Mikrochemische Technik, 2d ed. Pp. 68. Hamburg, Igoo.

Behrens, T. H.-Anleitung zur microchemischen Analyse der wichtigsten organischen Verbindungen. Hamburg, I895-1897.

Behrens, T. H.-Transl, by J. W. Judd. A manual of microchemical analysis with an introductory chapter by J. W. Judd. London, I894.

Behrens, W. - The microscope in botany. A guide for the microscopical investigation of vegetable substances. Translated and edited by Hervey and Ward. Pp. 466, illustrated. Boston, 1885 .

Behrens, W.-Tabellen zum Gebrauch, bei mikroskopischen Arbeiten. 3d edition. Pp. 237. Braunschweig, 1898 .

Behrens, W., Kossel, A., und Schiefferdecker, P.-Das Mikroscop und die Methoden der mikroskopischen Uuntersuchung. Pp. 3I5, I93 Fig. Braunschweig, 1889 t.

Boehm, A. A. und von Davidoff, M.-A text-book of Histology, including microscopic technique, from the $2 d$ German edition. Translated by H. H. Cushing and edited by G. C. Huber. Pp. 501, illustrated. Philadelphia and London, Igoo.

Boehm, A. und Oppel, A.-Taschenbuch der mikroskopischen Technik, 4th ed. with directions by Born for making wax models. Pp. 240. Mïnchen, I900.

Brewster, Sir David. - A treatise on the mikroscope. From the th ed. of the Encyc. Brit., with additions. Illustrated, 1837 .

Brewster, Sir David.-A treatise on optics. Illustrated. New edition. I,ondon, I853.

Browning, J.-How to work with the micro-spectroscope. I,ondon, I894. 
Bousfield, E. C.-Guide to photo-micrography. 2d ed. Illustrated. London, I892.

Carnoy, J. B. L,e Chanoine.-La Biologie Cellulaire; Etude comparée de la cellule dans les deux regnes. Illustrated (incomplete). Paris, I884. Structure and methods.

Carpenter, W. B.-The microscope and its revelations. 6th ed. Pp. 882, illustrated. London, and Philadelphia, r88r. Methods and structure.

Carpenter-Dallinger.-The microscope and its revelations, by the the late William B. Carpenter. 8 th edition, in which the ist seven and the $23 \mathrm{~d}$ chapters have been entirely re-written, and the text throughout reconstructed, enla1ged and revised by the Rev. W. H. Dallinger. 22 plates and nearly goo wood engravings. Pp. II8I. London and Philadelphia, I9oI.

Chamberlain, C. J.-Methods in plant histology, Illustrated. Chicago, I9oI.

Clark, C. H.-Practical methods in microscopy. Illustrated. Boston, I894.

Cooke, M. C.-One thousand objects for the microscope. Pp. I23. London, no date, 500 figures and brief descriptions of pretty objects for the microscope.

Crookshank, E. M.-Photography of bacteria. London and New York, 1887.

Cross and Cole.-Modern microscopy for beginners. Part I. The microscope and instructions for its use. Part II. Microscopic objects, how prepared and mounted. Illustrated. 2d edition, I,ondon, I895.

Czapski, Dr. Siegfried.-Theorie der optischen Instrumente nach Abbe. Illustrated. Breslau, I893.

Dana, J. D.-A system of mineralogy. Illustrated. 6th ed. New York, I892.

Daniell, A.-A text-book of the principles of physics. Illustrated, 3d ed. London, I895.

Daniell, A.-Physics for students of medicine. Illustrated. London and New York, I8g6.

Davis, G.-Practical microscopy. 3d ed. Illustrated. London, I895.

Dippe1, I.-Das Mikroskop und seine Anwendung. Illustrąted. Braunschweig, I898.

Dodge, Charles Wright.-Introduction to elementary practical biology ; a laboratory guide for high school and college students. New York, I894.

Eberth, C. J.-Friedlander's mikroskopische Technik zum gebrauche bei medicinischen und pathologische-anatomischen Untersuchungen. Pp. 359, illustrated. Berlin, I900.

E,bner, V. V.-Untersuchungen über die Ursachen der Anisotropie organischer Substanzen. Leipzig, I882. Large number of references.

Ellenberger, W.-Handbuch der vergleichenden Histologie und Physiologie der Haussåugethiere. 2d edition. Berlin, Igort.

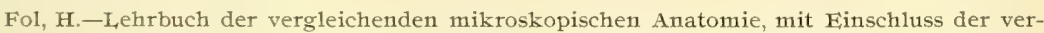
gleichenden Histologie und Histogenie. Illustrated (incomplete). Leipzig, I884. Methods and structure. *

Foster, Frank P.-An illustrated encyclopædic medical dictionary, being a dictionary of the technical terms used by writers on medicine and the collateral sciences in the Latin, English, French and German 1anguages. Illustrated, four quarto volumes, r888-r893.

Fraenkel und Pfeiffer.-Atlas der Bacterien-Kunde. Berlin, I889+.

Francotte, P.-Manuel de technique microscopique. Pp. 433, I10 Fig. Brussels, I886.

Francotte, P.-Microphotographie appliquée à 1'histologie, l'anatomie comparée et l'embryologie. Brussels, r886.

Frey, H.-The microscope and microscopical technology. Translated and edited by G. R. Cutter. Pp. 624, illustrated. New York, I880. Methods and structure.

Galt, H.-The microscopy of the starches illustrated by photomictographs. London, Igoo.

Gamgee, A.-A text-book of the physiological chemistry of the animal body. Part I, pp. 487 , 63 Fig. London and New York, 1880. Part, II, I893.

Gebhardt, W.-Die mikrophotographische Aufnahme gefärbter Präparate. Illustrated. München, I899.

Giltay, Dr. E.-Sieben Objecte unter dem Mikroskop. Einfuhrung in die Grundlehren der Miktoskopie. Leiden, 1893. This is also published in the Holland (Dutch) and French language.

Goodale, G. L. Physiological botany. Pp. $499+36$, illustrated. New York, r885. Structure and methods. 
Griffith and Henfrey.-The Micrographic Dictionary; a guide to the examination and investigation of the structure and nature of microscopic objects. Fourth edition, by Griffith, assisted by Berkeley and Jones. London, 1883 .

Gould, G. M.-The illustrated dictionary of medicine, biology and allied sciences. Illustrated, 5th ed., Philadelphia, 1900. This is recognized as the best single volume medical dictionary. It is especially satisfactory for the worker with the microscope.

Hager, H. und Mez. C.-Das mikroskop und Seine Anwendung. 8th ed., revised and enlarged, Pp. 335. Illustrated. Berlin, 1899.

Halliburton, W. D.-A text-book of chemical physiology and pathology. Pp. 874, ro4 illustrated. London and New York, I891.

Hanausek, T. F.-Lehrbuch der mikroskopischen Technik. Illustrated. Stuttgart, Igoo.

Harker, H.-Petrology for students; an introduction to the study of rocks under the microscope. Cambridge, I895.

Harting, P.-Theorie and algemeine Beschreibung des Mikroscopes. 2d ed. 3 vols. Braunschweig, I866.

Herzfeld, T, J.-The technical testing of yarns and textile fabrics with reference to official specifications. Translated by C. Salter. Illustrated. London, 1898 .

Hogg, J.-The microscope, its histony, construction and application. I5th edition, illustrated. Pp. 704. London and New York, I898. Much attention paid to to the polariscope.

Huber, G. C.-Laboratory work in histology, 3d revised and enlarged edition. Pp. 204, illustrated. Ann Atbor, I900.

James, F. L.,-Elementary microscopical technology. Part $\mathbf{I}$, the technical history of a slide from the crude material to the finished mount. Pp. Io7, illustrated. St. Louis, I887.

Jeserich, P.-Die mikrophotographie auf Bromsilbergelatine bei natlurlichem und Künstlichem Lichte. Figs. and Plates. Pp. 245. Berlin, I888.

Jüptner, Hanns Freiherr von.-Gründzuge der Siderologie, Erster Teil, Leipzig, Igoo. Full discussion of theory of solution, chemidal constitution, micrography, etc, of iron alloys and slags. Illustrated. Bibliography very complete. Part II not yet issued, "Will deal with relation between chemical constitution, mechanical treatment, microscopic structure, "etc.

Kahlden, C. von.-Technik der histologischen Unterschung pathologisch-anatomischer Präparate, 6th revised and enlarged edition. Pp. I74. Jena, I900.

Kaiser, W.-Die Technik des modernen Mikroskopes, 2d edition. Illustrated- Wien, Igor.

King, J.-The microscopist's companion. A popular manual of practical microscopy. Illustrated. Cincinnati, 1859.

Klément et Renard.-Réactions microchemiques à cristaux et leur application ẹn analyse qualitative. Pp. I26, 8 plates. Bruxelles, I886.

Kraus, G.-Zur Kentniss der Chlorophyllfarbstoffe. Stuttgart, I872.

Iankaster, E,-Half-hours with the microscope; a popular guide to the use of the microscope as a means of amusement and instruction. 20th ed. Pp. I50. London, 1898.

Latteux, P.-Manuel de technique microscopique. 3d ed. Paris, I887.

Le Conte, Joseph.-Sight-an exposition of the principles of monocular and binoculat vision. Pp. 275, illustrated. New York, I88x.

L,ee, A. B.-The microtomist's vade-mecum. A hand-book of the methods of microscopic anatomy. 5th ed. Pp. 532. Philadelphia, Igoo.

L.ehmann, C. G.-Physiological chemistry. 2 vols. Pp. $648+547$, illustrated. Philadelphia, I855.

L,ehmann, O.-Molekularphysik mit besonderer Berücksichtigung mikroskopischer Untersuchungen und Anleitung zu solchen, sowie einem Anhang über mikroskopische Analyse. 2 vols. Illustrated. Leipzig, I888-I889.

Leiss, C.-Die optischen Instrumente der Firma R. Fuess, deren Beschreibung. Justierung und Anwendung. Pp. 397, illustrated. Leipzig, I899.

Lockyer, J. N.-The spectroscope and its application. Pp. II7, illustrated. London and New York, 1873 
Inquer, I. MC. I.-Minerals in Rock Sections. Practical methods of identifying minerals in rock sections with the microscode.

M'Kendrick, J. G.-A text-book of physiology. Vol. I, general physiology. Pp. 5I6, illustrated. New York, ISS8.

Macé, E.-Les substances alimentaire étudies au microscope surtout au point de vue de leurs altérations et de leur falsifications. Illustrated. Paris, I8gr.

Macdonald, J. D.-A guide to the microscopical examination of drinking water. Illustrated. London, 1875. Methods and descriptions.

Maclunn, C. A.-The spectroscope in medicine. Pp. 325, illustrated. London, I885.

IIarktanner-Turneretscher, G.-Die Mikro-Photographie als Hilfsmittel naturwissenchaftlicher Forschung, Pp. 344, illustrated. Halle, a, S., I8go.

Martin, John H.-A manual of microscopic mounting with notes on the collection and examination of objects. $2 \mathrm{~d}$ ed. Illustrated. London, 1878 .

Mason, John J.-Minute structure of the central nervous system of certain reptiles and batrachians of America. Illustrated by permanent photo-micrographs. Newport, r879-82.

Matthews, C. G., and I,ott, F. E.-The microscope in the brewery and the malt house. Illustrated. London, I889.

Mayal1, John, Jr--Cantor lectures on the microscope, delivered before the society for the encouragement of arts, manufacturers and commerce. Nov.-Dec., I885. History of the microscope, and figures of many of the forms used at various times.

Meyer, A. Die Grundlagen und die Methoden für die mikroskopische Untersuchung von Pflanzenpulvern. Pp. 258, illustrated. Jena, Igor.

Moeller, J.-Leitfaden zu mikroskopisch-pharmakgnostischen Übungen. Pp. 336, illustrated. Wien, Igor.

Moitessier, A.-La photographie appliquée aux recherches micrographiques. Paris, I866.

Morel et Soulié.-Manuel de technique microscopique. Paris. I8g9.

Nägeli und Schwendener.-Das Mikroskop, Theorie und Anwendung desselben. 2d ed. Pp. 647, illustrated. Leipzig, I877.

Neuhauss, R.-Lehrbuch der Mikro-photographie. Pp. 266. Illustrated. 2d ed. revised. Braunschweig, 1898 .

Nichols, E. I. -The outlines of physics, Illustrated, N. Y., I897.

Nichols, E. I. and Franklin W. S.-The Elements of Physics; Iight and Sound. Pp. 20r, illustrated. New York and L,ondon, I897.

Pappenheim, A.-Grundriss der Farbenchemie zum Gebrauch bei mikroscopischen Arbeiten. Pp. 476 . Berlin, I9or.

Petri, R. J.-Das Mikróskop von seinen Anfängen bis zur jetzigen Vervollkommung für alle Freunde dieses Instiuments. Pp. 248. Illustrated. Berlin, I896.

Phin, J.-Practical hints on the selection and use of the microscope for beginners. 6th ed. Illustrated. New York, I8gi.

Posselt, E. A.-The structure of fibres, yarns and fabrics. Illustrated. Philadelphia and London, I89I.

Preyer, W.-Die Blutkrystalle. Jena, I87r. Full bibliography to that date.

Pringle, A.-Practical photo-micrography. Pp. I93, illustrated. New York, I8go.

Procter, R. A.-The spectroscope and its work. London, I882.

Quekett, J.-A practical treatise on the use of the microscope, including the different methods of preparing and examining animal, vegetable and mineral structures. Pp. 5I5, I2 plates, $2 \mathrm{~d}$ ed. London, 1852 .

Rafter, G. W-The Microscopical Examination of Potable Water. Pp. I60, New York, I892.

Ranvier, I. - Traité technique d'histologie. Pp. IIog, illustrated. Paris, 1875-I888. Structure and methods. Also German translation, I888.

Reeves, J. E.-A hand-book of medical microscopy, including chapters on bacteriology, neoplasms and urinary examination. Illustrated. Philadelphia, I894. 
Reference Hand-Book of the medical sciences. Albert H. Buck, editor. 8 quarto vols. Illustrated with many plates, and figures in the text. New York, 1885-1889. Supplement, I893. New edition, IgoI + .

Richatdson, J. G.-A hand-book of medical microscopy. Pp. 333, illustrated. Philadelphia, I87I. Methods and descriptions.

Robin, Ch.-Traité du microscope et desinjections, 2d ed. Pp. IIoI, illustrated. Paris, 1877. Methods and structure.

Roscoe, Sir Henry.-Lectures on spectrum analysis. 4th ed. London, I885.

Rosenbusch, K. H. F. translated by Iddings, P.-Microscopical physiography of the rock making minerals. Illustrated. New York, r889.

Ross, Andrew.-The microscope. Being the article contributed to the "Penny Cyclopædia." Republished in New York in 1877 . Illustrated.

Rusby, H. H. and Jeliffe, S. E.-Morphology and Histology of Plants designed especially as a guide to plant analysis and classification, and an introduction to pharmacognosy and vegetable physiol. Pp. 378, illustrated. New York, I899.

Rutherford, W.-Outlines of practical histology. 2d. ed. Illustrated. Pp. I94. L,ondon and Philadelphia, 1876. Methods and structure.

Sayre, L. E.-Organic Materia Medica and Pharmacognosy. An introduction to the vegetable kingdom and the vegetable and animal drugs. Chapters on insects injurious to drugs and on pharmacal botany. Pp. 220, Detroit, I880.

Schäfer, E. A.-A course of practical histology, being an introduction to the use of the microscope. Pp. 304, 40 Fig. Philadelphia, 1877. Methods.

Schellen, H.-Spectrum analysis, translated by Jane and Caroline L,assell. Edited with notes by W. Huggins. I3 plates, including Angström's and Kirchhoff's maps. London 1885 .

Schimper, A. F. W.-Anleitung zur mikroskopischen Untersuchungen der vegetabilischen Genussmittel. Pp. I58, illustrated. Jena, I900.

Schneider, A.-Microscopy and micro-techuique. Pp. I89, illustrated. Chicago, I89g.

Sedgwick and Wilson,-General biology, 2d ed. Illustrated. New York, I895.

Seiler, C.-Compendium of microscopical technology. A guide to physicians and students in the preparation of histological and pathological specimens. Pp. I30, illustrated. New York, I88I.

Silliman, Benj., Jr,-Principles of physics, or natural philosophy. 2d ed., rewritten. Pp. 7Io, 722 illustrations. New York and Chicago, 1860.

Spitta, E. J.-Photomicrography: $4^{\circ}$. Pp. I63. Illustrated by half-tone reproductions from original negatives. Text illustrations. London, I899.

Starr, Allen M., with the coöperation of Oliver S. Strong and Edward Leaming.-An atlas of nerve-cells. Columbia University Press, New York, I8g6. The atlas consists of text, diagrams, and some of the best photo-micrographs that have ever been published.

Sternberg, G. M.-Photo-micrographs, and how to make them. Pp. 204, 20 plates. Boston, I883.

Stoehr, S. P.-Text-book of histology including the microscopic technique, 3d American from 8th German edition. Translated by Dr. Emma Bilstein and edited by Dr. A. Schaper. Pp. 432, illustrated. Philadelphia, Igor.

Stokes, A.-Aquatic microscopy for beginners, or common objects from the ponds and ditches. Illustated. Portland, Conn., I8g6.

Suffolk, W. T.-On microscopical manipulation. 2d ed. Pp. 227, Illustrated. London, 1870.

Szymonowicz, L--Lehrbuch der Histologie-einschliesslich der mikroskopischen Technik. Illustrated. Würzburg, Igoo.

Thomas, Mason B., and Wm. R. Dudley.-A laboratory manual of plant histology. Illustrated. Crawfordsville, Ind., I894.

Trelease, Wm.-Poulsen's botanical micro-chemistry ; an introduction to the study of vegetable histology. Pp. II8, Boston, I884. Mehods. IS84.

Trutat, M.-La photographie appliquée à histoire naturelle. Pp. 225, illustrated. Paris, 
Valentin, G.-Die Untersuchung der Pflanzen und der Thiergewebe in polarisirtem Licht. L,eipzig, I86I.

Van Heurck, H-The microscope. Illustrated. London and New York, I898.

Vierordt. Die quantitative Spectralanalyse in ihrer Anwendung auf Physiologie, 1876.

Vog1, A. E.-Die wichtigsten vegetablischen Nahrungs und Genussmittel. Pp. 575., illustrated. Wien, 1899 .

Vogel, Conrad.-Practical pocket-book of photography. Pp. 202, Figs. London, 1893.

Vogel, H. W.-Practische Spectralanalyse irdischer stoffe; Anlertung zur Benutzung der Spectralapparate in der qualitativen und quantitativen chemische Analyse organischer und unorganscher Körper. 2d ed. Figs. Berlin I889.

Wall, O. A.-Notes on Pharmacognosy.-Pp. I80, iilustrated. St Louis, I897.

Weinschenk, E. Anleitung zum Gebrauch des Polarisationsmikroskopes. Pp. I23, illustrated. Freiburg. IgoI.

Wethered, M.-Medica1 microscopy. Pp. 406, Figs. I,ondon and Philadelphia, I892. I599.

Whipple, G. C.-The Microscopy of Drinking Water. Pp. xii + 300. Illustrated. New York,

Whitman, C. O.-Methods of research in microscopical anatomy and embryology. Pp. 255, illustrated. Boston, I885.

Wilder and Gage.-Anatomical technology as applied to the domestic cat. An introduction to human, veterinary and comparative anatomy. Pp. 575, 130 Fig. 2d ed. New York and Chicago, I886.

Wilson, Edmund, B., with the cöoperation of Edward Leaming.-An atlas of fertilization and karyokinesis. Columbia University Press, New York, 1895. This atlas marks an era in embryological study. It has admirable texts and diagrams, but the distinguishing feature is the large number of almost perfect photo-micrographs.

Wood, J. G.-Common objects for the microscope. Pp. I32. London, no date. Upwards of 400 figures of pretty objects for the microscope, also brief descriptions and direction for prepartions.

Wormly, T. G.-The micro-chemistry of poisons. 2d ed. Pp. 742, illus. Philadelphia, I885.

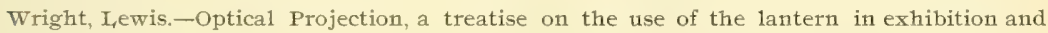
scientific demonstration. Pp. 425, illustrated. London, I89I. (Beginners will find this book very helpful).

Wythe, J. H.-The microscopist; a manual of microscopy and a compendium of microscopical science, 4th ed. Pp. 434, 252 Fig. Philadelphia, I880

Zimmermann, Dr. A.-Das Mikroskop, ein Leitfaden der wissenschaftlichen Mikroskopie. Illustrated. Leipzig und Wein, I895.

See also Watt's chemical dictionary, and the various general and technical encyclopedias.

PERIODICALS*

The American journal of anatomy (including histology, embryology and cytology). Baltimore, Igort.

The American journal of medical research, Boston, Igor + .

The American Journal of physiology. Boston, $1896+$.

The American journal of microscopy and popular science. Illustrated. New York, I876-I88I.

The American monthly microscopical journal. Illustrated. Washington, D. C., I880+ .

American naturalist. Illustrated. Salem and Philadelphia, I867t.

American quarterly microscopical journal, containing the transactions of the New Xork microscopical society. Illustrated. New York, $1878+$.

American microscopical society, Proceedings. I878-I894; Tuansactions, I895+.

*Note-When a periodical is no longer published, the dates of the first and last volumes are given; but if still being published, the date of the first volume is followed by a plus sign.

See Vol, XVI of the index Catalog of the Library of the Surgeon General's office for a full list of periodicals. 
Anatomischer Anzeiger. Centrablatt für die gesammte wissenchaftliche Anatomie. Amtliches Organ der anatomischen Gesellschaft. Herausgegeben von Dr. Karl Bardeleben. Jena, $I 886+$. Besides articles relating to the microscope or histology, a full record of current anatomical literature is given.

Annales de la société belge de microscopie. Bruxelles, $1874+$.

Archives d'Anatomie microscopique. Illustrated. Paris, I897. (Balbiani et Ranvier).

Archiv für miroscopische Anatomie. Illustrated. Bonn, I865+.

Centrablatt für Physiologie. Unter Mitwirkung der physiologischen Gesellschaft zu Berlin Herausgegeben von S. Exner und J. Gad. Leipzig and Wien, 1887+. Brief extracts of papers having a physiological bearing. Full bibliography of current literature.

English mechanic. London, I866 +. Contains many of the papers of Mr. Nelson on lighting, photo-micrography, etc.

Index medicus. New York, 1879t. Bibliography, including histology and microscopy.

International journal of micioscopy and popular science. London, I89o+.

Journal of anatomy and physiology. Illustrated. I,ondon and Cambridge, $1867 \div$.

Journal of Applied Microscopy and Laboratory methods. Illustrated. Rochester, N. Y. $1898 \div$

Journal de micrographie. Illustrated. Paris, I877-1892.

Journal of microscopy and natural science. L,ondon, $1885+$.

Journal of tine New York microscopical society. Illustrated. New York, $1885+$.

Journal of physiology. Illustrated. I,ondon and Cambridge, $1878+$.

Journal of the American chemical society. New York, 1879+.

Journal of the chemical society. L,ondon, I848+.

Journal of the royal microscopical society. Illustrated. London, $1878+$. Bibliography of works and papers relating to the microscope, microscopical methods and histology. It also includes a summary of many of the papers.

Journal of the Quekett microscopical club. London, I868+.

The Lens, a quarterly journal of microscopy, and the allied natural sciences, with the transactions of the state microscopical society of Illinois. Chicago, 1872-1873.

The Metallographist, a quarterly publication devoted to the study of metals with special reference to their physics, microstructure, their industrial treatment and application. Illustrated especially by photo-micrographs of metals and alloys. Boston, $1898+$.

The Microscope. Illustrated. Washington, D. C., I88I-I897.

Microscopical bulletin and science news. Illustrated. Philadelphia, I883+. The editor, Edward Pennock introduced the term "par-focal" for oculars (see vol, iii, p 3I).

Monthly microscopical journal. Illustrated, L,ondon, I869-1877.

Nature. Illustrated. London, I869t.

The Observer. Portland, Conn., I890-1897.

Philosophical Transactions of the Royal Society of London. Illustrated. L,ondon, $1665+$.

Proceedings of the American microscopical society, $1878+$.

Proceedings of the Royal Society. London, $1854+$.

Quarterly journal of microscopical science. Illustrated. London, $1853+$.

Science Record. Boston, r883-4.

Zeitschrift f. Angewandte. Mikroskopie. $1898+$.

Zeitschrift für Instrumentenkunde. Berlin, I88I + .

Zeitschrift für physiologische Chemie. Strassburg, $1877+$.

Zeitschrift für wissenschaftliche Mikroskpie und für mikroskopische Technik. Illustrated. Braunschweig. I884 +. Methods, bibliography and original papers.

Besides the above-named periodicals, articles on the microscope or the application of the microscope appear occasionally in nearly all of the scientific journals. One is likely to get references to these articles through the Jour. Roy. Micr. Soc. or the Zeit. wiss. Mikroskopie. Excellent articles on Photo-micrography occur in the special Journals and Annuals of Photography. 


\section{N D E X}

A

Abbe apertometer, 270-272.

Abbe camera lucida, $123-132$.

Abbe condenser or illuminator, 45-50.

Abbe's test-plate, method of using, 268270.

Aberration, chromatic, 4,5 ; by coverglass, 55 ; spherical, $4,5,268$.

Absorption spectra, I36-I39, I45-I 50 .

Acetelyne light, 37, 51, 229.

Achromatic condenser, 42-43, 256 ; objectives, II, 64 ; oculars, 22.

Achromatism, 12 .

Actinic focus, 226 ; image, $22 \mathrm{r}$.

Adjustable objectives, II, I2, 54-57 ; experiments, 54 ; and micrometry, I I 8 ; and photo-micrography, 234.

Adjusting collar, graduation of, 55 .

Adjustment, of analyzer, I5I ; coarse or rapid, and fine, $6_{3}$, Frontispiece ; of objective, II, I 2, 54; of objective for cover-glass, specific directions, 55 ; testing, 63 .

Aerial image, 30, 31, 230 .

Air bubbles, 94,95 .

Albumen fixative, Mayer's, I98.

Alcohol, absolute, I98; ethylic, I98; methyl, 198, 203.

Alcoholic dye, staining sections with, I89.

Alum solution, I98.

Amici prism, I34, I40.

Amplifier, Iog.

Amplification of microscope, IO3.

Analyzer, I4I, I5I.

Anastigmatic objective, 208, 2 I 2.

Angle, of aperture, 15, 16; of carbons for arc lamps, 25I-252; critical, 54.

Angström and Stokes' law of absorption spectra, 138 .

Angular aperture, 15, I6.

Anisotropic, ${ }^{5} 5$.

Apertometer, Abbe's, 270.

Aperture of objective, 15, 21, 270 ; angular, I5, I6 ; formula for, I6-I8; of illuminating cone, 45 ; numerical, 17 . 19, 270; numerical of condenser, 44 ; significance of, 20.

Aphengescope, 267.

Aplanatic cone, 46 ; objectives, II ; ocular, 22 ; triplet, 7 .
Apochromatic condenser, 42 ; objectives, I 2, 64, 214 .

Apparatus and material, I, 34, 90, IO3, I 22, I34, 205, 243 ; for micro-projection; 256, 257 ; for photography, 233 ; for photo-micrography, 205.

Appearances, interpretation, 90-102.

Arc lamp, 37, 229, 250-255; continuous current, 249.

Arrangement of condenser, 46 ; of lamp, bull's eye and microscope, 5 I ; minute objects, 204; serial sections, I 92 ; tissue for sections, I9I.

Artifacts, 9I.

Artificial illumination, $37,48,50$; for photo-micrography, 229.

Avoidance of diffusion currents, I8o; of distortion, I24.

Axial light, 36 ; experiments, 40 ; with Abbe illuminator, 48 .

Axial, point, 15 ; ray, 36 .

Axis, optic, 2, 3, Io; of illuminator, 49 ; secondary, $3,6,49$.

\section{B}

Back-ground for photographing, 209.

Back combination or system of objective, 9-I 2 .

Bacterial cultures, photographing, 24I242.

Balsam, 199; bottle, 183; mounting in, I83, I90; preparation of, I99; removal from lenses, 6I ; natural, Igo, I99; neutral, I99; removal from slides, I62; xylene, I99.

Bands, absorption, I37.

Base of microscope, Frontispiece.

Bath, water, 255.

Bed, camera, 207.

Benzin, removing from sections, 189 .

Biaxial crystals, I54.

Bibliography, 26, 33, 99, IOI, I2I, I33, I5O, I55, I57, I58, I59, I96, 204, 220, $240,242,251,258,267,273,274,282$.

Blocks for collodion, 178 ; for shell vials, I74.

Blood, absorption spectrum of, I46 ; circulation of with micro-projection, $257,264$.

Board, circulation, 280 .

Body of microscope, Frontispiece. 
Bottle for balsam, glycerin or shellac, I 72 ; reagent, I79.

Bowl, waste, $18 \mathrm{I}$.

Box, glass, I8I ; slide, I98.

Brownian movement, 99.

Bubble, air, 94, 95.

Bull's-eye, 5I, 217, 227; engraving glass for, 228 .

Burning point, 7,30 .

Buxton's photo-micrographic outfit, 237238.

Cabinet for microscopical preparations, I96, I97.

Calipers, micrometer, I64 ; pocket, I64.

Camera, bed, 207 ; for embryos, $2 \mathrm{II}$; for large, transparent sections, 2I5 ; photo-micrographic, 222, 225, 235 ; testing, 223 ; vertical, 205-2 IO.

Camera lucida, Abbe, I22-I32; Wollaston's, $107,125$.

Canada balsam, I99; mounting in, I83, I90; preparation of, I99; removal from lenses, $6 \mathrm{I}$; removal from slides, I62.

Carbol-xylene, 200.

Carbon-monoxide hemaglobin, spectrum of, 147 .

Carbons, for projection apparatus, 250255 ; adjusting, 25I ; angle, 25I ; kinds, 253; positive and negative, 252 ; size, 254 ; wear, 253.

Card, catalog, I96 ; centering, I69 ; inventory, $28 \mathrm{I}$.

Care of, eyes, 6I ; micro-projection apparatus, 265; microscope, mechanical parts, 59 ; optical parts, 6o ; negatives, $2 \mathrm{II}$; water immersion objec. tives, 58 .

Carmine to show currents and pedesis, 99 ; spectrum of, I48.

Carrier, objective, 259.

Castor-xylene clarifier, 200.

Cataloging, formula, I95; preparations, I94-I96.

Cedar-wood oil, bottle for, I99; for clearing, $184-185,200$; for oil immersion objectives, 200.

Cells, deep, thin, I68; isolated, preparation of, 175 ; mounting, 168 ; staining, 174 .

Cement, shellac, 203.

Cementing collodion, 20 .

Center, optical, 2, 3 .

Centering, and arrangement of illuminator, 43,47 ; card, 169 ; image of source of illumination, 44 ; the radiant, 255.

Centimeter rule, IO4, I33.
Central light, 36, 95 .

Chamber, moist, I 7 I.

Cheese-cloth for cleaning slides, I6I.

Chemical focus, I 2 ; rays, I2.

Chemistry, Micro-I 55 .

Chromatic, aberration, 4 ; correction, II, I2 ; correction, test for, 270.

Circulation of blood with micro-projection, 257, 264 ; board, 226.

Clarifier, 200 ; castor-xylene, 200.

Class demonstrations in histology and embryology, 243.

Cleaning back lens of objective, $6 \mathrm{I}$; homogeneous objectives, 59 ; micro-projection apparatus, 265 ; mixtures for glass, 165 ; sildes and cover-glasses, $161-16_{3}$; water immersion objectives, $5^{8}$.

Clearer, I73, I83, I90, 200.

Clearing mixture, preparation of, 200 ; tissues with cedar-wood oil, I84.

Clinical microscope, 243-245.

Cloudiness of objective and ocular, how to determine, 92 ; removal 60 .

Coarse adjustment of microscope, Frontispiece ; testing, 63 .

Cob-web micrometer, II 7 .

Collecting can, 28o

Collodion, 200 ; for coating glass rod, 97 ; cementing, 201 ; clarifying, 178 ; cotton, 200; for fastening sections to slide, I88; hardening, I78; method, I 76 ; thick, thin, $177,201$.

Color, correct photography, 217 ; correction, I2 ; images, $54,5^{8}$; law of, $\mathrm{x}_{3} 8$; production of, I54; screens, $218,220$.

Colored minerals, absorption spectra of, I49; specimens, photography of, $2 \mathrm{I} 8$.

Comparison prism, I4I, I42 ; spectrum, 142.

Compensating ocular, 24, 25.

Complementary spectra, I39.

Compound microscope, see under microscope.

Concave lenses, 3 ; mirror, use of, 37 .

Condenser, 4I-50 ; Abbe, 46, 48-49; achromatic, $42,43,227,256$; apochromatic, 42, 227; bull's-eye, 5I, 227 , 228 ; centering, 43,47 ; illuminating cone with, 45 ; lamp, for projection, 250 ; mirror for, 48 ; non-achromatic, 46 ; numerical aperture of, $44-46$; optic axis of, 43,47 ; for photo-micrograplay, 42, 227, 233 ; standard size for, 26,47 ; substage, 42 ; See also illuminator.

Condensing lens, 36 .

Cone, aplanatic, 46 ; illuminating, 45 .

Conjugate foci, 4 .

Construction of images, geometrical, 6 . 
Continuous, current arc lamp, 249 ; spectrum, 137.

Contoured, doubly, 97 .

Converging lens, $3^{-5}$; lens system, 9.

Convex lenses, 3-5.

Cooler, specimen, 257.

Correction, chromatic, or color, 5, I 2, 268270 ; cover-glass, $55^{-5} 5$; cover, tubelength for, 56-57; over and under, I 2.

Cotton, collodion, gun, or soluble, 200.

Counterstaining, 189-I9o.

Cover-glass, or covering glass, I62-I63; aberration by, 54; adjustment, specific directions, 55 ; adjustment for, in photo-micrography, 234 ; adjustment and tube-length, I2, I3, 55 ; anchoring, I70; cleaning, I62-I63; correction, 55,56 ; effect on rays from object, $\mathrm{I} 7,55$; gauges, I $64-165$; measurer, $164-165$; measuring thickness of, I63; non-adjustable objectives, table of thickness, I4; No. I, variation of thickness, I64; putting on, 94, I67; sealing, I69, I70; size of, I64, I62; thickness of, I3, I4, I63-165, I93; tube-length with, $13,56,57$; wiping, I63; with, serial sections, I93.

Crater of carbons, 252.

Critical angle, 54 .

Crystals, biaxial, depolarizing, 154 ; from frog for pedesis, Ioo.

Crystallization under microscope, 50, 157.

Crystallography, I55; list of substances for, $156-157$.

Currents, diffusion, avoidance of, I8o; in liquids, 98 .

Cutting sections, I78, I86, I9I-192.

\section{D}

Dark-ground illumination, $37,49-50$; with Abbe illuminator, 50 ; with mirror, 50 .

Daylight, lighting with, 35 .

Deck-plugs for collodion blocks, 178 .

Dehydration, I77, Igo.

Demonstration, microscope, 243-245; with micro-projection apparatus, 265.

Depolarizing crystals, 154 .

Designation, of oculars, 25; of wave length; I43.

Determination, of, field of microscope, 28 : equivalent focus, $272-273$; magnification, 103-IO9, 273; of working distance, 39.

Diamond, writing, 196.

Diaphragms and their employment, 36-50.

Diffraction, grating, 137 ; illusory appearances due to, IOI.

Diffusion currents, avoidance of, I8o.
Direct, light, 35; vision spectroscope I 34 .

Dispersing prism, 137 .

Dissecting microscope, $8,33,175,228$.

Dissociator, formaldehyde, $20 \mathrm{I}$; nitric acid, 203.

Distance, principal focal, 3. 30; standard at which the virtual image is measured, I09; working d. of simple microscope or objective, 39 ; working d. of compound microscope, II, 34 , 39-40.

Distinctness of outline, 96.

Distortion in drawing, avoidance of, 124.

Diverging lens, 3.

Double spectrum, I42 ; vision, IO3, IO5.

Doubly contoured, 97 ; refracting, 152.

Draw-tube, Frontispiece; pushing in, 38.

Drawing, with Abbe camera lucida, I29I3I ; board for Abbe camera lucida; I 29, I30; distortion, avoidance of, I24; embryograph for, I32 ; with microscope, I22; photographic camera for, $\mathrm{I}_{32}$; for photo-engraving, 273 ; scale and enlargement of, $\mathrm{I}_{2} \mathrm{I}$; with simple microscope, I32.

Dry objectives, IO, I6-I8; light utilized, I7 ; dry mounting, I67; numerical aperture, 16 ; dry plates, discovery by Maddox, 22I.

Dust, of living rooms, examination of, IOI ; on objectives and oculars, how to determine, 92 ; removal, 60.

Dye, general, staining with, I89; aqueous, I80, I89; alcoholic, I80, I89.

\section{$\mathrm{E}$}

Eccentric diaphragm, 44, 49-50.

Egg pippett, 28o.

Electric light, 37, 229, 235, 250.

Embryograph, I32

Embryos, camera for, 2 I I photographing, $2 \mathrm{II}-2 \mathrm{I} 4$; records of, $2 \mathrm{I} 3$.

Engraving glass for bull's-eye condenser, 228

Enlargements, 24I.

Eosin, 20I.

Epidiascope, 266-267

Episcope, 267.

Equivalent focal length or focus of objectives and oculars, I0, 25, 272 .

Erect image $\mathrm{I}$.

Ether, alcohol, 201 ; sulphuric, $20 \mathrm{I}$.

Ethylic alcohol, I98.

Examination of dust of the living rooms, bread crumbs, corn starch, fibres of cotton, linen, silk, human and animal hairs, potatoes, rice, scales of butterflies and moths, wheat, IOI. 
Experiments, Abbe condenser, 48 ; with Food, detection of adulteration in, 158 adjustable and immersion objectives, Form of objects, determination of, 93 .

54 ; compound microscope, 26 ; ho- Formal, 201.

mogeneous immersion objective, $5^{8}$; Formaldehyde, dissociator, $20 \mathrm{I}$; for isolalighting and focusing, 37 ; in microchemistry, I57; with micro spectroscope, I45; with micro-polariscope, I52; in mounting, I66; photo-micrography, 229 ; simple microscope, 6.

Exposure, of photographic plates, 232, 235,240 ; with color-screen 220.

Extraordinary ray of polarized light, I5o.

Eye and microscope, 6, 9, 32. tion, 173 .

Formula, for aperture, 16, I 7 ; for cataloging, 195 ; for refraction, 52-54.

Fraunhofer lines, I37.

Free, hand sections, 274 ; working distance, 40 .

Front combination or lens of objective, 9-II.

Frontal sections, r.92.

Eyes, care of, 6r ; muscae volitantes of, Ioo.

Eye-lens of the ocular, 22.

Eye-piece, 22 ; micrometer, I I4.

Eye-point, 7, 22, I23; of ocular, demonstration, 32.

Eye-shade, adjusting, 62 ; double, 62 .

\section{F}

Farrants' solution in mounting objects, I7I ; preparation of, $20 \mathrm{I}$.

Fibers, examination of, IOI ; textile, I58. Field, 28 ; with camera lucida, I07; illumination of $45,5 \mathrm{I}$; with orthoscopic ocular, 23; with periscopic ocular, 24; of view with microscope, 28, 29, I05, 123-125; size of, with different objectives and oculars, 28,29 .

Field-lens, of ocular, 22 ; action of, 32 ; dust on, 92.

Function of objective, 29-3I; of ocular, $3 \mathrm{I}$.

\section{G}

Gauge, cover-glass, I64-I65.

Gelatin, liquid, preparation of, 203.

Geometrical construction of images, 6 .

Glass, cleaning mixture for, 165 ; focusing, 209; ground, 29, 209; rod appearance under microscope, 96,$97 ;$ slides or slips, I6I, I62.

Glasses, opera, 262.

Glue, liquid, preparation of, 203

Glycerin, bottle for, 183 ; mounting objects in, order of procedure, I7O, 2OI ; removal, 6r.

Glycerin jelly, mounting objects in, order of procedure, I7O-I7I ; preparation of, 201.

Grating, diffraction, I37.

Filar, micrometer ocular, 23, 26; ocular micrometer, II 7, II8.

Filter, hot, I85.

Filtering balsam, etc., I85, I99.

Fine adjustment, Frontispiece; testing, 63.

Fir, balsam of, I99.

Fixative, albumen, Mayer's, I98.

Fixing, reagents for, I98; tissue, I76.

Focal distance, or point, principal, 30; length equivalent, Io.

Focus, 6 ; actinic, 226 ; chemical, I 2 ; conjugate, 4 ; equivalent, of objectives and oculars, IO, 25, 272-273; optical, I2; principal,3, 5; virtual, 3 ; visual, 226.

Focusing, 6, 34: adjustments, testing, 63; with compound microscope, 34 ; embryos, 213 ; experiments, 38 ; glass, 209 ; with high objectives, 38 ; with low objectives, 38 ; objective for micro-spectroscope, I 44; for photo-micrography, 206, 213, 230 ; scale for, 206 ; screen for photo-micrograpliy, 209 ; with simple microscope, 6, 34; slit of micro-spectroscope, I 45.

Ground glass, focusing screen, 209 ; preparation of, 29.

Gun cotton, 200.

\section{$\mathrm{H}$}

Half-tones from photo-micrographs, 232, 274.

Hardening collodion, I78; tissue, I76, I 83 .

Hematein, 203

Hematoxylin, 202 ; stained preparations, photographing, 2 I 8.

Hemoglobin spectrum, I47.

High school microscope, 64, 7I-89.

Histology, physiological, I96.

History of photo-micrography, 220.

Holder, lens, $7,217,228$; needle, 167 ; slide, I88.

Homogenous immersion objective, I6I9; cleaning, 59; experiments, 58; numerical aperture, I6-2I.

Homogenous liquid, II ; tester for, $5^{8}$ $27 \mathrm{I}$; vessel for, I99.

Horizontal camera, 230, 236-237.

Huygenian ocular, 22, 24, 32. 
I

Illuminating, cone (for condenser), aperture of, 45 ; objective, 13,238 ; power, $2 \mathrm{I}$.

Illumination, for Abbe camera lucida, I29; artificial, 37, 48, 50; artificial for photo-micrography, 229 ; centering image of source of, 44 ; with air and oil, 94, 95; dark ground, 49, 50 ; daylight, 35 ; of entire field, 5 I ; lamp for, 50 ; methods of, 35, 49; for micro-polariscope, I5I ; for microspectroscope, I44; oblique with air and oil, 94, 95; of opaque objects, I 44, 238 ; for photography, 208, 216, 229, 24I ; for photo-micrography, $230,233,238$; for projection, 250 ; for Wollaston's camera lucida, I 24.

Illuminator, 4I-50; vertical, I3, 238 . See also condenser.

Image, actinic, 22I ; aerial, 30, 230 ; centering i. of source of illumination, 44 ; color, 52, 58 ; inverted, real of objective, 30 ; of flame, 45 ; geometrical construction of, 6 ; and object, size and position, 5, 9, 108; rea1, 5, 9, $30-32$, IO 3 ; refraction, 52,58 ; retinal, $6,9,32$; swaying of, 48 ; virtual i. and standard distance at which measured, 6, rog.

Image-power of objectives, I8.

Imbedding; I 77,185 .

Immersion, fluid or liquid, $58,27 \mathrm{I}$; illuminator, 47 ; objective, II, 58-59.

Incandescence or line spectra, 137 .

Incident light, 35 .

Index, of refraction, 53 ; of medium in front of objective, I6-19.

Indicator ocular, 247.

Infiltration, collodion, I 77 ; paraffin, I84; paraffin dish for, 279 .

Ink for labels and catalogs, I95; for drawing, 274.

Interpretation of appearances under the microscope, 90-IO2.

Inventory card, 28I

Iris diaphragm, 82, 157 .

Irrigating with reagents, I7o.

Isochromatic plates, 217 .

Isolation, I73; with formaldehyde, I73; nitric acid, 175 .

Isotropic, I52.

$$
\mathrm{J}-\mathrm{K}
$$

Japanese filter or tissue paper, 60.

Jelly, glycerin, I7O-I 7I, 201.

Jena glass, 7I.

Jurisprudence, micrometry in, I 21.

Knife support, 277.
L

Labels and catalogs, I94-I96, 203.

Labeling microscopical preparations, I94 photographic negative, 2II; serial sections. 193.

Laboratory compound microscope, 64, $7 \mathrm{I}-89$.

Lamp, acetylene, 37, 5I, 229; condenser, 250 ; electric arc, 37, 229, 250-255; petroleum, $37,50-5 \mathrm{I}, 22 \mathrm{O}, 229$; spirit, I 83.

Lantern, magic, 249 ; slides, $24 \mathrm{I}$.

Law of color, ${ }_{13} 8$.

Lens, concave, 3 ; converging, 3 ; convex, 4 ; eye, 22 ; field, 32 ; holder, 7,175 , 217,228 ; paper, 60; system, 9; thick, 3 .

Lenses of micro-projection apparatus, cleaning, 265.

Letters in stairs, 93 ; for photo-engraving, 274.

Lettering oculars, 26.

Light, with Abbe illuminator, 48 ; acetylene, 37, 5I, 229; artificial, 37, 50, 229 ; axial, $36,40,48$; direct, 35 ; central, 36,40 ; electric, $37,229,250$; incident, 35 ; with mirror, 37 ; oblique, $36,4 \mathrm{I}, 48$; petroleum, 37, 50, 220, 229 ; for photo-micrography, 229; polarized, I5O ; reflected, 35 ; sun, 229; transmitted, 36 ; utilized with different objectives, I 7; for vertical illuminator, 239; wave length of, I42; Welsbach, 37, 229.

Lighting, 35; for Abbe camera lucida, I 29 ; artificial, 50; experiments, 37 ; with horizontal camera, 230 ; for micro-polariscope, I5I ; for micro-spectroscope, I44 ; with a mirror, 37,40 ; with daylight, 35,229 ; for photography, 208, 216; for photo-micrography, 229, 230; for vertical illuminator, 229.

Line spectrum, I37.

Liquid, currents in, 98 ; homogenous, II, $58,27 \mathrm{I}$.

\section{M}

Magic lantern, 249

Magnification of compensating oculars, 24 ; effect of adjusting objective, II 8 ; determination of, I03-IO9; expressed in diameters, ro3; initial or independent, 273; of microscope, IO3; in micro-projection, table, 262 ; of microscope with Abbe camera lucida, I3I; of microscope, compound, IO5; of microscope, simple, IO4 ; of photo- 
micrographs, determination of, 233 ; real images, IO3; table of, with ocular micrometer, III : with projection microscope, 262 ; varying with compound microscope, Io9; and velocity, 98.

Magnifier, tripod, 7, I04, 209.

Marker for preparations, 65-66.

Marking objects, $65^{-66,248}$; negatives, 2II, 2 I 4 ; objectives, 7I.

Masks for preparations, 264 .

Material and apparatus, I, 34, 90, IO3, I 23, I34, I56, I98, 205, 223, 243.

Measure, unit of, in micrometry, II2 ; of wave length, I43.

Measurer, cover-glass, I64-I65.

Measuring the thickness of cover-glass, 163.

Mechanical parts of compound microscope, 64 ; Frontispiece, 8 ; of microscope, care of, 59 ; testing, 63 .

Mechanical stage, $65,67-70,258,259$.

Megascope, 267.

Metallic surfaces, photography of, 235240 ; preparation of, 239.

Metallography, microscope in, I59.

Metals, examination of, I59, 235.

Met-hemaglobin, spectrum of, I36, 147 .

Methods, collodion, $176-183$; paraffin, I $83-$ I 91 .

Metric measures and equivalents, cover Ist p., I33.

Micro-chemistry, I55-I57 ; slides for, I6I.

Micro-metallography, objects for, 238.

Micrometer, IO3; calipers, I64 ; cob-web, II 7 ; filar m. ocular, II7, IIS ; filling lines of, Io6; lines, arrangement of ocular and stage, I20; lines, finding, I06; net, I28; object or objective, IO5; ocular or eye-piece, II4-I20; ocular, micrometry with, I 6 ; ocular, ratio, II9; ocular, valuation of, III, I14; ocular, varying valuation of, II8; for photo-micrography, 233 ; screw ocular, II7; stage, I05, I06: table of magnification, I I I.

Micrometry, definition, II 2, II4; with adjustable objectives, II 8 ; comparison of methods, II9-120; with compound microscope, II 2 ; and jurisprudence, I2I ; limit of accuracy in, I20; with ocular micrometer, II6 ; with simple microscope, I 2 ; remarks on, II 8 ; unit of measure in, I I 2.

Micro-millimeter, I 3

Micron, II 3 ; for measuring wave length of light, I43.

Micro-photograph, 220.

Micro-photography, distinguished from photo-micrography, 220.
Micro-planar objective, 21 2, 260.

Micro-polariscope, 100, I 50-I55.

Micro-polarizer, 150.

Micro-projection, 249-267 ; apparatus, 256-257 ; carbons for, 250-254; circulation of blood with, 257,264 ; condenser, 254, 256 ; current, 254 ; demonstration with, 265 ; magnification, 262 ; masks for specimens, 264 ; mechanical stages, 258-259; microscope for, 249; objectives and oculars, 259 ; pointer for, 265 ; preparations, 263 ; screen and screen distance, 261 ; specimen cooler, 257; stage, 258 ; stains for, 263; water-bath, 255 .

Microscope, definition, I ; amplification of, Io3 : clinical, 243 ; demonstration, 243 ; dissecting, 8 , 33, I 75, I76 228; care of, 59; eye and, 1, 6, 9; field of, 28, 29 ; focusing, 34 ; magnification, I03; for metallography, $159-160$; for microchemical analysis, 153 ; for opaque objects, $235^{-237}$; for photo-micrography, 225, 23I, 237 ; polarizing, I 5O; preparation, with erecting prism. I76; projection, 249-250 ; price of, 64, 7I ; putting an object under, 27 ; sun or solar, 249; screen, 59; stand for large, transparent objects, 2I5, 2I6 ; stand, for embryos, 212 ; traveling, 245-246.

Microscope compound, definition, 8; drawing with, I22 ; figures, frontispiece, 9, 7I-89, IO2, I53, I60, 237, 244-246; focusing, 38-39; for High schools, 64.7I; for laboratory, 64, 71-89; lamp for, 50; magnification or magnifying power, ro5; magnification and size of drawing with Abbe camera lucida, I3I; mechanical parts, 64: micrometry with, II2 ; optic axis of. 9, Io ; optical parts of, 9. 64 ; varying magnification, I09; working distance of, 39 ; testing, 63 .

Microscope, simple, definition, I ; experiments with, 6 ; figures, $6-8,33$, IO4, I $75,209,228,243$; focusing with, 34 ; magnification of, IO4; micrometry with, II2; working distance of, 34 ; Microscopic, objective, 9 ;obj ective low, attached to camera, 214 ; objects, drawing, I22; ocular, 22 ; slides or slips, I6I ; tube-length, $\mathrm{I}_{3}$, I4.

Microscopical preparations, cabinet for, I97; cataloging, I94 ; labeling, I94; mounting, I66-193.

Microtome, hand, 274-275 ; Minot's 275276 ; razor support for, $276-277$; sliding, 278 . 
Micro-spectroscope, I34-I5O ; adjusting, I39; experiments, I45 ; focusing, I44; focusing the slit, I39; lighting for, I44 ; objectives to use with, I44 ; reversal, apparent, of colors in, I34; slit, mechanism of, I35, I39.

Micrum, II3.

Mikron, II3.

Milk globules, to overcome pedesis of, 100

Minerals, absorption spectra of, I49.

Minot's microtomes, 275-276.

Minute objects, arrangement of 204.

Mirror, 9-II ; for Abbe illuminator, 48; of camera lucida, arrangement for drawing, I26; concave, use of, 37 ; dark ground illumination, 50 ; light with, central and oblique, $4 \mathrm{O}, 4 \mathrm{I}$; lighting with, 37 ; plane, use of, 37 .

Mixture, clearing, 200.

Models, wax, 274.

Moist, chamber, I7I.

Molecular movement, 99.

Monazite sand, spectrum of, I49.

Monnting cells, preparation of, I68; media and preparation of, I98 203; objects for polariscope, I5I ; permanent, 167 ; temporary, I66.

Mounting objects, dry in air, order of procedure, $167-168$; in glycerin, order of procedure, I7o; in glycerin jelly, order of procedure, I7o; in media miscible with water, I69; minute objects, 204; opaque objects, 239; permanent, I67; in resinous media. by drying or desiccation. order of procedure, 172 ; in resinous media, by successive displacements, order of procedure, 172 ; temporary, I66.

Movement, Brownian, or nolecular, 99.

Muscae volitantes, Ioo.

Muscular fibers, isolation of, I75.

\section{$\mathrm{N}$}

Natural balsam, I 99.

Needle-holder, I 67.

Negatives, labeling, 2Ir, 214; oculars, 22 ; rack for drying, 240 ; record of, 2I4; storing, 2I I, $2 \mathrm{I}_{4}$.

Net micrometer, 128.

Neutral balsam, I99.

Nicol prism, r5o.

Nitric acid, dissociator, 203.

Nomenclature of objectives, Io.

Non-achromatic condenser, 46 ; objectives, II.

Non-adjustable objectives, I 2 ; thickness of cover glass for, table, I4.
Normal salt solution, 203.

Nose-piece, 27,38 , 80; marking objectives on, $7 \mathrm{I}$.

Numerical aperture, of condenser, 44 ; of objectives, I6, 270; table of, I9.

Object, determination of form, 93 ; having plane or irregular outlines, relative position in a microscopical prepration, 92 ; and image, size of, Io, Io8 ; marking parts of, 65-66 ; marking position of, 248 ; micrometer, I05; mounting, 166 ; putting under microscope, 27; shading, 59; suitable for photo-micrography, 227 ; transparent with curved outlines, relative position in microscopic preparations, 94.

Objective, 9-I3, I2I ; achromatic, II ; adjustable, II, I2, 54 ; adjustable, micrometry with, I18; adjustable, photo-micrography with, 234 ; adjustment for, 54; aerial image of, 30 ; anastigmat, 208; aperture of, I5-22, 270 ; aplanatic, I1 ; apochromatic, I 2, 224; back combination of, IO, II ; carrier, 259; cleaning back lens of, $6 \mathrm{I}$; collar, graduated for adjustment, 56 ; cloudiness or dust, how to determine, 92 ; desiguation of, ro ; dry, IO, I7-J9, I2I; equivalent focus of, IO, $25,29,272$; field of, $28-29$; focusing for micro-spectroscope, I 44 ; front combination of, IO, II ; function of, 29 3I ; glass for, II-I3, 7I; high, focusing with, 38 ; homogeneous immersion, I7-I9, I2I ; homogeneous immersion, cleaning, 59 ; homogeneous immersion, experiments, 58 ; illuminating, I 3,238 ; image, power of, I8 ; immersion, II, I2I; index of refraction of medium in front of, $\mathrm{I} 7$, 19 ; initial or independent magnification of, 273 ; inverted, real image of, 30 ; for laboratory microscope, 64 ; lettering, Io; light utilized with, I 7 ; low, focusing with, 38 ; magnification of, 272 ; marking, by Krauss' method, $7 \mathrm{I}$; for micrometallography, 13, 159; micro-planar, 212, 260; to use with micro-polariscope, I5I ; microscopic, 9; to use with microspectroscope, I44; for micro-spectroscope, focusing. I44; nomenclature of, Io; non-achromatic, II ; nonadjustable, I2; non-adjustable, thickness of cover-glass for, table, I4; with nose piece, 38 ; numbering, Io; numerical aperture, I6-22, 270 ; oil 
immersion, II ; panto-chromatic, $\mathrm{I}_{3}$; para-chromatic, 13 ; for photography, 208, 2I2, 2I4, 224; for photo-micrography, 224; projection, I3, 212, 2I4, 259 ; putting in position and removing, 26 ; semi-apochromatic, I3; table of field, 29 ; terminology of, Io ; unadjustable, I2; variable, I3 ; visual and actinic foci of, in photo-micrography, 226: water immersion, I7-I9, 56 ; working distance of, 39-40.

Oblique light, 36 , 4I ; with Abbe illuminator, 48 ; with a mirror; 41,50 .

Ocular, various forms, 22-25; cloudiness, how to determine and remove, 60,92 ; equivalent focus of, $25,29,273$; eyepoint of, 32 ; field-lens, 32 ; filar or screw micrometer, 26, II7; focus, equivalent of, 25, 273 ; function of, $3 \mathrm{r}-33$; indicator, 67,247 ; iris diaphragm for, I57; lettering and numbering, 26 ; micrometer, micrometry with, II4-I2I ; parfocal, 24, 38 ; for photo-micrography, 224, 232, 235 ; pointer, 247 ; projection, 260 ; spectroscopic.134; standard size for, 26 ; table, effect on field, 29.

Oil, and air, appearances and distinguishing optically, 95 ; removal, 6I ; removal from sections, I79.

Oil-globules, with central and oblique illuminations, 95.

Oil immersion objectives, II.

Opaque objects, lighting, I44, 238 ; photography of, with microscope, 235240 ; projection of, 267.

Opera glasses, 262.

Optic axis, 2, ; of condenser or illuminator, 47 ; of microscope, Io.

Optical, bench, 237 ; center, 2 ; focus, I2 ; parts of compound microscope, 9,64 ; parts of microscope, care of, and testing, 6o, 63; section, 98 .

Order of procedure in mounting objects dry or in air, I67; in glycerin, 170 ; in glycerin jelly, I7O ; in resinnous media by desiccation, 172 ; in resinous media by successive displacement, 172 .

Ordinary ray, with polarizer, I50.

Orthochromatic plates, 217

Orthoscopic ocular, field with, 28 .

Outline distinctness of, 96 .

Oven paraffin, 279.

Over-correction, 5.

Oxy-hemoglobin, spectrum of, I38, I47.

\section{$P$}

Paper, bibulous, filter, lens, or Japanese for cleaning oculars and objectives, 60,180 ,

Paraffin, I85, 203; filtering, 185 ; infiltrating with, 185 ; dish for infiltrating, 279; imbedding in, I85, I86; method, 183 ; oven, 279; pail for melting, I84; removal from lenses, $6 \mathrm{I}$; removing from sections, $\mathrm{I} 88$.

Parfocal oculars, 24, 38 .

Parts, optical and mechanical of microscope, 8,64 ; testing, 63 .

Pedesis, 99 ; compared with currents, 99 ; to overcome, IOO; with polarizing microscope, 100 ; proof of reality of, IOO.

Penetrating power, 2I.

Penetration of objective, 2I.

Permanent mounting, 167 ; preparations of isolated cells, I 75 .

Permanganate of potash, absorption spectrum of, 136 , I46.

Petri dish, photographing bacterial cultures in, $24 \mathrm{I}$.

Petroleum light, 37, 229; as color screen, 220 .

Pharmacological products, examination of, 158 .

Photo-engraving, drawing for, 273 ; lettering for, 274.

Photographic, camera, 207; negatives, labeling, 2II, 2I4; objectives, 208 ; prints, $2 \mathrm{I}$.

Photography, back-ground for. 209; of bacterial cultures, 241-242 ; colorcorrect, 217 ; of colored objects, 218 ; compared with photo-micrography, 222 ; of embryos, 2II-2I4 ; focusing and exposure, 206, 213; indebtedness to photo-micrography, 220; of large transparent objects, 2I 4-2 I 6 ; lighting for, 208, 216; metallic objects, 235240 ; objectives for, 208, 212, 214; of objects in alcohol or water, 206 ; opaque objects, 235-240; plates for, 217 ; stage for, 2II; with rertical camera, 205-209, 225

Photo-micrograph, 220; determination of magnification for, 233 ; at 5-20 diameters, $212 ; 20-50$ diameters, 230 ; roo-2500 diameters, 233 ; of metallic surfaces, 235-240; objects suitable for, 227; of opaque objects, 235-240 ; prints of, 2 II ; plates for, 217 ; reproductions of, 232; with and without an ocular, 230-234.

Photo-micrographic, camera, 222, 225, 236 ; outfit, 236-237; stand, 23 I. 
Photo-micrography, 220-240 ; cover-glass correction, 234; apparatus for, 223 ; compared with ordinary photography, 222 ; condenser for, $42,226,227$; distinguished from micro-photography, 220 ; experiments, 229 ; exposure for, $2 \mathrm{I}_{3}, 220,232,235,240$; focusing for, $2 \mathrm{r} 3,2 \mathrm{I} 6$; foctusing screen for, 209 ; lighting, 229, 230, 233, 239; micrometer for, 233 ; objectives and oculars for, I3, 224, 232,235; vertical camera with, 2II, 222, 225; actinic foci in, 226 ; with and without ocular, $230,232,234$; record table for, 219.

Physiological histology, I96.

Picric-alcohol, 203.

Picro-fuchsin, I9o.

Pillar of microscope, Frontispiece.

Pin-hole diaphragm, 47.

Pippett, I79; egg, 280.

Plane mirror, use of, 37 .

Plates, color-correct, 217 ; exposure of, $2 \mathrm{I} 3,22 \mathrm{O}, 232,235.240$; isochromatic, or orthochromatic, 217 ; size of, 224.

Pleochroism, I52.

Pleurosigma angulatum, 4r.

Point, axial, I5 ; burning, 7 .

Pointer ocular, 247.

Polariscope, 140, I5o.

Polarized light, extraordinary and ordinary ray of, I50.

Polarizer and analyzer, I4O, I5I.

Polarizing microscope, pedesis with, roo.

Position of objects or parts of same object, 92 ; marking p., 248.

Positive oculars, IO, 22.

Power, of microscope, J03 ; illuminating, penetrating, resolving, of objective, I9-2I ; of ocular, 25.

Preparation of Canada balsam, Farrant's solution, glycerin, glycerin jelly,etc., I98-204.

Preparation, of clearing mixture, liquid gelatin and shellac cement, I98-204; of ground glass, 29 ; of metallic surfaces, 239 ; vials, I 74 .

Preparations, cataloging. I94-I96; cabinet for, I96-I97; 1abeling, I94; for microprojection, 263 ; permanent, of isolated cells, I75.

Price of American and foreign microscope, 7 I.

Principal, focus, 3, 5; focal distances, 3 , 30 ; optic axis, 2,5 .

Prism of Abbe camera lucida, I24, I27; Amici, I4O; comparison, I4I ; dispersing, I4I ; erecting, 176; Nicol, I5O; and slit of micro-spectroscope, mutual arrangement, I39; of Wollaston's camera lucida, I 25.
Prints, photographic, 2II.

Projection, apparatus, 249, 263-297; microscope, 249-266 ; see micro-projection ; objective, I3, 214, 259; ocular. $24,25,232,260$; opaque objects, 267 ; in photo-micrography, 232, 234.

Putting, on cover-glass, I67; an object under microscope, 27; an objective and ocular in position, 26, 27.

Pyroxylin, 200.

$$
\mathbf{Q}-\mathbf{R}
$$

Quadrant for camera lucida, I27, I28.

Radiant, centering, 255.

Ratio, ocular micrometer, I I g.

Razor and support, 276-277.

Reagent, bottle, I79; for fixing, I98; irrigation with, I70; for mounting, I98.

Real image, 5, 8, 9, 30; magnification, $\mathrm{IO}_{3}$, 262.

Record, of negatives, 2 I 4 ; table for photomicrography, 219.

Reflected light, 35 .

Reflection, total, 54.

Refraction, 52 ; images, 52,58 ; index of, 53; of medium in front of objective, 19.

Refractive, doubly, I52; highly, 97 ; singly, I52.

Relative position of objects, 92.

Resinous media, mounting objects in, order of procedure, by drying or desiccation, I72; by a series of displacements, I 72 .

Resolution and numerical aperature, 20.

Resolving power, 20.

Retinal image, $6,9$.

Revolving nose-piece, marking objectives on, $7 \mathrm{r}$.

Ribbon sections, I86-187; tray for, 187 .

\section{$\mathrm{S}$}

Sagittal sections, 192.

Salicylic acid, crystallization, 50.

Salt solution, normal, 203.

Scale, of drawing, I3I ; of sizes for photographing, 206; of wave lengths, I42.

Screen, color, 218-219; focusing s. for photo-micrography, 209; of ground glass, 29; for micro-projection, 26I ; for microscope, 59 .

Screw, society, 64; micrometer, 26, II 7.

Sealing cover-glass, I69, I70.

Searching ocular, 24.

Secondary axis, 3 .

Section, lifter, I8I-I82 ; optical, 98.

Sections, arrangement of tissue for, I9I ; clearing, I9o ; cutting, I 78 , I86; de- 
hydration of, I90; extending with water, I86; fastening to slide, I79, I87; frontal, I92 ; mounting, I83, I9O; removing benzine, oil and paraffin from, I79, I88; ribbon, I86; sagittal, I92 ; serial, I9I-I93 ; staining, 180,189 ; transferring, I79; thickness for micro-projection, 263 . Selenite plate for polariscope, I 54.

Semi-apochromatic objective, I3.

Serial sections, I9I-193; arranging and labeling, I92, I93; stage for, 67,69 ; thickness of cover-glass for, 193 .

Shell vials, I 74 .

Shellac cement, preparation of, 203 ; removal from lenses, $6 \mathrm{I}$.

Significance of aperture, 20.

Simple microscope, see under microscope. Sines, table of, $3 \mathrm{~d}$ page of cover.

Slides, I6r ; box for I98; cleaning, I6I ; holder for, I88-I89; lantern, 24I ; for micro-chemistry, I6I; tray for, I87. Sliding microtome, 278 .

Slips, I6I.

Slit mechanism of micro-spectroscope, I 35, I 39.

Society screw, 64 .

Sodium, lines and spectrum, I36-I37.

Solar spectrum or s. of sunlight, $136-137$.

Soluble cotton, 200.

Solution, alum, I98; Farrants', 201.

Specimen cooler, 257.

Spectral, colors, I38; ocular, I34, I39.

Spectroscope, direct vision, I34, I45.

Spectroscopic, examination of colorscreens, 220 ; ocular, I34.

Spectrum, I36-I50; absorption, 137 ; amount of material necessary and its proper manipulation, I45; analysis, I50 ; Angström and Stokes' law of, I 38 ; banded, not given by all colored objects, I48; of blood, I46; of carbon monoxide hemaglobin, $\mathrm{r} 47$; of carmine solution, I4S ; of minerals, I49; of colorless bodies, I48; comparison, I42 : complementary, I39 ; continuous, 137 ; double, $\mathrm{I}_{42}$; incandescence; I 37 ; line, I 37 ; met-hemaglobin, I 47 , monazite sand, I49; oxy-hemoglobin, I 38 , I 47; permanganate of potash, I 36 , I46; single-banded of hemaglobin, I3 8 , I47; sodium, I36, I37; solar, I 36 , I 37 ; two-banded of oxy-hemaglobin, 147 .

Spherical aberration, 4, 5; test for, 268 . Stage, Frontispiece, 65; mechanica1, 65, 67-70, 259; micrometer, 105; for micro-projection, 258 ; for photographing, 2I $\mathrm{I}$.
Stain, alcoholic, I8o, I89; aqueous, I80, I89; for micro-projection, 263.

Staining, cells, I74; dish, I90 ; sections, I80, I 89 .

Stand, of microscope, 65 ; photo-micrographic, $23 \mathrm{I}$; special for embryos, 2 I 2 ; special for large transparent objects, 2I5, 2I6.

Standard, distance (250 $\mathrm{mm}$.) at which the virtual image is measured, I09; screw, 64 ; size for condenser, 47 ; size for oculars, 26.

Stokes and Angström's law of absorption spectra, I 38 .

Storing negatives, 21 I ; preparations, I96. Substage, 86, Frontispiece.

Substances for crystallography, 156 .

Sulphonal with polarizer, I54.

Sulphuric ether, 201 .

Support for knife of microtome, 276-277.

Swaying of image 48.

Synthol, I98.

System, back, front, intermediate of lenses, IO, II ; crystal, I56 ; metric, cover Ist p., I33.

\section{T}

Table, for immersion fluid, 272 ; of magnification and valuation of ocular micrometer, III; magnification with projection microscope, 262 ; of tubelength and thickness of cover-glasses, I4 ; natural sines, third page of cover; of numerical aperture, I9; record, for photo-micrography, 219; size of fields, 29 ; testing homogeneous liquids, 272 ; of valuations of ocular micrometer, III ; weights and measures, $2 \mathrm{~d}$ page of cover.

Temporary mounting, 166.

Terminology of objectives, Io.

Test of chromatic and spherical aberration, 268-270.

Tester, cover.glass, I64-I65 ; for homogeneous liquids, $58,27 \mathrm{I}$.

Testing a camera, 223 ; a microscope and its parts, 63

Test-plate, Abbe's, method of using, 268.

Textile fibers, examination of, IOI, I 58.

Thickness, of cover-glass for non-adjustable objectives, table, I4; of serial sections, r93.

Tissues, arranging for sections, I9I ; fixing or hardening, 176,183 ; washing apparatus for, 280 .

Tolles-Mayall mechanical stage, 67 .

Transections, I92.

Transferring sections, I79.

Transmitted light, 36 . 
Tray for slides, I87.

Triplet, aplanatic, 7 .

Tripod, 7 , 104; base for microscope. 86 ; as focusing glass, 209.

Tube of microscope, Frontispiece.

Tube-length, I 3, I4, 56, for cover-glass adjustment, 56,57 ; importance of, 56 ; microscopical, I3, I4; of various opticians, table, I4; and optical combinations, IIS.

Turn-table, I68.

$$
\mathrm{U}-\mathrm{V}-\mathrm{W}-\mathrm{X}
$$

Unadjustable objectives, I 2 .

Under-correction, 5 .

Unit of measures, in micrometry, II2 ; of wave length, I43.

Valuation of ocular micrometer, Irf-II6; table, III.

Variable objective, I3.

Varying magnification of compound microscope, Iog.

Varying ocular micrometer valuation, II 8 .

Velocity under microscope, 98.

Vertical, camera, 205-2I0, 225: illuminator, $I_{3}, 238-239$.
Vials, preparation, 174 ; blocks for, 174.

Virtual image, $5,6,9,32$; standard distance at which measured, Iog.

Visibility with objectives, 20.

Vision, double, IO3, I05; microscopic, 2 r.

Washing apparatus for tissues, 280 .

Waste bowl, I8I.

Water immersion objective, I6-I9, 56; light utilized, I7; numerical aperture, I9

Water, bath, 235, 255; for immersion objectives and removal of, 56, 58 .

Wave length, designation of, r43; scale of, I 42 .

Wax models, 274 .

Weights and measures, see $2 \mathrm{~d}$ page of cover.

Welsbach light, 37, 229.

Wollaston's camera lucida, I07, 125 .

Work-room for photo-micrography, 224.

Work-table, position, etc., 62.

Working distance of microscope or objective, II, 34, 39-40.

Writing diamond, I96.

Xylene, I78, 200; balsam, I99; for removing oil, 179; removing from slides, I89.

Xylo1, German form of xylene, I78, 200. 



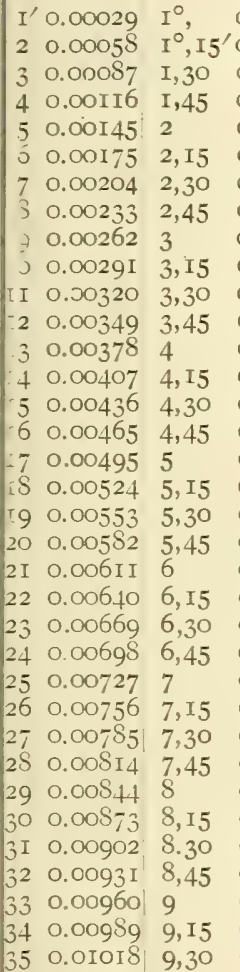

$\begin{array}{lll}36 & 0.01047 & 9,45\end{array}$ 370.01076 I0

$380.0 I_{105}$ IO, I5 $390.0 I_{3} 3410,30$ $400.01 \mathrm{II} 64$ IO, 45 $4 \mathrm{I} 0.0 \mathrm{IIg}$ I I

420.01222 IJ , I5 430.0125 I IX,30 440.01280 II, 45 450.01309 I2 460.01338 I 2, I5 470.01367 I2, 30 $0.0139612,45$ 490.01425 I3

500.01454 I 3, I 5 $5 \mathrm{I} \quad 0.0 \mathrm{I}_{4} \mathrm{~S}_{3} \mathrm{I}_{3}, 30$ $520.0 I_{5} I_{3}$ I 3,45 530.01542 I4

540.0157 I|I4, I5 $550.0160 \mathrm{O}$ I 4,30 $\begin{array}{lll}56 & 0.0162_{9} \mathrm{I} 4,45\end{array}$ 570.01658 I 5 580.01687 I 5, I5 $590.017 I_{6}$ I 5,30 $60 \quad 0.01745$ I 5,45 $0.0174516^{\circ}$,

$0.275643 I^{\circ}$,

$0.5150446^{\circ}$,

$0.719346 \mathrm{I}^{\circ}$

$0.8746276^{\circ}$

0.97030

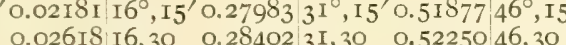
$0.0305416,45$ 0.03490 I 7 0.03926 I 7,15 0.04362 I 7,30 0.0479 .8 I 7,45 0.05234 IS 0.05669 I 8, I 5 0.06105 I 8,30 $0.0654018,45$ 0.06976 I9 0.074 I I I9, I5 0.07846 I9,30 $0.082 S_{\text {I I }} 9,45$ 0.0871620 0.09150120, I 5 $0.0958520,30$ o. IOOI9 20,45 O. I04532 2

o. I0887 2I, I5 O.II32O $2 \mathrm{I}, 3 \mathrm{O}$ O. I I $7542 \mathrm{I}, 45$ O.I2I $8 \rightarrow 22$

o. 1262022,15 o. I3O53 22,30 O. I $3485,22,45$ o. I39I 723 O. I 434923 , I 5 O. I $478 \mathrm{I} \mid 23,3 \mathrm{O}$ o. 1521223,45 O. 1564324 O. I6074:24, I5 O. I6505 24,30 \begin{tabular}{ll|l} 
O. I 6935 & 24,45
\end{tabular} O. 1736525

o. $1779425, I_{5}$ o. I $8224 \mid 25,30$ o. I8652 25,45 O. I 908 I 26

O. I 950926, I5 o. I 993726,30 $0.2036426,45$ 0. 2079127

o. 2 I 2 I 827, I 5 $0.21644,27,30$ 0. 2207027,45 0.2249528

0. 22920.28 , I5 $0.2334528,30$ $0.2376928,45$ 0.2419229

0.2461529, I 5 $0.2503829,30$ $0.2546029,45$ 0.2588230 $0.2630330,15$ $0.2672430,30$ 0,27 I 4430,45
0.28820 3I, 45 $0.292373^{2}$ $0.2965432,15$ 0.3007 I 32,30 0.30486 32,45 $0.30902 / 33$ $0.313 I 633, I 5$ $0.3173033,30$ 0.32 I 4433,45 0.3255734 o. $3296934, I_{5}$ 0.3338 I 34,30 $0.33792 \quad 34,45$ 0.3420235 0.346 I $235, I_{5}$ $0.35 \mathrm{O} 2 \mathrm{I} / 35,30$ 0. 35429135,45 0.3583736

0.3624436, I5 o. 3665036,30 $0.37056,36,45$ 0.3746 I 37 $0.3786537, I_{5}$ $0.3826837,30$ \begin{tabular}{ll|l|l|}
0.3867 & I & 37,45
\end{tabular} $0.39474 / 3^{8}$, I5 $0.3987538,30$ $0.4027538,45$ 0.4067439 $0.4107239,15$ $0.4146939,30$ $0.4186639,45$ $0.42262,40$ $0.4265740, I_{5}$ $0.4305 \mathrm{I} 4 \mathrm{O}, 3 \mathrm{O}$ $0.4344540,45$ $0.438374^{I}$

0.44229 II, I5 $0.446204 \mathrm{I}, 30$ 0.45 IO 4 I, 45 0.4539942

$0.4578742, I_{5}$ $0.4617542,30$ $0.4656 \mathrm{I} 42,45$ 0.4694743

\begin{tabular}{ll|l}
0.47332 & 43, I 5
\end{tabular} 0.477 I 643,30 $0.4809943,45$ $0.4848 \mathrm{I} 44$

0.4886244, I 5 $0.4924244,30$ o. 4962244,45 $0.50000 / 45$

$0.50377: 45,15$ $0.5075445,30$ 0.5 I I $29,45,45$ 0.3907338 0.5262 I 46,45 0.5299247 0.5336 I 47, I 5 $0.5373047,30$ $0.5409747,45$ o. 5446448 0.5482948, I 5 0. 55 I 9448,30 0. 5555748,45 0.559 I9 49 $0.5628 \mathrm{O} / 49$, I5 $0.5664 \mathrm{I} 49,30$ $0.5700049,45$ $0.5735^{8} 5^{\circ}$

O. 577 I 5 5O, I 5 $0.5807050,30$ $0.5842550,45$ $0.587795 \mathrm{I}$

0.59 I 3 I 5 I, I 5 0.59482 r, 30 0.59832 II, 45 0.60182152

$0.60529,52, I_{5}$ $0.6087652,30$ $0.6122 .252,45$ $0.6 I 56653$

0.6I909 53, I5 ० $6225 \mathrm{I} 53,30$ $0.6259253,45$ 0.6293254

0.6327 I 54, I 5 $0.6360854,30$ $0.6394454,45$ 0.6427955 0.646 I 255, I 5 $0.6494555,30$ 0. 6527655,45 0.6560656

$0.65935 .56, I_{5}$ $0.66262: 56,30$ $0.6658856,45$ 0.6691357 $0.6723757, I_{5}$ $0.6755957,30$ $0.67880157,45$ 0.6820058 0.685 I 8,58, I 5 $0.6883558,30$ 0.69I 5 I 58,45 0.6946659

0.6977959, I5 $0.7009159,30$ 0. 704 OI 59,45 0.707 I I 60 o. 7 IOI9 60, I5 $0.7132560,30$ $0.7163060,45$ $5^{\prime} 0.722366 \mathrm{I}^{\circ}$, I5 $5^{\prime} 0.8767376^{\circ}$, I5 $0.725376 \mathrm{I}, 3 \mathrm{O}$ $0.728376 \mathrm{I}, 45$ o. 7313562 o. $7343262, I_{5}$ $0.7372862,30$ $0.7402262,45$ o. 7431463 $0.7460663,15$ $0.7489663,30$ 0.75 I 8463,45 o. 7547,64 O. 7575664, I $_{5}$ 0.7604I 64,30 $0.7632364,45$ o. 7660465 0. 7688465 , I5 o. 77 I 6265,30 0.7743965,45 o. 777 I 566 o. 7798866, I 5 $0.7826166,30$ $0.7853266,45$ o. 788 oI 67

$0.7906967,15$ $0.7933567,30$ $0.7960067,45$ 0.7986468

0.80I 2568 , I5 $0.8038668,30$ $0.8064468,45$ \begin{tabular}{l}
0.8090269 \\
\hline
\end{tabular}

0.8 II57 69, I5 $0.8141269,30$ $0.8166469,45$ 0.8191570 0.82 I 6570,15 $0.824 I 370,30$ $0.8265970,45$ $0.829047 \mathrm{I}$

0.83 I4 7 7 I, I5 $0.833897 \mathrm{I}, 3 \mathrm{O}$ $0.836297 \mathrm{I}, 45$ 0.8386772

0.84 IO 472, I5 $0.8433972,30$ $0.8457372,45$ 0,8480573

0.8503573 , I 5 $0.8526473,30$ $0.8549 \mathrm{I} / 3,45$ 0.857 I 774

0.8594 I 74 , I 5 $0.86 \mathrm{I}_{3} 374,30$ $0.8638474,45$ 0.8660375 0.8682075, I 5 $0.8703675,30$ $0.8725075,45$

$0.8788276,30$
$0.8808976,45$ 0.8829577 0.8849977, I 5 0.887 OI 77,30 $0.8890277,45$ 0.89 IOI 78 $0.8929878,15$ $0.8949378,30$ $0.8968778,45$ 0.8987979 0.9007079, I 5 $0.9025979,30$ 0. 9044679,45 $0.9063 \mathrm{I} 80$

$0.9081480, I_{5}$ $0.9099680,30$ 0.9II76 80, 45 $0.913358 \mathrm{r}$

0.9153 I $8 I, I 5$ $0.917068 I, 30$ o. $918798 \mathrm{I}, 45$ 






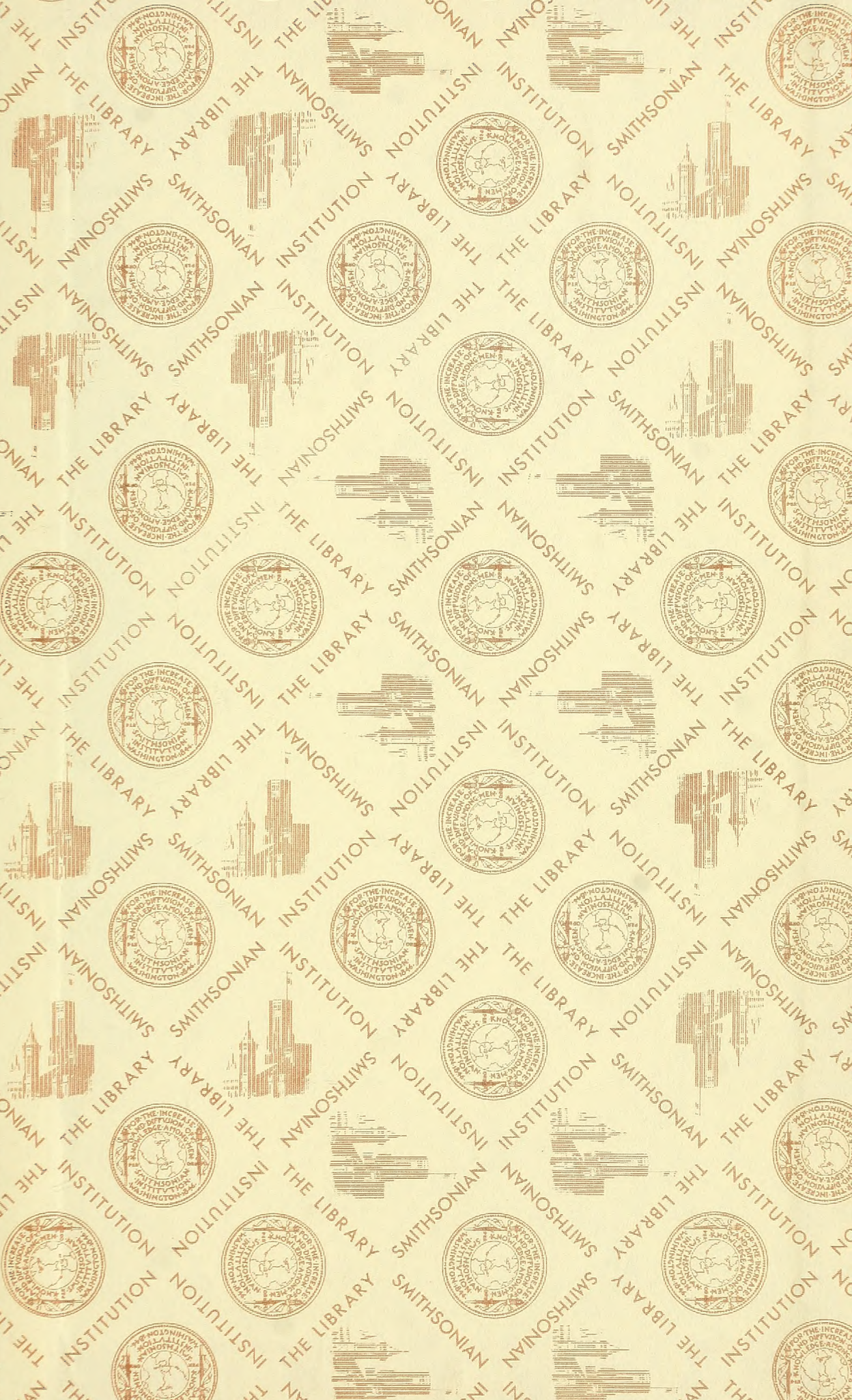


SMITHSONIAN INSTITUTION LIBRARIES

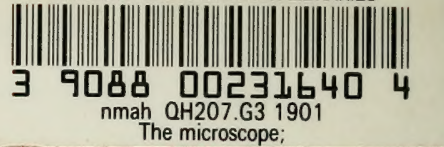

